Final Report

August 1, 1999

H.t.

JAU 052021

QSTI

\title{
Alcohol Flushing for Removing DNAPLs from Clay and Sand Layered Aquifer Systems
}

\author{
Work Performed Under Contract: \\ DE-AR21-96MC33083
}

\section{Submitted By:}

N.J. Hayden, P. Padgett, C. Farrell, J. Diebold, X. Zhou, M. Hood Department of Civil and Environmental Engineering University of Vermont, Burlington, VT 05405

Project Manager:

Karl Heinz Frohne

Principle Investigator:

Nancy J. Hayden 802-656-1924 (phone)

802-656-8446 (fax)

nhayden@emba.uvm.edu

Submitted To:

U.S. Department of Energy

Federal Energy Technology Center

PROCESSED FIOM BEST AMAILABLE COPY 


\section{DISCLAIMER}

This report was prepared as an account of work sponsored by an agency of the United States Government. Neither the United States Government nor any agency thereof, nor any of their employees, make any warranty, express or implied, or assumes any legal liability or responsibility for the accuracy, completeness, or usefulness of any information, apparatus, product, or process disclosed, or represents that its use would not infringe privately owned rights. Reference herein to any specific commercial product, process, or service by trade name, trademark, manufacturer, or otherwise does not necessarily constitute or imply its endorsement, recommendation, or favoring by the United States Government or any agency thereof. The views and opinions of authors expressed herein do not necessarily state or reflect those of the United States Government or any agency thereof. 


\section{DISCLAIMER}

Portions of this document may be illegible in electronic image products. Images are produced from the best available original document. 


\section{EXECUTIVE SUMMARY}

Alcohol flushing, also called cosolvent flushing, is a relatively new in-situ remediation technology that shows promise for widespread application to organic solvent contaminated sites. It has potential for removing the dense nonaqueous phase (DNAPL) source, located deep within the saturated zone, which is currently difficult, if not impossible, to remove. Alcohol flushing has the potential for dramatically decreasing cleanup times and total cleanup costs at DNAPL sites. Because of the ability to reuse the alcohol, alcohol flushing also has the potential to be highly cost effective.

One of the greatest impediments to any in-situ remediation technique, is subsurface soil heterogeneities and their effect on fluid flow of both the solvent and aqueous phases, DNAPL distribution and site characterization. Heterogeneities exist at all scales ranging from the pore scale to large-scale layering of geologic materials. Clays are of particular interest. At the pore-scale clays can dramatically affect the size and shape of pores thus affecting macroscopic properties such as permeability and porosity which influence fluid flow. In addition, these pore-scale changes can affect DNAPL distribution and trapping. Larger-scale clay layering can result in accumulation and trapping of DNAPL thus affecting fluid flow.

The overall goal of this project was to evaluate the application of in-situ alcohol flushing for remediating soils contaminated with dense nonaqueous phase liquids (DNAPLs) using laboratory studies. The economic, technical and environmental feasibility of this technique was also considered.

Homogeneous, clay-sand mixes and layered soil systems were packed in columns and different alcohol flushing scenarios were evaluated to determine the effect on DNAPL removal. The specific project tasks included: 1) characterization of alcohol-DNAPL interactions; 2) characterization of soils and layering; 3 ) column studies to evaluate various alcohol concentrations on DNAPL mobilization; 4) column studies to evaluated the effect of flow rate on DNAPL solubilization; 5) evaluation of alcohol reuse; 6) determination of process feasibility including economic and environmental concerns; and 7) recommendations for demonstration and evaluation.

Characterization of alcohol-DNAPL interactions included characterization of ternary phase behavior, interfacial tension, viscosity and density. Six ternary phase diagrams were determined for alcohol+water+solvent. The alcohols included isopropyl alcohol (IPA), ethanol, and methanol. The solvents included tetrachlorethylene (PCE) and trichloroethylene (TCE). Solubility of PCE and TCE were also determined at low alcohol concentrations. The higher molecular weight alcohol (IPA) had a much greater effect on the solubility of PCE and TCE than did either ethanol or methanol as evidenced by the greater single phase or miscible region of the ternary diagrams. IPA also had the greatest effect on the reduction of interfacial tension of PCE and TCE, and resulted in swelling of the TCE thus causing a significant decrease in density of TCE at higher alcohol concentrations. The addition of PCE and TCE at high aqueous phase IPA concentrations also resulted in decreases in aqueous-phase viscosity.

Characterization of soils and layering included grain size analysis, pressure-saturation relationships, permeability before and after establishing a residual DNAPL saturation, and porosity determinations. Two sandy materials and one clay soil were used in these studies. The medium-grained sand used 
was a uniform Ottawa white sand (40-60 mesh). A fine-grained sand was obtained by sieving a local sand to a uniform size. Clay soil was obtained from the Savannah River Site. The clay soil after being crushed and sieved consisted of about $70 \%$ red marine clay, primarily kaolinite, and silt. The sieved portion, henceforth called clay, was then mixed with the Ottawa sand at different clay fractions. Pressure-saturation relationships were determined for homogenous materials and for claysand mixtures of increasing clay fraction. A pore size distribution index was determined from the drainage portion of the pressure-saturation curve. Results showed that as the clay fraction increased the pore size distribution increased and the residual DNAPL saturation also increased. Residual DNAPL saturation in sand was on the order of $18 \%$ while that retained in a $20 \%$ clay-sand mix was close $40 \%$. Permeability of the homogeneous media was determined using a falling head or constant head method.

Layering in the columns was achieved by placing a divider in the middle of the column and slowly lifting this as different media were placed on either side of the divider. Layers consisted of the Ottawa white sand, the fine sand, a sand-clay mixture and the SRS clay. In place permeability was determined using a conservative tracer and the method of moments to determine the average linear velocity and pressure gradient measurements across the column. Using Darcy's law the hydraulic conductivity could be determined and adjusting for fluid viscosity and density the permeability could be determined. Generally, permeability determinations for each layer were similar to permeability measurements of the same media in homogeneous columns.

The chlorinated solvent displaced the water in homogenously-packed and layered columns. Two porous plates; one water-saturated and one treated with a hydrophobicizer then DNAPL saturated were used to displace water with the chlorinated solvent and subsequently imbibe water and drain the chlorinated solvent leaving a residual DNAPL in the column. This method was especially important in the layered columns to ensure a uniform residual saturation in the columns. The residual saturation was quantified in several ways. The first was a volumetric determination of the amount of DNAPL that went in during displacement and the amount that came out during water imbibition. The second method was to quantify the area under the breakthrough curve during alcohol flushing. The third method was used for the layered columns and involved the use of partitioning and conservative tracers and subsequent analysis of breakthrough curves. Results indicated that the partitioning tracer method provides reasonable results and is useful in this application.

Alcohol flushing of the columns was performed on homogeneous and layered columns to evaluate DNAPL mobilization. Trapping numbers were calculated at the onset of mobilization to compare to results of other researchers. Results showed that increasing amounts of clay in the clay-sand mixture resulted in increased residual saturation and that this residual was found to begin to mobilze at a range of trapping numbers below those reported in the literature for homogeneous well-sorted sand. This suggests that the potential to mobilize is greater in mixed-media and may be partially explained by the formation of multi-pore branched ganglia in the clay containing media as opposed to singlet formation in a well-sorted sand.

Some experiments performed using a two dimensional tank of layered media showed that mobilization of the DNAPL did occur but that it only mobilized distances on the order of centimeters 
and then was dissolved in the alcohol flushing solution. These results suggest that mobilization of DNAPL on a larger scale still needs further investigation and may not be fully represented by benchscale column experimentation.

Layered columns proved to be the greatest challenge of this project. The development of a layered column system was an evolving process and several different designs were used to gather data on permeability, residual saturation and alcohol flushing. Initially a system of manometers was used to measure pressure drops across the column. This was later switched to pressure transducers which were used for over a year. These were then determined to be too complex and 'noisy' for our purposes and manometers were used again. Determining residual saturation in these columns also went through several iterations. One method involved the destruction of the column and soil extraction. Another method involved using the area under the effluent curve. A third used the volumetric displacement of water or DNAPL and the fourth utilized partitioning tracers. All methods had their strengths and weaknesses. Sampling the layered columns was also difficult. Effluent from a single endport would mix the solutions from the two column sides so that the individual solutions could not be determined. The end port was split and had two outlets but there were flow problems and mixing associated with this method. Finally a system of side ports and side port sampling was developed and in the end seemed the most successful. Unfortunately, the project ran out before this system could be utilized to its fullest. However, considerable data were gathered from the various systems and showed that flushing in a layered system is as difficult as was originally thought. The more permeable layer cleaned out much more quickly than the less permeable layer because of the much higher flows going through the more permeable side even though a similar number of pore volumes of fluid was required to clean out the less permeable soil. Cleaning out the more permeable side resulted in even more flow going through that side due to the increase in permeability with the removal of the NAPL.

Dissolution of the residual DNAPL was the primary mechanism employed in the majority of the alcohol flushing experiments. Several different column scenarios were used with the layered columns to obtain the best representation of what was really happening in the columns. Single outlet endcaps, dual outlet endcaps and side port sample ports were used. The side port sampling ports provided the most reliable and informative results although the other methods were also useful. Results showed that alcohol flushing can be extremely beneficial in enhancing the removal of DNAPL due to its high ability for enhancing DNAPL solubility. Alcohol concentrations on the order of 40 and $50 \%$ isopropyl alcohol resulted in effluent PCE concentrations in the tens of thousands resulting in removal of the DNAPL in several pore volumes.

There was clearly evidence of tailing in the more permeable side long after the NAPL should have been cleaned out. This may be due to some mass transfer from the less permeable side to the more permeable media but a complete understanding of this was not obtained during the lifetime of this project.

The column results suggest that remediating DNAPL contaminated media is feasible but low permeability zones must be considered in determining the time to cleanup. For example, several pore volumes of alcohol may be sufficient to remove the contaminant but these several pore volumes must be experienced in all the media. According to our layered columns results, it took 5 to 10 pore 
volumes through the more permeable medium to achieve one pore volume through the fine medium.

Alcohol recovery using activated carbon proved to be very promising in that it was both technically and economically feasible. There was a decrease in PCE adsorption capacity on activated carbon with increasing alcohol concentrations. This could be compensated for by increasing the mass of the carbon dose in the recovery system.

In-situ flushing technologies are proving to be accepted technically, economically and by regulators, as evidenced by the increasing number of full-scale and demonstration systems. The Ground Water Remediation Technologies Analysis Center (GWRTAC) maintains a database of in-situ flushing studies both on-going and completed. The results of this project support the use of alcohol for in-situ flushing because of its ease of use, and recyclability. Soil heterogeneity is still a concern for the application of any in-situ technologies and this factor prevents them from working as effectively at all sites. Some strategies such as recirculation of alcohol and reduced flow rates where feasible may be helpful in making alcohol flushing more efficient. 


\section{TABLE OF CONTENTS}

Page Number

Executive Summary

Table of Contents 1

$1 \quad$ Introduction and Project Objectives 3

$\begin{array}{lll}2 & \text { Research Objectives } & 7\end{array}$

3 Characterization of alcohol-DNAPL interactions $\quad 8$

3.1 Introduction and Background

3.2 Methods

3.3 Results and Discussion

3.4 Summary

3.5 References

$4 \quad$ Characterization of soils and layering $\quad 20$

4.1 Introduction and Background

4.2 Methods

4.3 Results and Discussion

4.4 Summary

4.5 References

5 Effect of alcohol concentration on DNAPL mobilization

5.1 Introduction and Background

5.2 Methods

5.3 Results and Discussion

5.4 Summary

5.5 References

6 Effect of flow rate on DNAPL solubilization

6.1 Introduction and Background

6.2 Methods

6.3 Results and Discussion

6.4 Summary

6.5 References

$7 \quad$ Evaluation of alcohol reuse

7.1 Introduction

7.2 Background

7.3 Methods 
7.4 Results and Discussion

7.5 Summary

7.6 References

7.7 Statistical Analysis

8 Process feasibility

8.1 Introduction

8.2 Field Studies

8.3 Modeling

8.4 Economic Feasibility

8.5 Regulatory Feasibility

8.6 References

9 Recommendations for demonstration and evaluation. 
Alcohol flushing, also called cosolvent flushing, is a relatively new in-situ remediation technology that shows promise for removing organic solvents from the soil and groundwater. Soil and groundwater contamination from organic solvents and petroleum products is one of the most serious and widespread environmental problems of our time. Most of the DOE facilities and inactive sites are experiencing soil and groundwater contamination from organic solvents. These water immiscible solvents have entered the subsurface from leaking underground storage tanks and piping, and from past waste handling and disposal practices such as leaking lagoons, holding ponds and landfills. In many cases, they have traveled hundreds of feet down into the saturated zone. If left in the soil, these chemicals may pose a significant environmental and human health risk. Alcohol flushing has potential for application to spilled solvents located deep within the saturated zone which are difficult if not impossible to remove by current remediation strategies, thus, greatly expediting restoration time, reducing total remediation cost and reducing risk.

Organic solvents and oils that are immiscible with water are commonly referred to as nonaqueous phase liquids (NAPLs). The NAPLs that have a density greater than groundwater are often specifically referred to as dense nonaqueous phase liquids (DNAPLs). Common cleaning and extraction solvents, such as tetrachloroethylene (PCE) and trichloroethylene (TCE) are DNAPLs and are common contaminants at DOE sites. The Savannah River Site (SRS) may have up to several million pounds of PCE/TCE located in the subsurface environment (Westinghouse SRC 1992). Restoration of groundwater systems contaminated with DNAPLs is one of the most significant technical challenges at DOE sites today.

One current remediation strategy for contaminated groundwater involves pumping the groundwater and treating it above ground. Pump and treat strategies are generally only effective in removing the dissolved contaminants not in removing the DNAPL source. Due to the sparingly soluble nature of DNAPLs, such as PCE, relying on dissolution as a remediation strategy could take many decades, or much longer to achieve clean up. This will prove to be extremely costly in the long term and environmentally risky. Clearly a need exists for technologies that offer a faster, safer and cost effective means for restoring DNAPL sites.

Alcohol flushing or flooding was originally developed by the oil industries as a means to enhance oil recovery from oil bearing strata. Enhanced oil recovery (EOR) techniques also include: 1) water flooding, in combination with alkaline chemicals; 2) chemical flooding using surfactants; 3) thermal flooding; and 4) alcohol (cosolvent) flooding. These flooding processes all act to increase oil solubility, reduce oil viscosity and reduce oil-water interfacial tension in an attempt to enhance oil extraction. Recently many of the EOR techniques have been considered for environmental restoration of NAPL and DNAPL contaminated sites.

Chemical flooding using surfactants has been the subject of renewed research interest by the petroleum industry. Potential application of surfactant flushing for remediating contaminated soils and aquifers is also being explored (Fountain et al. 1996, Fountain et al. 1997, Pennel et al. 1993, Abdul et al. 1992). Several potential problems with surfactant flushing for environmental 
restoration are the possible interaction of the surfactants with the soil, difficulty in applying the correct surfactant dose so as not to cause clogging, and the fate of the surfactants if small amounts are left in the soil. A major problem with a surfactant flushing technique also seems to be the difficulty in reusing the surfactant solution which may lead to expensive operating costs.

Alcohol flooding or flushing holds greater potential than surfactant flushing as a widespread remediation technique for DNAPL contamination. It is also less problematic in the areas of reuse, concentration and fate in the environment. Either distillation or activated carbon adsorption, air stripping or some combination of these can be used to separate the DNAPL from the alcohol solutions. The recovery technique will depend on the mixture, ease and cost of operation. Activated carbon and air stripping are very common, fairly simple and relatively inexpensive treatment processes widely used in groundwater treatment. Adapting these for use in alcohol flushing will be straightforward. A slightly inaccurate calculation of alcohol concentration will not cause problems as is possible with surfactant flushing. Increasing concentrations of alcohol will only enhance solubility, thus enhancing DNAPL removal rates. Alcohols, such as ethanol, methanol and isopropyl alcohol, are also biodegradable. Ethanol, of food grade quality, is currently popular.

Alcohol flooding, however, has been investigated to a lesser extent than surfactant flushing, although, considerable laboratory work and pilot-scale studies suggest this to be a promising technique (Annable 1999, Rao et al. 1997, Hayden and Van der Hoven 1996, Augustijn et al. 1994, Brandes and Farley 1993, Boyd and Farley 1992). There is also support from various regulatory agencies in many of these studies which indicates acceptance, which is important for realistic implementation of this technology.

A general alcohol flushing system for environmental cleanup will have to include both injection and extraction wells. This is also common for other in-situ groundwater remediation technologies such as bioremediation or air sparging. Injection and extraction wells may be situated in a rectangular, oval or star-shaped patterns. The well depths vary and distance between wells will be based on projected capture zones. Sheet piling and hydraulic barriers have been used at many demonstration sites to maintain hydraulic control. Figure 1.1 shows a schematic of a in-situ alcohol flush. 


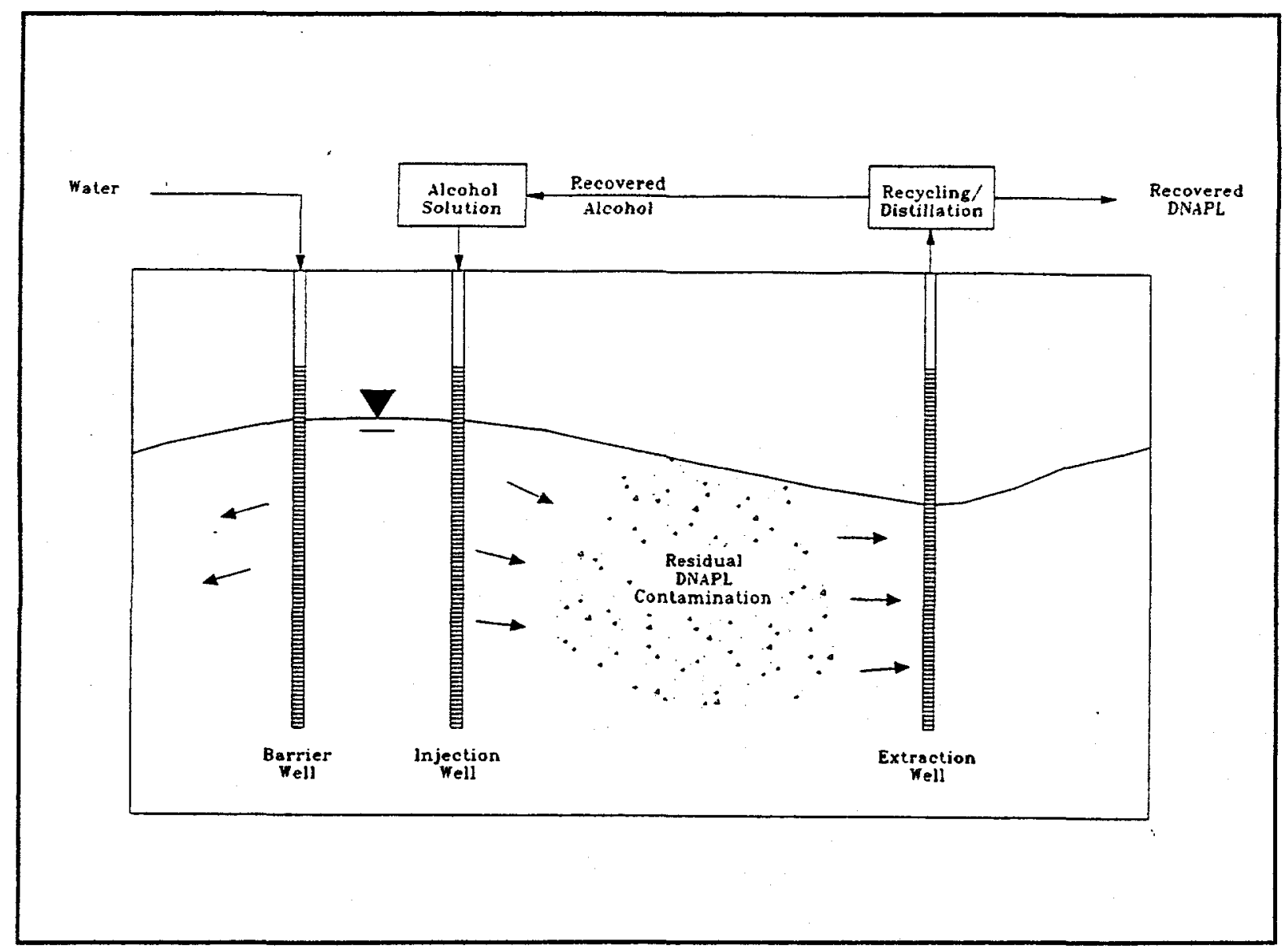

Figure 1-1. Schematic representation of a alcohol flushing strategy.

It is important to realize, that there is no one technology that will cost effectively remediate all our current contamination problems. Some technologies are better suited for certain applications than others. Cosolvent flushing has the potential to be another important remediation technology, especially for DNAPL source removal. It is not, however, appropriate for all remediation situations.

Table 1-1 qualitatively summarizes the advantages and disadvantages of some of the current and innovative technologies with alcohol flushing for remediating groundwater systems contaminated with DNAPLs. The summary provided in Table 1.1 supports the idea that different innovative technologies may be better suited to different situations and more than one technology may be needed for site remediation. 
Table 1-1. Comparison of remediation techniques, both current and innovative (field tested), with alcohol flushing for removing chlorinated DNAPLs from saturated soil environments.

\begin{tabular}{|l|l|l|}
\hline Technique & Advantages & Disadvantages \\
\hline Pump \& Treat & $\begin{array}{l}\text { Widespread application, proven } \\
\text { technology, relatively low capital } \\
\text { costs. }\end{array}$ & $\begin{array}{l}\text { No removal of DNAPL source, very } \\
\text { long operating times, long-term } \\
\text { pumping and treatment costs. }\end{array}$ \\
\hline Bioremediation & $\begin{array}{l}\text { Can reduce spread of soluble plume; } \\
\text { decreased cleanup time compared to } \\
\text { pump and treat. }\end{array}$ & $\begin{array}{l}\text { No removal of DNAPL source } \\
\text { within soil;chlorinated solvents } \\
\text { difficult to degrade; addition of } \\
\text { nutrients, methane, O and others often } \\
\text { need to be added; need injection and } \\
\text { extraction wells. }\end{array}$ \\
\hline Air Sparging & $\begin{array}{l}\text { Relies on air to volatilize } \\
\text { contaminants;horizontal wells may be } \\
\text { very efficient thus reducing cleanup } \\
\text { time compared to pump and treat. }\end{array}$ & $\begin{array}{l}\text { injection and extraction wells needed; } \\
\text { in combination with soil vapor } \\
\text { expensive; needs to be combined with } \\
\text { SVE. }\end{array}$ \\
\hline Alcohol Flushing & $\begin{array}{l}\text { Removes DNAPL source thus } \\
\text { decreasing cleanup cost and time, } \\
\text { reuse alcohol solution; alcohol is } \\
\text { biodegradable }\end{array}$ & $\begin{array}{l}\text { Injection and extraction wells } \\
\text { needed; relatively new and practical } \\
\text { limitations not known, }\end{array}$ \\
\hline
\end{tabular}

\section{$1.1 \quad$ References}

Abdul, A.S., T.L. Gibson, C.C. Ang, J.C. Smith and R.E. Sobczynski. 1992. In Situ Surfactant Washing of Polychlorinated Biphenyls and Oils from a Contaminated Site. Groundwater. 30(2): 219231.

Annable, M.D. 1999. Personal communication.

Augustijin, D.C.M., Jessup, R.E., Rao, P.S.C., and Wood,A.L., 1994. Remediation of Contaminated Soils by Solvent Flushing, Transport and Reactive Processes in Aquifers.

Boyd, G.R. and Farley, K.J., 1992. NAPL Removal from Groundwater by Alcohol Flooding: Laboratory Studies and Applications", Hydrocarbon Contaminated Soils and Groundwaters: Analysis, Fate, Environmental and Public Health Effects and Remediation.

Brandes, D. ; Farley, K.J. 1993. Importance of phase behavior on the removal of residual DNAPLs from porous media by alcohol flushing. Water Environment Research 65, 869-878.

Fountain, J.C., R.C. Starr, T. Middleton, M. Beikirch, C. Taylor and D. Hodge. 1996. A Controlled 
Field test of Surfactant-Enhanced Aquifer Remediation. Groundwater. 34(5): 910-916.

Fountain, J.C. 1997. The role of field trials in development and feasibility assessment of surfactantenhanced aquifer remediation. Water Environment Research. 69(2): 188-195.

Hayden, N.J. and Van Der Hoven, E.J., 1996. Alcohol flushing for enhanced removal of coal tar from contaminated soils, Water Environment Research, 68(7).

Jawitz, J.W., M.D. Annable, P.S.C. Rao and R.D. Rhue. 1998. Field Implementation of a Winsor Type I Surfactant/Alcohol Mixture for an In Situ Solubilization of a Complex LNAPL as a SinglePhase Microemulsion. Environ. Sci. Tech. 32(4):523-530.

Rao, P.S., Annable, M.D., Sillan, R.K., Dai, D., Hatfield, K., Graham, W.D., Wood, A.L., and Enfield, C.G., 1997. Field-scale Evaluation of In-Situ Cosolvent Flushing for Enhanced Aquifer Remediation, Water Resources Research, 33(12).

Pennel, K.D., Abriola, L.M., and Weber, W.J. Jr., 1993. Surfactant-Enhanced Solubilization of Residual Dodecane in Soil Columns. 1. Experimental Investigation", Environmental Sci. Tech. 27(12).

\section{Research Objectives}

This project focused on further expanding the feasibility, application and optimization of a new insitu remediation technology (alcohol flushing) for DNAPLs (PCE and TCE) in heterogeneous saturated soil systems. The primary goal was to determine the efficacy of alcohol flushing to enhance the removal of DNAPLs from a layered soil system and compare the overall feasibility of the various scenarios by also considering cost and time.

The specific project tasks included:

1) characterization of alcohol-DNAPL interactions;

2) characterization of soils and layering;

3) column studies to evaluate various alcohol concentrations on DNAPL mobilization;

4) column studies to evaluate the effect of flow rate on DNAPL solubilization;

5) evaluation of alcohol reuse;

6) determination of process feasibility including economic and environmental concerns; and

7) recommendations for demonstration and evaluation. 


\subsection{Introduction}

During an alcohol flushing operation the DNAPL and aqueous phases will undergo changes including solubility, interfacial tension, density and viscosity. In order to better understand enhanced solubilization during alcohol flushing, liquid-liquid equilibria must be characterized. Ternary-phase diagrams are a standard way of presenting the solubility characteristics of three-fluid phase systems in this case; water, alcohol and DNAPL. These diagrams have been used by petroleum and chemical engineers for decades and more recently by researchers investigating methods to remediate contaminated groundwater (Falta 1998, Imhoff et al.1995, Broholm and Cherry 1994, Brandes and Farley 1993).

In the case of a-residual DNAPL, which is trapped in soil pores by capillary forces, a reduction in the interfacial tension between the DNAPL and alcohol/aqueous phase could result in mobilization of the trapped DNAPL. Because of the large density differences between the two phases, this could mean a further downward migration of the DNAPL.

The capillary (Equation 1) and Bond numbers (Equation 2) are dimensionless numbers that have traditionally been used to relate the viscous forces (capillary number) and gravitational forces (Bond number) acting to mobilize a DNAPL blob to the capillary forces acting to trap it. Recently, researchers have used these in combination with the trapping number to characterize DNAPL trapping and remobilization during flushing experiments (Dawson and Roberts 1997, Pennell et al. 1996, Morrow et al. 1988). Pennell et al. (1996) develop and present the trapping number by performing a balance of the forces acting on a trapped spherical DNAPL blob (Equation 3).

$$
\begin{gathered}
N_{C}=\frac{q_{a q} \mu_{a q}}{\sigma_{a q} \cos \theta} \\
N_{B}=\frac{\Delta \rho g k k_{r w}}{\sigma_{a q} \cos \theta} \\
N_{T}=\sqrt{N_{C}^{2}+2 N_{C} N_{B} \sin \alpha+N_{B}^{2}}
\end{gathered}
$$

Where: $N_{T}=$ Trapping number $\mathrm{N}_{\mathrm{C}}=$ Capillary number 
$N_{B}=$ Bond number

$\mathrm{q}_{\mathrm{aq}}=$ Darcy flow of the aqueous phase

$\mu_{\mathrm{aq}}=$ viscosity of the aqueous phase

$\sigma_{\mathrm{aq}}=$ interfacial tension between the aqueous and nonaqueous phases

$\theta_{\mathrm{aq}}=$ contact angle of the wetting phase

$\Delta \rho=$ the difference in density between the nonaqueous and aqueous phases

$\mathrm{g}=$ gravitation constant

$\mathrm{k}=$ intrinsic permeability of the soil

$\mathrm{k}_{\mathrm{rw}}=$ ratio of the permeability of the soil with a DNAPL residual to the initial permeability

A reduction in the interfacial tension between the aqueous and nonaqueous phases through the use of an alcohol solution results in a reduction in the capillary forces holding the trapped DNAPL in place. This corresponds to an increase in $N_{C}$ and $N_{B}$ which means that the viscous and gravitational forces could now dominate and result in the remobilization of the blob. The viscosity of the aqueous solution is an important term in determining the viscous forces and the difference in density between the aqueous and nonaqueous phases is important in gravitational force term. Most researchers and environmental professionals believe a flushing approach which enhances solubility without mobilization of the DNAPL is the best one to utilize thus preventing the unwanted flow of DNAPLs to other regions of the aquifer. Understanding the forces involved in DNAPL mobilization, therefore, is important for the application of this technology.

The purpose of this study was to obtain liquid-liquid equilibria data for ternary diagrams and characterize the solubility enhancement, interfacial tension, density and viscosity of PCE and TCE/alcohol/aqueous systems. The need to characterize the behavior of DNAPLs in contact with various aqueous-phase solutions is critical for the successful application of alcohol flushing technology.

\subsection{Methods}

Chemicals. The chemicals were obtained by VWR Scientific and US Industrial Chemicals, with the following specifications; PCE and TCE (Reagent grade, $99.9 \%$ and $99.8 \%$ pure, respectively), Methanol and Isopropyl Alcohol (IPA) (HPLC grade, 99.8\% pure), Ethanol (Food Grade, 200 proof). Water was distilled using a Coming Mega-Pure ${ }^{\mathrm{TM}}$ System (MP-11A).

Ternary Phase Diagrams. Ternary phase diagrams were constructed from data collected, using the methods presented in Sayar (1991). The synthetic method provided the boundary between the single and two-phase regions and the analytical method provided the tie-lines. Temperature was held at $21.5^{\circ} \mathrm{C} \pm 1{ }^{\circ} \mathrm{C}$.

(a) Synthetic Method. Water and PCE or TCE mixtures were prepared by mass $( \pm 0.01 \mathrm{mg})$, placed in a constant temperature bath and titrated with alcohol until the two phases disappeared.

(b) Analytical Method. Alcohol, water and PCE or TCE mixtures were prepared by mass, equilibrated using a rotary mixer for greater than 24 hours, and centrifuged to separate the two phases. The composition of each phase was determined using a gas chromatograph and flame 
ionization detector (GC/FID). An automated headspace sampler was used to determine the mass of alcohol and PCE in the aqueous phase. The solvent phase was directly injected into the GC to determine the mass of alcohol dissolved in this phase. The water content of the solvent phase was determined by the Karl-Fischer method using an Accumet 150 coulometric $\mathrm{KF}$ automatic titrator (Denver Instrument Company, Arcada, CA). The mass fraction of immiscible solvent in the solvent phase and mass fraction of water in the aqueous phase was then determined by difference.

Solubility Enhancement: The solubility of PCE and TCE in various alcohol solutions, 0 to $50 \%$ $\mathrm{v} / \mathrm{v}$ initial alcohol concentration, was determined by placing $2 \mathrm{ml}$ of PCE or TCE in a $20 \mathrm{ml} \mathrm{crimp}$ cap vial containing 10 acid washed glass beads. The aqueous-alcohol solution was filled to the top to minimize head space, and the vial was crimp capped with a Teflon-lined septum. The solutions equilibrated on a rotary mixer $(2 \mathrm{rpm})$ for 24 hours, and centrifuged. Initial studies indicated this was sufficient time for equilibration. The aqueous phase was sampled and analyzed using a GC/FID with a headspace sampler to determine PCE or TCE concentrations. Five replicate vials were made for each solution.

Interfacial Tension: The interfacial tension of TCE and PCE in various aqueous-alcohol solutions was determined using the pendant drop technique (Ambwani and Fort 1979). The two-phase solution was allowed to equilibrate using a reciprocating shaker and then separate. A Rame-Hart model 100 goniometer (Rame-Hart, Inc. Mountain Lakes, NJ) was used to create and photograph the equilibrated TCE and PCE drops in water/alcohol mixtures. Six replicate drops were photographed and subsequently measured. Tables and the equation from Ambwani and Fort (1979) were then used to calculate interfacial tension. Temperature was held at $22^{\circ} \mathrm{C} \pm 2^{\circ} \mathrm{C}$.

Density and Viscosity: The aqueous-phase and solvent phase densities were determined gravimetrically for each phase in an equilibrated two phase mixture. Aqueous-phase viscosity for different equilibrated solutions was determined using a viscometer (Brookfield Dial Viscometer, Model LVT with UL Adapter $304 \mathrm{~S} / \mathrm{S}$, Stoughton, MA). Temperature was held at $22^{\circ} \mathrm{C} \pm 1^{\circ} \mathrm{C}$.

\subsection{Results and Discussion}

Ternary phase diagrams. Ternary phase diagrams for the six systems investigated are plotted in Figures 3.1-3.3. The higher molecular weight alcohol (IPA) has a much greater effect on solubility of PCE and TCE than does either ethanol or methanol as evidenced by the greater single phase or miscible region of the ternary diagrams.

The tie lines in all cases, except TCE/IPA/water have negative slopes. This indicates the preferential partitioning of the alcohol into the aqueous phase. In the TCE/IPA/water situation, the tie lines exhibit both positive and negative slopes. The negative sloped tie lines occur up to $55 \%$ IPA, by mass, and indicate preferential partitioning of IPA into the aqueous phase. Above $55 \%$, the slopes of the tie lines become positive, an indication of preferential partitioning of IPA into the TCE. This preferential partitioning by IPA results in a decrease of solvent density and is called swelling. 

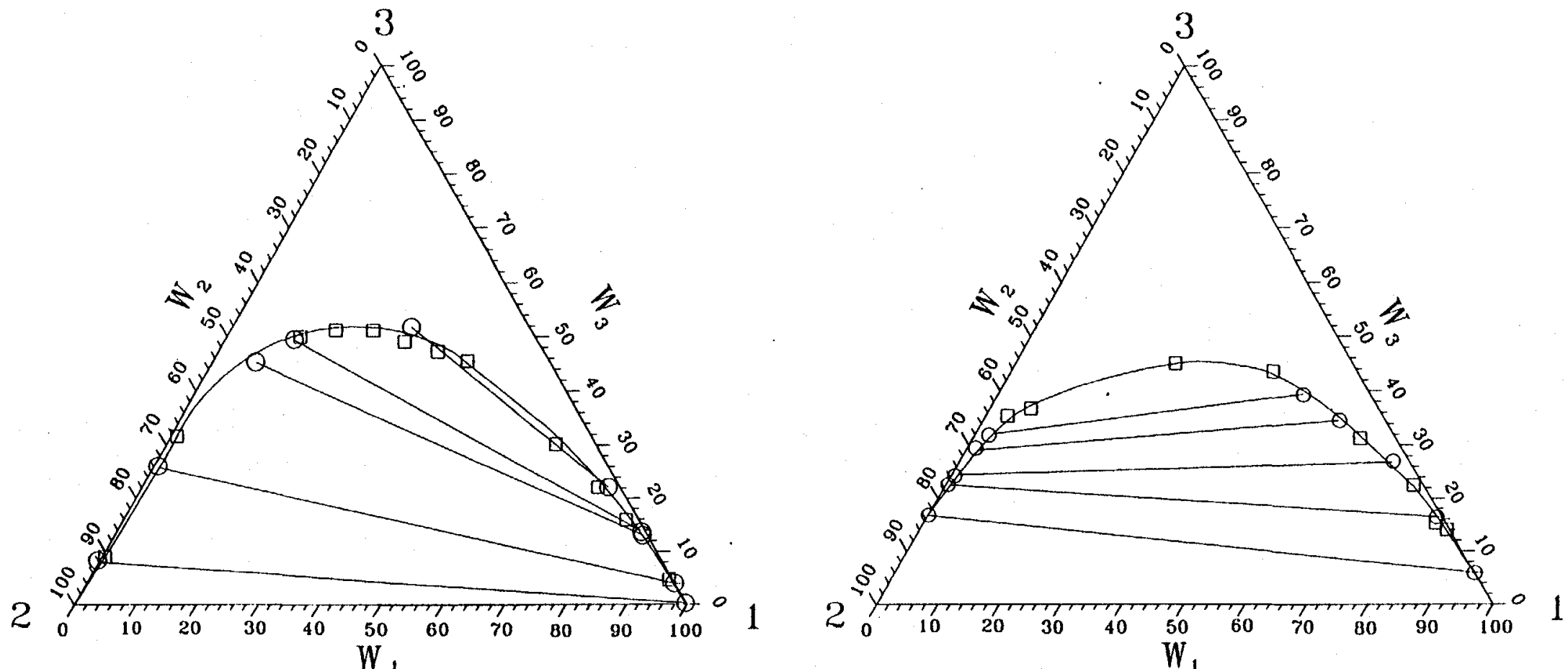

Figure 3.1 Ternary diagrams for experimental liquid-liquid equilibria: (left) tetrachloroethylene/water/isopropanol; (right) trichloroethylene/water/isopropanol (concentrations expressed as mass, $\% \mathrm{~W}_{\mathrm{i}}$ ). 


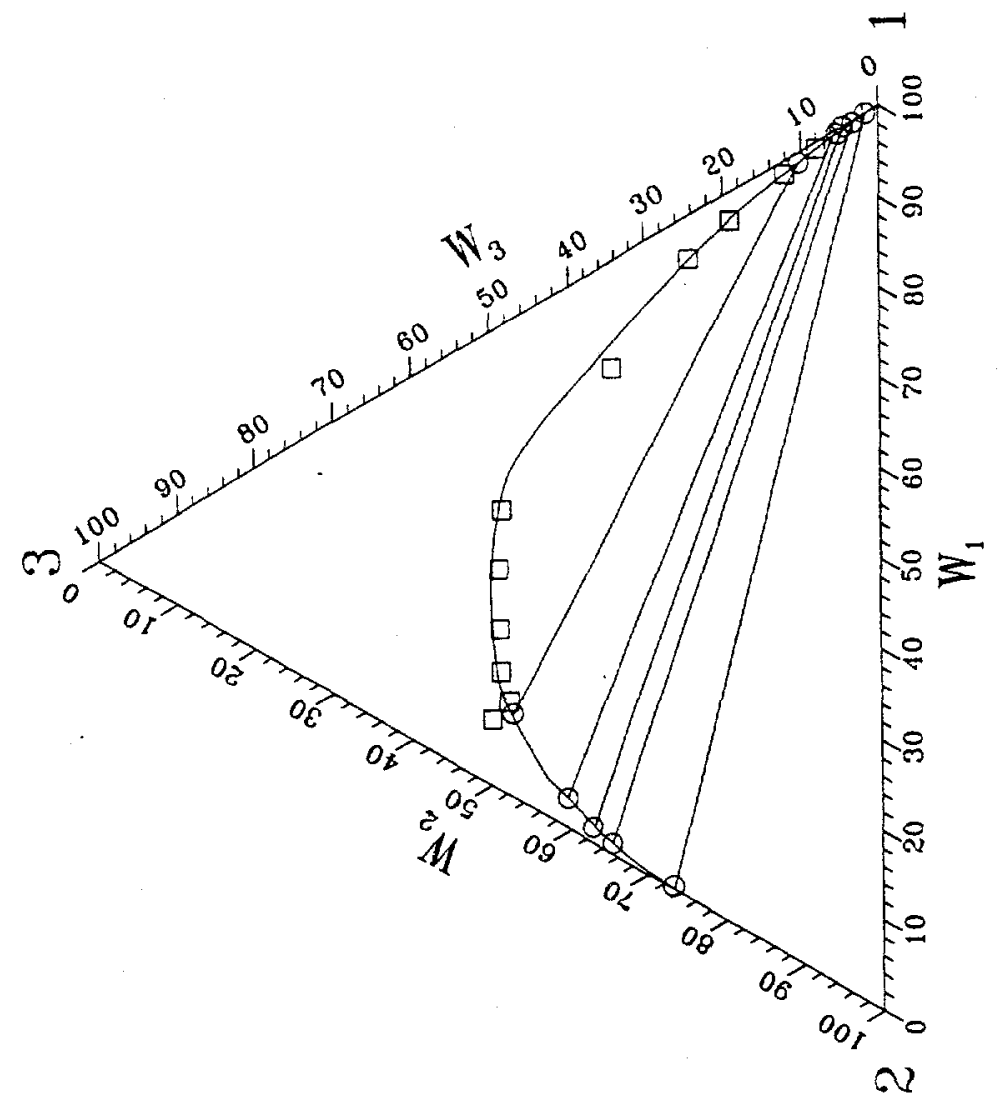

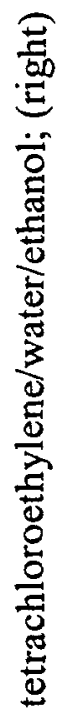

$\stackrel{\Xi}{\stackrel{5}{3}}$

苞兑

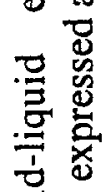

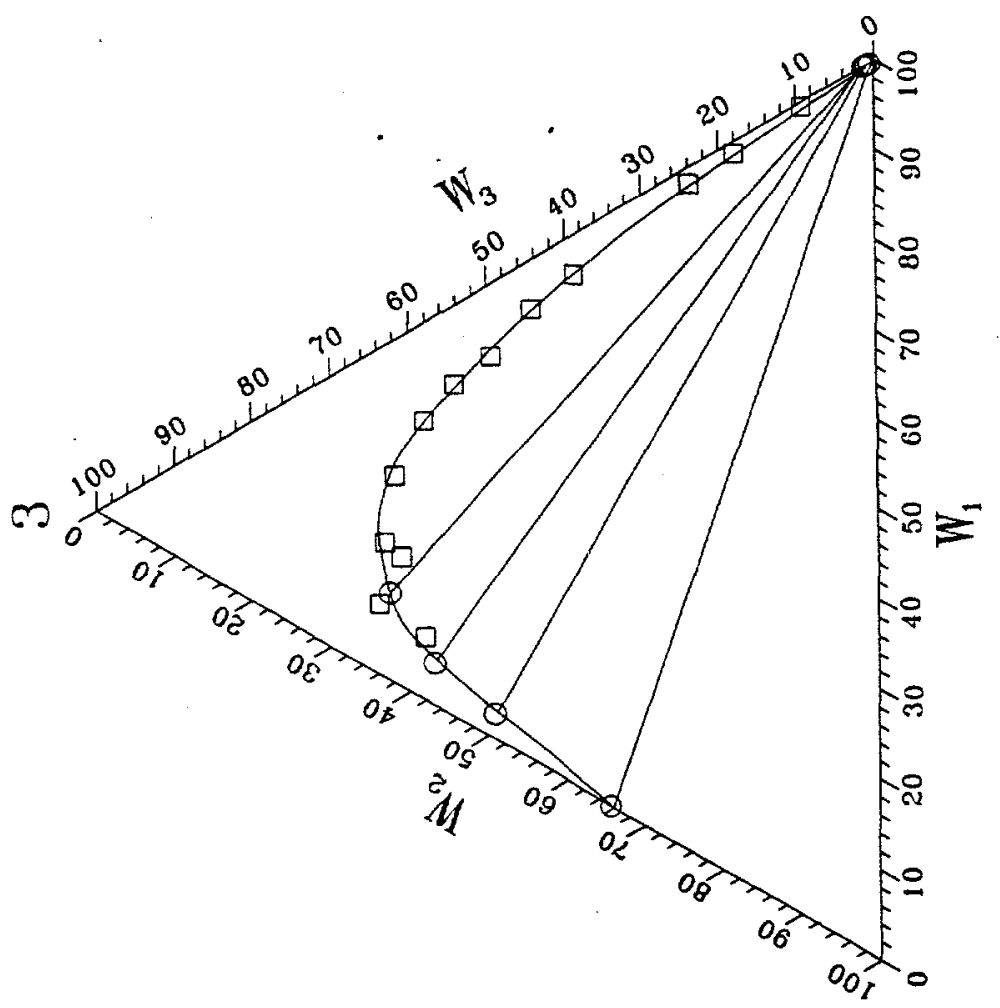

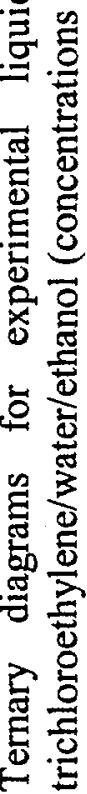

華 


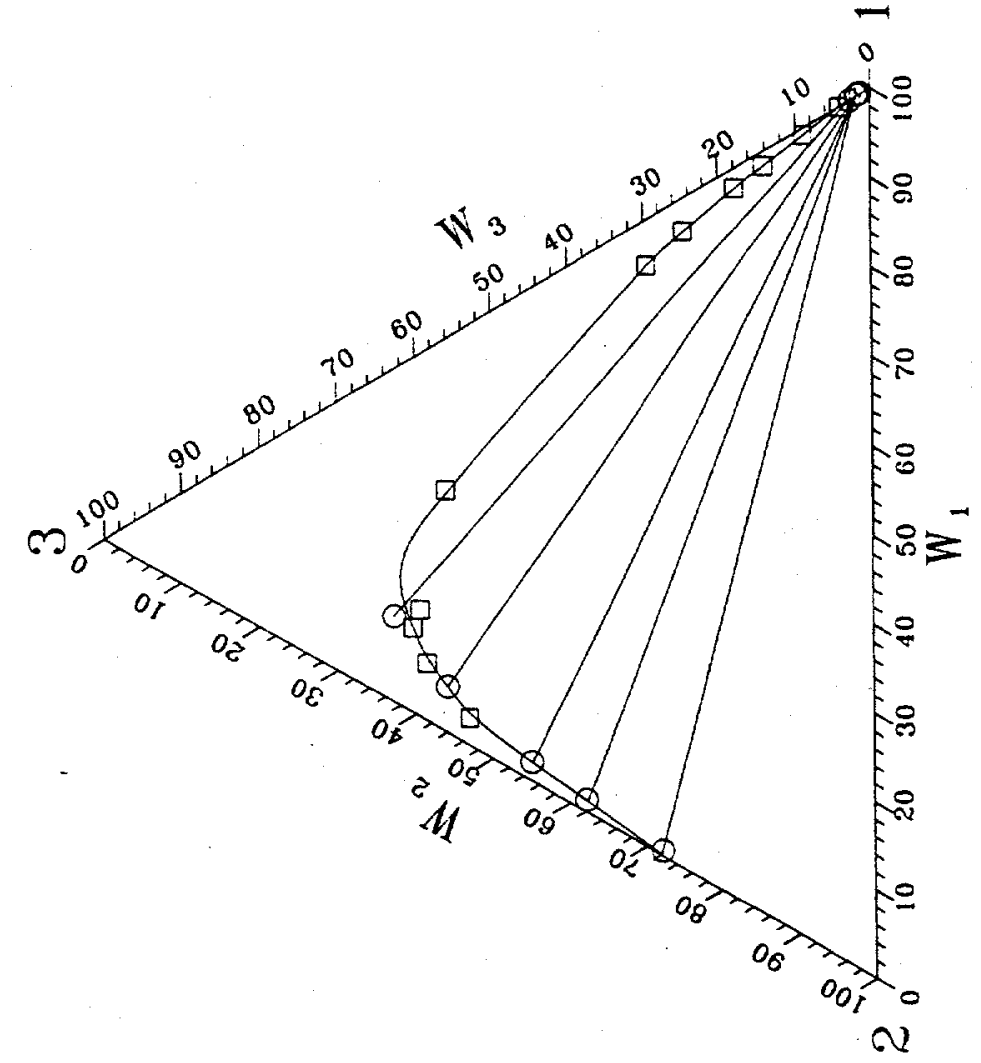

苟
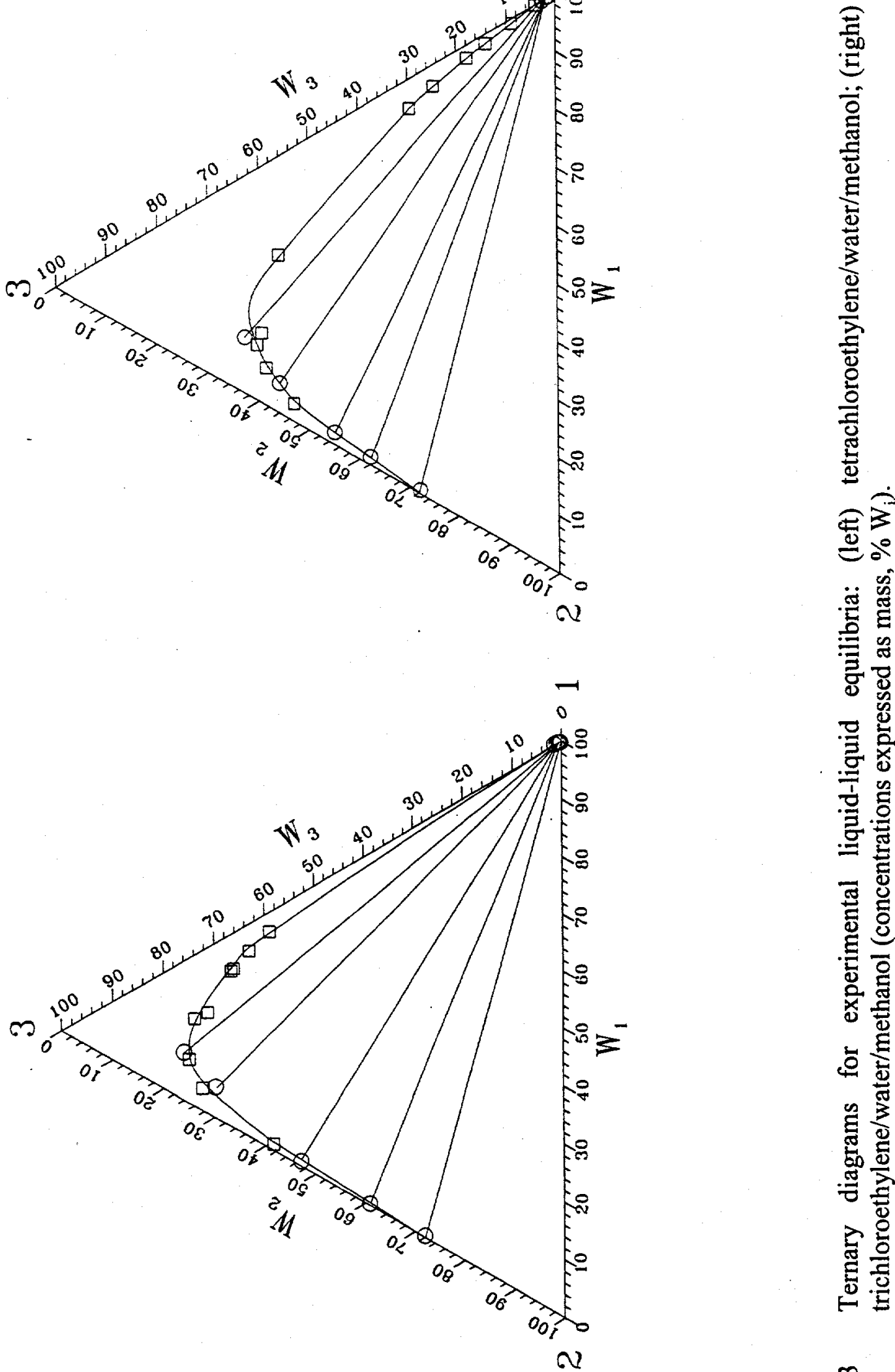

m 
Ternary diagrams for the TCE/IPA/water and PCE/IPA/water systems are in agreement with those reported by Brandes and Farley (1993). The ternary diagram of the PCE/methanol/water compare well with both the experimental and UNIQUAC model predictions reported by Imhoff et al. (1995). The ternary diagram for TCE/methanol/water compares well to that produced using an ESP numerical simulation as reported in Broholm and Cherry (1994). Ternary phase diagrams for the TCE/ethanol/water and PCE/ethanol/water systems could not be found in the literature.

Solubility Enhancement. At lower alcohol concentrations, it is impossible to read the solubility enhancement of PCE and TCE from the ternary diagrams. The enhancement of solubility at these lower alcohol concentrations (up to $30 \% \mathrm{v} / \mathrm{v}$ ), however, are also of interest for environmental application of this technology and are shown in Figure 3.4. As with the ternary diagrams, IPA had the greatest effect on enhancing the solubility of PCE at these alcohol concentrations. The relative increase in solubility enhancement was slightly greater with PCE than for TCE. For example, a solubility increase of approximately four times was measured for PCE at $30 \%$ compared to $0 \%$, while only an increase of 2.6 times was measured for TCE.

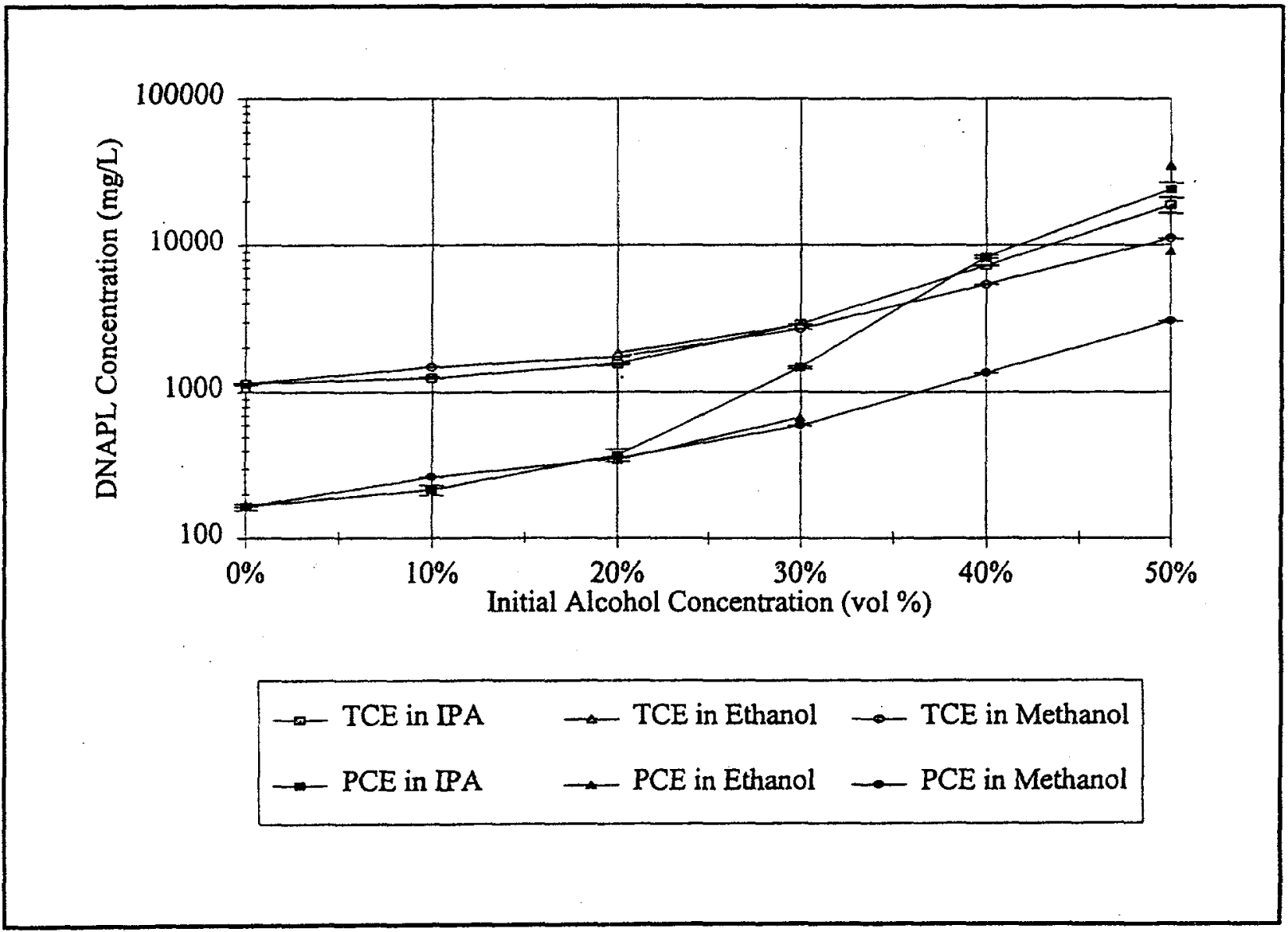

Figure 3.4 Solubility enhancement for PCE and TCE in various alcohol solutions 
Interfacial Tension. Interfacial tension of PCE and TCE in aqueous solutions decreased for increasing alcohol concentrations as shown in Figures 3.5 and 3.6. IPA was the most effective in reducing interfacial tension of the alcohols. This was expected based on the solubility results. TCE equilibrated with water had an average interfacial tension of 39 dynes/cm measured in this study ( 35 dynes/cm reported by Hunt et al. 1988) and PCE equilibrated with water had an average interfacial tension of 43 dynes $/ \mathrm{cm}$ measured in this study ( 45.8 dynes $/ \mathrm{cm}$ measured by Demond and Lindner 1993). Decreases in interfacial tension between chlorinated solvents and methanol showed about a $70 \%$ decrease at $30 \% \mathrm{v} / \mathrm{v}$. Interfacial tension of the two phases with IPA as the alcohol showed an exponential decline; with an overall decrease in interfacial tension of about an order of magnitude at $30 \%(\mathrm{v} / \mathrm{v})$ IPA.

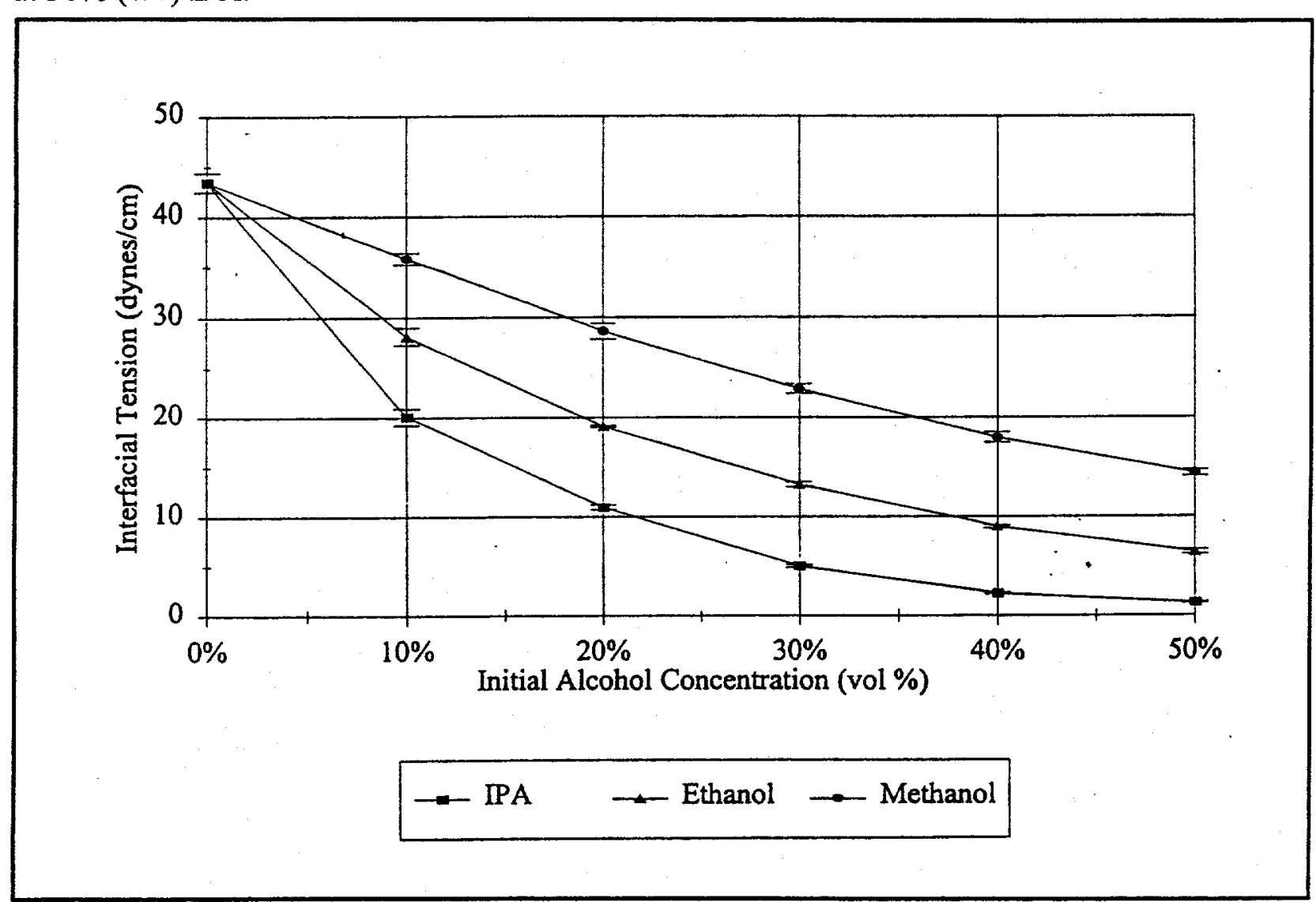

Figure 3.5 Interfacial tension of PCE with changing alcohol concentration.

Density. The changes in density of the DNAPL and the aqueous phase as a function of alcohol concentration are shown in Figure 3.7. There was very little density change in the PCE/IPA system even at high IPA concentrations. The density of the PCE phase went form $1.60 \mathrm{~g} / \mathrm{cm}^{3}$ to $1.49 \mathrm{~g} / \mathrm{cm}^{3}$. The density change in the TCE was dramatic in the IPA case going from $1.45 \mathrm{~g} / \mathrm{cm}^{3}$ to $1.04 \mathrm{~g} / \mathrm{cm}^{3}$. This phenomenon is called swelling and is also reflected in the slope of the tie lines in the ternary diagram. Minimal changes in TCE or PCE density were noted in the ethanol and methanol cases. 

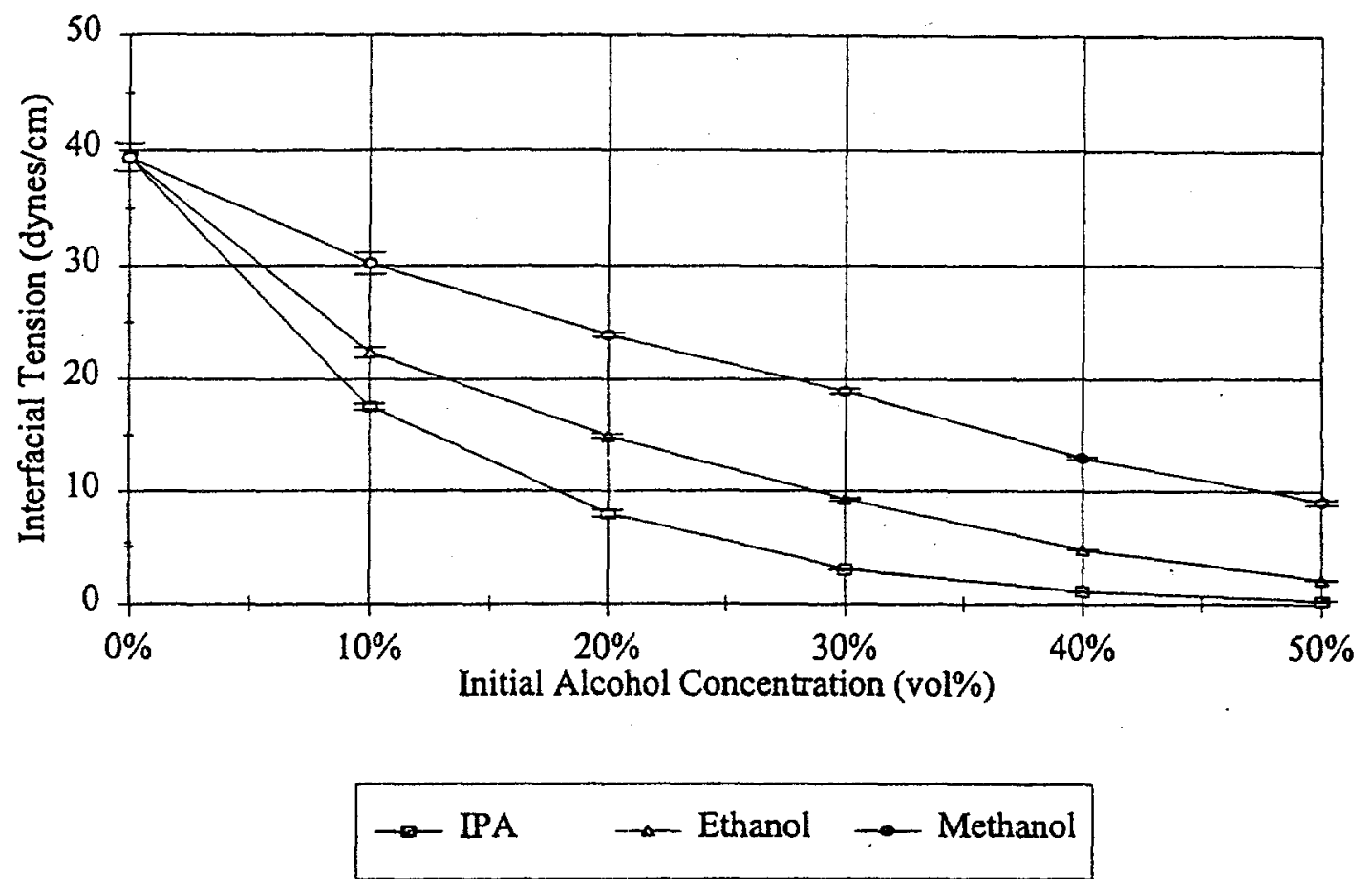

Figure 3.6 Interfacial tension of TCE with changing alcohol concentration.

Density changes of the aqueous phases with increasing alcohol concentrations were very similar for all alcohol systems. Because of this only one curve is shown.

Viscosity The aqueous-phase viscosity is affected by both its alcohol and chlorinated solvent concentrations. Viscosity diagrams are presented in Figure 3.8 and show both the effect of alcohol concentration on viscosity and the effect of the chlorinated solvent on viscosity. The dissolution of the chlorinated solvent did not affect viscosity until a relatively high alcohol concentration was reached. The concentration of chlorinated solvent was also high which caused a reduction of viscosity over that of water-alcohol systems. 


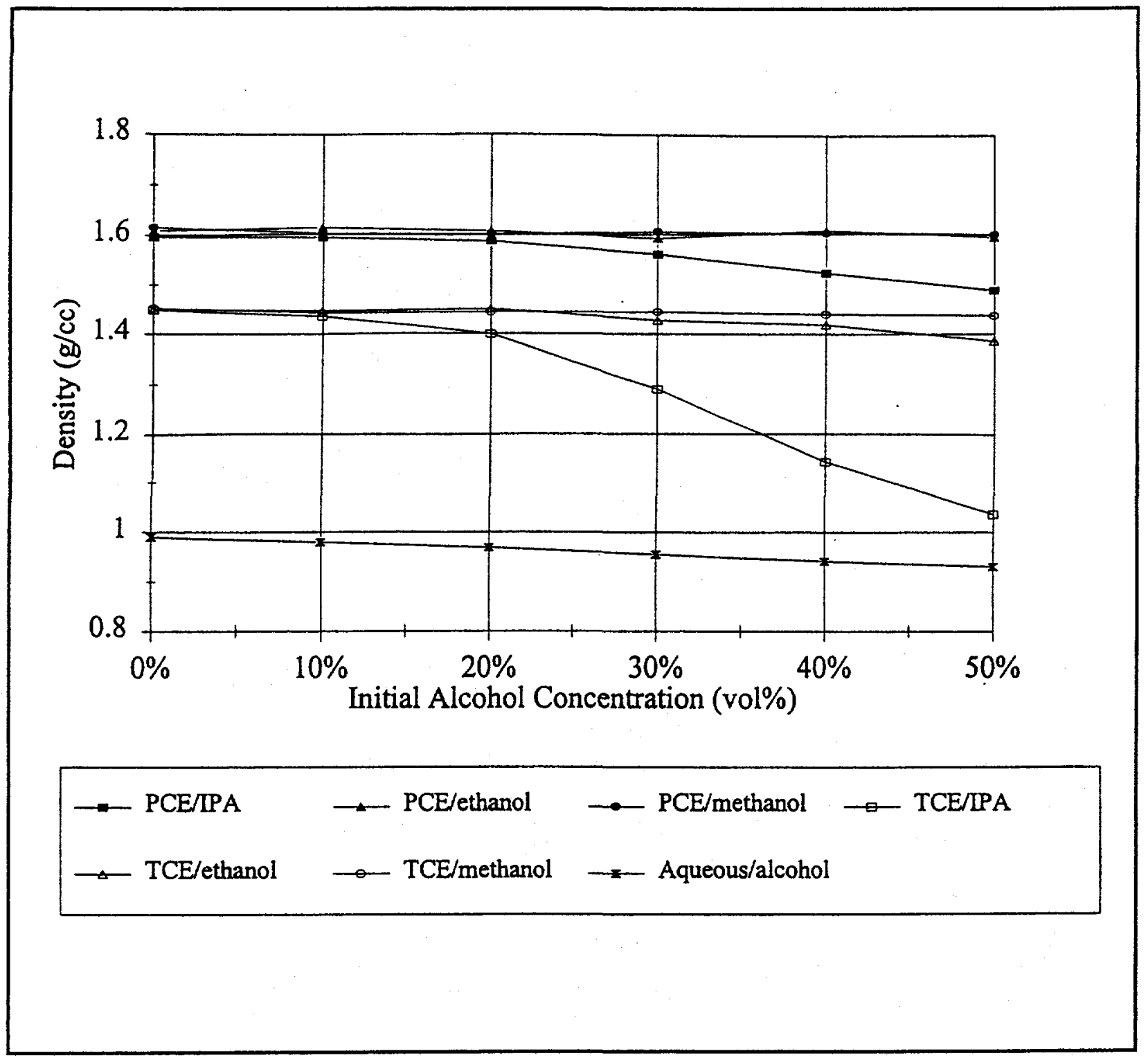

Figure 3.7 Density of various solutions as it relates to alcohol concentration. 


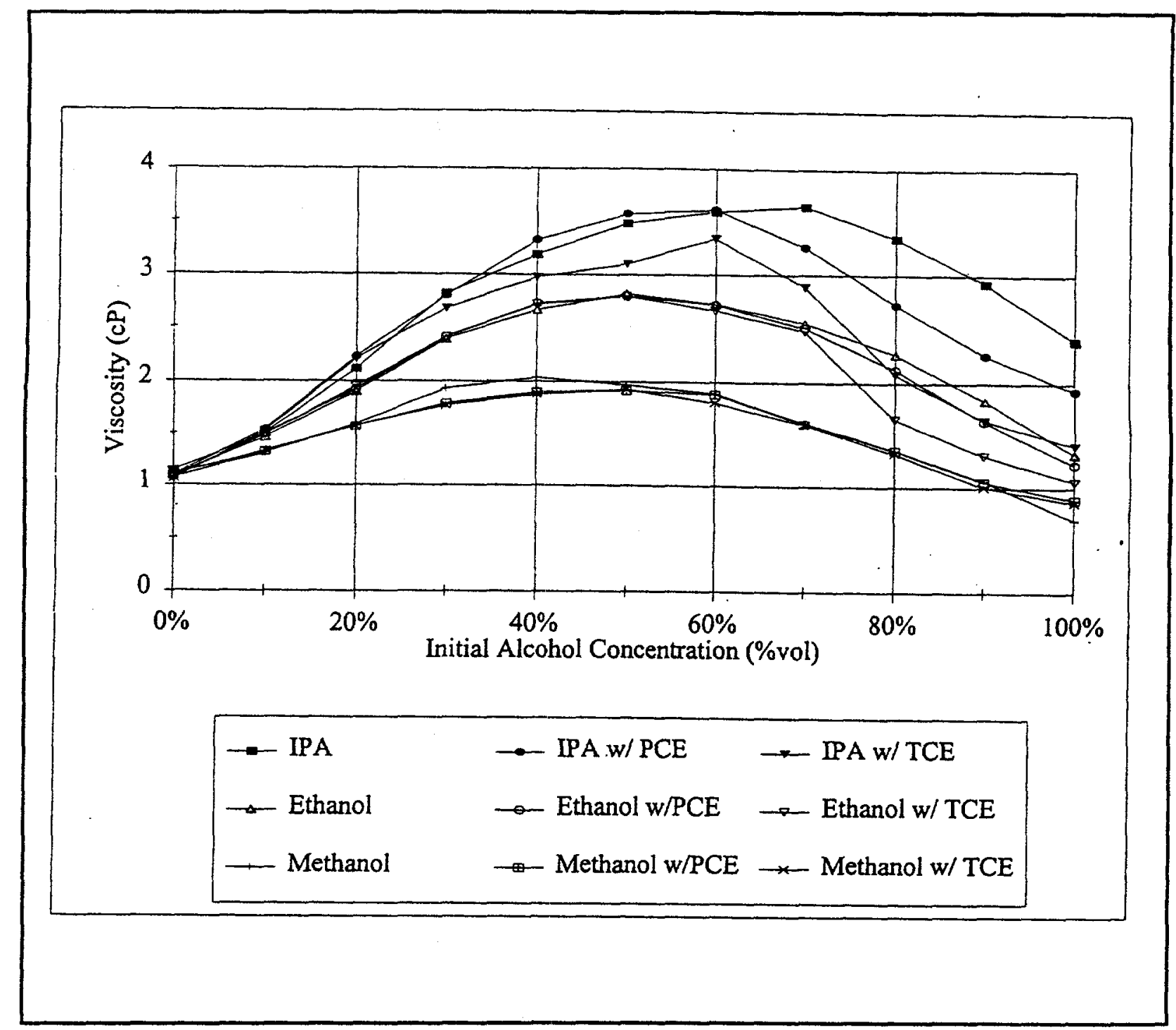

Figure 3.8 Viscosity of aqueous solutions as a function of alcohol concentrations.

\subsection{Summary}

The purpose of this study was to obtain liquid-liquid equilibria data for ternary diagrams and characterize the solubility enhancement, interfacial tension, viscosity and density of PCE and $\mathrm{TCE} /$ alcohol/aqueous systems for the purpose of using this information in alcohol flushing applications. An alcohol flushing strategy relies on enhanced solubilization without mobilization of the DNAPL. The results presented in this paper suggest that alcohol concentrations of $30 \%(\mathrm{v} / \mathrm{v})$ or greater are required in order to substantially enhance the solubility of either PCE or TCE. This corresponds, however, to a significant decrease in the interfacial tension between the DNAPL and aqueous phases which is important from the standpoint of potential mobilization of the trapped 
DNAPL.

\subsection{References}

Ambwani, D.S.; Fort, T.Jr. 1979. Pendant drop technique for measuring liquid boundary tensions. Surface and Colloid Science, , 2, 93-119.

Brandes, D. ; Farley, K.J. 1993. Importance of phase behavior on the removal of residual DNAPLs from porous media by alcohol flushing. Water Environment Research, 65, 869-878.

Broholm, K; Cherry, J.A. 1994. Enhanced dissolution of heterogeneously distributed solvents residuals by methanol flushing-a field experiment. Transport and Reactive Processes in Aquifers; Balkema, Rotterdam.

Dawson, H.E.; Roberts, P.V. 1997. Influence of viscous, gravitational and capillary forces on DNAPL saturation. Ground Water 35, 261-269.

Demond, A.; Lindner, A.S.; 1993. Estimation of interfacial tension between organic liquids and water. Environ. Sci. Technol. 27, 2318-2331.

Hunt, J.E.; Sitar, N.; Udell, K.S. 1998. Nonaqueous phase liquid transport and cleanup. 1. Experimental studies. Water Resources Research 24, 1259-1269.

Imhoff, P.T.; Gleyzer, S.N.; McBride, J.F.; Vancho, L.A.; Okuda, I.; Miller, C.T. 1996. Cosolventenhance remediation of residual dense nonaqueous phase liquids: experimental investigation. Environ. Sci. Technol. 29, 1966-1976.

Morrow, N.R.; Chatzis, I.; Taber, J.J. 1988. Entrapment and mobilization of residual oil in bead packs. SPE Reservoir Engineering, Soc. Of Petroleum Eng., August, 927-934.

Pennell, K.D; Pope, G.A.; Abriola, L.M. 1996. Influence of viscous and buoyancy forces on the mobilization of residual tetrachloroethylene during surfactant flushing. Environ. Sci. Technol. 30, 1328-1335.

Sayer, A.A. 1991. Liquid-liquid equilibria of some water + 2-propanol + solvent ternaries. $J$. Chem. Eng. Data 36, 51-65. 


\subsection{Introduction}

One of the major challenges in the field of groundwater remediation is the principle of heterogeneity. The variation in hydraulic conductivity $(\mathrm{K})$ or permeability $(\mathrm{k})$ of an aquifer is most often used to describe the degree of heterogeneity. In homogenous systems, hydraulic conductivities and, therefore, permeabilities are independent of location within the aquifer. In heterogeneous systems, however, these values change with respect to location within the aquifer.

Layered heterogeneous systems are fairly ubiquitous and are commonly found within sedimentary geological environments and in unconsolidated lacustrine and marine deposits (Freeze and Cherry, 1979). In layered systems, each layer making up the system is considered as being homogenous and, therefore having consistent hydraulic conductivity and permeability values throughout. This in itself is a simplification. However, when the system is considered as a whole, it is thought of as a heterogeneous system with different permeability values dependent upon location within the system.

Hydraulic conductivity is dependent upon both the soil matrix as well as the aqueous solution flowing though it. Permeability is understood to be a function solely of the porous media and is thus independent of aqueous fluid properties such as density and viscosity.

Hydraulic conductivities within actual aquifers in the field are commonly estimated using drawdown pumping tests, slug tests and tracer tests. A soil column permeameter makes for much more simplistic estimation of hydraulic conductivities in the laboratory. Essentially however, all methods for hydraulic conductivity determination utilize a rearrangement of Darcy's Law:

$$
K=\frac{Q d l}{A d h}
$$

where: $\mathrm{Q}$ is a known or measured flowrate; $\mathrm{A}$ is cross sectional area of the soil; $\mathrm{dl}$ is the length of the soil column; and dh is the known or measured differential pressure (in the field $\mathrm{dh} / \mathrm{dl}$ would be the measured hydraulic gradient). The permeability of the soil matrix can then be easily determined using Equation 2:

$$
k=\frac{K \mu}{\rho g}
$$

where: $\mu$ and $\rho$ are the aqueous phase viscosity and density, respectively; and $g$ is the gravitational constant.

There are numerous methods for the use of the permeameter in laboratory determination of hydraulic conductivity and subsequent permeability. Likewise, there are numerous methods for use in the field 
determination of these aquifer properties. However, because the focus of this research is on estimating permeability values within individual laboratory soil columns, only the laboratory methods will be discussed here. For additional information pertaining to field determination of hydraulic conductivities, references such as Freeze and Cherry (1979) and Bedient et al. (1994) provide sufficient overviews.

Simplistic methods such as the constant head and falling head permeameter tests provide adequate results for the hydraulic conductivities of porous media packed within soil columns. Klute (1965) indicates that the constant head test is best suited to soils with hydraulic conductivity values greater than $0.01 \mathrm{~cm} / \mathrm{min}(0.00017 \mathrm{~cm} / \mathrm{s})$, while the falling head test is more appropriately suited for more fine grained media with $\mathrm{K}$ values of less than $0.01 \mathrm{~cm} / \mathrm{min}$. The constant head test allows a calculation of $\mathrm{K}$ using Equation 1 by measuring the flowrate through the column (measured values of volume and time) forced through the column under a known hydraulic head. The falling head test uses measured values of time and the change in head in Equation 3 to determine a value of $\mathrm{K}$ :

$$
K=\frac{a L}{A t} \ln \left(\frac{H_{0}}{H_{1}}\right)
$$

where: $a$ is the cross sectional area of the water-filled standpipe; $L$ is the length of the column; $A$ is the cross sectional area of the column; $t$ is the time; and $\mathrm{H}_{0}$ and $\mathrm{H}_{1}$ are the initial and final heights of water within the standpipe.

While the constant and falling head methods have been found to provide accurate data in laboratory soil columns, it should be noted that they would require significant human oversight in order to obtain more than just discrete measurements of hydraulic conductivity and are considered insufficient in instances where it is desirable to continuously monitor changes in the permeability of a media.

The use of manometers is a potential method for allowing more continuous monitoring of the hydraulic conductivities and, therefore, permeabilities within soil columns. By placing a manometer at each end of the soil column, a measure of the differential pressure across the soil column can be made which can then be used in Equation 1 to arrive at a value for $K$. This method has been shown to provide fairly accurate results. However, similar to the methods of constant and falling head testing, the use of manometer for determination of $\mathrm{K}$ values requires significant oversight. In order to track any changes in $\mathrm{K}$ or $\mathrm{k}$, numerous differential pressure readings must be taken at fairly regular intervals.

Pressure transducers can also be used for estimation of K values. Pennel et al. (1996) successfully used pressure transducers to provide accurate permeability data in their soil columns. The transducer system is essentially an electronic manometer system, and the subsequent set up of the system is basically the same. Similar to the estimation of $\mathrm{K}$ with manometers, measured values of differential pressure are used in Equation 1 with known values of flowrate, cross sectional area and column 
length. The major difference between the two methods is the fact that the use of a transducer system allows for the collection of a significantly increased amount of data with very little human oversight. The use of pressure transducers can be, therefore, extremely effective in providing continuous monitoring of changing hydraulic conductivity and permeability data.

Transducers could also be used to determine permeability values for each media within a layered soil column. This is accomplished by measuring the flowrate through each media and measuring with transducers the differential pressure across each media. The hydraulic conductivities and permeabilities within each media of layered soil systems could also be monitored through modifications of the constant head test. However, as was described above, the problems of continuous human oversight would indicate that the use of pressure transducers may be more effective. A comparison of the systems available for permeability measurement in soil columns is summarized in Table 4.1 below.

\begin{tabular}{|l|l|l|}
\hline \multicolumn{2}{|c|}{ Table 4.1 Comparison of Various Permeability Measurement Systems } \\
\hline System & Advantages & Disadvantages \\
\hline \hline Constant Head & $\begin{array}{l}\text { - Fairly accurate data } \\
\text { - Widespread use and acceptance } \\
\text { - Ease of use }\end{array}$ & $\begin{array}{l}\text { - Discrete measurements only } \\
\text { - Only good for coarse media } \\
\text { - Human observational error }\end{array}$ \\
\hline Falling Head & $\begin{array}{l}\text { - Fairly accurate data } \\
\text { - Widespread use and acceptance } \\
\text { - Ease of use }\end{array}$ & $\begin{array}{l}\text { - Discrete measurements only } \\
\text { - Only good for fine media } \\
\text { - Human observational error }\end{array}$ \\
\hline Manometers & $\begin{array}{l}\text { - Fairly accurate data } \\
\text { - Easier for continual monitoring } \\
\text { - Good for different grain sizes }\end{array}$ & $\begin{array}{l}\text { - Human observational error } \\
\text { - Difficult for long-term continuous } \\
\text { monitoring }\end{array}$ \\
\hline Transducers & $\begin{array}{l}\text { - Very accurate data } \\
\text { - Best for continual monitoring } \\
\text { - Good for different grain sizes }\end{array}$ & $\begin{array}{l}\text { - More complex set-up and } \\
\text { operation }\end{array}$ \\
\hline
\end{tabular}

Bear (1972) alluded to the importance of ensuring that laminar flow conditions remain within the soil pores so that Darcy's Law remains linear and, therefore, valid. This is significant in the use of all of the methods discussed above for hydraulic conductivity determination.

The nature and degree of layered heterogeneity is most easily observed in differences in values of hydraulic conductivity and permeability between the different layers. As a result of these differences, the flowrates of aqueous solutions will be different through each media as well. In addition, the different permeabilities of the layered geologic environment are significant in terms of the degree and distribution of entrapped residual DNAPL saturation. The ultimate result of layered heterogeneity will be difficult in remediation of DNAPL using alcohol flushing once it has been entrapped as residual contamination. There appears to be an abundance of research in the literature pertaining to the alcohol flushing of DNAPLs from homogenous media (Boyd, 1991, Boyd and Farley, 1992, Brandes and Farley, 1993, Augustijin et al., 1994, Hayden and Van der Hoven, 1996, 
Imhoff et al., 1995, Rao et al., 1997). However, there seems to be a void in the literature with regard to flushing from heterogeneous media, especially that of layered heterogeneous soils. -

Non-aqueous phase liquids (NAPLs) are water-immiscible solvents and are classified as light nonaqueous phase liquids (LNAPLs) or dense non-aqueous phase liquids (DNAPLs) according to their densities relative to water. Typical DNAPL compounds include pesticides, herbicides and chlorinated solvents. While the physical and chemical properties of DNAPL compounds are understood to vary greatly, the principles governing the fate and transport of the DNAPLs are often similar.

The migration of DNAPL compounds and subsequent formation of a residually trapped separate phase are complex processes that warrant detailed explanations. When a DNAPL is released in sufficient quantity into the subsurface, the contaminant travels in a downward direction through the soil pores and fractures under the influences of gravity. If the quantity of DNAPL is especially large, some of the DNAPL will eventually reach the saturated zone. At this point, the displacement pressure must be overcome in order for the non-wetting NAPL to enter the water saturated zone and, therefore, continue migration. If the entrance pressure is exceeded, the release will subsequently displace water and continue its downward migration through the capillary fringe and water table, leaving behind a trail of residual contamination.

Residual NAPL contamination is understood to be non-continuous in nature (Wilson et al., 1990, Conrad et al. 1992). This is the main difference between mobile, bulk DNAPL contamination and residual phase DNAPL contamination. The entrapment process and the shape of the residual DNAPL blobs are controlled by capillary phenomena. Under the influence of gravity, the DNAPL drains the soil pores of water. The DNAPL blobs become discontinuous when the quantity of DNAPL moving through the soil media diminishes and the capillary forces within the soil pores become sufficiently strong enough to overcome the viscous and gravity forces of the diminishing DNAPL.

While the forces of gravity are most significant during the initial spill of DNAPL, forces of capillarity become very significant in immobilizing the DNAPL as the amount of DNAPL decreases and, thus, the gravitational force associated with the DNAPL dissipates. Capillary pressure is commonly defined as the difference between the non-wetting fluid pressure and the wetting fluid pressure. For a NAPL-water system with water as the wetting phase:

$$
P_{c}=P_{n}-P_{w}
$$

where: $P_{n}$ is the pressure of the NAPL and $P_{w}$ is the pressure of the water. Capillary pressure between a pair of immiscible fluids in a porous media is also commonly defined according to the Laplace Equation illustrated as Equation 5:

$$
P_{c}=\sigma\left(\frac{1}{r_{1}}+\frac{1}{r_{2}}\right)
$$


where: $\sigma$ is the interfacial tension between the two immiscible fluids and $r_{1}$ and $r_{2}$ are the radii of curvature in two directions of the interface between the two fluids. The Laplace Equation is often simplified by neglecting both the macroscopic geometry of the pore spaces and the minor gravitational force which acts to distort the interface shape by considering the porous media to be similar to a bundle of thin glass tubes of uniform radii. When these simplifications are made, the Laplace Equation can be simplified as:

$$
P_{c}=\frac{2 \sigma}{r_{p}} \cos \Theta
$$

where: $r_{p}$ is the characteristic pore radius of the media and $\Theta$ is the water solid contact angle in the presence of NAPL, indicative of the wettability of the NAPL-water system. (Wettability describes the preferential.coating of a solid surface in a two-fluid system. The concept of wettability is a rather complicated one and, as a result, a significant amount of research on this topic can be found in the literature. However, it should be noted that for the purpose of this research, a completely water-wet scenario was always assumed. Thus, the value of $\Theta$ would always be zero, and the final term in Equation 6 would reduce to a value of 1.0.)

Neglecting the contact angle term in a completely water-wet matrix, Equation 6 indicates that capillary pressures increase with increasing interfacial tensions and decreasing pore radii. Therefore, a NAPL-water pair with higher interfacial tension given the same soil conditions will result in a greater capillary pressure that will need to be overcome in order for the NAPL to enter the media. In addition, for particular NAPL-water pairs, a finer media with smaller pore radii will maintain higher capillary forces that will need to be overcome in order for a non-wetting fluid to enter the water-saturated media. These two implications of higher interfacial tensions and smaller pore radii will also have effects of increasing the difficulty of removing the NAPL once it infiltrates the porous media and becomes entrapped by capillary forces.

A significant amount of research has focused on the relationship between capillary pressure and nonaqueous phase liquid saturation of porous media. Empirical curves have been developed for numerous two-phase pairs in different soil types to illustrate the degree of saturation of NAPL or water by noting pressures within laboratory soil columns and volumes of water either displaced or returned to the column. In addition, numerous models are available which can generate pressuresaturation curves given various soil and fluid properties (Van Genuchten, 1980; Brooks and Corey, 1964). A typical pressure saturation curve is shown in Figure 4.1 below: 


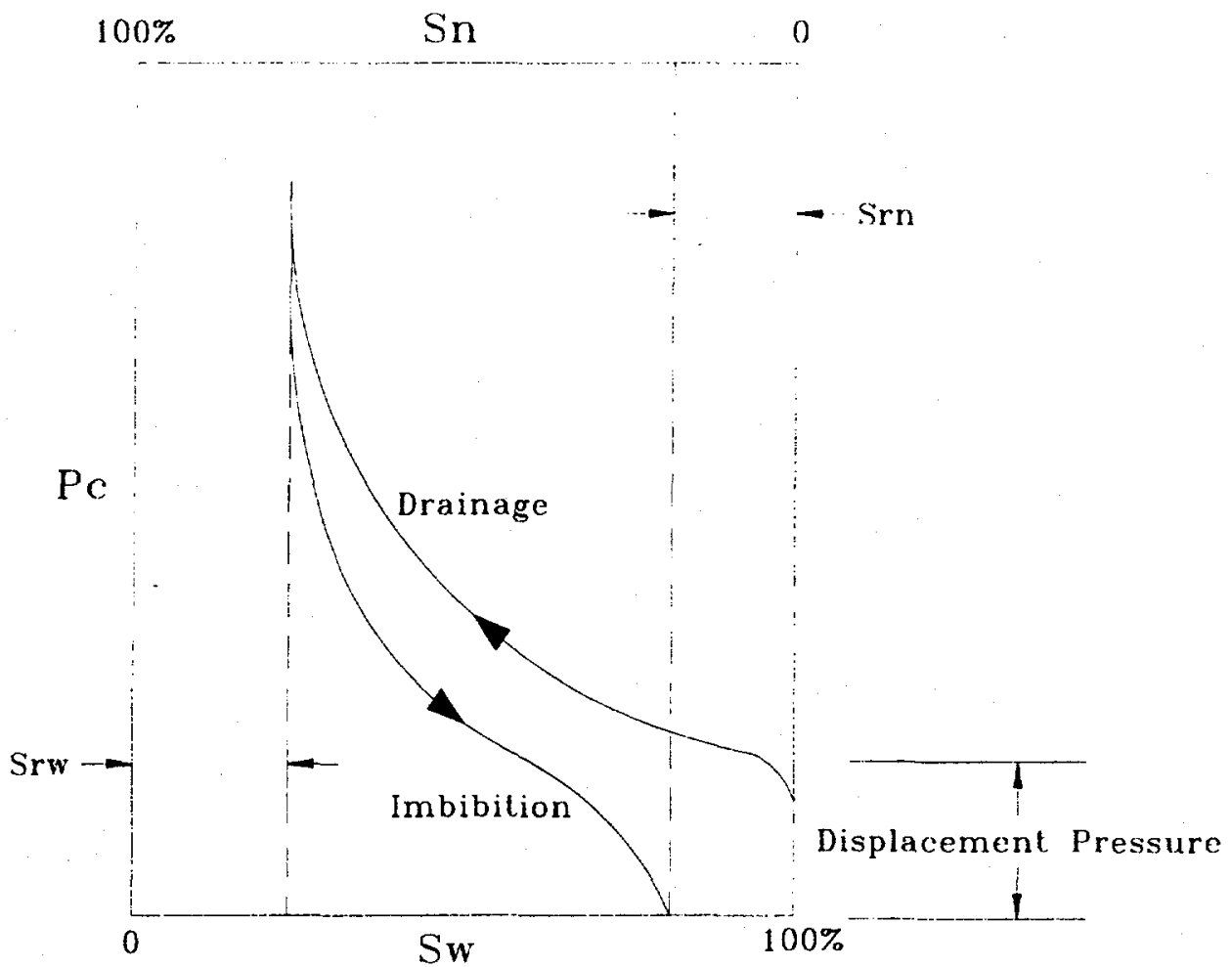

Figure 4.1 Hypothetical Pressure Saturation Curve

Figure 4.1 indicates that the initially water-wet soil matrix begins with a water saturation of $100 \%$. Drainage of the water phase occurs when the displacement capillary pressure mentioned above is overcome. At this point NAPL is allowed to infiltrate into the soil pores and, as a result, water saturation within the soil diminishes. At a certain point, it becomes increasingly difficult for the NAPL to displace additional water. This is because the water has been pushed out of all but the smallest pores and the only remaining water is either in these small pores or along the surfaces of the soil particles. At a certain point called the residual wetting fluid saturation $\left(S_{r w}\right)$, further increases in capillary pressure will have no effect on decreasing the water content of the soil matrix (Fetter, 1993). At this point, if the pressure from the NAPL is removed, the capillary pressure within the soil matrix will cause the wetting fluid to spontaneously imbibe into the matrix, subsequently displacing the NAPL. This is shown in Figure 4.1 as the lower curve. The imbibition of the wetting phase will continue until the capillary pressure within the matrix has been reduced to zero. As a result of hysteresis, the wetting phase saturation of the matrix will not usually return to $100 \%$. This disparity in final water saturation at zero capillary pressure is known as the irreducible, or residual NAPL saturation $\left(\mathrm{S}_{\mathrm{m}}\right)$. The quantity of residual saturation is measured as the volume of NAPL per unit void volume: 


$$
S_{r n}=\frac{V_{n w}}{V_{\text {voids }}}
$$

where: $V_{n w}$ is the volume of non-wetting NAPL remaining in the soil and $V_{\text {voids }}$ is the void volume of the sample.

As described in the above paragraphs and summarized by Mercer and Cohen (1990), the magnitude of the residual DNAPL saturation is dependent upon (1) the interfacial tension between the two immiscible fluids; (2) the media pore size; (3) the pore size distribution; (4) wettability; (5) gravitational forces; and, as will be shown later, (6) hydraulic gradients. For specific DNAPL/water pairs under similar hydraulic scenarios, it is apparent that the soil matrix properties are of principle importance in estimating the degree of residual saturation.

Different grain-sized soils will maintain different magnitudes of capillary pressure and therefore will have different values of residual saturation. In addition, the degree of sorting within the soil matrix will have a great impact upon capillary pressures and consequently, values of residual saturation. Chatzis et al. (1983) indicated that values of residual saturation increase with increasing pore aspect ratios (pore to throat size ratio) and increasing pore size distribution, or heterogeneity. Schwille (1988) indicated residual NAPL saturations measured in field-scale and laboratory experiments to be typically within the range of 10-35\% for saturated unconsolidated media. Mercer and Cohen (1990) report in their research that values for different NAPLs within saturated media generally range from 15 to $50 \%$.

The trail of residual contamination left behind from the migration of DNAPL is in the form of trapped NAPL singlets and ganglia, the size and shape of which depend mostly upon soil pore geometry (Powers et al., 1994). Chatzis et al. (1983) and Wilson and Conrad (1984) found that in soils with high aspect ratio, NAPL tends to "snap-off" within singular pore bodies, thus forming single pore blobs, or singlets. In addition, Powers et al. (1992) also found singlets to be more prevalent in their uniform, well-sorted homogenous soils.

NAPL blobs can also be trapped by a mechanism called by-passing. By-passing occurs when the flow of NAPL terminates and the subsequent flow of water through the porous medium becomes diverted into separate pores. Wilson and Conrad (1984) use the pore doublet model to illustrate the phenomenon of by-passing. The pore doublet model consists of two pores of different size. According to the definition of Darcy velocity $(\mathrm{q}=\mathrm{Q} / \mathrm{A})$, the velocity of the imbibing water is greater within smaller pores. This increased velocity causes the water to more easily displace NAPL in the smaller pores. The increased imbibition of water and displacement of NAPL in the smaller pores may cause the NAPL within the larger pores to become trapped. Powers et al. (1992) suggested that NAPL trapping from by-passing tends to occur more frequently in heterogeneous and more poorly sorted soils. When a number of pores are bypassed by the imbibing water, a more complex geometry of entrapped NAPL occurs. Branched ganglia are NAPL blobs that occupy numerous interconnected 
soil pores which were by passed when the advancing water front mobilized NAPL from surrounding smaller pores. Figure 4.2 is provided below as an illustration of both singlets formed by snap-off and branched ganglia formed by by-passing.

In the layered soil systems that will be studied within this research, two separate homogenous, well-

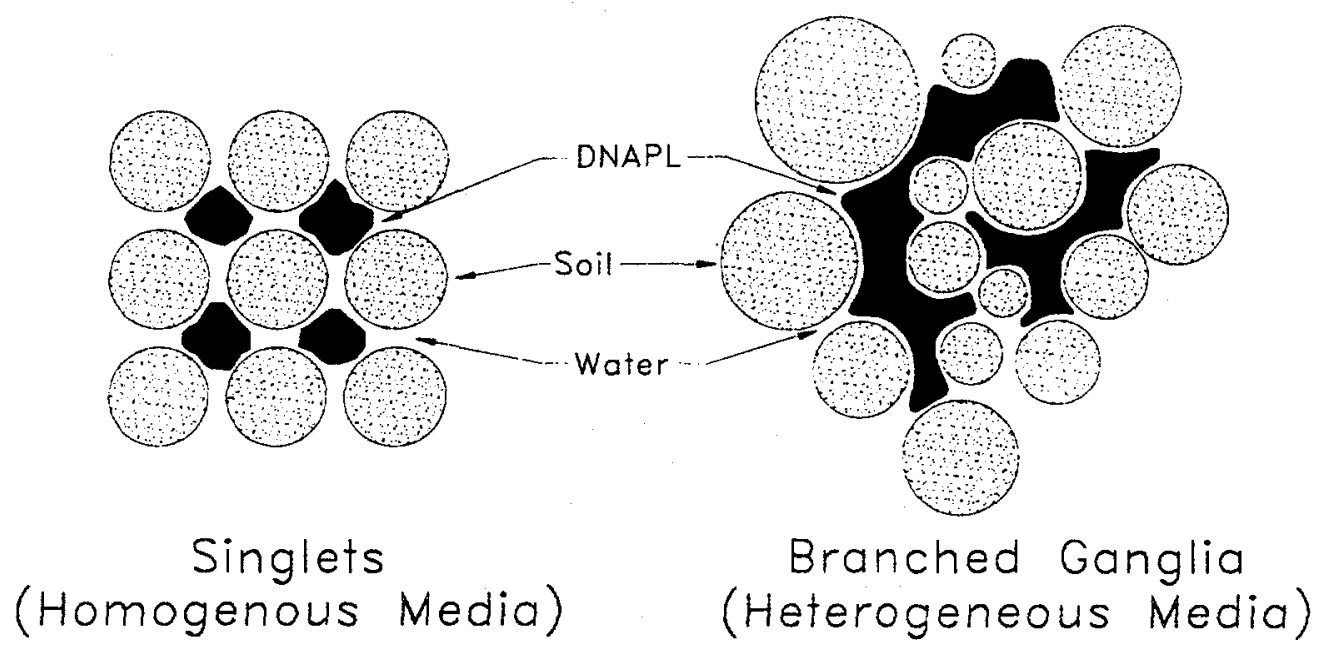

Figure 4.2 Illustration of Different Types of DNAPL Blobs

sorted soil media are used. The assumption of homogeneity within each layer can be tested through the use of column tracer tests such as those prescribed by Pickens and Grisak (1981). As a result of this supposed homogeneity, the principle of snap-off is most likely to be the dominant mechanism in terms of NAPL entrapment. This will have the effect of enabling the enhanced dissolution caused by the presence of the cosolvent solution to be more effective, as the specific surface area of singlets formed by snap-off is greater than that of the more complex ganglia which are typically formed by the mechanism of by-passing.

A significant amount of research has focused on the role of layered soil systems on the transient migration of DNAPL compounds (Schwille, 1988, Kueper et al., 1989, Anderson et al., 1992, Boyd and Farley, 1992, Tchelepi et al., 1993, Illangasakare et al., 1995, Roy et al., 1995). However, there appears to be a gap in the literature pertaining to the scenario of the remediation of DNAPLs from layered soil systems after residual entrapment has occurred.

When DNAPLs that are transported through the saturated zone under the force of gravity encounter lenses of differing permeability, pooling and subsequent longitudinal flow of the DNAPL often occurs. In the saturated zone, this is primarily due to the requirement of the DNAPL to overcome displacement capillary pressures in these lenses of differing permeability. In his studies, Kueper et al. (1989) used four different sands within a thin-walled, two-dimensional physical model to illustrate the implications of the layering of different soils on the transient migration of trichloroethylene (TCE). The results of the experiment showed that gravitational forces of the TCE overcame capillary forces in the coarse-grained media, and the NAPL was able to freely penetrate 
the coarse-grained soils. However, when the flow of NAPL intersected a lens of less permeable soil, pooling of the NAPL occurred. Kueper et al. (1989) reasoned that this phenomena was the result of a necessity of sufficient DNAPL pressure in order to overcome the higher displacement pressure of the finer-grained media.

Illangasakare et al. (1995) performed similar experiments using a thin-walled, two dimensional model with a DNAPL of 1,1,1-trichloroethane (TCA). While achieving similar results to those of Kueper et al. (1989) for the case of DNAPL flow through a coarse lens that encounters a fine lens, Illangasakare also show similar pooling for the scenario where a DNAPL migrating through a fine layer encounters a lens of more coarse-grained material. Again, a displacement, or entrance pressure needed to be exceeded when the DNAPL reached the previously unimpacted region of coarse material. However, Illangasakare (1995) did show that a lesser degree of pooling took place in the fine sand above the coarse layer in comparison with the pooling which took place in a coarse layer above a more fine-grained lens. This was considered indicative of the larger entrance pressure of the fine sand that needed to be overcome prior to movement of the DNAPL into the fine sand.

The impact of the pooling of migrating DNAPL above lenses of differing permeability presents a number of interesting implications for the removal of DNAPLs from multi-layered aquifers. As Brandes and Farley (1993) and Imhoff et al. (1995) indicate, the relatively continuous phase of DNAPL produced as a result of the pooling will more readily displace the residual droplets in the downgradient direction of flow in comparison to that of an aqueous phase alone. Thus, the DNAPL in the upper lens may by more readily displaced, leaving contamination behind in the lower lens. However, on the other hand, as a result of the pooling, the aqueous phase saturation may remain higher in the lower lens that has not yet been greatly impacted by the DNAPL. In this case, the greater aqueous phase relative permeability of the lower lens will allow a greater transmittance of a remedial solution. Thus a scenario of preferential flow through the lower lens may develop, leading to an incomplete remedial effort of the DNAPL within the upper lens.

An additional source of increased complexity caused by soil layering is the phenomenon of fingering. Fingering of a fluid often occurs in a porous medium as a result of density and/or viscosity differences between a migrating (displacing) fluid and a residual fluid. Heterogenous layers or lenses of differing permeability will exacerbate instabilities in the solvent front formed during the migration of DNAPL. The resulting fingers of DNAPL may have a significant impact upon the efficiency of an alcohol flushing procedure. The initial perturbation of the migrating front is often caused by an isolated non-uniformity or small-scale heterogeneity in the matrix structure (Boyd and Farley, 1992, Anderson et al., 1992). However, the presence of large scale heterogeneities may increase the potential for finger development. A commonly used measure of the potential for fingering is the mobility ratio. For an immiscible fluid pair, the mobility ratio is given as:

$$
M=\frac{k_{r d} / \mu_{d}}{k_{r} / \mu_{r}}
$$


where: $\mathrm{k}_{\mathrm{rd}}$ is the relative permeability of the displacing fluid; $\mu_{\mathrm{d}}$ is the viscosity of the displacing fluid; $k_{n}$ is the relative permeability of the resident fluid and $\mu_{r}$ is the viscosity of the resident fluid. Rearranging Equation 8 yields:

$$
M=\frac{k_{r d} \mu_{r}}{k_{r r} \mu_{d}}
$$

According to Tchelepi et al. (1993), stable front movement occurs when the value of $\mathrm{M}$ is less than 1.0 , while unstable flow which results in fingering will occur at values of $M$ greater than 1.0. DNAPL pooling above a lens of differing permeability will cause an increase in the relative permeability of the migrating DNAPL. In this scenario, the DNAPL is the displacing fluid. Thus, according to the equations above, an increase in the displacing phase relative permeability will affect an increase in the mobility ratio, thus increasing the likelihood of the occurrence of fingering.

The impact of finger development and the subsequent presence of fingers on the ultimate removal of DNAPL is considered to be significant. In a remediation effort, the flushed aqueous phase is the displacing fluid, while the DNAPL is the resident fluid. The migratory flow of DNAPL into fingers will cause a local pore-scale DNAPL saturation scenario. In this case, the aqueous phase will most likely by-pass these locally NAPL-saturated fingers and flow through pores principally occupied by water. Thus, the aqueous solution may never come into contact with a large amount of the DNAPL. In addition, where the water is able to contact the residual DNAPL, the relative permeability of the aqueous displacing fluid will slowly increase while that of the residual DNAPL will decrease due to dissolution effects. This results in an increasing value of $\mathrm{M}$, which causes greater instability of the water front and will result in further by-passing of the DNAPL. It can be presumed that the overall result of the presence of fingering will be a significant difficulty in obtaining sufficiently low concentrations of DNAPL. Roy et al. (1995) indicate that the presence of fingering generally limits the economic recovery of oil to 10 to $15 \%$ of the total volume present.

The presence of layers that cause pooling and fingering create a scenario where a totally homogenous, singlet-dominated residual may never actually be attained in both media of the layered system. However, the goal of the research is to evaluate the effects of soil layering on the removal of a residual DNAPL by alcohol flushing. Therefore, in order to effectively study this effect, the number of variables was reduced by not allowing any pooling or fingering issues to be present within the soil columns and allowing a homogenous residual saturation to develop on either side of the soil column prior to flushing activities.

\subsection{Methods}

Various soils were used in the laboratory experiments. One was an Ottawa white sand ranging in particle size from 0.0025 to 0.0042 millimeter diameter. One was a fine sand. The other was clay from the Savannah River Site. The clay from the site was received as tight dry clumps of soil. The clumps were ground by hand with a mortar and pestle, and the material was sieved. Clay material 
passing the 0.149 millimeter $(0.0059$ inch) screen was mixed with the Ottawa white sand. Mixtures contained $0,2,5,10$, and $20 \%$ clay by weight. Hydrometer analysis and $\mathrm{x}$-ray diffraction tests were performed to further characterize the clay from the Savannah River Site. In addition microscopic analysis using a stereomicroscope was used to visualize the structures formed by the sand/clay mixtures.

Four centimeter glass columns with stainless steel screens and endcaps were used in this study. Plexiglass sleeves provided support for the column endcaps, however the plexiglass did not contact any material inside the column. The glass column, screens, and endcaps were sealed with Viton orings.

Three columns were packed for each of the percent clay mixtures used. Columns were packed to a bulk density of $1.80 \mathrm{~g} / \mathrm{cm}^{3}$. The sand and clay mixture was packed into the column using a funnel and attached tube. The funnel and tube set-up reduced the likelihood of separation of grain sizes due to differences in settling velocities. Separation of grain size is often visible in heterogenous media if special packing methods are not employed. The packing material was carefully poured into the funnel, then the tube was placed in the bottom of the column. The tube was completely filled during packing of the column, to avoid material falling through the tube and separating by grain size. The tube was slowly lifted while packing the column, allowing the media to fill the column. Excess media mounded appreciably at the top of the column to achieve a bulk density of $1.80 \mathrm{~g} / \mathrm{cm}^{3}$. Therefore, the column was vibrated gently allowing the excess soil to evenly settle into the column.

After packing and sealing the column, carbon dioxide was introduced to the bottom of the column. Carbon dioxide replaces air present in the pore spaces. Carbon dioxide dissolves in water much more readily than air, therefore decreasing the amount of air entrapment during saturation of the column. Carbon dioxide was flushed through the columns at a pressure of 5 psi for approximately 30 minutes. After this, de-aired, distilled water was injected into the column from the bottom at a flowrate at or above $10 \mathrm{ml} / \mathrm{min}$. As soon as the column was saturated, the column was capped and stored overnight, allowing carbon dioxide to dissolve into the water and water to completely wet the soil grains. Ten to twelve hours later the column was flushed with de-aired, distilled water. This method appeared to provide an adequate saturation, with virtually no visible air pockets.

The pertinent information gathered on each column at this stage was bulk density and porosity. The bulk density, $\rho_{b}$, of the final packing was determined by weighing the column before packing, $\mathrm{W}_{\text {column }}$ and weighing the column after packing with media but before saturating with water, $\mathrm{W}_{\text {coltsand }}$. The volume of the column, $\mathrm{V}_{\text {column }}$, was determined by measuring the diameter with calipers, and measuring the depth of the column with a ruler. Bulk density is given by Equation, 10. The porosity, $\eta$, can then be calculated using the density, $\rho$, of the soil. Specifications for the Ottawa white sand indicate a density of $2.65 \mathrm{~g} / \mathrm{cm}^{3}$. This density is also assumed for all other porous aterials used in this study. The equation used to determine porosity is given in Equation 11 .

$$
\rho_{b}=\frac{W_{\text {col }+ \text { sand }}-W_{\text {column }}}{V_{\text {column }}}
$$


After packing and saturating the column, hydraulic conductivity tests were completed to determine the permeability of the media. Two methods, constant head tests and manometers, were used to determine the hydraulic conductivities of most columns. Hydraulic conductivity tests were performed on all columns both before and after emplacement of a PCE residual providing values

$$
\eta=1-\frac{\rho_{b}}{\rho}
$$

used in calculating the trapping numbers of the samples. However, only the results of the manometer tests were used in these calculations. The measurements required in the constant head test were difficult to obtain due to the set-up of the experiment and are, therefore, considered to have more error associated with them than the measurements taken for the manometer test. The constant head tests were performed in an effort to verify the results from the manometer tests.

Hydraulic conductivities determined by the manometer tests are based on Darcy's law of flow through porous media. Darcy's law states that the flow through a porous media is controlled by the conductivity of that media and the gradient of flow through the media. In this test of hydraulic conductivity, water flowed through the column at a predescribed flowrate, $Q$, and the gradient across the media was measured by manometers. The manometers were located at the bottom and top of the column and measured the difference in head, dh, across the length of media, $\mathrm{dL}$. Equation 12 provides the calculation used to determine the hydraulic conductivity based on the information gathered by the manometer test.

$$
K=\frac{Q}{A} \frac{d L}{d h}
$$

The permeability of the media was calculated from the hydraulic conductivity determined by the manometer test. Using the density, $\rho$, and kinematic viscosity, $v$, of water, hydraulic conductivity can be converted to permeability. This is shown in Equation 13. Permeabilities before and after NAPL emplacement were calculated in this manner.

$$
P=\frac{K}{v \rho}
$$

Column Set-up for enhanced dissolution studies

A soil column design that allowed for flexibility and interchangeableness as well as the prevention of leakage at elevated pressures was required. In addition, the use of glass columns was desired in order to be able to visually monitor the column at various stages of the experiment. The soil 
columns used in the homogenous column experiments were glass tubes $10 \mathrm{~cm}$ long and $5.4 \mathrm{~cm}$ in diameter. Figure 4.3 provided below is an illustration of the homogenous soil columns used for alcohol flushing experiments.

The glass columns were sealed at one end by a stainless steel end-cap and at the other end by either

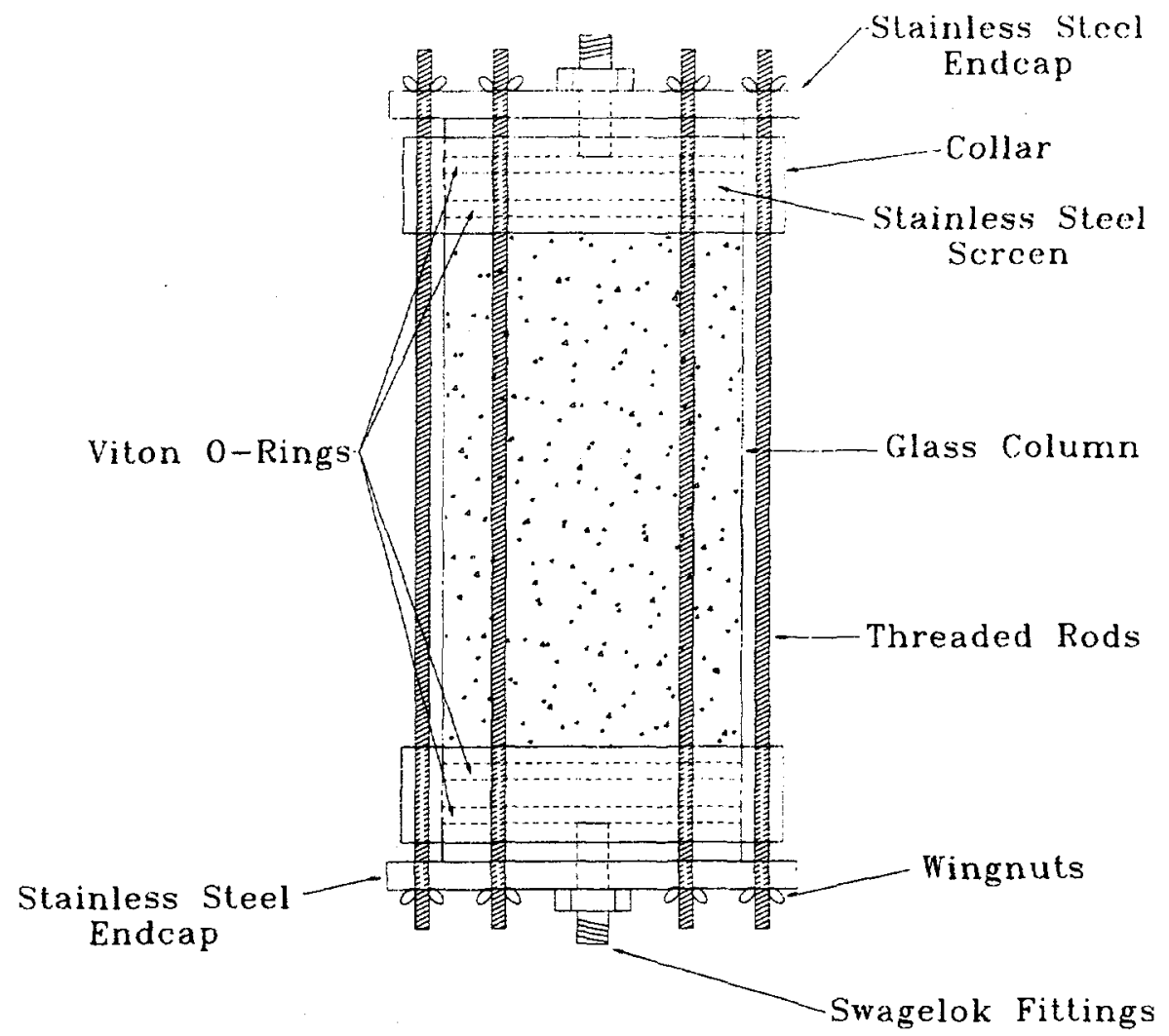

Figure 4.3 Homogenous Column Set-up

another stainless steel end cap or a specially constructed double-sided aluminum endcap. The two endcaps were connected to each other via threaded rods. Viton $\mathrm{O}$-rings were used at all connections between the column, stainless steel screens and end-caps. The end caps were tapped to permit attachment of Swage-lok ${ }^{\mathrm{TM}}$ pressure fittings that connected the soil columns to syringe pumps and cosolvent solution reservoirs.

Several different column designs were used for the heterogeneous column experiments, constructed double-outlet aluminum endcap. Initially a specially constructed double outlet aluminum endcap was used. This design was later replaced with a dual side port aluminum column. However, a majority of the experiments were done with the dual outlet system and this will be discussed .

The special endcap had two tapped outlet ports to which Swagelok ${ }^{\mathrm{TM}}$ pressure fittings were attached. The cap also had a small ridge which equally divided the cross sectional area and essentially allowed for the flow through the two different soil media to be separated. A schematic of the specially 
constructed endcap is provided as Figure 4.4.

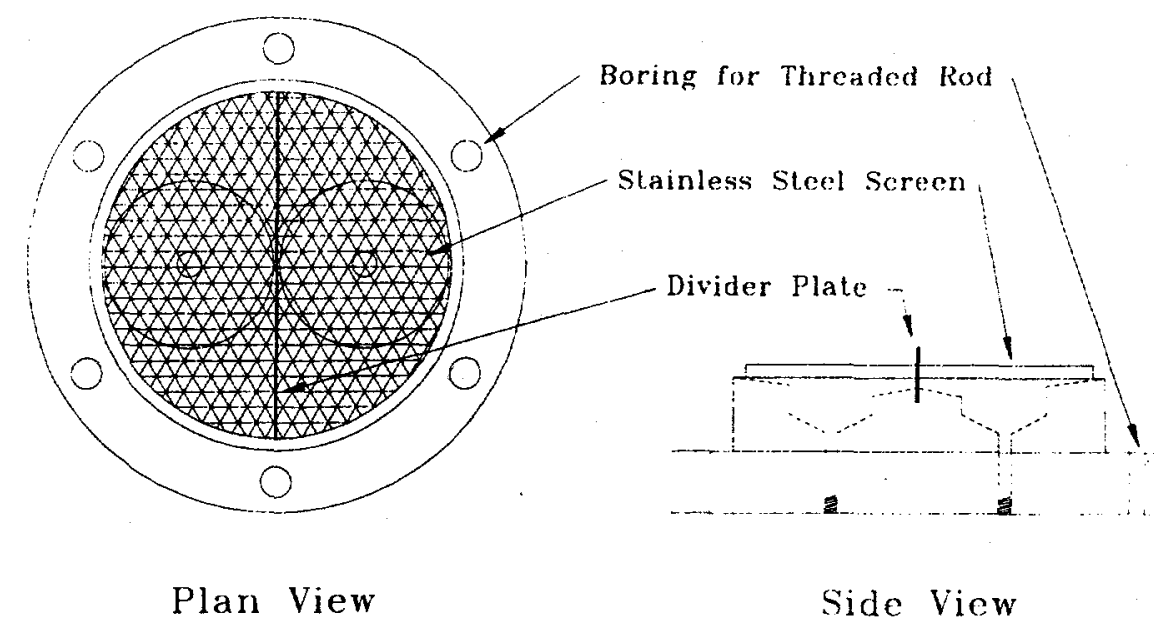

Figure 4.4 Schematic of Specially Constructed Double-Sided Aluminum Endcap

Figure 4.5 is an early version of the heterogeneous soil columns used for alcohol flushing experiments with a dual endcap. The later version used a single port endcap and two side ports and is shown in Figure 4.6. Sampling was performed at the side ports.

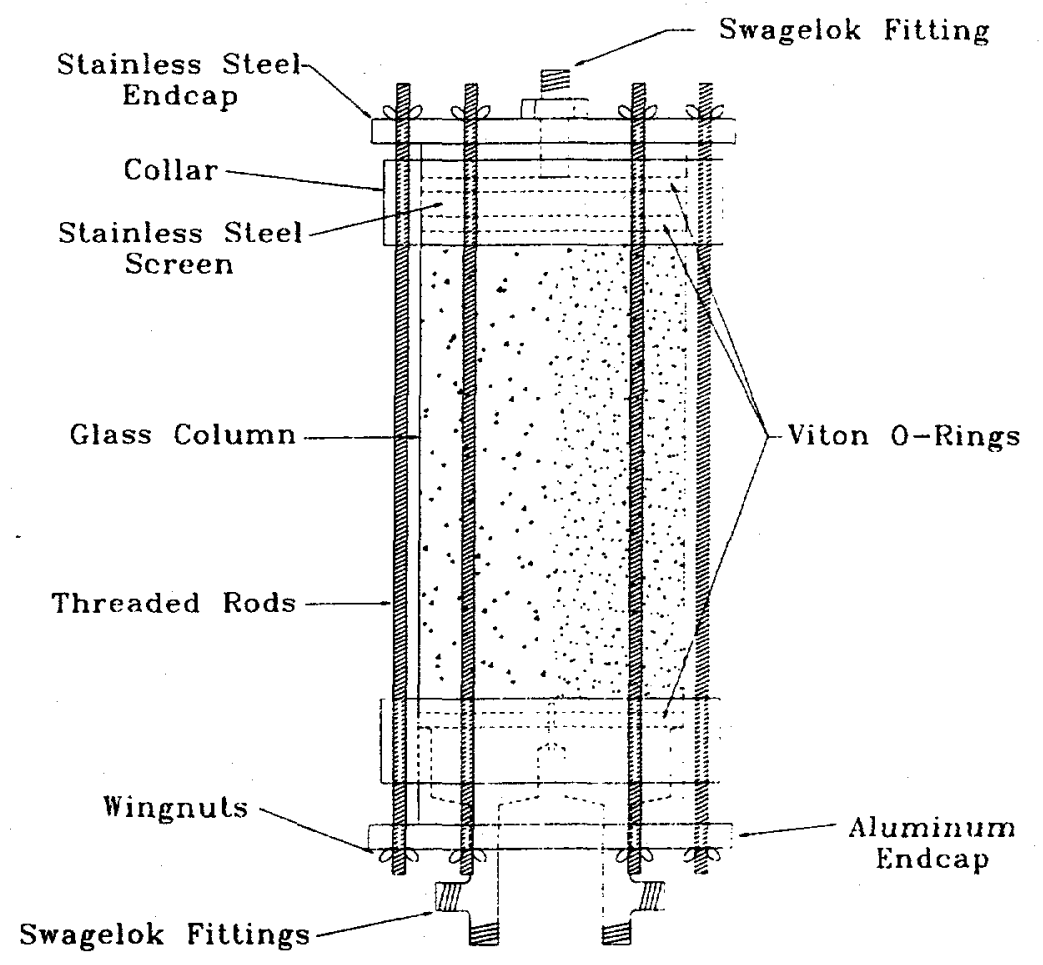

Figure 4.5 Heterogeneous Column Set-up 


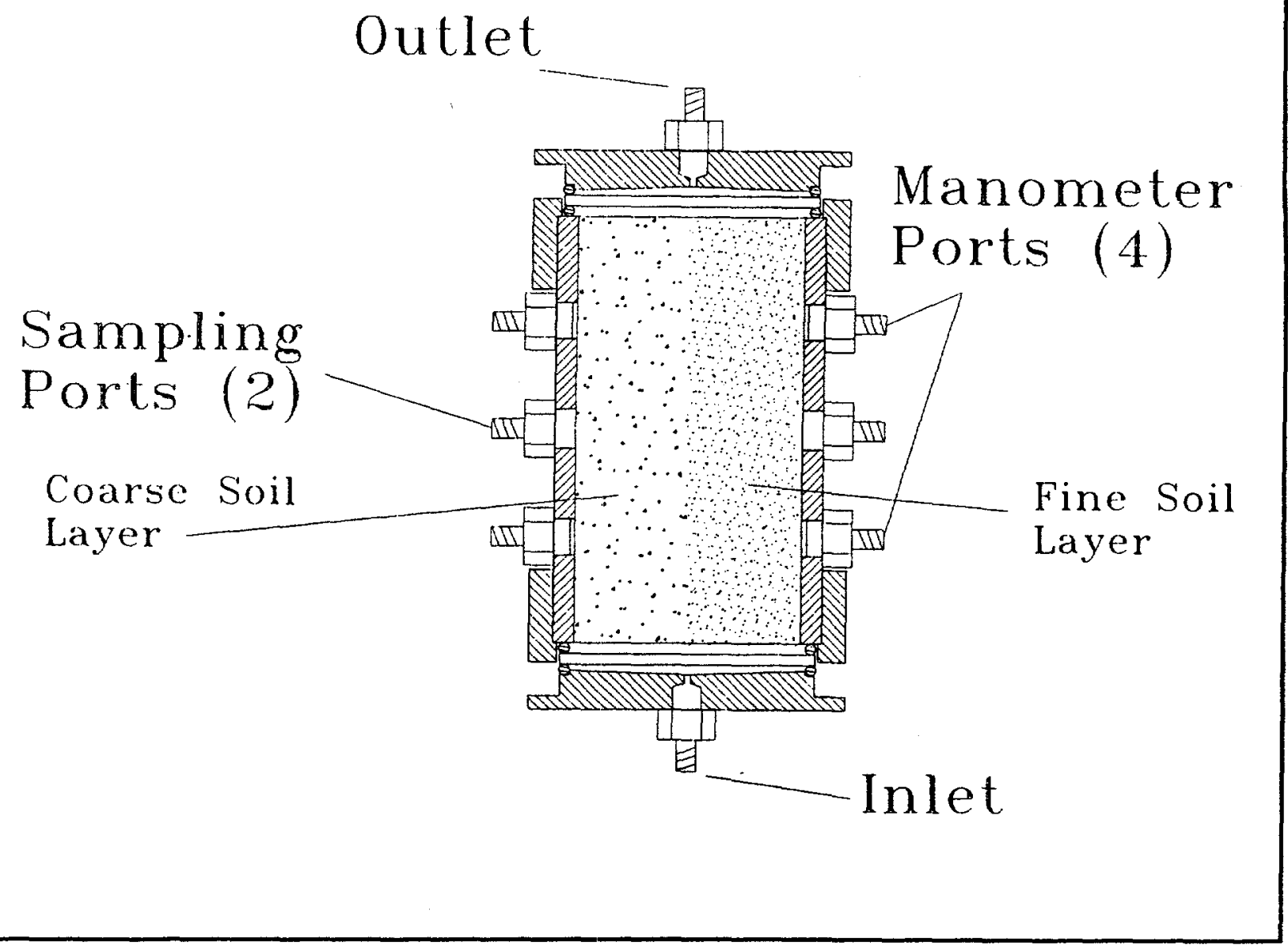

Figure 4.6 Revised column for layered soils showing modified side ports.

Homogenous Columns For Solubilization

The two media used for experimentation were a uniform medium white sand (Accusand 40/60, Unimin Corporation, Le Sueur, MN) and a uniform fine brown sand. The medium sand had an approximate grain size of $350 \mu \mathrm{m}$, with $98.0 \%$ by weight finer than $420 \mu \mathrm{m}$ and $0.3 \%$ finer than 250 $\mu \mathrm{m}$. The uniformity index of the medium sand was determined to be 1.25 . The fine sand was found to have an approximate grain size of $100 \mu \mathrm{m}$. The sieve analysis performed on the fine sand indicated $99.2 \%$ was finer than $150 \mu \mathrm{m}$ and $8.5 \%$ was finer than $53 \mu \mathrm{m}$. The uniformity index of the fine sand was 1.75 . These sizes were selected to represent materials firmly within the classification of sand according to ASTM (ASTM, 1980). According to the ASTM standard, both soils fall within the fine sand classification, with the medium sand at the upper end and the brown sand at the lower end of the classification. It was considered important that each was well sorted and 
homogenous with relatively no fines.

Homogenous columns were packed with sand under pressure using flexible rubber tubing attached to a catchment funnel to avoid the formation of horizontal striations that may have developed from grains of differing density being allowed to fall into the column under gravitational force. The sand was placed in the funnel and allowed to fill the entire length of the tubing. Sand was then filled into the column from the bottom to the top by continually lifting the funnel/tubing apparatus out of the column and allowing the sand to migrate as a continuum. After the majority of each sand had flowed into the column, the column was either tapped with a mallet or shaken using a high frequency vibrational shaker (Vortex Genie, Scientific Industries, Inc.) until the desired amount of sand was in place in the column. The use of these packing techniques resulted in consistent bulk density and porosity values for the homogenous columns. Following the packing of the sand, additional sand was placed in the column until a slight crown just above the level of the glass column was apparent. After a sufficient quantity of the media had been packed into the column, the column was capped with a fine stainless steel screen and a standard stainless steel endcap. The total mass of each medium actually placed into the column was then measured gravimetrically and recorded.

Soil parameters such as porosity, bulk density and pore volume were determined for each soil column. The bulk density of the soil within the column was determined by Equation 18 :

$$
\rho_{b}=\frac{M_{s}}{V_{T}}
$$

where: $M_{s}$ is the mass of soil in the column and $V_{T}$ is the total volume of the column. The pore volume, or void volume, was then determined by the following formula:

$$
V_{v}=V_{T}-V_{s}
$$

where: $V_{s}$ is the volume of sand occupying the column. $V_{s}$ was calculated by dividing the mass of soil $\left(\mathrm{M}_{\mathrm{s}}\right)$ within the column by the density of sand, $2.65 \mathrm{~g} / \mathrm{cm}^{3}$. Finally, the porosity $(\eta)$ could be calculated by using Equation 20:

$$
\eta=\frac{V_{v}}{V_{T}}
$$

\section{Heterogeneous Columns For Solubilization}

In the layered column experiments, the two sand media were packed so that each media occupied $50 \%$ of the available column volume. The packing technique for the layered columns with the specially constructed endcaps described above and the side port columns was similar to that described for the homogenous columns, with the following exceptions. Two sets of funnel/tubing apparati were used so that the column could be filled with both media simultaneously. A thin 
aluminum plate was placed into the column to divide the column into two equal lengthwise volumes. The plate was sized to form a seal along the column walls and along the ridge of the double-sided endcap at the bottom of the column. A calculation was performed prior to placing the sand into each funnel in order to determine the mass of sand required to attain similar bulk densities of each media with those in the homogenous columns. The funnel/tubing apparati were then slowly raised so that both sand media flowed as a continuum into each half of the column. After the majority of each sand had flowed into the column, the thin aluminum plate was slowly removed and the sands were packed by tapping the column continuously until a slight crown was formed above the glass rim of the column. (Packing with the vibrational shaker was attempted on one heterogeneous column, however, the high frequency vibration caused the sands to rotate about the column and slightly distort the line of division between the two sands. Thus the vibrational packing method was deemed unsatisfactory for the remainder of the heterogeneous columns.) The columns were then sealed with fine stainless steel screens and standard stainless steel endcaps. The total masses of each media actually placed into each side of the column were measured. Similar to the homogenous columns, bulk soil parameters were then calculated using the actual masses of each media in the layered column. All parameters were calculated under the assumption that each media occupied exactly $50 \%$ of the column volume. Discrepancies from this assumption were noted in the fact that calculated porosity and bulk density values for the layered columns were observed to deviate slightly from those of the homogenous columns.

\section{Dispersion Analysis}

A series of dispersion tests were run on both homogenous and heterogeneous columns in order to compare dispersivity values between the media within the homogenous columns and the relatively homogenous material on each side of the layered columns. The tests were run on one homogenous column of each material and one heterogeneous column with the thought that a comparison of the results for dispersivity between the two homogeneous and one heterogeneous column would provide a good indication of the degree of homogeneity actually within each media.

The method outlined by Pickens and Grisak (1981) for laboratory column testing of dispersivity was followed. In this method, a continuous injection of tracer was performed and the effluent concentrations are monitored until completion of the breakthrough curve was obtained. The method used a rearrangement of the one-dimensional advective dispersion equation written as:

$$
\frac{C}{C_{o}}=0.5\left[\operatorname{erfc}\left(\frac{1-U}{2\left(U D_{L} / v_{x} L\right)^{1 / 2}}\right)\right]
$$

where: $U$ is the number of pore volumes; $D_{L}$ is the coefficient of hydrodynamic dispersion; $v_{x}$ is the linear velocity; $L$ is the length of the column; $C$ is the concentration of tracer; and $C_{0}$ is the initial concentration of tracer. All parameters were known except the concentration (C), which was measured, and the coefficient of hydrodynamic dispersion $\left(D_{\mathcal{L}}\right)$, which was the variable to be determined. The concentration of tracer in the effluent, $C$, was measured at numerous pore volumes and the value of $C / C_{0}$ was plotted as a function of $\left[(U-1) / U^{1 / 2}\right]$ on a linear probability axis. If the data 
plot as a straight line, they may be assumed to be normally distributed and, thus, the form of the advective-dispersion equation above is valid. The value of $D_{L}$ is related to the slope of the straight line and can be found using Equation 18 below:

$$
D_{L}=\left(\frac{v_{x} L}{8}\right)\left(J_{0.84}-J_{0.16}\right)^{2}
$$

where: $\mathrm{J}_{0.84}$ is the value of $\left[(\mathrm{U}-1) / \mathrm{U}^{1 / 2}\right]$ when $\mathrm{C} / \mathrm{C}_{\mathrm{o}}$ is 0.84 and $\mathrm{J}_{0.16}$ is the value of $\left[(\mathrm{U}-1) / \mathrm{U}^{1 / 2}\right]$ when $\mathrm{C} / \mathrm{C}_{0}$ is 0.16 . From the definition of the coefficient of hydrodynamic dispersion, the value of $\mathrm{D}_{\mathrm{L}}$ was then used to determine the value of dispersivity:

$$
D_{L}=\alpha_{L} \nu_{x}+D^{*}
$$

or,

$$
\alpha_{L}=\frac{D_{L}-D^{*}}{v_{x}}
$$

where: $D^{*}$ is the effective diffusion coefficient and $\alpha_{\perp}$ is the value of dispersivity. The effective diffusion coefficient is the product of the molecular diffusion coefficient of the tracer and a tortuosity factor $(\omega)$. In these experiments, bromide was used as the tracer. The molecular diffusion coefficient was found to be $1.9 \mathrm{E}^{-5} \mathrm{~cm}^{2} / \mathrm{sec}$ (CRC Handbook, 60th Edition, 1980) while the tortuosity factor was assumed to be 0.7 , a value found by Perkins and Johnson (1963) to be applicable for soil column studies where uniform sands are used.

Bromide was chosen as the tracer because of its availability, its nonreactive nature and its ease of detection using an ion-selective probe device. Saturated $10 \mathrm{~cm}$ columns were injected with a continuous source of bromide at a concentration of $1000 \mathrm{mg} / \mathrm{L}$ in an upflow direction at $10 \mathrm{ml} / \mathrm{hr}$ using a calibrated syringe pump. This concentration was selected in order to maximize the accuracy of the ion selective probe while minimizing the effects of density and viscosity gradients. The upflow flush also assisted in minimizing the development of unstable flow as a result of density and viscosity gradients. The homogenous columns were equipped with double-sided endcaps on the top of the column in order to remain consistent with the tracer experiment conducted on the heterogeneous column. Effluent samples were collected from each port using a 2-ml dedicated syringe which was attached to a three way tee. The effluent was forced into the syringe by switching the direction of the tee during sample collection. Approximately $1.5 \mathrm{ml}$ of effluent was collected every 15 minutes in each syringe from both sides of the column. Exactly $1.0 \mathrm{ml}$ of sample was then diluted with distilled water to $25 \mathrm{ml}$ using a volumetric flask. The diluted sample was then analyzed for bromide concentration using an ion selective probe (Beckman Instruments $\$ 12 \mathrm{pH} / \mathrm{ISE}$ Meter, Fullerton, CA). All column tracer tests and effluent analyses were performed at $21 \pm 1^{\circ} \mathrm{C}$. 


\section{Permeability Measurements}

Following packing activities (and actually before dispersion analyses), the columns were saturated in order to simulate water-wet saturated porous media. The columns were first flushed in an upflow direction with carbon dioxide $\left(\mathrm{CO}_{2}\right)$. The $\mathrm{CO}_{2}$ flowed into the column at a pressure of approximately 6 pounds per square inch (psi) for a duration of 30 minutes. Following $\mathrm{CO}_{2}$ flushing, the column was saturated with de-aired water. The de-aired water was flushed upward into the column at a flowrate of $10 \mathrm{ml} / \mathrm{min}$ using a calibrated syringe pump (Harvard Apparatus, Model 22). Flushing continued until complete saturation was obtained, noted by the breakthrough of water from the upper endcap. The column was then left to equilibrate overnight, allowing any trapped $\mathrm{CO}_{2}$ left behind after the flush to dissolve into the water.

Once all of the air had been vacated and the column had been adequately saturated with water, initial permeability tests were performed. Initial permeability measurements were conducted using three different methods on the first two columns. Constant head and falling head permeability tests were run for comparison with the results obtained with a newly designed permeability measurement system. The newly designed method utilized differential pressure transducers to continuously monitor the differential pressures across the columns. After about 1 year of use, we went back to the older version because it proved reliable and easier to use.

Two wet-wet 0-5 inch differential pressure transducers (Model PX-154-005DI, Omega Engineering, Stamford, CT) were used to experimentally determine differential pressures across the soil columns. For four of the six homogenous columns, water was injected into the top of the column in a downflow direction using the calibrated syringe pump, and the differential pressure was monitored with a single transducer. (Aqueous solutions were always passed through the columns in a downflow direction). This method was employed as these four columns used single-port endcaps on both sides.

During conductivity testing for the two homogenous columns that had double sided endcaps on the bottom of each column, water was pulled downward through both ports of the double sided endcap simultaneously using a syringe pump operating in withdrawal mode. For these columns, both transducers were employed to monitor the differential pressures across both sides of the homogenous column. The main advantage with using transducers was the ability to continuously monitor the differential pressure in the columns throughout the alcohol flushing experiments. While this feature was not entirely necessary for initial or final conductivity measurements, the use of transducers was advantageous during the flushing experiments, as it was postulated that the differential pressures across the soil columns would diminish with flushing time as a result of the continual removal of residual phase DNAPL. Therefore, it was possible to show that hydraulic conductivities and therefore permeabilities of the porous media increased throughout the alcohol flush. Other advantages of the use of differential pressure transducers include the lack of observational error and increased accuracy of the instruments over manometers and constant or falling head methods or manometers. For these reasons, it was concluded that a more accurate differential pressure measurement system using transducers should be developed. 
Initial System Design and Configuration for Solubilization Experiments

A system using two 0-5 inch wet-wet differential pressure transducers were excited with 24 volt direct current (VDC) power transformers. Fluctuations in the excitation source were minimized through the use of a power protection strip, although minor variations in the magnitude of input voltage were considered to be insignificant due to the fact that the units are capable of being powered by voltages of between 18 and $30 \mathrm{VDC}$. The transducers output a current of between 4 and 20 milliamperes $(\mathrm{mA})$ depending upon the magnitude of difference in pressure being monitored on each side of the transducer membrane. A 12-bit analog to digital converter (A/D) was used to convert the output signal of the transducers into an appropriate range of bits so that the data could be input into a computer file. Since the A/D board converts voltage to bits, the current output of each transducer was converted to voltage using a $250 \mathrm{ohm}$ resistor. Thus, in accordance with Ohm's Law, the output range of the transducers being sent to the $A / D$ was between one and five volts. A computer program was used to calculate the value of differential pressure in inches of water at specified intervals based on the number of bits being sent from the $\mathrm{A} / \mathrm{D}$. The data was subsequently stored in a file which could be imported into a spreadsheet for graphing and data analysis purposes. Table 4.2 summarizes the data conversion process from actual pressure difference across the transducer membrane to the data being stored in the appropriate file within the computer.

\begin{tabular}{|c|c|c|c|c|}
\hline \multicolumn{2}{|c|}{ Table 4.2 Summary of Data Conversion from Transducer to Output File } \\
\hline $\begin{array}{c}\text { Actual Pressure } \\
\text { Across Membrane } \\
\text { (in H2O) }\end{array}$ & $\begin{array}{c}\text { Transducer } \\
\text { Current Output } \\
\text { (mA) }\end{array}$ & $\begin{array}{c}\text { Input to A/D } \\
\text { Converter } \\
\text { (volts) }\end{array}$ & $\begin{array}{c}\text { Output from 12- } \\
\text { bit AD Converter } \\
\text { (bits) }\end{array}$ & $\begin{array}{c}\text { Value of Diff. } \\
\text { Pressure in File } \\
\text { (in H2O) }\end{array}$ \\
\hline 0.0 & 4.0 & 1.0 & 819.0 & 0.0 \\
\hline 1.0 & 7.2 & 1.8 & 1474.4 & 1.0 \\
\hline 2.0 & 10.4 & 2.6 & 2129.8 & 2.0 \\
\hline 3.0 & 13.6 & 3.4 & 2785.2 & 3.0 \\
\hline 4.0 & 16.8 & 4.2 & 3440.6 & 4.0 \\
\hline 5.0 & 20.0 & 5.0 & 4096.0 & 5.0 \\
\hline
\end{tabular}

The transducers were connected at each end of the column as shown in Figure 4.7. 


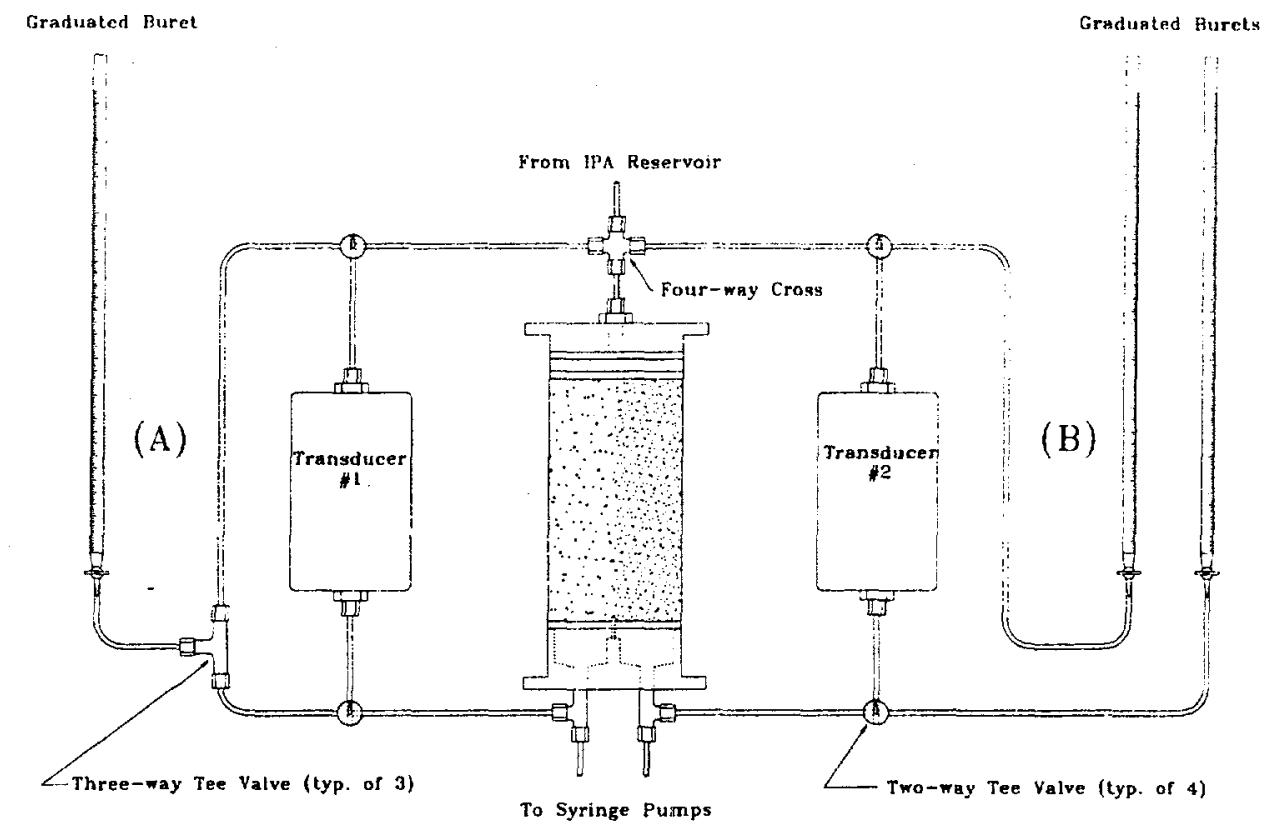

Figure 4.7 Transducer system set up.

Figure 4.6 Transducer System Set-up

(A) System for Checking Zero

(B) System for Checking Gain

Figure 4.7 illustrates the differential monitoring system being used on a heterogeneous column. For the homogenous column experiments, only one transducer was employed, essentially employed as shown on either side of Figure 4.7. The high pressure port of each transducer was connected to a 4-way cross or three-way tee fitting on the upper endcap of the columns. The low pressure ports were connected to the lower endcaps by way of three-way tees. This connection scheme was done because water flushing was performed in a downflow direction through the column. All connections were made with Swagelok ${ }^{\mathrm{TM}}$ pressure fittings.

A single buret was connected to the high and low pressure ends of each transducer by way of a tee for zeroing purposes. Transducers are known to drift slightly over time and occasionally need to be recalibrated, or re-zeroed. As is shown in Figure 4.7(A), the buret was connected to each end of the transducers by two-direction tee valves. During calibration, water runs continuously from the buret to each side of the transducer membrane. If necessary, the zeroing potentiometer in the transducer was adjusted until a reading of 1.0 volts or 4 milliamperes is read on a volt/current meter which is attached to the transducer output terminal. The transducers were checked at least once daily during flushing activities in order to ensure that the units stayed properly zeroed.

At the initiation of flushing activity for each test, the gain of the transducers was checked. This was done in order to ensure that the zero to five inch range of the units remained consistent. In order to 
accomplish this task, a second buret was used on each transducer. The first buret remained connected to the high pressure port, while the second buret was connected to the low pressure port (Figure 4.7(B)). Water was placed in the second buret to a level exactly five inches below that of the buret connected to the high pressure port. If necessary, the gain potentiometer in the transducer was then slightly adjusted until a reading of 5 volts or 20 milliamperes is recorded on the voltage/current meter. The second buret was then unattached from the system and the low pressure port was reconnected to the tee and, thus, the original single buret. The voltage or current of the zeroed system was then rechecked to ensure that the unit was still properly zeroed. Following zeroing and calibration activities, the positions of the upper and lower two-way tee valves are changed so that the transducer is connected to the soil column (see Figure 4.7). This system was finally abandoned in the final year because of complexity and noise in the data.

The data collected by the transducers at prescribed intervals over the course of the alcohol flushing period was written to a computer print file. The file was then imported into a spreadsheet program for subsequent plotting. The data were plotted on each graph and illustrated differential pressure on the ordinate and the number of pore volumes of cosolvent flushed through the column on the abscissa. With the known flowrate imposed by the syringe pump, a simple manipulation of Darcy's Law allowed an easy calculation of the hydraulic conductivity of the soil:

$$
K=\frac{Q d l}{A d h}
$$

where: $\mathrm{Q}$ is the known flowrate; $\mathrm{dl}$ is the length of the soil column; $\mathrm{A}$ is cross sectional area of the soil; and dh is the experimentally measured differential pressure. At least three different flow rates were imposed on the column and an average value of initial hydraulic conductivity was determined. The permeability of the soil matrix was then determined using Equation (below):

$$
k=\frac{K \mu}{\rho g}
$$

where $\mu$ and $\rho$ are the aqueous phase viscosity and density, respectively, and $g$ is the gravitational constant.

It should be noted that during alcohol flushing experiments, the values of viscosity and density had to be altered in order to represent values for the appropriate alcohol flushing solution. A dial viscometer (Brookfield Engineering Laboratories, Inc., Stoughton, MA) was used to perform experiments to determine values of viscosity for various percent IPA solutions and effluent solutions containing PCE. The results of studies performed by Hayden and Noyes (1995) were used to provide indication of density changes due to the alcohol/PCE/water solution. All experiments were performed at $22 \pm 2^{\circ} \mathrm{C}$.

It should also be noted that as the results generated by the constant and falling head methods were found to be consistent with those of the transducer method, the use of transducers was considered 
appropriate for use on all subsequent columns and analysis of hydraulic conductivity by constant and falling head methods was discontinued.

\section{Establishment of Residual PCE Saturation}

Following initial permeability testing, the process for attaining residual PCE saturation was commenced. Figure 4.8 provides an illustration of the apparatus used for the establishment of residual PCE saturation within the soil columns.

In order to attain a more uniform residual saturation, 1-bar maximum pressure (air-water) porous

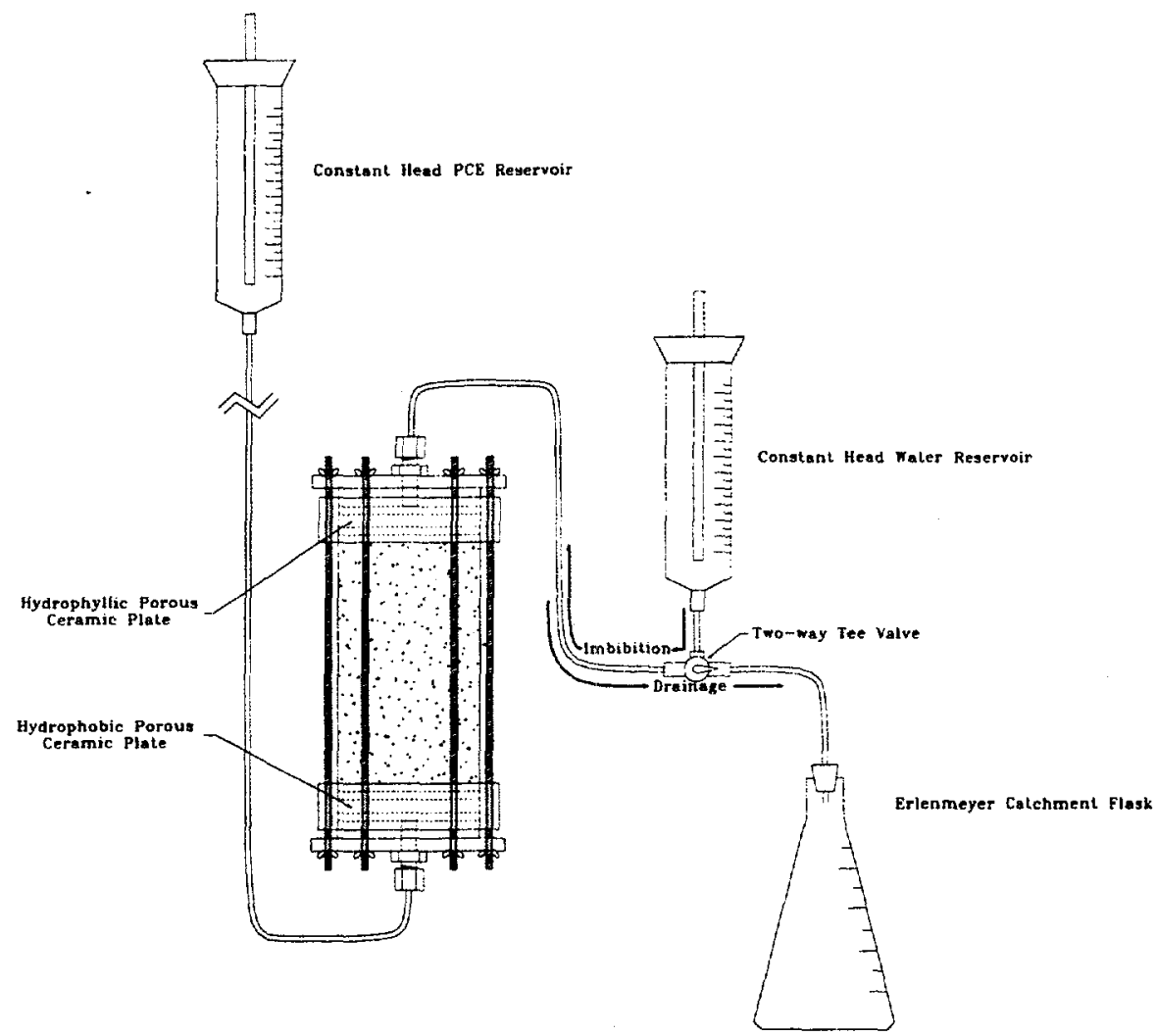

Figure 4.8 Schematic of System Used for Establishment of Residual Saturation

ceramic plates (Soil Moisture Equipment Corporation, Goleta, CA) were placed at each end of the column. On each column, one hydrophobic and one hydrophillic plate was used. A portion of the ceramic plates were made hydrophobic by coating the plates with a hydrophobicizing agent (Glassclad 18, United Chemical Technologies, Inc., Bristol, PA) prior to using them in the column experiments.

During the initial drainage cycle, PCE was flushed into the column from the bottom through the hydrophobic porous plate. The upward flush was performed in order to minimize the effects of viscous fingering. The PCE flowed upward through the column under pressures ranging from 105 
to 250 millibar. Water was subsequently displaced from the soil pores by the migrating PCE and was allowed to flow through the hydrophillic ceramic plate and into a vacuum flask. The hydrophillic plate also prevented any PCE from migrating out of the column and, thus, allowed for a more complete PCE displacement. This was especially helpful in the case of the layered soil columns, where the PCE entrance pressure was much lower in the larger-grained medium sand. The lower entrance pressure caused the medium sand layer to be more rapidly saturated with PCE. The presence of the hydrophillic plate prevented breakthrough and by-passing, and a uniform PCE saturation of the fine sand layer was eventually obtained.

The water drainage cycle was run for a period of 48 hours in the 10-cm homogenous columns. Following completion of the drainage cycle, the amount of water collected in the catchment flask (See Figure 4.8) was measured gravimetrically as well as volumetrically. As shown in Figure 4.8, a two-way tee valve was placed in the outlet tubing. The tee valve was used to switch the connection of the column with the collection flask to the constant head water reservoir. The water reservoir and associated tubing was filled with a known amount of de-aired water prior to the drainage cycle so that no air would be allowed to migrate into the soil column upon commencement of imbibition. Once the tee was switched, spontaneous imbibition occurred in a downward direction through the column, as the soil pores within the column were under a significant capillary pressure. Because the goal of the imbibition process was to return the system to a state of zero (gage) pressure, a pressure head was not used during the imbibition process, and the water reservoir was placed at an equal height with respect to the middle of the soil column. During the imbibition cycle, the hydrophobic plate was responsible for the creation of a uniform residual saturation within the soil media, as only PCE was allowed to pass through the plate and out of the column. After a period of 48 hours, the total amount of water imbibed back into the column was noted by monitoring the level of water in the syringe reservoir.

\section{Quantification of Residual Saturation}

The first method for estimating the residual saturation of each homogenous column was performed by noting the volumes of water drained from and then subsequently imbibed into the column. This method is valid provided there are no losses from the system. Losses may have been the result of evaporation, slight leakages in the column or possibly the infiltration of water into the hydrophobic plate and PCE into the hydrophillic plate, although attempts were made to minimized these losses. Equation 23 shown below was used to estimate the amount of PCE entrapped in the column.

$$
V_{\text {residual }}=V_{\text {drain }}-V_{\text {imbibe }}
$$

Equation 23 simply states that the residual volume of PCE within the column is equal to the difference between the amount of water drained from the column and the amount of water imbibed into the column. The residual saturation was then calculated using Equation 24:

$$
S_{r}=\frac{V_{\text {residual }}}{V_{v}}
$$


where $V_{v}$ is the void volume of the soil within the column.

Another method of estimating residual saturation based on volumetric measurements was performed by analyzing the effluent PCE concentrations during alcohol flushing activities. Effluent samples were analyzed during the course of the alcohol flushing experiments to measure PCE concentration, as will be described in greater detail in subsequent sections. The total mass of PCE in the effluent was determined by integrating the effluent concentrations over flushing time (or number of pore volumes flushed). The mass value was converted to a volume of PCE by dividing by the density of PCE. Equation 24 was used to estimate the residual saturation. The success of this method in providing an accurate measure of the residual saturation is dependent upon the removal of PCE by the process of dissolution. Therefore, the mass of any PCE that is mobilized or left in the soil following flushing activities had to be accounted for. Any mobilization which took place during the flush was quantified volumetrically with micro syringes. In columns where contamination was known to persist following completion of flushing activities, extraction tests were performed in order to quantify the remaining contamination. For these extraction tests, soil was transferred to a 1-liter Erlenmeyer flask. Following transfer of the soil, $500 \mathrm{ml}$ of isopropyl alcohol (IPA) was immediately placed in the flask with the soil. The flask was then stoppered and the solution was shaken for a period of 30 minutes using a mechanical vibrational shaker (Eberbach Corp., Ann Arbor, MI). At least three samples were taken from the supernatant liquid after the sand had been allowed to settle in the flask. The samples were diluted by placing either 10,50 or $100 \mu \mathrm{l}$ of sample into $5 \mathrm{ml}$ of distilled water, depending upon the expected concentration of PCE in the IPA solution. The diluted samples were then analyzed for headspace concentration on a calibrated gas chromatograph. Following sample collection, the supernatant was carefully poured off and the remaining liquid within the flask was evaporated at $105^{\circ} \mathrm{C}$. The dry sand was then weighed and the ratio of mass of PCE per mass of soil was calculated. The total amount of PCE left within the soil was then determined by calculating the product of the mass ratio and the original mass of soil used within the column.

Similar to the method outlined previously, the overall residual saturation of each soil column was quickly and easily estimated by noting the volumes of water drained out of and then subsequently imbibed back into the column. However, while this method was useful in providing an estimation of the total amount of NAPL within the soil column, it was not suitable for estimating the individual NAPL saturations within each layer of the column.

An analysis of the residual saturation within the heterogeneous columns was also performed by analyzing the effluent PCE concentrations during alcohol flushing activities. The total mass of PCE collected via the enhanced dissolution from the alcohol flush was then determined by integrating the effluent concentrations over flushing times (or number of pore volumes flushed), and the residual saturation was determined for each media by converting the mass of PCE to an equivalent volume and dividing that volume by the calculated pore volume within each media.

Soil leaching studies were performed on two heterogeneous columns (H-7 and H-8) prior to any alcohol flushing in order to better understand the actual residual saturation within each separate 
media. In this study, two soil samples were collected from each media at one centimeter intervalsover the $10-\mathrm{cm}$ length of the column. A total number of 40 samples (20 from each media) were placed in 20-ml headspace vials and $10 \mathrm{ml}$ of IPA was placed in the vial with the soil in order to extract the PCE from the soil. The vials were immediately crimp-capped and rotated overnight at 1 revolution per minute (RPM) using a small, specially constructed rotisserie. The samples were then centrifuged at 1500 RPM (International Equipment Corporation, Model HN-SII, Needham Heights, MA) for 12 minutes in order to separate the soil from the supernatant liquid containing the PCE. Aqueous samples were then diluted and analyzed using the gas chromatograph to estimate the mass of PCE associated with the appropriate mass of soil. Saturation values were estimated for each sample by multiplying the mass to mass ratio by the total mass of soil on each side of the column, dividing the result by the density of PCE to obtain a volumetric estimation of PCE, and then finally dividing the volume of PCE by the estimation of pore volume within each media to arrive at an estimation of the percent saturation of PCE. A weighted average of all of the 20 samples collected from each media was then calculated to provide the estimation of the total residual saturation of each media.

The partitioning tracer test was also performed in order to obtain a better understanding of the different saturations within each media of the heterogeneous columns. Partitioning tracer tests were performed on three of the heterogeneous columns. The tests consisted of injecting a tracer solution that contained one non-reactive, or conservative tracer, and two reactive tracers into the column containing PCE at residual saturation. The theory of the partitioning tracer test indicates that the conservative tracer will flow through the column unencumbered while the two reactive tracers will partition into the trapped PCE. When water containing low or no concentrations of the tracer follows the tracer through the column, the tracer will begin to partition back into the aqueous phase in an effort to satisfy the equilibrium condition specified by the equilibrium coefficient. The partitioning tracers will continue to move between the NAPL and aqueous phases, resulting in a lower net rate of flow in comparison with that of the non-reactive tracer. The magnitude of the delay in breakthrough for the reactive tracer is a function of the partition coefficient and the saturation of PCE. Thus, a comparison of the breakthrough curves for the reactive and non-reactive tracers allows for an estimation of the amount of residual PCE trapped in the soil.

During the test, a slug of tracer with known masses of reactive and non-reactive tracers was injected into the soil column. Effluent samples were collected at fairly regular intervals in order to determine the concentration of each tracer exiting the column. Breakthrough curves were subsequently constructed with the effluent information, and the method of moments was used to determine the centroid of the breakthrough curve, or the mean residence time of the tracer in the column. The mean residence times of the reactive and non-reactive tracers were then used along with the partitioning coefficient in Equation 25 to arrive at an estimation of the residual PCE saturation within a particular soil.

$$
S_{n}=\frac{t_{p}-t_{n}}{(K-1) t_{n}+t_{p}}
$$


In Equation $25, S_{n}$ is the residual saturation, $t_{p}$ is the mean residence time of the partitioning, or reactive tracer, $t_{n}$ is the mean residence time of the non-reactive, or conservative tracer and $K$ is the partition coefficient for PCE. Theoretical development and applicability information pertaining to the use of Equation 25 is found in Jin et al. (1995) and Linnemeyer (1997).

The partitioning tests were attempted in two different fashions. Bromide was used as the conservative tracer and 4-methyl 2-pentanol and 2,4-dimethyl 3-pentanol were both used as reactive tracers for each method. In the first trial, the tracers were injected into the bottom of Column $\mathrm{H}-7$ and allowed to flow upward through the column. Two single port endcaps were used for this test and a single effluent sample was collected from the top of the column. A $0.5 \mathrm{ml}$ portion of the sample was collected and diluted 10:1 in distilled water for headspace analysis of the two reactive tracers using the gas chromatograph (Hewlett-Packard 5890 Series II). The remainder of the sample (usually between 0.5 and $2 \mathrm{ml}$ ) was diluted up to $25 \mathrm{ml}$ with distilled water and analyzed for bromide ion concentrations using an ion-selective electrode meter ( $\phi 12 \mathrm{pH} / \mathrm{ISE}$ Meter, Beckman Instruments, Inc., Fullerton, CA). It was theoretically expected that the breakthrough curves for all of the tracers would exhibit secondary peaks, denoting both the initial breakthrough of each tracer from the medium sand and then the secondary breakthrough from the fine sand. This method, while more simple to execute, was found to provide in concise results with respect to the saturation values for each media. Therefore, a second method was attempted for Columns $\mathrm{H}-9$ and $\mathrm{H}-10$.

In the second round of partitioning tracer tests, the tracers were flushed in a downflow direction under a hydraulic head and effluent samples were collected from below the double-sided endcap at the bottom of the column. Initially, the outlets of the double-sided endcap were both desaturated at a pressure of 5 millibar (below the drainage pressure of both media) so that an equal condition of zero pressure would be present at the terminus of each soil at the screen. This was performed to avoid any preferential flows that might develop as a result of siphon effects. The ports were then sealed off to produce a static condition within the column. The water in the dead space of the single port endcap at the top of the column was then vacated and replaced with an equal volume of tracer. The column was then attached to a constant head permeameter tank. The differential head was adjusted to provide an optimal flowrate that was high enough to transmit flow through each media yet low enough to provide a maximum detention time for the tracers to be in the column. An adequate detention time was considered important so that the tracers would have sufficient contact time with the NAPL. The tracer solution contained a bromide concentration of $500 \mathrm{mg} / \mathrm{L}$. The 4methyl 2-pentanol and 2,4 dimethyl 3-pentanol concentrations were 320.8 and $331.6 \mathrm{mg} / \mathrm{L}$, respectively. Effluent samples were collected throughout the test in separate vials and analyzed for tracer concentrations in the same manner as that described above. This method appeared to provide more accurate results in terms of the saturation found within each media of the layered columns.

\subsection{Results and Discussion}

Sieve analyses were completed for the sand used in these experiments. The sieve analysis indicated that soil grains were distributed between $0.25 \mathrm{~mm}$ and $0.50 \mathrm{~mm}$ with a uniformity coefficient of 1.28. Figure 4.9 graphically shows the results of the sieve analysis for the Ottawa white sand. 
The clay soil used in these experiments was obtained from the Savannah River Site. The clay was delivered in large clumps, with approximate dimensions of 2 to $4 \mathrm{~cm}$. The clay was crushed using

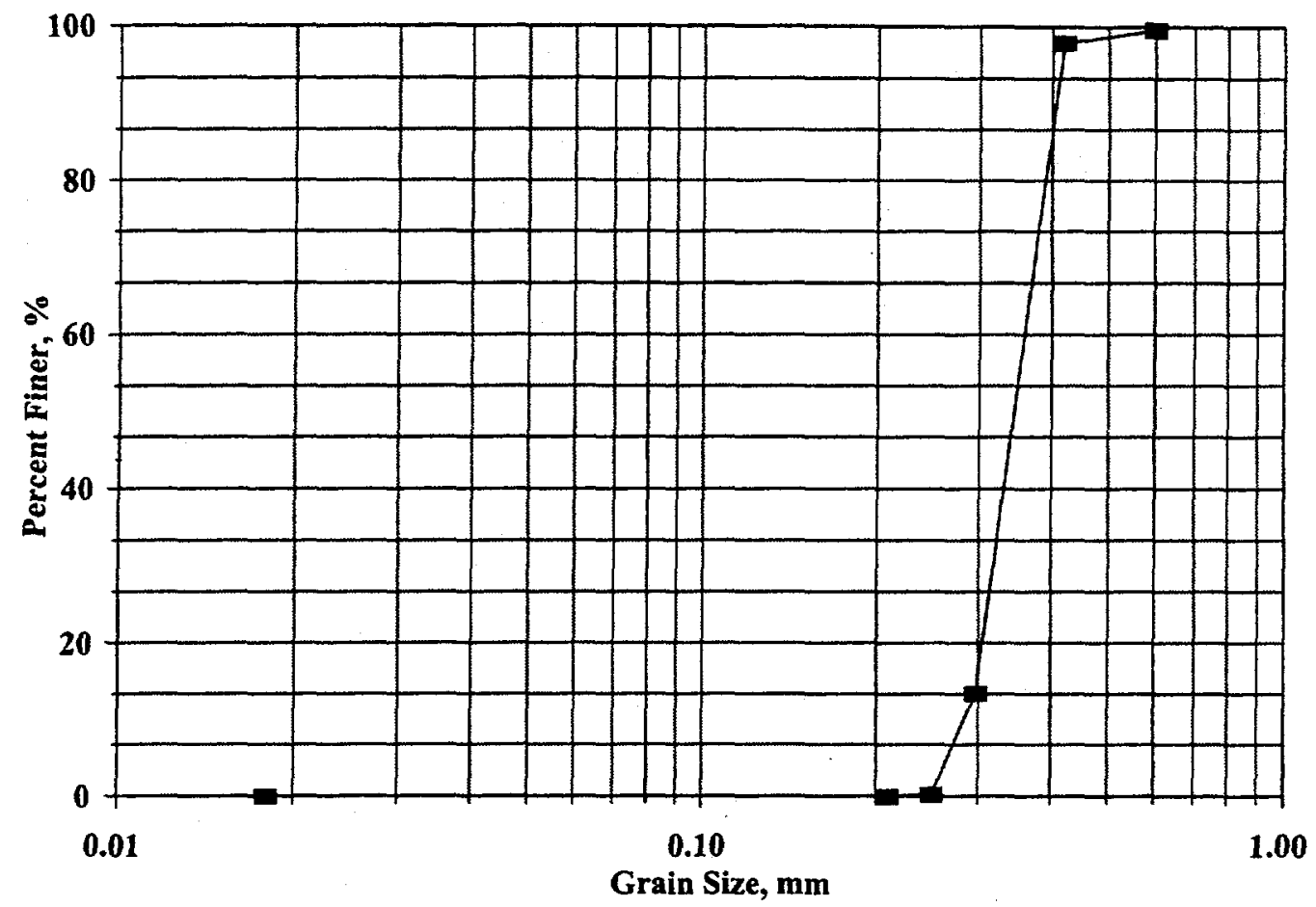

Figure 4.9 Grain size distribution for Ottawa white sand.

a morter and pestle and the portion passing the No. 100 sieve (or less than $0.149 \mathrm{~mm}$ ) was used in the experiments. Characterization of this clay soil was performed using $x$-ray diffraction and hydrometer analyses. The $\mathrm{x}$-ray diffraction analysis indicated that material obtained from the Savannah River Site was predominantly kaolinite. Shrinking and swelling are not as likely to occur in this clay as in a montmorillonite or even an illite clay. The hydrometer analysis results are presented in Figure 4.10 and indicate that approximately $80 \%$ of the SRS clay soils were finer than $0.01 \mathrm{~mm}$ and approximately $73 \%$ were finer than $0.002 \mathrm{~mm}$. Therefore, approximately $27 \%$ of the clay soil used was not clay by definition. However, this soil is called "clay" or "clay soil" to differentiate it from sand. The data from the hydrometer analyses were combined with the sand sieve analysis to develop the grain size distribution for each soil mixture used in the experiments. Results of these combinations are presented in Figure 4.11.

The grain size distributions of the different mixtures appear very similar in these graphs, however, at the pore level varying clay content from $0 \%$ to $20 \%$ results in visible differences. These differences were evident by viewing and photographing soil mixtures using a stereomicroscope. It is noticeable that only a few pores are present in this slide, it is conceivable that some of the pore space is occupied by clay and the an uneven distribution of clay could result in a varied pore size distribution throughout the media. 


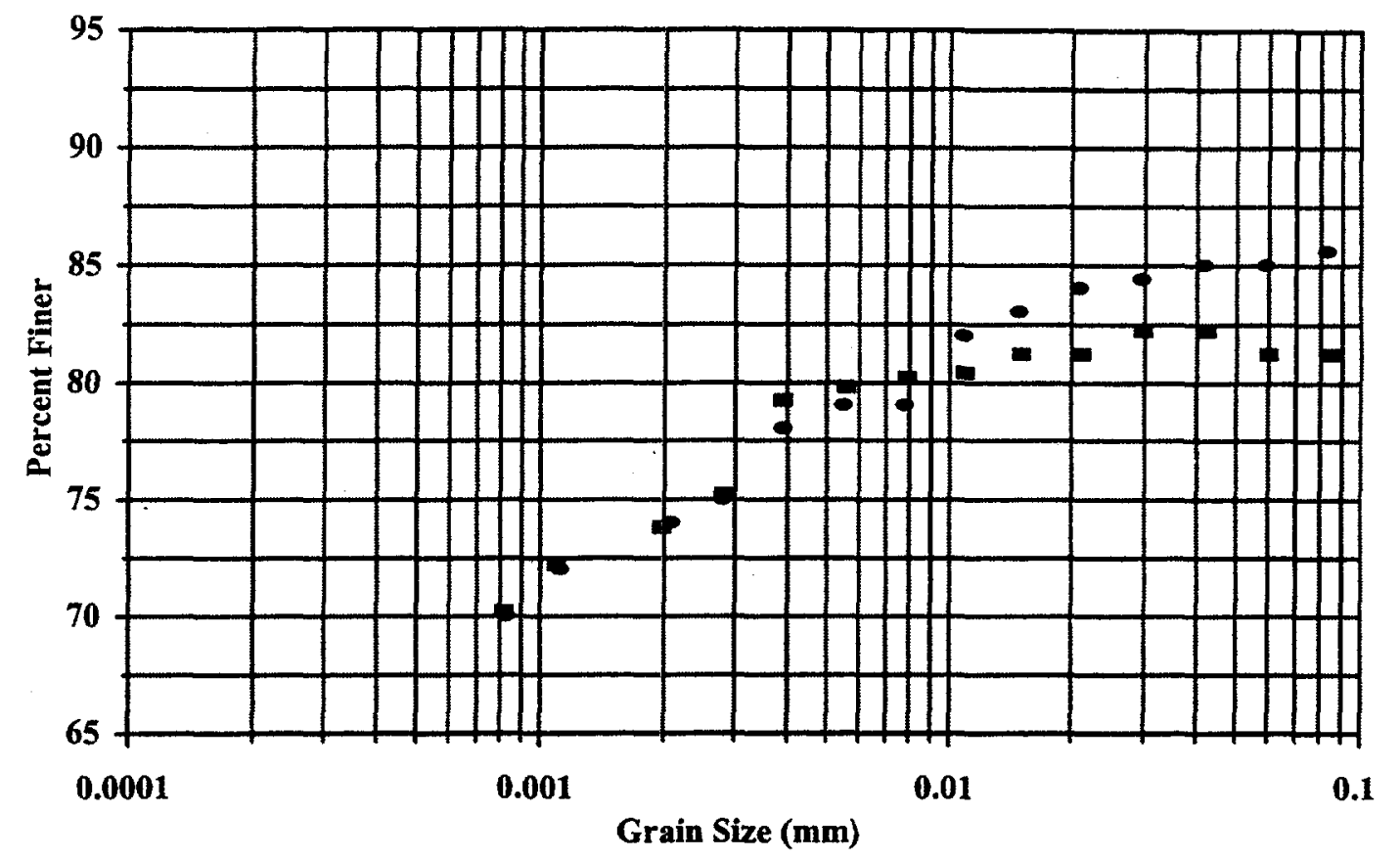

- SRS Clay-Sample\#1 - SRS Clay-Sample\#2

Figure 4.10 Results of hydrometer analysis of SRS clay.

Microscopic observation of the soil mixtures provided valuable information regarding the structure and possible behavior of the clay mixtures. The clay particles appeared to cling or clump at the sand surface in all of the mixtures. For low clay content mixtures, such as the $2 \%$ and $5 \%$ clay soils, the clay particles were less well-distributed on the sand particles. At the higher clay contents of $10 \%$ and $20 \%$, the clay appeared to coat the sand grains. This type of attachment to soil grains has been observed by previous researchers (Wan and Wilson, 1992, Wilson and Mace, 1992). Samples of wet soil taken from a column indicated that the clay particles were contained in clumps at various locations on the soil grains, instead of uniformly coating the sand grain. The change in clay distribution seems to indicate that clay particles are migrating in the column with the movement of water, and perhaps PCE through the soil. When water was applied to dry soil mixtures, some of the clay appeared to move from the sand grain surface to the air-water interface, and travel along this interface. In addition, a few clay particles appeared to be spinning, or moving rapidly. This type of behavior has also been observed by researchers examining colloidal particle behavior in porous media. Wan and Wilson (1992) observed that colloidal particles adhered to interface between DNAPL and water. If this phenomena is occurring in our soils, then the interaction of clay at the interface of trapped DNAPL blobs could affect the trapping mechanisms as well as mobilization of those blobs. Further research investigating the effect of clay on the PCE-water interface could provide valuable information for predicting behavior of PCE in clay-containing porous media. 
In addition to the clay-sand mixtures used in this study, a set of experiments was completed on a fine sand-coarse sand mixture column. This column contained $5 \%$ fine sand passing the No. 100 sieve, similar to the clay separation technique, and the same Ottowa white sand as in the clay columns. The results of this column, F5-1, are presented with the $5 \%$ clay column results. The behavior of this column is compared to the $5 \%$ clay columns later in this chapter.

Hydraulic conductivity was determined for the packed soil columns both before and after the PCE residual was established. Prior to introducing PCE to the column, the hydraulic conductivities appeared fairly consistent for the same soil mixtures. Table 4.4 provides the results of the hydraulic conductivity measurements as well as the porosity and bulk density measurements for the columns. Figure 4.12 graphically shows the relationship between the percent clay and hydraulic conductivity. Hydraulic conductivity was found to decrease with increasing clay content. This is to be expected because as the amount of clay increases in the soil, the soil pores become smaller and movement of water through the soil is more difficult. The values of hydraulic conductivity obtained from the soil packings were higher than the values of conductivity reported for clay soils at SRS (Looney et al., 1992). However, the difficulties of working with high percent clay soils prevented experimentation of columns containing SRS clay soils only. Instead, by using lower percent clay soils, we hope to understand the effect of percent clay on the behavior of PCE and perhaps extrapolate PCE behavior for sites like the Savannah River Site.

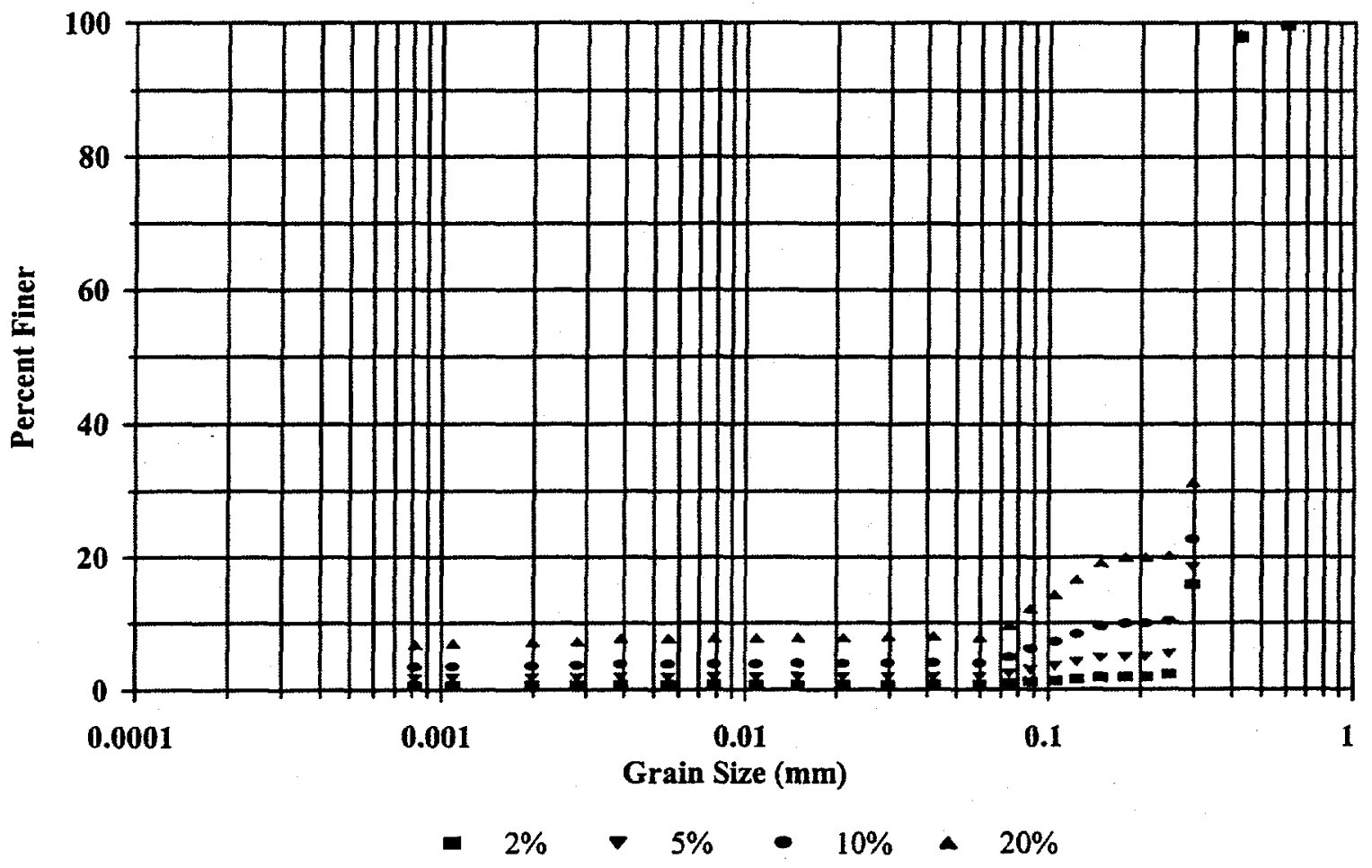

Figure 4.11 Grain size distributions for all sand-clay mixtures. 


\begin{tabular}{|c|c|c|c|c|}
\hline Column & Media & Porosity & $\begin{array}{l}\text { Bulk Density } \\
\left(\mathrm{g} / \mathrm{cm}^{3}\right)\end{array}$ & $\begin{array}{l}\text { Hydraulic Conductivity } \\
\text { (cm/s) }\end{array}$ \\
\hline $\mathrm{C} 0-1$ & white sand & 0.322 & 1.80 & $1.61 \times 10^{-2}$ \\
\hline $\mathrm{CO}-2$ & white sand & 0.320 & 1.80 & $1.46 \times 10^{-2}$ \\
\hline $\mathrm{CO}-3$ & white sand & 0.320 & 1.80 & $1.46 \times 10^{-2}$ \\
\hline $\mathrm{C} 2-1$ & $2 \%$ clay & 0.320 & 1.80 & $1.32 \times 10^{-2}$ \\
\hline $\mathrm{C} 2-2$ & $2 \%$ clay & 0.320 & 1.80 & $1.69 \times 10^{-2}$ \\
\hline $\mathrm{C} 2-3$ & $2 \%$ clay & 0.325 & 1.79 & $1.14 \times 10^{-2}$ \\
\hline$C 5-1$ & $-5 \%$ clay & 0.321 & 1.80 & $1.29 \times 10^{-2}$ \\
\hline $\mathrm{C} 5-2$ & $5 \%$ clay & 0.321 & 1.80 & $9.38 \times 10^{-3}$ \\
\hline $\mathrm{C} 5-3$ & $5 \%$ clay & 0.321 & 1.80 & $8.32 \times 10^{-3}$ \\
\hline $\mathrm{C} 10-1$ & $10 \%$ clay & 0.322 & 1.80 & $9.13 \times 10^{-3}$ \\
\hline $\mathrm{C} 10-2$ & $10 \%$ clay & 0.321 & 1.80 & $7.19 \times 10^{-3}$ \\
\hline $\mathrm{C} 10-3$ & $10 \%$ clay & 0.321 & 1.80 & $4.50 \times 10^{-3}$ \\
\hline $\mathrm{C} 10-4$ & $10 \%$ clay & 0.308 & 1.83 & $7.42 \times 10^{-3}$ \\
\hline $\mathrm{C} 20-1$ & $20 \%$ clay & 0.321 & 1.81 & $8.09 \times 10^{-4}$ \\
\hline $\mathrm{C} 20 \mathrm{a}-2$ & $20 \%$ clay & 0.321 & 1.80 & $5.87 \times 10^{-3}$ \\
\hline C2Oa-3 & $20 \%$ clay & 0.321 & 1.80 & $5.45 \times 10^{-3}$ \\
\hline F5-1 & $5 \%$ fine sand & 0.321 & 1.80 & $1.43 \times 10^{-2}$ \\
\hline
\end{tabular}




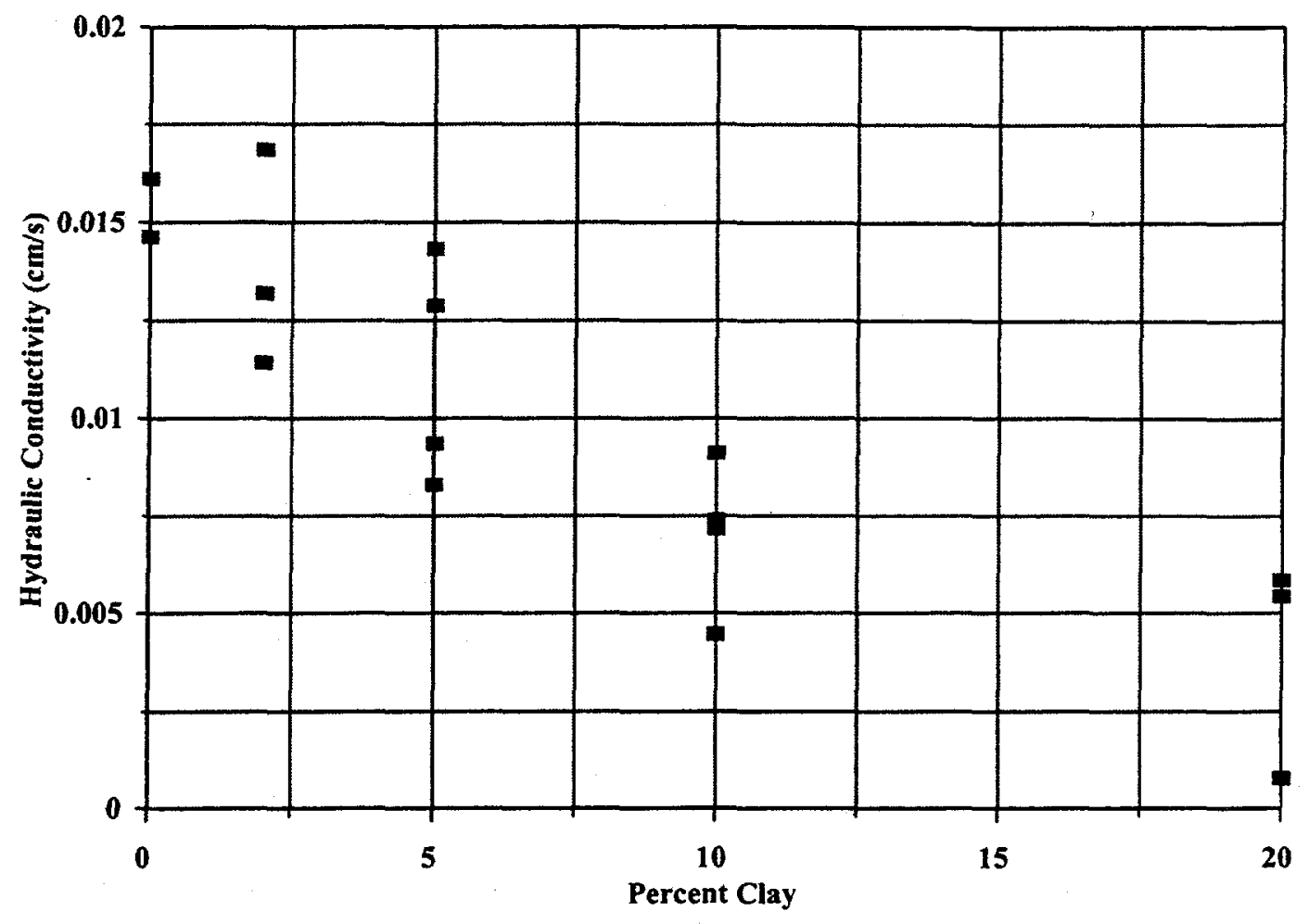

Figure 4.12 Effect of clay on the hydraulic conductivity of all packed columns.

The values of hydraulic conductivity appear to vary consistently with percent clay, as shown in Figure 4.12 . The decrease in hydraulic conductivity between $0 \%$ clay and $20 \%$ clay is approximately 1.5 orders of magnitude. For hydraulic conductivity, this change is not significant, however, it indicates that some structural difference may exist at the pore scale. This pore scale difference is likely to affect the behavior of PCE in the system.

It should be noted that the $20 \%$ clay columns contain two different types of packings, and therefore, represent two different situations. The $20 \%$ clay column with a lower permeability was packed similarly to the lower percent clay columns, with clay passing the 100 sieve or particles less than $0.149 \mathrm{~mm}$. The other two $20 \%$ clay columns were packed with crushed clay, but not sieved clay, and therefore, contained larger clay clumps. These clumps acted differently than the smaller clay particles. This difference will be visible again when viewing the capillary pressure-saturation curves.

Columns Used in Solubilzation Experiment

The medium sand columns were packed to bulk densities of approximately $1.8 \mathrm{~g} / \mathrm{cm}^{3}$ while the finer 
brown sand columns was packed to a maximum of approximately $1.65 \mathrm{~g} / \mathrm{cm}^{3}$. The porosities of the medium sand columns were found to be $0.33,0.33$ and 0.34 , respectively, while those of the fine sand columns were found to be $0.38,0.39$ and 0.40 , respectively.

Table 4.5 is provided below as a summary of the various soil parameters, packing techniques and column configurations used during experimentation with the homogenous columns.

\begin{tabular}{|c|c|c|c|c|c|}
\hline \multicolumn{2}{|c|}{ Table 4.5 Summary of Experimental Homogenous Columns } \\
\hline Column & $\begin{array}{c}\text { Sand } \\
\text { Type }\end{array}$ & $\begin{array}{c}\text { Compaction } \\
\text { Technique }\end{array}$ & Bulk Density & Porosity & Endcap Type \\
\hline W-1 & medium & tap & 1.77 & 0.33 & $\begin{array}{c}\text { Stainless steel single } \\
\text { port end cap }\end{array}$ \\
\hline W-2 & medium & tap & 1.76 & 0.33 & $\begin{array}{c}\text { Stainless steel single } \\
\text { port end cap }\end{array}$ \\
\hline W-3 & medium & vibration & 1.75 & 0.34 & $\begin{array}{c}\text { Aluminum double- } \\
\text { sided endcap }\end{array}$ \\
\hline F-2 & fine & tap & 1.62 & 0.39 & $\begin{array}{c}\text { Stainless steel single } \\
\text { port end cap }\end{array}$ \\
\hline F-3 & fine & tap & 1.63 & 0.38 & $\begin{array}{c}\text { Stainless steel single } \\
\text { port end cap }\end{array}$ \\
\hline F-4 & fine & vibration & 1.58 & 0.40 & $\begin{array}{c}\text { Aluminum double- } \\
\text { sided endcap }\end{array}$ \\
\hline
\end{tabular}

\section{Heterogeneous Columns}

Similar to the homogenous columns, bulk soil parameters were then calculated using the actual masses of each media in the layered column. All parameters were calculated under the assumption that each media occupied exactly $50 \%$ of the column volume. Discrepancies from this assumption were noted in the fact that calculated porosity and bulk density values for the layered columns were observed to deviate slightly from those of the homogenous columns. Table 4.6 is provided below as a summary of the various soil parameters, packing techniques and column configurations used during experimentation.

Table 4.6 Summary of Experimental Heterogeneous Columns

\begin{tabular}{|c|c|c|c|c|c|}
\hline \multirow{2}{*}{ Column } & \multirow{2}{*}{$\begin{array}{c}\text { Compaction } \\
\text { Technique }\end{array}$} & \multicolumn{2}{|c|}{ Bulk Density } & \multicolumn{2}{c|}{ Porosity } \\
\cline { 3 - 6 } & Medium sand & Fine Sand & Medium sand & Fine Sand \\
\hline
\end{tabular}




\begin{tabular}{|c|c|c|c|c|c|}
\hline H-6 & vibration & 1.75 & 1.55 & 0.34 & 0.41 \\
\hline H-7 & tap & 1.78 & 1.58 & 0.33 & 0.40 \\
\hline H-8 & tap & 1.78 & 1.55 & 0.34 & 0.42 \\
\hline H-9 & tap & 1.78 & 1.60 & 0.33 & 0.40 \\
\hline H-10 & tap & 1.84 & 1.61 & 0.30 & 0.40 \\
\hline H-1 & tap & 1.80 & 1.6 & 0.34 & 0.40 \\
\hline H-4 & tap & 1.80 & $1.810 \%$ clay & 0.32 & 0.32 \\
\hline
\end{tabular}

The relative success of the layered media packing was shown by the results of a dispersion test which was performed on two homogenous columns and one heterogeneous column. The results of the dispersion tests were compared in an attempt to see how closely the dispersion and, thus, the homogeneity, of each media within the layered columns compared to the value for that media in homogenous columns. The tests were performed on homogenous columns W-3, F-4 and heterogeneous column H-6. All columns were equipped with double port endcaps at the top and, thus, effluent tracer samples were collected in duplicate for all columns. Therefore, two values of dispersivity were determined for each homogenous column, one from the left side of the column and one from the right side of the column.

The data on linear probability scale plot sufficiently as straight lines, indicating the advectiondispersion equation to be correct. The results for the calculated values of hydrodynamic dispersion $\left(D_{\mathcal{V}}\right)$ and dispersivity $\left(\alpha_{x}\right)$ for the three columns are summarized in Table 4.7.

Table 4.7 Hydrodynamic Dispersion and Dispersivity Values

\begin{tabular}{|c|c|c|c|c|c|c|}
\hline Column & $\begin{array}{c}\text { Left Side } \\
\mathrm{D}_{\mathrm{L}} \\
\left(\mathrm{cm}^{2} / \mathrm{sec}\right)\end{array}$ & $\begin{array}{c}\text { Right Side } \\
\mathrm{D}_{\mathrm{L}} \\
\left(\mathrm{cm}^{2} / \mathrm{sec}\right)\end{array}$ & $\begin{array}{c}\text { Average } \mathrm{D}_{\mathrm{L}} \\
\left(\mathrm{cm}^{2} / \mathrm{sec}\right)\end{array}$ & $\begin{array}{c}\text { Left Side } \alpha_{\mathrm{x}} \\
(\mathrm{cm})\end{array}$ & $\begin{array}{c}\text { Right Side } \\
\alpha_{\mathrm{x}} \\
(\mathrm{cm})\end{array}$ & $\begin{array}{c}\text { Average } \alpha_{\mathrm{x}} \\
(\mathrm{cm})\end{array}$ \\
\hline $\mathrm{W}-3$ & $4.12 \mathrm{E}^{-5}$ & $3.13 \mathrm{E}^{-5}$ & $3.63 \mathrm{E}^{-5}$ & 0.080 & 0.052 & $\mathbf{0 . 0 6 6}$ \\
\hline $\mathrm{F}-4$ & $2.52 \mathrm{E}^{-5}$ & $3.35 \mathrm{E}^{-5}$ & $2.94 \mathrm{E}^{-5}$ & 0.040 & 0.068 & $\mathbf{0 . 0 5 4}$ \\
\hline $\mathrm{H}-6^{*}$ & $7.89 \mathrm{E}^{-5}$ & $8.40 \mathrm{E}^{-5}$ & $\mathbf{8 . 1 5 E}^{-5}$ & 0.093 & 0.121 & $\mathbf{0 . 1 0 7}$ \\
\hline
\end{tabular}

* Left Side $=$ Fine sand, Right Side $=$ Medium Sand

The results appear to be relatively accurate in comparison with the dispersivity value of approximately $0.035 \mathrm{~cm}$ obtained by Pickens and Grisak (1981) for a fine sand with a mean grain size of $0.20 \mathrm{~mm}$ (a value between the sizes of the two media used in this research) and a uniformity coefficient of 2.3. However, the values obtained here and by Pickens and Grisak (1981) were both found to be much lower than the dispersivity values for two fine sands reported by Klotz et al. (1979). Dispersivities reported there for sands of mean grain sizes of 0.17 and 0.35 (fairly consistent 
with the fine and medium sands) were found to be 0.205 and $1.01 \mathrm{~cm}$, respectively. The reason for these higher dispersivities was not one of a significantly higher uniformity coefficient, as each media reported by Klotz et al. (1979) had uniformity coefficients of 1.81 and 1.13, respectively. Klotz et al. (1979) used a different tracer test method in which a slug of tracer was injected and centroid methods were used to estimate a center of gravity for the concentration-time distribution. Since these results correspond fairly well with the values indicated by the literature in which the methods for the study were obtained, the data seem reasonable.

The results indicate that the values of hydrodynamic dispersion and dispersivity of each media in the heterogeneous column were slightly higher than their respective dispersivities when packed alone in homogenous columns. This may be due to some mixing at the boundary of the two layers during packing and/or some mixing at the each end of the column during saturation activities. The mixing at the endcaps was the result of one of the two sands basically spreading into the other half and occurred when the plates and screens were switched during saturation processes. The mixing along the boundary of the two media occurred during packing activities as small amounts of each media may have swept around the removable divider plate upon emplacement of the dry sands into each side of the column.

\section{Permeability Monitoring System}

A system for continuous monitoring of differential pressure in order to determine permeability was designed using differential pressure transducers. The integrity of the system was initially proven in a comparison of permeability results using the transducer system with constant and falling head permeability methods. The results of the permeability tests for the first two homogenous columns are summarized in Table 4.8 below.

\begin{tabular}{|c|c|c|}
\hline & \multicolumn{2}{|c|}{ Table 4.8 Comparison of Intrinsic Permeability values } \\
\hline Transducer System & Column F-2 & Column F-3 \\
\hline Constant Head & $1.39 \mathrm{E}^{-8}$ & $1.29 \mathrm{E}^{-8}$ \\
\hline Falling Head & $1.58 \mathrm{E}^{-8}$ & $1.14 \mathrm{E}^{-8}$ \\
\hline Average & $1.49 \mathrm{E}^{-8}$ & $\mathrm{n} / \mathrm{a}$ \\
\hline
\end{tabular}

$\mathrm{n} / \mathrm{a}=$ not attempted

The relatively consistent permeability values shown in Table 4.8 provide an indication of the integrity of permeability data obtained through the use of the transducer system.

In addition to illustrating the benefit of using the transducer system for determining intrinsic permeability, it was considered important that the system provide accurate permeability data on a continuous basis during alcohol flushing experiments. While the transducer system was successful in providing continuous, unattended data, it was also observed to be extremely sensitive. Figure 4.13 
illustrates the continuously monitored differential pressure during an alcohol flushing experiment.

The continuous differential pressure data appear in Figure 4.13 to be very noisy and disjointed. In
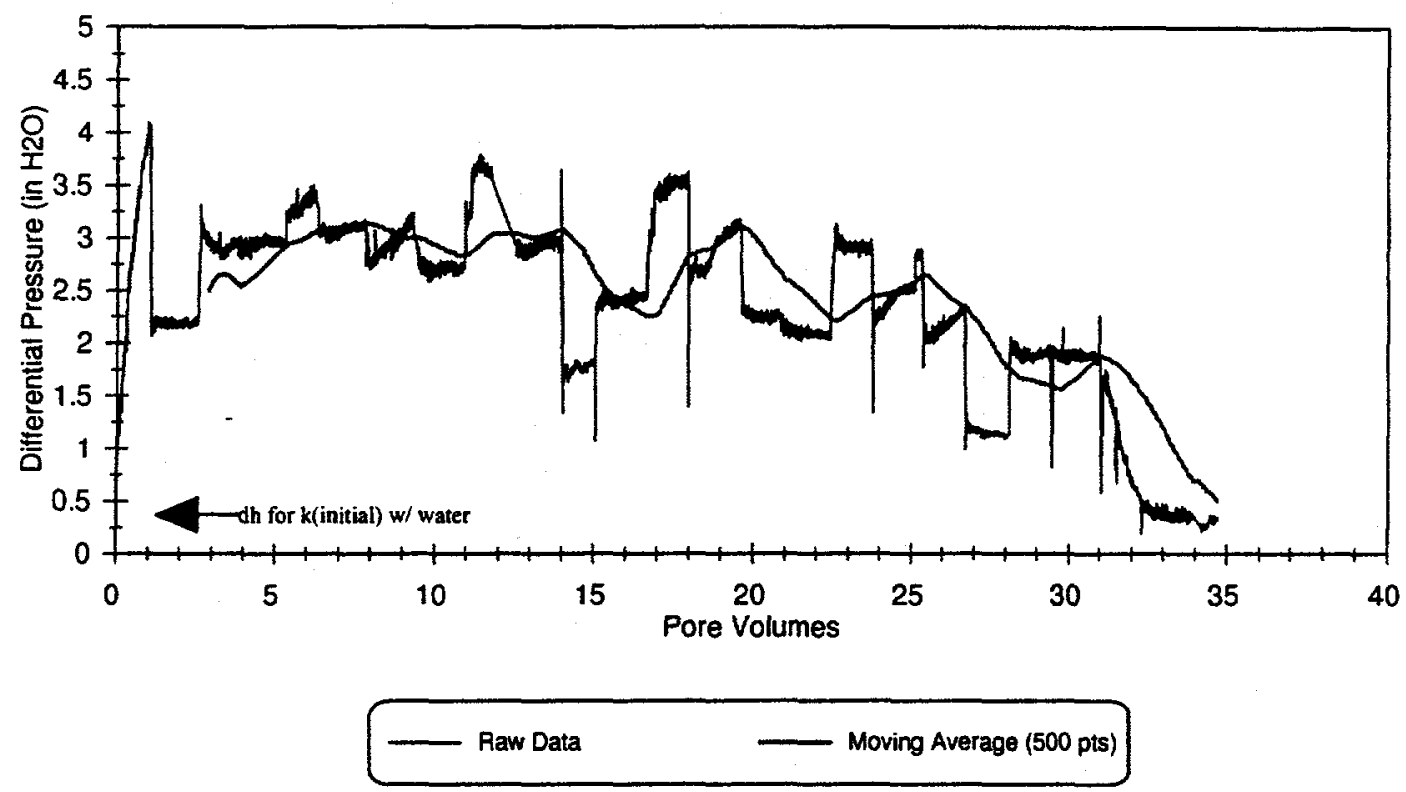

Figure 4.13 Differential Pressures During Column F-2 Flush

an attempt to try to smooth the somewhat noisy data, a moving average of 500 points is included on Figure 4.12. However, despite the 500-point moving average, the data still fluctuate. The reason for the fluctuations was observed to be attributed to the changing of syringes every 12 to 14 hours (approximately every 1.5 pore volumes). The reattachment of the syringes seemed to cause a pressurization of the column, resulting in a drastic change in differential pressure across the column. This pressurization appeared to have two different results, in that at times the overall differential pressure across the column would be observed to increase after the syringe change while at other times the effect would be a decrease in differential pressure.

The final differential pressure values shown in Figure 4.12 provide further illustration of the reliability of the transducer system for permeability measurement. The back calculated differential pressure values corresponding to the intrinsic permeability of the media in Column F-2 were attained when the flushing solution was changed back to water at the end of the flushing experiment. In addition, while the data do appear to be somewhat disjointed, the apparent overall downward trend in differential pressure indicates the success of the continuous differential pressure (and, therefore, permeability) monitoring in illustrating the removal of residual DNAPL.

Table 4.9 provides a summary of the intrinsic (before residual DNAPL saturation) permeability values for the experimental columns. 


\begin{tabular}{|c|c|c|c|c|}
\hline \multirow{2}{*}{ Column } & Sand Type & $\begin{array}{c}\mathbf{k} \\
\left(\mathrm{cm}^{2}\right)\end{array}$ & Sand Type & $\begin{array}{c}\mathbf{k} \\
\left(\mathbf{c m}^{2}\right)\end{array}$ \\
\hline \hline $\mathrm{F}-2$ & fine & $1.49 \mathrm{E}^{-8}$ & & \\
\hline $\mathrm{F}-3$ & fine & $1.22 \mathrm{E}^{-8}$ & & \\
\hline $\mathrm{F}-4$ & fine & $2.24 \mathrm{E}^{-8}$ & & \\
\hline Average & fine & $1.65 \mathrm{E}^{-8}$ & & \\
\hline $\mathrm{W}-1$ & & & medium & $2.45 \mathrm{E}^{-7}$ \\
\hline $\mathrm{W}-2$ & & & medium & $2.75 \mathrm{E}^{-7}$ \\
\hline $\mathrm{W}-3$ & & & medium & $2.34 \mathrm{E}^{-7}$ \\
\hline Average & & & medium & $2.51 \mathrm{E}^{-7}$ \\
\hline \hline $\mathrm{H}-6$ & fine & $1.23 \mathrm{E}^{-7}$ & medium & $2.85 \mathrm{E}^{-7}$ \\
\hline $\mathrm{H}-9$ & fine & $1.60 \mathrm{E}^{-8}$ & medium & $3.33 \mathrm{E}^{-8}$ \\
\hline H-10 & fine & $1.60 \mathrm{E}^{-8}$ & medium & $3.35 \mathrm{E}^{-8}$ \\
\hline Average & fine & $1.60 \mathrm{E}^{-8 *}$ & medium & $3.34 \mathrm{E}^{-8 *}$ \\
\hline
\end{tabular}

*=Disregarding flawed H-6 permeability values

Limitations of the initial layered column and flow system became apparent during the heterogeneous column study. The permeability data for Column $\mathrm{H}-6$ illustrate this fact. The intrinsic permeabilities of the two media were shown during the homogenous column experiments to differ by approximately one order of magnitude. While the mixing issue might have been partly responsible for more similar intrinsic permeability values between each media in the layered Column $\mathrm{H}-6$, the extremely elevated permeability data for the fine sand indicated that a certain degree of by passing of the fine material within the layered column must have occurred. In other words, a smaller percentage of the flow was actually moving through the fine sand in comparison to that within the medium sand.

As a result of the difficulty in determining an exact amount of total flow through each media, a separate flushing method was devised for the final two heterogeneous columns, H-9 and H-10. For these columns, the effluent was allowed to drip out each side of the column under a forced hydraulic head. The downflow drip method allowed the actual amount of flow through each media to be quantified by collecting effluent from each side of the column separately. Thus, a more accurate measure of the permeability was made because both the differential head and the flowrate were known.

The results of the intrinsic permeability data for Columns H-9 and H-10 indicate the presence of the previously-mentioned soil mixing issue. The permeability data for the fine sand are fairly consistent with that of the homogenous columns. However, the permeabilities of the medium sand are slightly lower as the result of the slight intrusion of the fine media. 
While the permeability data for Columns H-9 and H-10 were considered accurate, the effluent concentration data generated through the use of this drip method suggested that there were losses due to volatilization which took place during sample collection. As briefly mentioned earlier, because of the difficulties with these columns, the system with side ports was developed.

\section{Quantification of Residual Saturation}

The results of the two different methods for quantifying residual PCE saturation in the homogenous columns are summarized in Table 4.10.

\begin{tabular}{|c|c|c|c|}
\hline \multicolumn{2}{|c|}{ Table 4.10 Residual Saturation Values for Homogenous Columns } \\
\hline Column & Sand Type & $\begin{array}{c}\text { Volumetric } \\
\text { Estimation }\end{array}$ & $\begin{array}{c}\text { Estimation by } \\
\text { Effluent Analysis* }\end{array}$ \\
\hline \hline F-2 & fine & $17.8 \%$ & $17.5 \%$ \\
\hline F-3 & fine & $22.8 \%$ & $19.5 \%$ \\
\hline F-4 & fine & $15.8 \%$ & $12.8 \%$ \\
\hline W-1 & white & $10.5 \%$ & $14.2 \%$ \\
\hline W-2 & white & $18.6 \%$ & $22.5 \%$ \\
\hline W-3 & white & $9.6 \%$ & $9.8 \%$ \\
\hline
\end{tabular}

*=Estimation for Columns F-4 and W-3 include extraction test data for PCE left in column

The saturation estimates are observed to be fairly consistent between the two methods. Error can be attributed to both methods of estimation. Error in the data from the volumetric method of estimation can be found in the volumetric measurements of water, evaporation losses over the course of drainage and imbibition and the possibility of water entering the hydrophobic plate or PCE being forced into the hydrophillic plate. Error associated with the estimation of residual PCE by effluent analysis can be attributed to slight volatilization of effluent upon sampling, sample handling and preparation error and the analytical error $(<10 \%)$ associated with the headspace sampler and gas chromatograph.

With the exception of the residual saturation data for Column W-2, which will be discussed below, the residual saturations of the fine sand were shown to be slightly greater than those of the medium sand. This conclusion appears appropriate considering the larger pore volumes and smaller pore sizes of the fine sand columns as well as the greater capillary forces acting to trap the NAPL blobs.

Column W-2 exhibited higher residual saturations than would be expected based on the other two medium sand columns. PCE was observed within small pockets along the glass column wall. These voids developed as a result of the flipping of the column during saturation activities. These pockets 
appeared to have had larger pore sizes than the remainder of the column and, as a result, the column looked as if some areas had trapped a greater amount of NAPL than others. It appears as if there was in fact a larger residual saturation in Column W-2, but that it was attributed to the formation of void spaces in the column which were formed during column set-up activities and not present in any of the other homogenous columns.

Without considering the data of Column W-2, the average residual saturation values for the fine sand and medium sand were found to be approximately $18 \%$ and $11 \%$, respectively. These values for residual saturation appear to be reasonable in consideration of the previous work of Chatzis et al. (1983), Schwille (1988), Mercer and Cohen (1990). However, it should be noted that the two soils selected for use in this research were both very homogenous and not that greatly different in terms of grain size $(350 \mu \mathrm{m}$ and $100 \mu \mathrm{m}$ average grain size, respectively).

\section{Heterogeneous Columns}

Residual saturations were attained in five heterogeneous columns according to the methods outlined earlier. Similar to the results of the homogenous column study, a slightly different amount of NAPL is entrapped by each different soil type. Thus, the initial step of the heterogeneous column study was to accurately quantify the actual amount of PCE in each media within the layered soil column. The volumetric method of estimation which was described in detail as part of the homogenous column study was ineffective in providing information as to the different NAPL saturations on each side of the column. The method of effluent analysis provided adequate results for Columns H-9 and H-10 where cosolvent flow through each media was effectively separated. However, in Column $\mathrm{H}-6$, where issues of short-circuiting were apparent, the effluent data was not especially accurate. The soil leachate tests performed on Columns $\mathrm{H}-7$ and $\mathrm{H}-8$ provided a fairly accurate indication of the amount and distribution of the residual NAPL, but the columns had to be sacrificed in order to obtain these data. The partitioning tracer tests (PTT) performed on Columns $\mathrm{H}-9$ and $\mathrm{H}-10$ were also found to provide mostly accurate NAPL saturations in each media within the layered columns. The method was considered effective as it was non-intrusive, however, as with the other methods it had limitations, including its apparent sensitivity. The results of the different analyses for quantification of residual saturation in the heterogeneous columns are summarized in Table 4.11 below.

\begin{tabular}{|c|c|c|c|c|c|c|}
\hline \multicolumn{1}{|c|}{ Table-4.11 Residual Saturation Values for Heterogeneous Columns } \\
\hline & \multicolumn{3}{|c|}{ Fine Sand Data } & \multicolumn{3}{c|}{ Medium sand Data } \\
\hline Column & $\begin{array}{c}\text { Estimation } \\
\text { by PCE } \\
\text { Removed }\end{array}$ & $\begin{array}{c}\text { Estimation } \\
\text { by PTT }\end{array}$ & $\begin{array}{c}\text { Estimation } \\
\text { by } \\
\text { Extraction } \\
\text { Test }\end{array}$ & $\begin{array}{c}\text { Estimation } \\
\text { by PCE } \\
\text { Removed }\end{array}$ & $\begin{array}{c}\text { Estimation } \\
\text { by PTT }\end{array}$ & $\begin{array}{c}\text { Estimation } \\
\text { by } \\
\text { Extraction } \\
\text { Test }\end{array}$ \\
\hline H-6 & $14.93 \%$ & n/a & n/a & $5.40 \%$ & n/a & n/a \\
\hline H-7 & n/a & n/a & $15.86 \%$ & n/a & n/a & $15.06 \%$ \\
\hline
\end{tabular}




\begin{tabular}{|c|c|c|c|c|c|c|}
\hline $\mathrm{H}-8$ & $\mathrm{n} / \mathrm{a}$ & $\mathrm{n} / \mathrm{a}$ & $18.73 \%$ & $\mathrm{n} / \mathrm{a}$ & $\mathrm{n} / \mathrm{a}$ & $14.32 \%$ \\
\hline $\mathrm{H}-9$ & $17.6 \%$ & $16.02 \%$ & $\mathrm{n} / \mathrm{a}$ & $14.30 \%$ & $14.54 \%$ & $\mathrm{n} / \mathrm{a}$ \\
\hline $\mathrm{H}-10$ & $17.39 \%$ & $9.33 \%$ & $\mathrm{n} / \mathrm{a}$ & $13.26 \%$ & $13.89 \%$ & $\mathrm{n} / \mathrm{a}$ \\
\hline
\end{tabular}

$\mathrm{n} / \mathbf{a}=$ not applicable

As shown in Table 4.11, the saturation values were observed to be slightly greater in the fine sand compared to that of the medium sand. Similar to the homogenous column results, some curious results were observed in the heterogeneous column data. The apparent outlying values of residual saturation in the medium sand of Column H-6 estimated by effluent analysis and the fine sand of Column $\mathrm{H}-10$ estimated by partitioning tracer tests. Without considering these curious values, mean values for the fine sand and medium sand residual saturations were found to be approximately $17 \%$ and $14 \%$, respectively.

Of interest is the apparent increase in the mean residual saturation value of $14 \%$ for the medium sand in the heterogeneous columns in comparison with the mean residual saturation value of $11 \%$ found in the homogenous columns. This increase is most likely the result of the previously described soil mixing issues within the white sand of the heterogeneous columns.

Saturation estimates were observed to be fairly consistent between the three methods, with the exception of the aforementioned problems with Columns H-6 and H-10. Error can be attributed to all three methods of estimation and will be discussed in each of the following sections.

An attempt to quantify the residual saturation within the two media of the heterogeneous columns was performed by analyzing the effluent from each side of the column for Columns H-6, H-9 and $\mathrm{H}-10$. For all of these columns with the exception of $\mathrm{H}-6$, in which the PCE was able to be removed completely by the alcohol flush, the effluent sample analysis was used in conjunction with an extraction test to determine the total amount of PCE flushed out and the amount remaining following

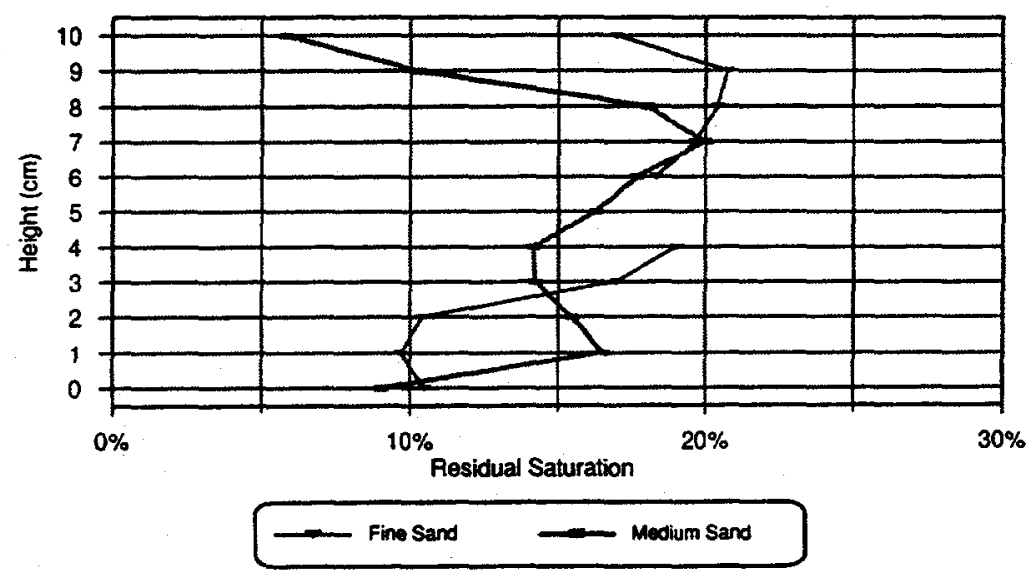

Figure 4.14 Column H-7 Residual PCE Diģ̧ibution 


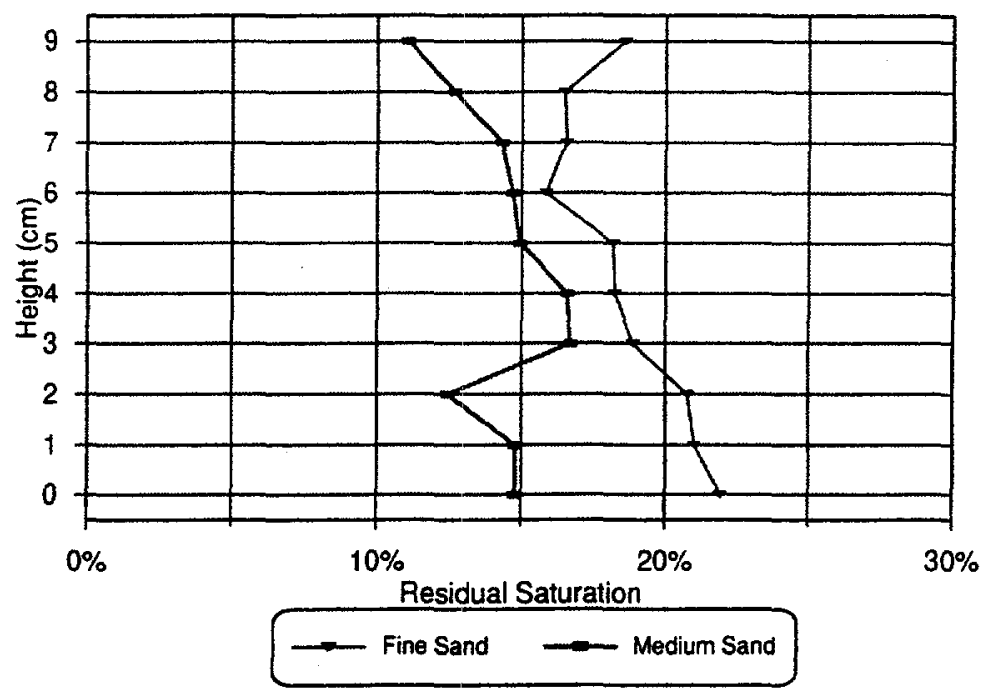

Figure 4.15 Column H-8 Residual PCE Distribution

completion of flushing. These data were combined with the effluent data to arrive at total masses of PCE originally within each media.

Columns H-7 and H-8 were packed, brought to residual PCE saturation and then sacrificed by performing soil extraction tests in order to obtain additional information on the degree of residual saturation within each media. The results pertaining to the total residual PCE saturations for each media within Columns $\mathrm{H}-7$ and $\mathrm{H}-8$ are included in Table 4.11. The distribution of residual NAPL within each column is summarized in Figures 4.14 and 4.15.

As shown in Figure 4.14, a number of samples for Column H-7 were lost as the result of overdilution of the aqueous samples. This problem was rectified for the remainder of the samples and for the remainder of the extraction tests on other columns. The data in Figures 4.14 and 4.15 do, however, still appear to illustrate a trend. It appears from the results of each media with the possible exception of the fine sand in Column H-8 that some degree of loss occurred near the ends of the columns. This loss was most likely attributable to volatilization of the PCE when the porous plates and screens were switched and, thus, the soil was exposed to the atmosphere.

Partitioning tracer tests were performed on Columns H-9 and H-10 to further evaluate the residual saturations within each media. The PTT was considered for use as it has been described by previous researchers (Jin et al., 1995, Linnemeyer, 1997) as fairly accurate in determining saturations of nonaqueous phase liquids and it is also a non-invasive and non-destructive method. Thus, following determination of the residual within each media, the column was still in adequate condition to allow 
for alcohol flushing experiments. The principles involved in the PTT are summarized earlier. A detailed sensitivity analysis for the PTT method can be found in Linnemeyer (1997). The results of the test for Column H-9 are provided in Figure 4.16.

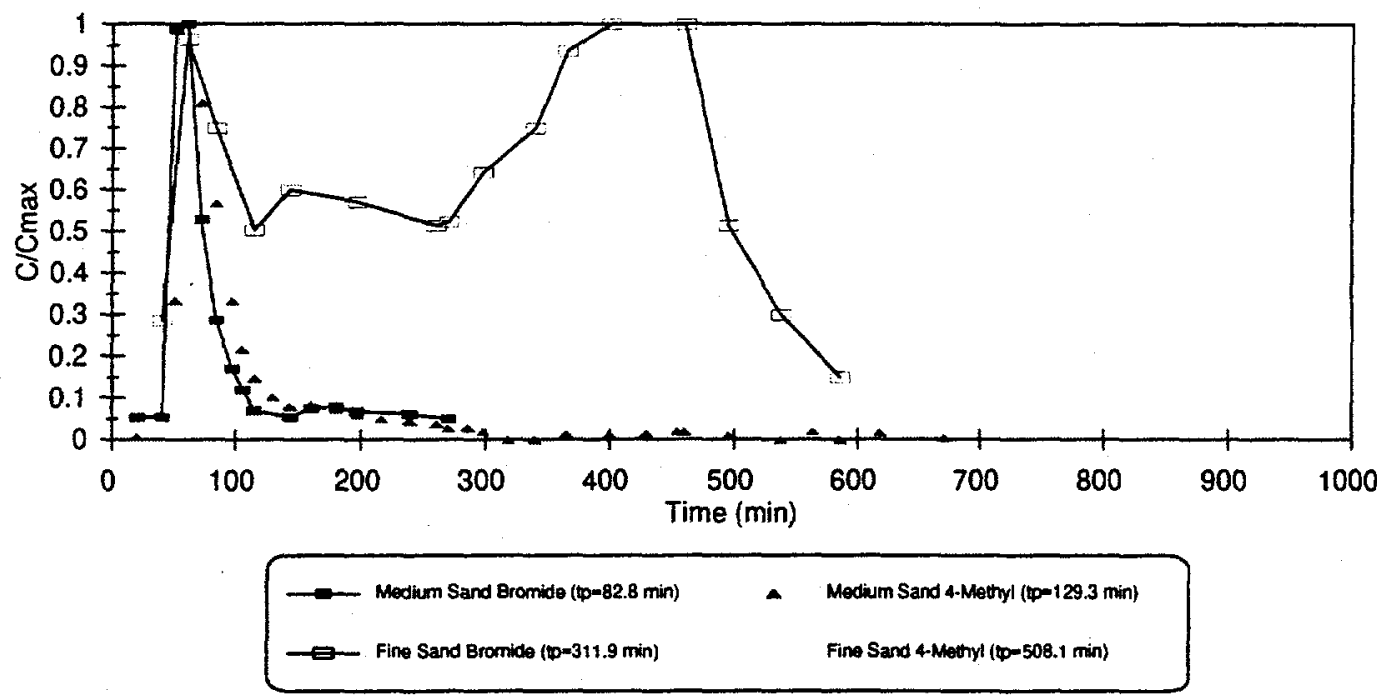

Figure 4.16 Column H-9 Partitioning Tracer Test Results

The method of moments was used to calculate the center of mass times indicated for each tracer on Figure 4.16. The centers of mass, or the mean residence times for the conservative tracer (bromide) and the reactive tracer (4-methyl 2-pentanol), were then used in Equation 29 along with the equilibrium partition coefficient for PCE to calculate the value of residual saturation in each media. As shown in Figure 4.16, this method appears to be more accurate for the medium sand, as the breakthrough curves for each tracer are much more narrow and well defined. However, it should also be noted that as a result of these narrow curves, the average retention times are much closer together, thus making a slight deviation in either value more significant to the overall saturation value. The data for the fine sand appear to be somewhat variable. The main aspect of the fine sand data that appears to be different from that of the medium sand is the fact that double peaks are noted for each tracer. This is most likely the result of interference with the breakthrough curve of the medium sand. While the interference peaks are certainly apparent for the fine sands, it is interesting to note the very minor secondary peaks for the medium sand shown on Figures 4.16. These slight secondary humps are probably the result again of interference, this time in the medium sand by the fine sand.

It should also be noted that data obtained for the 2,4 dimethyl 3 pentanol reactive tracer was not used in estimation of residual saturation. This was due to the fact that over the course of the test (greater than 16 hours) the breakthrough curve for 2,4 dimethyl 3 pentanol was never able to be completed 
for the fine sand.

Figure 4.16 illustrates the potential for error in estimation of residual saturation using the PTT. It can be seen that the breakthrough curve for each tracer is delineated by a somewhat limited amount of data. in addition, the flowrates through each media had to be somewhat elevated in order to effectively obtain all of the tracer mass within one approximate 18 hour period. This may have resulted in an insufficient contact time for each tracer with the NAPL. As a result of these sources, a slight degree of error was attributed to the overall estimation of residual saturation by the PTT.

The data for the PTT for Column $\mathrm{H}-10$ are provided on Figure 4.17.
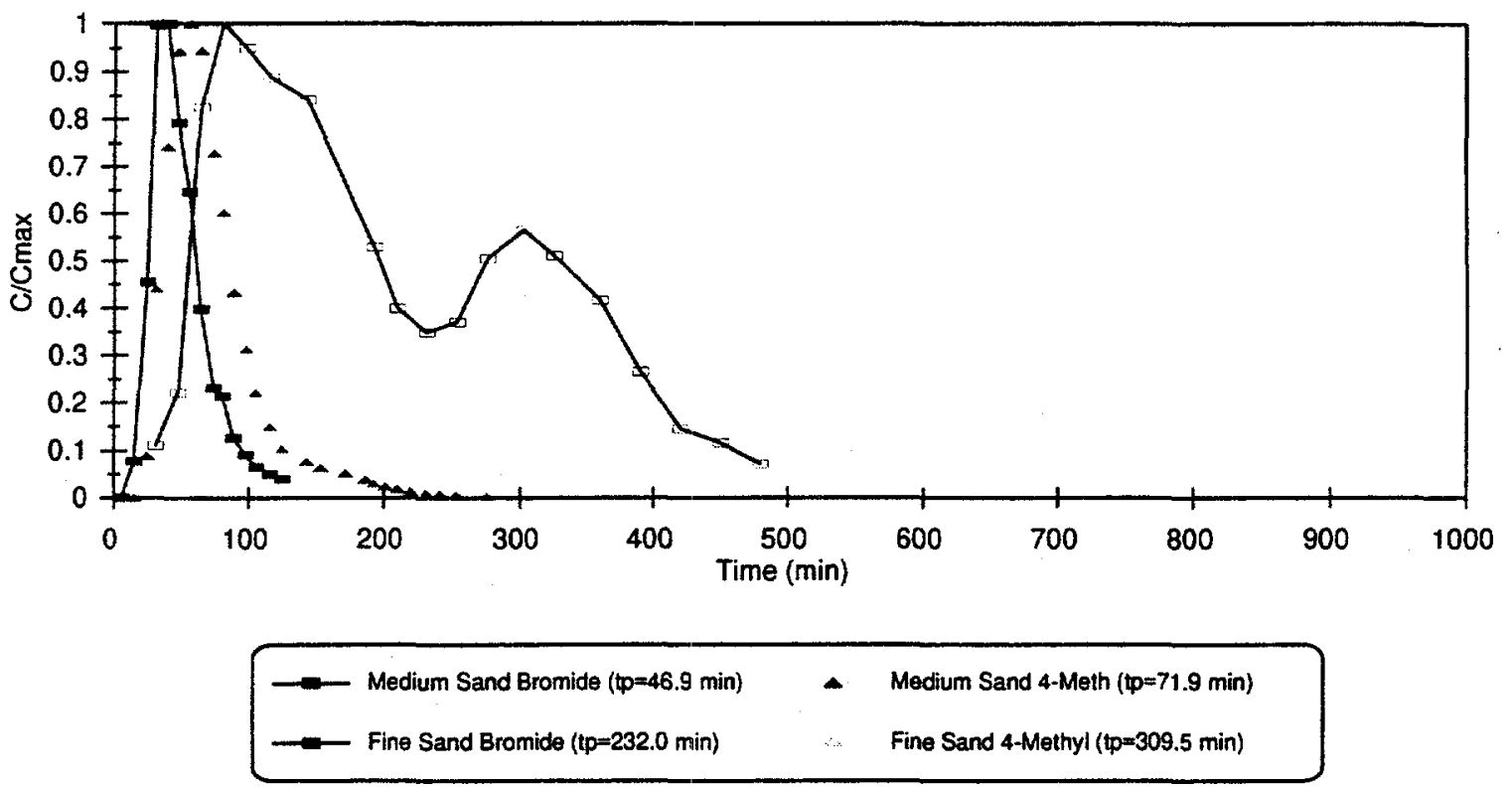

Figure 4.17 Column H-10 Partitioning Tracer Results

The breakthrough curves for Column H-10 appear to be somewhat smoother than those of Column $\mathrm{H}-9$. It is also interesting to note that the secondary peaks for the fine sand within Column $\mathrm{H}-10$ are lower than those for Column H-9. Furthermore, the secondary peaks are not the locations where $\mathrm{C} / \mathrm{C}_{\max }$ reaches values of 1.0 . This indicates that the majority of the tracers came through under the influence of the medium sand and that only a minor amount actually came through the fine sand later. This is most likely due to the slightly faster flow rates recorded during the PTT for H- 10 . Average flow rates of 27.4 and $9.5 \mathrm{ml} / \mathrm{hr}$ were recorded for the white and fine sands in column $\mathrm{H}$ 10 , respectively. These were higher than the flow rates of 22.9 and $5.8 \mathrm{ml} / \mathrm{hr}$ for each media of Column H-9. The result of these expedited rates in Column H-10 was most likely an insufficient contact time for the tracers, especially that within the fine sand, where the majority of the tracers were flushed through with the medium sand. This insufficient contact time most likely led to a significant amount of error in the estimation of residual saturation for the fine sand in Column H-10. 
This fact is shown clearly on Table 4.11, as the estimation of residual saturation in the fine sand of Column $\mathrm{H}-10$ was found to be much lower than would be expected.

Figure 4.18 shows the PTT results from $\mathrm{H}-4$ with the side ports.

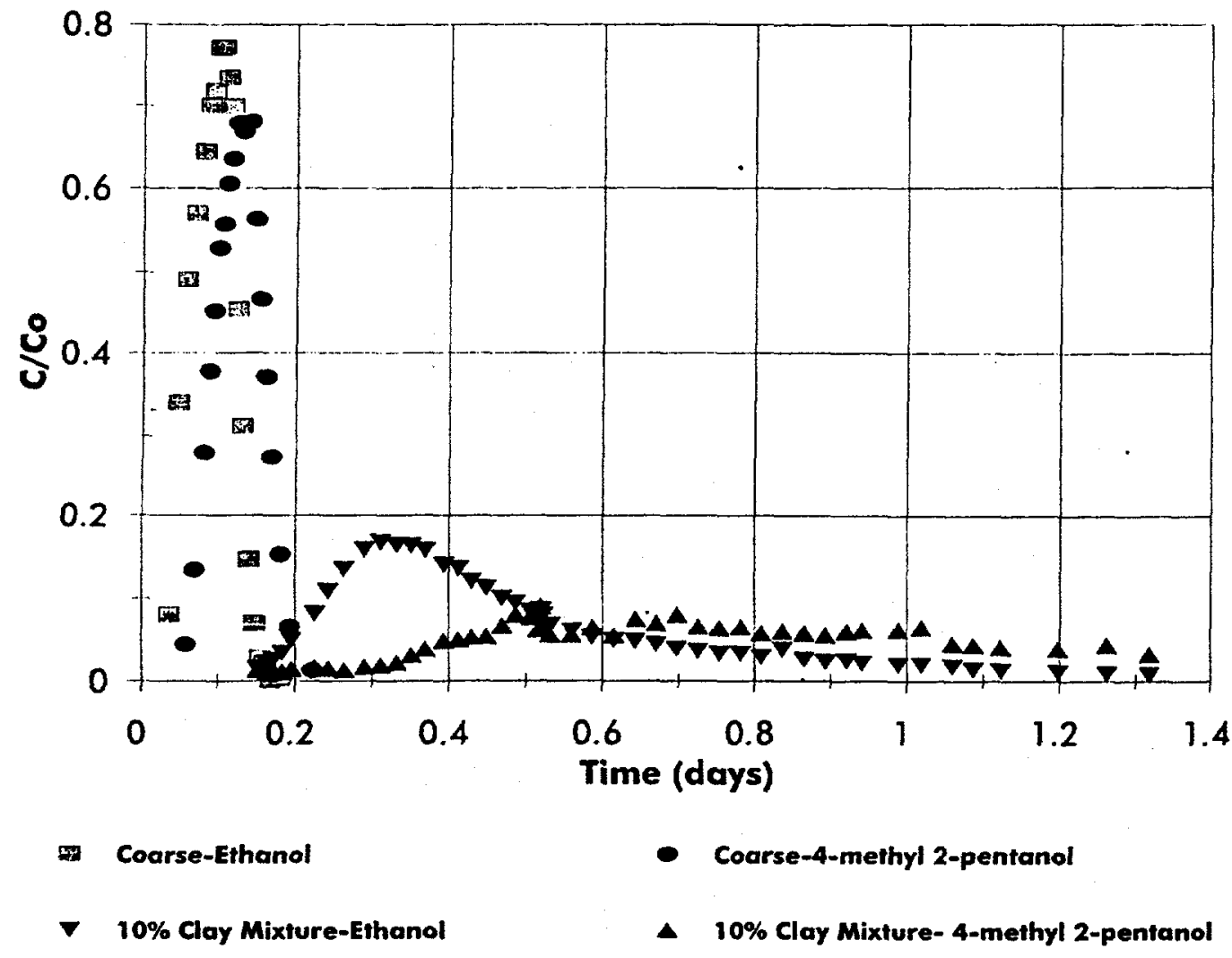

Figure 4.18. Column H-4 partitioning tracer results from side port sampling.

\subsection{Summary}

A system to continuously monitor permeabilities in soil columns was initially designed and developed. The system consisted of two wet-wet differential pressure transducers which transmitted differential pressure data to a computer file. Pressure data in the file was converted to permeability using Darcy's law and a consideration of the aqueous properties of the solution flowing through the columns. This method was found to be complex and sensitive to syringe changes on the pump ans therefore was discontined. A side prot method of sampling with manometers to determine the prssure drop across columns was used.

The degree of homogeneity within each medium of the columns packed in layered fashion was evaluated by comparing dispersivity results of homogenous and layered heterogeneous columns. The results of the dispersion tests indicated that a slight degree of mixing occurred in the layered columns during preparatory activities. These findings were bolstered by (1) the apparent lower permeabilities of the medium sand in the layered columns as compared to those of the homogenous columns and 
(2) direct observations made upon decommissioning of the layered columns.

Differences in the amount of residual saturation within each medium were noted. Residual saturations for the medium and fine sands in the homogeneous columns were $11.0 \%$ and $17.7 \%$, respectively. Residual saturations of these same media in the layered heterogeneous columns were found to be $14.3 \%$ and $17.1 \%$, respectively. The difference in residual saturation values for the medium sand between homogenous and layered columns was attributed to the slight degree of heterogeneity in the layered columns. Estimations of residual saturation were made in the homogenous columns with volumetric measurements of the amount of water drained and subsequently imbibed and integration of effluent data during the course of flushing. Integration of effluent data, partitioning tracer tests, and soil extraction tests were performed in order to provide sufficient indication of the residual saturation on each side of the layered columns. All of these methods were found to provide reasonable indications of residual saturation and all were found to compare favorably with one another, indicating that each method was satisfactory.

Investigation into the degree of reduction in permeabilities due to the residual PCE saturation was performed. Permeabilities were found to decrease by one half order of magnitude in the fine sand and by only a factor of two in the medium sand. The minimal decrease in permeability in the medium sand illustrates the shortcomings of its use in later flushing experiments where increases in permeability were expected. Permeability values were then used in calculations of the total trapping number $\left(\mathrm{N}_{\mathrm{T}}\right)$, which provides indication of the degree of residual entrapment. The trapping number gave an indication of the optimal concentration of alcohol flushing solution that could be used in order to maximize the solubilization of the PCE while avoiding unwanted mobilization of the residual. Trapping number calculations indicated that a maximum $40 \%$ IPA solution could be used on the medium sand in order to minimize the likelihood of mobilization. Due to the lower permeabilities of the fine sand, mobilization was never a possibility at the IPA concentrations used during this research.

Evaluation of the degree of residual PCE removal from homogenous columns was successfully illustrated using permeability data in the homogenous fine sand columns. Permeabilities were shown to increase from values shown for residual saturation up to initial intrinsic values as the result of the removal of residual DNAPL by alcohol flushing. This upward trend in permeability data was more difficult to visualize in the medium sand hornogenous columns due to the minimal change in permeability. The use of a finer medium or a medium of known heterogeneity ( 5 or $10 \%$ clay) may have caused a greater change in permeability that would have more accurately illustrated the degree of remediation with permeability data. Data from the final two homogenous columns indicated the need for continuous differential pressure monitoring, as the discreetly collected data was found to be insufficient in eliminating the effects of the inherent noise in the bench-scale system.

\subsection{References}

American Society for Testing and Materials, "Natural Building Stones; Soil and Rock," Annual Book 
of ASTM Standards, Part 19, Philadelphia, PA, 1980.

Anderson, M.R., Johnson, R.L., and Pankow, J.F., "Dissolution of Dense Chlorinated Solvents into Ground Water: 1. Dissolution from a Well-Defined Source", Ground Water, Vol. 30, No.2, March- April 1992.

Augustijin, D.C.M., Jessup, R.E., Rao, P.S.C., and Wood,A.L., "Remediation of Contaminated Soils by Solvent Flushing", Transport and Reactive.Processes in Aquifers, 1994.

Bear, J., Dynamics of Fluids in Porous Media, New York, NY: American Elsevier, 1972.

Bedient, P.B., Rifai, H.S., and Newell, C.J., Groundwater Contamination, Transport and Remediation, Englewood Cliffs, NJ: Prentice Hall, 1994.

Boyd, G.R., "Factors Influencing Nonaqueous Phase Liquid Removal from Groundwater by Alcohol Flooding", Ph.D. dissertation, Clemson University, SC 1991.

Boyd, G.R. and Farley, K.J., "Residual NAPL Removal from Groundwater Using Alcohol Flooding", Trans. American Geophysical Union, Vol 71, No. 17, 1990.

Boyd, G.R. and Farley, K.J., "NAPL Removal from Groundwater by Alcohol Flooding: Laboratory Studies and Applications", Hydrocarbon Contaminated Soils and Groundwaters: Analysis, Fate, Environmental and Public Health Effects and Remediation, 1992.

Brandes, D. and Farley, K.J., "Importance of Phase Behavior in Remediation of DNAPL Contaminated Aquifers by Alcohol Flooding", Water Environment Federation, Vol. 65, 1993.

Brooks, R.H. and Corey, A.T., "Hydraulic properties of porous media", Hydrology Paper No. 3. Civil Engineering Department, Colorado State University, 1964.

Chatzis, I., Morrow, N.R., and Lim, H.T., "Magnitude and detailed structure of residual oil saturation", Society of Petroleum Engineering Journal, 1983.

Conrad, S.H., Wilson, J.L., Mason, W.R., and Peplinski, W.J., "Visualization of Residual Organic Liquid Trapped in Aquifers", Water Resources Research, Vol. 28, No. 2, February, 1992.

CRC Handbook of Chemistry and Physics, 60th Edition, Weast, R.C., Ed., Boca Raton, FL: CRC Press, Inc., 1980.

Fetter, C.W., Contaminant Hydrogeology, Upper Saddle River, NJ: Prentice Hall, 1993.

Freeze, R.A., and Cherry, J.A., Groundwater, Englewood Cliffs, NJ: Prentice Hall, 1979. 
Fried, J.J., Muntzer, P., and Zilliox, L., "Ground-water pollution by transfer of oil hydrocarbons, Ground Water, Vol 17, No. 6, 1979.

Hayden, N.J. and Van Der Hoven, E.J., "Alcohol flushing for enhanced removal of coal tar from contaminated soils.", Water Environment Research, Vol 68, No. 7, 1996.

Hayden, N.J. and Linnemeyer, H.C., "Investigation of Partitioning Tracers for Determination of Residual Coal Tar Saturations", Submitted for publication, 1997.

Hofman, J.A.M.H. and Stien, H.N., Colloids and Surfaces, 1991.

Hunt, J.R. Sitar, N., and Udell, K.S., "Nonaqueous Phase Liquid Transport and Cleanup: 1: Analysis of Mechanisms", Water Resources Research, Vol 24, No. 8, August 1988.

Illangasakare, T.H., Ramsey, J.L., Jensen, K.H. and Butts, M.B., "Experimental Study of Movement and Distribution of Dense Organic Contaminants in Heterogeneous Aquifers", Journal of Contaminant Hydrology, Vol. 20, 1995.

Imhoff, P.T., Gleyzer, S.N., McBride, J.F., Vancho, L.A., Okuda, I., and Miller, C.S. "CosolventEnhanced Remediation of Residual Dense Non-Aqueous Phase Liquids: Experimental Investigation", Environmental Science and Technology, Vol. 29, No. 8, 1995.

Imhoff, P.T., Thyrum, G.P, and Miller, C.T., "Dissolution fingering during the solubilization of nonaqueous phase liquids in saturated porous media; 2: Experimental observations", Water Resources Research, Vol 32, No. 7, 1996.

Jin, M., Delshad, M., Dwarakanath, V., McKinney, D.C., Pope, G.A., Sepehrnoori, K., Tilburg, C.E. and Jackson, R.E., "Partitioning tracer test for detection, estimation, and remediation performance assessment of subsurface nonaqueous phase liquids", Water Resources Research, Vol 31, No. 5, 1995.

Klotz, D., Seiler, K.P., Moser, H., and Neumaier, F., "Dispersivity and Velocity Relationship from Laboratory and Field Experiments", Journal of Hydrology, Vol 45, 1979.

Klute, A., "Laboratory Measurement of Hydraulic Conductivity of Saturated Soil", Methods of Soil Analysis, Part 1, ed. C.A. Black, Madison, WI: American Society of Agronomy, 1965.

Kokal, S.L., Maini, B.B., and Woo, R., "Flow of Emulsions in Porous Media", In Emulsions: Fundamentals and Applications in the Petroleum Industry, Schramm, L.L. Ed, Washington, DC: American Chemical Society, 1992.

Kueper, B.H., Abbott, W., and Farqhar, G., "Experimental Observations of Multiphase Flow in Heterogeneous Porous Media", Journal of Contaminant Hydrology, Vol 5, 1989. 
Kueper, B.H. and McWhorter, D.B., "The Behavior of Dense, Nonaqueous Phase Liquids in Fractured Clay and Rock", Ground Water, Vol. 29, No. 5, September-October 1991.

Linnemeyer, H.C., "Determination of Coal Tar Saturation Using the Partitioning Interwell Tracer Test", Masters Thesis, University of Vermont, 1997.

Mackay, D.M., Roberts, P.V., and Cherry, J.A., "Transport of organic contaminants in groundwater", Environmental Science and Technology, Vol 19, No. 5, 1985.

McAuliffe, C.D., Journal of Petroleum Technology, 1973.

Mercer, J.M. and Cohen, R.M., "a Review of Immiscible Fluids in the Subsurface: Properties, Models, Characterization and Remediation", Journal of Contaminant Hydrology, Vol 6, 1990.

Miller, C.T., Poirier-McNeill, M.M. and Mayer, A.S., "Dissolution of Trapped Non-aqueous Phase Liquids: Mass Transfer Characteristics", Water Resources Research, Vol. 26, No. 11, November, 1990.

Nkedi-Kizza, P., Rao, P.S.C., and Hornsby, A.G., "Influence of Organic Cosolvents on Leaching of Hydrophobic Organic Chemicals through Soils", Environmental Science and Technology, Vol.21, No. $11,1987$.

Okuda, I., McBride, J.F., Gleyzer, S.N., and Miller, C.T., "Physicochemical Transport Processes Affecting the Removal of Residual DNAPL by Nonionic Surfactant Solutions", Environmental Science and Technology, Vol. 30, No. 6, 1996.

Padgett, P.K., "Mobilization of Residual PCE During Alcohol Flushing of a Clay-Containing Porous Media", Masters Thesis, University of Vermont, 1998.

Pennel, K.D., Abriola, L.M., and Weber, W.J. Jr., "Surfactant-Enhanced Solubilization of Residual Dodecane in Soil Columns. 1. Experimental Investigation", Environmental Science and Technology, Vol. 27, No. 12, 1993.

Pennel, K.D., Pope, G.A., and Abriola, L.M., "Influence of Viscous and Buoyancy Forces on the Mobilization of Residual Tetrachloroethylene during Surfactant Flushing", Environmental Science and Technology, Vol. 30, No. 4, 1996.

Perkins, T.K. and Johnson, O.C., "a review of diffusion and dispersion in porous media", Society of Petroleum Engineers Journal, Vol. 3, 1963.

Pickens, J.F. and Grisak, G.E., "Scale-Dependent Dispersion in a Stratified Granular Aquifer", Water Resources Research, Vol 17, No. 4, 1981. 
Powers, S.E., Celso, O.L., Abriola, L.M., and Weber, W.J., Jr., "Theoretical Study of the Significance of Nonequilibrium Dissolution of Nonaqueous Phase Liquids in Subsurface Systems", Water Resources Research, Vol 27, No. 4, April, 1991 $\checkmark$

Powers, S.E., Abriola, L.M., and Weber, W.J., Jr., "An Experimental Investigation of Non-Aqueous Phase Liquid Dissolution in Saturated Subsurface Systems: Steady State Mass Transfer Rates", Water Resources Research, Vol 28, No. 10, October 1992.

Powers, S.E., Abriola, L.M., and Weber, W.J., Jr., "An Experimental Investigation of Non-Aqueous Phase Liquid Dissolution in Saturated Subsurface Systems: Transient Mass Transfer Rates", Water Resources Research, Vol 30, No. 2, February, 1994.

Powers, S.E., Abriola, L.M., Dunkin, J.C., and Weber, W.J., Jr., "Phenomenological models for transient NAPL-water mass-transfer processes", Journal of Contaminant Hydrology, Vol 16, 1994.

Rao, P.S., Lee, L.S. and Pinal, R., "Cosolvency and Sorption of Hydrophobic Organic Chemicals", Environmental Science and Technology, Vol 24, 1990.

Rao, P.S., Annable, M.D., Sillan, R.K., Dai, D., Hatfield, K., Graham, W.D., Wood, A.L., and Enfield, C.G., "Field-scale Evaluation of In-Situ Cosolvent Flushing for Enhanced Aquifer Remediation", Water Resources Research, Vol 33, No. 12, 1997.

Roy, S.B., Dzombak, D.A., and Ali, M.A., "Assessment of in-situ solvent extraction for remediation of coal tar sites: Column studies", Water Environment Research, Vol 67, No. 1, 1995.

Schwille, F, Dense Chlorinated Solvents in Porous and Fractured Media, Chelsea, MI: Lewis Publishers, 1988.

Soo, H. and Radke, C.J., "The Flow Mechanism of Dilute, Stable Emulsion in Porous Media", Ind. Eng. Chem. Fundam., Vol. 23, 1984.

Tchelepi, H.A., Orr, F.M., Rakotomala, N., Salin., D., and Woumeni, R., "Dispersion, permeability, heterogeneity, and viscous fingering: Acoustic experimental observations and particle tracking simulations", Physical Fluids, Vol. 5, No. 7, July, 1993.

Van Genuchten, M.T., "a Closed-form Equation for Predicting the Hydraulic Conductivity of Unsaturated Soils", Soil Science Society Am. Journal, Vol 44, 1980.

Wilson, J.L. and Conrad, S.H., "Is Physical Displacement of Residual Hydrocarbons a Realistic Possibility in Aquifer Restoration?", Department of Geoscience and Geophysical Research Center, New Mexico Institute of Technology and Mining, 1984.

Wilson, J.L., Conrad, S.H., Mason, W.R., Peplinski, W., and Hagan, E., "Laboratory Investigation 
of Residual Liquid Organics from Spills, Leaks and Disposal of Hazardous Waste in Groundwater", US EPA, EPA/600/6-90/004, April, 1990.

Wood, A.L., Bouchard, D.C., Brusseau, M.L. and Rao, P.S.C., "Cosolvent Effects on Sorption and Mobility of Organic Contaminants in Soils", Chemosphere, Vol. 21, Nos. 4,5, 1990. 


\section{$5 \quad$ Effect of Alcohol Concentration on DNAPL Mobilization}

\subsection{Introduction and Background}

As a NAPL spill flows from the surface to the subsurface, it travels as a separate liquid phase in the vadose and saturated zones resulting in multi-phase flow. Multi-phase flow is dependent on many variables including the pressures, densities, viscosities, and interfacial tensions of the fluids present in the system. In the case of LNAPLs, the NAPL generally moves as thin films in the vadose zone with a some portion generally residing on the water table surface. The floating NAPL may move with the prevailing groundwater flow. DNAPLs, which move in the same fashions as LNAPLs in the vados zone, differ in that they will continue to move downward into the saturated zone and are not impeded by the water table. As they migrate downward by gravitational forces, a large enough pressure may exist to overcome the entry pressure of a pore and displace the water from the pore.

Depending on the size of the spill, the DNAPL may dissipate as a residual saturation or pool on an impermeable soil layer. It is important to note that the direction of DNAPL flow is generally governed by gravity and may differ significantly from the prevailing direction of ground water flow. This research is specifically concerned with the situation where a DNAPL has penetrated and established a residual saturation in the saturated zone.

The three major forces controlling the behavior of DNAPL in the saturated zone are capillary forces, viscous forces, and buoyancy or gravity forces. The capillary force is defined as the difference in cohesive forces within a liquid phase and the adhesive forces between two adjoining fluid phases (Adamson, 1990). In a soil matrix, this is expressed as the pressure difference between the advancing fluid and the receding fluid. The maximum capillary force in porous media is usually found in pore throats, where the radius of curvature of the fluid is minimized. The capillary pressure is a function of the interfacial tension, $\sigma$, between the two adjoining fluids, the wetting characteristics of the soil, and the geometry of the pore as represented by Equation 1. This equation is based on Young's equation and represents the general case for a capillary tube where the radii of curvature are the same in all directions (Adamson, 1990).

$$
P_{c}=\frac{2 \sigma \cos \theta}{r_{t}}
$$

where $P_{c}$ is capillary pressure

$\sigma$ is interfacial tension

$\theta$ is the contact angle

$r_{t}$ is the pore throat radius 
In Equation 1, $\theta$ describes the contact angle between the solid and wetting fluid, and is a measure of the affinity of the liquid for the solid. Considering a two-phase system where PCE is present in the saturated zone, the wetting fluid is water and the non-wetting fluid is PCE. Three general wetting conditions exist for such a two-phase system; a fluid is either wetting, intermediate wetting, or non-wetting. The specific condition is defined by the angle of contact that the fluid makes with the solid surface of the porous media. Small contact angles, less than 90 degrees indicate a water wet condition, where as contact angles greater than 90 degrees indicate a non-wetting fluid (Adamson, 1990). When the contact angle is zero degrees, the surface is completely coated by the wetting fluid. This condition prevails in the saturated zone, where pore spaces are completely waterfilled. When a DNAPL, such as PCE, enters the pore space, displacing water, a thin film of water remains between the DNAPL and the soil grain.

One reason the wetting condition of contaminated aquifers is assumed to completely water wet is the initial water-wet condition before the invasion of NAPL. The soil grains are coated with water before the NAPL enters the pore, and to break the water film surrounding soil grains requires a highly hydrophobic NAPL, such as coal tar (Powers and Tamblin et al., 1995). Work conducted by Wilson et al. (1990) indicates that soils containing residual DNAPL saturations remain completely water-wet.

Another reason that the water-wet condition can be considered a good assumption is the effect of the rough soil surface on the wetting characteristics of the soil. The rough surfaces of soil grains can have an effect on the contact angle (Adamson, 1990) and hence the wettability of a liquid in a twophase system. When the contact angle is less than 90 degrees, roughened surfaces result in an even lower contact angle. However, when the contact angle is greater than 90 degrees, a roughened surface will cause an even higher contact angle. The contact angle of a smooth surface and a rough surface have been related by surface area, where $r$ is the ratio of the real surface area to the smooth surface area. This ratio is always greater than 1 . The contact angle of the roughened surface is then given by the following equation:

$$
\cos \theta_{\text {rough }}=r \cos \theta_{\text {smooth }}
$$

Therefore, a water-wet soil grain with a contact angle close to but slightly greater than 0 degrees is likely to behave as a completely water-wet surface with a contact angle equal to 0 degrees.

The DNAPL blobs, therefore, which are trapped in pores remain completely separated from the soil grain by a water film. The existence of this water film throughout the porous media, insures that the aqueous phase is continuous everywhere. Conversely, trapped DNAPL in porous media exists in discontinuous blobs which are formed by the interplay of forces acting on the DNAPL as it flows through the soil.

As DNAPL travels through the saturated portion of the aquifer, it enters pore bodies and displaces the majority of the water contained in the pore. However, before entering the pore body, the DNAPL must overcome the pore entry pressure, $P_{e}$, caused by the interfacial tension of the two phases. Due 
to the three dimensionality of the soil system, the advancing fluid may find multiple pathways into one pore. Each of these pathways into the pore represents a pore throat leading to or from the pore body. The capillary pressure is determined by the largest throat radius associated with that pore. From Equation 1, the pressure acting on the water is inversely proportional to the radius of the pore. Therefore, larger pores require less pressure for DNAPLs to enter. When pressure imposed by the DNAPL at the fluid-fluid interface is greater than the pore entry pressure, water is displaced and DNAPL enters the pore. It is possible, therefore, that small pores are not invaded when larger pores are available for entry. This can lead to selective pathways or fingering within the media.

The DNAPL continues its downward migration in the saturated zone by entering pores in this manner. An impermeable layer can impede or halt DNAPL migration and cause a pool to develop. An impermeable layer would constitute a layer of soil whose pore throats are sufficiently small to prevent the DNAPL from overcoming the $\mathrm{P}_{\mathrm{e}}$ and entering the pores. If an impermeable layer is not encountered and the release of DNAPL is discontinued, the DNAPL will disperse through the soil in trapped blobs.

As a DNAPL flows through porous media the geometry of the pore spaces can cause dissociation of the flow in the DNAPL resulting in discontinuous trapped blobs of DNAPL. Entrapment of DNAPLs is complex and is dependent on properties of the soil matrix, the aqueous phase, and the DNAPL itself. Two major mechanisms can be used to explain trapping of a DNAPL in porous media. These are snap-off and by-passing (Chatzis et al., 1983, Wilson et al., 1990).

Snap-off occurs when the DNAPL exits a pore throat which is much smaller than the preceding pore body. At the pore throat a blob of NAPL is pinched off and separated from the continuous DNAPL flow (Chatzis et al., 1983, Wilson et al., 1990). Snap-off occurs in pores with a high pore body-tothroat size ratio, called the pore aspect ratio. Snap-off tends to create single pore blobs or singlets (Chatzis et al., 1983, Wilson et al., 1990) as opposed to larger blobs that comprise many pores. Powers et al. (1992) found that singlets were more prevalent in their uniform soils which contained pores of similar size with similar pore aspect ratios. Having adjacent pores that are similar in size prevents large fluctuations in pore velocity and hence, prevents the formation of blobs occupying multiple pores.

The by-passing mechanism of trapping occurs when the flow of DNAPL is diverted through two adjacent pores of varying size. When the DNAPL recedes from the pores, water imbibes into them with a higher velocity in the smaller pore. This faster flow causes water to rapidly displace DNAPL in the small pore, and cut-off or by-pass the larger pore. This by-passing action traps DNAPL in the larger pore (Chatzis et al., 1983, Wilson et al., 1990). By-passing is more likely to occur in large pores of heterogeneous soils because heterogeneous soils often have adjacent pores of differing sizes. By-passing can also occur on a larger scale in heterogeneous soils, encompassing multiple pores or lenses of soil, forming blobs referred to as branched ganglia. Powers et al. (1992) found that multi-pore size ganglia tend to form in non-uniform or poorly-sorted soils.

Entrapment of a DNAPL in the aquifer material reduces the pore space available for ground water 
flow. The volume of DNAPL trapped in the pores spaces expressed as a percentage of the total pore volume is generally referred to as the residual DNAPL saturation. Residual DNAPL saturations can range from one or two percent to as high as fifty or sixty percent. The amount of DNAPL trapped and the distribution of the DNAPL are dependant on a variety of factors. The composition of soil and its pore structure appear to greatly influence the residual DNAPL saturation. Wilson et al. (1990) indicate that porosity can affect residual saturation, however, the permeability of the soil does not affect the residual saturation providing that the groundwater velocity is low. This means that at low groundwater velocities soils with similar geometry but different permeabilities will have a similar residual saturation. This is reasonable considering that a high porosity is indicative of small pore structures, including the pore throats which affects the capillary pressure or main trapping force.

\section{Pore-scale Heterogeneities Caused by Clay}

Based on the relationship between soil pore geometry and blob geometry, it appears that soil heterogeneities caused by clay content will affect the establishment and distribution of the residual DNAPL saturation. Unfortunately, to date little research exists on the effect of clay on the formation and distribution of a residual DNAPL saturation. Considering the unique properties of clay such as clay-organic interactions and the colloidal nature of clay particles, it is difficult to postulate the effect of heterogeneities caused by clay solely on studies where heterogeneous sands were used.

The presence of non-polar, hydrophobic solvents has been shown to cause slight shrinkage in clays used for landfill liners (Green et al., 1983). The slight shrinkage of clays can cause cracking in the clay soil, and may be attributed to migration of water out of the clay lattice and into the surrounding bulk liquid (Green et al., 1983). Cracking caused by clay shrinkage leads to dramatic increases in the hydraulic conductivity of the clay. Storey and Pierce (1988) determined that increasing concentrations of a liquid hydrocarbon, methanol, increased the attraction of the clay particles, thus increasing hydraulic conductivity. From these studies, it seems that the complex electrostatic and structural properties of clays cause clay particles in the presence of organic contaminants to behave differently than fine sands or silts.

The colloidal nature of clay particles introduces another way in which clays in the aquifer may affect the behavior of DNAPLs in the porous media. Wan and Wilson (1994) showed that colloidal clay particles can adhere to the gas-water interface in glass micromodels. They noted that some unknown attractive force exists between a hydrophilic particle, such as clay, and the gas interface. In their experiments, they were able to dislodge colloids attached to the solid-liquid interface of the pore walls, but were unable to dislodge most particles attached to the gas-water interface. This result is not as easily explained and Wan and Wilson (1994) present two hypotheses. One hypothesis is that the solvation or hydration force between the gas bubble and the hydrophilic particle is attractive. Solvation explains the special molecular structure that develops in the first few layers of molecules surrounding the gas-water interface, in the case of hydration, the molecules and the bulk liquid are water. Another hypothesis developed by Wan and Wilson (1994) is that the combination of kinetic energy and the force attracting particles to the gas interface causes the particles to rupture the interface and become held by interfacial forces. If this same effect occurs with other NAPLs, it is 
possible that the adherence of colloids at the interface will change its interfacial properties, hence affecting DNAPL trapping, dissolution, and mobilization. Wan and Wilson (1994) demonstrated the complex colloidal interactions with various phases in the subsurface. This coupled with the general lack of research into the effects colloids and clay interactions on DNAPL behavior prevent full understanding of the effects that clay will have on the mobilization of DNAPL in the saturated zone during an alcohol flush.

\section{$\underline{\text { Pore Size Distribution }}$}

It is anticipated that the clay content of a soil can significantly affect the pore sizes and the distribution of pore sizes in a porous medium. The heterogeneity of the soil will change with varying clay content. The level of heterogeneity could potentially affect the pore size distribution. As the amount of clay in the soil increases more smaller pores are present which leads to an overall change in pore size. Characterization of the pore sizes and pore size distribution is required to assess the effects of clay on DNAPL behavior. Depending on the specific clay species, little interaction between the clay and DNAPL may indicate that concepts applying to heterogenous sand media are applicable when considering DNAPL behavoir. In which case, characterization of the pore sizes and the pore size distribution will provide knowledge about the topology and distribution of DNAPL blobs.

Capillary pressure-saturation relationships provide one method for determining pore size distribution. A two-phase capillary pressure-saturation test must be conducted on the soil of concern. The two phases used in the test are not important as long as the densities and interfacial tension between the two fluids are known. The test is completed by introducing a non-wetting fluid to a water-wet porous media with positive pressure on the non-wetting fluid, or a negative pressure on the wetting fluid. The non-wetting fluid, for example a DNAPL, acting under pressure enters the soil by displacing some pore water. This stage of the test is called drainage. After some maximum pressure is applied where little or no additional pore water exits the soils, the pressure is decreased and water re-enters the pores displacing the DNAPL. This stage of the test is called imbibition. During imbibition of water back into the porous media and the retreat of the DNAPL, snap-off and by-passing cause the formation of discontinuous DNAPL blobs, thus creating a residual saturation in the soil.

By slowly increasing the pressure applied to the system, discrete measurements of capillary pressure and its corresponding change in saturation can be obtained. These measurements combine on a graph of capillary pressure versus saturation to form the capillary pressure-saturation curve. The test of the relationship of capillary pressure and saturation is conducted in a soil column, where capillary pressure is measured using Equation 3.

$$
P_{c}=P_{n w}-P_{w}
$$


As the capillary pressure applied to the DNAPL-water interface increases, smaller pores with higher entry pressures are invaded. Therefore, as the capillary pressure increases, the overall water saturation decreases in the region. The largest pores have the lowest entry pressure and are desaturated first. No drainage occurs until the entry pressure of the largest pore is applied to the DNAPL-water interface. This minimum entry pressure is referred to as the bubbling pressure. Water saturation will decrease as increases in capillary pressure cause smaller pores to drain. This continues until no more water exits the system and the remaining water is contained in tiny pores or in the small water films which exist between the DNAPL and soil grains. The saturation at this point is called the irreducible water saturation. The thin film of water is interconnected and allows water to continue to flow through the soil at a greatly reduced hydraulic conductivity. Generally, soils with smaller pores exhibit larger irreducible water saturations due to the relative amount of water in thin films compared with the volume of water drained from the pore.

The soil column now contains non-wetting fluid and an irreducible saturation of wetting fluid. In order to establish a residual saturation of non-wetting fluid, or examine the hystersis properties of the media, the pressure applied to the interface of the two fluids is decreased to obtain the imbibition curve. The imbibition curve describes the influx of water back into the pores. The imbibition mimics the drainage curve but is slightly offset due to hystersis in the system. Hystersis is caused by the residual DNAPL blobs trapped in the pores preventing the water saturation from completely recovering to its initial state.

The drainage portion of the capillary pressure-saturation relationship is used to indicate the pore size distribution of soils. In the case of a soil with uniform pore sizes, the entry pressure for all the pores is very similar. Therefore, the pressure required to drain the pores to the irreducible water saturation is just slightly higher than the entry pressure. In a soil with non-uniform pore sizes, as the pressure increases, smaller pores desaturate only slightly change the overall saturation of the soil. In this case, a large pressure difference exists between initial desaturation, or entry pressure, and the pressure required to achieve an irreducible water saturation. One difference between these curves is the slope of the middle portion or straight-line portion of the graph. This difference is due to the distribution of pore sizes, therefore, the straight-line portion of the graph can be used to describe the pore size distribution of the soil.

Researchers such as Brooks and Corey (1966) and van Genuchten (1980) have developed mathematical models to fit data from a capillary pressure-saturation test. The models yield fitting parameters that in some cases can be used to describe properties of the soil. Brooks and Corey (1966) developed a method of examining the pore size distribution of a soil using a transformed plot of the data. In this method, the $\log$ of the water content, $\theta$ is plotted versus the log of the capillary pressure, $P_{c}$. The negative of the slope of the straight line portion of this graph is called the pore size distribution index, $\lambda$. As the value of $\lambda$ increases, the pore size distribution becomes increasingly smaller. Brooks and Corey (1966) show that homogeneous sands have $\lambda$ values of approximately 2 , while more heterogeneous media have values of $\lambda$ closer to 0.5 . The equation relating water content to the capillary pressure developed by Brooks and Corey is presented in Equation 4 along with the expression for effective saturation in Equation 5. 


$$
\Theta=\Theta_{r}+\left(\Theta_{s}-\Theta_{r}\right)\left(\frac{\Psi}{h_{b}}\right)^{-\lambda}
$$

where:

$\Theta=$ volumetric water content

$\Theta_{s}=$ volumetric water content at saturation

$\Theta_{r}=$ irreduciblewater content

$\psi=$ matricpotential, or capillarypressure

$h_{b}=$ bubbling pressure

$\lambda=$ experimentallydeterminedpore sizedistribution index

van Genuchten (1980) developed fitting parameters to describe the capillary pressuresaturation curve. From these parameters, $\mathrm{n}$ is dependent on the slope of the straight-line portion of the pressure-saturation curve. As the slope of the straight-line portion of the curve increases, $n$ increases indicating a larger pore size distribution. The equations presented in Equations 5-9 represent the van Genuchten estimation of pressure-saturation curves. In these equations, $\theta_{\mathrm{r}}$ and $\theta_{\mathrm{s}}$ are soil moisture properties, and $\mathrm{m}$ is estimated from the slope of the pressure-saturation curve, $\mathrm{S}_{\mathrm{p}}$.

$$
\begin{gathered}
S_{e}=\frac{S_{w}-\Theta_{r}}{1-\Theta_{r}} \\
\text { where: } \\
S_{e}=\text { effective saturation } \\
S_{w}=\text { saturation ratio, or } \Theta / \Theta_{s} \\
\text { for }\left(0<S_{p} \leq 1\right) \quad m=1-\exp \left(-0.8 S_{p}\right) \\
\text { for }\left(S_{p}>1\right) \quad m=1-\frac{0.5755}{S_{p}}+\frac{0.1}{S_{p}^{2}}+\frac{0.025}{S_{p}^{3}} \\
n=\frac{1}{(1-m)}
\end{gathered}
$$




$$
\begin{gathered}
\alpha=\frac{1}{h_{p}}\left(2^{1 / m}-1\right)^{1-m} \\
\Theta=\Theta_{r}+\frac{\Theta_{s}-\Theta_{r}}{\left[1+(\alpha \psi)^{n}\right]^{m}}
\end{gathered}
$$

These models are useful in this application because they provide a reproducible and reliable method of determining pore size distribution. When applying these models to characterize the pore size distribution, the method developed by Brooks and Corey (1966) provides a quantifiable value representing pore size distribution. This quantity, $\lambda$, can be used to compare the pore size distributions of a variety of soils, and perhaps to relate the characteristics of DNAPL trapping and mobilization in those soils. As stated earlier, little research exists relating grain size distribution and hence pore size distribution to DNAPL mobilization in the saturated zone. Currently existing predictive models for the mobilization of DNAPL were developed considering a homogeneous media. The addition of heterogeneity, especially clay, is likely to affect the manner in which these models are used.

\section{Mobilization of DNAPL}

Predictive models for mobilization of NAPLs in the subsurface have been used for many years by the petroleum industry. Recently, Pennell et al. (1996) and Dawson and Roberts (1997) developed models specifically for the mobilization of DNAPLs in the saturated zone. In addition, Hunt et al. (1988a) developed a method for determining the critical blob length where mobilization will occur in homogeneous media. These models are based on the models previously developed by petroleum engineers for mobilization and recovery crude oil, an LNAPL.

A reduction in NAPL saturation by mobilization has been predicted for crude oil in a sandstone aquifer using a dimensionless number called the Capillary Number, $\mathrm{N}_{\mathrm{c}}$. The Capillary Number is defined as the ratio of the viscous forces to the capillary forces (Perry and Chilton, 1972), and is given in Equation 10.

$$
N_{C}=\frac{q_{a q} \mu_{a q}}{\sigma_{a q} \cos \theta}
$$


In this equation, $q_{a q}$ is the darcy velocity, $\mu_{\mathrm{aq}}$ is the viscosity of the aqueous solution phase, $\sigma_{\mathrm{aq}}$ is the interfacial tension between the aqueous and non-aqueous phases, and $\theta$ is the contact angle which is considered to be $180^{\circ}$ for a water-wet system. Water flooding studies conducted by the petroleum industry have determined that NAPL saturation is strongly dependent on the interfacial tension between the two fluids and the wettability of the solids with respect to the NAPL, as measured by the contact angle (Batycky, 1981, Holm, 1986). Generally, these studies determined that water floods can not attain Capillary Numbers large enough to greatly decrease NAPL saturation. NAPL desaturation generally occur at values of $\mathrm{N}_{\mathrm{c}}$ greater than $1 \times 10^{-5}$. Therefore, without chemically altering the Capillary Number, it is not feasible to mobilize NAPLs at a residual saturation.

Few studies in the petroleum industry studied the movement of DNAPLs in the subsurface due to gravitational forces (Morrow and Songkran, 1981; Morrow et al. 1988). In these studies, gravitational forces became more significant in movement of DNAPLs when the Capillary Number (or effect of viscous forces) is low. The gravitational force is compared to the viscous force by using a dimensionless number called the Bond Number. The Bond Number is the ratio of gravitational forces to capillary forces (Perry and Chilton, 1973), and is given in Equation 11.

$$
N_{B}=\frac{\Delta \rho g r^{2}}{\sigma_{a q}}
$$

In this equation, $\Delta \rho$ is the difference in density between the two phases ie. $\left(\rho_{\text {NAPL }}-\rho_{a q}\right), g$ is the gravitational constant, and $r$ is the particle radius.

In their analysis of oil entrapment in glass bead columns, Morrow et al. (1988) used this form of the Bond Number containing the radius of packing material rather than the permeability. However, because this form of the Bond Number is derived on a microscopic scale, and the capillary number is derived on a macroscopic scale, Morrow et al. (1988) found it necessary to multiply the Bond Number by 0.001412 in order to compare the two numbers. Others have been able to derive the Bond Number on a macroscopic scale using permeability instead of using the particle radius. These forms of the Bond Number are presented in Equation 12 (derived by Dawson and Roberts, 1997) and Equation 13 (derived by Pennell et al., 1996). In these equations $k$ is permeability, $\mathrm{k}_{\mathrm{rw}}$ is the ratio of the permeability with a NAPL residual to the original permeability, and $\mathrm{n}$ is porosity.

$$
N_{B}=\frac{\Delta \rho g(k / n)}{\sigma_{a q}}
$$


The residual saturation of NAPL will likely affect both the porosity and permeability. Because Equation 2-13 addresses the condition of the soil after NAPL emplacement it is attractive with this application and was chosen to be used in this work.

$$
N_{B}=\frac{\Delta \rho g k k_{r w}}{\sigma_{a q} \cos \theta}
$$

The same three forces, capillary, viscous, and gravity forces, that act on flowing DNAPL influence the trapped DNAPL blob. Unlike the case where the DNAPL is flowing, the forces acting on a trapped DNAPL blob keep the system in static equilibrium. This means that for every force acting on the blob, there is an equal and opposite force.

Pennell et al. (1996) neglected viscous forces in their development of a model to describe trapping because shear forces are not substantial compared to the other forces acting in this system. Hence the main forces considered are the capillary forces at the pore throats and the gravitational force. The force balance is presented in analytical terms in Equation 14. In this equation, the capillary pressures acting at the top and bottom of the blob are functions of the interfacial tension between the DNAPL and the aqueous phase.

$$
\begin{aligned}
& 0=P_{C R}\left(\pi r_{b}^{2}\right)-P_{C A}\left(\pi r_{b}^{2}\right)-\rho_{o} g\left(\pi r_{b}^{2}\right) \Delta l \sin \alpha \\
& P_{C R}=\text { receding capillarypressure } \\
& P_{C A}=\text { advancing capillarypressure } \\
& \pi r_{b}^{2}=\text { area ofDNAPLblob normal to vectorl } \\
& \rho_{o}=\text { density oforganicorDNAPL } \\
& g=\text { gravitational constant } \\
& \pi r_{b}^{2} \Delta l=\text { approximated DNAPL blob volume } \\
& \alpha=\text { angle between the horizontal axis and the direction offlow }
\end{aligned}
$$

Using this relationship in conjunction with the force balance and Darcy's law of flow, Pennell et al. (1996) developed a theoretical model for a total trapping number, $\mathrm{N}_{\mathrm{T}}$. The trapping number is a dimensionless number that describes the potential for DNAPL mobilization in the pore. Assumptions made while developing this model include similar advancing and receding contact angles, and isotropic medium. Considering the current applications of this model are mostly laboratory columns, these assumptions appear valid. However, in an aquifer it is unlikely that no 
hystersis will occur or that the aquifer will be isotropic.

The model developed for mobilization incorporates the Capillary Number, $\mathrm{N}_{\mathrm{C}}$, and the Bond Number, $\mathrm{N}_{\mathrm{B}}$. The trapping number equation is given in Equation 15 using the dimensionless Capillary and Bond Numbers. Simplifications of the trapping number made by assuming horizontal and vertical flow are given in Equations 16 and 17.

$$
N_{T}=\sqrt{N_{C}^{2}+2 N_{C} N_{B} \sin \alpha+N_{B}^{2}}
$$

When flow is horizontal and $\alpha=0$ :

$$
N_{T}=\sqrt{N_{C}{ }^{2}+N_{B}{ }^{2}}
$$

Then flow is vertical and $\alpha=90^{\circ}$, in the direction of the buoyancy force:

$$
N_{T}=\left|N_{C}+N_{B}\right|
$$

The capillary number, $N_{c}$, in the above equations explains the viscosity forces acting on the blob in relation to the capillary forces. Viscous force enters into the trapping number model when Darcy's law is used to explain the change in pressure across the blob. Therefore, the viscous force acting on the blob is dependent on Darcy's flow, $\mathrm{q}_{\mathrm{w}}$, and hence, the media permeability and the system pressure gradient. The viscosity force describes the force on the blob due to the flowing aqueous phase. The capillary number is therefore an indicator of the magnitude of the force due to flow versus the forces holding the blob in place. Essentially, the viscosity force attempts to move the blob while the capillary force attempts to hold it in place. The bond number, $\mathrm{N}_{\mathrm{B}}$, explains the gravitational force on the blob in relation to the capillary force. The buoyancy force or gravitational force is a function of the density difference between the two fluids. The gravitational force attempts to pull the DNAPL blob vertically down while the capillary force resists and attempts to hold the blob in place.

Pennell et al. (1996) found mobilization of PCE in homogenous Ottawa white sand during surfactant flushing at values of trapping number greater than $2 \times 10^{-5}$. The results yielded large changes in saturation for four groups of homogenous sand varying in grain size. This indicates that grain size does not appear to affect the trapping number at mobilization for sands, as long as the material is fairly homogeneous. Both vertical and horizontal displacements were observed in their study. Horizontal displacement studies indicated that particle size distribution affected PCE mobilization. PCE continued to mobilize at trapping numbers greater than $2 \times 10^{-5}$, however, the result of the mobilization was different in the well-graded material. In a well-graded material, Pennell et al. (1996) observed PCE migration on a $45^{\circ}$ angle, where as in the well-sorted material, the PCE migrated directly downward appearing to pool at the bottom of the column. Pennell et al. (1996) concluded that the trapping number model developed using permeability in the Bond Number 
worked well and may be appropriate for general application after further laboratory and field-scale studies.

Pennell et al. (1996) used three different surfactants in their flushing experiments to vary interfacial tensions. Our work considers the effect of alcohol on mobilization of PCE at a residual saturation. The results obtained by Pennell et al. (1996) are likely similar to what is expected to be observed for alcohol since the mobilization mechanisms are similar. The only difference is the development of micellular solutes. However, micellular solubilization is expected to affect dissolution more than mobilization. Also, in their study, Pennell et al. (1996) minimized formation of micelles by injecting less than 2 pore volumes of each surfactant solution into the columns.

A similar model to the Pennell et al. (1996) model is presented by Dawson and Roberts (1997). These models for mobilization are based on the specific situation where the DNAPL residual is present in the media as singlets. This assumption appears valid for homogeneous media, however, studies discussed earlier indicate that heterogeneous media may contain a DNAPL residual in the form of multi-pore blobs or ganglia. It appears that the previously presented models may not be appropriate for predicting mobilization in heterogeneous media.

Hunt et al. (1988a) developed a model to predict the critical DNAPL length required to enter a pore (overcome the pore entry pressure) for a continuous DNAPL phase or a multi-pore blob. The length term is important here because DNAPL can have an appreciable pressure contribution due to head. For example, a ganglia that is vertically oriented may have a pressure head at the bottom of the ganglia where the trapping is occurring that is many pore lengths long. This situation is represented in Figure 5.1. The critical blob length is dependent on the pore geometry, the DNAPL density, and the interfacial tension between the two phases. The equation for critical blob length is given in Equation 18.

$$
L_{\max }=\frac{2 \sigma_{o w}}{r_{t} g \Delta p} \text { where } r_{t}=\text { radius of pore throat }
$$


The equation for critical blob length appears to be a ratio of the capillary forces to the gravitational forces, or the inverse of the Bond Number. However, this form of the Bond Number is slightly different than the Bond Number presented in Morrow et al. (1988) because the radius used here is the pore throat radius, not the particle radius. Using Equation 2-16, it is possible to determine the maximum blob length trapped by a pore with a pore throat radius, $r_{r}$. The actual length of the ganglia trapped by the pore throat is likely to be less than the maximum length calculated with Equation 16 . However, a range and distribution of blob sizes can be determined based on the distribution of pore throat sizes. A variety of methods can be used to determine the average pore throat radii or distribution of pore throat radii in a porous media. The pore throat can be estimated from grain size as 0.077 times the particle diameter assuming a rhombohedral packing of spherical media (Berg, 1975). Using this estimation of pore throat size, Hunt et al. (1988a) determined that for trichloroethylene (TCE) in soil, the critical length decreased an order of magnitude for an order of magnitude increase in particle size. Therefore, it seems that knowledge of the porous media pore size distribution could be critical to understanding and predicting mobilization.

The models developed by the petroleum industry and refined by Pennell et al. (1996) and Hunt et al. (1988a) have been used to predict mobilization of DNAPL in porous media. By changing the system, however, the potential for mobilization is also changed. The initial subsurface conditions of pore topology, pore size distribution, grain size, aqueous phase and DNAPL properties, and groundwater flow patterns create a specific trapping number that is indicative of the residual saturation achieved in that media. To mobilize the blobs trapped by the initial subsurface system, the system must be changed. It is possible to change the subsurface system by imposing a groundwater gradient, hence changing the flowrate and velocity, or altering the aqueous phase and changing the interfacial tension between the two phases. These are the most common and easily implemented methods for mobilizing trapped DNAPL.

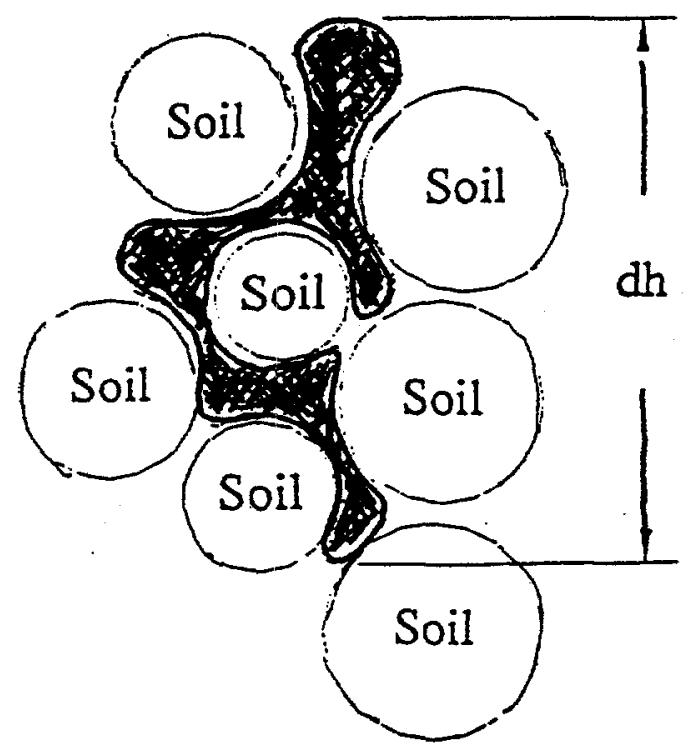

Figure 5.1 Multi-pore ganglia with head component of force at the interface. 


\section{Alcohol Flushing}

Alcohol flushing is considered a possible DNAPL remediation technique due to its ability to lower interfacial tension between the DNAPL and the aqueous phase (Boyd and Farley, 1990). Lowering interfacial tension between the DNAPL and aqueous phase also means a significant increase in dissolution of the DNAPL into the surrounding aqueous phase. However, it may cause the DNAPL to mobilize. Generally, the goal of an alcohol remediation scheme in treating a DNAPL residual is to enhance dissolution without mobilizing the DNAPL. Mobilization of a significant portion of the DNAPL could cause it to migrate to an undesirable or unknown location.

Understanding the magnitude of the enhanced dissolution by alcohol flushing explains why this technology is considered important and viable for DNAPL remediation. An interfacial tension(IFT) of 43.5 dynes $/ \mathrm{cm}$ between water and PCE with an aqueous phase solubility of $162 \mathrm{mg} / \mathrm{L}$, and an IFT of 1.32 dynes $/ \mathrm{cm}$ at a $50 \%$ isopropyl alcohol solution was determined. The aqueous phase solubility of PCE in this solution must be characterized using a ternary diagram because the system has entered the one phase flow regime. However, the solubility could be as high as $100,000 \mathrm{mg} / \mathrm{L}$. This represents a $97 \%$ decrease in interfacial tension and almost a 200 fold increase in solubility. Based on this, sites that were estimated to take 100 years to clean up by dissolution could take less than one year to remediate using alcohol flushing. However, other factors that may limit the effectiveness of an alcohol flush must also be considered when evaluating this technology.

A major consideration in alcohol flushing is adequate contact between the NAPL blobs and the alcohol solution. When a NAPL blob is occupying the majority of space in a pore, only the thin aqueous phase films along the soil grains are available for dissolution. Both equilibrium and nonequilibrium mass transfer can occur across these films depending on the flowrate of the aqueous phase (Powers et al., 1991; Powers et al., 1992; Hunt et al., 1988b). Flow in the films surrounding DNAPL blobs can be significant especially considering the hydraulic gradient imposed during a flushing scheme.

Powers et al. (1992) developed a phenomenological model for the steady state mass transfer of contaminant from the DNAPL to the aqueous phase. Using column experiments they found that the rate of dissolution was dependent on the distribution of entrapped NAPL and the aqueous phase velocity. The mass transfer model was developed using the grain size and grain size distribution of the media to estimate the distribution of the entrapped NAPL. Some laboratory-scale studies support the local equilibrium assumption (Miller et al., 1990; Fried et al., 1979). However, other experimental and field studies indicate that mass transfer from the DNAPL to the aqueous phase is a non-equilibrium process (Hunt et al., 1988b; Powers et al., 1991). Several hypotheses of the rate limiting condition have been suggested. These hypotheses include:

1. rate limited mass transfer between the films;

2. physical by-passing of the contaminated region on both macroscopic and microscopic scales due to the low aqueous phase permeability; or,

3. non-uniform flow of the aqueous phase due to soil heterogeneities. 
Rate-limited mass transfer from the DNAPLs to the aqueous phase will decrease the effectiveness of alcohol flushing schemes. Some of the hypotheses affecting the dissolution of a trapped DNAPL blob may also affect the potential to mobilize the blob. By-passing of the contaminated regions of the aquifer will prevent enhanced dissolution and mobilization.

Overall, it appears that alcohol flushing is a promising technology for NAPL remediation (Boyd and Farley, 1990; Brandes and Farley, 1993; Imhoff et al., 1995; Hayden and Van der Hoven, 1996). However, mobilization of DNAPLs pose the risk of spreading contamination and reducing the effectiveness of this technology. Understanding and characterizing this factor is necessary prior to widespread use of alcohol flushing as a DNAPL remediation scheme.

\subsection{Methods}

Capillary pressure-saturation relationships were determined for at least three columns of each sand/clay mixture studied. The main purpose was to characterize the pore size distribution of the porous media in the column. The second function of the capillary pressure-saturation test was to establish a residual saturation of $\mathrm{PCE}$ in the column media, although a complete $\mathrm{P}_{\mathrm{C}}-\mathrm{S}$ test is not required to establish a residual.

The methodology for the tests was based on the Tempe cell pressure-saturation test but with some modifications, including the glass/stainless steel columns and ceramic pressure plates on both sides. A pressurized reservoir provided PCE to the system, and draining water was collected in a burette. The PCE flowed into and out of the bottom of the column, while the water flowed into and out of the top of the column. A schematic of the capillary pressure-saturation setup is shown in Figure 5.2.

The test was performed by applying increasing increments of air pressure to the PCE reservoir. The air pressure was supplied at $110 \mathrm{psi}$ by the building air compressor. Two pressure regulators were used to control the pressure delivered to the column. The first regulator dropped the pressure to 10 psi, the second regulator provided variable air pressure to the PCE reservoir. The pressure applied to the reservoir varied from 0 mbars to 250 mbars.

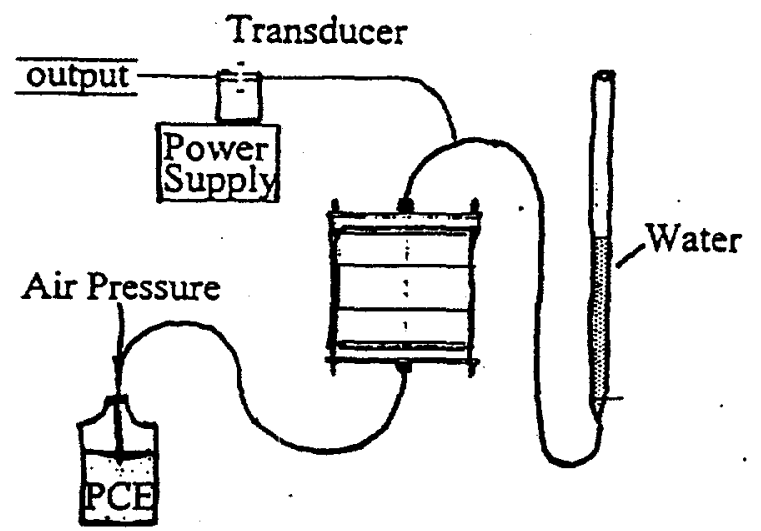

Figure 5.2 Set-up of capillary pressure-saturation test. 
For each pressure applied, water was allowed to drain out of the column, and then was measured in the burette. The pore water drained from the column indicated the reduction in water saturation at the applied capillary pressure. The saturation, $\mathrm{S}$, was determined using Equation 20 . This equation is based on the pore volume of the column, PV, and the assumption that initially all the pores are filled with water. The capillary pressure is then plotted versus the saturation to give the capillary pressure-saturation curve.

$$
S=\left(1-\frac{V_{\text {drained }}}{P V}\right) * 100
$$

In standard capillary pressure-saturation tests, a test cell requires 12 to 24 hours to come to equilibrium with the applied pressure. To speed up the test, some capillary pressure-saturation tests were completed using transducers (PX170 Series from Omega). The transducers were placed on the water drainage line from the column to the burette, and measured the pressure observed by the pore water. Once the majority of the pore water had drained, or the change in the volume drained from the column was less than 0.1 milliliter per hour, the burette was closed, and the pressure was measured by the transducers. This pressure indicated the pressure of the pore water or PCE/water interface at that time, which was usually slightly below the applied pressure.

The ceramic plates were used on both sides of the column to assure that all pores were subjected to the applied pressure. Use of plates on both sides of the column has not been done by other researchers, but it allows establishment of the better residual saturation. The ceramic plates have an entry pressure much lower than the soil entry pressures and thus prevents breakthrough of PCE when water is draining or breakthrough of water when the PCE is draining. The ceramic plates had a rated entry pressure of 1 bar for air entry into water saturated plates. For entry of PCE, the entry pressure into the plate is lower due to the difference in interfacial tensions between water-PCE and water-air. The interfacial tension of PCE/water is about $60 \%$ the interfacial tension of air/water, therefore, the maximum pressure that could be applied to the PCE in the system was about 0.6 bars or 600 mbars. The soils appeared to approach an irreducible water saturation at 250 mbars for all media.

The ceramic plate used to pass PCE into and out of the column was hydrophobized using Glassclad 18 manufactured by United Chemical Technologies, Inc. The Glassclad 18 was applied to both sides of each ceramic plate used for PCE. The plates were only treated once, unless water breakthrough occurred. Before placing the plates on the columns, they were soaked in PCE for 12 to 24 hours to wet the surfaces. The combination of hydrophobizing and wetting the plate with PCE rendered the plate effective for excluding water from flowing completely through the column.

Although some problems occurred, overall, the methodology used to determine capillary pressuresaturation relationships worked well. Some columns developed leaks on the water end of the column, leading to some overestimation of the water saturation in the column. Occasionally, 
breakthrough of the ceramic plate would occur, meaning that PCE would flow through the plate and hence through the column. When possible, in these instances, data points up to breakthrough were used to determine a capillary pressure-saturation relationship. The main reason for breakthrough seemed to be inadequate wetting of the plates prior to introduction of the other fluid. Therefore, water plates were allowed to sit on the columns, attached to a water reservoir, for twelve to twentyfour hours prior to the introduction of PCE.

\section{Determining Pore Size Distribution}

The pore size distribution of a media can be determined using direct methods or indirect measurement techniques. An example of a direct method is a scanning electron microscope where a cross-section of the media is photographed and pores sizes are measured from the photograph. Using data from a capillary pressure-saturation test is an indirect method of measuring pore size distribution and was used to understand the pore size distribution of these soils. The slope of the middle or straight-line portion of the capillary pressure-saturation curve indicates the distribution of pores. A uniform pore size distribution has a shallow slope or horizontal line. This indicates that the entry pressure of all the pores is the same. A well distributed population of pore sizes is indicated by a steep or vertical line. Figure 5.3 provides a sample of a uniform and non-uniform pore size distribution.

A pore size distribution index can be used to numerically quantify the pore size distribution using capillary pressure-saturation data. Brooks and Corey (1966) developed the pore size distribution index, $\lambda$, as the negative slope of the straight line portion of the graph of the log of the capillary pressure versus the log of saturation. As the value of the index increases, the pore size distribution becomes more uniform. Values of 2 are typical for sands and media with non-uniform pore sizes can have values of $\lambda$ as low as 0.5 . This index was calculated for all capillary pressure-saturation tests completed in this study.

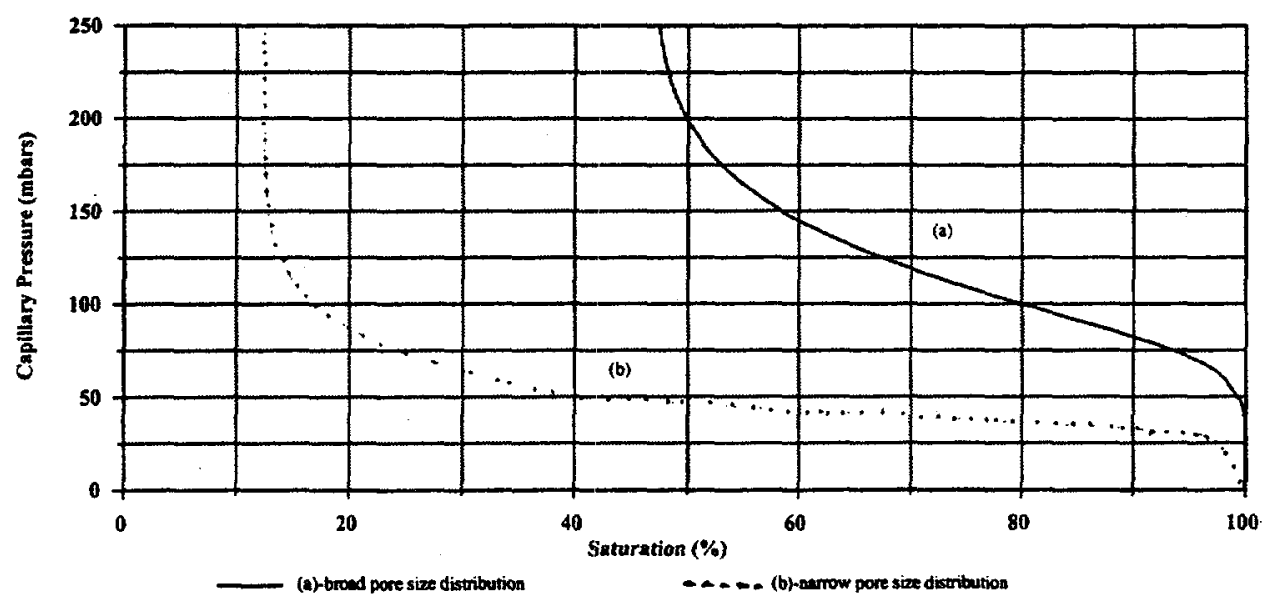

Figure 5.3 $\mathrm{P}_{\mathrm{c}}$-S curves for (a) broad pore size distribution and (b) narrow pore size distribution. 


\section{Establishing a Residual}

A discontinuous, residual PCE saturation was established in all of the columns as a result of the capillary pressure-saturation tests. The goal of all the tests was to apply PCE to the column at a pressure of 250 mbars. This was not always achieved because the seals on either side of the PCE plate leaked. If the leak was sufficient, no PCE would enter the column. Leaks usually developed three to five days into the test at the higher pressures. All tests achieved a pressure of at least 100 mbars. After completely draining at a pressure of 250 mbars, the pressure on the PCE reservoir was removed and the PCE was allowed to drain while water imbibed into the column. The PCE drained for 1 to 2 days until no more water entered the column. The remaining PCE was assumed to exist as discontinuous blobs in the media. At this point the plates were removed and screens were placed back on the ends of the columns. Water was flushed downward through the soil column to assure that the PCE was discontinuous and trapped. A second hydraulic conductivity test was then performed and the column was flushed with an alcohol solution.

Due to small but significant leaks and losses, the residual saturation of the columns could not be determined using either the volume of water that entered and exited the column, or the volume of PCE that entered and exited the column. The water volumes were not accurate because it appeared that even though the hydrophobicized plate prevented water breakthrough, they may have absorbed some water. Therefore, it appeared that more water entered the column during imbibition than exited the column during drainage. The PCE volumes could not be used because the seals at the hydrophobicized plate inevitably leaked slightly and prevented accurate measurement of the PCE entering the column. It is important to note that even small errors in the volumetric amounts of water or PCE entering/exiting the column could lead to high percent errors. An error of 1 milliliter in PCE in the column could translate to a $33 \%$ error in the residual saturation. The volumetric methods work better on large columns where small errors in volume measurement do not translate to large errors in estimation of residual saturation.

Because the residual saturation could not be determined using the volumes, it was determined by quantification of the column effluent during alcohol flushing. In the two-phase region of flow, it seemed reasonable to assume that the effluent contained a saturated PCE concentration. According to the ternary diagram for PCE, water and isopropyl alcohol, given in Figure 3-, one phase flow can occur at approximately $45 \%$ IPA. The ternary diagram describes the state of a one-phase or twophase solution containing isopropyl alcohol, PCE and water. The area above the curved line in the diagarm represents the area of a one phase solution. Therefore, for alcohol flushing solutions of 40 to 100 percent gas chromatography (GC) analysis was required to determine the concentration of PCE in the effluent. The GC samples were analyzed using a Hewlett Packard Model 19395 headspace sampler and Hewlett Packard Model 5890 Series II Gas Chromatograph with flame ionization detector.

The residual saturation of each column was estimated based on a combination of solubility data, concentration data, and the volume flushed through the column. The values of PCE solubility in 
various solutions of isopropyl alcohol presented in Chapter 3 (Figure 3.4) were used.

\section{Alcohol Flush}

The flow rate used in the alcohol flushing experiments was $10 \mathrm{~cm}^{3} / \mathrm{hr}$. In the columns, this flowrate translated to a linear velocity of $1.146 \mathrm{ft} /$ day. This exceeds the reported velocity of $0.225 \mathrm{ft} /$ day in the M-area of the Savannah River Site by a factor of five (Looney et al., 1992), however, an increased flowrate and hence linear velocity would be anticipated in the field due to the induced gradient created during flushing between wells.

After determining permeability by flushing water up through the column, the trapping number for the column was determined based on the permeabilities and percent alcohol to be used. An appropriate alcohol solution was chosen so that no mobilization would occur $\left(\mathrm{N}_{\mathrm{T}}>2 \times 10^{-5}\right)$. This alcohol solution was usually 30 percent isopropyl alcohol by volume. To begin the alcohol flush, at least three pore volumes of water were flushed down the column, in the direction of gravity, using a Harvard Apparatus syringe pump set at $10 \mathrm{~cm}^{3} /$ hour. This initial water flush insured that the PCE saturation was trapped at the viscosity forces imposed by the $10 \mathrm{~cm}^{3} /$ hour flowrate. Then a syringe containing the isopropyl alcohol solution to be used, based on the trapping number, was placed in the pump and connected to the column. At least three pore volumes of the alcohol mixture passed through the column at $10 \mathrm{~cm}^{3} /$ hour. If any mobilization occurred, the volume of mobilized PCE was measured for that alcohol treatment. Column effluent was collected in $100 \mathrm{ml}$ vials. The mobilized PCE blobs were extracted from the vials and measured using a 100 microliter syringe.

After mobilization of PCE for that alcohol treatment ceased, or at least three pore volumes were pumped through with no mobilization evident, de-aired, distilled water was re-introduced to the column for at least three pore volumes before the next alcohol treatment. This ensured that no interaction between the tail of the previous treatment and the front of the current treatment interfered with mobilization observations. The amount of dispersion along the front through the $4 \mathrm{~cm}$ column was considered to be very low based on other studies conducted using similar soils and the same flowrates (Farrell, 1998). The next alcohol treatment used was usually $10 \%$ greater than the previous flush. This increase in percent alcohol continued until appreciable mobilization had occurred in the column. Each flush was completed with approximately three pore volumes of $100 \%$ isopropyl alcohol to completely clean out the column. This final effluent was sampled as were all other effluents at $40 \%$ or greater alcohol concentration to determine the amount of PCE originally in the column.

\subsection{Results and Discussion}

\section{Capillary Pressure-Saturation Relationships}

Capillary pressure-saturation relationships were determined for the majority of the columns. The data obtained from the tests were plotted and are shown in Figures 5.4-5.8 for each soil mixture used. A fitted line based on the model developed by van Genuchtan (1980) is also provided in these graphs 
- The data are easily modeled using this well-established technique, and are considered to provide reasonable information about the capillary pressure-saturation relationships of these soils.

Problems occurring at the end of some of the tests contributed to errors in determining the irreducible water saturation, $\Theta_{\mathrm{r}}$ as shown in Figures 5.4, 5.5 and 5.6. Leaks on the drainage side of the experiment caused water to escape the system instead of being measured as part of the saturation. Many of these leaks occurred in the early experiments and were due to failure of previously used orings. When the source of the problems was determined, new o-rings were used for each capillary pressure-saturation test. However, the important part of the capillary pressure-saturation relationship for determining the pore size distribution index is the straight-line portion of the curve. Therefore, the errors at the high capillary pressure were not as much of a concern, if the data still provided information about the pore size distribution.

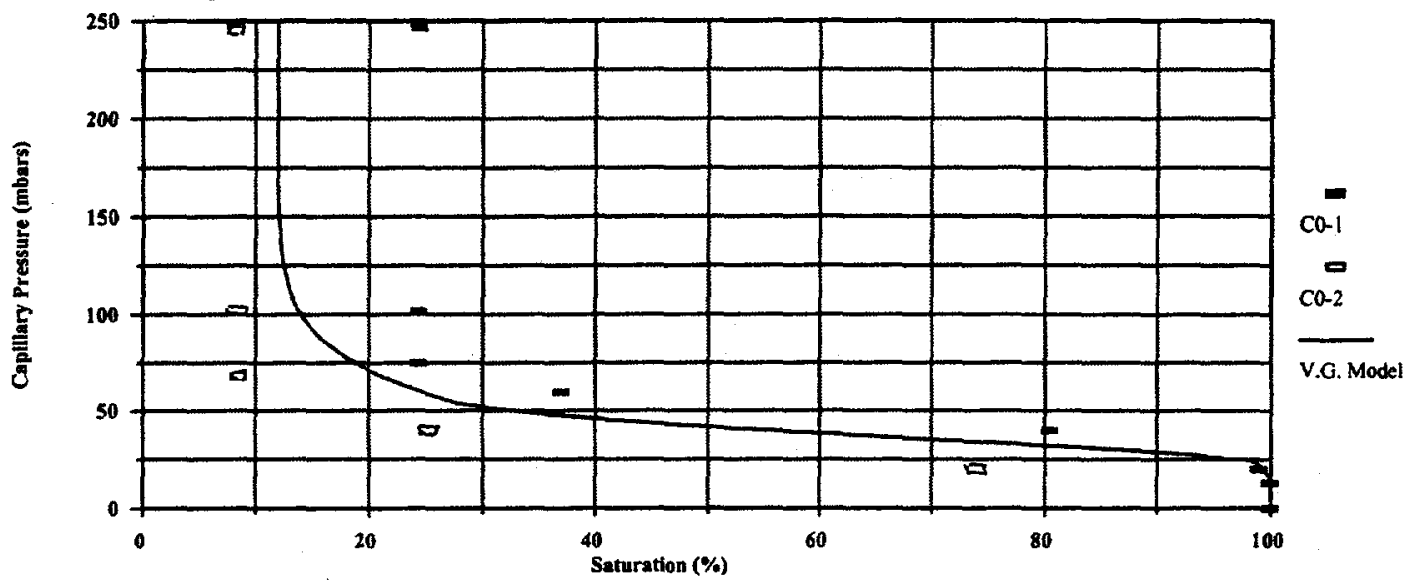

Figure 5.4 Capillary pressure-saturation relationships (PCE-water) for columns containing Ottowa white sand.

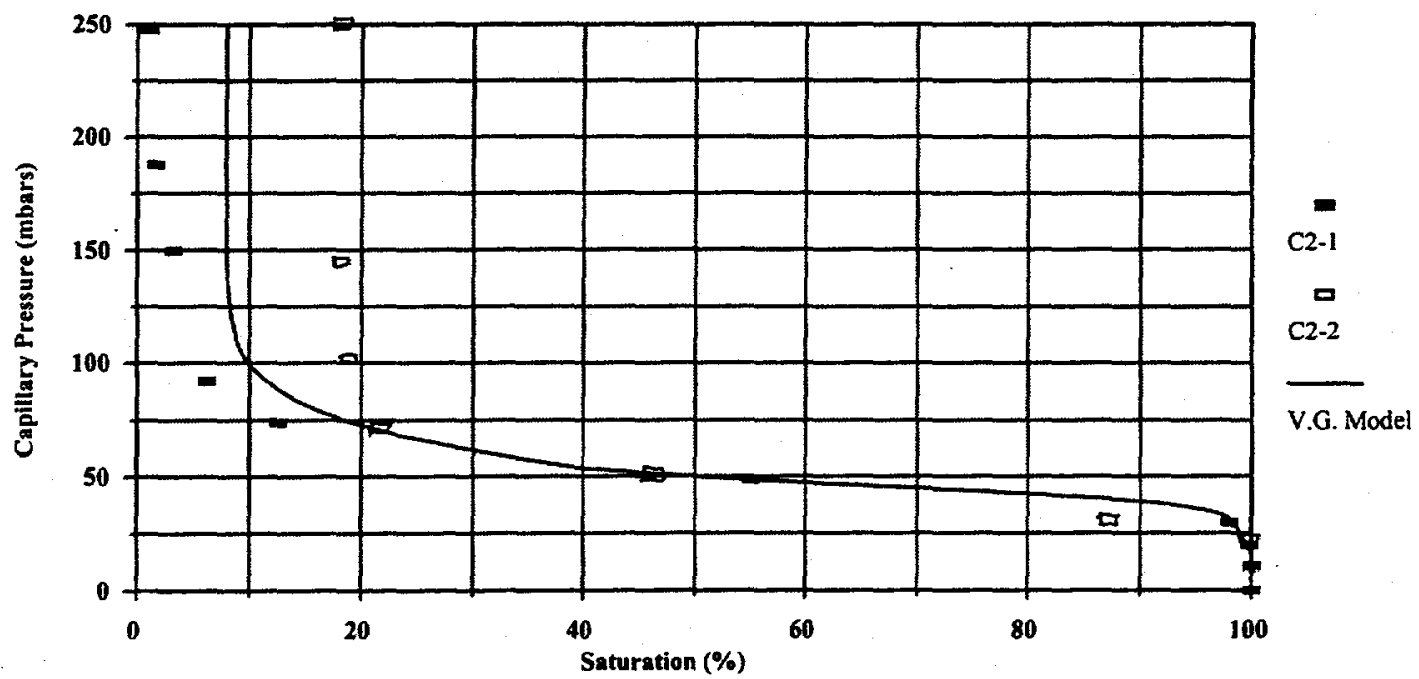

Figure 5.5 Capillary pressure-saturation relationships (PCE-water) for the columns containing $2 \%$ clay-sand mixture. 


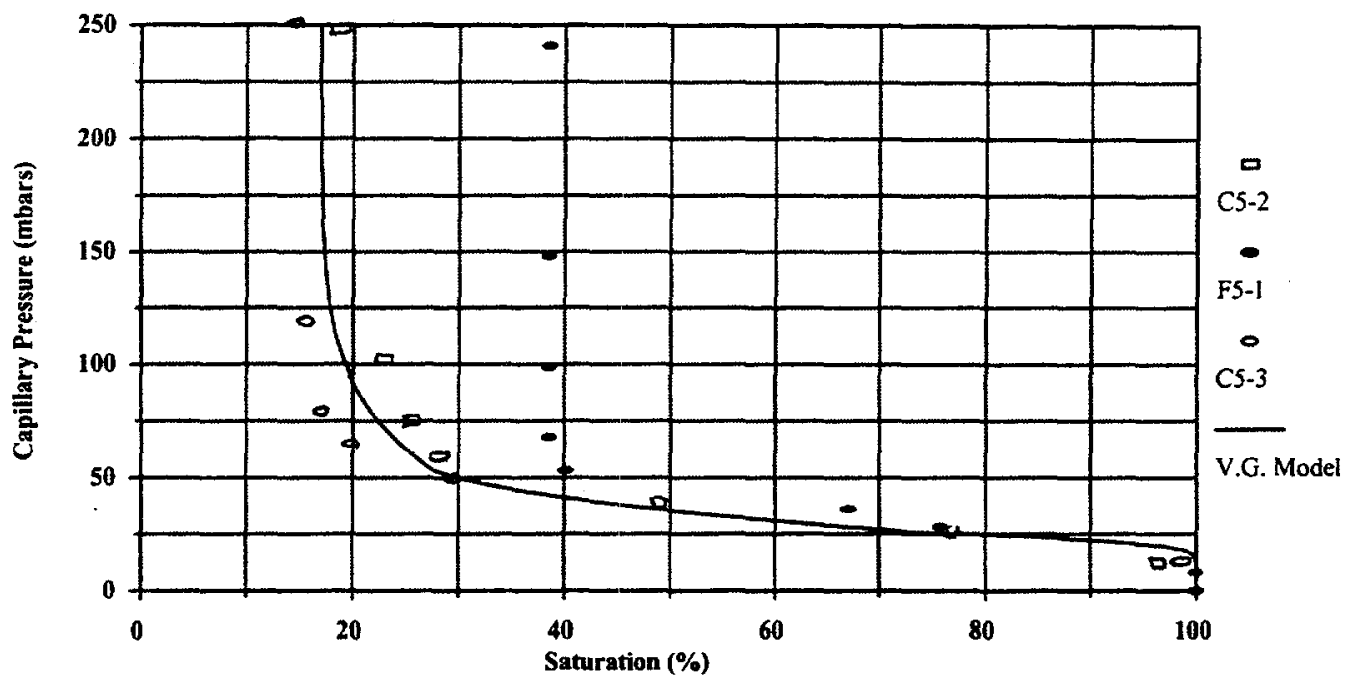

Figure 5.6 Capillary pressure-saturation relationships (PCE-water) for the columns containing $5 \%$ clay-sand mixture and $5 \%$ fine sand-sand mixture (F5-1).

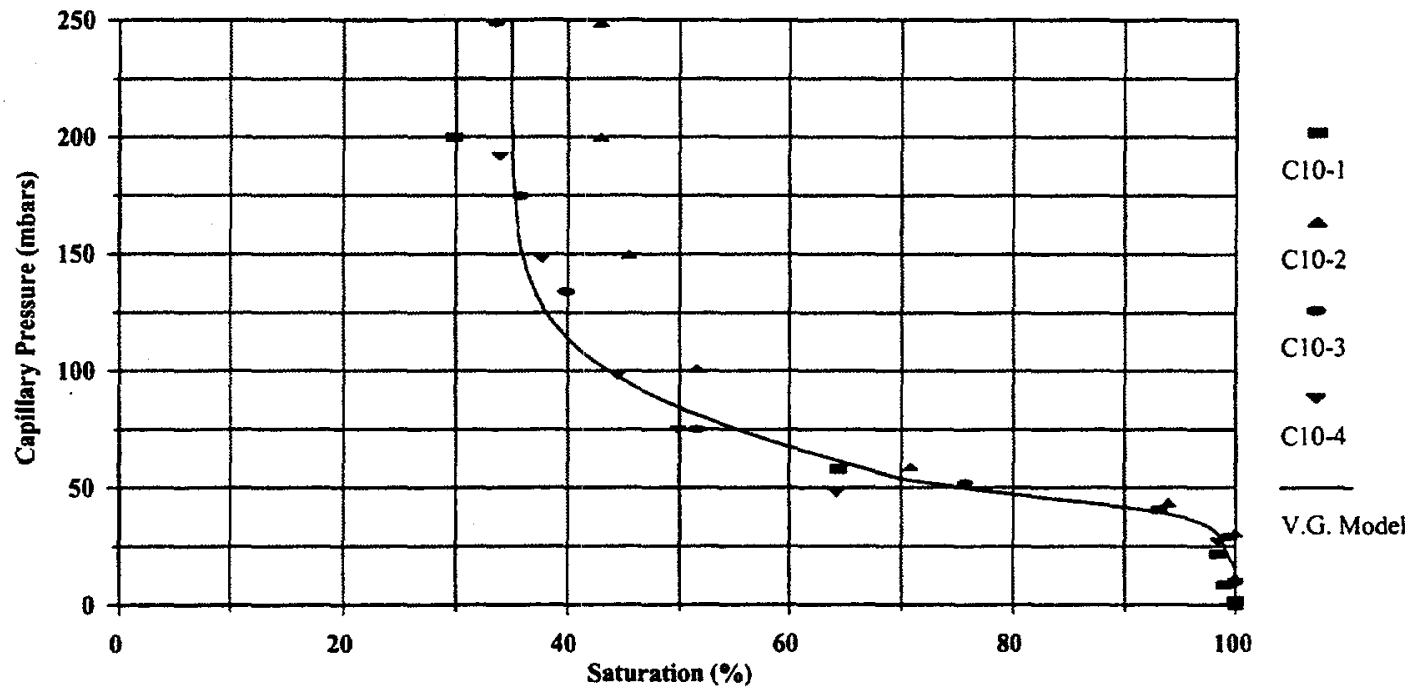

Figure 5.7 Capillary pressure-saturation relationships (PCE-water) for the columns containing $10 \%$ clay-sand mixture. 
Note that columns C20a-1 and C20a-2 shown in Figure 5.8 are packed differently, and hence, yield a different $\mathrm{P}_{\mathrm{C}}-\mathrm{S}$ relationship. It is also interesting to note that the entry pressure of the soils was generally similar regardless of clay content. This indicates that some pores are not changed by the presence of clay, or that the clay is not evenly distributed throughout the media. However, it does appear that the presence of the clay increases the irreducible water saturation of the media, increasing from about $10 \%$ in the $0 \%$ material to about $40 \%$ in the $20 \%$ clay material. One possible explanation for this is the thicker water films that develop as a result of the clay in the pores. Another explanation is that colloidal clay particles may move and clump in pore throats increasing the capillary pressure and making drainage of pores more difficult. Clay is also highly hydrophilic, even kaolinite clay, which may increase the adsorption/retention of water.

The capillary pressure-saturation curves also indicate that the pore size distributions vary for the soil mixtures. The pore size distribution is determined based on the slope of the straight-line portion of the curve.

\section{Pore Size Distribution}

The pore size distribution index, $\lambda$, developed by Brooks and Corey (1966) was determined for each capillary pressure-saturation relationship. The index is defined as the negative slope of the straight line portion of the $\log$ capillary pressure versus saturation curve. Values of the pore size distribution index are presented in Table 5.1. Typically materials with a homogenous pore size distribution have a $\lambda$ value of 2.0 , while more heterogenous pore size distributions have $\lambda$ values closer to 0.5 (Corey,

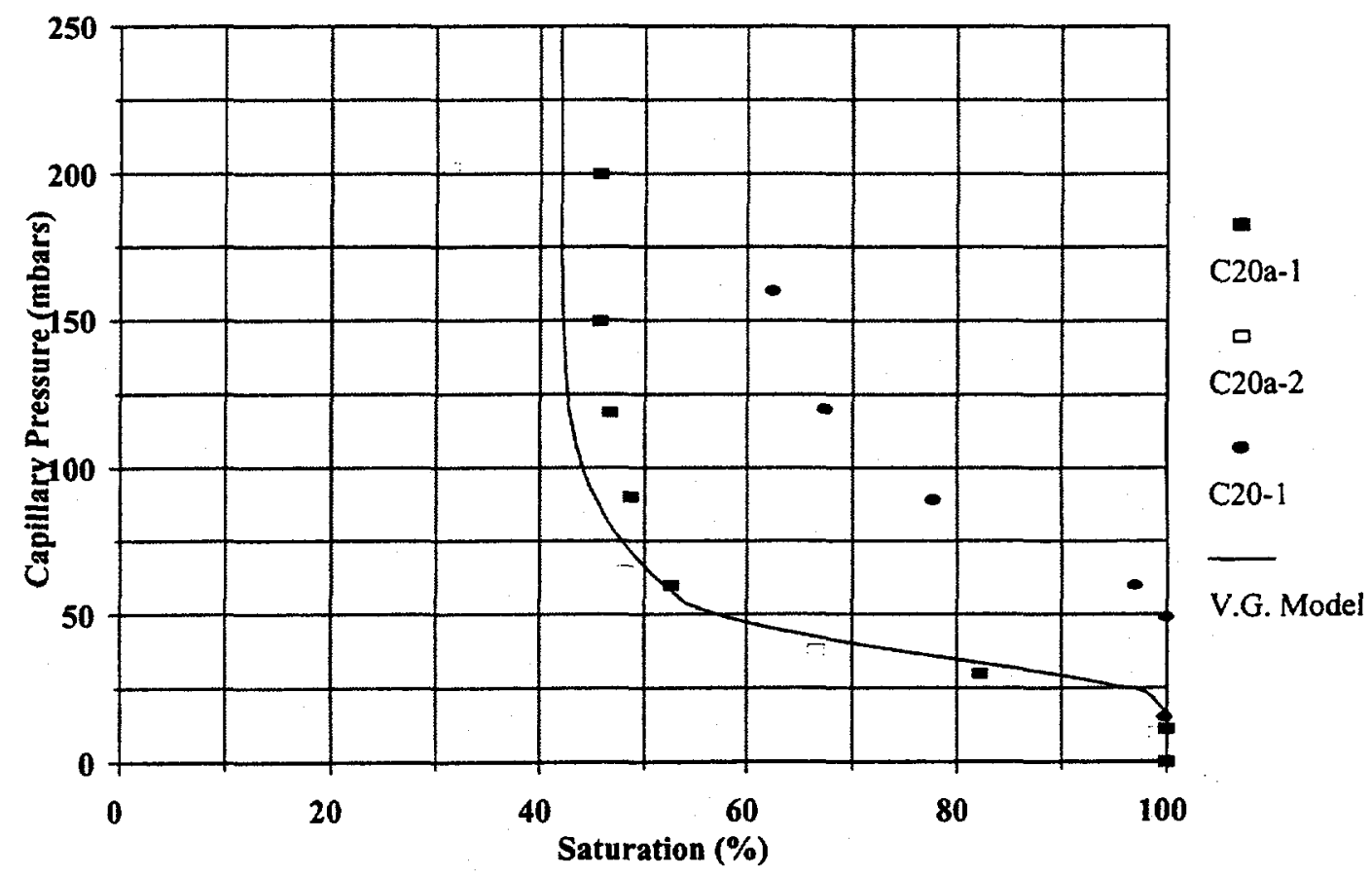

Figure 5.8 Capillary pressure-saturation relationships (PCE-water) for the columns containing $20 \%$ clay-sand mixture. 
1994).

\begin{tabular}{|c|c|c|}
\hline \multicolumn{2}{|c|}{ Table 5.1 Pore Size Distribution Index } \\
\hline Column & Media & Pore Size Distribution Index \\
\hline $\mathrm{C} 0-1$ & white sand & 1.97 \\
\hline $\mathrm{C} 0-2$ & white sand & 1.91 \\
\hline $\mathrm{C} 0-3$ & white sand & ND \\
\hline $\mathrm{C} 2-1$ & $2 \%$ clay & 1.73 \\
\hline $\mathrm{C} 2-2$ & $2 \%$ clay & 3.1 \\
\hline $\mathrm{C} 2-3$ & $2 \%$ clay & $\mathrm{ND}$ \\
\hline $\mathrm{C} 5-1$ & $5 \%$ clay & $\mathrm{ND}$ \\
\hline $\mathrm{C} 5-2$ & $5 \%$ clay & 0.94 \\
\hline $\mathrm{C} 5-3$ & $5 \%$ clay & 1.50 \\
\hline $\mathrm{C} 10-1$ & $10 \%$ clay & $\mathrm{ND}$ \\
\hline $\mathrm{C} 10-2$ & $10 \%$ clay & 0.6 \\
\hline $\mathrm{C} 10-3$ & $10 \%$ clay & 0.61 \\
\hline $\mathrm{C} 10-4$ & $10 \%$ clay & 0.55 \\
\hline $\mathrm{C} 20-1$ & $20 \%$ clay & 0.5 \\
\hline $\mathrm{C} 20 \mathrm{a}-2$ & $20 \%$ clay & 0.43 \\
\hline $\mathrm{C} 20 \mathrm{a}-3$ & $20 \%$ clay & 0.73 \\
\hline $\mathrm{F} 5-1$ & $5 \%$ fine sand & 1.06 \\
\hline
\end{tabular}

ND-Not Determined

The pore size distribution of the media changed with the clay/sand mixture. As the clay content in the medium increased, the variety pore sizes also increased. This was the expected result based on the earlier discussion of the clay distribution and its effect on the soil pore structure. It is likely that the clay coats the sand grains and decreases the size of some pores. This leads to an increased pore size distribution and hence lower value of $\lambda$. Figure 5.9 provides a graph showing the relationship between $\lambda$ values and the percent clay. The data here generally behave as expected with decreasing values of $\lambda$ as the percent clay increased. Some scatter is likely due to the small number of points available to determine $\lambda$. Using only a few points, 3-5 in this case, causes a higher likelihood of error in determining $\lambda$.

After fitting the capillary pressure-saturation data with the van Genuchtan model, the line generated from the model was used to determine a value of $\lambda$ for each media tested. In Figure 5.10, these five values of $\lambda$ were plotted against percent clay. These data are also indicative of the expected trend 
that as the percent clay increases, the value of $\lambda$ decreases.

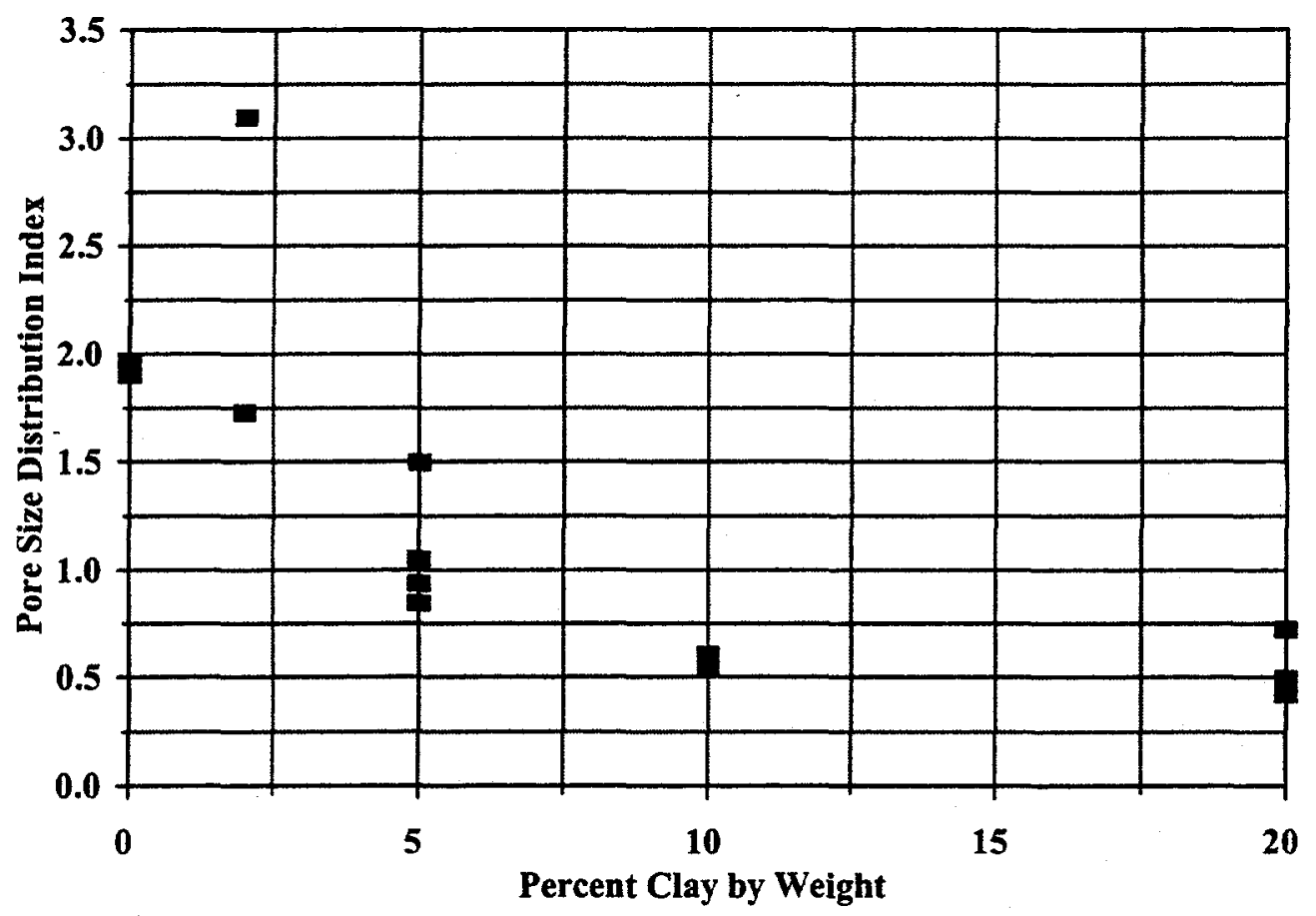

Figure 5.9 Effect of percent clay on the experimentally determined pore size distribution index.

The actual pore throat sizes can be estimated for each percent clay from the specific pressure values associated with saturation. Based on the sizes of the pore throats, blob sizes of trapped PCE can also be estimated. This will be further discussed in the next section. 


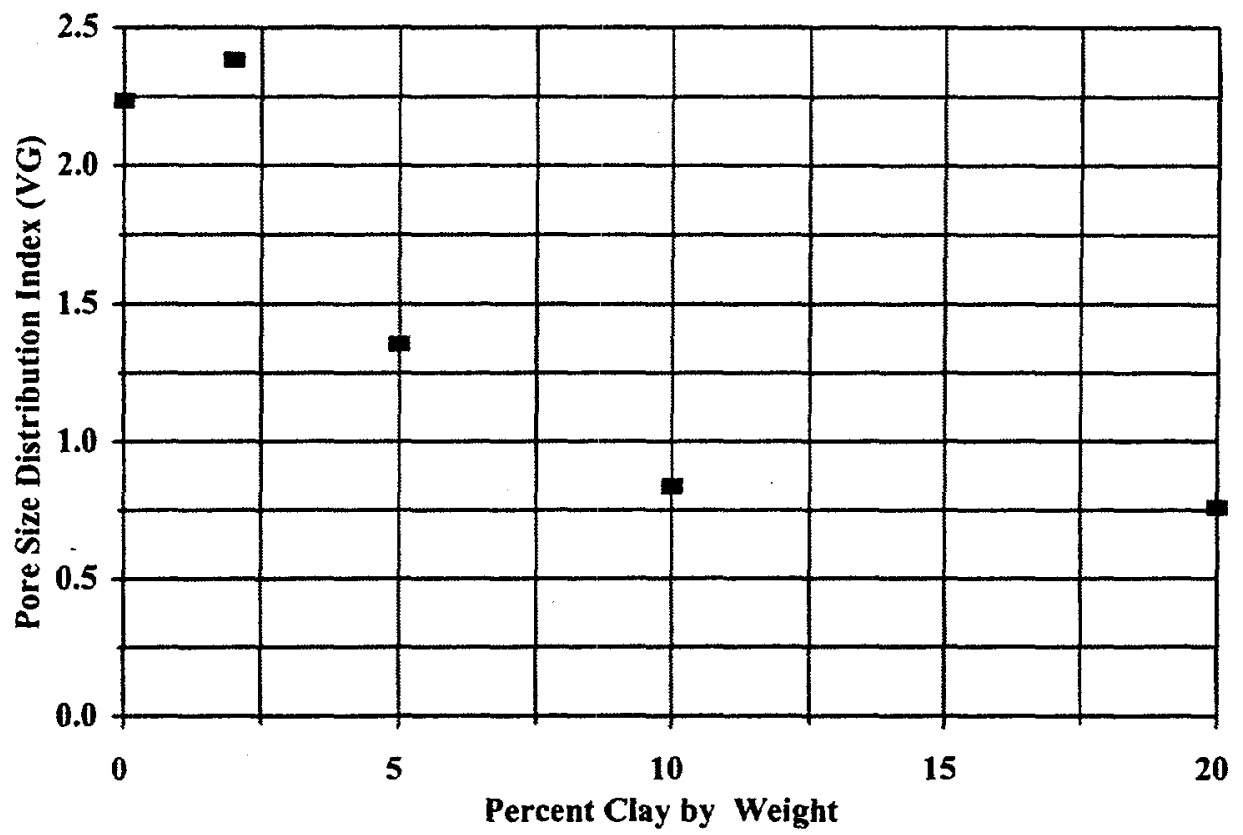

Figure 5.10 Effect of percent clay on the pore size distribution as determined by the van Genuchten model.

\section{Properties of Media With PCE Residual}

A PCE residual was established in the columns as a result of the capillary pressure-saturation tests. The presence, size, and shape of trapped PCE in the pores considerably affects hydraulic conductivity of the aqueous phase through the soil. In addition, the size and shape of the blobs will affect viscosity forces on the blob and the contact between the PCE phase and aqueous phase possibly limiting mobilization of the blob or the effectiveness of an alcohol flush. Characterization of the residual saturation, including size and shape of the trapped PCE blobs is, therefore, critical when considering hydraulic conductivity, mobilization, and the effectiveness of alcohol flushing.

Table 5.2 provides the residual saturation values determined for each column. These results are based on the assumption of equilibrium dissolution for the two-phase flow region and GC analysis of column effluent during alcohol flushing in the one-phase flow region.

\begin{tabular}{|c|c|c|}
\hline \multicolumn{3}{|c|}{ Table 5.2 Residual Saturation } \\
\hline Column & Media & Residual Saturation \\
\hline $\mathrm{C} 0-1$ & white sand & 17.88 \\
\hline
\end{tabular}




\begin{tabular}{|c|c|c|}
\hline & Table 5.2 Residual Saturation \\
\hline Column & Media & Residual Saturation \\
\hline $\mathrm{C} 0-2$ & white sand & 15.90 \\
\hline $\mathrm{C} 0-3$ & white sand & 13.43 \\
\hline $\mathrm{C} 2-1$ & $2 \%$ clay & 14.29 \\
\hline $\mathrm{C} 2-2$ & $2 \%$ clay & 5.28 \\
\hline $\mathrm{C} 2-3$ & $2 \%$ clay & 28.91 \\
\hline $\mathrm{C} 5-1$ & $5 \%$ clay & 43.75 \\
\hline $\mathrm{C} 5-2$ & $5 \%$ clay & 24.70 \\
\hline $\mathrm{C} 5-3$ & $5 \%$ clay & 37.51 \\
\hline $\mathrm{C} 10-1$ & $10 \%$ clay & 40.46 \\
\hline $\mathrm{C} 10-2$ & $10 \%$ clay & 20.91 \\
\hline $\mathrm{C} 10-3$ & $10 \%$ clay & 30.85 \\
\hline $\mathrm{C} 10-4$ & $10 \%$ clay & 43.90 \\
\hline $\mathrm{C} 20-1$ & $20 \%$ clay & 44.62 \\
\hline $\mathrm{C} 20 \mathrm{a}-2$ & $20 \%$ clay & 28.76 \\
\hline $\mathrm{C} 20 \mathrm{a}-3$ & $20 \%$ clay & 33.73 \\
\hline F $5-1$ & $5 \%$ fine sand & 15.33 \\
\hline
\end{tabular}

Figure 5.11 provides information about the affect of the amount of clay on the residual PCE saturation. It seems that as the amount of clay in the media increases, the residual PCE saturation also increases. Mace and Wilson (1992) also observed this in their studies of 3\% montmorillonite clay in a Selvitta sand. They noted an increase in the residual saturation of the organic liquid, Soltrol, from $16.9 \%$ in $0 \%$ clay Selvitta Sand to $30.3 \%$ in $3 \%$ clay in Selvitta Sand. Based on their results, Mace and Wilson (1992) postulated that clay particles detach from the sand surface and adhere to the NAPL-water interface. The colloidal clay particles then travel along this interface with the moving liquids and become compressed into pore throats. This causes an increase in capillary pressure and hence an increase in bypassing (Mace and Wilson, 1992). An increase in the incidence of by-passing may also affect the blob sizes and shapes formed in the media. More multi-pore ganglia could affect the potential to mobilize the blobs. This will be discussed further when mobilization of blobs is considered.

When viewing the graph of residual saturation versus percent clay provided in Figure 5.11, it appears that each of the clay mixtures has a wide range of residual saturations. This could be due to errors during experimentation. However, this large variation was not observed for the $0 \%$ clay columns. 
It seems feasible that the clay in the media is reacting or interacting with the PCE NAPL causing a large variation in residual saturation. Based on work completed by Wan and Wilson (1992) and Mace and Wilson (1992), it seems plausible that interactions between the clay and NAPL could affect NAPL behavior and, hence, the residual saturation and the distribution, ie. size and shape, of the residual PCE established in the column.

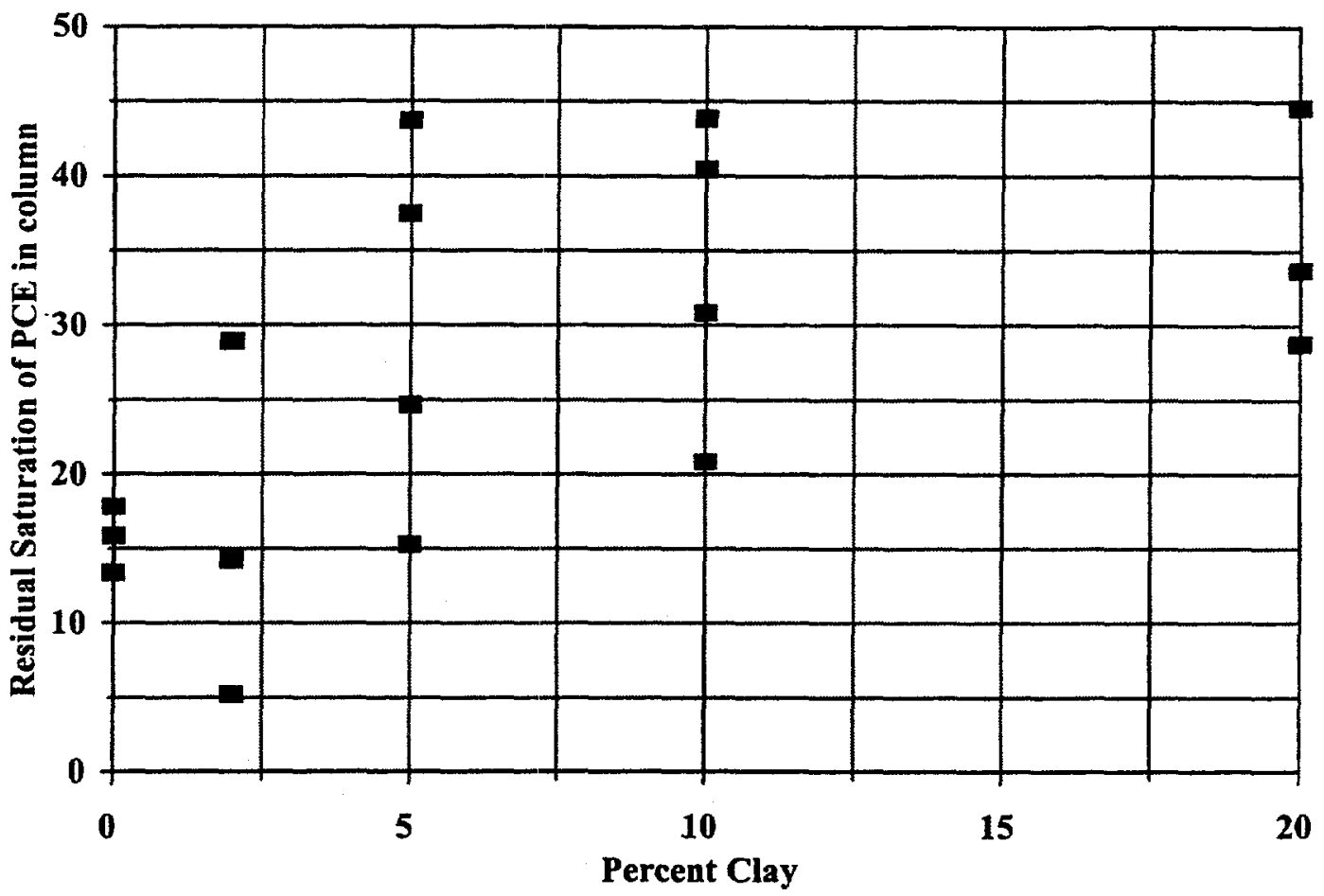

Figure 5.11 Residual saturation of PCE in the soil as a function of percent clay.

The size of PCE blobs is considered to affect mobilization according to work completed by Hunt et al. (1988a). In addition, the shape of the PCE blob could alter the affect of viscosity forces on the blob. To find correlation between blob size and shape, and mobilization, there must be some method of characterizing blob size and shape. Blob size and shape can be inferred by grain size and pore size distribution. Direct methods of measuring blob size and shape were too involved and too costly for this project.

The capillary pressure-saturation tests provided values of pore sizes by calculating a pore throat radius assuming the cross-section of the pore throat was circular. PCE blob size was calculated using the critical trapping length developed by Hunt et al. (1988a). In their study, Hunt et al. (1988a) determined a critical length which describes the maximum length of the trapped blobs. Therefore, for a specific capillary pressure, a maximum length of blob can exist. The saturation that coincides 
with that capillary pressure is an indicator of the number of pores that can contain blobs of that critical length. The actual number of blobs of that length is lower, and can be estimated based on the residual saturation. This method of attempting to quantify blob sizes is admittedly crude, but does provide some characterization of the PCE blobs in the columns. Figures 5.12 through 5.15 provide graphs of pore throat size distribution and blob size distribution for each of the percent clay mixtures.

These estimations of blob size and pore size are based on the interfacial tension of PCE and water. Wan and Wilson (1992) suggest that clay particles adhered to the interface of NAPL blobs may affect the interfacial properties of the NAPL. If this is the case in these columns, the above analysis of pore size and blob size may be invalid. However, it is unclear whether these interactions are actually occurring. Further analysis is required to determine if any appreciable change in interfacial tension occurs as a result of clay particles adhering to the interface of NAPL and water.

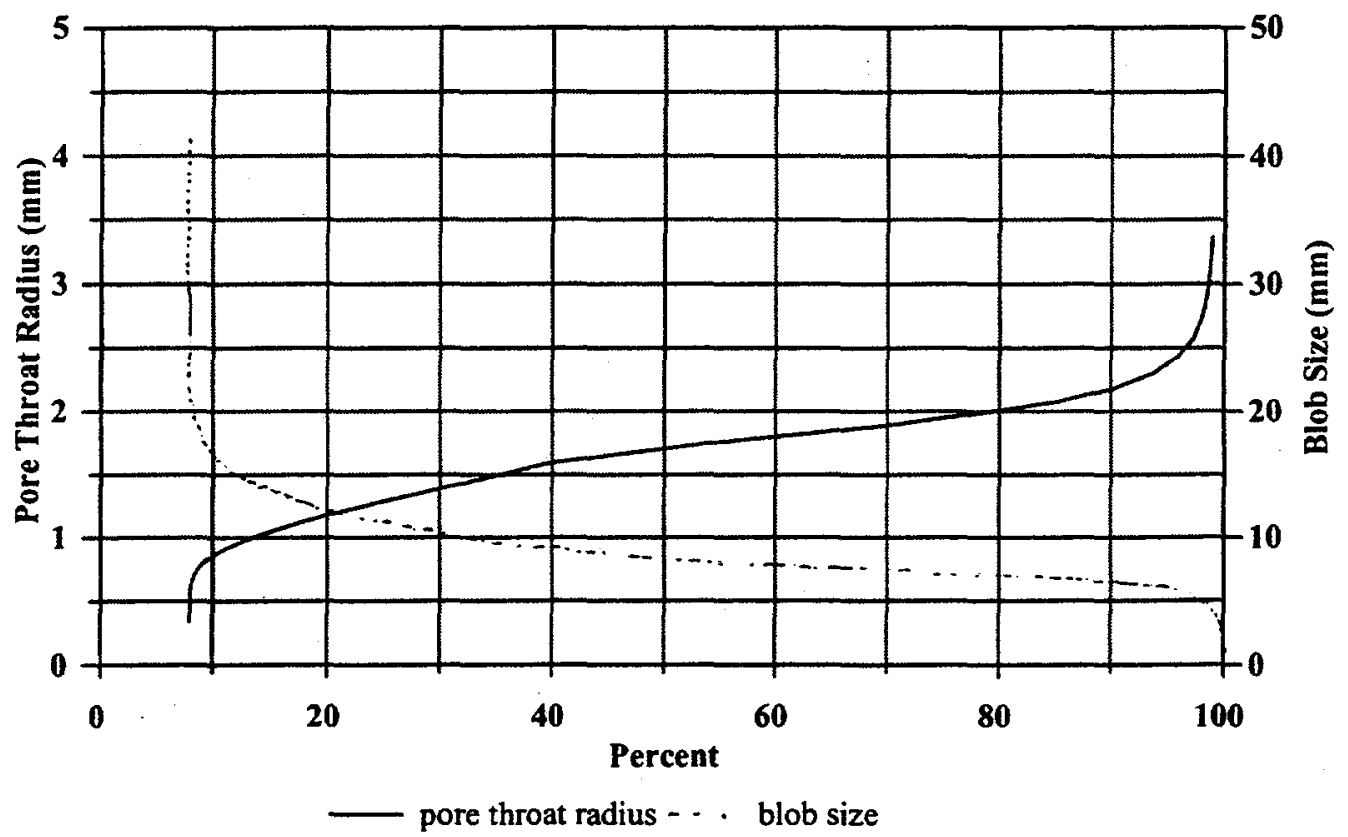

Figure 5.12 Pore throat radius and blob size distributions for $2 \%$ clay.

Figure 4.10 Pore throat radius and blob size distribution for $0 \%$ clay. 


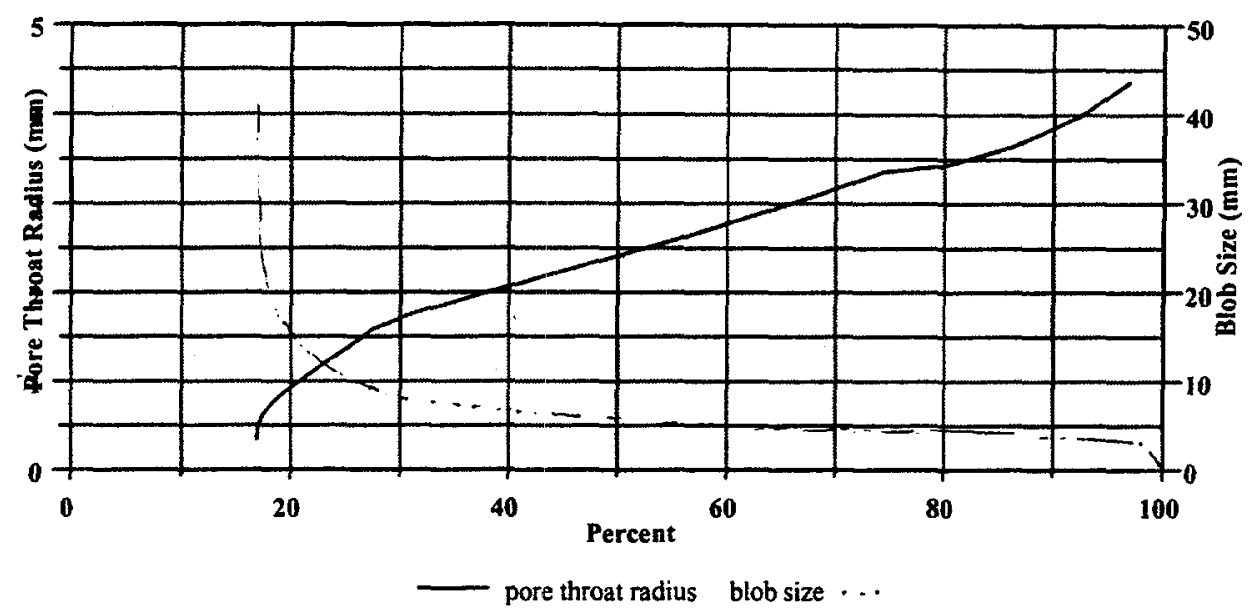

Figure 5.13 Pore throat radius and blob size distributions for $5 \%$ clay.

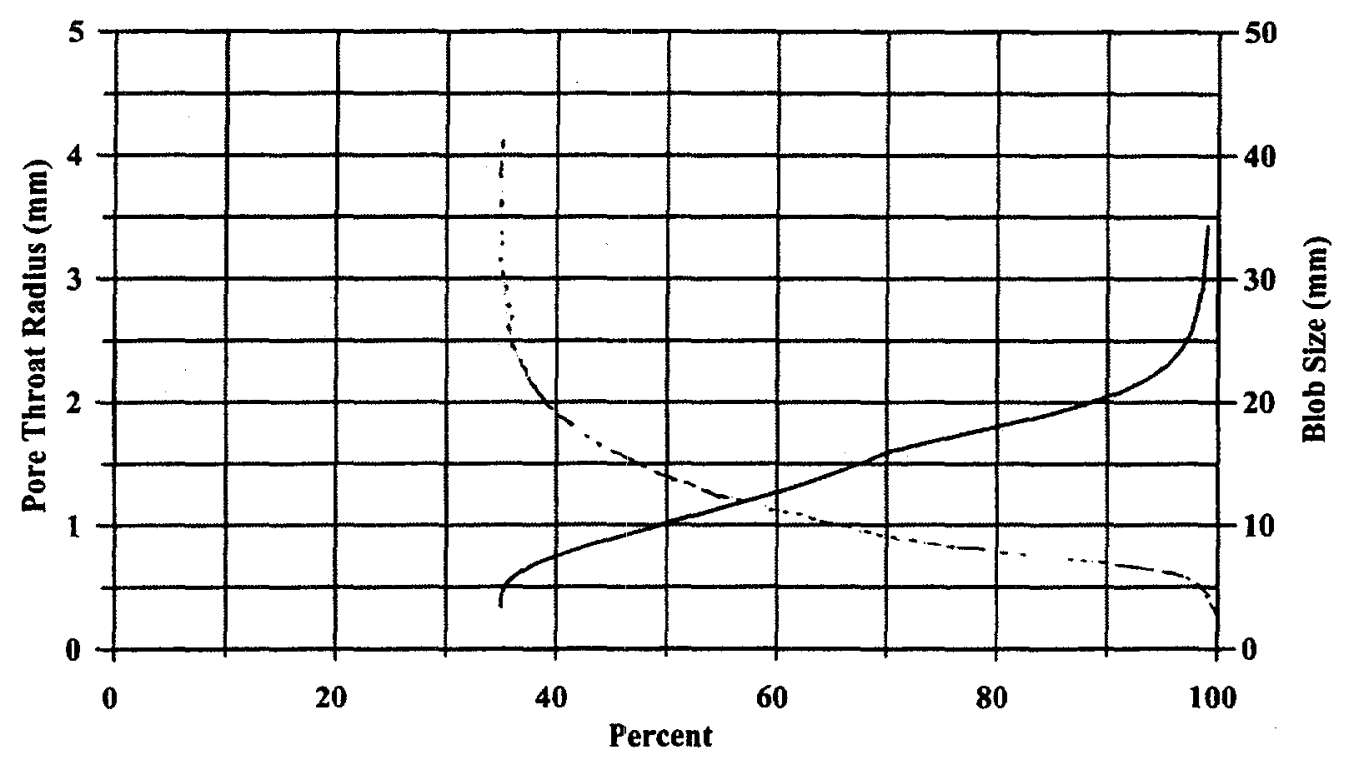

- pore throat radius $\cdots$ blob size

Figure 5.14 Pore throat radius and blob size distribution for $10 \%$ clay. 


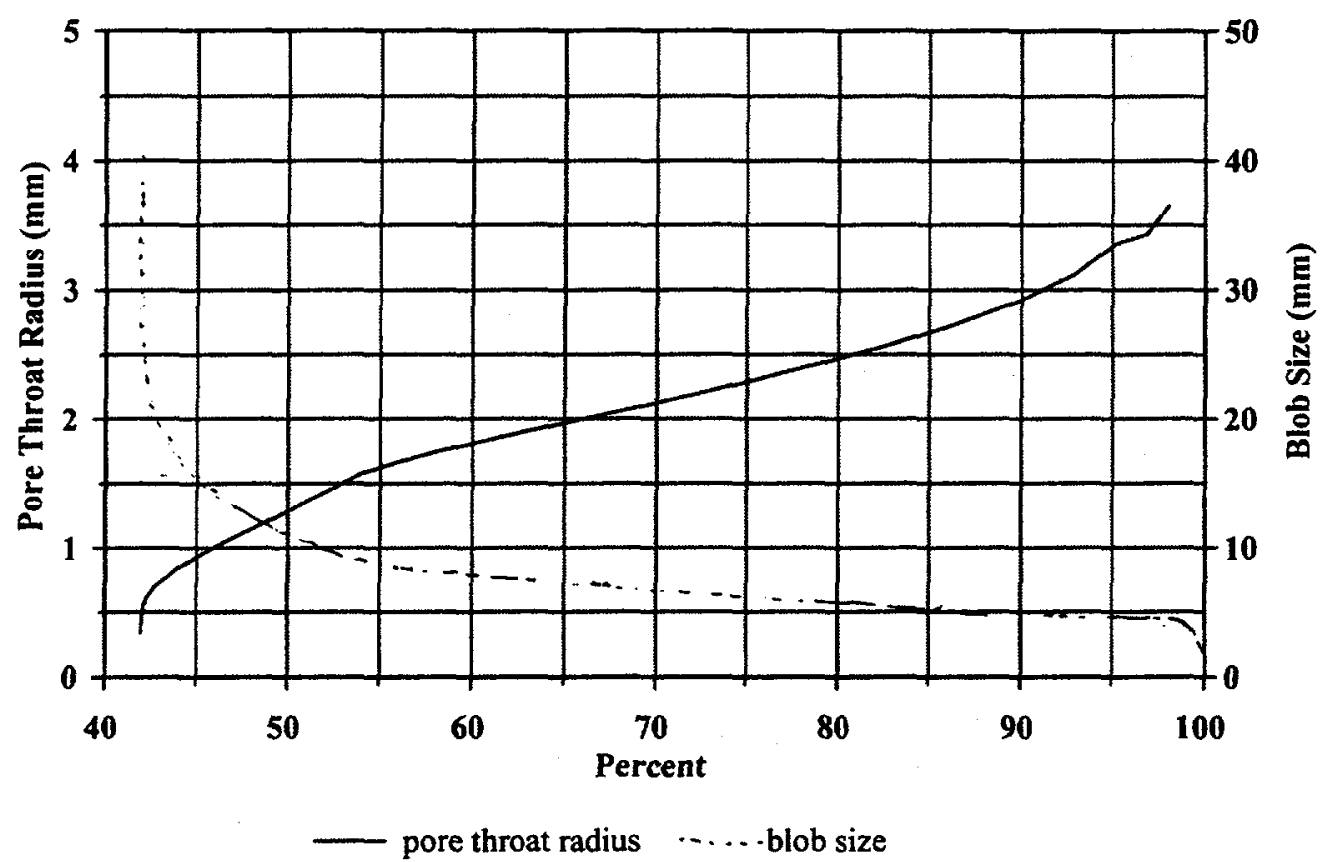

Figure 5.15 Pore throat radius and blob size distributions for $20 \%$ clay.

Another method of determining pore sizes and hence blob sizes is to use grain size analysis data. Berg (1975) determined that the distribution of pore throat radii can be estimated as 0.077 times the particle diameter, assuming a rhombohedral packing of spherical media. Using this information, along with the grain size analysis completed for the soils used in this study, the pore throat radii can be estimated along with the resulting blob sizes. It appears that Berg's method underestimates the pore sizes, or that the capillary pressure-saturation data overestimates the pore sizes. Because Berg's method is an idealized model based on spherical grains and a rhombohedral packing is likely less indicative of this soil than the experimentally determined pore sizes based on the capillary pressuresaturation relationship.

As previously stated, the size and shape of PCE blobs will affect the hydraulic conductivity of the soil. Therefore, hydraulic conductivity tests were conducted after PCE emplacement, $\mathrm{K}_{\mathrm{PCE}}$. These tests determined that the hydraulic conductivity changed in most of the columns. The results are presented in Table 5.4, along with $\mathrm{K}$ values for the clean soil and the relative ratio of $\mathrm{K}_{\mathrm{PCE}}$ to $\mathrm{K}$. Statistical analysis (non-parametric Wilcoxon rank test) showed that for the white sand alone, or $0 \%$ clay, the hydraulic conductivities before and after PCE emplacement were equal. We know that is not true theoretically, but the limitations or errors present in the experiment were greater than the difference in hydraulic conductivities. The other clay/white sand mixtures behaved as expected, with 
a lower hydraulic conductivity in the column when a PCE residual saturation was present. As percent clay increased, both $\mathrm{K}$ and $\mathrm{K}_{\mathrm{PCE}}$ decreased. The difference in $\mathrm{K}$ values in the presence of a residual PCE between different media seems to decrease with increasing clay content.

\begin{tabular}{|c|c|c|c|c|}
\hline Column & Media & $\begin{array}{c}\text { Hydraulic } \\
\text { Conductivity } \\
(\mathrm{cm} / \mathrm{s})\end{array}$ & $\begin{array}{c}\text { Hydraulic } \\
\text { Conductivity } \\
\text { w/PCE } \\
(\mathrm{cm} / \mathrm{s})\end{array}$ & $\mathrm{K}_{\mathrm{PCE}} / \mathrm{K}$ \\
\hline $\mathrm{C} 0-1$ & white sand & $1.61 \times 10^{-2}$ & $5.53 \times 10^{-3}$ & 0.343 \\
\hline $\mathrm{C} 0-2$ & white sand & $1.46 \times 10^{-2}$ & $1.21 \times 10^{-2}$ & 0.824 \\
\hline $\mathrm{C} 0-3$ & white sand & $1.46 \times 10^{-2}$ & $1.32 \times 10^{-2}$ & 0.903 \\
\hline $\mathrm{C} 2-1$ & $2 \%$ clay & $1.32 \times 10^{-2}$ & $7.23 \times 10^{-3}$ & 0.547 \\
\hline $\mathrm{C} 2-2$ & $2 \%$ clay & $1.69 \times 10^{-2}$ & $9.60 \times 10^{-3}$ & 0.567 \\
\hline $\mathrm{C} 2-3$ & $2 \%$ clay & $1.14 \times 10^{-2}$ & $3.50 \times 10^{-3}$ & 0.306 \\
\hline $\mathrm{C} 5-1$ & $5 \%$ clay & $1.29 \times 10^{-2}$ & $1.19 \times 10^{-3}$ & 0.092 \\
\hline C5-2 & $5 \%$ clay & $9.38 \times 10^{-3}$ & $3.23 \times 10^{-3}$ & 0.344 \\
\hline $\mathrm{C} 5-3$ & $5 \%$ clay & $8.32 \times 10^{-3}$ & $3.42 \times 10^{-3}$ & 0.412 \\
\hline $\mathrm{C} 10-1$ & $10 \%$ clay & $9.13 \times 10^{-3}$ & $2.25 \times 10^{-4}$ & 0.025 \\
\hline $\mathrm{C} 10-2$ & $10 \%$ clay & $7.19 \times 10^{-3}$ & $2.31 \times 10^{-3}$ & 0.322 \\
\hline $\mathrm{ClO-3}$ & $10 \%$ clay & $4.50 \times 10^{-3}$ & $1.42 \times 10^{-3}$ & 0.315 \\
\hline $\mathrm{C} 10-4$ & $10 \%$ clay & $7.42 \times 10^{-3}$ & $1.07 \times 10^{-3}$ & 0.144 \\
\hline $\mathrm{C} 20-1$ & $20 \%$ clay & $8.09 \times 10^{-4}$ & $4.15 \times 10^{-5}$ & 0.051 \\
\hline $\mathrm{C} 20 \mathrm{a}-2$ & $20 \%$ clay & $5.87 \times 10^{-3}$ & $1.05 \times 10^{-3}$ & 0.178 \\
\hline $\mathrm{C} 20 \mathrm{a}-3$ & $20 \%$ clay & $5.45 \times 10^{-3}$ & $7.51 \times 10^{-4}$ & 0.138 \\
\hline F5-1 & $5 \%$ fine sand & $1.43 \times 10^{-2}$ & $3.13 \times 10^{-3}$ & 0.218 \\
\hline
\end{tabular}

Figure 5.16 provides a graphical representation of this phenomena. Figure 5.16 also indicates that the difference in $\mathrm{K}$ values before and after PCE emplacement within each media increases as the clay content increases. Meaning that as the clay content increases, the change in $\mathrm{K}$ with the introduction of PCE into the column increases. 


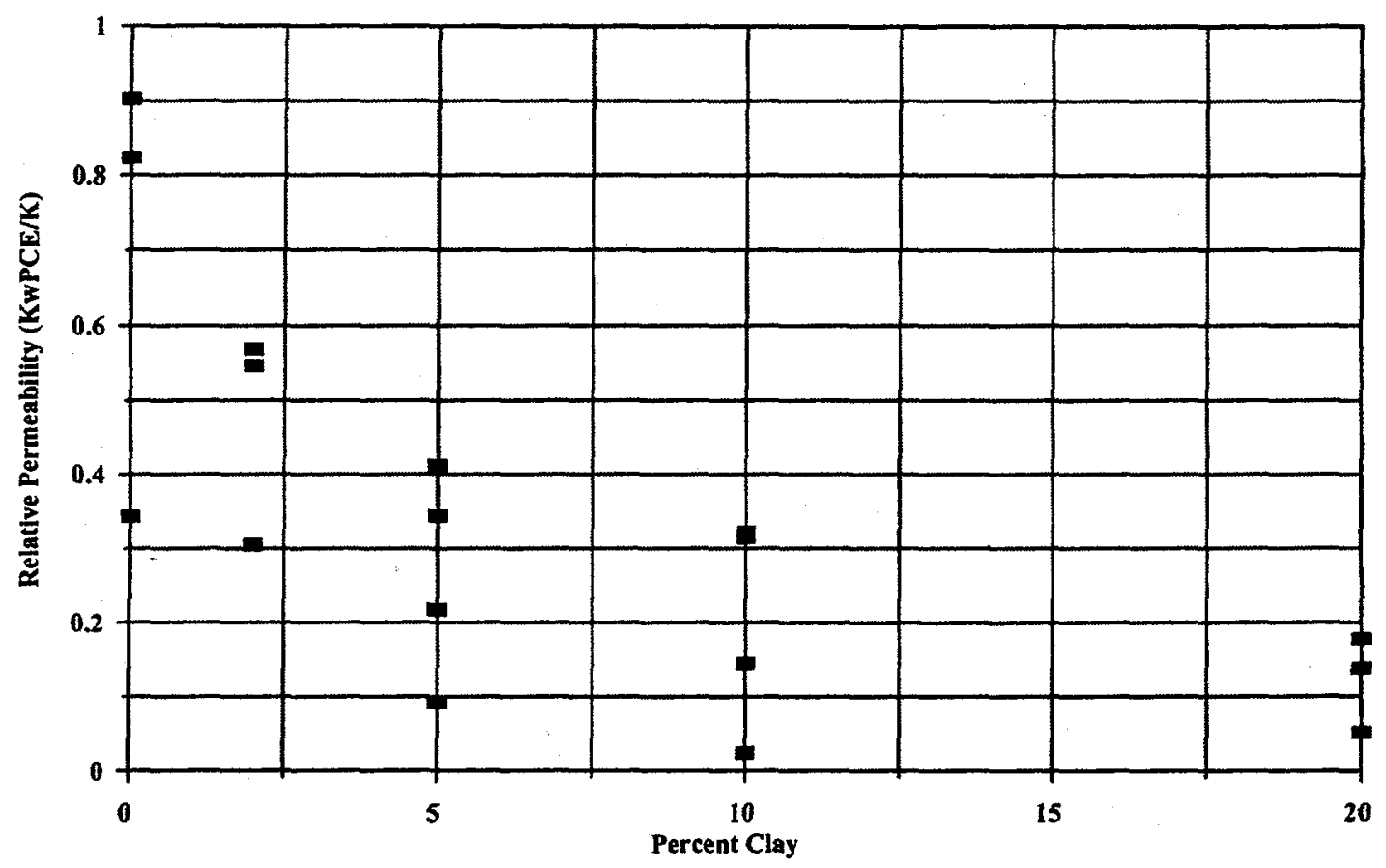

Figure 5.16 Effect of clay content on the relative permeability of the soil with a PCE residual.

\section{Mobilization}

Mobilization of the residual PCE was achieved in all of the columns by flushing with alcohol solutions of varying concentrations. The alcohol concentration where significant mobilization was noted was used to determine the trapping number at mobilization. Significant mobilization indicates that more than a few microliters of PCE NAPL were observed in the effluent. Occasionally, a few microliters of NAPL located somewhere other than the soil, such as on the screen or port, would mobilize. Little to none of this "excess" PCE NAPL is considered to have come from the soil due to the effective residual saturation established by using two ceramic plates during PCE imbibition and drainage. By considering the trapping number, the results observed from these experiments can be compared to previous work. Deviations noted in these results are explained by the structure of the soil and the presence of the clay. 


\section{Trapping Number}

Mobilization was noted for a wide variety of trapping numbers. This fact counters earlier hopes that one trapping number value may be used to characterize the potential for mobilization of a residual PCE saturation. Aquifer material is often heterogeneous, and most work completed on mobilization of DNAPLs to date deals with homogenous material (Pennell et al., 1996, Dawson and Roberts, 1997). In this study, the degree of heterogeneity was shown to affect mobilization in media containing a clay/sand mixture. Figure 5.17 provides a graph depicting the trapping number at mobilization for mixtures of $0,2,5,10$, and 20 percent clay in homogenous white sand. Note that as the percent clay increases, the change in trapping number for mobilization decreases or appears to approach some minimal trapping number. Therefore, designing a remediation scheme where the trapping number does not exceed this "safe" trapping number is appealing. However at this low trapping number, generally the interfacial tension is similar to that of water and PCE so the alcohol flushing scheme may not provide adequate remediation incentive. Meaning that the dissolution may not increase significantly at an interfacial tension where mobilization will not occur. Therefore, it appears that site specific information is necessary to achieve optimal remediation without promoting mobilization. Because of this fact, a new model accounting for heterogeneity and other soil

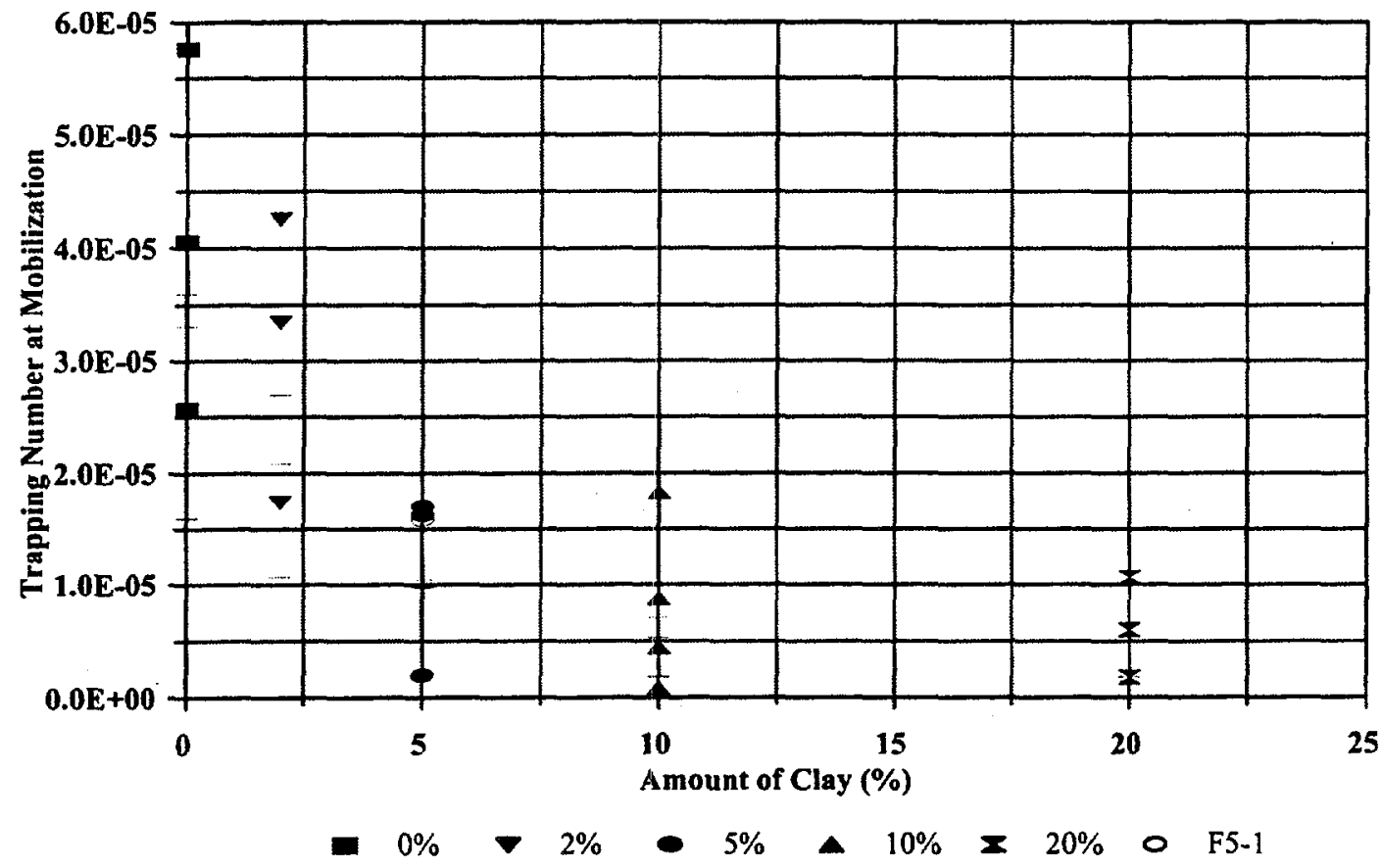

Figure 5.17 Mobilization as a function of percent clay.

properties seems necessary to predict mobilization. The dashed lines below the data points indicate the highest trapping number measured where mobilization did not occur. Therefore, mobilization could have occurred at any alcohol concentration between the one that resulted in the dash and the 
one that resulted in mobilization.

Based on the necessity to further characterize mobilization, trapping number was plotted against a variety of soil properties. The soil properties used were hydraulic conductivity, and hence permeability, both before and after PCE emplacement, residual PCE saturation, and pore size distribution.

The hydraulic conductivity of the media with a PCE residual appeared to impact the trapping number at which mobilization would occur. Hydraulic conductivities before and after PCE emplacement are used to determine the trapping number, hence trapping number is dependant on soil permeability and residual saturation. As the hydraulic conductivity of the media with a PCE residual decreases, the likelihood for mobilization increases, or the trapping number at mobilization decreases. This is illustrated in Figure 5.18. These data appear to be fairly linear and a regression analysis of the data provided a correlation coefficient of 0.95 .

Pennell et al. (1996) reported permeability values for one of the homogeneous sands used in their study, $100-120$ mesh $(0.125 \mathrm{~mm}-0.149 \mathrm{~mm})$ Ottawa sand. These permeability values result in hydraulic conductivities with PCE residual of $0.00194 \mathrm{~cm} / \mathrm{s}$. Using this value, one would predict mobilization at approximately $1 \times 10^{-5}$. However, Pennell et al. (1996) reported mobilization occurring at a trapping number closer to $2 \times 10^{-5}$. This discrepancy could be due to a variety of factors. The most likely reasons include colloidal interactions of the clay particles in the media used,

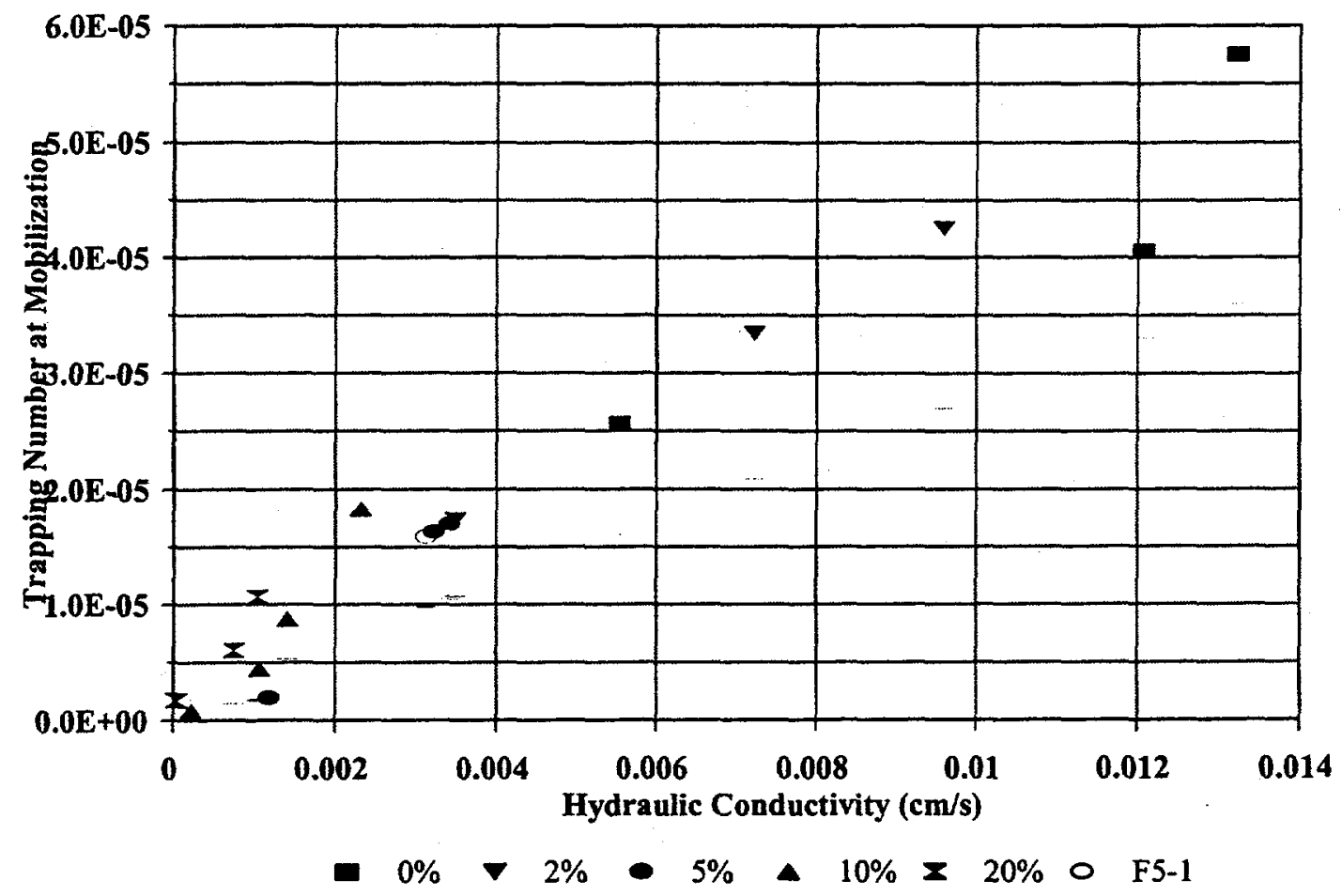

Figure 5.18 Mobilization as a function of the hydraulic conductivity with a PCE residual. 
differences in using surfactants as opposed to iso-propyl alcohol, or the method of establishing a residual. Comparing the results reported in Pennell et al. (1996) based on the change in permeability, or relative permeability appeared to provide a better correlation.

The relationship between the change in hydraulic conductivity, or relative $\mathrm{K}$, and mobilization is shown in Figure 5.19. The relationship appears fairly linear below a relative $\mathrm{K}$ of approximately 0.5 or $50 \%$. A regression analysis of this data was performed and the obtained correlation coefficient was 0.75 indicating a linear relationship may exist. Above a relative $\mathrm{K}$ of 0.5 , the value of the trapping number at mobilization is approximately $3.0 \times 10^{-5}$ to $4.0 \times 10^{-5}$. By plotting the trapping number on a log scale, a slightly better correlation coefficient of 0.80 is obtained for data points below a $\mathrm{K}_{\mathrm{PCE}} / \mathrm{K}$ ratio of 0.50 . A graph of this is provided in Figure 5.20.

The data reported by Pennell et al. (1996) fit nicely on Figures 5.19. This is understandable, because the relative permeability is less dependant on soil type than using only a hydraulic conductivity value. Pennell et al. (1996) reported a relative permeability for 100-120 mesh Ottawa white sand of 0.37 . With this value of relative permeability and Figure 5.19, the trapping number where mobilization would be expected is just below $2 \times 10^{-5}$. This correlates with the reported findings.

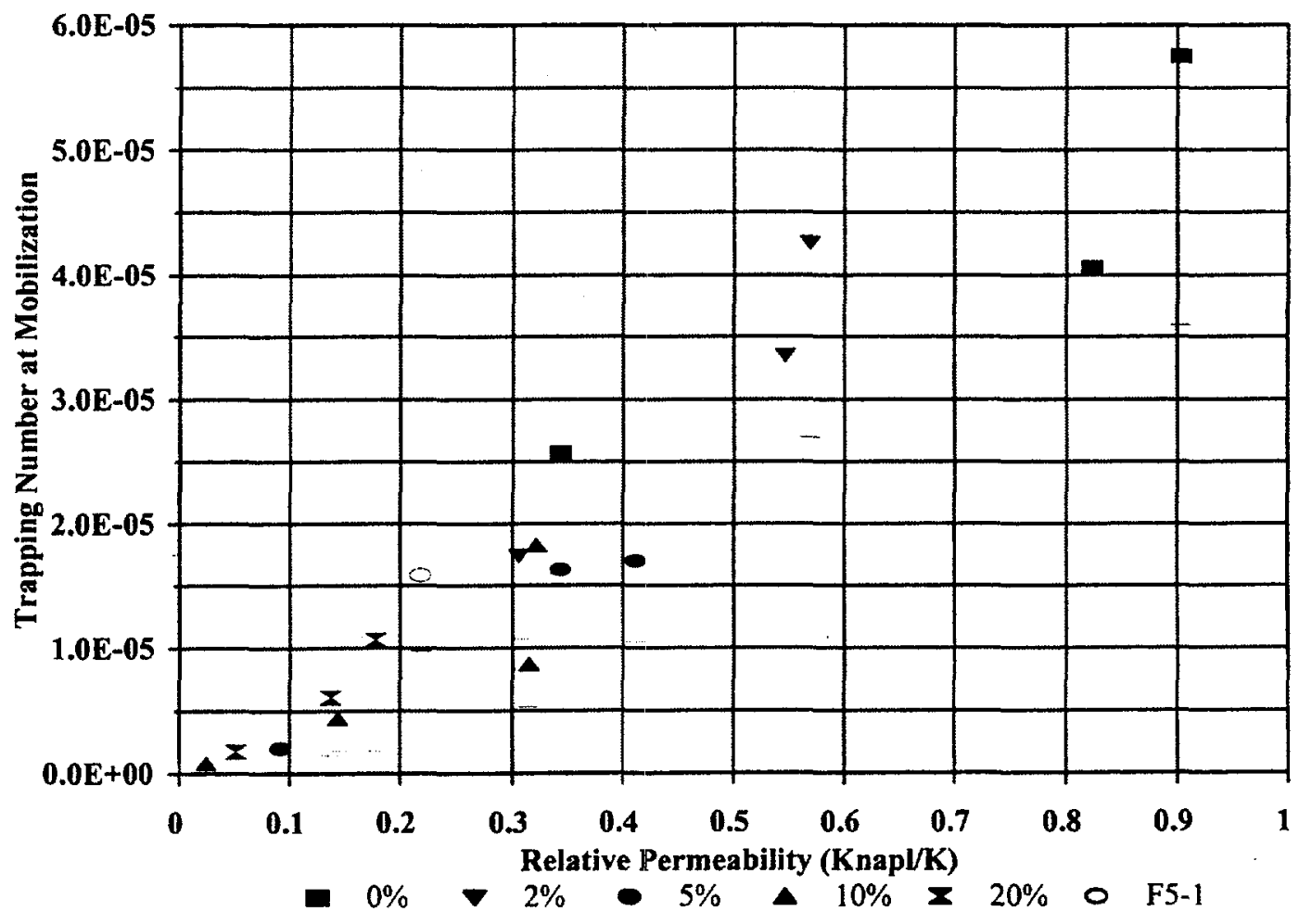

Figure 5.19 Mobilization as a function of relative permeability. 
Considering Figures 5.19 and 5.20 it seems that a larger difference in hydraulic conductivities before and after PCE contamination is indicative of a greater potential for mobilization. However, without knowledge of the hydraulic conductivity prior to contamination, Figures 5.19 and 5.20 may not provide applicable information, therefore, when applying to a specific site Figure 5.18 relating hydraulic conductivity and onset of mobilization may be more useful. The hydraulic conductivity data appear to provide information that can easily be modeled using a linear fit model. This indicates that the hydraulic conductivity may provide adequate information to determine the likelihood for mobilization in these soils. However, other parameters may provide a more accurate predication of

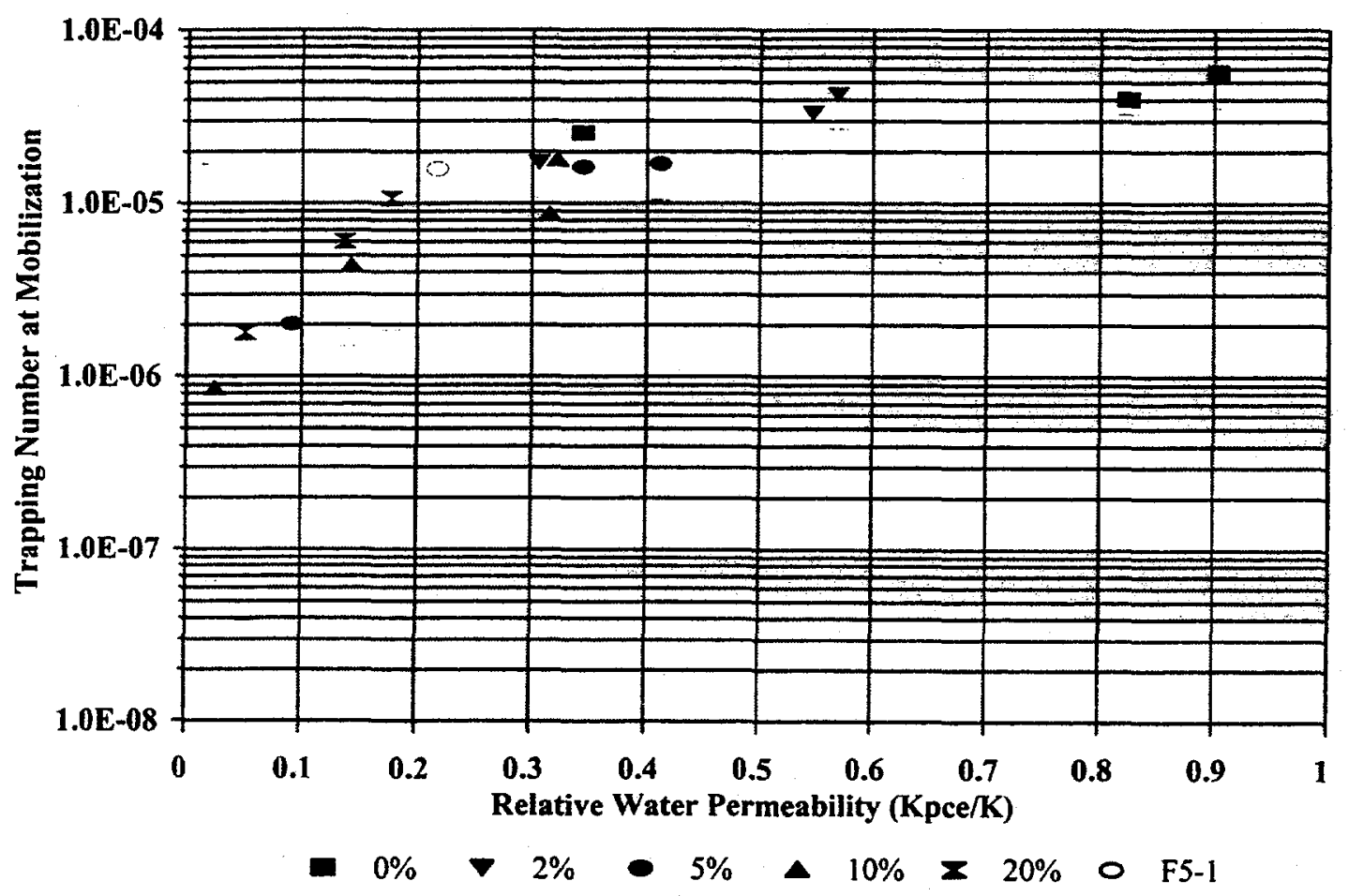

Figure $5.20 \mathrm{Log}$ of the trapping number as a function of relative permeability.

mobilization in these soils.

Figure 5.21 shows that the residual saturation of PCE in the soil column negatively correlates with the trapping number at mobilization. In other words, as the residual saturation increases, the trapping number at mobilization decreases or the potential for mobilization increases. It can be surmised that this result is due to the soil mixture. Soil mixtures containing a higher percent of clay are more heterogenous, and hence may contain more multi-pore ganglia PCE blobs. Hunt et al. (1988b) showed that these blobs are easier to mobilize in a one dimensional column flush than singlets due to the added head component of the PCE ganglia. 


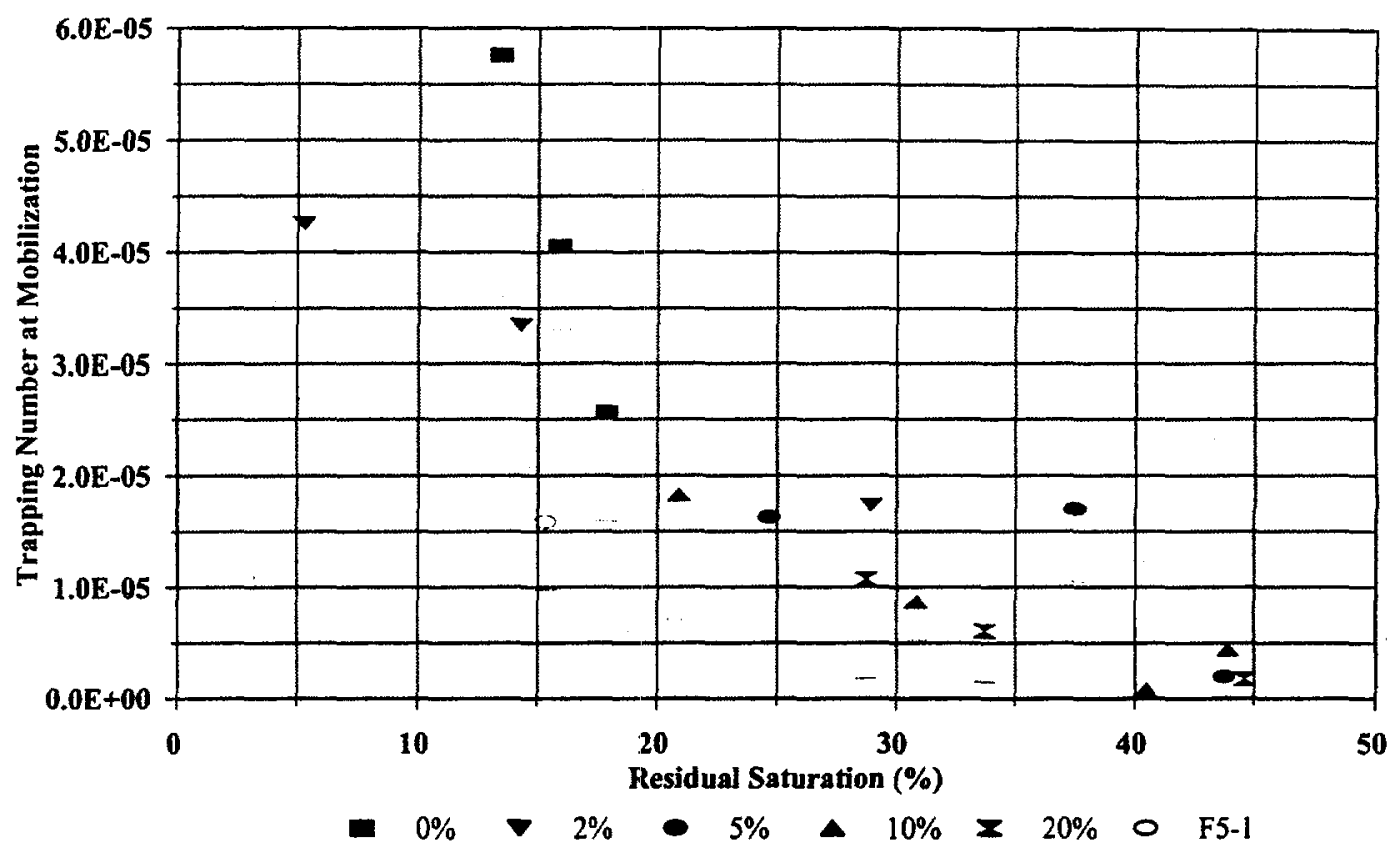

Figure 5.21 Effect of residual saturation on mobilization of PCE.

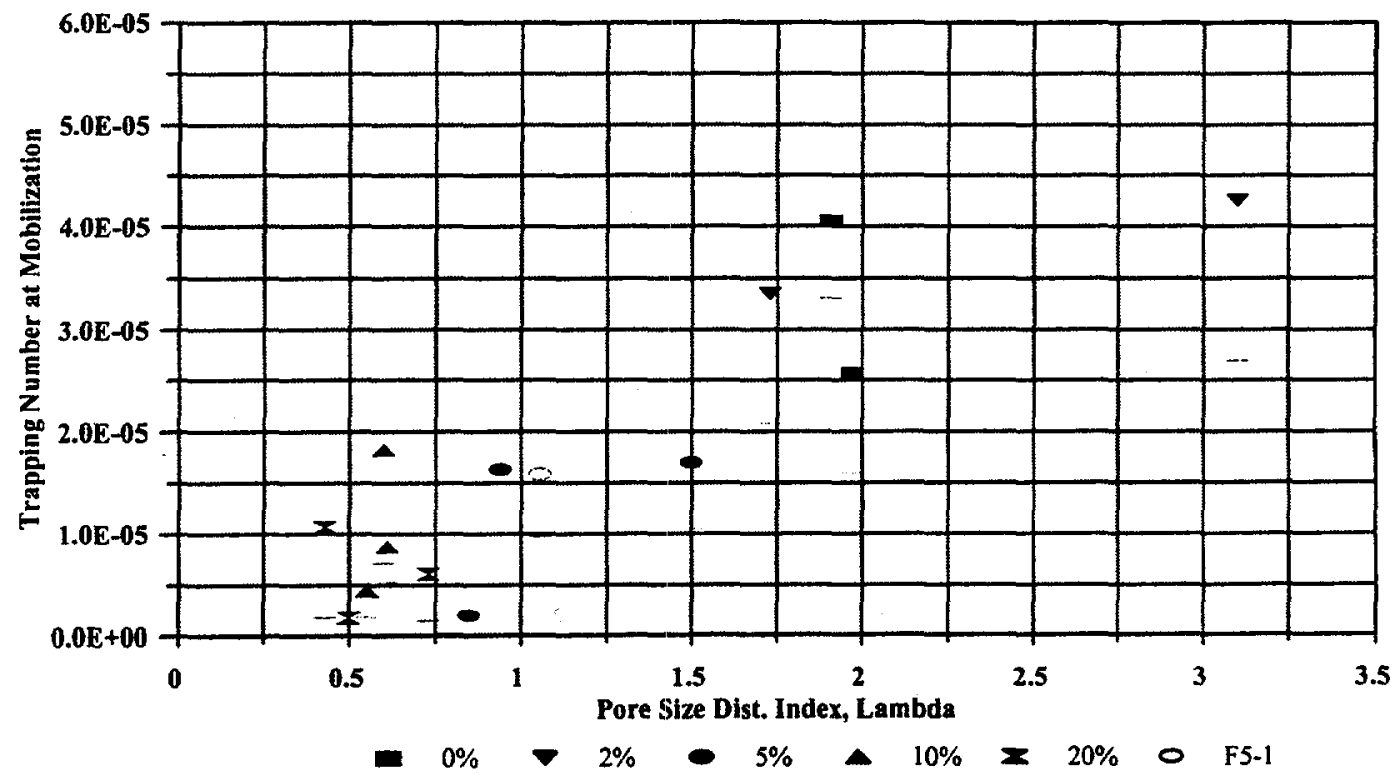

Figure 5.22 Trapping number at mobilization as a function of pore size distribution. 
At the higher values of residual saturation, it appears that the data indicate a linear relationship between residual saturation and the trapping number mobilization. At lower values of trapping number, it appears that a range of trapping numbers may suffice to predict mobilization. As seen before when comparing hydraulic conductivity data and mobilization, the range is $3.0 \times 10^{-5}$ to 4.0 $\times 10^{-5}$. It appears that some of the $5 \%$ clay columns may be outliers if a linear relationship in fact exists. Errors in determining residual saturation may account for scatter observed here, or again, clay interactions may make it difficult to establish similar residual saturations in columns containing the same media. The residual saturations reported in the work completed by Pennell et al. (1996) ranged from $10 \%$ to $24 \%$. From Figure 5.21, mobilization would be expected at trapping numbers of 1.5 $\times 10^{-5}$ to $3.5 \times 10^{-5}$. Again, this correlates fairly nicely with the values of trapping number where mobilization was noted by Pennell et al. (1996).

\section{Effect of Clay on Mobilization}

The actual length of the blob ganglia were estimated for this study from pressure saturation data. Based on these data, the higher percent clay mixtures contained longer blobs. Considering a head component when predicting mobilization, it seems a slight change in interfacial tension would be more likely to mobilize these longer blobs. This situation is not accounted for in current predictive models.

Current mobilization models consider a homogeneous media therefore, the models are based on mobilization of a singlet. The potential presence of multi-pore ganglia would, therefore, require a new model. However, by applying the trapping number model, the effect of the ganglia on mobilization can be examined. As previously discussed, one method of characterizing blob sizes is to use pore size distribution. Therefore, the trapping number at mobilization was plotted against the pore size distribution index in Figure 5.22. Although an overall trend of decreased mobilization was noted with a decrease in pore size distribution, the data appear too scattered to develop any type of predictive model. The general trend noted here was expected considering that a more homogeneous or decreased pore size distribution (higher $\lambda$ value) is less likely to have multi-pore ganglia and more likely to have singlets. Another possible explanation for the scatter is the clay interaction. If clay particles move during the PCE imbibition and drainage, the pore size distribution values determined by the capillary pressure-saturation curve may not be indicative of the final pore structure of the media. Another possibility is that the capillary pressure-saturation relationship is indicative of the pore structure but as Wan and Wilson (1992) postulated more by-passing can occur in the presence of colloidal clay particles. So, the potential for mobilization due more multi-pore ganglia may increase regardless of the pore structure. Also, as previously mentioned, the potential for clay particles to affect the interfacial tension between the two phases could affect mobilization results.

In the interest of understanding whether the results seen here are due to clay chemistry and structure, or just due to the particle size, a 5\% fine sand column was analyzed. The results of this column have been presented with the clay mixture columns. It appears that this column reacted fairly consistently with the clay mixture columns. Some variation did exist in places, however, it is difficult to conclude whether variation was due to the presence of sand or experimental error. However, it is 
clear that the $5 \%$ fine sand column did not react similarly to the $0 \%$ clay column, indicating that particle size, and perhaps colloids, can at least partially explain the phenomena noted in these experiments. This stands to reason considering that the clay used was kaolinite, which tends to be less chemically reactive than other clays. A montmorillonite clay, which is highly reactive, may cause very different results than the kaolinite clay. A study using montmorillonite clay would be interesting and would provide a basis for comparison of clay reactivity and structure on the behavior of PCE in clay containing porous media. Green et al. (1983) found that dissolved chlorinated compounds in landfill leachate caused collapse of the clay interlayer precipitating cracking of clay liners. If this similar type of phenomena occurs in naturally-occurring montmorillonite clays, movement of contaminants may be governed by cracks in the clay as in fractured flow through bedrock.

\subsection{Summary}

The main objective of this portion of the research was to determine the effect of the SRS clay on mobilization of PCE at a residual saturation while flushing with an isopropyl alcohol solution. Based on the results of the experiments, it is evident that the presence of clay does affect mobilization. Using the trapping number to quantify the potential for mobilization, it was noted that the potential to mobilize increased with increasing clay content. This means that for the SRS site, where these clays are found, alcohol flushing may pose a greater risk of mobilization of residual PCE than in homogenous sand aquifers. However, the soils studied here contained $20 \%$ clay or less; the SRS site soils may contain considerably higher amounts of clay.

Mobilization of residual PCE in clay-containing soils was due to the change in interfacial tension during alcohol flushing. It is unclear why a positive correlation exists between the potential to mobilize and the amount of clay, however, it could be due to either one or both of the following reasons:

1) the presence of clay causes a more heterogeneous pore structure leading to more multi-pore ganglia and, hence, a head component that would aid mobilization in the vertical direction; or,

2) colloidal clay particles adhere to the PCE-water interface and alter the interfacial properties causing mobilization to occur more readily.

The first reason was examined by determining the effect of percent clay on the pore size distribution. It appears that soils containing higher amounts of clay contained a broad range of pore sizes. As previously discussed, this causes more by-passing than in a media with a narrow pore size distribution. It also, therefore, causes the formation of multi-pore ganglia. A narrow pore size distribution favors formation of small blobs, such as singlets or doublets. The clay affect other soil properties, such as permeability, however, the affect on the pore size distribution seemed most interesting from the perspective of mobilization of a PCE residual.

Another interesting relationship was shown between the amount of clay in the media and the residual saturation established in that media. It appeared that as the percent of clay in the media increased, the residual saturation increased. However, the data indicated a much greater variability in the 
residual of the clay containing columns than in the residual of the white sand only column. This could be due to clay interactions at the PCE-water interface. Additional experiments would be necessary to determine if a difference in variability really exists between the columns that contain clay and those that do not contain clay.

Another objective of this research was to address development of a model to predict mobilization of a PCE residual in a media containing SRS clay. Based on the data obtained it seems that one of the current predictive models, the trapping number, can be used with a modification in interpretation. To date, a trapping number of $2 \times 10^{-5}$ has been used to indicate mobilization in homogeneous soils. Using a media containing less than $20 \%$ SRS clay along the hydraulic conductivity of the soil with the PCE residual, a trapping number where mobilization will occur can be predicted. Prediction of the trapping number at mobilization then provides the critical interfacial tension where mobilization will likely occur. From this information a remediation scheme can be designed so that the interfacial tension of the system is maintaing above the critical interfacial tension.

This research indicated that the presence of SRS clay in the media increased the likelihood for mobilization of residual PCE. It is hypothesized that the pore size distribution of the clay-containing media favored formation of multi-pore blobs that mobilize at higher interfacial tensions than singlets due to the head component. Additionally, interactions of the clay at the interface of the PCE and water may have caused or enhanced mobilization in these soils.

\subsection{References}

Adamson, A.W. 1990. Physical Chemistry of Surfaces. Wiley, Inc. New York.

Berg, R.R. 1975. Capillary pressures in stratigraphic traps. American Association of Petroleum Geology Bulletin. v. 59, pp. 939-956.

Brandes, D., and K.J. Farley. 1993. Importance of phase behavior on the removal of residual DNAPLs from porous media by alcohol flooding. Water Environment Research. v. 65, no. 7, pp. 869-878.

Boyd, G.R., and K.J. Farley. 1990 Factors influencing nonaqueous phase liquid removal from groundwater by the alcohol flooding technique. Summary Report:Concepts in Manipulation of Groundwater Colloid for Environmental Resoration. Manteo, North Carolina.

Brooks, R.H., and A.T. Corey. 1966. Properties of porous meida affecting fluid flow. Journal of Irrigation and Drainage Division, Proceedings ASCE., v. 92, IR 2, pp. 61-88.

Chatzis, I., N.R. Morrow, and H.T. Lim. 1983. Magnitude and detailed structure of residual oil saturation. SPE Journal. v. 23, no. 2, pp. 311-325.

Corey, A.T. 1994. Mechanics of Immiscible Fluids in Porous Media. Water Resources Publications. Colorado.

Dawson, H. E., and P.V. Roberts. 1997. Influence of viscous, gravitational, and capillary forces on DNAPL saturation. Ground Water. v. 35, no. 2, pp.261-269.

Freeze, R.A., and D.B. McWhorter. 1997. A Framework for Assessing Risk Reduction Due to DNAPL Mass Removal from Low-Permeability Soils. Ground Water. v. 35, no. 1, pp. 
111-123.

Fried, J.J., P. Muntzer, and L. Zilliox. 1979. Ground-water pollution by transfer of oil hydrocarbons. Ground Water. v. 17, no. 6, pp. 1921-1925.

Green, W.J., G.F. Lee, R.A. Jones, and T. Palit. 1983. Interaction of clay soils with water and organic solvents: implications for the disposal of hazardous wastes. Environmental Science and Technology. v. 17, pp. 278-282.

Hayden, N.J, and E.J. Van der Hoven. 1996. Alcohol flushing for enhanced removal of coal tar from contaminated soils. Water Environment Research. v. 69, no. 7, pp.1165-1171.

Hayden, N.J. 1997. Monthly Report to Department of Energy. Project no. ??

Hunt, J.R., N. Sitar, K.S. Udell. 1988a. Nonaqueous Phase Liquid Transport and Cleanup 1. Analysis of Mechanisms. Water Resources Research. v. 24, no. 8, pp. 1247-1258.

Hunt, J.R., N. Sitar, K.S. Udell. 1988b. Nonaqueous Phase Liquid Transport and Cleanup 1. Experimental Studies. Water Resources Research. v. 24, no. 8, pp. 1259-1269.

Imhoff, P.T., S.N. Gleyzer, J. F. McBride, L.A. Vancho, I. Okuda, and C.T. Miller. 1995. Cosolvent-enhanced remediation of residual dense nonaqueous phase liquids: experimental investigation. Environmental Science and Technology. v. 29, no. 8, pp.1966-1976.

Looney, B.B., J. Rossabi, and D.M. Tuck. 1992. Assessing DNAPL Contamination, A/M-Area, Savannah River Site: Phase I Results. Westinghouse Savannah River Company. Aiken, South Carolina.

Mackay, D.M., and J.A. Cherry. 1989. Groundwater contamination: pump and treat remediation. Environmental Science and Technology. v. 23, no. 6, pp. 2017-2029.

Miller, C.T., M.M. Poirier-McNeill, and A.S. Mayer. 1990. Dissolution of trapped nonaqueous phase liquids: mass transfer characteristics. Water Resources Research. v. 26, no. 11, pp. 2783-2796.

Morrow, N.R., I. Chatzis, and J.J. Taber. 1988. Entrapment and mobilization of residual oil in bead packs. SPE Reservoir Engineering, Society of Petroleum Engineers. August. pp. 927-934.

Morrow, N.R., and B. Songkran. 1981. Effect of viscous and buoyancy forces on nonwetting phase trapping in porous media. In: Surfactant Phenomena in Enhanced Oil Recovery. Plenum Press. New York. pp. 387-411.

Pennell, K.D., G.A. Pope, and L.M. Abriola. 1996. Influence of visocous and bouyancy forces on the mobilization of residual tetrachloroethylene during surfactant flushing. Environmental Science and Technology. v. 30, no. 4, pp.1328-1335.

Perry, P.H. and C.H. Chilton. 1973. Chemical Engineer's Handbook. McGraw-Hill.

Powers, S.E., L.M. Abriola, and W.J. Weber. 1992. An experimental investigation of nonaqueous phase liquid dissolution in saturation subsurface system: steady state mass transfer rates. Water Resources Research. v. 28, no. 10, pp. 2691-2705.

Powers, S.E., C.O. Loureiro, L.M. Abriola, and W.J. Weber. 1991. Theoretical study of the significance of nonequilibrium dissolution of nonaqueous phase liquids in subsurface systems. Water Resources Research. v. 27, no. 4, pp. 463-477.

Powers, S.E., M.E. Tamblin. 1995. Wettability of porous media after exposure to synthetic gasolines. Journal of Contaminant Hydrology. v. 19, pp. 105-125. 
Storey, J.M, and J.J. Pierce. 1989. Influence of changes in methanol concentration on clay particle interactions. Canadian Geotechnical Journal. v. 26, pp. 57-63.

Van Genuchtan, M.T. 1980. A closed-form equation for predicting the hydraulic conductivity of unsaturated soils. Soil Science Society of America Journal. v. 44, pp.892-898.

Wan, J., and J.L. Wilson. 1994. Visualization of the role of the gas-water interface on the fate and transport of colloids in porous media. v. 30, no. 1, pp. 11-23.

Wilson, J.L., S.H. Conrad, W.R. Mason, W. Peplinski, and E. Hagan. 1990. Laboratory Investigation of Residual Liquid Organics from Spills, Leaks, and the Disposal of Hazardous Wastes in Groundwater. U.S. Environmental Protection Agency. EPA/600/690/004. 


\subsection{Introduction and Background}

There are three principal mechanisms for the removal of residual phase DNAPLs: enhanced dissolution; mobilization by reduction of interfacial tensions; and mobilization by selective alcohol partitioning into the NAPL. In a remedial flushing scheme, dissolution is considered more advantageous than either form of mobilization, as unwanted downward mobilization that could potentially move the contaminant out of the remedial capture zone would not be a factor. Therefore, it appears that optimal remedial alcohol flushing scenarios would strive to maximize enhancements in dissolution while ensuring against the effects of mobilization.

A significant amount of research has focused on the dissolution that occurs as the result of the interphase mass transfer between immiscible aqueous solutions and organic liquids at residual saturation in porous media (Miller et al., 1990, Powers et al., 1991, Powers et al., 1992, Powers et al., 1994, Imhoff et al., 1996). The most common concept pertaining to the mass transfer between two immiscible phases is that the rate of interphase mass transfer is a function of a concentration gradient driving force and an interfacial area between the two phases. The general form of the mass flux equation can be written as an approximation of Fick's first law:

$$
J=K\left(C_{s}-C\right)
$$

where: $\mathrm{J}$ is the solute mass flux between a solid, vapor or immiscible liquid phase to the aqueous phase; $\mathrm{K}$ is a mass transfer coefficient; $\mathrm{C}_{\mathrm{s}}$ is the aqueous phase concentration when the aqueous phase is in thermodynamic equilibrium with the immiscible phase; and $\mathrm{C}$ is the aqueous phase solute concentration in bulk solution.

The result of the addition of an alcohol or cosolvent to the aqueous solution is an increase in the value of $\mathrm{C}_{\mathrm{s}}$ in the equation for Fick's Law. Thus, the concentration gradient driving force is increased by the presence of the cosolvent, that results in an increased rate of mass transfer from the NAPL to the aqueous phase.

Certain authors indicate the importance of the interfacial area between the two fluids by incorporating a specific surface area term in the interphase mass transfer equation. Powers et al. (1994) indicate that the total mass transferred across the interface is the product of the flux of the species (as approximated by the product of the mass transfer coefficient and the concentration gradient between the bulk aqueous phase and the concentration which would be in thermodynamic equilibrium with the organic phase) and the specific interfacial area across which mass transfer can occur:

$$
S=K a_{o}\left(C_{s}-C\right)
$$


where: $\mathrm{S}$ is the solute mass transferred between phases per unit time and volume of the medium and $a_{0}$ is the specific surface area, or the NAPL-water interfacial area per unit volume of the medium.

Equation 2 indicates the relative importance of the NAPL blob size and shape. Because a NAPL singlet would have a significantly higher specific surface area than a NAPL ganglion, the more spherical NAPL singlet found within a homogenous media would be more readily removed by dissolution processes in comparison with a branched ganglia found in a more heterogeneous media.

Experimental work performed by Miller et al. (1990) using toluene as a representative NAPL in several different porous media indicated that the mass transfer between the NAPL and aqueous phases is strongly related to both the aqueous phase velocity and the NAPL saturation. These findings illustrate the importance of sufficient contact time and interfacial area for adequate mass transfer to occur. More specifically, Powers et al. (1992) outlined that the rate coefficients and, therefore, overall mass transfer will be limited when (1) aqueous phase velocities are high (short contact times), (2) NAPL saturations are low, (3) NAPL is distributed as large, multi-pore blobs with relatively small specific surface areas and (4) when the mass fraction of soluble species within the NAPL is relatively small. Thus, it is apparent that as the result of the differing pore sizes and geometries in layered soil systems, the rate of mass transfer and therefore, the amount of time required for successful clean-up of the residual NAPL by enhanced dissolution may be quite variable among the different layers. Additional problems for complete remediation may develop with regard to the development of preferential flow paths during flushing. For instance, if one specific lens of soil material is cleaned out by more rapidly dissolving away the residual, the alcohol may continue to more easily flow through that media, thereby leaving the other media to remain contaminated with NAPL.

There has been considerable research into the actual applicability of the assumption of equilibrium between two immiscible phases and, therefore, the use of simplistic models such as the approximation of Fick's first law. Field data from contaminated NAPL sites frequently indicate that contaminant concentrations in groundwater are lower than their corresponding equilibrium values (Mackay et al., 1985; Mercer and Cohen, 1990). Explanations used to support these field observations include the presence of a rate-limited mass transfer, physical by-passing of contaminated regions due to relative permeability effects and/or aquifer heterogeneities, and the presence of multiple components in some NAPLs. However, it is important to note that numerous authors have supported the validity of the local equilibrium assumption for single component NAPLs in homogenous media in laboratory scale experimental studies, such as those being performed in this research (Fried et al., 1979; Miller et al., 1990; Anderson et al., 1992). As an example, Miller et al. (1990) showed mass transfer rates for toluene-water systems within numerous one-dimensional soil columns packed with different sized glass beads to range from 179 to $6604 \mathrm{~d}^{-1}$. These relatively high coefficients indicated that equilibrium in these systems was reached very quickly (in Miller's (1990) work, equilibrium was reached between 0.00015 and 0.00559 days, or between 0.2 and 8.0 minutes). From these results, it appears that interphase mass transfer may be successfully represented using simplistic models such as the modified Fick's Law in laboratory column investigations where the passage of one pore volume of solution may take a number of hours. 
The work of Imhoff et al. (1995) illustrated the solubility of a certain solute to be dependent upon the amount of cosolvent present in solution. At sufficiently high cosolvent concentrations, a NAPL may even become completely miscible with the solvent mixture. The use of ternary diagrams aids in the estimation of solubilities and therefore the degree of dissolution enhancement for different solute-solvent-cosolvent mixtures. Ternary diagrams are commonly used in the petroleum engineering field to illustrate two-phase systems consisting of oil, water and a particular cosolvent (or in some cases, a surfactant). Ternary diagrams are also used by the chemical engineering field to represent two phase systems of water, water-miscible solvent and water-immiscible solvent in solvent recovery processes.

Figure 6.1 is provided below as an illustration of a ternary diagram for a PCE-water-isopropyl alcohol (IPA) system.

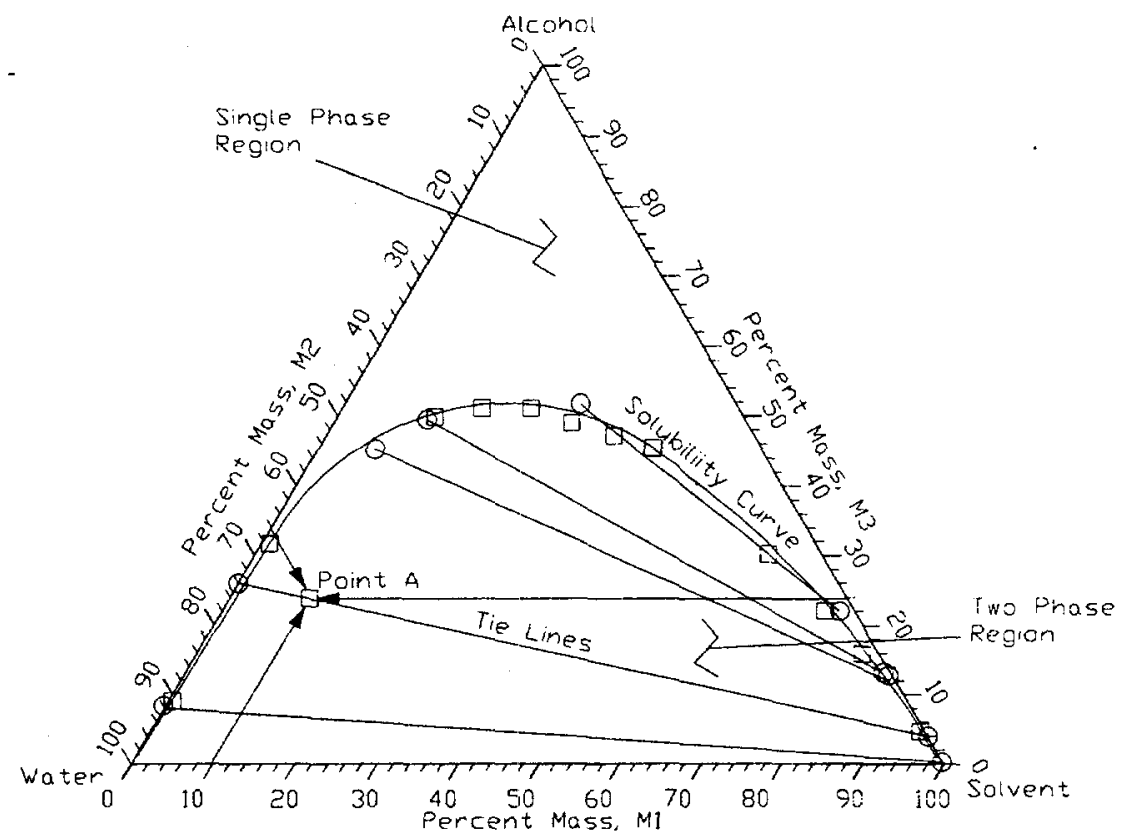

Figure 6.1 Typical Ternary Diagram

Each apex of the triangle shown in Figure 6.1 represents a pure component. All possible ternary compositions fall within the interior regions of the ternary diagram. The sides of the ternary diagram represent binary mixtures in which the component at the opposite apex is not present. For example, Point A on Figure 6.1 indicates a composition of $66 \%$ (by mass) water, $10 \%$ solvent, and $24 \%$ alcohol. The solubility curve on the inside of the diagram represents a boundary between the regions of one single and two separate phases. As is shown in Figure 6.1, a single phase will exist above the solubility curve, while the ternary system will contain two separate phases in the region below the solubility curve. Because two separate phases are present for Point $\mathrm{A}$, two different compositions are possible. These two compositions are represented by the endpoints of the tie line which passes through Point A. For example, for Point A shown on Figure 6.1, there are two endpoints illustrated 
for the tie line. The composition of the aqueous phase for Point $\mathrm{A}$ is indicated by the endpoint of the tie line to the left of the ternary diagram and, thus, closer to $100 \%$ water. The endpoint of the tie line toward the $100 \%$ solvent point at the right side of the ternary diagram illustrates the oleic composition for Point A.

The degree of dissolution of DNAPL into the aqueous phase is shown on the ternary diagram by the distance of the solubility curve from the alcohol/water side of the triangle. Where the solubility curve is close to the alcohol/water side of the triangle, the solubility of DNAPL in the aqueous phase is small. Larger aqueous phase solubilities can be obtained as the solubility curve diverges from the alcohol/water side of the ternary diagram. This is the desired location within which to operate during a remedial operation where enhanced dissolution is sought to be maximized. In practice, however, care must be taken not to operate at a location on the ternary diagram that is too close to the solubility curve, as dispersive mixing at the alcohol front may cause the alcohol concentration to diminish which could result in the existence of two separate phases when the overall composition falls below the solubility curve (Brandes and Farley, 1993).

As shown in Figure 6.1, the tie lines are shown to have a negative slope for the particular ternary diagram which was used for illustrative purposes. Alcohols such as IPA which have negatively sloping tie lines will more readily partition into the aqueous phase rather than the oleic, or nonaqueous phase. Larger, higher molecular weight alcohols which are less polar will be shown in a ternary diagram to have positively sloping tie lines. These alcohols will partition into the oil phase and have the effect of swelling the NAPL.

Alcohols such as IPA, which favor partitioning into the aqueous phase, will more effectively enhance the dissolution of the trapped NAPL blobs. This is due to the fact that the alcohol concentration remains high in the aqueous phase, and the NAPL blobs do not swell but rather diminish in size, thereby increasing the specific surface area required for enhanced dissolution. The nature of IPA as an alcohol which more effectively dissolves residual NAPL was one of the reasons that it was selected for use in the research herein.

\section{Emulsification Issues}

As a result of the often complex phase behavior of the ternary system of cosolvent, DNAPL and water, NAPL removal cannot be solely attributed to dissolution and mobilization processes. A third, and often significant mechanism is that of the transport of NAPL in water-based macro emulsions.

Okuda et al., (1996) defined macro emulsions as small droplets of NAPL which are suspended in the aqueous phase which do not coalesce rapidly. They provide definitions of three forms of NAPL in typical water-NAPL-surfactant systems. The first form is that of the mobilized NAPL, which is in the form of coalescible droplets of NAPL and released from the soil media when capillary forces acting on the residual NAPL are overcome by viscous and/or gravitational forces. The second form of NAPL within the ternary system is that of solubilized NAPL. The solubilized NAPL is defined by Okuda et al. (1996) as either dissolved NAPL in the aqueous phase or micro emulsions that are transparent and easily transported in the aqueous phase due to their relatively small sizes of less than 
$1 \mu \mathrm{m}$. Finally, Okuda et al. (1996) provide information pertaining to the third form of NAPL in a ternary mixture. Macro-emulsified NAPL are described as thermodynamically unstable polydispersed NAPL droplets of greater than $1 \mu \mathrm{m}$ in size whose transport behavior can be significantly different from the bulk fluid.

In general, macro emulsions are considered thermodynamically unstable because they will eventually separate into two phases. However, this separation may take weeks or months and, therefore, they can often be considered as existing in pseudo-equilibrium with the aqueous phase, or as being stable. In addition, the presence of surfactants and/or cosolvents aids in achieving emulsion stability as a result of the reduction in interfacial tensions and the formation of rigid films on the surface of the droplets (Kokal et al., 1996).

The formation of these macro emulsions during surfactant or cosolvent flushing is important because: (i) macro emulsions may have an effect upon the permeability of the aquifer media (ii) macro emulsions are mobile and, therefore, their movement should be controlled during a remediation exercise and (iii) the formation of the macro emulsions may affect the solubilization kinetics of the DNAPL.

McAuliffe (1973) was the first researcher to propose the concept of a permeability reduction in porous media as a result of emulsion flow. He postulated that since the macro emulsions were typically of similar size to that of the pore throat radii, the emulsion droplets would eventually plug many of the throats, leading to a permeability reduction. Soo and Radke (1984) confirmed that a significant permeability reduction in porous media is derived from the retention of emulsion drops by the soil pores in a process they termed straining capture of the oil droplets. They also observed that emulsion droplets smaller than the pore throats were also trapped by crevices and pockets and sometimes on the surface of the porous media. Kokal et al. (1996) summarize that two factors control the permeability reduction in porous media from macro emulsions: (i) the total volume of droplets retained by the soil and (ii) the effectiveness of the droplets in restricting the flow, which is most directly the result of droplet size.

\subsection{Methods}

\section{Alcohol Flushing Experiments}

Following the establishment of an adequate residual saturation in each column and the quantification thereof, the columns were prepared to be flushed with an alcohol solution. The major objective of this research was to evaluate the efficacy of alcohol flushing in removing DNAPLs from each layered soil. A secondary objective was to investigate the effect of alcohol flushing on the permeability of the soil matrix to aqueous solution as an indicator of the degree of clean-up within the soil media as a result of flushing. In other words, it was proposed that as the NAPL was flushed from the soil, the permeability could be shown to increase. Thus, experiments were conducted to monitor both the degree of PCE removal as well as the degree of permeability increase in the soil columns. These objectives were completed by analyzing effluent PCE concentrations and 
monitoring differential pressures across the soil columns throughout the duration of the experiments.

The alcohol used for this research was iso-propyl alcohol (IPA). IPA was chosen over methanol and ethanol primarily because of its greater impact on the dissolution of PCE. In addition, IPA will not preferentially partition from the water into the PCE. Thus, swelling of the nonaqueous phase is not an issue with IPA.

The methods of Pennell et al. (1996) were followed in order to estimate the maximum percent alcohol solution that could be used with each soil media so as to maximize the enhancement of PCE dissolution while avoiding the effects of mobilization. Following this method, the permeabilities of the soil media to aqueous solution both prior to and following residual saturation with PCE were used in an estimation of the total trapping number, $\left(\mathrm{N}_{\mathrm{T}}\right)$. In the case of the two media employed during this work; an IPA concentration of $40 \%$ volumetrically was initially used in all but one homogenous column. In that one column, an initial IPA concentration of $30 \%$ was used in an attempt to totally avoid mobilization. An initial IPA concentration of $40 \%$ was used for all heterogeneous columns. The IPA concentration was typically increased to $50 \%$ after a significant amount of PCE had been dissolved from the soil media in order to expedite the completion of flushing activities.

In four of the homogenous column experiments, the alcohol solution was injected into the column in a downflow direction from the top of the column using the syringe pump. As siphon effects would have been a problem if this injection technique had been used for the heterogeneous columns, a special syringe pump with withdrawal capability was initially used to pump the solution from a reservoir down through the column and into the two syringes for the first heterogeneous column. In order to generate data using consistent methods with this heterogeneous column, two additional homogenous columns were flushed in a downward direction using the special syringe pump with withdrawal capability. For these three columns, the cosolvent solution was essentially pulled through the column and through the double sided endcaps.

However, the downflow pumping method was observed to have flaws, as the short circuiting of the fine material was observed through overly rapid clean-up times of the medium sand and unrealistic permeability results for both the fine and the medium sands. This short circuiting effect will be described in detail later. As a result, a different method was attempted in order to obtain more accurate measurements of permeability and effluent PCE concentrations. A gravity-induced downflow system was used. In this system, a state of zero pressure was maintained at the very bottom of the column, where the soil media was in contact with the screen on the double-sided endcap. A differential head driving force was imposed on the system through the use of a constant head reservoir. The rate of flow through each media was then measured by allowing the effluent to flow into separate collection vials over a prescribed time interval. The previously encountered siphon effect problem was avoided by drilling out the ports on the double-sided endcap to sizes such that the formation of a droplet would not clog the port. Thus, the location of zero pressure was at equal heights on each side of the endcap. 
A summary of the flushing scenarios and the methods of IPA solution introduction into each column is provided in Table 6.1 below.

Table 6.1 Summary of Alcohol Flushing Scenarios

\begin{tabular}{|c|c|c|c|}
\hline Column & $\begin{array}{c}\text { Method of Introducing IPA } \\
\text { Solution }\end{array}$ & Initial \% IPA & Final \% IPA \\
\hline \hline W-1 & downflow syringe injection & 40 & 50 \\
\hline W-2 & downflow syringe injection & 40 & 50 \\
\hline W-3 & downflow syringe withdrawal & 30 & 40 \\
\hline F-2 & downflow syringe injection & 40 & 50 \\
\hline F-3 & downflow syringe injection & 40 & 50 \\
\hline F-4 & downflow syringe withdrawal & 40 & 40 \\
\hline H-6 & downflow syringe withdrawal & 40 & 50 \\
\hline H-9 & gravity-driven downflow injection & 40 & 50 \\
\hline H-10 & gravity-driven downflow injection & 40 & 50 \\
\hline
\end{tabular}

\section{Effluent Sample Collection}

Effluent samples were collected throughout the column flushing experiments from stainless steel two-way tee valves which were located immediately below the column in the effluent tubing apparatus. The tubing apparatus either ran directly into an effluent catchment vessel in instances where the alcohol solution was injected from the top of the column or the withdrawal syringe pumps in the cases where the alcohol solution was pumped through the column. Figure 6.2 is provided below to illustrate the effluent sample collection procedure for the homogenous columns. 


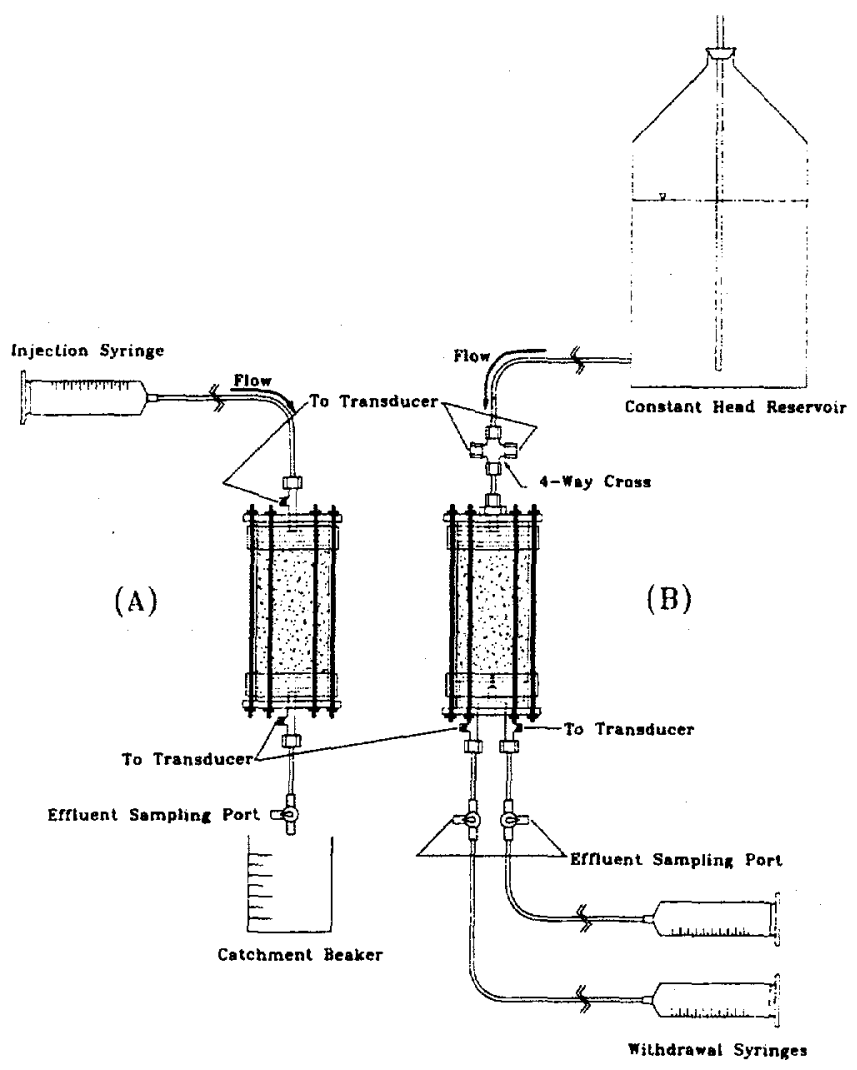

Figure 6.2 Schematic Illustrating Homogenous Column Flushing Scenarios

(A) Columns with Single Port Lower Endcaps

(B) Columns with Double Port Lower Endcaps

At the time of sample collection, the stop-cock on the two way tee was switched so that the ternary alcohol/PCE/water solution was able to flow to the edge of the valve outlet. In columns W-1, W-2, $\mathrm{F}-2$ and F-3, this was accomplished by allowing the syringe pump to pump the effluent to the outlet after switching the valve. In the case of columns W-3 and F-4, the withdrawal syringe pumps were temporarily shut off immediately before the valve was switched. The valve was carefully opened and the effluent solution was allowed to flow by gravity toward the edge of the outlet portion of the valve. The stop-cock was shut immediately when the effluent solution was apparent at the edge of the valve. This was done to avoid against solution being lost from the system. In all columns, once the effluent reached the edge of the valve opening, the sample was collected. Microliter syringes $(10,50$ and $100 \mu \mathrm{l})$ were used to collect the effluent samples from the inner reaches of the valve outlet. Samples were collected from this inner location in order to minimize PCE losses due to volatilization. Different volumes of sample were collected depending upon the expected concentration of PCE in the effluent. The collected sample was then injected into a $20 \mathrm{ml}$ vial containing $5 \mathrm{ml}$ of distilled water. The vial was subsequently crimp-capped and placed in a 
refrigerator at $\pm 4^{\circ} \mathrm{C}$ prior to headspace analysis. Following sample collection, the stop-cock on the valve was manipulated so that the solution within the open portion of the valve would be flushed out of the effluent line, thus allowing the sample port to be dry until the time of the next sample.

Following the termination of flushing activities, the soil was analyzed for any remaining PCE. Remaining PCE within the homogenous columns was quantified following the procedures outlined. All sampling procedures were conducted at $22 \pm 2^{\circ} \mathrm{C}$.

\section{Heterogeneous Columns}

Effluent samples were collected in two different fashions for the heterogeneous columns of the earlier column design and through side ports in the later design. For Column H-6, effluent samples were collected from stainless steel two-way tee valves which were located immediately below the column in the effluent tubing apparatus which ran directly into the withdrawal syringe pumps. For Columns $\mathrm{H}-9$ and $\mathrm{H}-10$, the effluent was allowed to drip out of each side of the column and into a catchment vessel. Figure 6.3 is provided below to illustrate each effluent sample collection procedure.

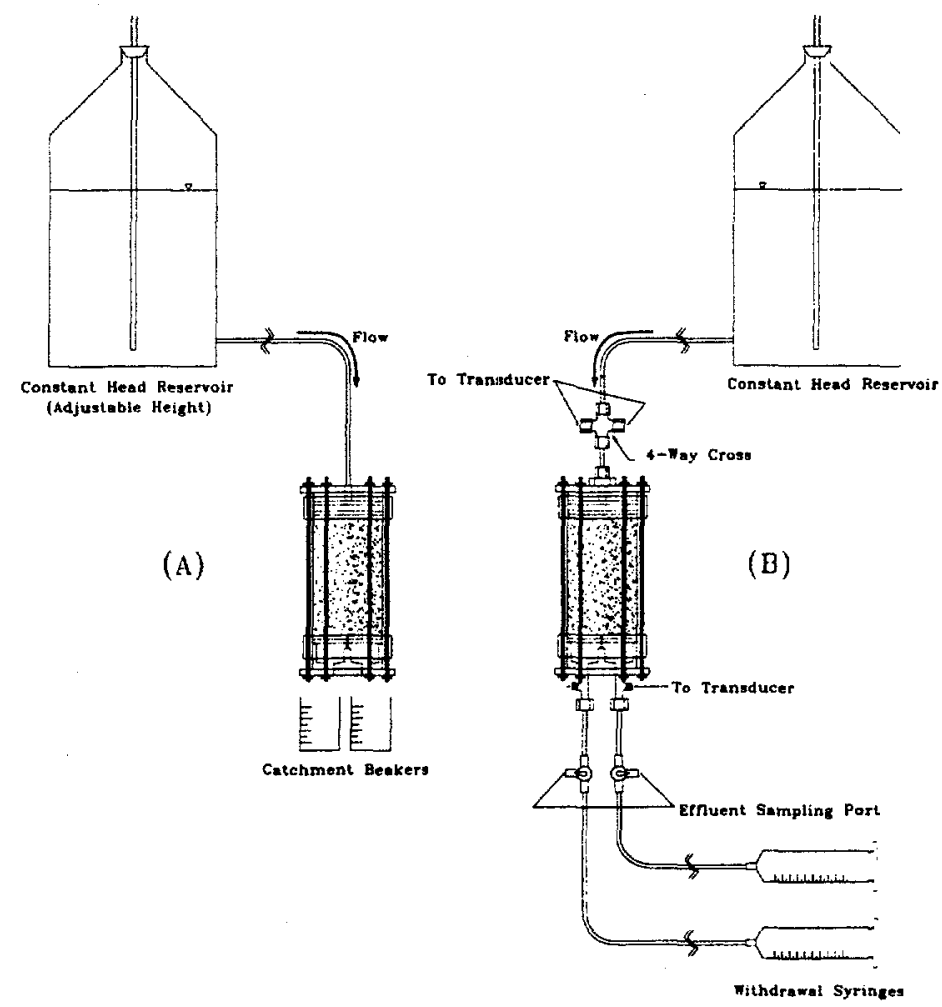

Figure 6.3 Schematic Illustrating Heterogeneous Column Flushing Scenarios

(A) Columns $\mathrm{H}-9$ and $\mathrm{H}-10$

(B) Column H-6 
The method used for sample collection for Column H-6 was exactly the same as that described for Columns W-3 and F-4 above. For Columns H-9 and H-10, the effluent flowed under gravitational head through each media and into two separate catchment beakers below the column. This method was employed so that a more accurate measure of the flowrate through each media could be obtained. For consistency purposes, the sample effluent was allowed to drip for exactly one minute from each media. Microliter syringes ( 20 and $50 \mu 1$ ) were then used to collect the effluent samples from the catchment vessels. Different volumes of sample were collected depending upon the expected concentration of PCE in the effluent. The collected sample was then injected into a $20 \mathrm{ml}$ vial containing $5 \mathrm{ml}$ of distilled water. The vial was subsequently crimp-capped and placed in a refrigerator at $\pm 4^{\circ} \mathrm{C}$ prior to headspace analysis.

The soil was again analyzed for any remaining PCE following the termination of flushing activities. Each media from Column H-6 was extracted, prepared and analyzed in the same fashion as that described for Columns F-4 and W-3 and described in detail in Section 3.6.1b. This use of this method allowed each media to be totally cleaned out. The soils within Columns $\mathrm{H}-9$ and $\mathrm{H}-10$ were analyzed using the same incremental sampling technique that was used to determine residual saturations in Columns $\mathrm{H}-7$ and $\mathrm{H}-8$. This method was used for Columns $\mathrm{H}-9$ and $\mathrm{H}-10$ because of the fact that flushing was terminated after the medium sand had been cleaned out and before complete remediation of the fine sand. Thus, the use of this method allowed estimations of both the total amount of PCE left in each media as well as the remaining distributions of NAPL.

\section{Gas Chromatograph Sample Analysis}

The analysis of the effluent samples and the soil leachate samples was conducted with a gas chromatograph (Hewlett Packard Model 5890 Series II) with a flame ionization detector (FID) and a 30 meter DB624 glass capillary column (J\&W Scientific). An automated headspace sampler (Hewlett Packard 19395A) was used in conjunction with the gas chromatograph for all sample analysis. The gas chromatograph utilized helium as the carrier gas at a flowrate of $16 \mathrm{ml} / \mathrm{min}$. The injector temperature was $200^{\circ} \mathrm{C}$ and the detector temperature was $300^{\circ} \mathrm{C}$. The method developed to analyze the PCE content of the headspace above each sample is summarized in Table 6.2 below:

\begin{tabular}{|l|l|}
\hline \multicolumn{2}{|c|}{ Table 6.2 Summary of GC Method for Headspace Analysis } \\
\hline Parameter & Value \\
\hline \hline Initial Temperature & $60^{\circ} \mathrm{C}$ \\
\hline Hold & $2 \mathrm{~min}$ \\
\hline Ramp & $45^{\circ} \mathrm{C} / \mathrm{min}$ \\
\hline Final Temperature & $180^{\circ} \mathrm{C}$ \\
\hline Hold & $2 \mathrm{~min}$ \\
\hline
\end{tabular}


Calibration stock standards were prepared according to the procedure outlined in United States Environmental Protection Agency (USEPA) Method 602 (USEPA, 1986). Reagent grade methanol was used as the solvent for the stock standards. A glass-ground $10 \mathrm{ml}$ volumetric flask was filled with approximately $9.8 \mathrm{ml}$ of methanol using a $10 \mathrm{ml}$ glass syringe. The flask was then capped and weighed on a five place balance. Approximately $100 \mu \mathrm{l}$ of PCE was then added to the volumetric flask using a $100 \mu$ l glass syringe. The flask was then recapped and weighed again to determine the mass of PCE injected into the flask. Finally, the $10 \mathrm{ml}$ flask was filled to volume. The solution was mixed by inverting the flask several times and the contents were then poured into a $10 \mathrm{ml}$ headspace vial. The headspace vial was immediately crimp capped with a Teflon-coated septum, covered with white adhesive tape to mitigate light infiltration, and stored in darkness at $0^{\circ} \mathrm{C}$. Secondary stock solutions of 10:1 and 100:1 dilutions were prepared from the initial stock solutions. All stock solutions were prepared monthly and checked against the old stock solutions. The new stock solutions were considered acceptable if they were found to be within $5 \%$ of the old stock solutions.

A calibration curve was generated on a daily basis during GC use. At least five calibration standards were generated by taking known volumes of the stock standard solutions and placing them in $5 \mathrm{ml}$ of distilled water. The standards were generated to bracket the expected range of PCE concentrations in the actual samples. At least one linear regression was performed on the calibration points, with PCE concentration as the independent variable and response as the dependent variable. The percent error of each calibration point from the least squares estimate of the regression line was calculated. The calibration was considered appropriate if the majority of the least squares estimates of concentration of the calibration standards were within $10 \%$ and none were of by more than $15 \%$ of the known values. Check standards were run after every ten samples. Again, if the majority of the regression-calculated check standard concentrations were within $10 \%$ and no greater than $15 \%$ of their actual values, the analytical run was considered acceptable.

\subsection{Results}

It was important to fully characterize columns prior to flushing to understand both the amount of residual DNAPL trapped in each media as well as the degree to which the NAPL is trapped. The magnitude of trapping will have a significant effect upon the removal of the DNAPL by alcohol flushing. The methods of Pennel et al. (1996) were employed in order to quantify the degree of trapping and, therefore, to develop a flushing scenario that would maximize mass removal rates while avoiding. NAPL mobilization.

The theory behind the trapping number $\left(\mathrm{N}_{\mathrm{T}}\right)$ method was previously discussed. If the intrinsic permeability of a specific media and the effective permeability of that media at residual saturation to the aqueous phase are known, a calculation of the trapping number and, thus a quantification of the degree of NAPL entrapment can be made (assuming that parameters intrinsic to the NAPL-water pair such as interfacial tension, density and viscosity are also known). Intrinsic permeabilities (k) and permeabilities after NAPL saturation $\left(\mathrm{k}_{\mathrm{n}}\right)$ were calculated and the results are provided in Table 6.3 . 
Table 6.3 Permeability Values for All Experimental Columns

\begin{tabular}{|c|c|c|c|c|c|c|}
\hline Column & Sand Type & $\begin{array}{c}\mathbf{k} \\
\left(\mathbf{c m}^{2}\right)\end{array}$ & $\begin{array}{c}\mathbf{k}_{\mathbf{n}} \\
\left(\mathbf{c m}^{2}\right)\end{array}$ & Sand Type & $\begin{array}{c}\mathbf{k} \\
\left(\mathbf{c m}^{2}\right)\end{array}$ & $\begin{array}{c}\mathbf{k}_{\mathbf{n}} \\
\left(\mathbf{c m}^{2}\right)\end{array}$ \\
\hline \hline F-2 & fine & $1.49 \mathrm{E}^{-8}$ & $5.74 \mathrm{E}^{-9}$ & & & \\
\hline F-3 & fine & $1.22 \mathrm{E}^{-8}$ & $5.71 \mathrm{E}^{-9}$ & & & \\
\hline F-4 & fine & $2.24 \mathrm{E}^{-8}$ & $6.08 \mathrm{E}^{-9}$ & & & \\
\hline $\mathrm{W}-1$ & & & & medium & $2.45 \mathrm{E}^{-7}$ & $8.53 \mathrm{E}^{-8}$ \\
\hline $\mathrm{W}-2$ & & & & medium & $2.75 \mathrm{E}^{-7}$ & $1.22 \mathrm{E}^{-7}$ \\
\hline $\mathrm{W}-3$ & & & & medium & $2.34 \mathrm{E}^{-7}$ & $1.12 \mathrm{E}^{-7}$ \\
\hline $\mathrm{H}-6$ & fine & $1.23 \mathrm{E}^{-7}$ & $1.10 \mathrm{E}^{-7}$ & medium & $2.85 \mathrm{E}^{-7}$ & $1.77 \mathrm{E}^{-7}$ \\
\hline $\mathrm{H}-9$ & fine & $1.60 \mathrm{E}^{-8}$ & $7.64 \mathrm{E}^{-9}$ & medium & $3.33 \mathrm{E}^{-8}$ & $2.90 \mathrm{E}^{-8}$ \\
\hline $\mathrm{H}-10$ & fine & $1.60 \mathrm{E}^{-8}$ & $6.06 \mathrm{E}^{-9}$ & medium & $3.35 \mathrm{E}^{-8}$ & $1.64 \mathrm{E}^{-8}$ \\
\hline
\end{tabular}

$\mathrm{k}=$ intrinsic permeability

$\mathrm{k}_{\mathrm{N}}=$ effective permeability at residual NAPL saturation

Figure 6.4 provides a graphical illustration of the differences in permeability values as a result of the residual NAPL saturation. In Figure 6.4, fine sand permeability data are illustrated in the bars on the left side of the graph. Data from the medium sand are presented on the right side of the graph. The data from Column H-6 were not included in Figure 6.4 due to a lack of confidence in permeability values as a result of the by-passing issue.

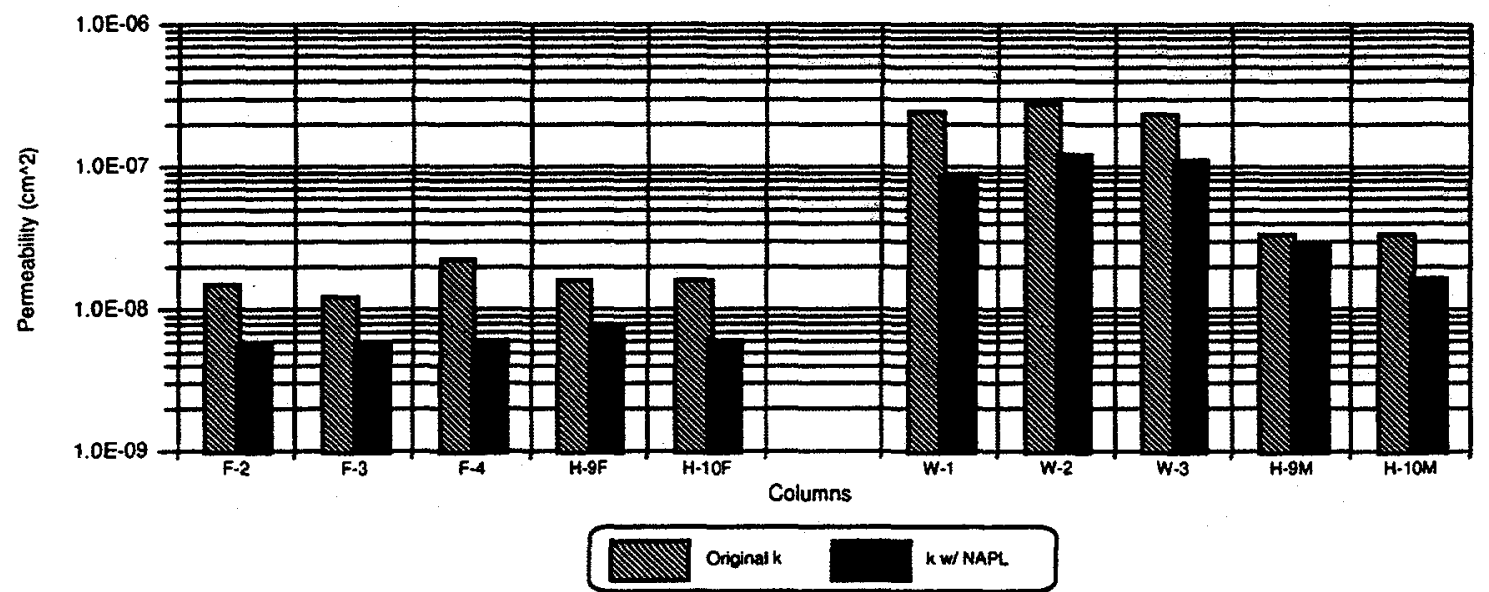

Figure 6.4 Permeabilities Before and After Residual PCE Saturation

As shown in both Table 6.3 and Figure 6.4, the result of the residual saturation in the fine sand is a 
lowering of the permeability by nearly one half order of magnitude. The effect is observed to be slightly less in the medium sand.

The permeabilities of the medium sand media of the heterogeneous columns are lower both before and after NAPL saturation in comparison with the values in the homogenous columns. This is most likely due to the previously mentioned soil mixing issue in the layered columns. There does not appear to be a similar effect in the fine media of the heterogeneous columns.

The experimentally determined permeabilities before and after residual NAPL saturation were used with experimentally measured values for viscosity, density and interfacial tension in the calculation of the trapping number. The trapping number provides an estimation of the degree to which the residual NAPL is held within the soil pores. The trapping number was used to determine the maximum percent IPA solution that could be used in order to remediate the residual DNAPL by dissolution while avoiding unwanted mobilization. According to the research of Pennel et al. (1996), the critical range of trapping numbers required to initiate mobilization falls within the range of $2 \mathrm{E}^{-5}$ to $5 \mathrm{E}^{-5}$ in homogenous sand.

\section{Homogenous Columns}

Effluent PCE concentrations provided an indication as to the degree of PCE remediation within the column and also served as a secondary indicator of the total mass of PCE actually entrapped at residual saturation. Three homogenous columns of each soil media were initially studied. The effluent concentration data for Column F-2 is provided here as an illustration of the typical results from the flushing of the homogenous columns.

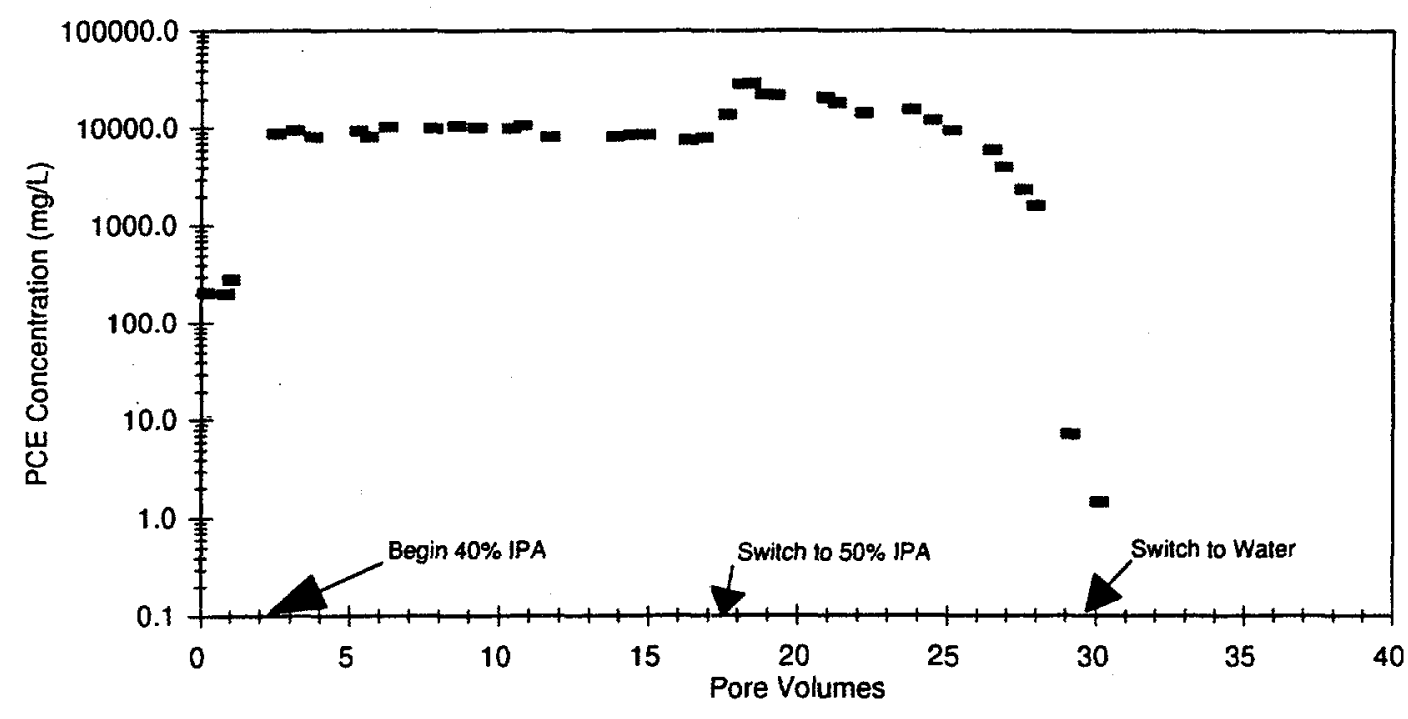

Figure 6.5 Column F-2 Effluent PCE Concentrations

Figure 6.4 illustrates the benefit of using alcohol flushing in the remediation of residual phase 
DNAPLs. The effluent PCE concentration data show the PCE to be removed down to a concentration of $1 \mathrm{mg} / \mathrm{L}$ after approximately 30 pore volumes of a $40 \%$ and then $50 \%$ IPA solution was flushed through the column. In comparison, the removal of this amount of DNAPL by water flooding alone would have required approximately 1,400 pore volumes. Thus, the remediation time was reduced by a factor of nearly 50 through the use of dissolution-enhancing alcohols. The benefit of the use of alcohol flushing can also be seen in the effluent PCE concentrations. At $40 \%$ IPA, the effluent concentrations of PCE are approximately $10,000 \mathrm{mg} / \mathrm{L}$, or 50 times greater than the solubility value of $200 \mathrm{mg} / \mathrm{L}$ for PCE in water. A $50 \%$ IPA solution can increase solubility values by a factor of greater than 150 .

For consistency with other experimental columns, Column F-2 was initially flushed with a $40 \%$ alcohol solution. In order to expedite the remediation process, the IPA solution was switched to a $50 \%$ solution after approximately 16.5 pore volumes. The effluent PCE concentrations appear to be consistent in consideration of the ternary diagram for the PCE/IPA/water system.

An IPA/water ratio of around $40 / 60$ corresponds to a PCE content of around 1.5 to $2 \%$ by mass in the single-phase region. The PCE content would vary, depending upon the exact makeup of the ternary mixture. For example, the makeup could be $39.0 \%$ IPA, $59.5 \%$ water, $1.5 \%$ PCE or $39.0 \%$ IPA, $59.0 \%$ water, $2 \%$ PCE, etc. A $1.5 \%$ PCE content by mass within the aqueous phase would correspond to an aqueous phase concentration of slightly less than $10,000 \mathrm{mg} / \mathrm{L}$, considering the density of PCE of $1.63 \mathrm{~g} / \mathrm{ml}$. A $2 \%$ PCE content by mass would result in an aqueous phase concentration of slightly more than $12,000 \mathrm{mg} / \mathrm{L}$. Likewise, the $P C E$ concentrations resulting from the flush of 50\% IPA solution appear to be reasonable as well. The ternary diagram illustrated in Chapter 3 indicates that PCE amounts of up around 7 percent would be plausible. Thus, dilute aqueous PCE concentrations of up to approximately $40,000 \mathrm{mg} / \mathrm{L}$ would appear to be appropriate for the 50\% IPA flushing scenario. However, ternary phase diagrams from Chapter 3 indicate the solubility curve between the single and two-phase regions to be slightly further away from the alcohol/water leg of the triangle. This means there are a greater number of possible ternary mixtures and thus aqueous phase PCE concentrations.

Each of the remaining columns was flushed in a similar fashion. The results of all but two columns appeared to be consistent with each other and with the concentrations that would have been expected in consideration of the ternary diagram. The effluent concentrations from Column W-3 and F-4 were found to be slightly lower than those of the other four homogenous columns. The reason for this discrepancy was not clear, but there were differences in endcaps between Columns W-3 and F-4 and the remainder of the homogenous columns. These two columns used the specially constructed aluminum endcaps while the other four columns had stainless steel endcaps at each end of the column, perhaps resulting in some slight variation. Silicone was used to hold the screens and divider plate in place on the double-sided endcap and may have contributed to the apparent minor losses due to adsorption. It appeared to take longer for the column to reach equilibrium effluent concentrations at the initiation of flushing, and effluent concentrations were observed to tail off at a more gradual rate at the conclusion of flushing for Columns W-3 and F-4. The gradual tailing shown in these data may have been indicative of the desorption of PCE from the silicone into the passing aqueous solution. 


\section{Heterogeneous Columns}

Column H-6 was flushed in the same way as Columns W-3 and F-4, via withdrawal syringe pumps. As a result of the problems of by-passing with this method, the final two heterogeneous columns, $\mathrm{H}-9$ and $\mathrm{H}-10$ (H-7 and H-8 were sacrificed), were flushed by allowing the alcohol solution to flow downward through each media under a natural hydraulic gradient.

While syringe withdrawal method was successful in completely removing all of the residual DNAPL from each media, the issue of by-passing that could not be quantified was again apparent upon analysis of the effluent results (see Figure 6.5). The effluent concentration data for each media within Column H-6 is shown in Figure 6.5.

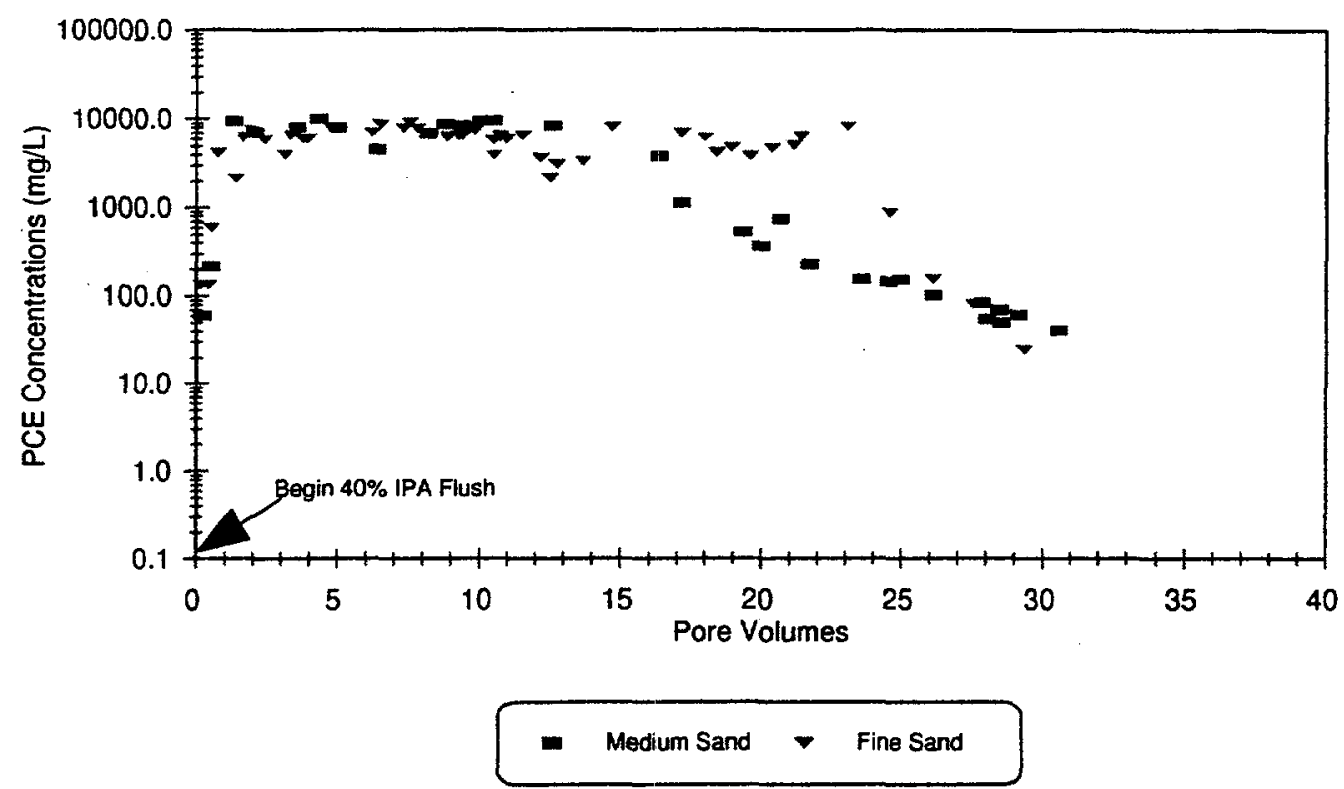

Figure 6.5 Column H-6 Effluent PCE Concentrations

On a normalized pore volume scale, both media should have been observed to be cleaned out within approximately the same number of pore volumes if the flow through each media was actually the same. Thus, it appears that a greater flowrate passed through the medium sand. Additional support for the by passing issue is shown in the nature of the down slope of the effluent concentration curve for the medium sand. In homogenous systems, when all of the NAPL was removed, the effluent concentrations dropped precipitously. The effluent concentrations curve for the medium sand in Figure 6.5 seems to indicate some sort of interference with the fine material, as it is shown to slope very gradually. The possibility of the desorption of PCE from the silicone on the lower endcap was brought to attention in the discussion of the results from Columns F-4 and W-3. This may have also been a slight factor in Column H-6, but it is more likely that the majority of the tailing can be attributed to an influence with the fine media.

The distribution of DNAPL within the medium sand of the layered columns may also have an 
influence on the tail of the effluent concentration curve for the medium sand shown in Figure 6.5. The medium sand was shown by dispersion analyses, permeability data and direct observation to be slightly mixed with some of the fine grained media. This mixing may have also been exacerbated by the vibrational packing technique used for Column H-6. As a result of the mixing between sand layers, some of the DNAPL within this media may have been trapped as branched ganglia. This type of NAPL distribution is quite different than the singlet-dominated distribution thought to be prevalent within the homogeneous columns and the fine media within the layered columns. Branched ganglia would be more resistant to remediation by dissolution-enhanced IPA flushing, as the specific surface area of the branched ganglia is much lower than that of singlets.

Effluent concentrations for each media during the $40 \%$ IPA flush at the beginning of the experiment appear to be appropriate in consideration of the ternary diagram at the beginning of the treatment. It should be noted, however, that the concentrations of PCE are slightly lower than those of the homogenous columns with stainless steel endcaps. The initial PCE concentrations are more similar to those of Columns W-3 and F-4 as Column H-6 had the specially constructed endcap which contained silicone caulking. The concentrations are lower than would be expected for a $50 \%$ IPA flush. The reason for this may be due to the fact that the medium sand is completely cleaned out of PCE and the effluent is effectively being diluted.

While the method used for flushing Column H-6 had problems, the method used for Columns H-9 and $\mathrm{H}-10$ was also found to be problematic with regard to the effluent analysis. The effluent results of Column H-9 are shown in Figure 6.6.

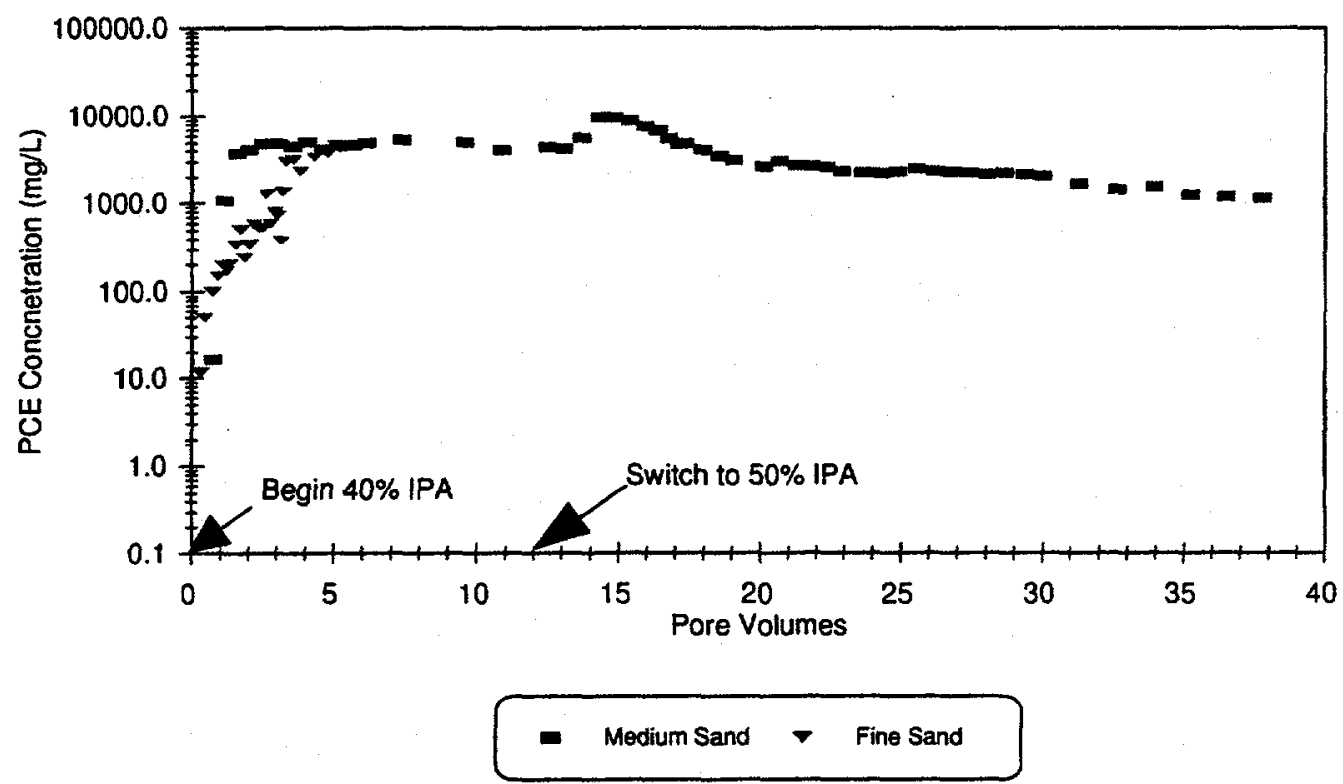

Figure 6.6 Column H-9 Effluent PCE Concentrations

The method of sample collection that was used caused the effluent to be exposed to volatilization and therefore, caused the concentration data to be much lower than that shown in previous 
experiments is illustrated on Figure 6.6. Typical values for effluent PCE concentrations during a flush with $40 \% \mathrm{IPA}$ are around $10,000 \mathrm{mg} / \mathrm{L}$. Typical effluent concentrations for a $50 \% \mathrm{IPA}$ flush are approximately $40,000 \mathrm{mg} / \mathrm{L}$. .

A volatilization test was performed during the alcohol flushing activities for Column H-9. During this test, a saturated ternary solution of PPA, water and PCE was allowed to drip from a constant head source at the same rate as effluent from the column. The flowrate, height of fall, and time for sample collection were identical to those of the flushing experiment. Additionally, the effluent samples were diluted in the exact same manner and analyzed on the gas chromatograph during sample analysis for the actual alcohol flush effluent samples. In order to quantify the volatilization loss, a sample was collected from inside the constant head reservoir containing the ternary solution and two effluent samples were collected after the solution had been allowed to drip into a catchment vessel for a period of one minute. This process was repeated ten times during the experiment. The results of the study indicated a volatilization loss of approximately $45 \%$.

The loss was used in the estimation of the residual saturation by effluent analysis. In other words, the integration for the mass of PCE removed from the column was performed and the resultant value was corrected for volatilization loss. These values compared favorably to the values obtained via the partitioning tracer test (PTT). However, a closer analysis of the corrected individual effluent data indicated that some additional source of loss remained, as the corrected concentration values were still observed to be slightly low.

The flowrate during the flush of Column $\mathrm{H}-9$ had to be somewhat elevated to both complete the test within one long day and obtain adequately sized samples from each media in minimal time frames. (Flushing could not be intermittently discontinued because of problems which may have arisen as a result of diffusion from zones of higher contamination to zones of lower contamination within the column.) It was postulated that this elevated flowrate may not have allowed equilibrium concentrations to develop during the flush. In order to check this postulation, a homogenous column was packed and brought to residual PCE saturation following the methods outlined earlier. The column was a $10 \mathrm{~cm}$ glass column with stainless steel endcaps, exactly the same as Columns W-1 and W-2. The column was flushed with a $40 \% \mathrm{IPA}$ solution at a flowrate of $30 \mathrm{ml} / \mathrm{hr}$, which was the approximate flowrate for the alcohol flush of Column H-9. Eight effluent samples were collected at one hour increments after approximately one pore volume of solution had been flushed through the column. The effluent concentrations were slightly lower than those of Columns W-1 and W-2, indicating that the elevated flowrate may, in fact, have contributed to a loss of mass during the alcohol flush. The loss was found to be approximately $10 \%$.

While the effluent data of Columns $\mathrm{H}-9$ and $\mathrm{H}-10$ may not be representative of the actual effluent concentrations exiting the column, the overall trend of the data is very interesting. Similar to Column H-6, the gradual slope of the data is different than results shown for homogenous columns, where effluent data declines sharply upon removal of the last bit of NAPL. The gradual slope of the medium sand data in Figure 6.6 suggests an influence from the fine media. As the medium sand becomes clean, contamination from the fine sand appears to be migrating into the medium sand side. Thus, the effluent data seem to be indicating that in layered heterogeneous columns it might be 
possible to obtain the complete remediation of both media if enough time is allowed and a sufficient amount of alcohol solution is used.

The effluent data for Columns $\mathrm{H}-9$ and $\mathrm{H}-10$ indicate that the fine media in each column was never successfully cleaned of residual PCE. Of interest are the extremely low effluent concentrations for $40 \%$ and $50 \%$ IPA flushes. As the flowrate through the fine sand only averaged approximately 5 $\mathrm{ml} / \mathrm{hr}$, the reason for the low effluent concentrations could not have possibly been one of insufficient time for adequate mass transfer between the NAPL and aqueous phases. The reason must have been an increased rate of volatilization in comparison with the medium sand effluent, which was most likely due to the lower flowrate. The lack of enhanced removal from the fine material of each layered column was partly the result of an increased ratio of flow through the medium sand to that in the fine sand $\left(\mathrm{Q}_{\mathrm{M}} / \mathrm{Q}_{\mathrm{F}}\right)$. The increase in this ratio was due to the expedited clean-up of the medium sand which caused more of the flushing solution to flow through the medium sand. Data supporting preferential flow is shown by the data in Figure 6-7.

In order to quantify the amount of PCE remaining in the fine media as well as to check on the

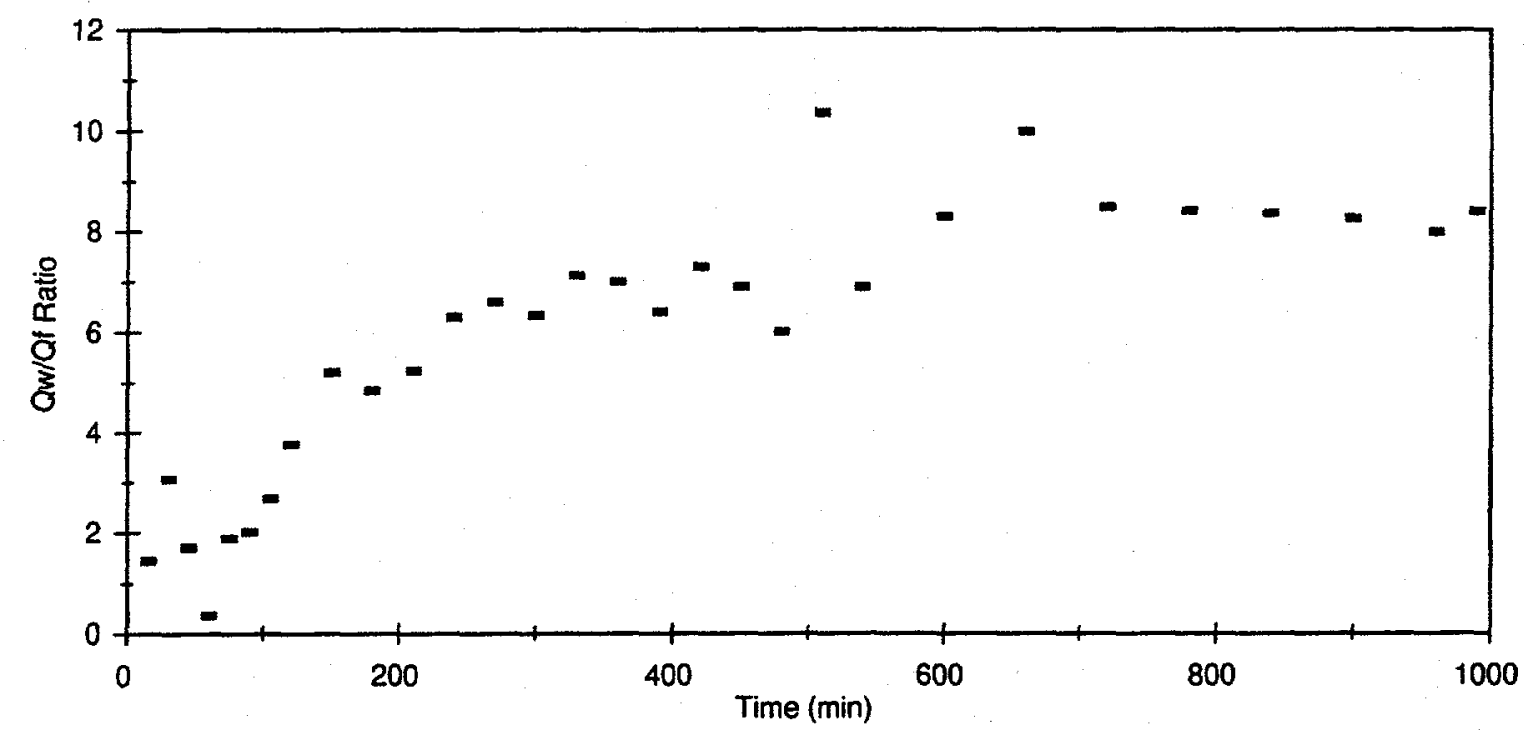

Figure 6-7 Flowrate Ratio for Column H-9

possibility of any PCE remaining in the medium sand, extraction tests were performed following the conclusion of the alcohol flushes of Columns H-9 and H-10. The method was the same as that described for the extraction tests of Columns $\mathrm{H}-7$ and H-8 which were performed to quantify the residual PCE saturation within those two columns. The only exception to the method was that for the extraction tests for Columns H-9 and H-10, three soil samples of each medium (instead of two) were collected for each one-centimeter lift. The results of the extraction tests indicated both the total amount of residual saturation remaining in each medium as well as the final distribution of the NAPL. The tests indicate that residual saturations of $17.26 \%$ and $17.32 \%$, or nearly all of the PCE 
initially thought to be within the fine media of H-9 and H-10, respectively (see Table 4.6) was still located in the columns. The distribution of the NAPL in the fine sand of Column H-9 is indicated in the bar graph of Figure 6.8.

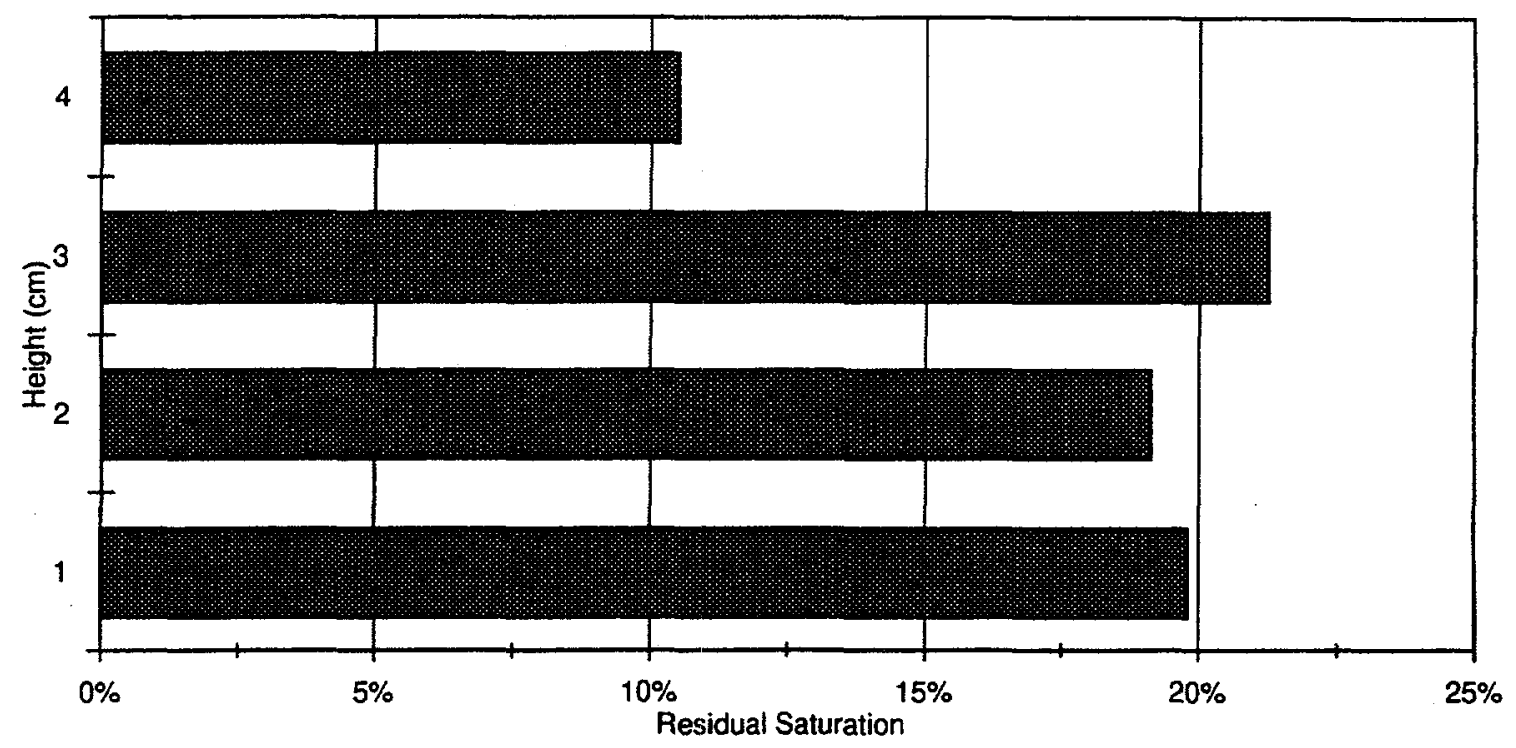

Figure 6.8 Residual Saturation Remaining in Column H-9 Fine Sand After Flush

As shown by Figure 6.8, there appears to be a much lower amount of PCE in the upper onecentimeter lift in comparison with the other depths. This is most likely due to the fact that the small amount of PCE that was removed from the column would have been removed form the upper portion of the medium due to the downflow method of column flushing.

As was stated above, an extraction test was also performed for the medium sand. The results of this test indicated that an overall residual saturation of $0.16 \%$ remained in the medium sand. Thus, it was concluded that the medium sand was effectively cleaned of residual PCE contamination.

The results of the final extraction tests performed for Column $\mathrm{H}-10$ indicated that the medium sand contained only $0.36 \%$ saturation at the conclusion of flushing activities. Thus, it appears that the medium sand in Column $\mathrm{H}-10$ was also successfully cleaned by the IPA flush. The results of the final distribution of $\mathrm{PCE}$ within the fine media of Column $\mathrm{H}-10$, however, appear to be contradictory to those of Column $\mathrm{H}-9$ and, therefore, warrant discussion. The distribution of residual NAPL in the fine media of Column H-10 is shown in Figure 6.9. 


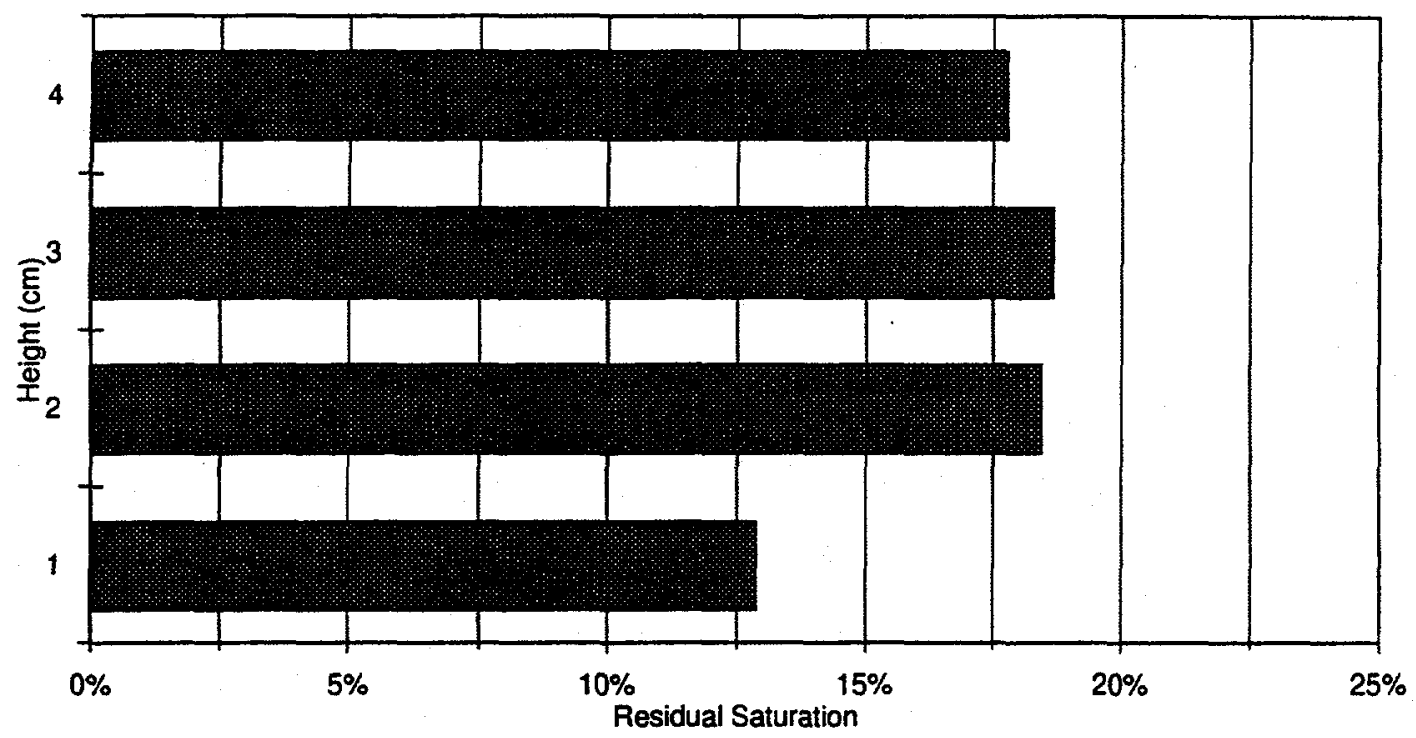

Figure 6.9 Residual Saturation Remaining in Column H-10 Fine Sand After Flush

The smaller remaining residual saturation in the lower regions of Column $\mathrm{H}-10$ is most likely the result of breakthrough of the water plate by PCE which was observed during drainage processes. This unintended breakthrough most likely caused portions of the soils near the water plate to not become totally saturated with PCE. As a result, much less NAPL was left in residual form following imbibition processes. The water plate was located at the lower portion of the column in Figure 6.9.

\section{Permeability Analyses During Flushing}

Differential pressures across the soil column were also monitored throughout the duration of the alcohol flushing experiments using transducers. The differential pressure data were used to estimate hydraulic conductivities through a rearrangement of Darcy's Law. Subsequent permeability values were calculated from hydraulic conductivity values using Equation 9:

$$
k=\frac{K \mu}{\rho g}
$$

where: $\mathrm{k}$ is the permeability; $\mathrm{K}$ is the hydraulic conductivity; $\mu$ and $\rho$ are the viscosity and density of the aqueous phase, respectively; and g is the gravitational constant. Equation 30 indicates the fact that the permeability of a matrix is contingent solely on the soil media itself, as it considers the viscosities and densities of different solutions being passed through the media. Table 6-6 provides experimental data indicating values of viscosity and density used in calculation of effective permeability values. 


\begin{tabular}{|c|c|c|}
\hline \multicolumn{3}{|c|}{ Table 6-6 Viscosity and Density Values for Various Aqueous Solutions } \\
\hline Solution & Viscosity (cP) & Density $(\mathrm{g} / \mathrm{m})^{*}$ \\
\hline Water & 1.1 & 1.00 \\
\hline IPA & 2.4 & 0.79 \\
\hline $30 \%$ IPA & 2.8 & 0.96 \\
\hline $40 \%$ IPA & 3.2 & 0.95 \\
\hline $50 \%$ IPA & 3.5 & 0.93 \\
\hline PCE & 1.7 & 1.63 \\
\hline $30 \%$ IPA w/ PCE & 3.0 & 0.96 \\
\hline $40 \%$ IPA w/ PCE & 3.3 & 0.94 \\
\hline $50 \%$ IPA w/ PCE & 3.6 & 0.93 \\
\hline
\end{tabular}

$\mathrm{n} / \mathrm{a}=$ not available

* = from Hayden (1998)

It was anticipated that the permeability data would provide a secondary indication as to the degree of remediation of the residual NAPL. The effective permeabilities of the media should continuously increase until being returned to initial, intrinsic values upon complete removal of the NAPL. The data from Column F-2 provide a good illustration of the permeability data collected during alcohol flushing.

As shown in Figure 6.10 and was indicated in Table 6-6, the initial permeability (k) value for the fine sand in Column F-2 was $1.49 \mathrm{E}^{-8} \mathrm{~cm}^{2}$ while the permeability of the column at residual saturation $\left(\mathrm{k}_{\mathrm{n}}\right.$ or $k(n)$ ) was found to be $5.74 \mathrm{E}^{-9} \mathrm{~cm}^{2}$. The permeability data presented in Figure 6.10 show that the column was eventually returned to its original intrinsic permeability value as a result of alcohol flushing.

The permeability data are shown to be somewhat noisy. The main reason for this noisiness is the sensitivity of the pressure transducers. As was discussed, the differential pressure transducers were found to be highly sensitive. Issues such as the slight pressurization of the columns upon syringe changing may have caused slight alterations to overall differential pressure across the soil column.

Another reason for the varying differential pressure data may have been the formation of macro emulsions. The potential for permeability reduction as a result of the formation of macro-emulsions has been well documented (McAuliffe, 1973, Soo and Radke, 1984, Kokal et al., 1996). Essentially, these smaller intermediate-phase droplets become mobilized by the aqueous solution and act to clog pore throats and potential pathways of flow for the cosolvent solution, thus causing elevated values 

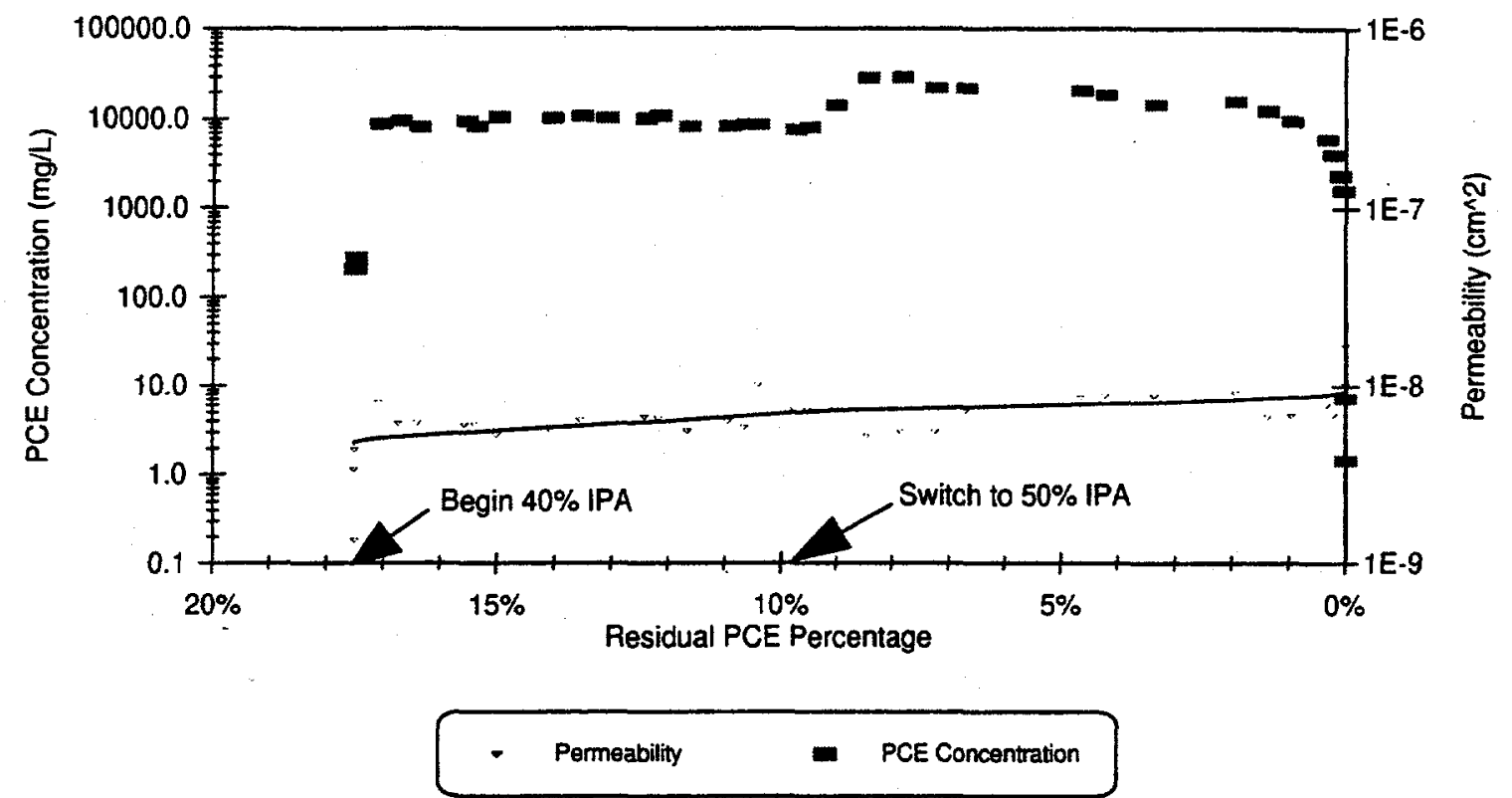

Figure 6.10 Column F-2 Permeabilities and Concentrations vs. Remaining $S_{R}$

of differential pressure. Pink-colored macro emulsions were first noted in the effluent stream from F-2 at approximately 3 pore volumes. However, it is most probable that these emulsions developed previous to their observation at 3 pore volumes. In most columns, the macro-emulsions were observed throughout the duration of the alcohol flush.

Figure 6.10 is included as a final indication of the effect of the removal of DNAPL on the permeability of the media. Figure 6.10 illustrates the permeabilities and PCE concentrations as functions of the remaining residual saturation within the column.

As the remaining residual saturation within the column was calculated with effluent PCE concentration data, only permeabilities which corresponded to those effluent sampling times could be plotted. However, data from a regression which was run on all of the permeability data were included in order to illustrate more clearly the apparent upward trend in permeability values over the course of the alcohol flushing. Similar to Figure 6.10, the data indicate an increase in permeability from the $\mathrm{k}_{\mathrm{n}}$ value of approximately $5 \mathrm{E}^{-9} \mathrm{~cm}^{2}$ to the original $\mathrm{k}$ value of approximately $1 \mathrm{E}^{-8} \mathrm{~cm}^{2}$. Of significant interest is the fact that the data shown on Figure 6.11 indicate that the greatest increase in permeability appears to occur at the beginning of the flush, as the slope of the permeability versus saturation curve is shown to be slightly greater at the beginning of the test before becoming more gradual toward the end. Figure 6.11 also indicates the advantage of using a higher percent alcohol solution whenever possible, as half of the initial saturation was observed to be removed during the $50 \%$ IPA flush over 12 pore volumes compared to 17 pore volumes for the $40 \%$ flush.

Column F-3 was the second fine sand column analyzed. Similar to Column F-2, it can be seen by the permeability data presented in Figure 6.11 that Column F-3 was eventually returned to its original intrinsic permeability value as a result of alcohol flushing. Similar to the data for Column 
F-2, the data are shown to be somewhat noisy. The most likely cause of this fluctuating data was the pressurization of the column during syringe changing. Additional reasons for the noisy data may have included the presence of macro emulsions.

Figure 6.11 is provided as a final indication of the effect of the removal of DNAPL on the permeability of the media within Column F-3 and is an illustration of the changes in permeability with decreasing residual saturation.

Similar to Figure 6.10, the data in Figure 6.11 indicate an increase in permeability from the $k_{n}$ value

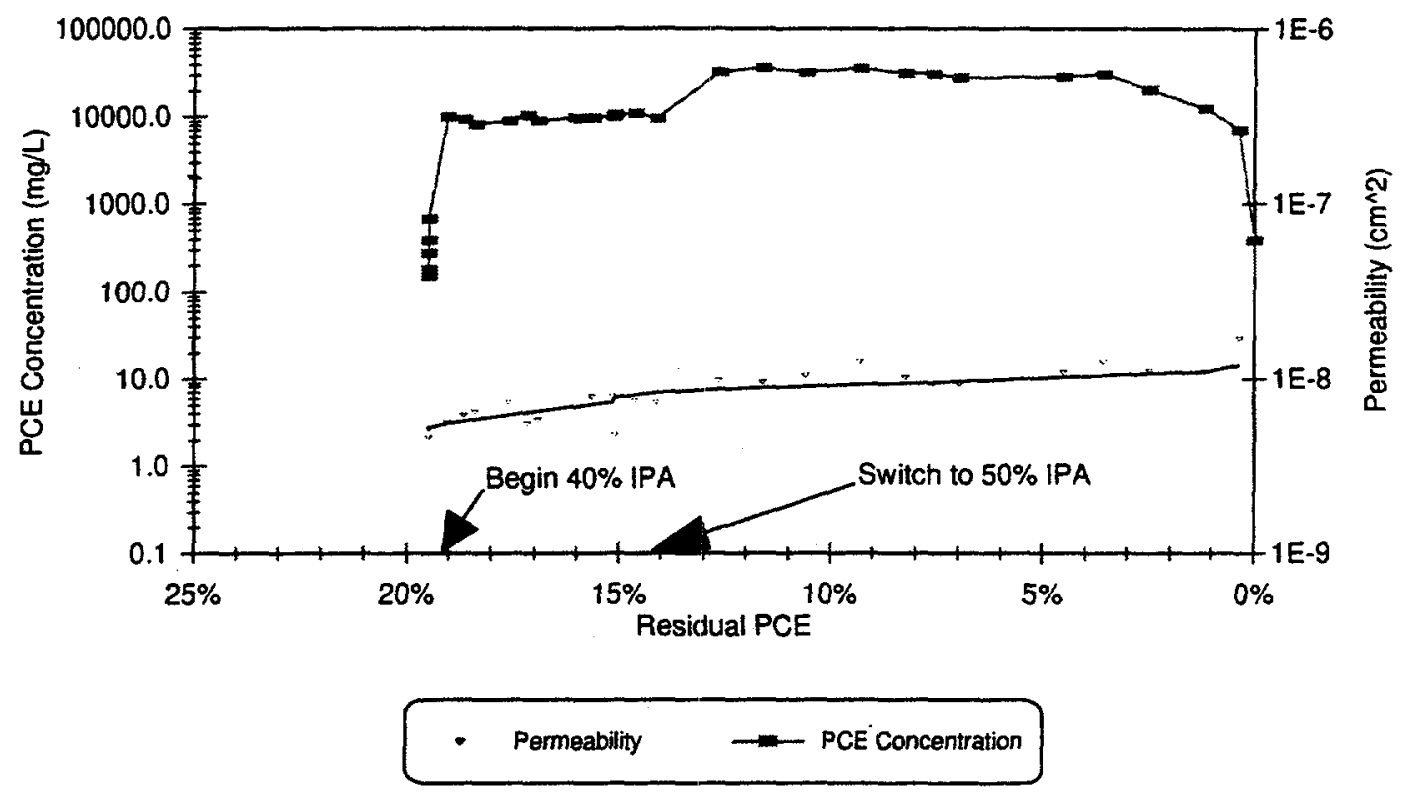

Figure 6.11 Column F-3 Permeabilities and Effluent Concentrations

of approximately $5 \mathrm{E}^{-9} \mathrm{~cm}^{2}$ to the original $\mathrm{k}$ value of approximately $1 \mathrm{E}^{-8} \mathrm{~cm}^{2}$. Again, there appears to be a greater increase in permeability at the beginning of the flush, as the largest increase in permeability is shown to occur between the initial residual saturation value of $19.5 \%$ to approximately $15 \%$ residual saturation. The permeabilities are shown to level off during the latter portions of the test. Figure 6.11 again indicates the advantage of using a higher percent alcohol solution whenever possible, as greater than $60 \%$ of the initial saturation was observed to be removed during the initial $50 \%$ IPA flush. This is of interest as the $50 \%$ flush was only run for approximately $45 \%$ of the total number of pore volumes.

The permeability data for Column F-4 indicate the advantage of continuous monitoring of differential pressures during alcohol flushing. The differential pressures within Column F-4 were only monitored with the transducer setup on an intermittent basis, as Columns F-4 and W-3 (both with double sided end-caps) were run simultaneously. In order to minimize the amount of differential pressure data and because four pressure transducers were not available, the intermittent readings were attempted. The results of the flushing of Column F-4 are shown Figure 6.12: 
It appeared that the flushing of this column was terminated before total clean up had been achieved.
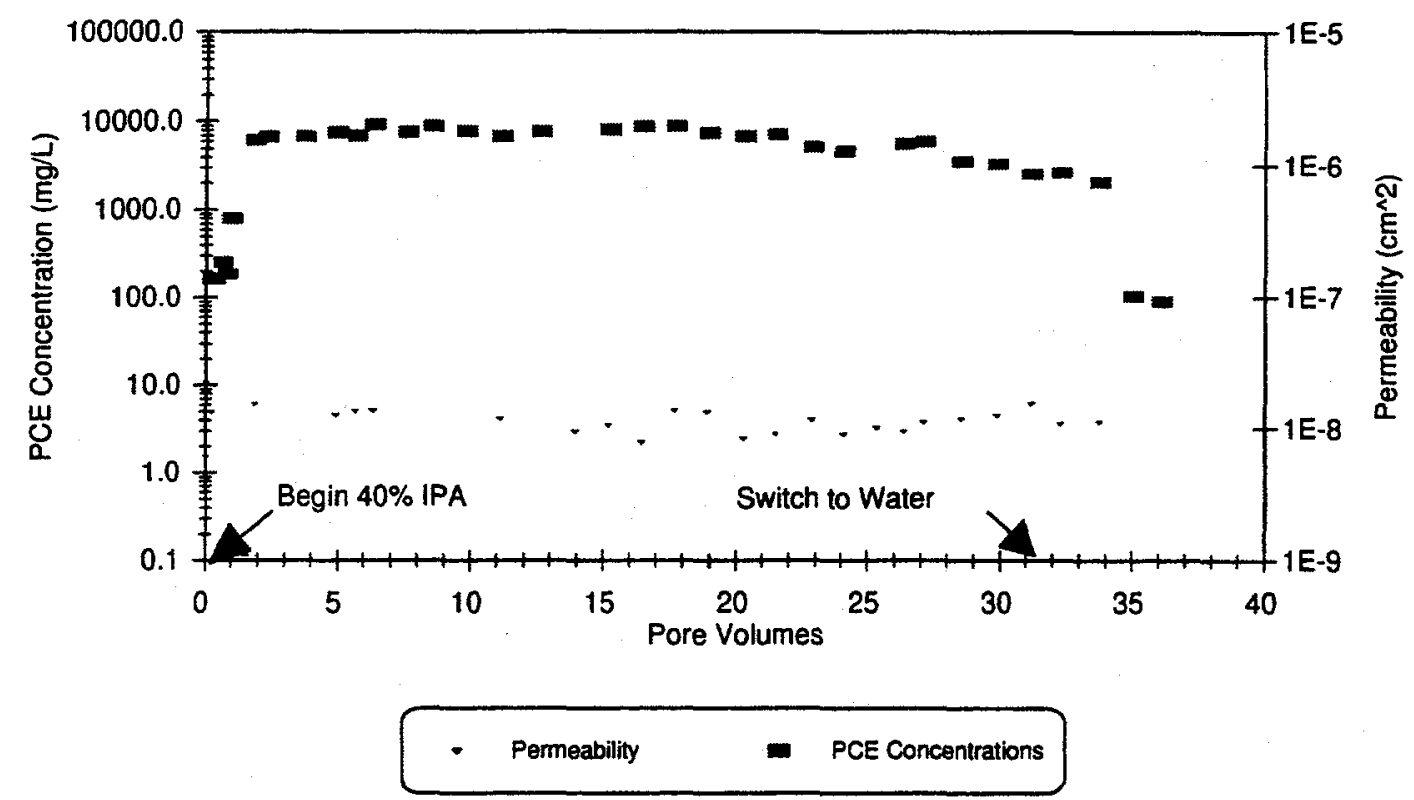

Figure 6.12 Column F-4 Permeabilities and Effluent Concentrations During Flushing

This is evidenced by the lack of a steep downward slope on the effluent concentration curve. (The last two effluent concentrations shown on Figure 6.12 were collected during the final water flood. Therefore, these two concentrations should be considered inaccurate.) An extraction test was performed on this column at the conclusion of flushing activities. It is interesting to note that while the effluent concentrations still appear a bit high at the end of the flush, nearly all of the PCE was removed by flushing. This conclusion is substantiated by the fact that only 0.04 grams of PCE were found to remain in the column as a result of the extraction test. It is likely that this last bit of contamination persisted near the outlet port at the bottom of the column and thus, caused the effluent concentrations to remain high. Column F-4 was flushed solely with a 40\% IPA solution, which is the main reason that after 35 pore volumes, a slight amount of $\mathrm{PCE}$ still persisted within the column.

The original permeability value for the fine sand in Column F-4 was $2.24 \mathrm{E}^{-8} \mathrm{~cm}^{2}$ while the permeability of the column at residual saturation was found to be $6.08 \mathrm{E}^{-9} \mathrm{~cm}^{2}$. Permeability values are shown on Figure 6.12 to consistently lay between these two values without illustrating any kind of definite trend. If anything is shown by the permeability data in Figure 6.12 , it is that the permeabilities seem to immediately jump to original intrinsic values $\left(2.24 \mathrm{E}^{-8} \mathrm{~cm}^{2}\right)$. Even if a preferential flow path did develop, it would certainly not occur before the transmittance of one pore volume of flushing solution. Thus, the permeability data of Column F-4 illustrate the need for continual and abundant differential pressure monitoring during flushing activities. When the changes between $k$ and $k_{n}$ are fairly slight (one half order of magnitude for the fine sand columns), it is important to collect a significant amount of data in order to illustrate a trend. In doing so, outlying data points will be more apparent in comparison with the remainder of the data. 
The initial permeability value for the Ottawa medium white sand in Column W-1 was $2.45 \mathrm{E}^{-7} \mathrm{~cm}^{2}$, while the permeability of the column at residual saturation was found to be $8.53 \mathrm{E}^{-8} \mathrm{~cm}^{2}$. The permeability data of Column W-1 illustrate the short comings of the use of this particular sand in these experiments. Firstly, the magnitude of the reduction in permeability as a result of NAPL entrapment was shown to be only a factor of two. This reduction was considered minimal in comparison with the half order of magnitude reduction in permeabilities for the fine sand columns. Secondly, the permeability data appears to be a bit more noisy for the medium sand than for the fine sand. Of interest is the fact that the noise appears to increase at higher values of permeability. While all of the data appear noisy for the reasons of column pressurization during syringe changes and the possible formation of macro-emulsions, the noise at the end of the test is considered to have a greater impact upon the permeability data. This is due to the same problem that plagued the latter portions of the permeability data for Column F-3. Slight variations at low values of differential pressure have a more significant impact on percent error than they would at greater values. As the values of differential pressure were observed to stay fairly low throughout the alcohol flushing of the medium sand columns, this type of medium white sand was probably too coarse for use in these experiments.

The permeability data for Column W-1 do not provide an indication that the column was returned to its original intrinsic permeability value as a result of alcohol flushing. A potential explanation for this lies in the fact that a low level of confidence is attributed to all values of permeability calculated with the differential pressure data. This is due to the fact that low values of differential pressure were used in all cases for the medium sand data, and that slight errors in these low differential pressure values would result in a miscalculation of permeability. Therefore, the true initial permeability may well have been 7 to $9 \mathrm{E}^{-8} \mathrm{~cm}^{2}$, and not the value of $2.45 \mathrm{E}-7 \mathrm{~cm}^{2}$ calculated initially.

While there are problems with the exact permeability values of the medium sand columns, the data indicate an overall upward trend in permeability values. As a final indication of the effect of the removal of DNAPL on the permeability of the media within Column W-1, permeabilities and PCE concentrations were plotted versus the remaining residual saturation within the column. Figure 6.13 is provided as an illustration of the changes in permeability with decreasing residual saturation. 


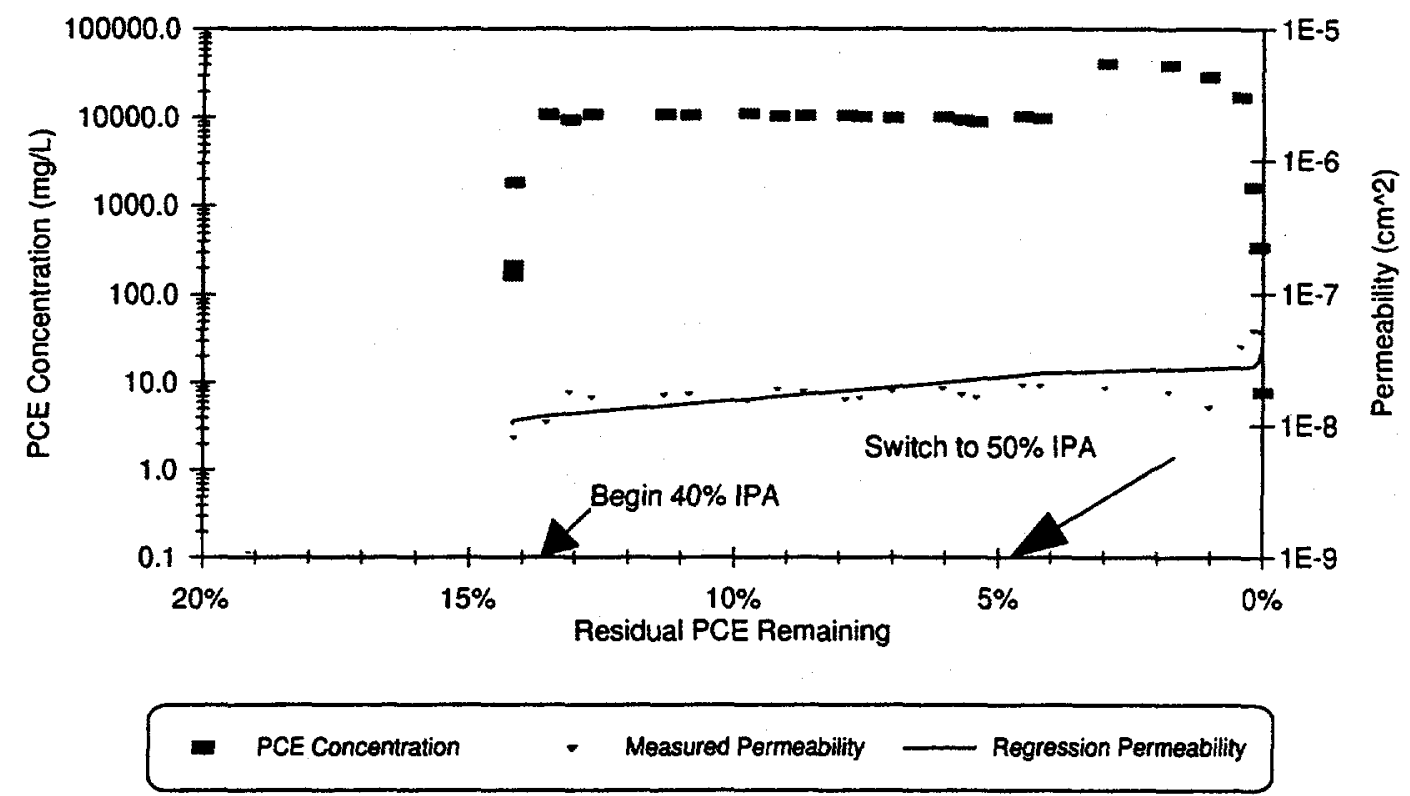

Figure 6.13 Column W-1 Permeabilities and Effluent Concentrations vs. $S_{R}$

This data shown on Figure 6-14 appear to be fairly linear. However, data from a regression that was run on all of the permeability data was included in order to illustrate more clearly the apparent upward trend in permeability values over the course of the alcohol flushing. Column W-2 provided similar data to that of Column W-1.

Run simultaneously with Column F-4, Column W-3 also used an aluminum double-sided endcap at the bottom in order to be consistent with the layered columns that would be run in the heterogeneous column study. The results of the alcohol flushing of Column W-3 indicated (1) the shortcomings of the use of this medium-grained sand as the difference between intrinsic and residual NAPL permeabilities was too slight and (2) the issue of not obtaining a sufficient amount of differential pressure data to buffer outlying data points.

\section{Heterogeneous Columns}

Similar to the results of the effluent concentration study, the results of the permeability analyses for Column H-6 provide indication of the method used in flushing Column H-6. As was done with Columns W-3 and F-4, differential pressures were not continuously monitored during the flush of Column H-6. The discreet pressure monitoring was performed in order to minimize the amount of data. It was also done in an attempt to minimize the error associated with the low differential pressure measurements of the medium sand. Thus, while the column flush was run at $5 \mathrm{ml} / \mathrm{hr}$ on each side, the flowrate was increased to $1 \mathrm{ml} / \mathrm{min}$, or $60 \mathrm{ml} / \mathrm{hr}$ for approximately 2 to 4 minutes for differential pressure measurement. In running at these slightly elevated flowrates, it was anticipated that the differential pressure data would be higher and therefore slightly more accurate than that 
shown for the homogenous medium sand columns. However, the problems of sparse data associated with incremental differential pressure monitoring that were discussed for Columns W-3 and F- 4 were also observed in the permeability data for Column H-6. The results of the flushing of the medium sand within Column H-6 is shown as Figure 6.14 while the results for the fine sand are provided in Figure 6.15.

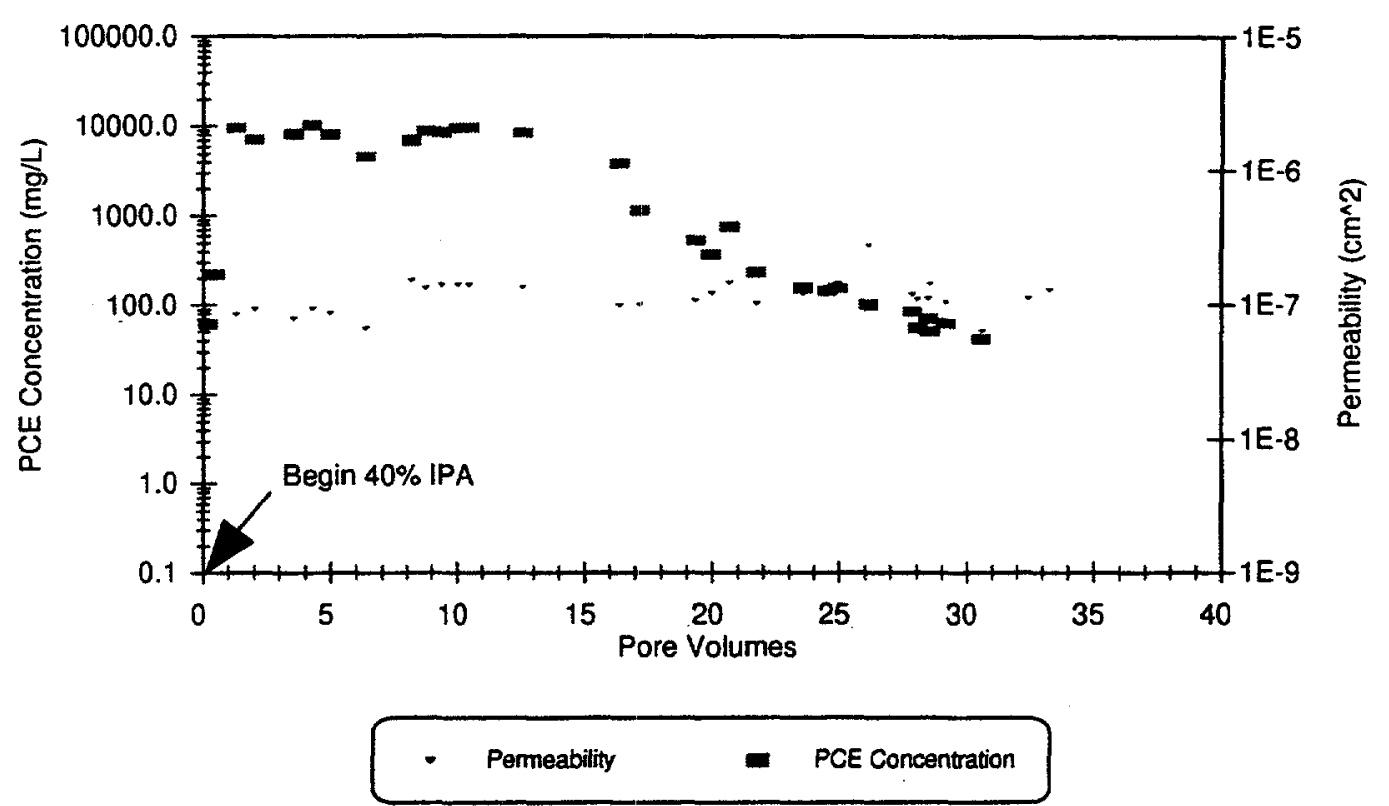

Figure 6.14 Column H-6 Medium Sand Permeabilities and Effluent Concentrations 
The data for the medium sand appear to illustrate a slight upward trend throughout the flush. This conclusion is weakened, however, by the apparent lack of a sufficient amount of data. More troubling are the permeability data for the fine sand. If anything, the data shown on Figure 6-16 seem to indicate a downward trend in permeability throughout the flush.

The actual initial and final permeability values for each medium within Column H-6 are not completely understood. This is due to the issue of by passing and the fact that the actual flowrates through each media were not exactly known. The values are skeptical because the calculations assumed that exactly $5 \mathrm{ml} / \mathrm{hr}$ was being pumped through each media. The somewhat suspect results produced from experimentation with Column H-6 indicated that a different method had to be developed in order to more accurately draw conclusions as to the behavior of the layered columns upon subjection to remediation by dissolution-dominated alcohol flushing.

Columns $\mathrm{H}-9$ and $\mathrm{H}-10$ were packed in $4 \mathrm{~cm}$ columns instead of the $10 \mathrm{~cm}$ models used previously for all other columns. The shorter columns were used in order to expedite clean-up times from a few weeks down to a day. This switch was possible as transducers were no longer being used to monitor differential pressure. Therefore, a maximum column length was not required in order to increase

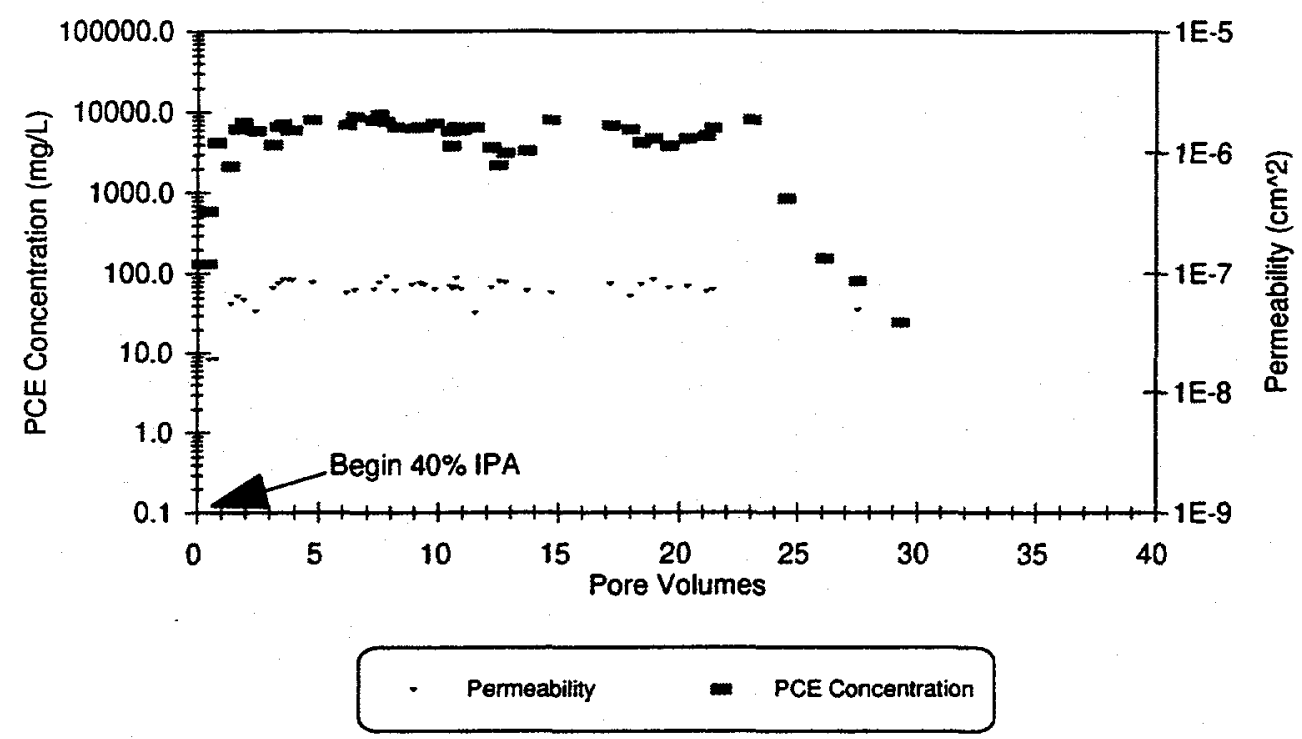

Figure 6.15 Column H-6 Fine Sand Permeabilities and Effluent Concentrations

differential pressure values. As was reported above, Columns $\mathrm{H}-9$ and $\mathrm{H}-10$ were flushed in a downflow direction by allowing the alcohol solution to flow through the column under a hydraulic head. The flowrate of effluent from both media was measured and used in conjunction with the known head to calculate hydraulic conductivities and, thus, permeabilities. These values were found to be slightly greater in accuracy as they were derived from raw data that was not manipulated in any way. The results of the permeability values before and after NAPL entrapment in each media were 
presented earlier.

While the effluent concentration data was negatively affected by the use of the drip method for flushing Columns $\mathrm{H}-9$ and $\mathrm{H}-10$, the permeability data was found to be fairly accurate. The permeability data for the medium sand within Column H-9 are provided in Figure 6.16 and illustrate a slight yet definite upward trend in permeability values during the course of the flush. Furthermore, the values of permeability appear to increase from values of approximately $2 \times 10^{-8} \mathrm{~cm}^{2}$ to approximately $4 \times 10^{-8} \mathrm{~cm}^{2}$. In order to better view the trend, Figure 6.17 is included with a smaller permeability scale.

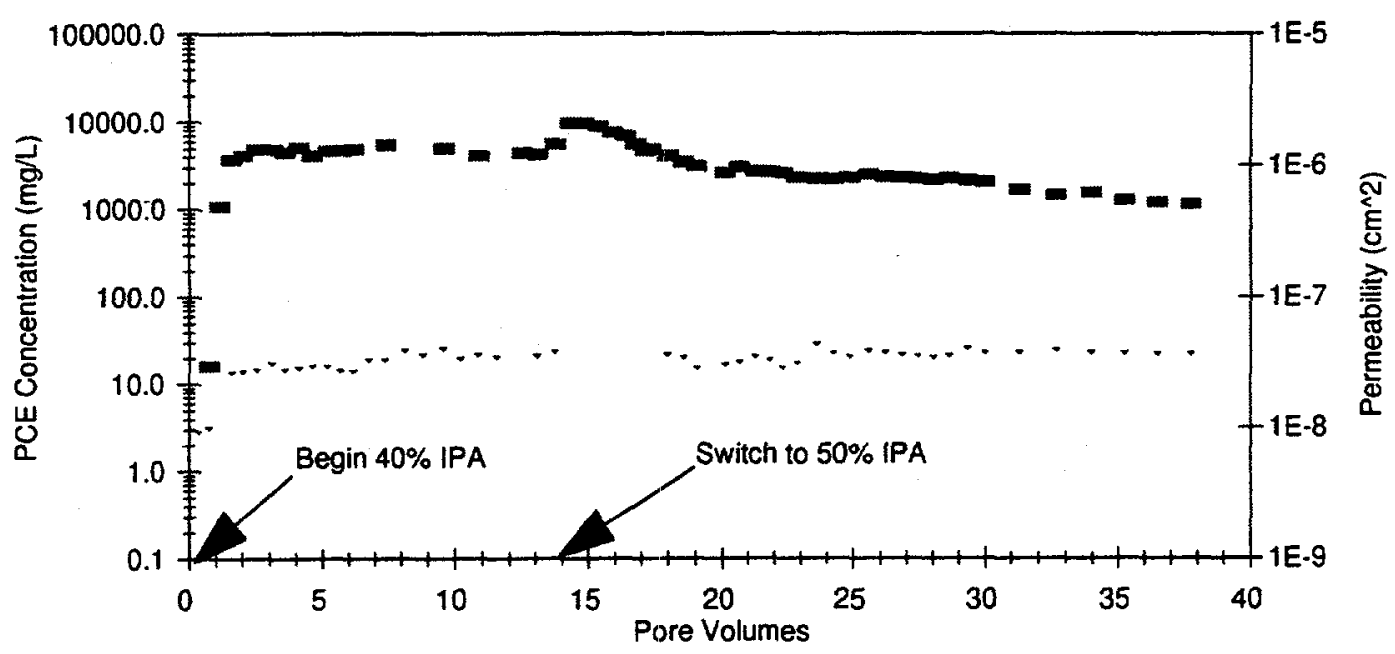

- Permeability PCE Concentration

Figure 6.16 Column H-9 Medium Sand Permeabilities and Effluent Concentrations 


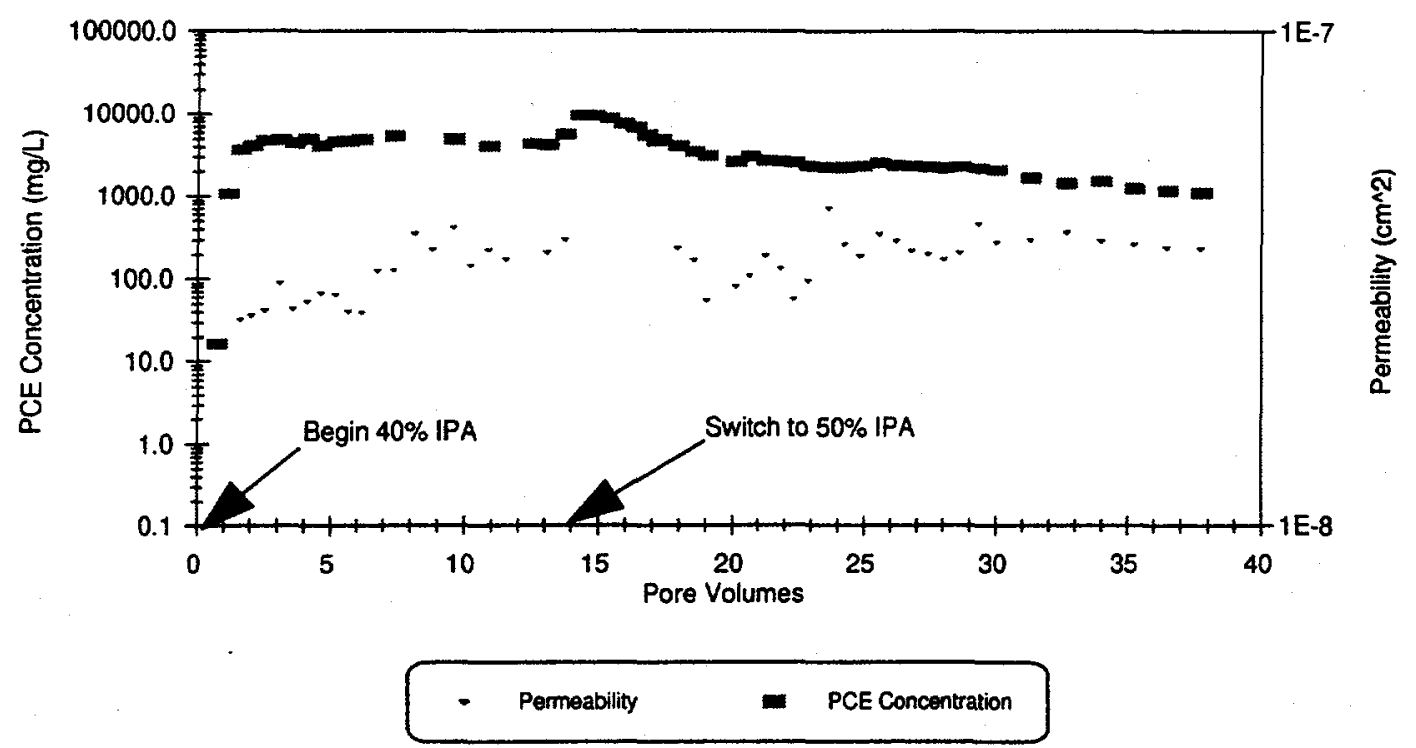

Figure 6.17 Column H-9 Medium Sand Permeabilities and Effluent Concentrations Expanded Permeability Scale

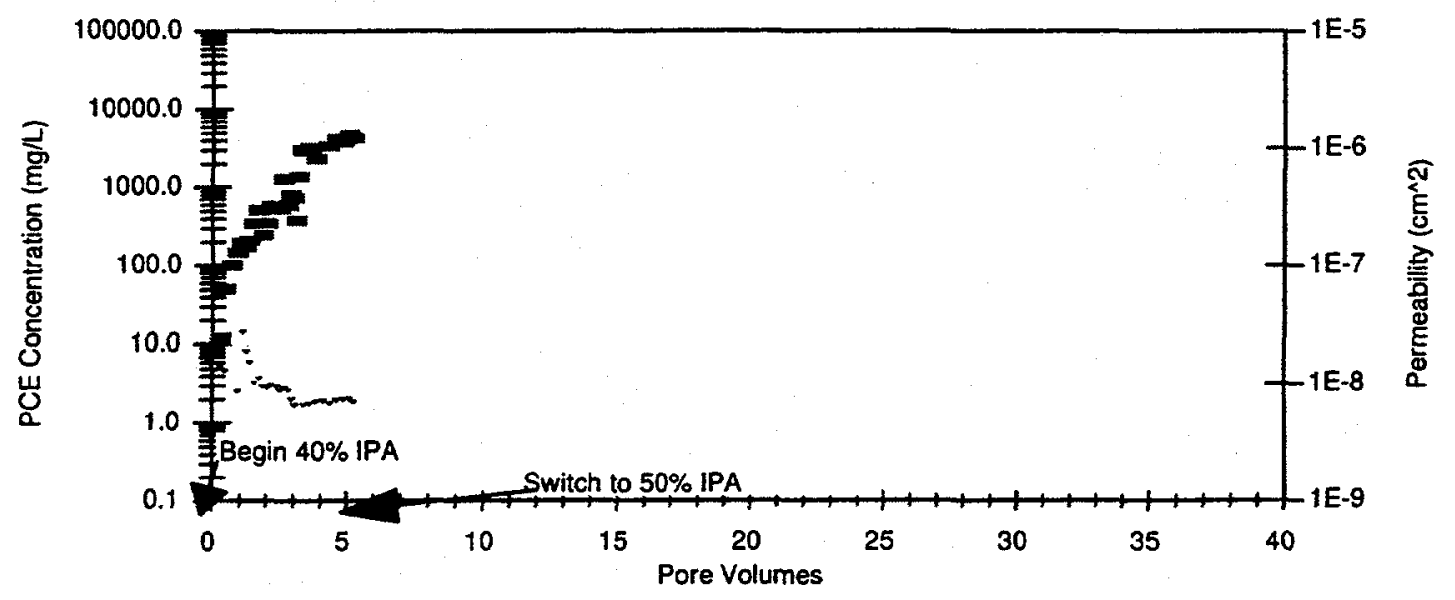

- Permeability - PCE Concentration

Figure 6.18 Column H-9 Fine Sand Permeabilities and Effluent Concentrations 
to Figures 6.16 and 6.17 , the upward trend in permeabilities seems to end at approximately 25 pore volumes, at which point the permeabilities appear to remain fairly constant. At this point most all of the NAPL has been dissolved away from the medium sand. In addition, the greatest increase in permeability values appears to occur within the first 10 pore volumes. This is consistent with data from the homogenous columns which showed the greatest increase in permeability at the initiation of the experiment.

The data pertaining to the permeabilities within the fine media of Column $\mathrm{H}-9$ are presented in Figure 6.18. Similar to the effluent data, the permeability data in Figure 6.18 indicate that the fine media within Column H-9 was never successfully cleaned. These data remain fairly low after approximately two pore volumes. Furthermore, these permeabilities appear to be relatively close to the $k_{n}$ value of approximately $8 \times 10^{-9} \mathrm{~cm}^{2}$ for the fine sand within Column H-9. However, close inspection of the permeability data indicates a very slight upward trend. While it doesn't appear from the effluent data that much NAPL is being removed from the fine media, the very slight increase in permeability may be attributed to the apparent removal of NAPL from the fine media by the alcohol solution flowing through the medium sand. It should be noted, however, that the amount of permeability data able to be collected for the fine media within Column H-9 may not be sufficient enough to provide firm conclusions as to increasing trends. It should also be noted that the slightly elevated values of permeability for the data points between one and two pore volumes are the result transition issues between an aqueous phase of all water and one of $40 \%$ IPA.

The permeability data for each media within Column $\mathrm{H}-10$ was also found to be much more reliable as a result of the use of the drip method of alcohol flushing. The permeability values for the medium sand in Column H-10 appear to show a more rapid increase to the value of intrinsic permeability in comparison with that of Column H-9. This is likely the result of the development of preferential pathways within the medium sand. In addition, the fact that such a large increase in permeability appears to occur at the initiation of the experiment is consistent with data from the homogenous columns which showed the greatest increase in permeability at the initiation of the experiment.

Similar to the results for the fine media in Column H-9, the fine media within Column $\mathrm{H}-10$ was also never successfully cleaned. The permeability data is shown to again remain fairly constant and relatively close to the $k_{N}$ value of approximately $6 \mathrm{E}^{-9}$ after approximately two pore volumes.

Results from columns with the two side ports is shown in Figure 6.19. 
Column H-4, Both Soils .

$\mathrm{q}=0.42 \mathrm{ft} /$ day

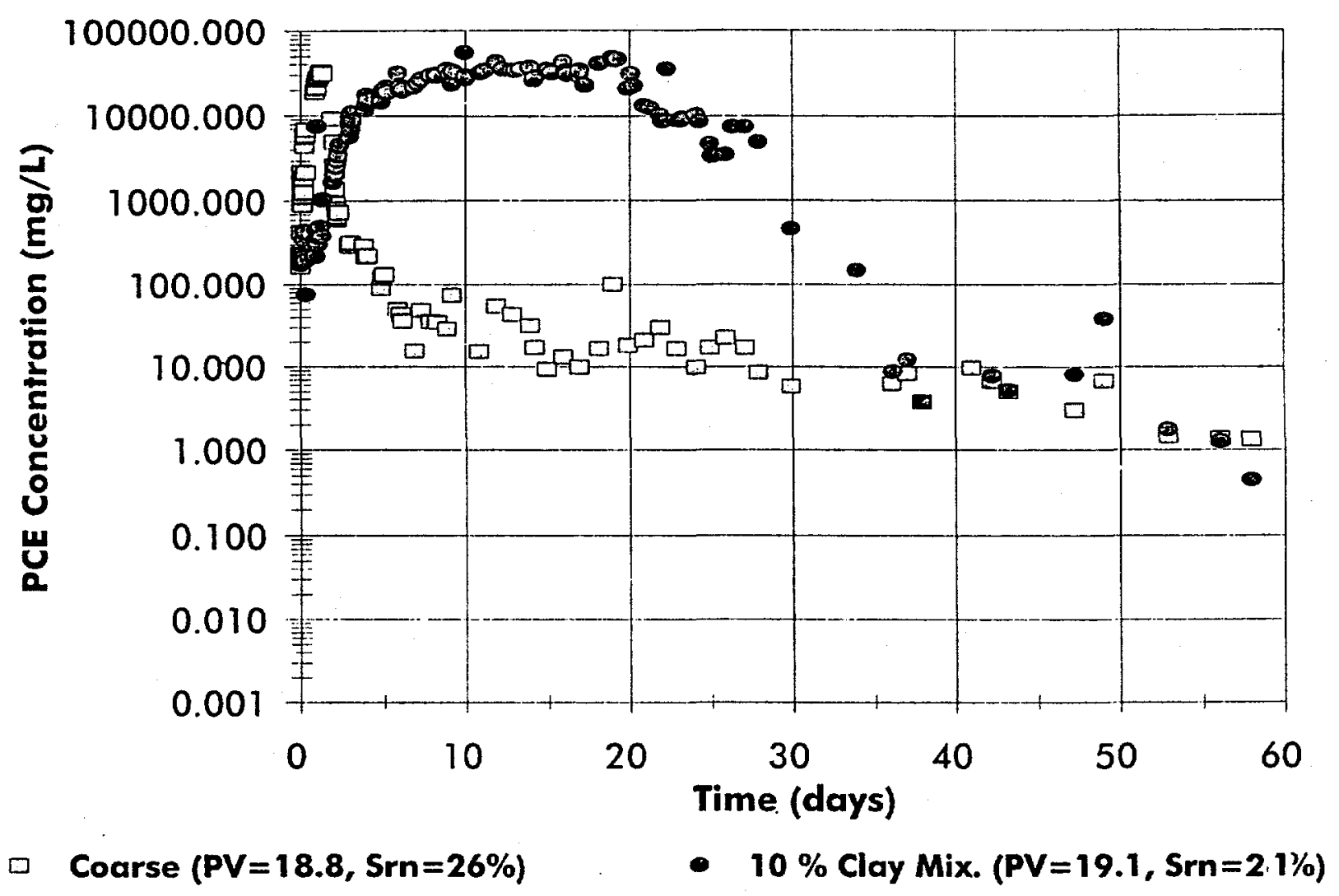

Figure 6.19 Results from two layer column with sampling from side ports.

\subsection{Summary}

Effluent concentration data were found to provide a good indication of the degree of PCE removal from the homogenous columns. Expedited removal of PCE due to enhanced dissolution was shown in all columns as mass removal times were decreased by nearly two orders of magnitude. Volatilization issues were not apparent in the majority of the homogenous columns and effluent concentrations were found to be consistent with those expected in consideration of the ternary system of alcohol, water and PCE. Minor losses were apparent in two of the homogenous columns as the result of the presence of silicone on the specially constructed endcap.

The results of the heterogeneous column study provided experimental illustration of the difficulty 
of achieving complete remediation of layered soil systems, as the medium sand within each column was consistently cleaned of residual PCE contamination while the PCE within the finer material was observed to be substantially more difficult to remove. Permeability data collected during experimentation with heterogeneous soil columns indicated this difficulty, as increasing permeability values were observed within the medium sand and stagnant, consistently low permeability values were seen in the finer sand. Effluent concentration data and flowrate ratio data provided further indication of the preferential clean up of the more coarse-grained media within the layered systems.

\subsection{References}

Anderson, M.R., Johnson, R.L., and Pankow, J.F., "Dissolution of Dense Chlorinated Solvents into Ground Water: 1. Dissolution from a Well-Defined Source", Ground Water, Vol. 30, No.2, MarchApril 1992.

Boyd, G.R. and Farley, K.J., "Residual NAPL Removal from Groundwater Using Alcohol Flooding", Trans. American Geophysical Union, Vol 71, No. 17, 1990.

Boyd, G.R. and Farley, K.J., "NAPL Removal from Groundwater by Alcohol Flooding: Laboratory Studies and Applications", Hydrocarbon Contaminated Soils and Groundwaters: Analysis, Fate, Environmental and Public Health Effects and Remediation, 1992.

Brandes, D. and Farley, K.J., "Importance of Phase Behavior in Remediation of DNAPL Contaminated Aquifers by Alcohol Flooding", Water Environment Federation, Vol. 65, 1993.

Fried, J.J., Muntzer, P., and Zilliox, L., "Ground-water pollution by transfer of oil hydrocarbons, Ground Water, Vol 17, No. 6, 1979.

Imhoff, P.T., Gleyzer, S.N., McBride, J.F., Vancho, L.A., Okuda, I., and Miller, C.S. "CosolventEnhanced Remediation of Residual Dense Non-Aqueous Phase Liquids: Experimental Investigation", Environmental Science and Technology, Vol. 29, No. 8, 1995.

Imhoff, P.T., Thyrum, G.P, and Miller, C.T., "Dissolution fingering during the solubilization of nonaqueous phase liquids in saturated porous media; 2: Experimental observations", Water Resources Research, Vol 32, No. 7, 1996.

Kokal, S.L., Maini, B.B., and Woo, R., "Flow of Emulsions in Porous Media", In Emulsions: Fundamentals and Applications in the Petroleum Industry, Schramm, L.L. Ed, Washington, DC: American Chemical Society, 1992.

Mackay, D.M., Roberts, P.V., and Cherry, J.A., "Transport of organic contaminants in groundwater", Environmental Science and Technology, Vol 19, No. 5, 1985.

Miller, C.T., Poirier-McNeill, M.M. and Mayer, A.S., "Dissolution of Trapped Non-aqueous Phase Liquids: Mass Transfer Characteristics", Water Resources Research, Vol. 26, No. 11, November, 
1990.

Okuda, I., McBride, J.F., Gleyzer, S.N., and Miller, C.T., "Physicochemical Transport Processes Affecting the Removal of Residual DNAPL by Nonionic Surfactant Solutions", Environmental Science and Technology, Vol. 30, No. 6, 1996.

Pennel, K.D., Pope, G.A., and Abriola, L.M., "Influence of Viscous and Buoyancy Forces on the Mobilization of Residual Tetrachloroethylene during Surfactant Flushing", Environmental Science and Technology, Vol. 30, No. 4, 1996.

Powers, S.E., Celso, O.L., Abriola, L.M., and Weber, W.J., Jr., "Theoretical Study of the Significance of Nonequilibrium Dissolution of Nonaqueous Phase Liquids in Subsurface Systems", Water Resources Research, Vol 27, No. 4, April, 1991.

Powers, S.E., Abriola, L.M., and Weber, W.J., Jr., "An Experimental Investigation of Non-Aqueous Phase Liquid Dissolution in Saturated Subsurface Systems: Steady State Mass Transfer Rates", Water Resources Research, Vol 28, No. 10, October 1992.

Powers, S.E., Abriola, L.M., and Weber, W.J., Jr., "An Experimental Investigation of Non-Aqueous Phase Liquid Dissolution in Saturated Subsurface Systems: Transient Mass Transfer Rates", Water Resources Research, Vol 30, No. 2, February, 1994.

Powers, S.E., Abriola, L.M., Dunkin, J.C., and Weber, W.J., Jr., "Phenomenological models for transient NAPL-water mass-transfer processes", Journal of Contaminant Hydrology, Vol 16, 1994.

Soo, H. and Radke, C.J., "The Flow Mechanism of Dilute, Stable Emulsion in Porous Media", Ind. Eng. Chem. Fundam., Vol. 23, 1984. 


\subsection{Introduction}

Reuse of the alcohol solution is important for reducing the overall cost of the remediation scheme and well as reducing the volume of hazardous waste generated from the remediation process. Some typical liquid separation technologies for volatile organic compounds include solvent extraction, distillation, reducing solubility so that the contaminant becomes a separated immiscible phase and adsorption. The feasibility of these technologies depends on the characeristics of the compounds of interest. Table 7-1 shows various properties for PCE, TCE and alcohols (based on Verschueren (1983)).

Table 7-1 Properties of PCE, TCE, IPA, ethanol and water

\begin{tabular}{|c|c|c|c|c|c|}
\hline Property & PCE & TCE & IPA & Ethanol & Water \\
\hline Molecular Formula & $\mathrm{C}_{2} \mathrm{Cl}_{4}$ & $\mathrm{HC}_{2} \mathrm{Cl}_{3}$ & $\mathrm{H}_{7} \mathrm{C}_{3} \mathrm{OH}$ & $\mathrm{H}_{5} \mathrm{C}_{2} \mathrm{OH}$ & $\mathrm{H}_{2} \mathrm{O}$ \\
\hline Molecular Structure & $\begin{array}{l}\mathrm{Ci} \\
\mathrm{Cl}, \mathrm{C}=\mathrm{C}^{\prime} \mathrm{Cl}\end{array}$ & $\mathrm{Cl}, \mathrm{C}=\mathrm{C}^{\prime}$ & 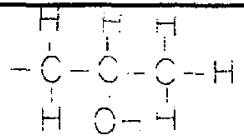 & 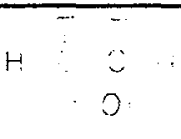 & - \\
\hline Molecular Weight & 165.83 & 131.5 & 60.1 & 46.07 & 18 \\
\hline Molecule Size & Small & Small & Small & Small & Small \\
\hline Melting Point (@1 atm) ${ }^{\circ} \mathrm{C}$ & -22.7 & -87 & $-86 /-89$ & $-114 /-117$ & 0 \\
\hline Boiling Point (@1 atm) ${ }^{\circ} \mathrm{C}$ & 121.4 & 86.7 & 82.4 & 78.4 & 100 \\
\hline Polarity & Nonpolar & Nonpolar & Polar & Polar & Polar \\
\hline Density $\left(\mathrm{g} / \mathrm{ml} @ 1 \mathrm{~atm}, 20^{\circ} \mathrm{C}\right)$ & 1.626 & 1.46 & 0.785 & 0.789 & 0.999 \\
\hline Water Solubility (mg/1@ I latm) 25 & 160 & 1100 & Miscible & Miscible & - \\
\hline
\end{tabular}

Solvent extraction is a process in which an immiscible solvent, such as petroleum ether, benzene, hexane or some other organic solvents are used to recover the constituents of interest (Sawyer et. al., 1994). Even though solvent extraction may be an effective and economical means to recover organic solutes from inorganic solvents like water, it is much more difficult and less feasible to separate PCE from an alcohol solution.

Distillation is a process of separation based on the difference in composition between a liquid and the vapor formed. Simple distillation is effective in the separation of liquids from nonvolatile solids, as in the distillation of pure water from salt water, or recovery of an organic solvent from mixtures with nonvolatile substances. Due to the fact that the components of the alcohol flushing extraction solution (alcohol, PCE and TCE) are volatile and that the boiling points for the compounds are similar, it may be difficult to separate them into their pure phases. 
Perry and Weissberger (1965) reported that an azeotrope occurs during the distillation process of a ternary system composed of alcohol, water and PCE. An azeotrope is a point at which the system forms a maximum or minimum boiling point of the mixture. The distillates, therefore, will remain in the same fraction ratio mixture and hence will not undergo further separation. Many binary and a few ternary systems have been studied in terms of the azeotrope of those systems, and information is available from many general sources. An example of a binary system of interest is IPA and water. This system forms an azeotrope and boils at $80.3^{\circ} \mathrm{C}$ at atmospheric pressure. The distillate remain in the mixture of $87.8 \%$ IPA and $12.6 \%$ water. The ternary system of IPA/water/PCE was not found in the literature but the related ternary system of propanol/PCE/water forms an azeotrope and boils at $81^{\circ} \mathrm{C}$. The distillates remain in the ratio of $12.5 \%$ water, $20.8 \%$ propanol, and $66.7 \% \mathrm{PCE}$. The property of propanol/PCE/water ternary system implies that: (1) the azeotrope boiling point of the system is very close to $83^{\circ} \mathrm{C}$, the boiling point of propanol, the alcohol component of the ternary system; and (2) a PCE concentration of $66.7 \%$ is much higher than that will be encountered in waste from alcohol flushing SRS and even from any other real practice. As a result, there is no possibility for PCE or propanol to be separated from this ternary system by simple distillation. A much more complicated scheme would be needed to "break" the azeotrope if distillation is used. This may not be practical because of the high cost and complexity of such a system.

As stated earlier, PCE solubility increases dramatically when the alcohol volumetric concentration is greater than $30 \%$ in solution. Therefore, changing the mixture composition leading PCE to be a separate phase may be a way to partially separate PCE from an alcohol solution. For example, adding water to bring a solution IPA concentration from $60 \%$ down to $50 \%$ or $30 \%$ may reduce the corresponding PCE solubility from about $132,000 \mathrm{mg} / \mathrm{L}$ down to about $30,000 \mathrm{mg} / \mathrm{L}$ or $1,600 \mathrm{mg} / \mathrm{L}$, almost 4.4 or 82.5 times reduction, respectively. Then extra PCE forms a separate phase and sink to the bottom of the solution due to its higher density. The leftover solution with a much lower PCE concentration can then undergo further treatment. Although this method enlarges the total waste volume, it reduces the PCE strength in solution dramatically and eases the further PCE separation. The overall process of this method, therefore, may be cost-effective.

Granular activated carbon adsorption is one of the best available technologies for removing target synthetic organic chemicals from the polluted drinking water sources (US EPA, 1986). Two market research organizations, Freedonia Group and SRI International, assert that the demand for activated carbon will increase by $5 \%$ per year between 1995 and 2000 , to a total 400 million pounds, due to increases in water treatment and solvent recovery uses (Chemical and Engineering News 1996).

The essence of carbon treatment of contaminated fluids is to utilize activated carbon's superb adsorptive capacity to remove adsorbable impurities from the fluids. This can be achieved using an activated carbon bed or other activated carbon configurations. Organic compounds with low solubility and low or non- polarity, such as PCE and TCE, can be adsorbed by activated carbon, and those with high solubility and polarity, such as ethanol, IPA, are usually not highly adsorbed. The partition coefficient (K) was used by Luehrs et al. (1996) as limiting adsorption coefficient 
Activated carbon adsorption uses activated carbon as the adsorbent to accumulate the molecules of other materials onto its surface. Activated carbon adsorption has a wide application in environmental engineering. Cohesion and adhesion forces, ranging from strong valence bonds to the van der Waals forces are responsible for adsorption. These forces can be loosely classified into three categories: (1) chemical forces, such as the covalent forces in molecules of $\mathrm{H}_{2}$ and $\mathrm{H}_{2} \mathrm{O}$; (2) electrostatic forces; and (3) physical forces. Based on the adsorption forces, adsorption can generally be classified into the following three patterns.

- Chemical adsorption or chemisorption, adsorption in which the adsorbate undergoes a chemical reaction with the adsorbent. Chemical adsorption processes exhibit a high energy of adsorption, and thus are favored by high temperatures.

- Exchange or electrostatic adsorption, adsorption results from the electrostatic attraction to charged sites at the interface. The valence and the molecular size of the adsorbate are important in determining the order of the preference for electrostatic adsorption by a particular adsorbent. Electrostatic adsorption dominates in activated carbon adsorption of metal ions.

- Physical adsorption occurs as a result of van der Waals forces. In physical adsorption, the adsorbed molecules are not affixed to a specific site at the surface but are free to undergo transitional movement at the interface. Hence, the molecules adsorbed in this way can easily be desorbed. Adsorption of this type is sometimes referred to as "ideal" adsorption. It is usually predominant at low temperatures. The adsorption of organic compounds, particularly nonploar organic compounds, by activated carbon is a typical example of physical adsorption.

Activated carbon is a common adsorbent using in many water and wastewater treatment systems. The distinctive feature of activated carbon is its high internal porosity and associated large specific surface area. This is a critical factor in the adsorption process. The surface area of macropores and micropores of the activated carbon granules contribute to the total surface area, however, the system of macropores generally contributes little to the total surface area. The macropores function mainly as channels through which solute molecules diffuse or pass into the system of micropores. The specific surface area of activated carbon typically ranges from 500$1400 \mathrm{~m}^{2} / \mathrm{g}$, and some carbons have been known to have a specific surface area up to $2500 \mathrm{~m}^{2} / \mathrm{g}$ providing a large amount of adsorption sites.

A diagrammatic conception of an activated carbon granule and its adsorption of impurities from a passing liquid are shown in Figure 7-1. Figure 7-2 shows an expanded view of an internal pore (micropore) of an activated carbon granule. 


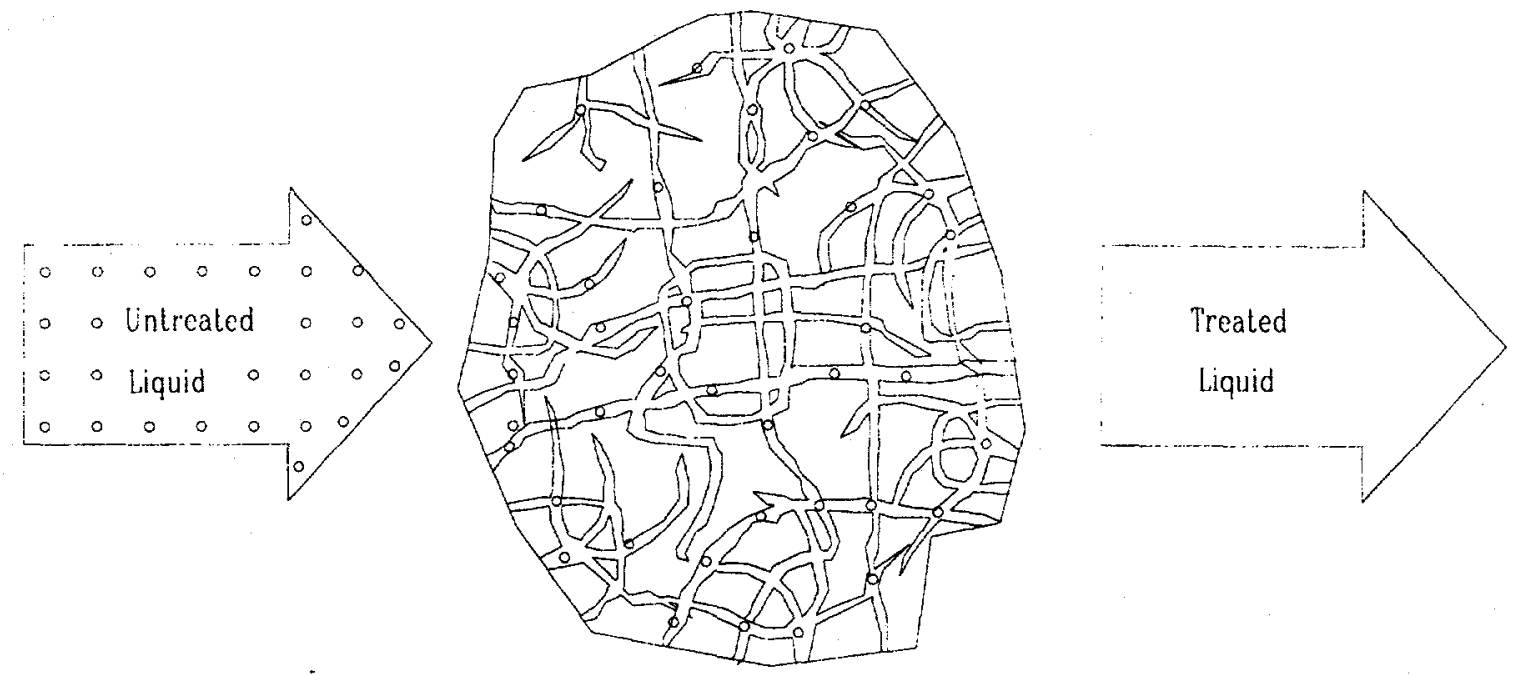

Figure 7-1 Diagrammatic conception of an activated carbon granule and its adsorption

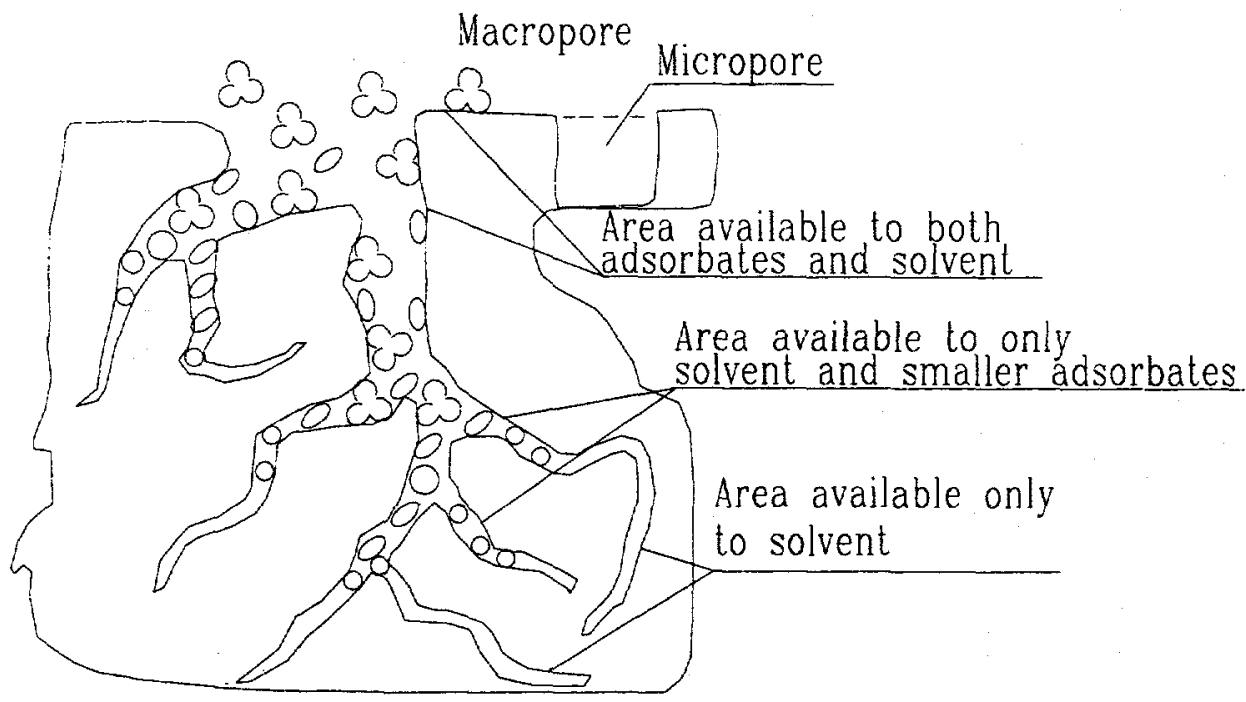

Figure 7-2 Diagrammatic conception of an activated carbon internal pore

The primary driving force for adsorption may be a consequence of lyophobic (solvent dislike) character of the solute relative to the particular solvent, or of a high affinity of the solute for the solid. For the majority of systems encountered in water and wastewater treatment practice, adsorption results from the combined action of these two forces, i. e., hydrophobicity (water dislike) of the solute, and the affinity of the solute for the adsorbent. The degree of solubility is by far the most significant factor in determining the intensity of the first of the two driving forces. In this context, the "degree" of the solubility can be thought of as the extent of chemical compatibility between the solvent and the solute. 
Lin et al. (1995) demonstrated that solubility, polarity and molecular weight of the adsorbate play a very important role in the adsorption process. The affinity of an adsorbate, which is strongly associated with the polarity of the adsorbate and the adsorbent, is the same physical item as previously explained between absorbent (activated carbon) and adsorbate. Generally, the affinity of organic compounds for activated carbon is proportional to their molecular weight, and the carbon portion of the compounds.

Solubility reflects the compatibility between the solute and the solvent. A solute with a higher solubility usually has a higher attraction for or compatibility with the solvent and is harder to be separated from the solvent. Weber et al. (1983) pointed out that an inverse relationship can be anticipated between the extent of adsorption of a solute and its solubility from which adsorption occurs. This trend has been shown in many early experiments (Holmes and Mckelvey 1928, Hanson and Craig 1954).

Lin et al. (1995) demonstrated that the adsorption of organic compounds by activated carbon is complicated by the interaction and complex phenomena of molecular weight, polarity and solubility. Their study showed that the adsorption capacity of activated carbon for four organic materials, acetone, IPA, phenol, and tetrahydrofuran, follows the order of tetrahydrofuran $>$ acetone $>$ phenol > IPA. It can be found that, among the four, acetone, miscible with water and with the smallest molecular weight and highest polarity, has a higher adsorption capacity than that of phenol, which has the lowest polarity and largest molecular weight. On the other hand, IPA has a higher polarity and a smaller molecular weight than phenol, but has a lower adsorption capacity.

In a single water system, most nonpolar organic compounds including PCE and TCE are immiscible with water and termed hydrophobic. Thus there is a strong adsorption driving force dispelling PCE and TCE molecules away from water molecules and making them susceptible for adsorption to an appropriate adsorbent, such as activated carbon.

Adsorption is inversely proportional to solute solubility and directly proportional to the solute concentration. In other words, a higher solute concentration results in a higher adsorption driving force, adsorption rate and adsorption capacity.

A competition usually exists in a system of multiple solutes. The adsorption capacity of a component is typically proportional to its single partial adsorption capacity, yielding less adsorption capacity for this component in a multiple solute system. But in some cases, the interaction between the solutes may result in an environment that is favorable for adsorption of one or all components in the system yielding a higher adsorption capacity than in a single solute system.

Liquid adsorption systems may be classified into four systems; (1) single sorbate and single solvent, (2) multiple sorbates and single solvent, (3) single sorbate and mixed solvents, and (4) multiple sorbates and mixed solvents. Type (3) and (4) represent the adsorption involved in the 
separation of PCE from an alcohol solution. The existing theories, and engineering aspects of carbon adsorption of organic chemicals come from the recent practical application of this technology. However, these applications have treated trace or low concentration of chemicals in a single solvent of water, wastewater and gases. The cosolvent system, such as water and alcohol solution may dramatically enhance the affinity of the organic solute for the solvent. Thus a high affinity makes the organic solute much more soluble in the water and alcohol solution than in water alone. Simple extrapolation of existing data and theories for adsorption from single aqueous system to an alcohol and water solution system may be neither feasible nor desirable.

The impact of solvent on the adsorption could be caused by; (1) adsorbability of the solvent for activated carbon, (2) affinity of the solvent for the solute, (3) other factors such as temperature, $\mathrm{pH}$ and the dissolved oxygen of the solvent.

The essence of the separation of PCE from its alcohol solution using activated carbon relies on the adsorptive capacity differential between the PCE and alcohol in the adsorption system. Thus alcohol, even if not very adsorbable, can compete with the PCE for adsorption sites on the activated carbon.

As mentioned previously, in a water, alcohol and PCE system, the presence of alcohol enhances the compatibility between PCE and water-alcohol phases. This may reduce the PCE adsorption capacity depending on the alcohol concentration. The enhanced compatibility, however, may result in a higher PCE concentration, which forms a higher adsorption driving force for PCE molecules onto the adsorbent.

The increase in viscosity of the solution as a result of the presence of IPA could reduce the mobility of solute molecules in the internal pores of the activated carbon and thus decreases the adsorption rate.

IPA and ethanol are typical useful alcohols for alcohol flushing. IPA may have several technical merits over ethanol such as IPA solution has a higher PCE solubility. Ethanol, however, also has some other practical advantages over IPA.

The extraction solution from an alcohol flushing a PCE or TCE contaminated site like SRS would contain polar compounds (water and alcohol), non-polar compounds (PCE and TCE), trace metal ions $\left(\mathrm{Mg}^{2+}, \mathrm{Ca}^{2+}\right.$ and $\mathrm{Fe}^{2+}$ ) and some dissolved oxygen. The typical temperature for the extracted flushing solution would be in the range of 15 to $20^{\circ} \mathrm{C}$, which is equal to the typical temperature for the groundwater. The $\mathrm{pH}$ for the flushing extraction solution would be on the order of 6 to 8 . These parameters of the flushing extraction solution may also have some effect on adsorption.

Adsorption equilibrium is typically described using the adsorption isotherm, which shows the distribution of adsorbate between solid $\left(q_{e}\right)$ and solution phases $(C)$ at equilibrium. Figure 7-3 shows three typical adsorption isotherms ---- favorable, linear and unfavorable adsorptions. When the $\mathrm{q}_{\mathrm{e}}$ reaches a value of $\mathrm{Q}^{0}$ (adsorption capacity), $C$ reaches $C s$ (saturation concentration) 
and no more adsorption occurs. Most carbon adsorptions of organics from water are favorable or the type I adsorption shown in Figure 7-3.

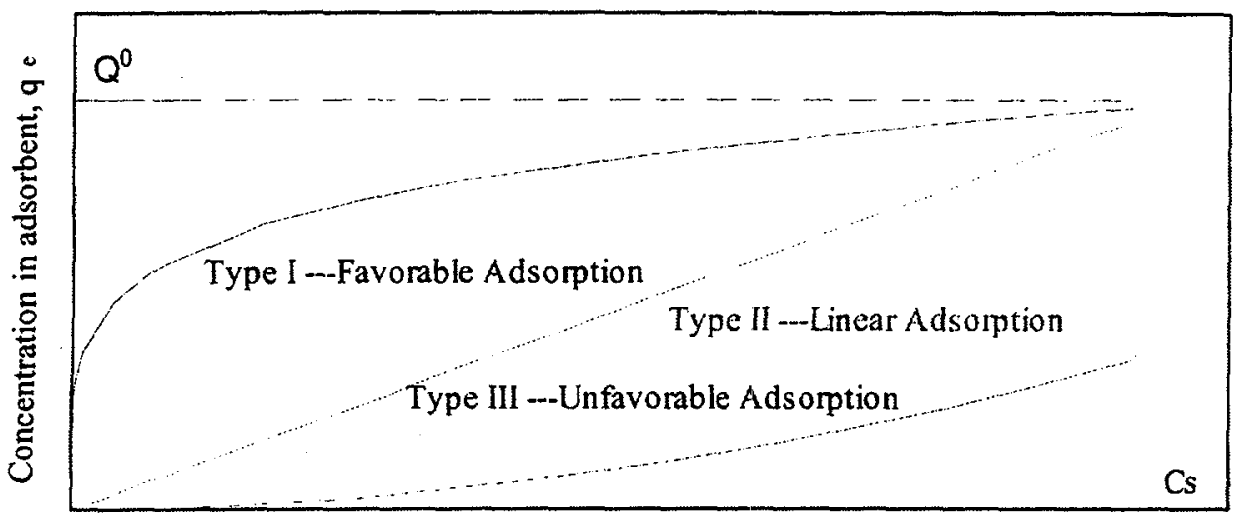

Concentration in solution, $\mathrm{C}$

Figure 7-3 Diagram of typical equilibrium isotherms

Typical adsorption models describing the adsorption isotherm include the Freundlich, Langmuir, and Brunauer, Emmett, Teller (BET) models. Each of these models has different assumptions.

For a very high solute concentration solid-liquid system, the multilayer adsorption may occur, therefore the isotherm can be expressed by BET.

The Langmuir adsorption isotherm (describing the type I adsorption shown in Figure 7-3) and its linear form could be expressed in Equations (1) and (2).

$$
\begin{aligned}
q_{e} & =\frac{Q^{0} b C}{(1+b C)} \\
\frac{1}{q_{e}} & =\frac{1}{Q^{0}}+\left(\frac{1}{b Q^{0}}\right)\left(\frac{1}{C}\right)
\end{aligned}
$$

Where $\mathrm{q}_{\mathrm{e}}=$ solute concentration in adsorbent $(\mathrm{mg} / \mathrm{g})$

$\mathrm{Q}^{0}=$ the mass of solute adsorbed per unit of weight of adsorbent in forming a complete monolayer on the surface

$b=a$ constant related to the energy or net enthalpy, $\Delta H$, of adsorption,

$\left(b \circ e^{-} \Delta^{H / R T}\right), b$ also determines the shape of the adsorption curve

$\mathrm{C}=$ aqueous solute concentration at equilibrium $(\mathrm{mg} / \mathrm{l})$.

The Freundlich isotherm model is an empirical isotherm model and has the advantage of simplicity and preciseness (Weber 1972). It has also been found to be extremely useful in most treatment applications. The Freundlich isotherm model and its logarithmic equation form are expressed in Equations (3) and (4). The logarithmic form of the Freundlich isotherm model could also be termed the linear Freundlich isotherm and is typically depicted in Figure 7-4. The linear 
Freundlich isotherm is often used in practice because it can greatly simplify the data processing. Thus this model was used to process the adsorption data involved in this research.

$$
\begin{aligned}
& \mathrm{q}_{\mathrm{e}}=\mathrm{K}_{\mathrm{F}} \mathrm{C}^{1 / \mathrm{n}} \\
& \log \mathrm{q}_{\mathrm{e}}=\log \mathrm{K}_{\mathrm{F}}+1 / \mathrm{n} \log \mathrm{C}
\end{aligned}
$$

Where: $K_{F}=$ Constant ( $y$ intercept of line shown in Figure 7-4), related to adsorption capacity, $\mathrm{Q}^{0}$ $1 / \mathrm{n}=$ Constant (the slope of the line shown in Figure 7-4), related adsorption intensity similar to $b$ in the Langmuir adsorption model $\mathrm{q}_{\mathrm{e}}(\mathrm{mg} / \mathrm{g})$ and $\mathrm{C}(\mathrm{mg} / \mathrm{L})=$ the same meanings as indicated above

A stronger adsorption with a higher adsorption capacity, $\mathrm{Q}^{0}$, has a higher value of $\mathrm{K}_{\mathrm{F}}$. Adsorption capacity might be defined as the adsorption level at which no more adsorption occurs. $1 / \mathrm{n}$ determines the type and the shape of an adsorption isotherm. In Figure 7-4, it could be found that the $1 / n$ has a value of less than 1 for a favorable adsorption and that the smaller the $1 / n$, the stronger the adsorption at a certain $\mathrm{C}$ and the steeper the shape of the isotherm at the lower $\mathrm{C}$ zone.

An isotherm is typically determined using batch studies and can be used to determine the adsorption capacity, as well as other adsorption characteristics. With the carbon adsorption parameters and the required effluent solute concentration predetermined, an estimation of how much contaminated solution could be treated with a carbon batch reactor or column bed could be estimated, although this is not a simple extrapolation. Cooper and Alley (1990) found that the activated carbon bed capacity for adsorption of gaseous fluid would seldom exceed $30-40 \%$ of that indicated by an equilibrium isotherm. Therefore, the bed capacity for the liquid solute may be less than $30 \%$ of that determined by an equilibrium isotherm due to the solvent effect.

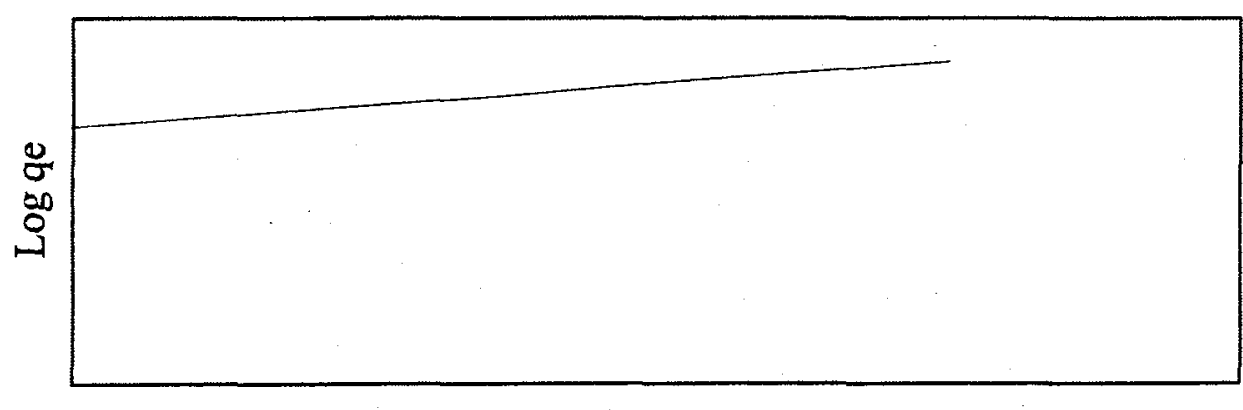

$\log C$

Figure 7-4 A hypothetical linear Freundlich isotherm

Weber and Bernardin (1983) described that the activated carbon adsorption of organic chemicals is comprised of four consecutive significant steps: (1) bulk transport, (2) film transport, (3) intrapore transport, and (4) adsorption attachment. These four steps are depicted in Figure 7-5. 
Among the four steps, the second and/or the third steps may exert major resistance to the solute molecule movement, and therefore are considered to be adsorptive rate-limiting steps.

The first step is a process in which solute or adsorbate molecules transport through the bulk solution to the surface of an activated carbon granule. This step usually occurs quickly and is considered to be a rapid step and not a rate-limiting step.

The second step or film-diffusion step is when the adsorbate molecule transfers through the adsorbate "film" surrounding the outer surface of the activated carbon particle. The third step or pore diffusion step is the diffusion of solute molecules within the internal pore of an activated carbon particle. The time for accomplishing these two steps and determining which one is the rate-limiting step largely depends on the flow conditions of the solution in contact with activated carbon. In a batch reactor with a high degree of agitation or mixing, turbulent flow predominates and prevents the formation of thick adsorbate film. In this case, film diffusion is not the ratelimiting step. Conversely, for a continuous flow system, such as a fixed bed of activated carbon, the film diffusion step will be the rate-limiting step for most normal flow rates (Weber, 1972).

Upon reaching an available site on the activated carbon surface, the adsorbate molecule binds itself there. Compared to the previous three steps, this step occurs almost instantaneously.

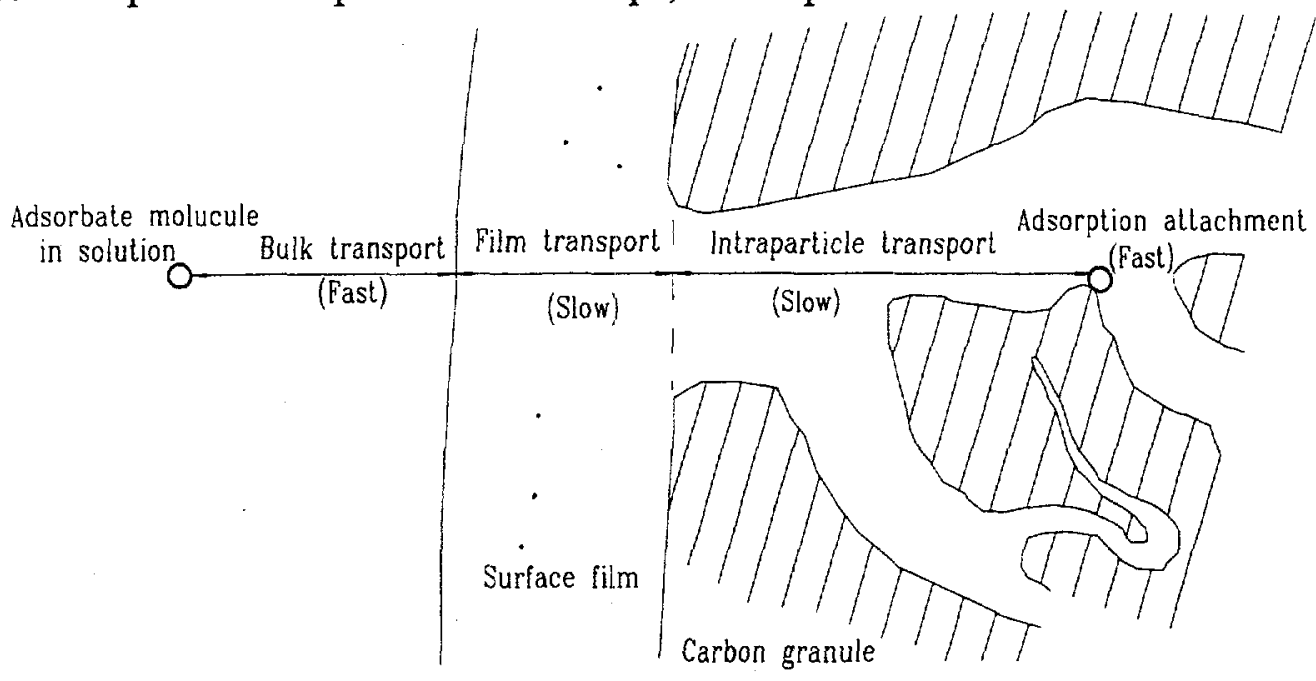

Figure 7-5 Schematic of the significant mass transfer steps in adsorption

The effluent solute breakthrough curve is generally applied to describe the adsorption occurring in a column adsorber. Solute breakthrough curves are defined as a profile of aqueous solute concentration with time or the solution passing through a column adsorber. One of the important parameters that could be determined from the solute breakthrough curve is the mass transfer zone. The mass transfer zone is defined as a length of the column adsorber bed over which adsorption occurs and the adsorbate concentration in the effluent varies from the influent concentration to zero or a some specific value, which is called breakpoint concentration.

As the column carbon bed continues to operate, the mass transfer zone moves down the column. Figure 7-6 shows a typical adsorption breakthrough pattern for column bed adsorption associated 
with a steady flow in the column. When a predetermined concentration appears in the effluent, breakthrough has occurred. This predetermined concentration is also called breakpoint concentration --- $C_{b}$. At this point, the effluent quality no longer meets treatment objectives. Then the carbon in the column typically needs replacing with new or reactivated carbon. The spent activated carbon can either be regenerated or disposed of. When the carbon becomes so saturated with the contaminants that they can no longer be adsorbed, the carbon is said to be spent $\left(C_{e}=C_{0}\right)$.

An adequate contact time for batch reactor is also needed for column carbon bed to reach the adsorption equilibrium. The contact time is closely related to empty bed contact time, which has value of the ratio of the effective volume of the mass transfer zone to its hydraulic loading rate. This contact time is usually less than the contact time needed for a batch reactor to treat the solution with the same initial adsorbate concentration. This is because the activated carbon in the activated carbon bed is in contact with the feed solution, which has high concentration. This results in a higher driving force for adsorption, which produces a shorter period of time required to reach the adsorption equilibrium in the column bed. Hassler (1974) pointed out that the superficial contact time (contact time) should be on the order of 20 to 60 minutes.

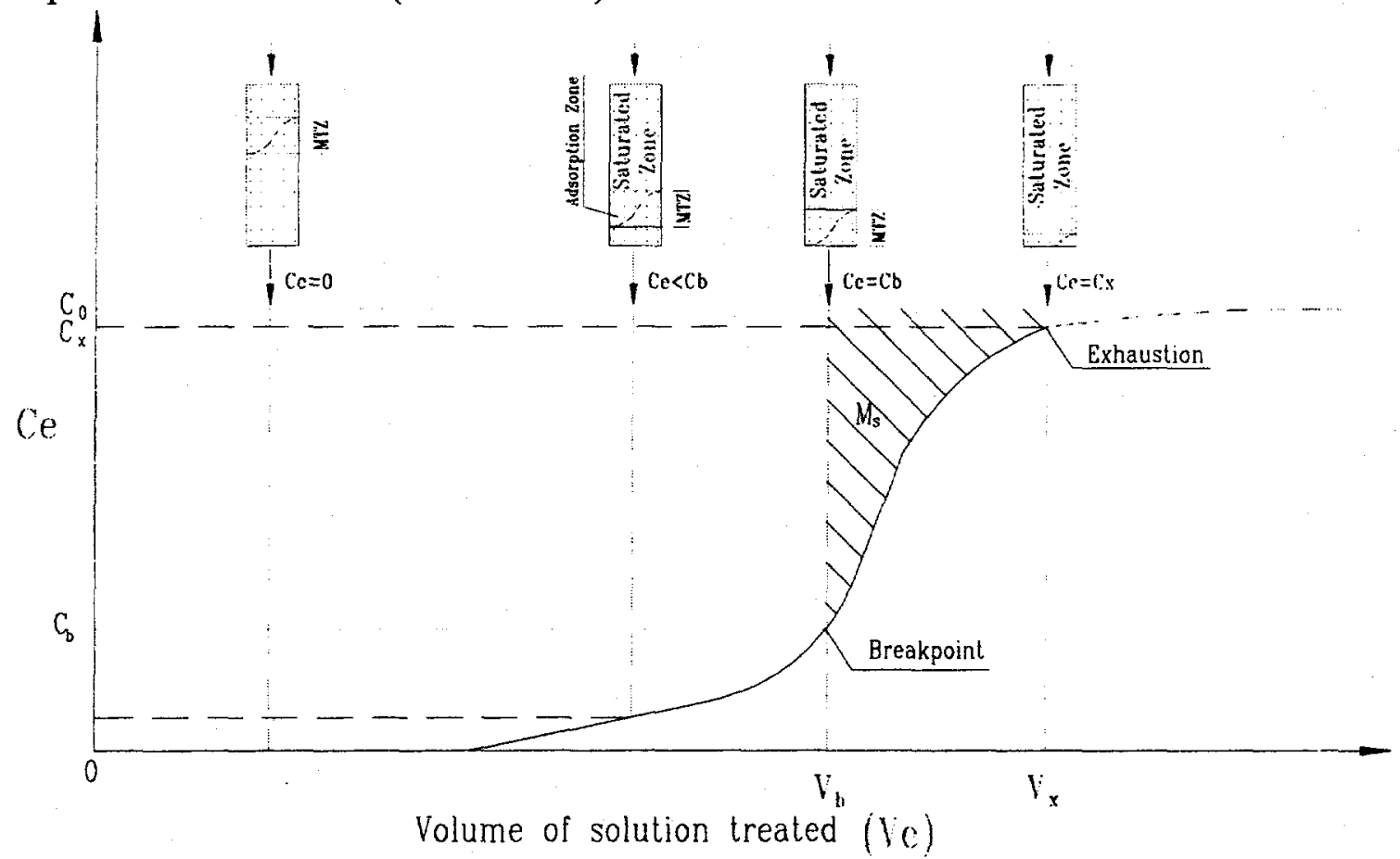

Figure 7-6 Schematic representation of the movement of the mass transfer zone and the resulting breakthrough curve

Another particular aspect of the carbon bed is the linear velocity of the liquid passing the bed. A bed passed by a flow at a higher linear velocity typically treats more solution at the same contact time. Typical linear velocity ranges from 1.25 to $5 \mathrm{~m} / \mathrm{hr}\left(0.5\right.$ to $\left.2.0 \mathrm{gpm} / \mathrm{ft}^{2}\right)$. The activated carbon bed needs to contain at least one mass transfer zone, otherwise the activated carbon in the column adsorber operates less effectively and efficiently. 
In column reactors, the carbon at the inlet of the carbon bed is continuously in contact with the concentrated influent solution. The concentration in the solution in contact with a given layer of carbon in a column changes very slowly under this condition. Thus high uptake levels of adsorbate by the carbon could be achieved. Therefore carbon column is wildly used in the real practice in environmental engineering. Granular activated carbon is used in conjunction with the column bed because of its low hydraulic head loss.

The amount of the extraction solution from an alcohol flushing remediation practice may not be very large. Thus the fixed column carbon bed may be more cost effective than pulsed carbon beds because the pulsed carbon beds are more complicated and higher in capital cost, even though they are a little bit more efficient. Typical patterns of the fixed carbon beds are schematically shown in Figure 7-7.

Because of the small flow rate of the extraction solution from an alcohol flushing remediation, two carbon beds in series would be a good choice. Each bed could operate individually to treat the solution adequately. The operation of this carbon bed configuration could be: (1) The influent is completely treated in the first carbon bed and then flows out of the system. (2) When first carbon bed is exhausted, the influent is shifted to the second carbon bed. The first carbon bed would be disconnected and replaced with fresh carbon, and then restored back to the operation line.
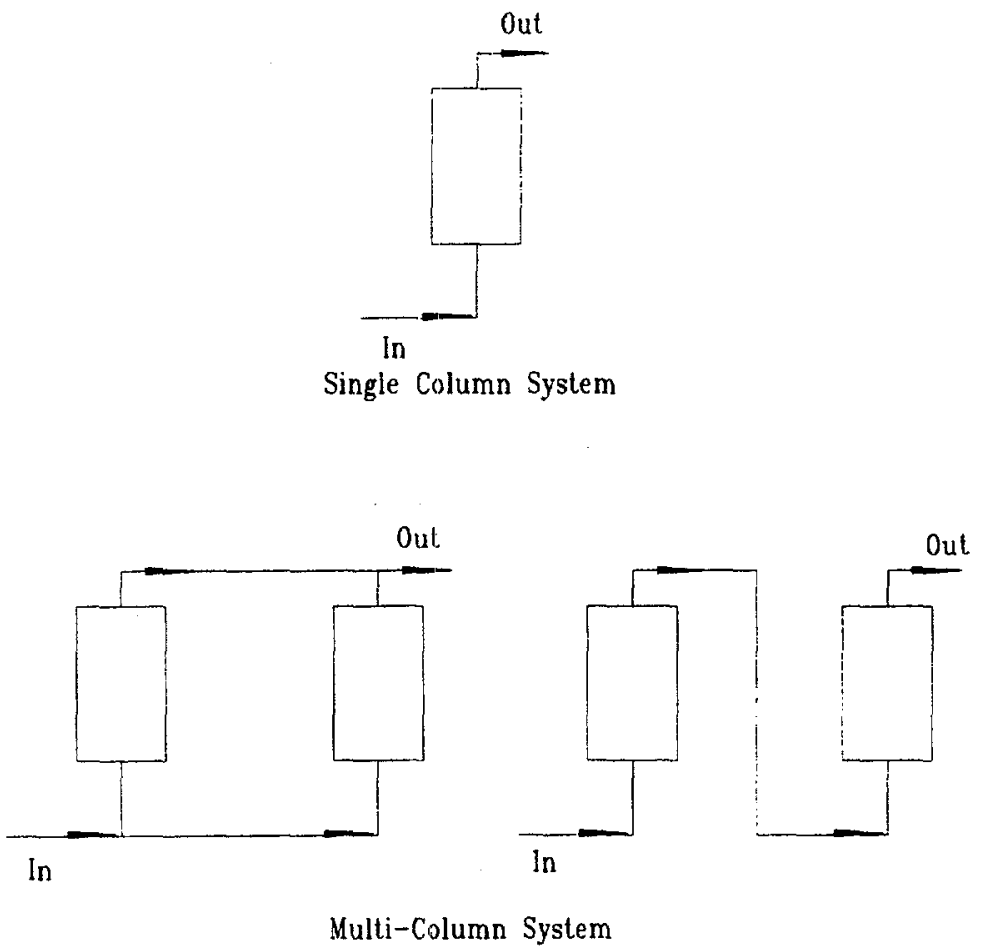

Figure 7-7 Schematic of the fixed carbon bed 


\subsection{Methods}

The representative materials used in this study were water, IPA, PCE and activated carbon. The research was implemented through a three-stage investigation including isotherm studies, column studies and an economical evaluation for the practical application of this technology.

The activated carbon used in this research was Filtrasorb-400, which is a granular activated manufactured by Calgon, Corporation, Pittsburgh, PA. The physical properties of Filtrasorb-400, as provided by the supplier (Fuller, 1998), are listed in Table 7-3.

Table 7-3 Activated carbon physical properties

\begin{tabular}{|c|c|}
\hline Parameter & Value \\
\hline Surface area & $1050-1200 \mathrm{~m}^{2} / \mathrm{g}$ \\
\hline Pore volume & $0.94 \mathrm{ml} / \mathrm{g}$ \\
\hline Particle density & $1.82 \mathrm{~g} / \mathrm{ml}$ \\
\hline Uniformity coefficient (Max) & 1.9 \\
\hline Effective size & 0.55 to $0.75 \mathrm{~mm}$ \\
\hline Sieve size, US Sieve Series, Weight \% & 5 \\
\hline Larger than No. 12 (opening of 1.4 mm or 0.056 inches) (Max) & 4 \\
\hline Smaller than No. 40 (opening of 0.42 mm or 0.0165 inches) & \\
\hline
\end{tabular}

The Filtrosorb- 400 granular activated carbon was produced through activation in natural water at room temperature, and it is a neutral or nonpolar activated carbon.

Chemicals used in this study are listed in Table 7-4.

Table 7-4 Description of chemicals used in this research

\begin{tabular}{|c|c|c|}
\hline Chemicals & Parameters & Supplier \\
\hline $\begin{array}{l}\text { Tetrachloroethylene } \\
\text { (PCE) }\end{array}$ & $\begin{array}{c}\text { Baker Analyzed }, \\
\text { Reagent, Assay } 99.9 \%\end{array}$ & Mallinckrodt Baker, Inc., Phillipsburg, NJ 08865 \\
\hline $\begin{array}{l}\text { Trichloroethylene } \\
\text { (TCE) }\end{array}$ & $\begin{array}{c}\text { Safemor@, } 8633 \text {, Assay } \\
99.8 \% \text {, }\end{array}$ & Mallinckrodt Baker, Inc., Paris, Kentucky 40361 \\
\hline Isopropanol (IPA) & $\begin{array}{c}\text { ARఠ, Analytical } \\
\text { reagent, Assay } 100 \%\end{array}$ & Mallinckrodt Baker, Inc., Paris, Kentucky 40361 \\
\hline Ethanol & Dehydated-200 Proof & $\begin{array}{c}\text { Packaged by McCormick Distilling Co., DSP-CT- } \\
18 \text { Brookfield, Connecticut, Distributed by } \\
\text { McCormick Distilling Co., Inc., DSP-MO-5 Weston, } \\
\text { Missouri } 64098 \\
\end{array}$ \\
\hline Methanol & $\begin{array}{l}\text { MX 0485-7, GR, } \\
\text { Anlydrous, Assay } \\
99.8 \%\end{array}$ & $\begin{array}{l}\text { EM Science, A Division of EM Industries, Inc., } 480 \\
\text { S. Democrat Road, Gibbstown, NJ } 08027\end{array}$ \\
\hline Distilled water & Produced by a distiller & Corning, Mega-Pure ${ }^{\mathrm{TM}}$ System \\
\hline
\end{tabular}


The isotherm studies were conducted using batch experiments. The impact of the IPA concentration, PCE concentration, activated carbon dose and the combination of these three variables on adsorption were investigated. The experimental data were analyzed using the linear Freundlich isotherm model. Adsorption parameters were determined based on this model. The adsorption parameters were used to estimate solution volume treatment capacity for the amount of the activated carbon packed in a carbon column for the column experiments.

TCE, another common chlorinated contaminant, was also studied to estimate its batch adsorption parameter in an IPA and water solvent system. Ethanol, another possible alcohol, was used to estimate the batch adsorption parameters of PCE from an ethanol-water system.

A range of alcohol and PCE concentrations was used to conduct the isotherm studies to simulate a range of possible solution scenarios in the field. The variables of interest in the study were alcohol (IPA or ethanol) and chlorinated compounds (PCE and TCE), and activated carbon. The overall summary for these studies are listed below:

(1) The IPA concentrations used were $0,15,30$ and $60 \%$ (volumetric concentration), ethanol concentration used was $30 \%$;

(2) The maximum PCE or TCE concentration used was slightly less than the solubility in its respective alcohol solution. The maximum PCE and TCE concentrations used are shown in Table 7-5.

Table 7-5 Maximum PCE and TCE concentration

\begin{tabular}{|c|c|c|}
\hline Alcohol (Vol. \%) & $\begin{array}{c}\text { Maximum PCE } \\
\text { concentration }(\mathrm{mg} / \mathrm{L})\end{array}$ & $\begin{array}{c}\text { Maximum TCE } \\
\text { concentration }(\mathrm{mg} / \mathrm{L})\end{array}$ \\
\hline IPA 0\% & 120 & $/$ \\
\hline IPA 15\% & 240 & $/$ \\
\hline IPA 30\% & 1,000 & 3,000 \\
\hline IPA 60\% & 100,000 & $/$ \\
\hline Ethanol 30\% & 500 & $/$ \\
\hline
\end{tabular}

(3) The activated carbon dose was determined from previous tests such that the isotherm curve covers the equilibrium solution PCE concentration from 0.1 up to 0.8 of its initial solution concentration.

(4) A contact time of more than 3-week was generally used for the isotherm studies except those were indicated to determine the appropriate time for these experiments.

The "bottle point" method was used to conduct the isotherm studies, and it is depicted in Figure 7-7. 

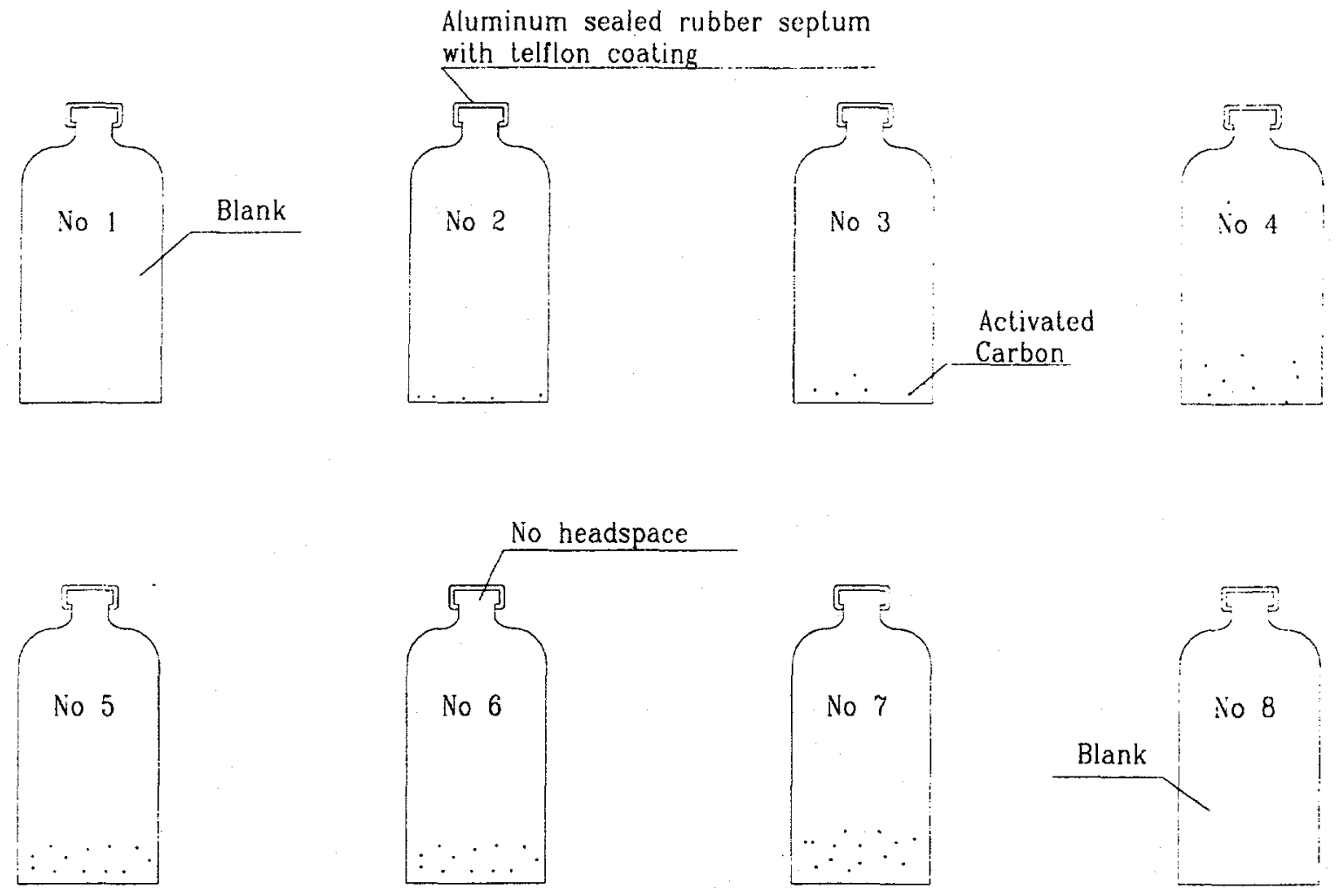

Figure 7-7 Schematic of batch reactors

Note: No 5 repeals No 6 .

A total of 8 bottles, each with a volume of $60 \mathrm{~mL}$, were used for each batch test. Six bottles were used as activated carbon reactors while the other 2 bottles were used as blanks to check any losses that might be occur. One blank bottle was filled with solution before the filling of the 6 activated carbon bottle reactors. The second blank bottle was filled last among the 8 bottles. Bottle No. 5 was a duplicate for the bottle No. 6 . The activated carbon dose in the activated carbon reactors increased in the order of No. 2 to No. 7. Each bottle was fully filled with solution. All eight bottles were capped with aluminum caps in conjunction with rubber septum with Teflon coating.

The activated carbon as received from supplier was sieved with a No \# 40 sieve (with opening of $0.42 \mathrm{~mm}$ or 0.0165 inches). The activated carbon retained by the No \# 40 sieve was washed with distilled water. The washed carbon was placed into a glass beaker. The glass beaker with washed carbon was dried in an oven set at $110^{\circ} \mathrm{C}$ for 24 hours. The dry carbon was stored in a dessicator until use.

The PCE solution for each experiment was mixed in a 1,000-mL glass volumetric flask. 24 hours was tested necessary for the PCE solution to get thoroughly mixed. A sample was taken from the thoroughly mixed parent solution in the $1,000 \mathrm{~mL}$ flask and was saved in a headspace vial (borosillicate glass vial with aluminum cap in conjunction with rubber septum with Teflon coating) for determining the initial concentration. The rest of solution in the flask was delivered 
into the blank bottles and bottles containing activated carbon. The loaded bottles were wrapped with opaque paper to reduce light penetration and the light effect that may have on the reaction. Then those bottles were mounted on a rotary shaker rotating at 6 revolutions per minute. The mixing time or contact time was from about 2 to 30 days. Since normal temperature variations generally have only minor effects on adsorption, the ambient temperature for isotherm studies was $21^{\circ} \mathrm{C} \pm 1^{\circ} \mathrm{C}$.

After mixing, the bottles were placed on a counter for at least 5 hours to allow the fine carbon particles to settle. Solution samples from each bottle and blank were taken and measured with a gas chromatograph.

3.2.1.1

Adsorption systems of different combinations of the three primary variables of IPA, PCE and carbon dose may yield different adsorption isotherms and adsorption parameters. Therefore isotherm studies were conducted over a series of batch experiments.

(1) The effect of IPA on the adsorption

The effect of alcohol (IPA) was investigated using 4-batch isotherm tests. The initial PCE concentration and carbon doses were held the same and the IPA concentration varied. The value of the three constituents is shown in Table 7-6. All isotherm studies were conducted using the "bottle point" method, and the volume of the glass bottle is of $60 \mathrm{ml}$.

Table 7-6 Parameters of adsorption system for alcohol effect on adsorption

\begin{tabular}{|l|c|}
\hline \multicolumn{1}{|c|}{ Parameter } & Value \\
\hline Initial PCE concentration $(\mathrm{mg} / \mathrm{L})$ & 130 \\
\hline IPA concentration $(\%)$ & $0,15,30$, and 60 \\
\hline Activated carbon dose $(\mathrm{mg})$ & $3.5,6.4,10.8,14.7,14.5,18.7$ \\
\hline
\end{tabular}

(2) Determination of the carbon dose

The linear Freundlich isotherm, which expresses the relationship between the PCE concentration in the adsorbent and the solution phases, assumes that an appropriate carbon dose is used. The appropriate carbon dose, an important factor, was investigated using two tests. In the first test, an adsorption solution with a $60 \%$ IPA and a PCE concentration of $120 \mathrm{mg} / \mathrm{L}$ was used. In the second, the adsorption was conducted with a solution at an IPA of $30 \%$ and a PCE concentration of $7.05 \mathrm{mg} / \mathrm{L}$. The parameters of the two systems are listed in Table 7-7.

Table 7-7 Parameters of the adsorption system for determining carbon dose

\begin{tabular}{|l|c|c|}
\hline \multicolumn{1}{|c|}{ Parameter } & Value & Value \\
\hline IPA concentration (\%) & 60 & 30 \\
\hline Initial PCE concentration (mg/L) & 120 & 7.05 \\
\hline Activated carbon dose (mg) & 26 to 6600 & 3 to 765 \\
\hline
\end{tabular}

(3) Effect of initial PCE concentration 
The effect of the initial PCE was investigated using three tests in which the $60 \%$ IPA and the carbon dose range were held the same. A different PCE concentration was used for each of the tests. The parameters of the adsorption systems are shown in Table 7-8.

Table 7-8 Parameters of the adsorption for the effect of initial PCE concentration

\begin{tabular}{|c|c|c|c|}
\hline Parameter & Value & Value & Value \\
\hline IPA concentration (\%) & 60 & 60 & 60 \\
\hline $\begin{array}{r}\text { Initial PCE concentration } \\
(\mathrm{mg} / \mathrm{L})\end{array}$ & 1000 & 10000 & 100000 \\
\hline Activated carbon dose (mg) & 1260,3000, & 1260,3000, & 1260,3000, \\
& 6000,9000, & 6000,9000, & 6000,9000, \\
& 9000,13200 & 9000,13200 & 9000,13200 \\
\hline
\end{tabular}

(4) Effect of varied initial PCE and IPA concentrations

The effect of varied initial PCE and IPA concentrations was investigated using a set of tests, in which IPA and its corresponding PCE concentrations varied and the range of carbon dose was chosen such that the equilibrium PCE concentration ranged from 0.1 to 0.8 times the initial PCE concentration. The values of those parameters are shown in Table 7-9.

Table 7-9 Experimental factors

\begin{tabular}{|c|c|c|c|c|c|c|c|}
\hline Parameter & \multicolumn{4}{|c|}{ Value } & \multicolumn{3}{|c|}{ Value } \\
\hline IPA (\%) & \multicolumn{4}{|c|}{0} & \multicolumn{3}{|c|}{15} \\
\hline PCE (mg/L) & 100 & 70 & 110 & 65 & 240 & \multirow{2}{*}{$\frac{160}{51,88}$} & 160 \\
\hline Carbon (mg) & \multirow{2}{*}{\multicolumn{2}{|c|}{$\frac{4,6,11}{12 \mathrm{~d}}$}} & \multicolumn{2}{|c|}{15,15 , } & 6,20 & & 88,122 \\
\hline Contact time & & & $22 \mathrm{~d}$ & $30 \mathrm{~d}$ & $28 \mathrm{hr}$ & $12 \mathrm{~d}$ & $30 \mathrm{~d}$ \\
\hline Parameter & & Value & & & & Value & \\
\hline IPA (\%) & & 30 & & & & 60 & \\
\hline PCE $(\mathrm{mg} / \mathrm{L})$ & 1000 & 950 & 760 & & 99000 & 101000 & 108000 \\
\hline Carbon (mg) & $\begin{array}{c}22, \\
103,\end{array}$ & $\begin{array}{l}207, \\
389,\end{array}$ & $\begin{array}{l}389, \\
540 \\
\end{array}$ & & $\begin{array}{l}1254, \\
2962\end{array}$ & $\begin{array}{l}6005 \\
9069\end{array}$ & $\begin{array}{c}9065, \\
13167\end{array}$ \\
\hline Contact time & $40 \mathrm{hr}$ & $19 \mathrm{~d}$ & $30 \mathrm{~d}$ & & $40 \mathrm{hr}$ & $12 \mathrm{~d}$ & $30 \mathrm{~d}$ \\
\hline
\end{tabular}

(5) Effect of the contact time

The effect of contact time on adsorption was incorporated in the previous part of the batch experiments. The contact time used varied from 30 hours to 30 days. This effect was investigated by holding the IPA and the range of carbon dose the same and varying the contact time. The parameters for the experiments for this purpose are also shown in Table 7-10. There was a small variation in initial PCE concentration of the solution because of the difficulty in keeping it constant among different trials.

(6) PCE adsorption from ethanol 
This adsorption was conducted over a system composed of $30 \%$ ethanol, $360 \mathrm{mg} / \mathrm{L}$ PCE (less than its solubility of $700 \mathrm{mg} / \mathrm{L}$ in $30 \%$ ethanol solution), and the range of the carbon dose of 3.7 to $540 \mathrm{mg}$.

(7) TCE adsorption from IPA solution

TCE adsorption was conducted over a system composed of $30 \%$ IPA, initial TCE concentration of $2503 \mathrm{mg} / \mathrm{L}$ (less than its solubility of $3000 \mathrm{mg} / \mathrm{L}$ in $30 \%$ IPA solution), and the range of the activated carbon dose of 3.5 to $630 \mathrm{mg}$.

\section{Column studies}

In the column experiments, the influent solution with different IPA and initial PCE concentrations was applied to pass the carbon column. The breakthrough curve for each column study was determined based on the batch study data. The breakthrough curves were used to determine the impact of alcohol and PCE concentration on the adsorption, which occurred in the carbon column. The carbon column capacity for recovery of IPA was determined based on the column experimental results.

Column tests were used to investigate: (a) the mass transfer zone; (b) the effect of the presence of IPA on adsorption of PCE onto activated carbon and the PCE migration in the mass transfer zone; and (c) the activated carbon treatment capacity of PCE or alcohol recovery capacity as an estimate for the operation of a scale-up column activated carbon adsorber.

The column systems are shown in Figures 7-8 and 7-9. Figure 7-8 shows the details of the column setup, which is composed of a glass column with $3.1 \mathrm{~cm}$ inside diameter; two aluminum end-caps; two O-rings (Viton®, Small Parts, Inc., Miami Lakes, FL 33014-0650); tubings (Teflon $\circledast$, Small Parts, Inc., Miami lakes, FL 33014-0650); stainless fittings (Swagelock®, Crawford Fitting Company, Solon, Ohio 44139). In order to reduce the column end effect, $1 \mathrm{~cm}$ of the column was left carbon-free between the carbon bed and the fitting connectors at both of the inlet and outlet of the column. The total glass column was $10 \mathrm{~cm}$ in length and the effective activated bed length in the column was $8 \mathrm{~cm}$, which was 2.58 times the column inside diameter of $3.1 \mathrm{~cm}$. The overall column underwent a 2-day PCE adsorption test beforehand and the results showed that the column did not adsorb PCE. 


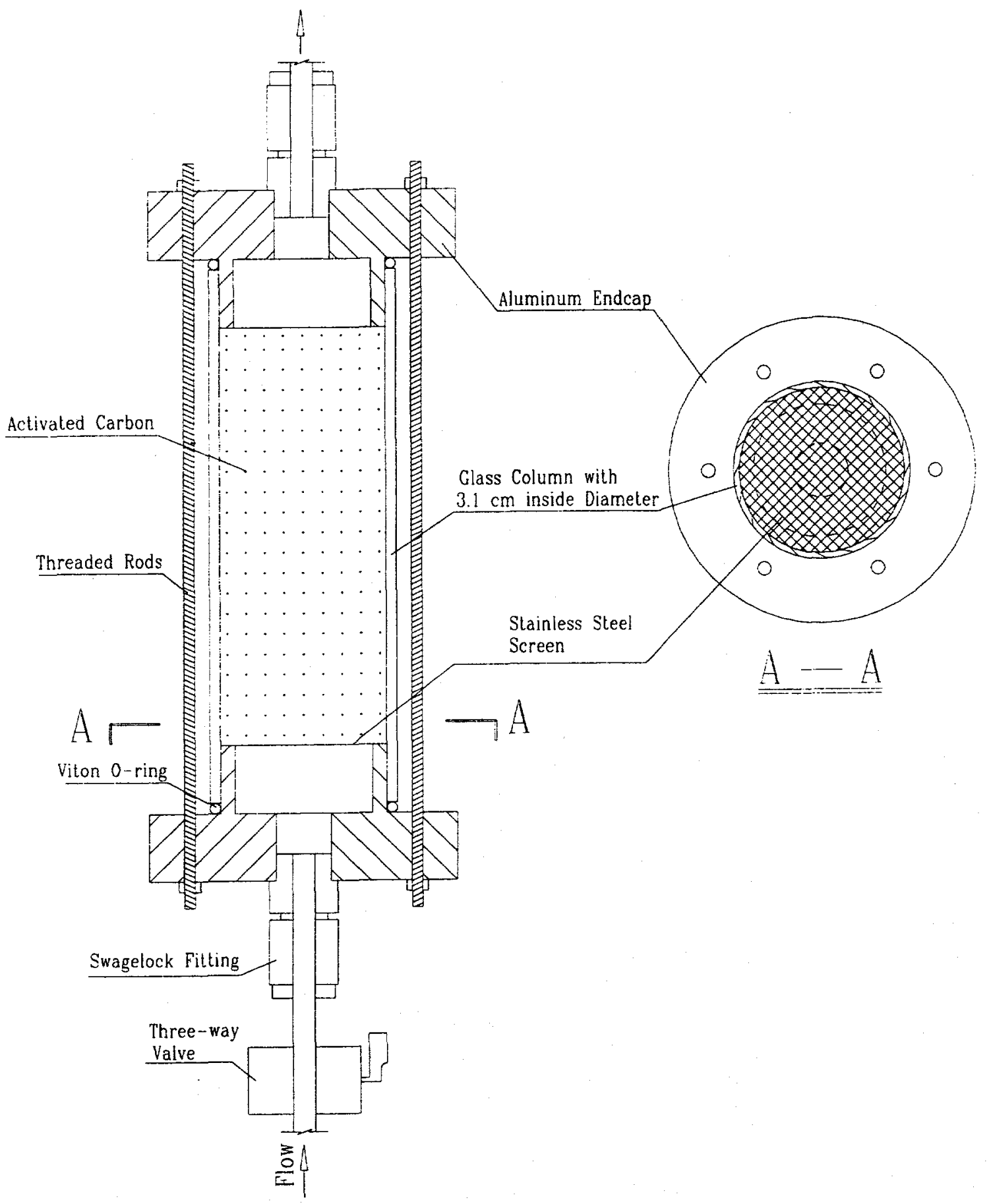

Figure 7-8 Column Setup 


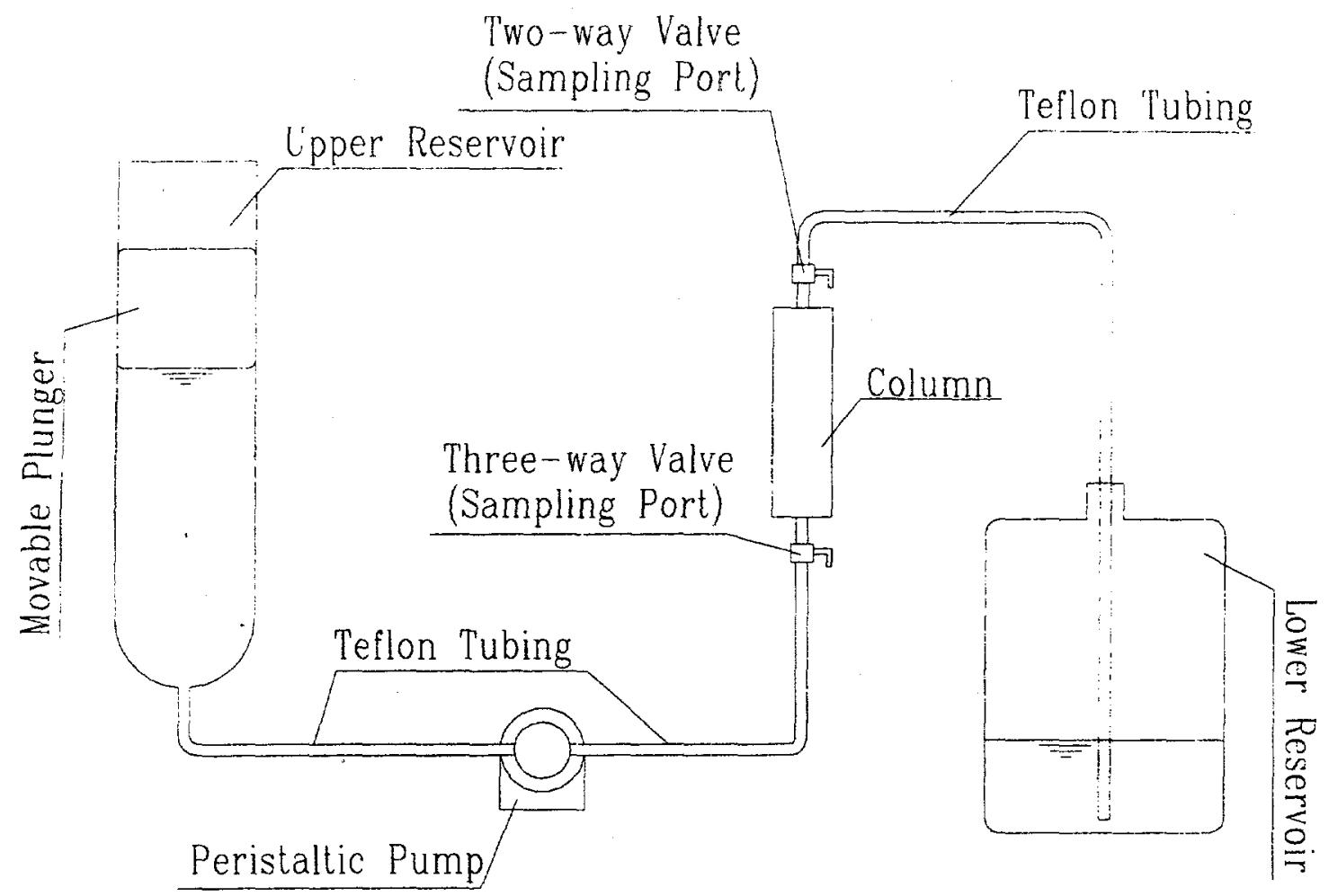

Figure 7-9 Schematic of the Column System Setup

Figure 7-9 shows the overall column system, which consisted of the following: (1) an upper reservoir with a movable plunger in to prevent PCE and IPA from evaporating. The plunger was made of a glass bottle wrapped with aluminum foil; (2) a lower glass reservoir for collecting the effluent; (3) a peristaltic pump for injecting the solution into the carbon column bed; (4) an activated carbon bed column setup shown in Figure 7-8; and (5) a three-way stainless steel valve and a stainless steel two-way valve (Popper \& Sons, Inc., Hyde Park, New York) installed at the inlet and outlet of the activated carbon column setup for sampling the influent and effluent, respectively.

The sampling program, such as sampling time interval, the ratio for influent sample to the effluent samples was determined in a test conducted beforehand. The detailed information is described in the column study procedure.

The activated carbon as received from the supplier was sieved with a No \# 40 sieve. The carbon retained on the No \# 40 sieve was gathered and soaked in distilled water for at least 48 hours in order to dispel the air trapped in the intrapores of the carbon. The soaked activated carbon was packed into a glass column fully under distilled water. The packed column was flushed with distilled water upward for at least 24 hours. The PCE solution flow rate into column bed was set with the peristaltic pump during this distilled water flushing step. Column tests were conducted in an ambient temperature of $21 \pm 1^{\circ} \mathrm{C}$. 
The volume of the influent solution for the column test was estimated based on the amount of activated carbon packed in the column bed and the adsorption capacity of the activated carbon determined in batch isotherm studies for this kind of solution. The influent solution was mixed in 4-L amber glass bottles. From the previously tests, a mixing time of 24-hour was found to be needed to mix the influent solution thoroghly. Once the influent solution was prepared, a 10-mL sample was first taken into a $10-\mathrm{mL}$ headspace vial. This sample was then stored in a refrigerator until measured with a gas chromatograph. The rest of the mixed solution was poured into the upper reservoir, from which the solution was pumped into activated carbon column in a upflow.

Effluent samples and influent samples (about $1 / 3$ of effluent samples) were taken with a $10-\mathrm{mL}$ syringe and stored in $10-\mathrm{mL}$ headspace vials. The vials were stored in a refrigerator until measured. The column test for each solution case was terminated when the effluent concentration was approximately equal to or larger than the exhaust concentration $C_{x}$, which was set to be about $90 \%$ of the average column influent concentration.

The time for effluent sampling and the flow rate were recorded so that the volume of solution passing through the column could be calculated. Column experiments were mainly conducted using $30 \%$ and $60 \%$ IPA solution. The parameters of the solution for the column experiments are listed in Table 7-10.

Table 7-10 Parameters of the column experiments

\begin{tabular}{|c|c|c|c|c|}
\hline $\begin{array}{c}\text { IPA } \\
(\text { Vol. \%) }\end{array}$ & $\begin{array}{c}\text { PCE } \\
(\mathrm{mg} / \mathrm{L})\end{array}$ & $\begin{array}{c}\text { Flow rate } \\
(\mathrm{ml} / \mathrm{min})\end{array}$ & $\begin{array}{c}\text { Superficial } \\
\text { velocity }(\mathrm{m} / \mathrm{hr})\end{array}$ & $\begin{array}{c}\text { Empty bed } \\
\text { retention time } \\
(\mathrm{min})\end{array}$ \\
\hline 30 & 300 & 2.5 & 0.199 & 24 \\
\hline 30 & 1,000 & 2.5 & 0.199 & 24 \\
\hline 30 & 1,000 & 6 & 0.477 & 10 \\
\hline 60 & 100,000 & 1 & 0.079 & 60 \\
\hline 60 & 100,000 & 4 & 0.318 & 15 \\
\hline
\end{tabular}

The value of the superficial velocity or hydraulic loading rate listed in Table 7-10 was set based on criteria of, (a) the arrival of the adsorption equilibrium in the column, (b) the similar values used by the early researchers, and (c) the specific situation involved in this experiment.

\section{Analytical method}

All samples taken from the experiments were measured using a gas chromatograph (GC), Hewlett Packard Model 5890 Series II, which is connected with a 30-meter DB624 glass capillary column (J\&W Scientific) and a flame ionization detector (FD) or a 75-meter DB-VRX glass capillary column (J\&W Scientific) and a photo ionization detector (PID), 4430, (C) Copyright 1988 O.I•Corporation, at a lamp intensity of 5 . An automated headspace sampler (Hewlett Packard 19395) was used in conjunction with the GC for all sample analysis. 
Calibration stock standards were prepared according to the procedure outlined in US EPA Method 602 (US EPA, 1986). Several duplicate original stocks were made. Several identical samples were taken from each duplicate, and then measured by a GC. The stocks were accepted when each response for a duplicate fell within $5 \%$ to their average. Stocks were updated monthly and they were checked against the previous ones at the same strength.

Prior to the sample concentration measurement, a calibration curve with concentrations bracketing the entire sample concentrations was generated by plotting the GC measured responses of stock solution samples of known volumes against their concentrations or masses involved. At least five points were used for generation of a calibration curve. Least square regression over a part of or the whole calibration range was conducted to determine the calibration parameters. Check standards were run about every ten samples. The maximum holding time for samples in the refrigerator was 2-day for isotherm studies and 3-day for column study.

\subsection{Results}

\section{Batch isotherm study}

The PCE concentration in solution was measured with a gas chromotograph, and the concentration in carbon phase was calculated by the difference method shown in Equation (5).

$$
q_{e}=\frac{V_{\text {Bottle }}\left(C_{\text {initial }}-C_{\text {final }}\right)}{M_{A C}}
$$

Where: $\mathrm{q}_{\mathrm{e}}=$ PCE concentration in solid activated carbon

$\mathrm{V}_{\text {bottle }}=$ Volume of the bottle

$\mathrm{C}_{\text {initial }}=$ Initial solution concentration corrected for the blank concentration

$\mathrm{C}_{\text {final }}=$ Finial aqueous concentration in each carbon bottle

$\mathrm{M}_{\mathrm{AC}}=$ Mass of activated carbon in bottles

The PCE concentration in carbon and solution phases were modeled using the linear Freundlich isotherm. The linear Freundlich isotherm uses a least square regression method to determine the adsorption parameters. The adsorption parameters were considered acceptable when the regression coefficient $R^{2}$ had a value equal to or greater than 0.81 ensuring a good likelihood between the two regression variables, aqueous concentration $C$ and solid concentration $q_{e}$ at adsorption equilibrium.

Statistical analysis (T test) was applied to compare the linear Freundlich isotherm parameters for a set of the same or similar adsorptions with different contact times for an IPA concentration case associated. The statistical analysis was conducted using the slope $(1 / \mathrm{n})$ and intercept $\left(\mathrm{K}_{\mathrm{F}}\right)$ of the linear Freundlich isotherm. The statistical identity between any two data sets of an IPA 
concentration case was tested by meeting the criterion that both slope $(1 / n)$ and intercept $\left(K_{F}\right)$ are statistically equal to each other. The confidence interval used for the statistical analysis was $95 \%$.

If all data sets for experiments of one IPA concentration case were statistically identical, the average of all those adsorption parameters was taken to be final adsorption parameters. If the data sets were not statistically identical, a review of the data sets and experimental processes was conducted to determine the appropriate data set to determine the final adsorption parameters.

Adsorption parameters were used to investigate the alcohol concentration, activated carbon dose, adsorbate concentration and the combination of the three factors' effect on the adsorption capacity of the activated carbon. Based on the adsorption parameters determined by isotherm studies, the treatment capacity of activated is expressed in Equation (6) for the linear Freundlich isotherm: Because the PCE concentration in solution passing the carbon above adsorption mass transfer zone in a column bed, $\mathrm{C}_{0}$ instead of $\mathrm{C}_{\mathrm{b}}$ was plugged into the right side of the Equation (2).

$$
V=\frac{K_{F} C_{b}^{1 / n}}{\left(C_{0}-C_{b}\right)} \times M
$$

Where: $M=$ the amount of the activated carbon,

$C_{0}=$ initial adsorbate concentration,

$\mathrm{C}_{\mathrm{b}}=$ adsorbate concentration at break point, which was determined in column test to be $10 \mathrm{mg} / \mathrm{L}$ for $30 \% \mathrm{IPA}$ and $1000 \mathrm{mg} / \mathrm{L}$ for $60 \%$ IPA solutions,

$\mathrm{V}=$ the volume of solution whose solute (adsorbate) concentration drops from $C_{0}$ to $C_{b}$ by $M$ of activated carbon, and all other items stand for the same as those described earlier.

Batch isotherm studies were conducted to investigate the effect of varying IPA concentration on PCE adsorption. Isotherm results for solutions with an initial PCE concentration of approximately $130 \mathrm{mg} / \mathrm{L}$ and $0,15,30$ and $60 \%$ IPA are shown in Figure 7-11. The $\mathrm{x}$-axis of Figure 7-11 was normalized to the initial PCE concentration, $\mathrm{C}_{0}$, so that all the isotherms could be visually compared.

Figure 7-11 shows that favorable adsorption patterns occurred in the 0 and $15 \%$ IPA solutions. An unfavorable adsorption pattern occurred in the $30 \%$ IPA solution, and almost no adsorption trend could be found for the adsorption which occurred in the $60 \%$ IPA solution. The PCE concentration in the carbon phase, $q_{e}$, ranges from 300 to $700 \mathrm{mg} / \mathrm{g}$ for the adsorption which occurred in the $0 \%$ IPA solution and $\mathrm{q}_{\mathrm{e}}$ decreases with the IPA concentration in the adsorption solution. 


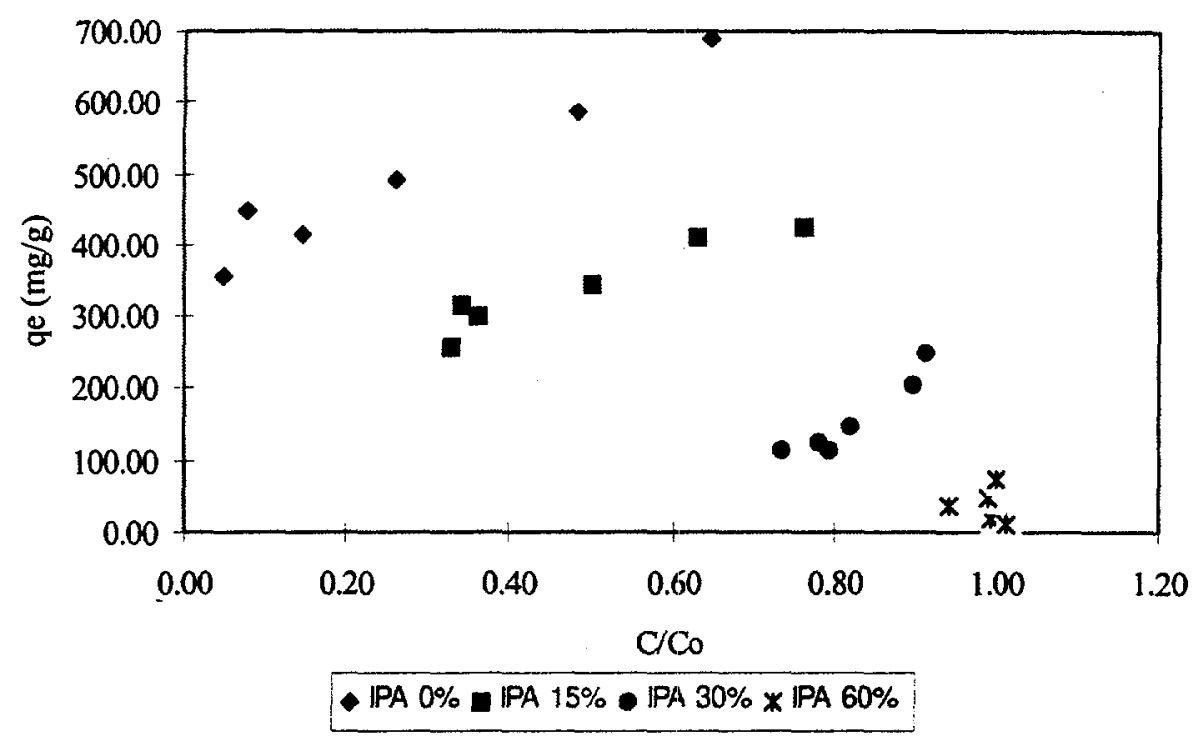

Figure 7-11 Adsorption isotherms for different IPA concentrations

In the four adsorption solutions, the carbon dose range and the initial PCE concentration were kept the same and only the IPA concentration varied. Thus it could be indicated that the PCE adsorption decreasing with the IPA concentration was caused by the presence of IPA. The presence of IPA enhanced the PCE solubility in the solution and thereafter enhanced the compatibility between the PCE molecules and the solvent phase. This enhanced compatibility made the PCE molecules harder to be adsorbed by activated carbon molecules.

Figure 7-11 also shows that the adsorption, which occurred in the 0\% IPA solution, is relatively stronger than that in the 15\% IPA solution. This indicates that the negative effect of the $15 \%$ IPA concentration on PCE adsorption is not adequate to alter the PCE adsorption pattern from a favorable pattern to an unfavorable pattern in this solution. This is because the PCE solubility of $280 \mathrm{mg} / \mathrm{L}$ in a $15 \%$ IPA solution is not much higher than that of $160 \mathrm{mg} / \mathrm{L}$ in water alone. In the $30 \%$ IPA solution, the PCE solubility is $1,600 \mathrm{mg} / \mathrm{L}$, which reduced the adsorbability of the PCE for carbon dramatically. Thus an unfavorable and weak PCE adsorption was observed in this solution.

The PCE of solubility of $132,000 \mathrm{mg} / \mathrm{L}$ in $60 \%$ IPA solution is 825 times the PCE solubility in water. This super high PCE solubility in 60\% IPA solution may give rise to a "disappearance" in PCE adsorbability for the carbon and "no PCE adsorption" was observed in this solution. Other factors possibly for this "no PCE adsorption" also exist. The competition of IPA molecules due to its high concentration for adsorption sites may interfere with the PCE adsorption. The involvement of a small PCE concentration and the difficulty in measurement of the PCE concentration in this solution subject the final results to a high possibility in large error due to even a slight experimental error. The low activated carbon dose was also of concern. If a much higher carbon dose had been used in such a solution, this may have offset the reduction in PCE 
adsorbility due to the high IPA concentration. As a result, PCE adsorption may be observed. This will be further discussed in the next section.

The Freundlich model was used to fit the data from experiments and is shown in Figure 7-12 using the linear transformation of the raw adsorption isotherm. This involved taking the logarithmic value of the PCE concentrations in both the carbon and solution phases. Transformation of the Freundlich isotherm was previously discussed. The linear regression was performed on the data shown in Figure 7-12 to determine model parameters and these are shown in Figure 7-13 and also listed in Table 7-11.

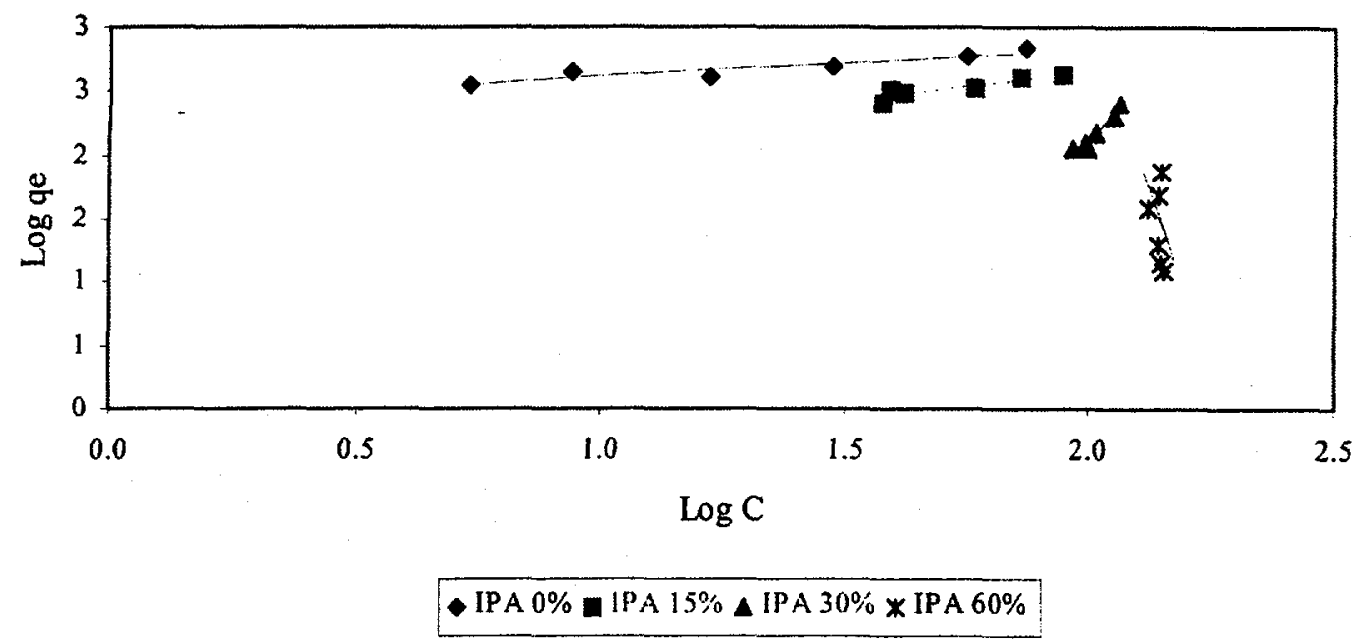

Figure 7-13 Linear Freundlich isotherms for different IPA concentrations

Table 7-11 Adsorption parameters of the Freundlich isotherms

\begin{tabular}{|c|c|c|c|}
\hline \multirow{2}{*}{ IPA (\%) } & \multicolumn{3}{|c|}{ Value } \\
\cline { 2 - 4 } & $\log \mathrm{K}_{\mathrm{F}}$ & $\mathrm{K}_{\mathrm{F}}$ & $1 / \mathrm{n}$ \\
\hline 0 & 2.40 & 249 & 0.22 \\
\hline 15 & 1.63 & 42 & 0.52 \\
\hline 30 & -5.34 & $4.56 \times 10^{-6}$ & 3.73 \\
\hline 60 & 23.76 & $5.75 \times 10^{23}$ & -10.37 \\
\hline
\end{tabular}

Figure 7-13 shows that the PCE adsorption occurring in the $0 \%$ and $15 \%$ IPA solutions could be modeled very well using the linear Freundlich isotherm. The PCE adsorption in the 30\% IPA solution also seems to be modeled well using the linear Freundlich isotherm. The fitness of the linear Freundlich isotherms for these adsorption data are supported by the fact that those isotherms have very high regression $R^{2}$ value of greater than 0.81 . The $R^{2}$ of 0.138 for the PCE adsorption at $60 \%$ IPA solution implies that these adsorption data could not be modeled well using the linear Freundlich isotherm due to the reasons discussed above. 
Table 7-11 quantitatively shows the effect of IPA on the PCE adsorption using the parameters of the linear Freundlich isotherms. The relationships between $\mathrm{K}_{\mathrm{F}}$ and adsorption $1 / \mathrm{n}$ and IPA concentration (up to $30 \%$ because "no PCE adsorption" occurred in the $60 \%$ IPA solution) are shown in Figures 7-13 and 7-14.

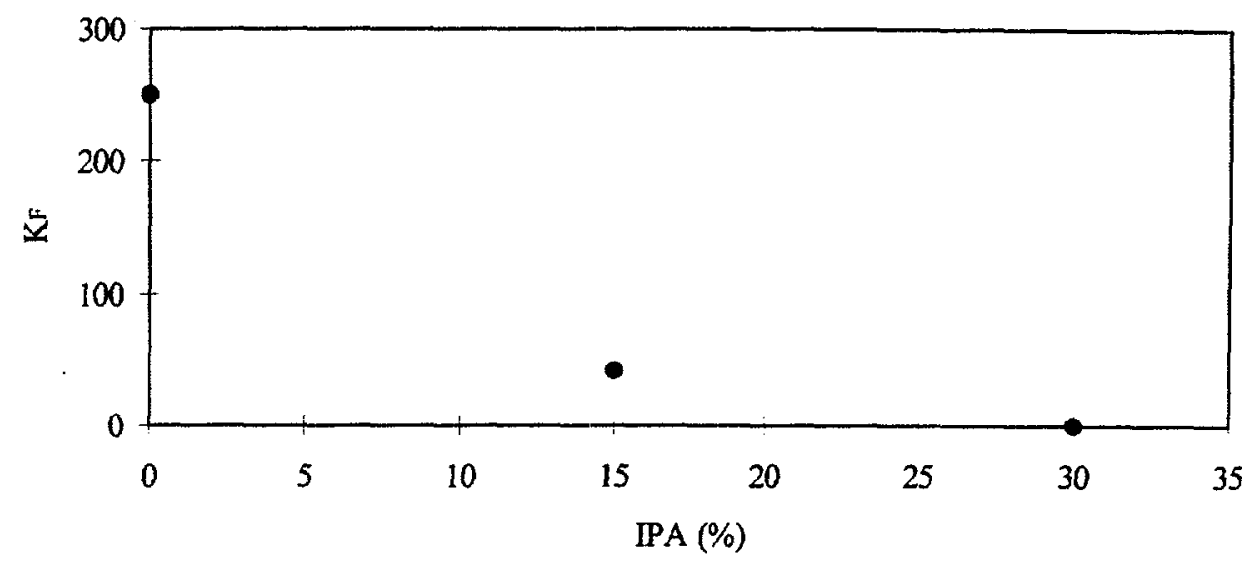

Figure 7-13 Relationship between $K_{F}$ and IPA concentration

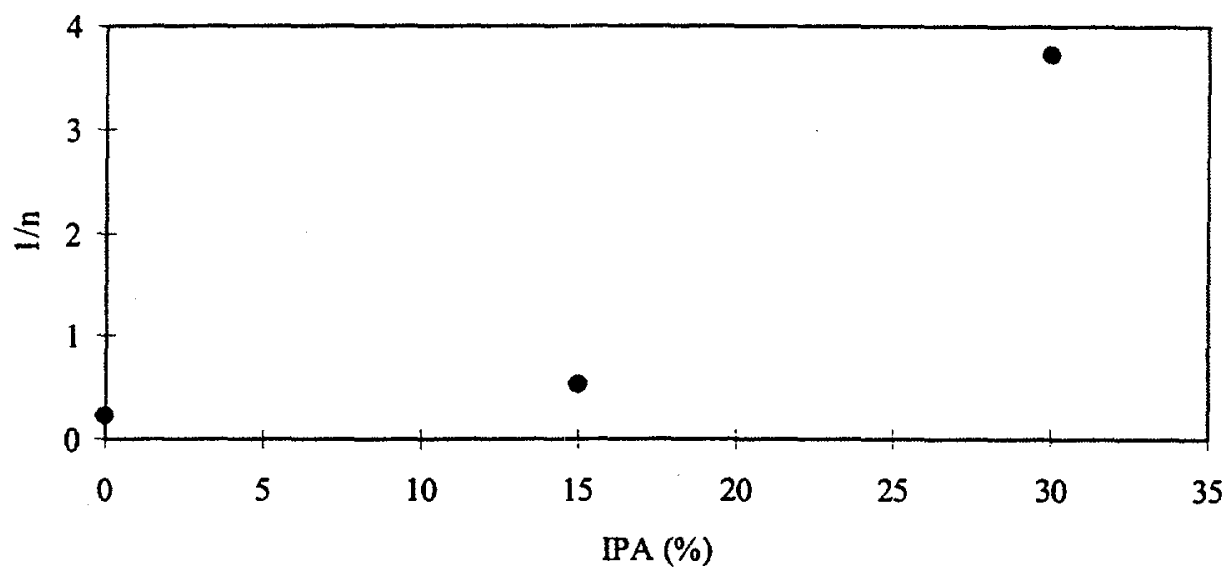

Figure 7-14 Relationship between 1/n and IPA concentration

For a solution with no greater than $30 \% \mathrm{IPA}$, the $\mathrm{K}_{\mathrm{F}}$ decreases exponentially from a $\mathrm{K}_{\mathrm{F}}$ of 250 for the $0 \% \mathrm{IPA}$ solution to almost 0 for the $30 \% \mathrm{IPA}$ solution. A higher decreasing rate in $\mathrm{K}_{\mathrm{F}}$ could be observed in the low IPA solutions than in the high IPA solutions as shown in Figure 7-13. As indicated before, $\mathrm{K}_{\mathrm{F}}$ is proportional to the adsorption capacity. Thus an inverse relationship between the adsorption capacity and the IPA concentration could be concluded. The tremendous $\mathrm{K}_{\mathrm{F}}$ value for the $60 \%$ IPA solution was resulted purely from the mathematical regression and does not reflect the true adsorption occurred. The adsorption constant $1 / n$ increases exponentially with the IPA concentration. This indicates that the adsorption intensity becomes weaker in the 
higher IPA solution because a high value of $1 / \mathrm{n}$ is associated with a weak adsorption. The $1 / \mathrm{n}$ of -10.37 also does not reflect the true PCE adsorption intensity observed in the $60 \%$ IPA solution.

Summarizing the effect of IPA on the adsorption of PCE ends up with a conclusion that the adsorption of PCE by carbon is affected negatively by the IPA concentration.

The previous results of PCE isotherm at $60 \%$ IPA suggested that the activated carbon dose was not appropriate in this study. If the increase in IPA concentration does affect PCE adsorption, then a higher carbon dose may be needed to obtain a strong adsorption. This was investigated by varying the activated carbon dose range using $60 \%$ IPA solution with a PCE concentration of approximately $120 \mathrm{mg} / \mathrm{L}$.

The relationship between the equilibrium PCE concentration normalized to $\mathrm{C}_{0}$ and the mass of the activated carbon is shown in Figure 7-15.

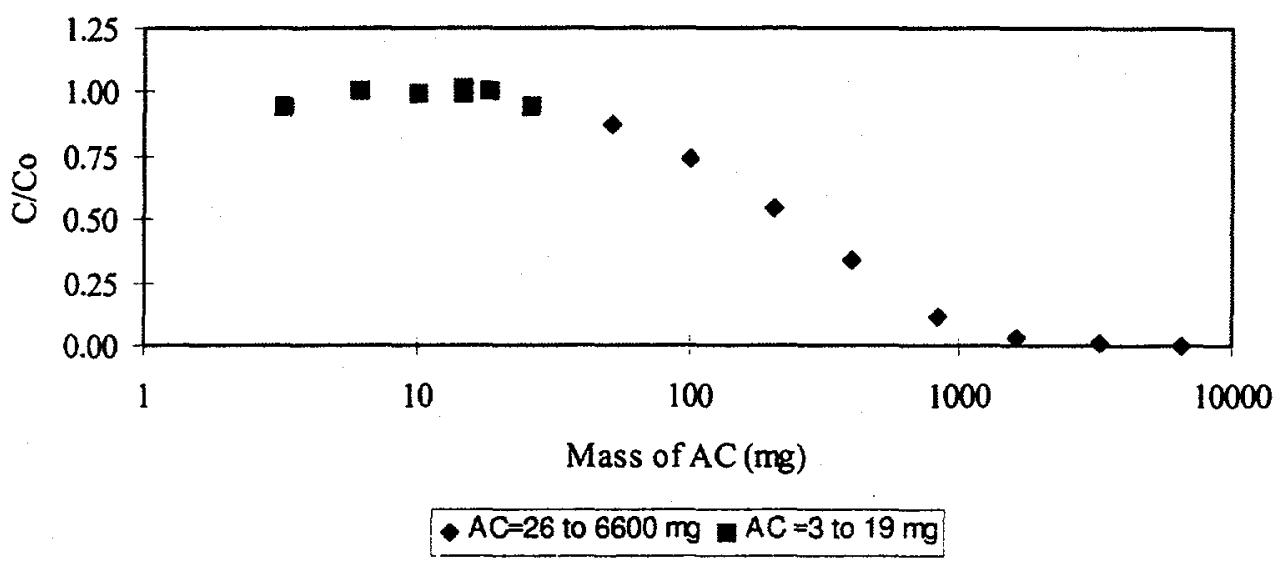

Figure 7-15 $\mathrm{C} / \mathrm{C}_{0}$ versus mass of $\mathrm{AC}$ for $60 \%$ IPA solutions

The square dots in Figure 7-15 are those data shown in Figure 7-11 for the 60\% IPA case. Figure 7-15 shows that the curve could be divided into three distinct zones, an upper flat zone, an effective adsorption zone (the linearly sloped part of the curve between mass of AC of about 18 to $800 \mathrm{mg}$ ), and a. low flat zone.

When the carbon dose is very small, for example less than $18 \mathrm{mg}$ in $60 \mathrm{ml}$ solution or $0.30 \mathrm{mg}$ carbon per liter solution, the corresponding part of the curve is horizontal. This upper flat part of the curve seems to indicate that "no PCE adsorption occurs", which was discussed in the previous section. On the contrary, the PCE adsorption should occur strongly, which could be illustrated by the subsequent effective adsorption zone. The sloped region of the graph shows that it was the small carbon dose that caused an "imperceptible" change in PCE concentration in the earlier $60 \%$ IPA results. This "imperceptible" change in PCE concentration could be corrected by using the logarithmic value of $y$-axis of Figure 7-15 and shown in Figure 7-16. 


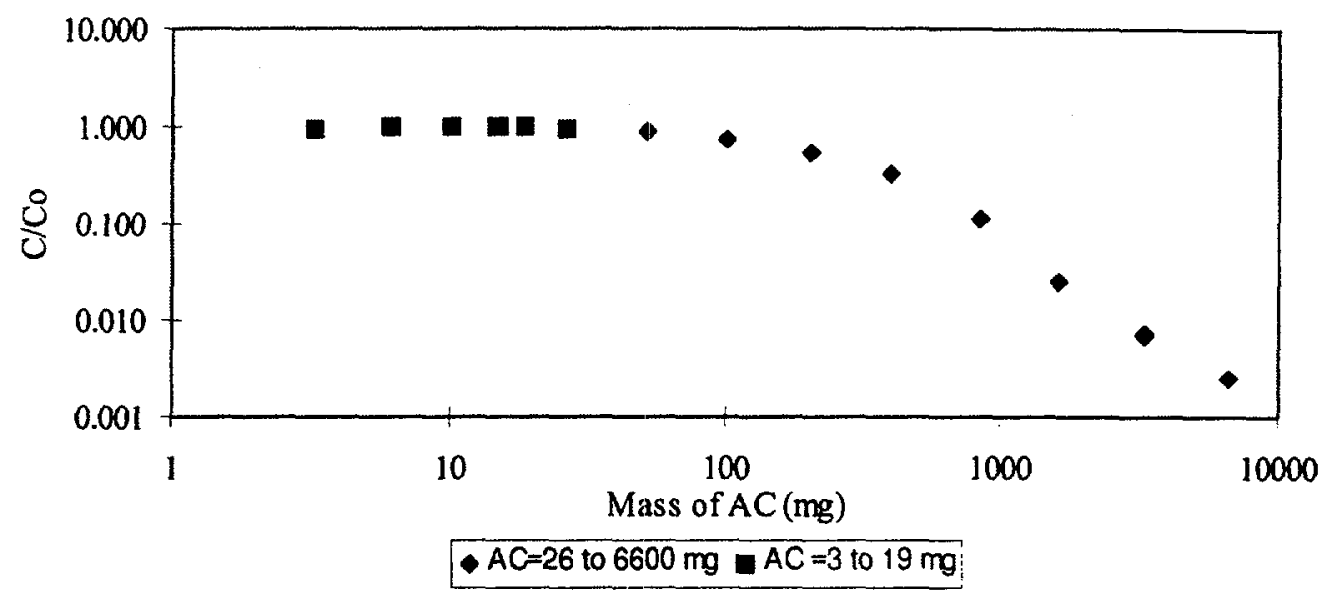

Figure 7-16 $\mathrm{C} / \mathrm{C}_{0}$ versus mass of $\mathrm{AC}$ for $60 \%$ IPA solutions (modified)

Compared with Figure 7-15, Figure 7-16 has a more slurred reading in the $C / C_{0}$ at its higher value zone $\left(\mathrm{C} / \mathrm{C}_{0}\right.$ greater than 0.1$)$, and a more distinct reading in the zone with $\mathrm{C} / \mathrm{C}_{0}$ of less than 0.1 . The typical interest from the curve shown in Figure 7-15 probably lies in the investigation of the $\mathrm{PCE}$ concentration varying from $\mathrm{C} / \mathrm{C}_{0}$ of 1 to 0.1 . Thus the type shown in Figure 7-15 rather than that in Figure 7-16 was for this research.

The effective adsorption zone may imply that the negative effect of the IPA concentration on PCE adsorption could be corrected by a larger carbon dose which provides with more adsorption sites. Under this scenario, although the carbon may not be very efficient, the sufficient amount of carbon adsorption sites could adsorb a significant amount of PCE molecules yielding a noticeable change in the PCE concentration in the solution phase.

The relationship curve shown in Figure 7-15 becomes horizontal again when the carbon dose is greater than $2000 \mathrm{mg}$. This is probably due to the fact that almost all of the PCE molecules have been adsorbed onto the carbon surface when the carbon dose is greater than $2000 \mathrm{mg}$. Then the further adsorption becomes weaker and weaker because of the very low PCE concentration and if any adsorption, no noticeable change in PCE concentration, C, could be observed because of the further extremely small adsorption. This feature also explains the overlap of the tail part of the relationship with the $x$-axis shown in Figure 7-15. The amount of activated carbon in excess of $2000 \mathrm{mg}$ could only exert a small part of its adsorption function and capacity. Thus from an economical point of view, it may not be cost-effective for the activated carbon dose to be above a certain value. However, an overdose may be needed to meet an extremely high requirement in the allowable effluent concentration.

Figure 7-17 shows that the adsorption isotherm over the effective adsorption zone is favorable and strong. Comparing Figure 7-17 with Figure 7-11 shows that the negative effect of IPA on the PCE adsorption can be corrected by increasing the carbon dose. 


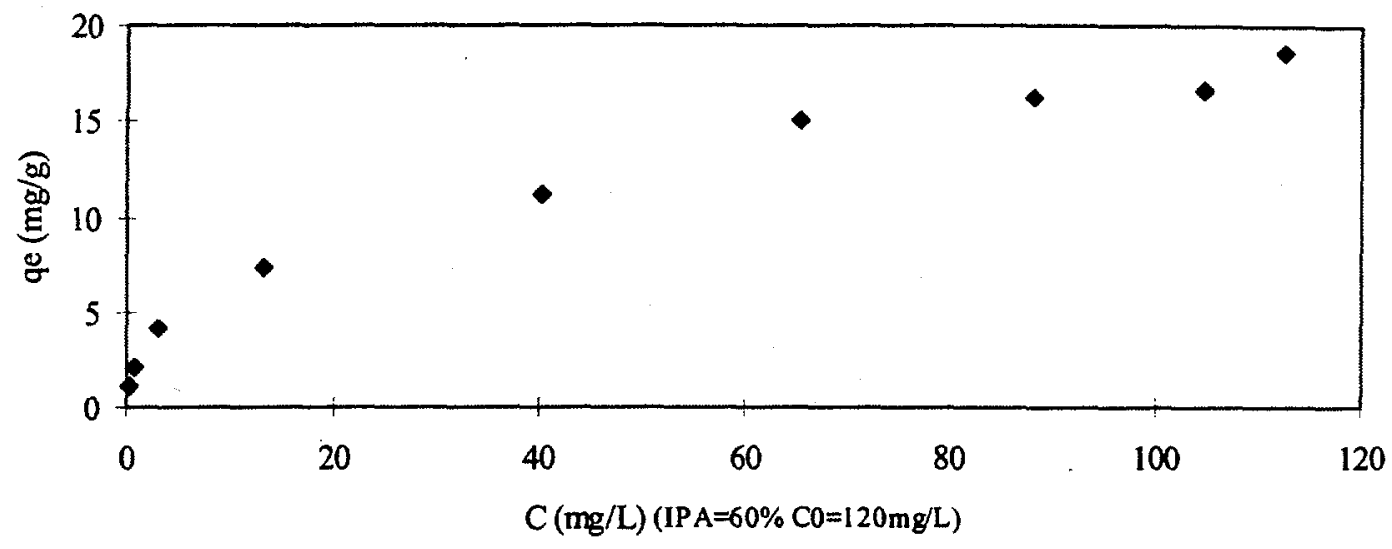

Figure 7-17 Adsorption isotherm for a 60\% IPA solution

The linear Freundlich isotherm over the effective adsorption zone was developed and is shown in Figure 7-18. The effective adsorption can be modeled well using the linear Freundlich isotherm, which is supported by the high $R^{2}$ of 0.9932 also shown in Figure $7-18$. The $K_{F}$ and $1 / n$ values are 2.14 and 0.457 , respectively. Comparing those adsorption parameters with those of the previous PCE adsorptions, this PCE adsorption is weaker than the adsoprtions in $0 \%$ and $15 \%$ IPA solutions but stronger than that in the 30\% IPA solution. This suggests that an optimal carbon dose for the $30 \%$ IPA solution should also be explored.

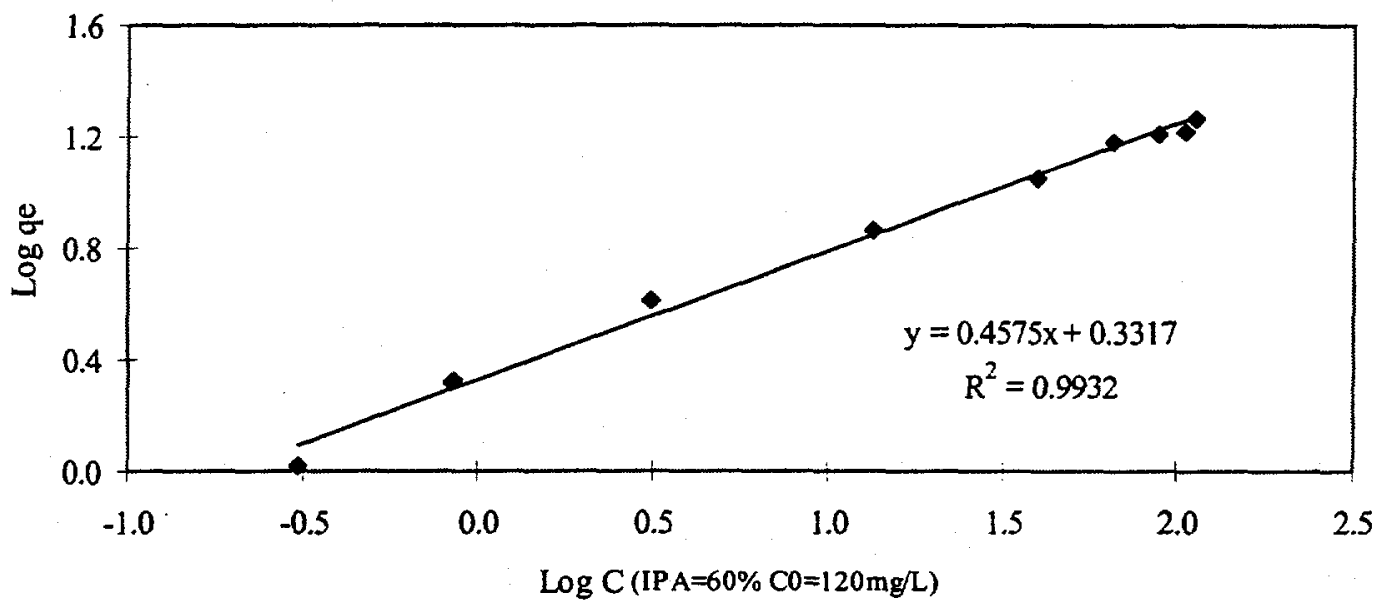

Figure 7-18 Linear Freundlich isotherm for a $60 \%$ IPA solution

Figures 7-19 to 7-21 show that a similar adsorption feature could be obtained for the $30 \%$ IPA solution as that for the $60 \%$ IPA solution. Figure 7-19 shows that the effective adsorption zone occurs over the carbon dose range of about 3 to $80 \mathrm{mg}$. Figure 7-20 shows that a favorable and strong adsorption occurs over the effective adsorption zone. Figure 7-21 shows that $K_{F}$ and $1 / n$ values are 10.59 and 0.473 , respectively. Even this solution has an initial PCE concentration of $7.05 \mathrm{mg} / \mathrm{L}$, which is lower than that of previous $30 \%$ IPA solution with an initial PCE 
concentration of $130 \mathrm{mg} / \mathrm{L}$, the former solution has higher adsorption parameters, $K_{F}$ and $1 / \mathrm{n}$, than the later solution. This is because the previous $30 \%$ IPA solution involved a lower and inappropriate carbon dose resulting in a weak adsorption, which may not be modeled well using the linear Freundlich isotherm.

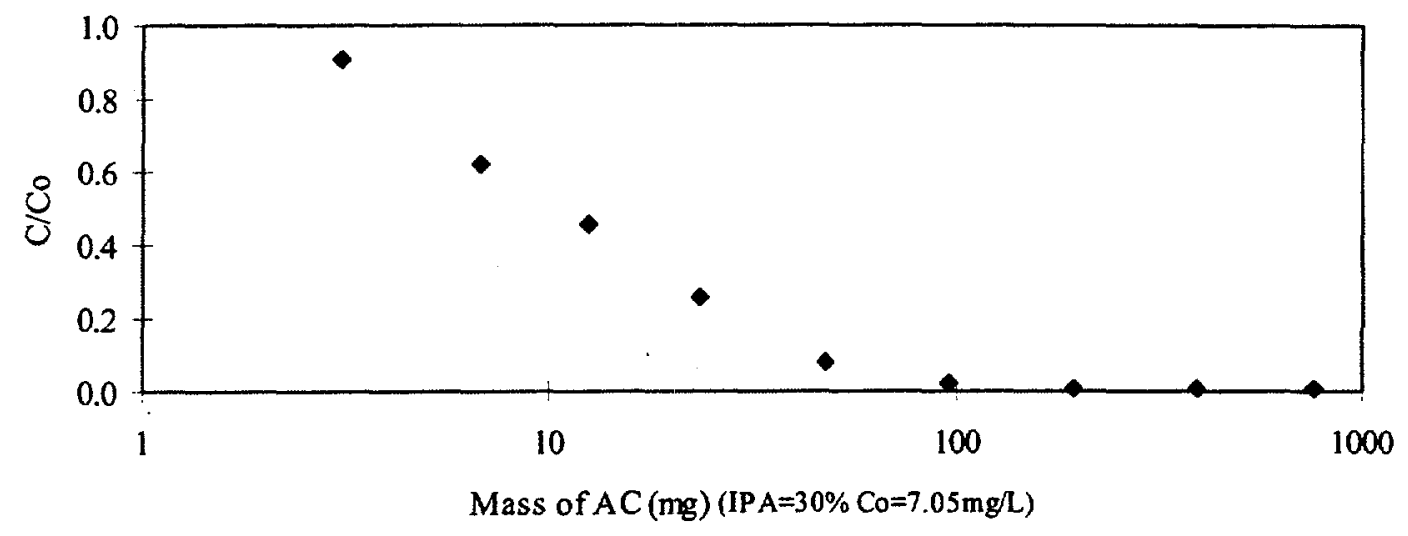

Figure 7-19 $\mathrm{C} / \mathrm{C}_{0}$ versus mass of $\mathrm{AC}$ for a $30 \%$ IPA solution

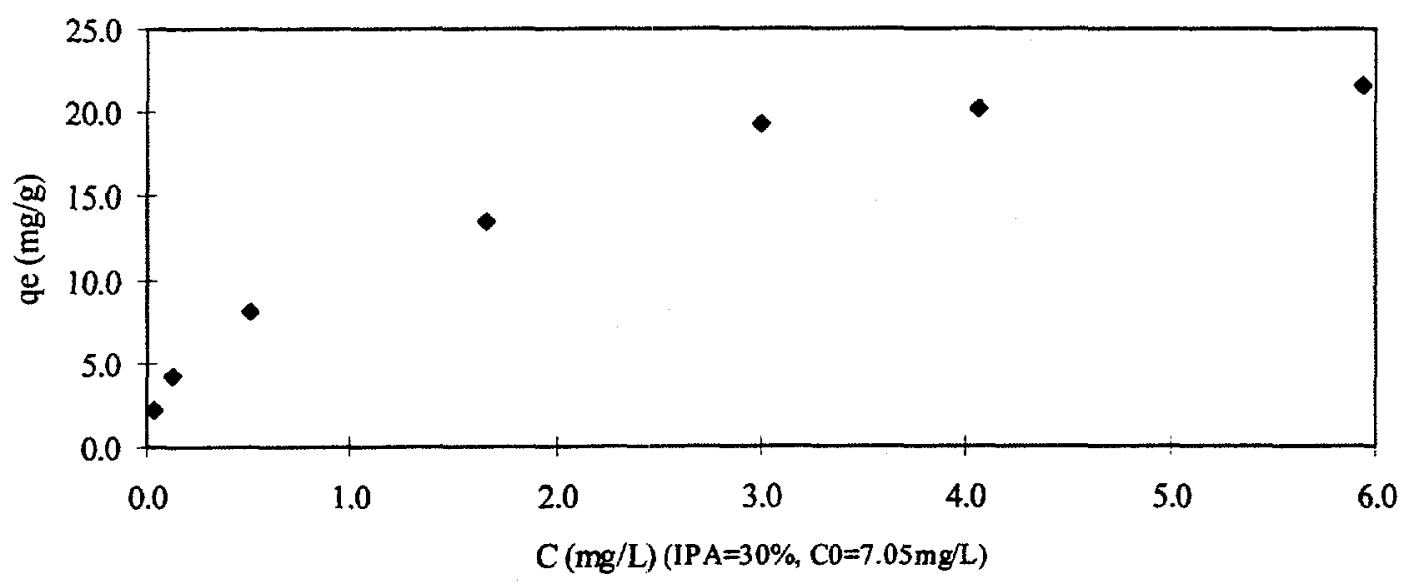

Figure 7-20 Adsorption isotherm for a 30\% IPA solution 


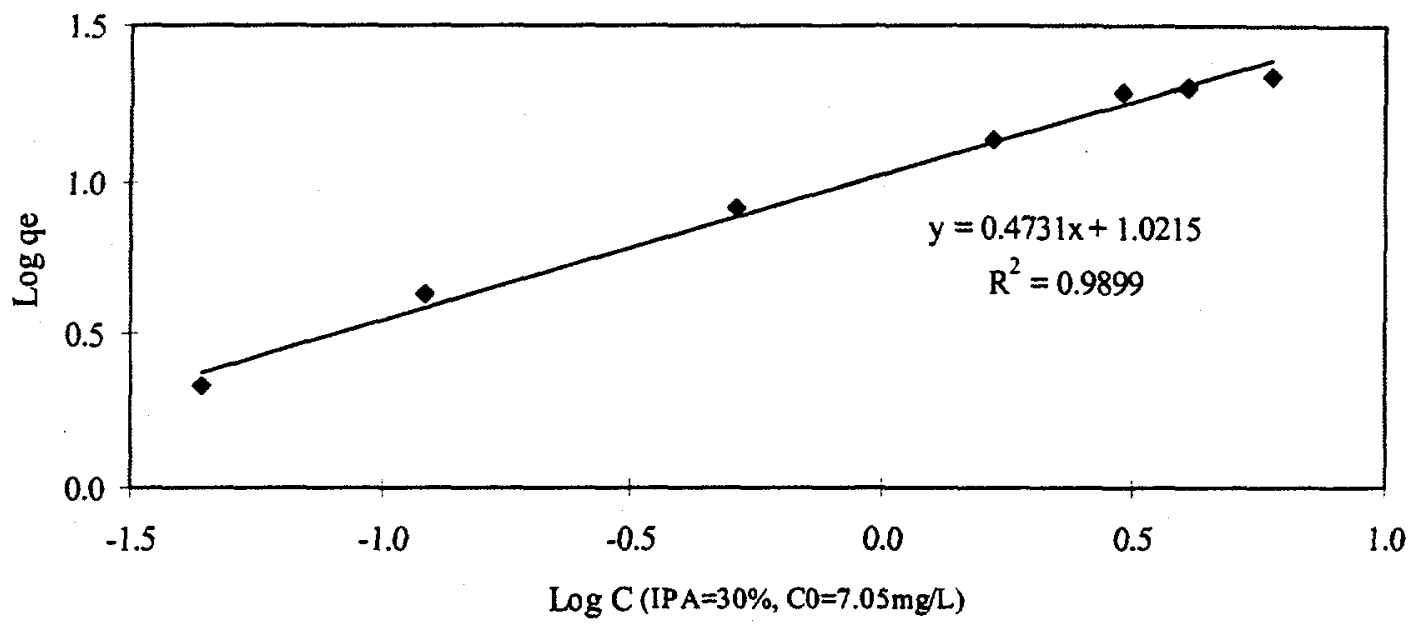

Figure 7-21 Linear Freundlich isotherm for a 30\% IPA solution

Other conclusions from these two tests could also be drawn. An effective adsorption could be obtained even from a solution with a very high IPA concentration, $60 \%$, or in a $30 \%$ IPA solution with a very low initial PCE concentration, $7.05 \mathrm{mg} / \mathrm{L}$, if an appropriate activated carbon dose is used for the adsorption. This is supported by the maximum PCE $\mathrm{q}_{\mathrm{e}}$ of about $20 \mathrm{mg} / \mathrm{g}$ shown in Figure $7-18$, or $25 \mathrm{mg} / \mathrm{g}$ shown in Figure 7-20. The value of $\mathrm{q}_{\mathrm{e}}$ of $20 \mathrm{mg} / \mathrm{g}$ is relatively low but still in the range of 2 to $1183 \mathrm{mg} / \mathrm{g}$ for practical value of $\mathrm{q}_{\mathrm{e}}$ surveyed by Cheremisinoff and Morresi (1978). The linear Freundlich isotherm might be used to model adsorption over a solution with an appropriate ratio among its IPA concentration, initial PCE concentration and the carbon dose. This is a little different from the traditional statement about the linear Freundlich isotherm, which states that the Freundlich model, as an empirical model, is valid over moderate solute concentrations.

The effect of the initial PCE concentration was examined using an additional three batch experiments. The relationship between the relative equilibrium solution concentration and the activated carbon dose for each of the additional experiments is shown in Figures 7-22. In order to have a clear view of $C / C_{0}$ at the zone of $C / C_{0}<0.1$, a modified Figure 7-22 is shown in Figure 723. 


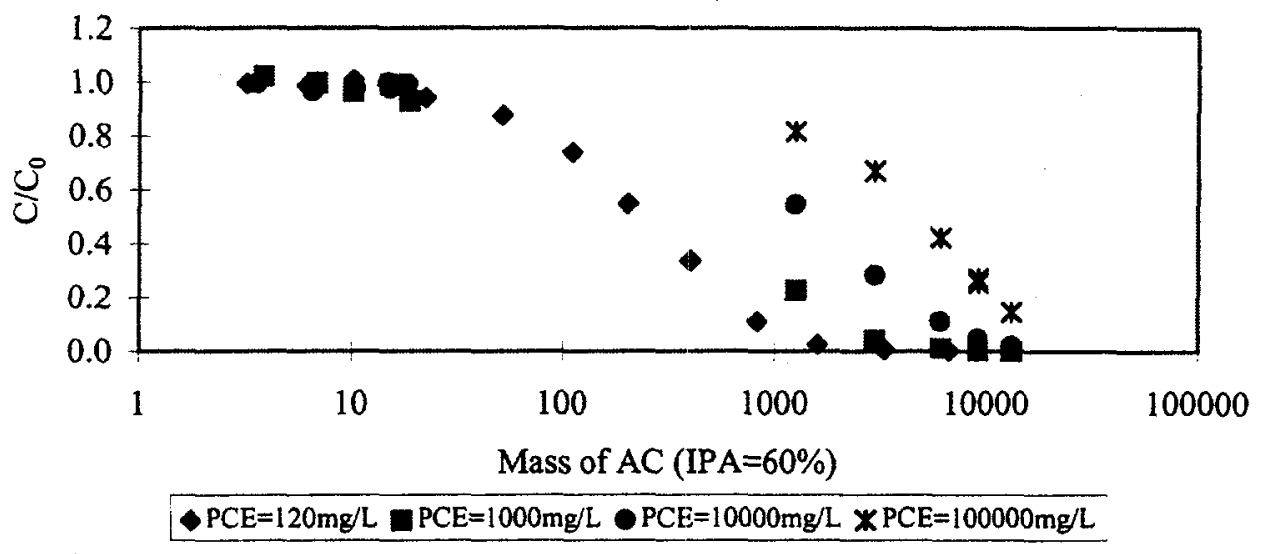

Figure 7-22 $\mathrm{C} / \mathrm{C}_{0}$ versus mass of $\mathrm{AC}$ for three $\mathrm{PCE}$ concentrations

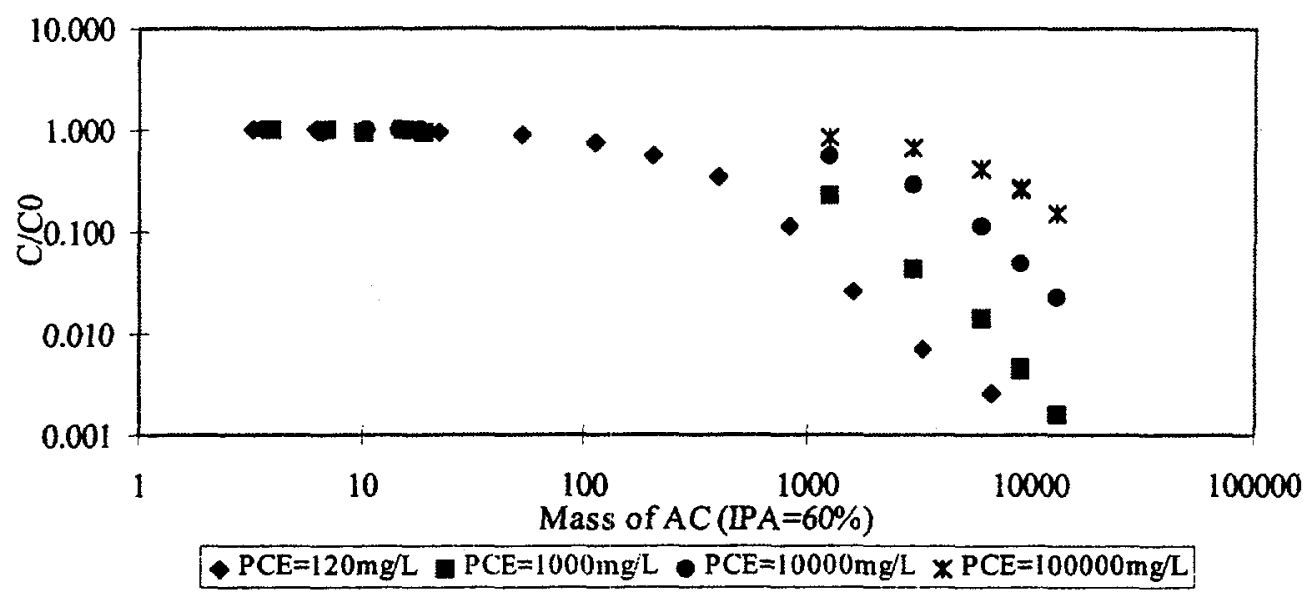

Figure 7-23 $\mathrm{C} / \mathrm{C}_{0}$ versus mass of $\mathrm{AC}$ for three $\mathrm{PCE}$ concentrations (Modified)

These results show that the ratio of the carbon dose, $\mathrm{C}_{\mathrm{AC}}$, to the initial PCE concentration, $\mathrm{C}_{0}$, over the effective adsorption zone ranges from 0.1 to $111 \mathrm{mg} / \mathrm{mg}$ and this ratio decreases with the PCE concentration, which indicated that a lees carbon dose is needed for an adsorption occurring in solution with a higher the initial PCE concentration.

Table 7-12 Effective activated carbon dose range

\begin{tabular}{|c|c|c|c|}
\hline $\begin{array}{c}\text { PCE } \\
\left(\mathrm{C}_{0}\right)(\mathrm{mg} / \mathrm{L})\end{array}$ & $\begin{array}{c}\text { Effective } \mathrm{AC} \\
\left(\mathrm{M}_{\mathrm{AC}}\right)(\mathrm{mg})\end{array}$ & $\begin{array}{c}\text { Effective } \mathrm{AC} \text { Concentration } \\
\left(\mathrm{C}_{\mathrm{AC}}\right)(\mathrm{mg} / \mathrm{L})\end{array}$ & $\mathrm{C}_{\mathrm{AC}} / \mathrm{C}_{0}(\mathrm{mg} / \mathrm{mg})$ \\
\hline 120 & 18 to 800 & 333 to 13,333 & 2.78 to 111 \\
\hline 1,000 & 70 to 3,000 & 1,167 to 50,000 & 1.2 to 50 \\
\hline 10,000 & 250 to 9000 & 4,167 to 150,000 & 0.42 to 15 \\
\hline 100,000 & 600 to 20,000 & 10,000 to 333,333 & 0.1 to 3 \\
\hline
\end{tabular}




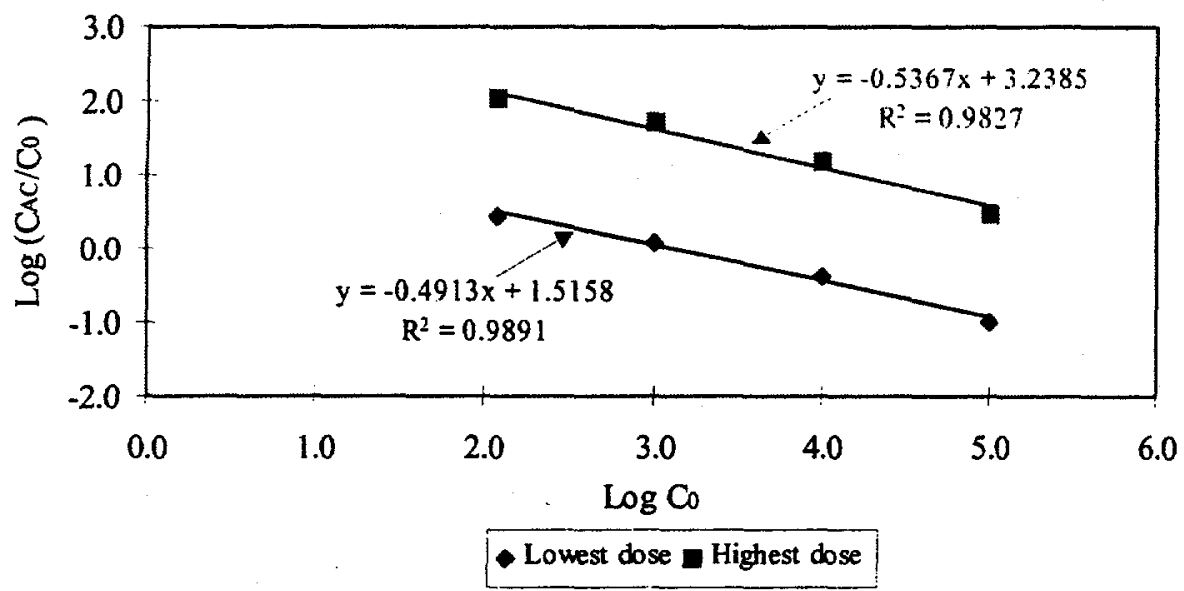

Figure 7-24 Transformed Ratio of $C_{A C} / C_{0}$ versus $C_{0}$ in $60 \%$ IPA solutions

The four highest and lowest ratios of $C_{A C} / C_{0}$ listed in last column of Table 7-12 and the initial PCE concentrations, $\mathrm{C}_{0}$, were transformed into logarithmic values. The relationship between the highest $\log \mathrm{C}_{\mathrm{AC}} / \mathrm{C}_{0}$ and lowest $\log \mathrm{C}_{\mathrm{AC}} / \mathrm{C}_{0}$ for each solution and its respective $\log \mathrm{C}_{0}$ were developed and shown in Figure 7-24. An inverse linear relationship was found between the ratio of the $\log C_{A C} / C_{0}$ versus the $\log C_{0}$ shown in Figure 7-24. This linear relationship was supported by the high $R^{2}$ value greater than 0.9 . The closeness between the two-regression slopes of -0.4913 and -0.5367 reveals that there is a parallelism between the two linear trends, and that indicates that all effective adsorption doses fall in the band formed by the two straight lines shown in Figure 7-24.

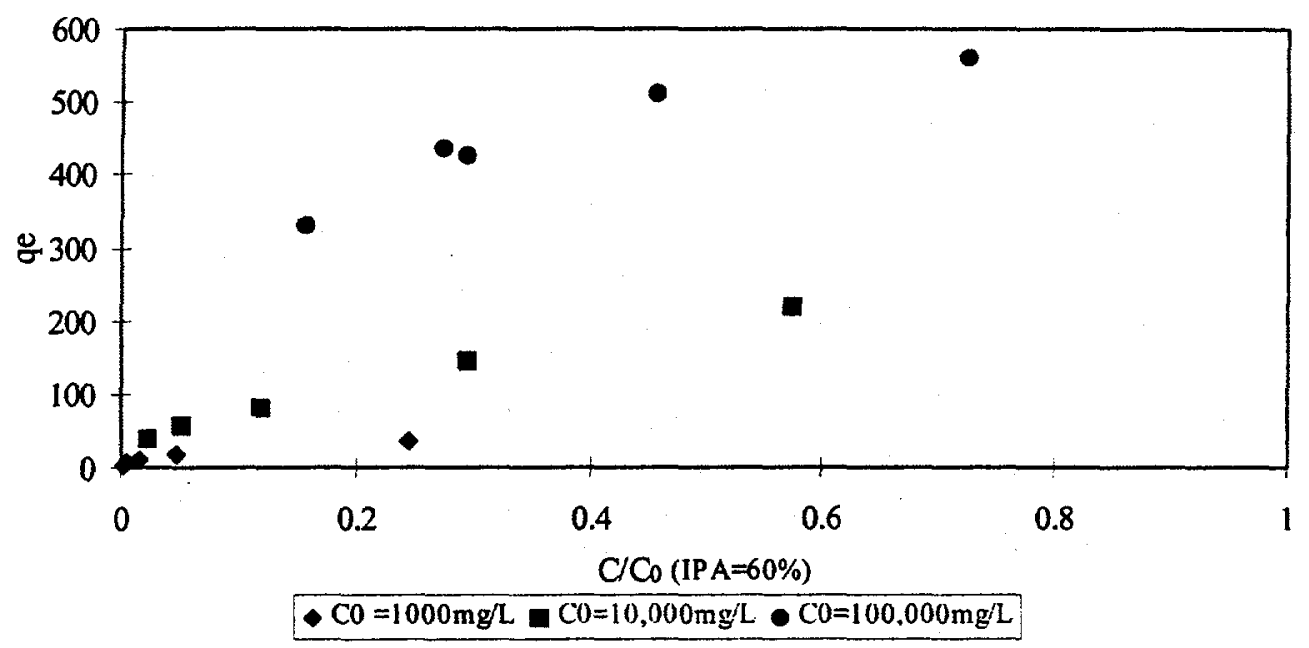

Figure 7-25 Adsorption isotherms within a range of initial PCE concentrations

The adsorption isotherms over the effective adsorption zones for the three tests are shown in Figure 7-25, which shows that these adsorptions are favorable and strong. The linear Freundlich isotherms for these cases are shown in Figure 7-26. The linear Freundlich isotherm for each of four cases has a very high $R^{2}(>0.97)$, which indicates those adsorptions are well modeled using the linear Feundlich model. 


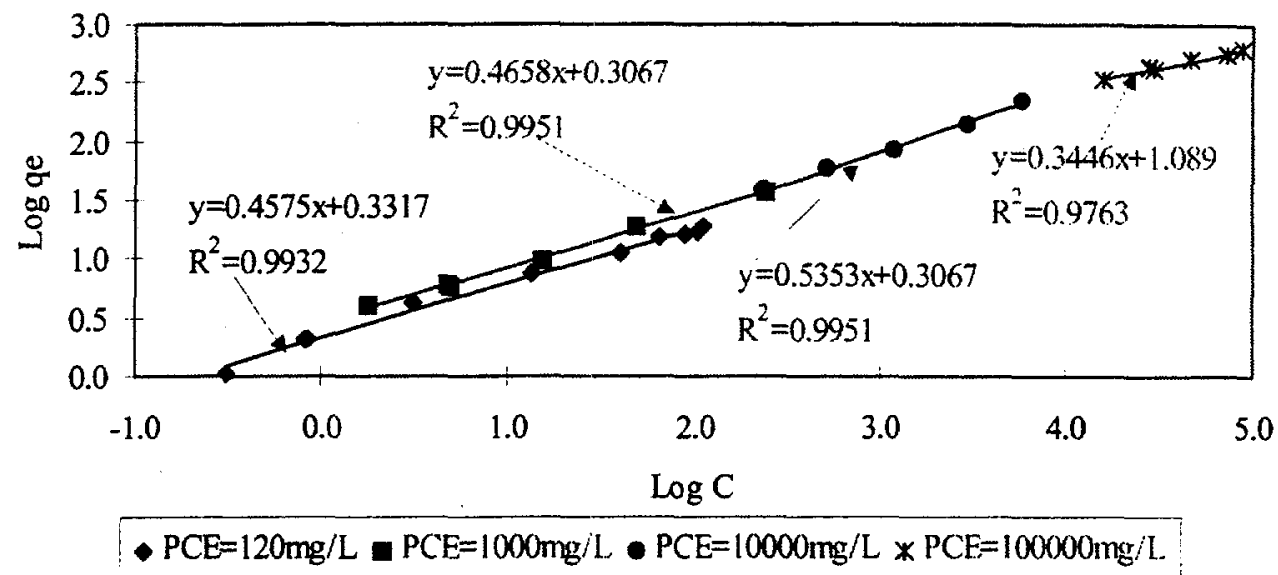

Figure 7-26 Linear Freundlich isotherms three PCE concentrations

Table $7-13 \mathrm{~K}_{\mathrm{F}}$ and $1 / \mathrm{n}$ from the Freundlich model for the $60 \%$ IPA solutions

\begin{tabular}{|c|c|c|c|}
\hline PCE $C_{0}(\mathrm{mg} / \mathrm{L})$ & $\log \mathrm{K}_{\mathrm{F}}$ & $\mathrm{K}_{\mathrm{F}}$ & $1 / \mathrm{n}$ \\
\hline 120 & 0.331 & 2.14 & 0.457 \\
\hline 1,000 & 0.426 & 2.667 & 0.466 \\
\hline 10,000 & 0.306 & 2.023 & 0.535 \\
\hline 100,000 & 1.089 & 12.274 & 0.345 \\
\hline
\end{tabular}

The $\mathrm{K}_{\mathrm{F}}$ and $1 / \mathrm{n}$ listed in Table 7-13 shows that the adsorption parameters are quite close to each other for the solutions with a $\mathrm{C}_{0}$ of less than $10,000 \mathrm{mg} / \mathrm{L}$. In the $60 \%$ IPA solution, the $\mathrm{K}_{\mathrm{F}}$ has a much higher value and a lower $1 / \mathrm{n}$. Statistical analysis over the parameters listed in Table 7-13 shows that there is no significant statistical deference among the $K_{F}$ and $1 / n$ for three low initial PCE concentration cases. The $K_{F}$ and $1 / \mathrm{n}$ for the case at PCE $C_{0}$ of $100,000 \mathrm{mg} / \mathrm{L}$ are significantly different from the previous three low initial PCE concentration cases. These features in $K_{F}$ and $1 / n$ indicate that: (a) the effect of $C_{0}$ on the adsorption parameters is not very significant when it is less than $10,000 \mathrm{mg} / \mathrm{L}$; and (b) much higher adsorption parameters were observed in a solution with $\mathrm{C}_{0}=100,000 \mathrm{mg} / \mathrm{L}$. 


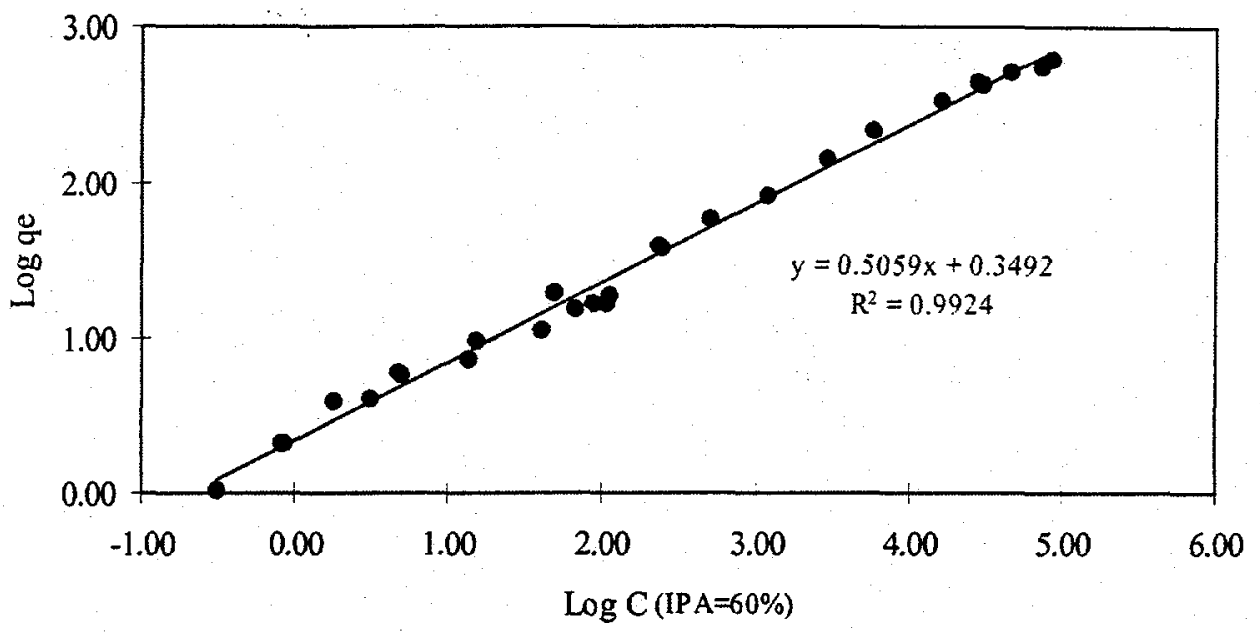

Figure 7-27 Overall linear Freundlich isotherm for 60\% IPA experiments

The linear Freundilich isotherm was also used to fit the all data for 60\% IPA case and the results is shown in Figure 7-27. A high $R^{2}$ of 0.99 indicates that the linear Freundlich isotherm seems to fit the all adsorption data regardless of the initial PCE concentration involved. However, cautions need to be taken when applying these regression parameters. This could be seen from the fact that a $q_{e}$ of about $200 \mathrm{mg} / \mathrm{L}$ could be calculated based on these regression parameters. This $q_{e}$ seems to be irrational, because a $q_{e}$ of about $700 \mathrm{mg} / \mathrm{L}$ was obtained from three batch experiments shown in next section and from the two column tests. Thus it may be wise to use the adsorption parameters obtained from regression over the data for the high initial PCE concentration solutions.

The batch experiments were repeated for each alcohol concentration for several different contact times (equilibrium retention time). Part of the reasons for this experimental scenario was to investigate if there was a difference in adsorption results due to different solution mixing and part of the reasons for this experimental scenario was to investigate if the adsorption results could be repeated for similar adsorption systems.

The adsorption isotherms over the effective adsorption zones for the four IPA concentration cases are shown in Figures 7-28 through 7-31. All of these isotherms show that the adsorption is strong and favorable. These results also show that there is generally good reproducibility among the experiments and that a period of time of 1 or 2 days is a sufficient contact time for equilibrium to take place. 


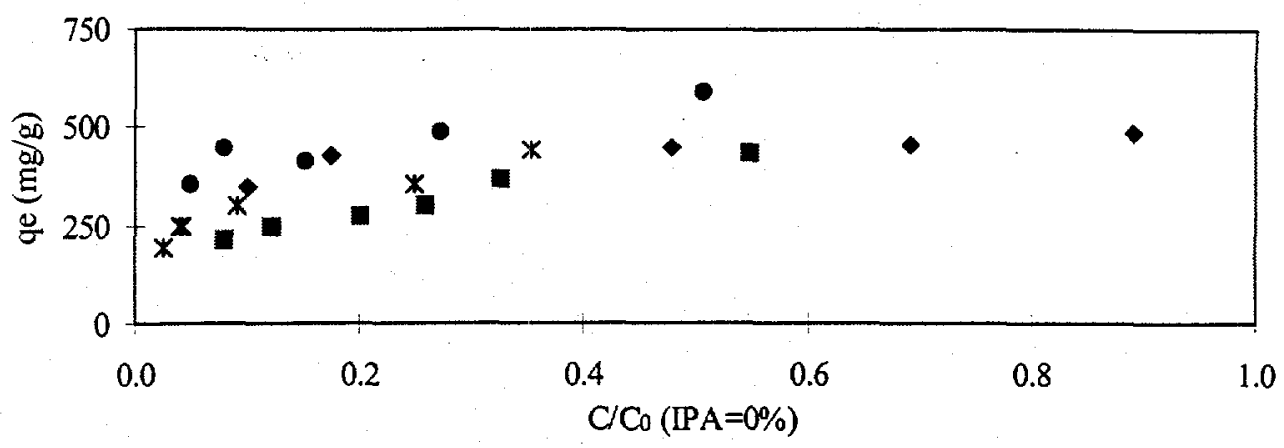

- $28 \mathrm{hr} \mathrm{CO}=100 \mathrm{mg} / \mathrm{L} \quad \square 12 \mathrm{~d} \mathrm{CO}=77 \mathrm{mg} \mathrm{L} \quad-22 \mathrm{~d} \mathrm{Co}=110 \mathrm{mg} / \mathrm{L} \quad \times 30 \mathrm{~d} \mathrm{C} 0=65 \mathrm{mg} / \mathrm{L}$

Figure 7-28 Adsorption isotherms for 0\% IPA

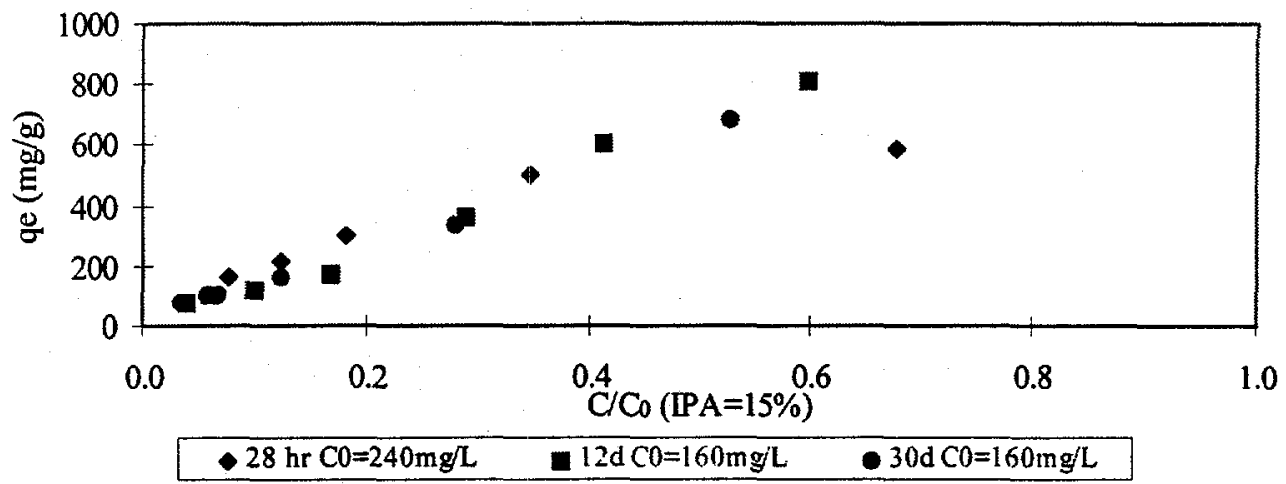

Figure 7-29 Adsorption isotherms for 15\% IPA

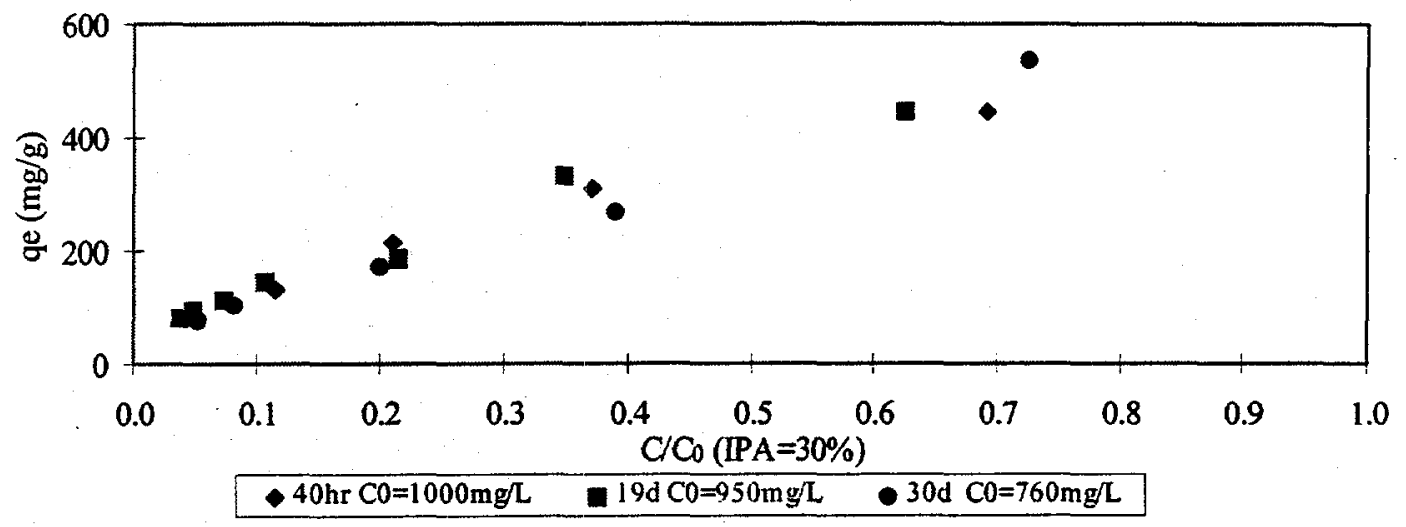

Figure 7-30 Adsorption isotherms for 30\% IPA 


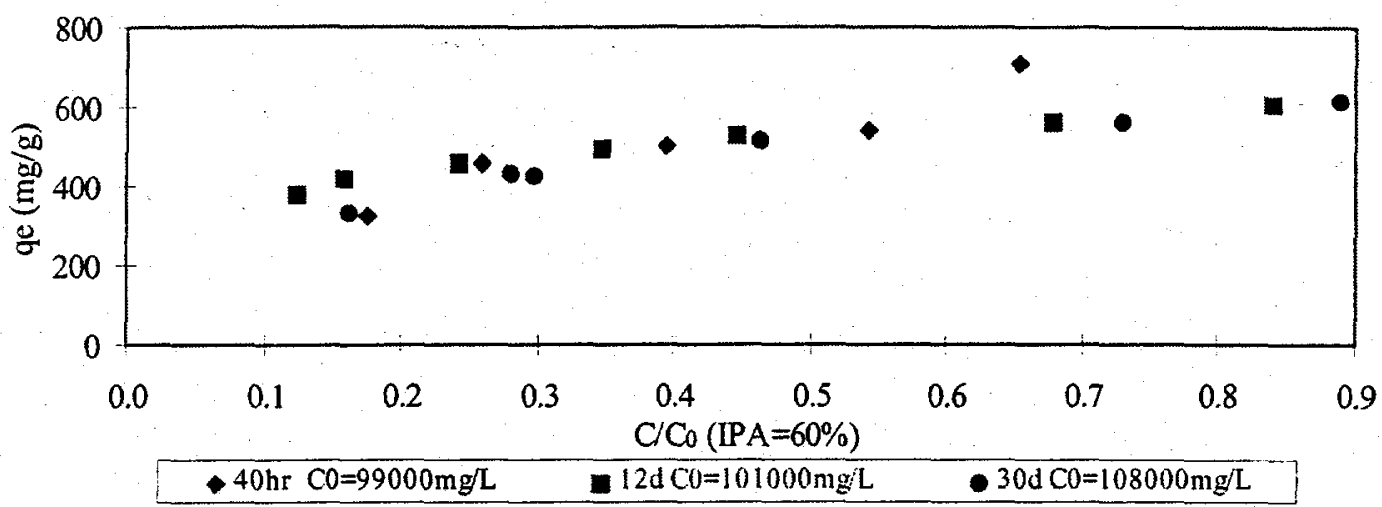

Figure 7-31 Adsorption isotherms for 60\% IPA

The detailed statistical analysis and comparison results are shown in Appendix A. The statistical analysis showed that no significant differences exist among the isotherms at a confidence level of $95 \%$ except for the $60 \%$ IPA case. The reasons for those occurred in the 60\% IPA case are not clear but several ideas come to mind and they will be discussed in detail later. Because no significant differences exist among isotherms for cases with less than or equal to $30 \% \mathrm{IPA}$, an overall linear Freundlich isotherm was used to model the adsorption over all data points for each of those IPA cases. The overall linear Freundlich isotherms for those adsorptions were developed and are shown in Figures 7-32 through 7-35.

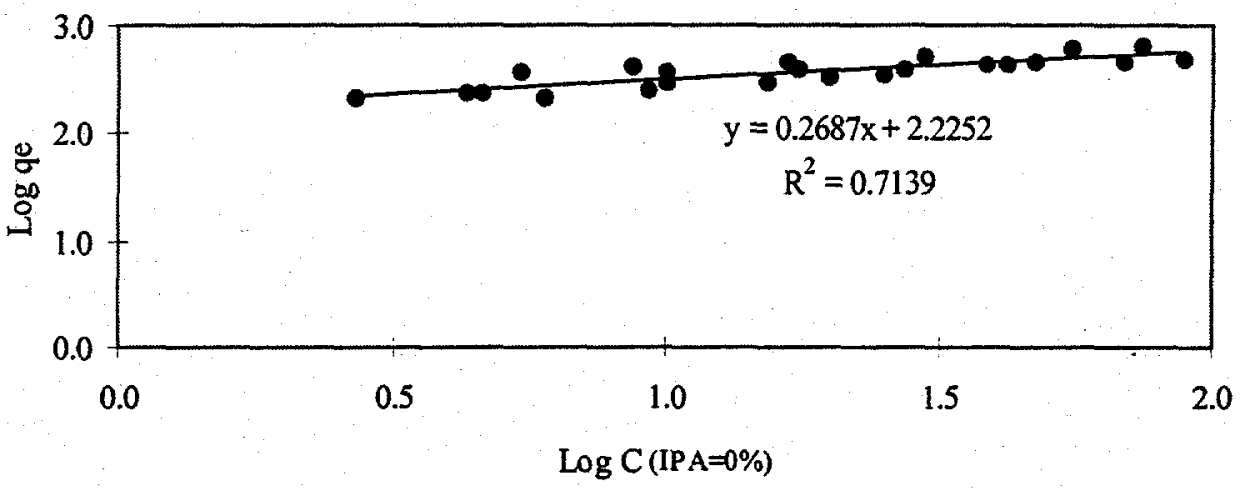

Figure 7-32 Overall linear Freundlich isotherm for 0\% IPA

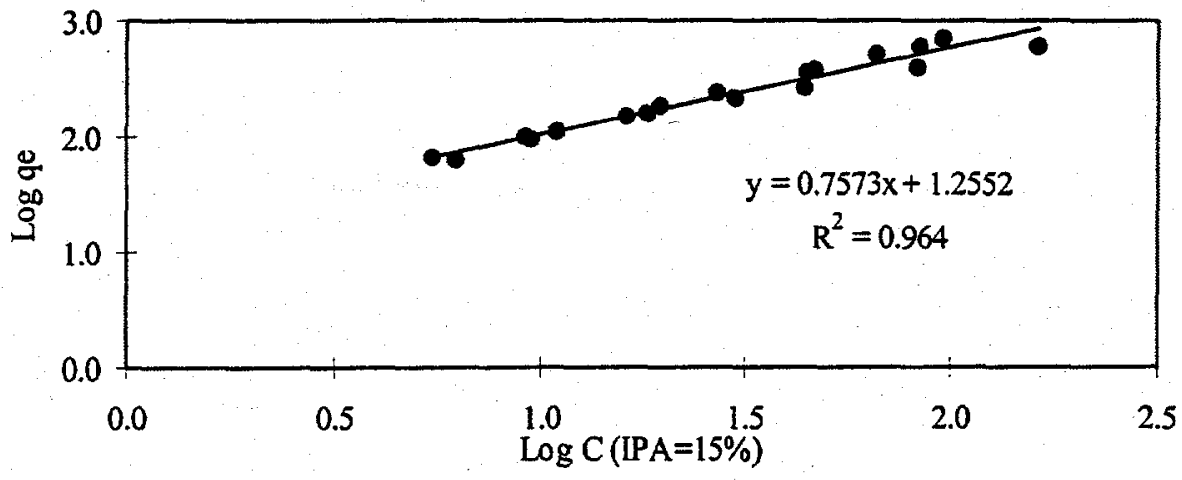

Figure 7-33 Overall linear Freundlich isotherm for 15\% IPA 


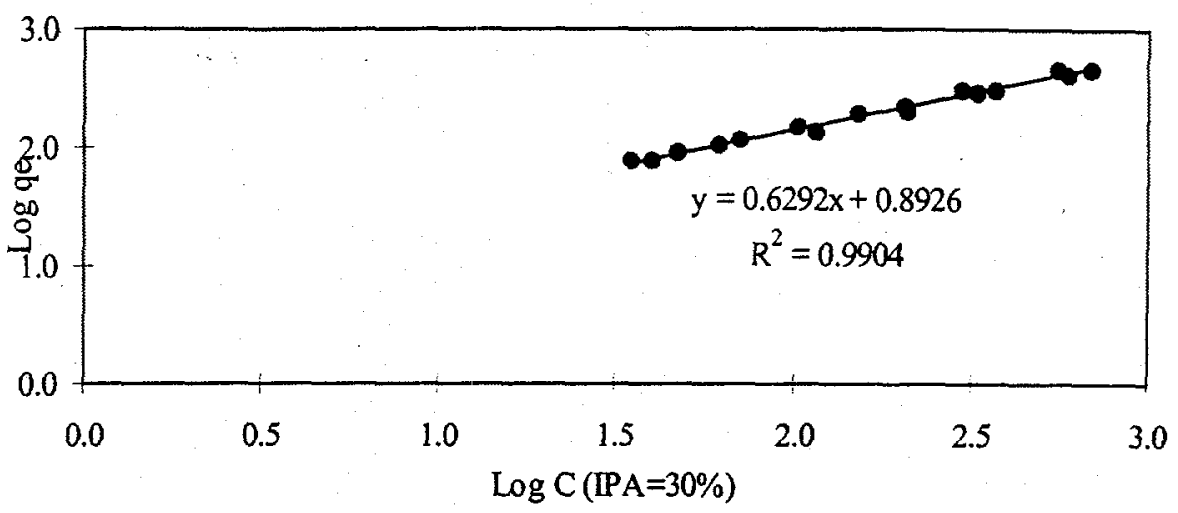

Figure 7-34 Overall linear Freundlich isotherm for 30\% IPA

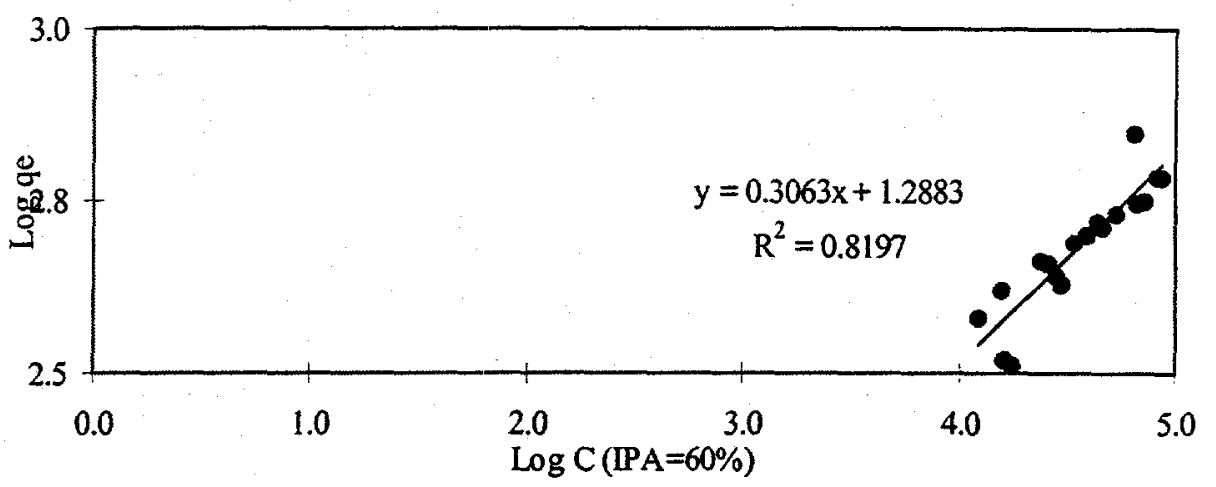

Figure 7-35 Overall linear Freundlich isotherm for 60\% IPA

Figures 7-32, 7-33 and 7-34 show that all the three adsorptions are modeled well using the linear Freundlich isotherm, though the regression $\mathrm{R}^{2}$ of 0.714 for the $0 \%$ IPA solution case is a little bit low. This is due to a greater variability stemmed from the low PCE concentration involved in the experiments. This variability made the experimental results more vulnerable and sensible to the experimental errors. However, these parameters determined from the Freundlich isotherm model could be applicable for future similar PCE adsorption.

Figure 7-35 shows a slight curvilinear isotherm pattern occurred when combining all the data for the $60 \%$ IPA solution case. This slight curvilinear isotherm pattern may echo the simple curvilinear isotherm pattern shown in Figure 7-31. This slight curvilinear isotherm pattern may also be the cause for the statistical analysis results that there are significant differences among the isotherms for the $60 \%$ IPA case. There are some possible reasons for this kind adsorption. The linear Freundlich isotherm may not be applicable to a solution with a very high initial solute concentration like the PCE concentration of $100,000 \mathrm{mg} / \mathrm{L}$ involved in this study. This is because the Freundlich model, as an empirical model, was developed over the adsorption occurred in solutions with moderate solute concentration, which consists with the statement indicated by Weber (1972) that the Freundlich isotherm model is valid in a solution with a moderate solute solution. A more likely explanation is the error associated with the data at the ends of the isotherms. The tail of the isotherm at high $\log q_{e}$ and high $\log C$ corresponds to the lowest amount of carbon and the smallest amount of change of concentration in the bottle. Therefore any 
small measurement error in either of the mass of carbon or the initial or final concentration of PCE can have a significant impact on the outcome. The low $\log q_{e}$ and $\operatorname{low} \log C$ points on the isotherm correspond to the largest amount of carbon and the largest amount of change of concentration in the bottle. Therefore the outcome at this end of the isotherm is also more vulnerable to the experimental errors. There is also a slightly greater analytical error associated with low solution concentrations which may have contributed to the greater data variability in this region. Because of the rare occurrence of this type of adsorption in actual system and because of the high $R^{2}$ of 0.82 for these data, the parameters resulting from this linear Freundlich isotherm was tentatively used to estimate and predict the PCE adsorption in the $60 \%$ IPA solution for the column tests.

The adsorption parameters from the linear Freundlich isotherms for all of the four IPA cases are summarized in Table 7-14. The relationships between adsorption parameters of $K_{F}$ and $1 / n$ and IPA concentration were developed and shown in Figures 7-36 and 7-37.

Table 7-14 Adsorption parameters for PCE in various \% alcohol solutions

\begin{tabular}{|c|c|c|c|}
\hline $\operatorname{IPA}(\%)$ & $\log \left(\mathrm{K}_{\mathrm{F}}\right)$ & $\mathrm{K}_{\mathrm{F}}$ & $1 / \mathrm{n}$ \\
\hline 0 & 2.23 & 170 & 0.27 \\
\hline 15 & 1.26 & 18.2 & 0.76 \\
\hline 30 & 0.89 & 7.8 & 0.63 \\
\hline 60 & 1.29 & 19.5 & 0.31 \\
\hline
\end{tabular}

Figures 7-36 and 7-37 show that the $\mathrm{K}_{\mathrm{F}}$ value decreases with the IPA concentration of not greater than $30 \%$ in a solution. This trend is similar to that revealed by the earlier tests investigating the effect of IPA concentration. This seems reasonable because a higher IPA concentration results in a higher PCE solubility and a lower adsorbability. A larger $\mathrm{K}_{\mathrm{F}}$ was observed for the $60 \% \mathrm{PPA}$ case than that for the $30 \%$ IPA case. This could be the result of the much higher initial PCE concentration was involved in the $60 \%$ IPA case and this high initial PCE concentration enhances the adsorbability. This enhanced adsorbability outdoes the negative effect from the high IPA concentration, thus a higher $\mathrm{K}_{\mathrm{F}}$ or higher adsorption capacity was obtained. The $1 / \mathrm{n}$ value increases with IPA concentration up to $15 \%$ IPA greatly, and then it decreases with the IPA concentration at a much more gentle slope. This trend differs from that revealed earlier. The possible reasons for this trend in $1 / \mathrm{n}$ are the adsorption in this solution was less sensitive than that in $0 \%$ IPA solution because of the IPA effect. In the solutions with IPA greater than $15 \%$, the negative effect of IPA was outdone by the increase in initial PCE concentration, thus a more sensitive adsorption to initial PCE concentration occurred in the $30 \%$ and $60 \%$ solutions. Likewise a most sensitive adsorption occurred in the 60\% IPA solution. 


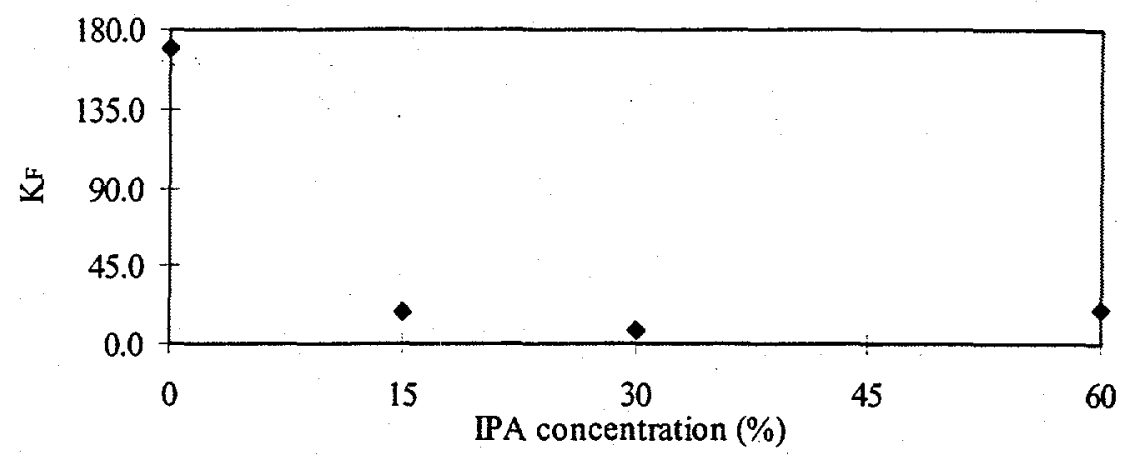

Figure 7-36 $\mathrm{K}_{\mathrm{F}}$ versus IPA concentration for varied IPA and PCE concentrations

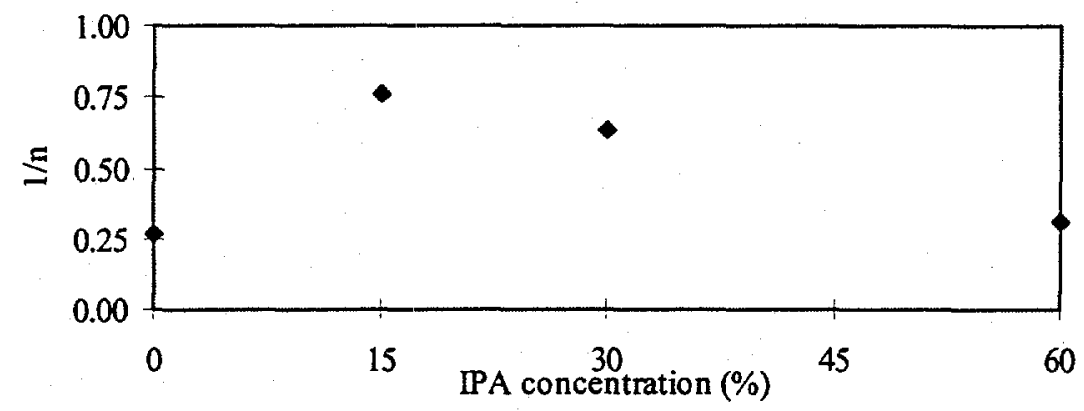

Figure 7-37 1/n versus IPA concentration for varied IPA and PCE concentrations

Adsorption of TCE, another common chlorinated contaminant, from IPA solution was briefly investigated to approximately estimate its adsorption parameters. Compared to PCE, TCE has a solubility of $1100 \mathrm{mg} / \mathrm{L}$, which is higher than PCE of $160 \mathrm{mg} / \mathrm{L}$, in water, and it also has a higher solubility in a alcohol solution. The experimental results are shown in Figures 7-38 through 7-40. Figure 7-38 shows that an effective adsorption zone existed for the TCE adsorption onto activated carbon from 30\% IPA solution. Over this effective adsorption zone a strong and favorable adsorption pattern was revealed and shown in Figure 7-39.

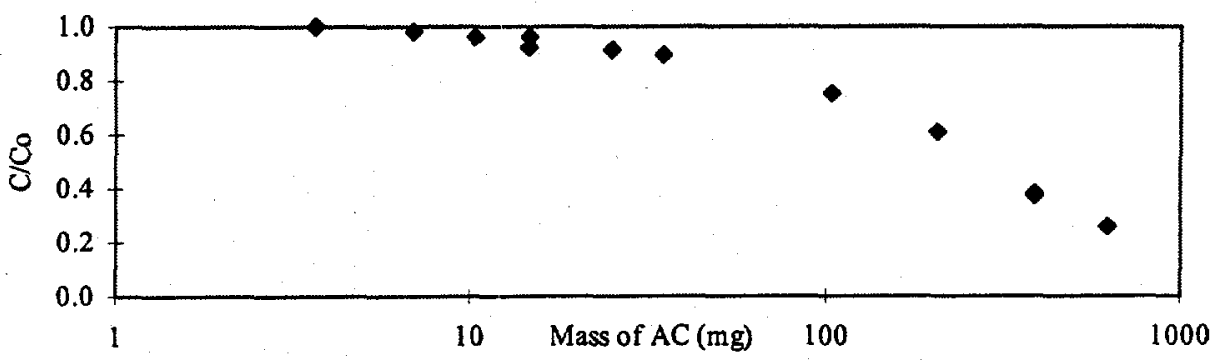

Figure 7-38 $\mathrm{C} / \mathrm{C}_{0}$ versus mass of $\mathrm{AC}$ for TCE from 30\% IPA solution 


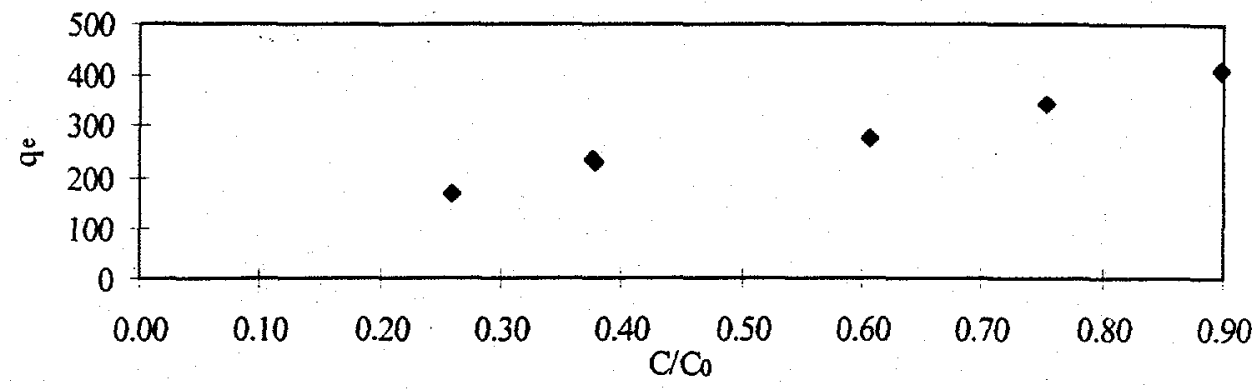

Figure 7-39 Adsorption isotherm for TCE from 30\% IPA solution

The TCE concentration in carbon $\left(q_{\mathrm{e}}\right)$ ranges from about 120 to $400 \mathrm{mg} / \mathrm{g}$, which is also very high. Figures 7-40 shows that the linear Freundlich isotherm modeled the TCE adsorption data over the effective adsorption zone very well, which implies that carbon adsorption of TCE from another similar IPA concentration solution could be predicted by the parameters from the linear Freundlich isotherm revealed by this test.

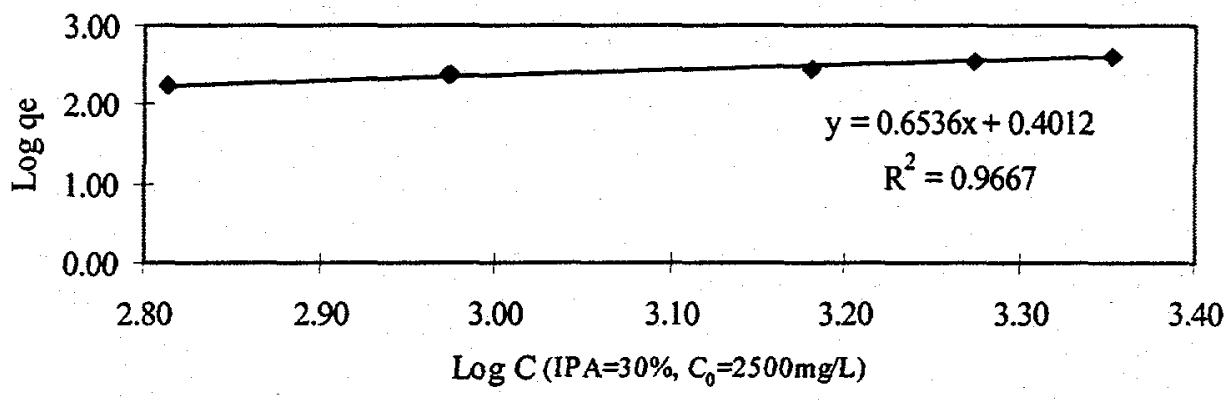

Figure 7-40 Linear Freundlich isotherm for TCE from $30 \%$ IPA solution

The $\mathrm{K}_{\mathrm{F}}$ of 4.49 for TCE is less than the $\mathrm{K}_{\mathrm{F}}$ of 7.8 for PCE from $30 \%$ IPA solution listed earlier in Table 7-11, even though the PCE concentration of about $1000 \mathrm{mg} / \mathrm{L}$ in its solution is less than the half concentration of TCE of $2500 \mathrm{mg} / \mathrm{L}$ in this solution. This trend reflects that TCE, having a higher solubility than PCE, has a lower adsorbability than PCE for carbon. The $1 / \mathrm{n}$ of 0.6536 is close to the value of 0.63 for PCE adsorption shown in Table 7-14. Due to the fact that there is a sort of molecular polar similarity between TCE and PCE, the adsorption of TCE and PCE from the same alcohol concentration solution may have a similar feature. Thus the adsorption of TCE by activated carbon from other IPA concentration solutions might be projected approximately from the PCE adsorption from IPA solution discussed previously.

The use of ethanol flushing solution has several advantages over IPA. The primary advantage is that there is less environmental concern about using it as exhibited by a much higher MCL for ethanol. A batch study was conducted to briefly investigate the PCE adsorption parameters from $30 \%$ ethanol solution with an initial PCE concentration of $360 \mathrm{mg} / \mathrm{L}$. The effective PCE adsorption zone was found in Figure 7-41, the strong and favorable PCE adsorption pattern was shown in Figure 7-42, and the linear Freundlich isotherm over the effective adsorption zone is shown in Figure 7-43. 


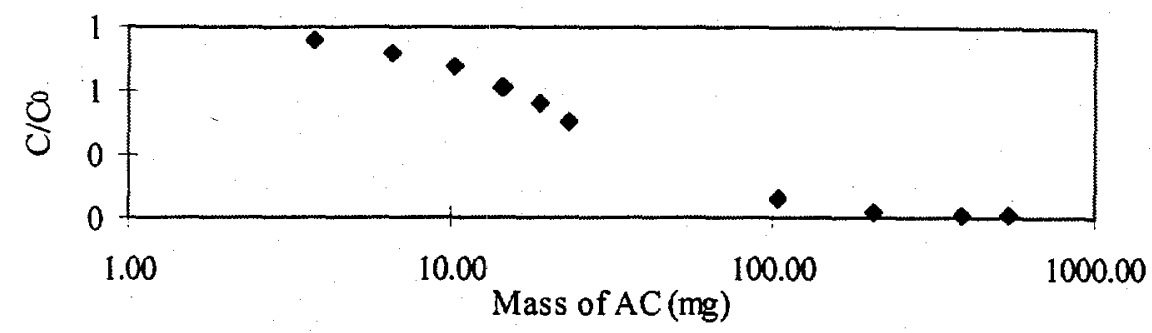

Figure 7-41 C/ $\mathrm{C}_{0}$ versus mass of $\mathrm{AC}$ for $\mathrm{PCE}$ from a $30 \%$ ethanol solution

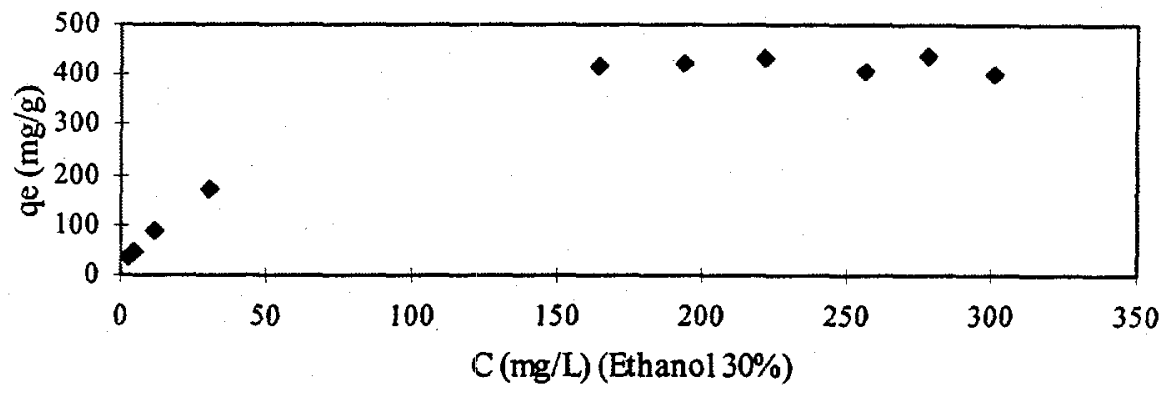

Figure 7-42 Adsorption isotherm for PCE from a 30\% ethanol solution

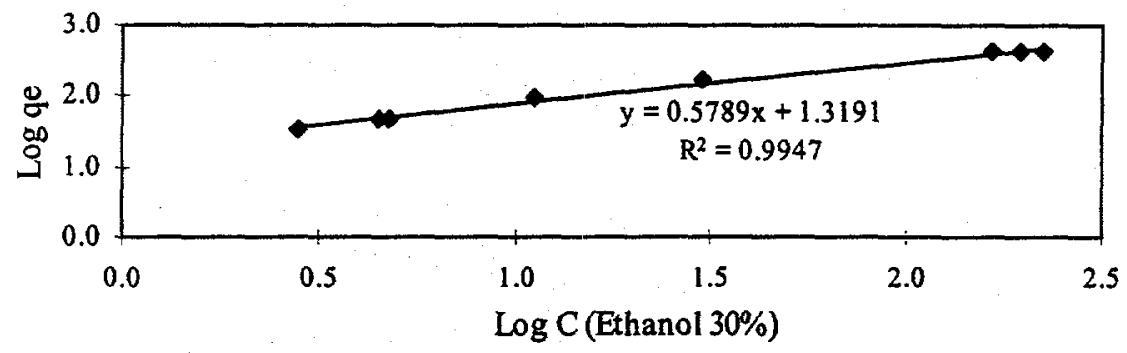

Figure 7-43 Linear Freundlich isotherm for PCE from a 30\% ethanol solution

The PCE concentration in carbon ranges from about 40 to $430 \mathrm{mg} / \mathrm{g}$, which is also very high. The linear Freundlich isotherm modeled the PCE adsorption over the effective adsorption zone very well.

The $\mathrm{K}_{\mathrm{F}}$ of 20.8 for this adsorption is higher than that of 7.8 for the PCE adsorption from a $30 \%$ IPA solution, which is listed in Table 7-14. The reason for this higher $\mathrm{K}_{\mathrm{F}}$ value is due to the lower PCE solubility of $500 \mathrm{mg} / \mathrm{L}$ in $30 \%$ ethanol solution, though this $30 \%$ ethanol solution has a lower initial PCE concentration of $360 \mathrm{mg} / \mathrm{L}$ than that of about $1,000 \mathrm{mg} / \mathrm{L}$ in the previous $30 \%$ IPA solution. The $1 / \mathrm{n}$ of 0.5789 for this solution is slightly less than that of 0.62 for the PCE from the $30 \%$ IPA solution. 
The linear Freundlich isotherms for various solutions are summarized in Figure 7-44, which shows the value of $\log \mathrm{q}_{\mathrm{e}}$ for all of the linear Freundlich isotherms ranges from 1.5 to 2.8 or $\mathrm{q}_{\mathrm{e}}$ ranges from 31.6 to $631 \mathrm{mg} / \mathrm{g}$, a high adsorption $\mathrm{q}_{\mathrm{e}}$. The isotherm for $30 \%$ IPA solution has the least diversity in data points while the isotherm for $0 \%$ IPA has the greatest diversity.

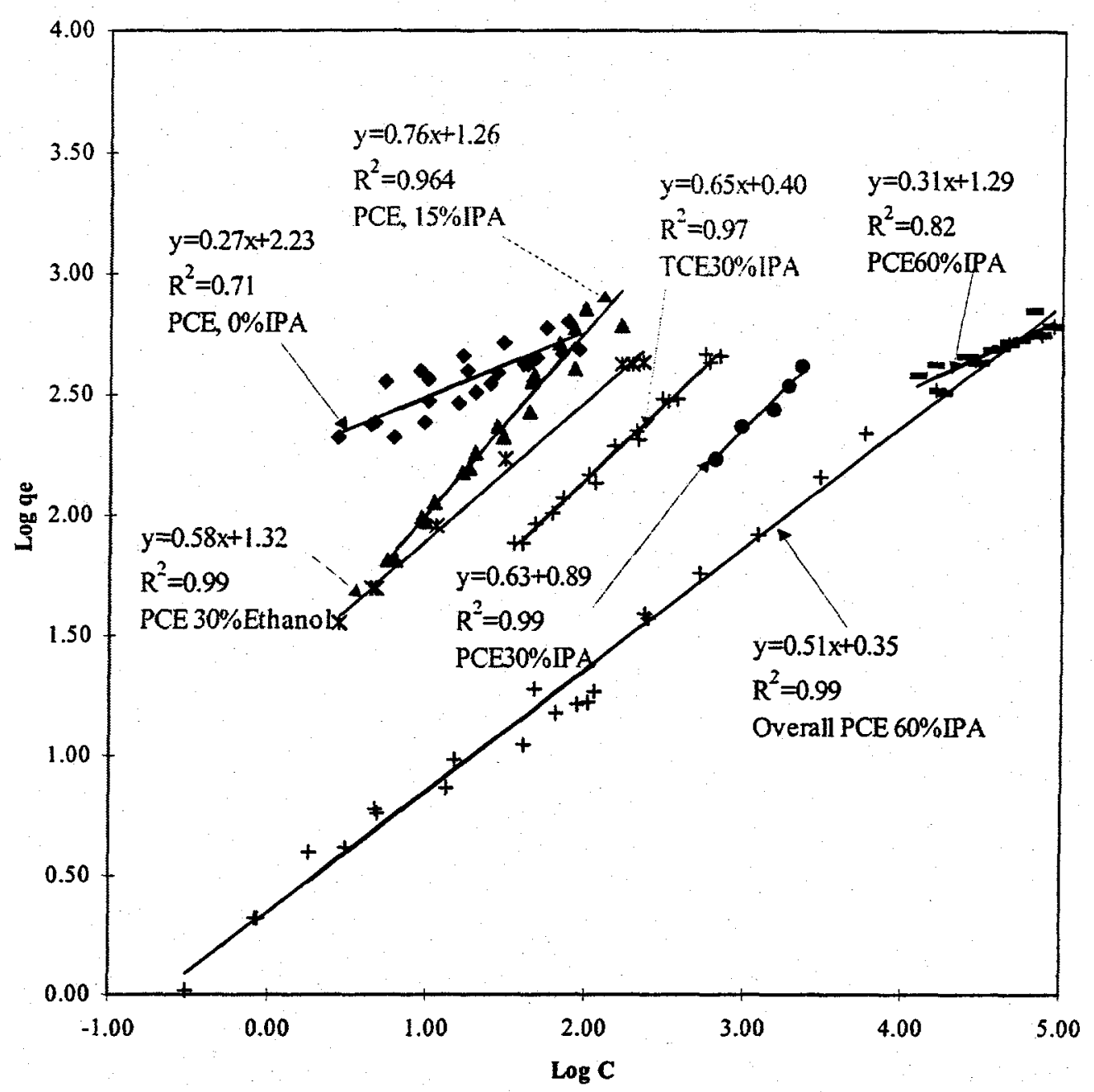

Figure 7-44 Summary of the Linear Freundlich isotherms of isotherm studies

One of the purposes of batch isotherm studies is to determine the parameters for carbon column studies, which were conducted using solutions composed of PCE and $30 \%$ or $60 \%$ IPA solvent. The treatment capacity of carbon for PCE from $30 \%$ and $60 \%$ IPA solutions were estimated based on the batch study results and the equation (2). The treatment capacities are listed in Table 7-15. The mass of activated carbon loaded in the column was about of $34 \mathrm{~g}$. The feed PCE concentrations were assumed and are also listed in Table 7-15 too. Table 7-15 shows that a very high treatment capacity for PCE from a solution with a less than $30 \%$ IPA concentration, and that a low capacity for solution with $60 \%$ IPA and an initial PCE concentration of $100,000 \mathrm{mg} / \mathrm{L}$ were found. 
Table 7-15 Feed PCE concentrations and the treatment capacities

\begin{tabular}{|c|c|c|c|}
\hline IPA (\%) & $\begin{array}{c}\text { Feed PCE } \\
(\mathrm{mg} / \mathrm{L})\end{array}$ & $\begin{array}{c}\text { Designed effluent } \\
\text { PCE }(\mathrm{mg} / \mathrm{L})\end{array}$ & Volume of solution (L) \\
\hline \multirow{3}{*}{30} & \multirow{3}{*}{750} & 0.2 & 26.939 \\
\cline { 3 - 4 } & & 0.1 & 23.946 \\
\cline { 3 - 4 } & & 0.05 & 22.685 \\
\hline \multirow{3}{*}{60} & \multirow{3}{*}{100000} & 0.01 & 21.769 \\
\cline { 3 - 4 } & & 0.2 & 0.277 \\
\cline { 3 - 4 } & & 0.1 & 0.246 \\
\cline { 3 - 4 } & & 0.05 & 0.233 \\
\cline { 3 - 4 } & & 0.01 & 0.224 \\
\hline
\end{tabular}

\section{Column tests}

Column tests were conducted using 30\% and $60 \%$ IPA solutions. Column tests were used to determine the IPA effect on the carbon adsorption of PCE in a carbon column bed, and to determine the column bed operating parameters for guidance to the operation of a scale-up adsorber. The total volume of the PCE solution for the column tests was estimated based on the value listed Table 7-15 for each IPA concentration solution.

Breakthrough curves were used to obtain or determine; (1) mass transfer zone, (2) the unit activated carbon treatment capacity for solution or alcohol recovery capacity, and (3) the effect of the presence of IPA on adsorption of PCE on activated carbon.

As outlined previously, the activated carbon column bed needs to contain at least one mass transfer zone in order to operate carbon bed effectively and efficiently. Practically it is possible to reduce the PCE concentration in the effluent solution using activated carbon down to a very low level. From the view point of recovery and reuse of alcohol for alcohol flushing remediation, seeking a very low column effluent PCE concentration, such as $5 \mu \mathrm{g} / \mathrm{L}$, as the carbon column effluent breakpoint concentration $C_{b}$ may not be cost-effective. On the other hand, a reasonable $C_{b}$, such as a value less than PCE solubility of $160 \mathrm{mg} / \mathrm{L}$ in water, could be a good option. For this research, the $C_{b}$ was chosen to be $10 \mathrm{mg} / \mathrm{L}$, which is typical PCE concentration in the PCE contaminated ground water. As to IPA 60\% solution flushing case, because the feed PCE concentration of about $100,000 \mathrm{mg} / \mathrm{L}$ is such high that a relative concentration of 0.1 and $1 \%$ of the feed concentration ( 100 and $1,000 \mathrm{mg} / \mathrm{L}$ ) used in these experiments were considered to be the breakpoint concentration $\left(\mathrm{C}_{\mathrm{b}}\right)$.

Typically the breakthrough curve plotted from the experiment data has a long tail at the high effluent concentration zone. Thus the column test was terminated when the $C_{x}$ (exhaustion concentration) was equal to or above $90 \%$ percent of $\mathrm{C}_{0}$ (feed PCE concentration). Therefore, the primary adsorption zone, which is almost the same as the mass transfer zone, was utilized to determine the breakthrough curve parameters and to estimate the length of the mass transfer zone. The primary adsorption zone is defined as that part of the bed over which there is a concentration increase from $C_{b}$ to $C_{x}$. The primary adsorption zone was assumed to be of constant 
length, $\delta$. The method to calculate the two parameters was presented in Equations (5) and Equation (6).

Two experiments at flow rates of $1 \mathrm{ml} / \mathrm{min}$ and $4 \mathrm{ml} / \mathrm{min}$ were conducted. The breakthrough curves for the two experiments are shown in Figure 7-45. The concentration normalized to the average influent feed PCE concentration $\left(\mathrm{C}_{0}\right)$ was used as $y$-variable. The $\mathrm{x}$-variable represents the solution volume passing through the carbon column per gram carbon in the column. The influent concentration is also shown in Figure 7-45.

The influent concentrations throughout both experiments are quite close to the feed PCE concentration of $100,000 \mathrm{mg} / \mathrm{L}$. Losses of PCE were minimized by the experimental setup and the sampling procedure, although some variation in influent concentration does exist. When comparing the two curves, there is a greater spread of the data at the high rate flow. This is expected because of main transfer issues that occurred in the column. At higher flow rate, the PCE does not have time to diffuse into the carbon before it is swept to further down the column. This increases the active zone of adsorption and this is reflected in higher concentration, the earlier in the effluent, and the longer time to reach breakthrough. As a result, the length of the adsorption zone increases and a breakthrough curve with a smaller slope would be observer. When $\mathrm{C} / \mathrm{C}_{0}=1$ was reached, the solution volume is $12 \mathrm{~mL} / \mathrm{g}$ for the low rate flow and $17 \mathrm{~mL} / \mathrm{g}$ for the high rate flow.

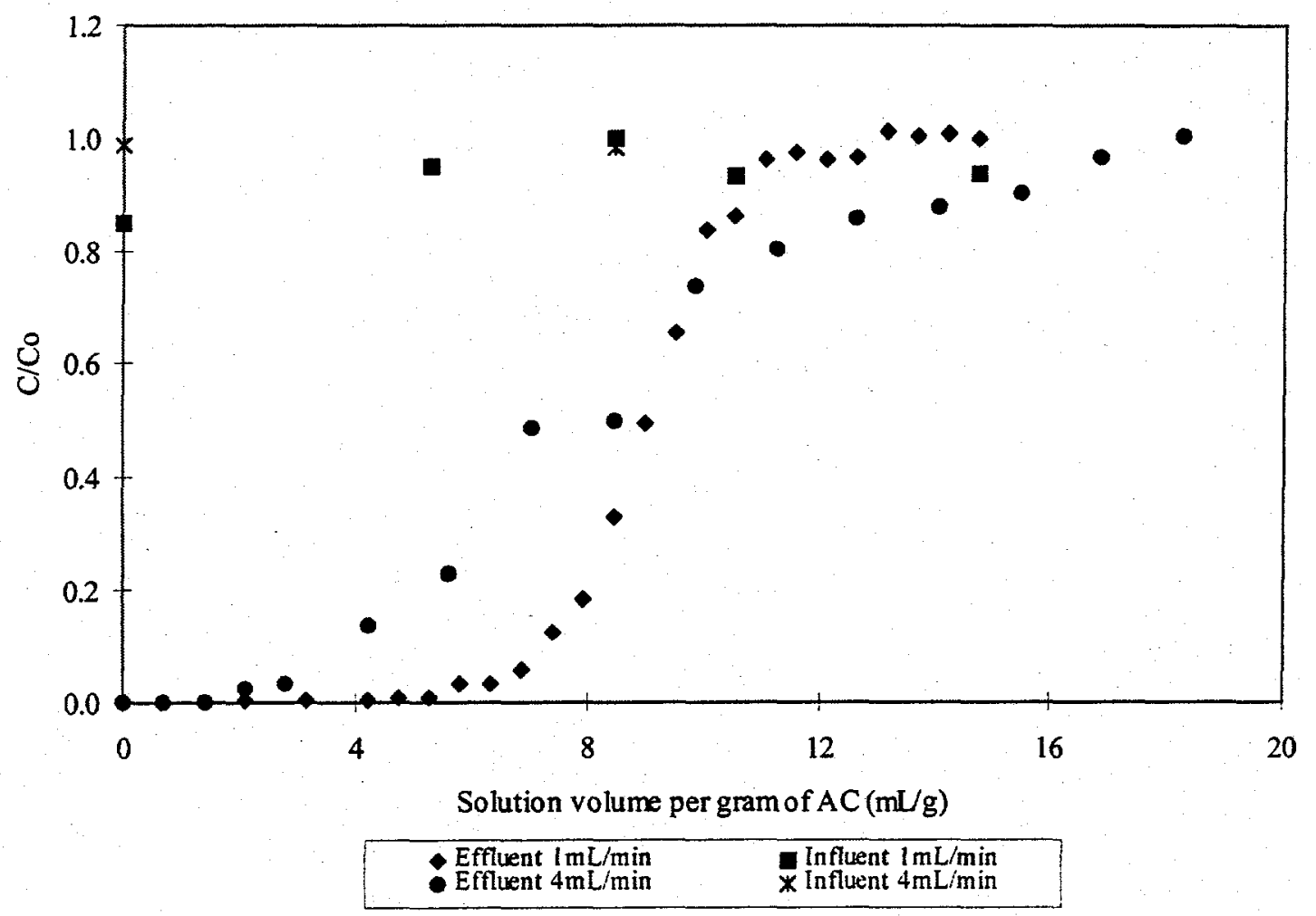

Figure 7-45 Breakthrough curves for $60 \%$ IPA flow 
The points of $\mathrm{C} / \mathrm{C}_{0}=0.5$ for the two curves occurred roughly at $7.8 \mathrm{~mL} / \mathrm{g}$ for the high rate flow and $8.3 \mathrm{~mL} / \mathrm{g}$ for the low rate flow. Because the carbon bed pore volume of about $0.1 \mathrm{~mL} / \mathrm{g}$ is small compared to about $7.8 \mathrm{~mL} / \mathrm{g}$ at $\mathrm{C} / \mathrm{C}_{0}=0.5$, the hydraulic dispersion has a very small effect on these adsorption breakthrough curves. The difference of $0.5 \mathrm{~mL} / \mathrm{g}$ between the two points of $\mathrm{C} / \mathrm{C}_{0}=0.5$ is very small compared to the total solution volume of more than $12 \mathrm{~mL} / \mathrm{g}$ at the point of $\mathrm{C} / \mathrm{C}_{0}=1$. This difference of $0.5 \mathrm{~mL} / \mathrm{g}$ may be attributed to the experiment error or it might be related, to some extent, to the contact time or retention time of the experiments, because the high rate flow has a smaller retention time.

The mass transfer zone definition and the method to calculate the length of the mass transfer zone are shown in Chapter 2. The length of the mass transfer zone was calculated using Equation (2-3) and (2-4) and the results are listed in Table 7-16. The $C_{b}$ and $q_{e} @ E P$ as shown in Table 716 denote the PCE concentration in solution at the breakpoint (BP) and in carbon at exhaustion point (EP) on the breakthrough curve shown in Figure 7-6. The $\mathrm{q}_{\mathrm{e}}{ }^{*}$ denotes the PCE concentration in carbon when the PCE concentration is equal to the feed PCE concentration of $100,000 \mathrm{mg} / \mathrm{L}$ and it was calculated using Equation (1) based on the batch study results.

As shown in Table 7-16, the mass transfer zone and the alcohol recovery capacity are affected by the breakpoint concentration, $C_{b}$, of the solution. The higher the $C_{b}$, the shorter the mass transfer zone, and the higher the alcohol recovery capacity. This is because the higher $\mathrm{C}_{b}$, the smaller amount of PCE that needs adsorbing, and a smaller amount of the carbon needed. It looks a high value of $C_{b}$ of 100 and $1000 \mathrm{mg} / \mathrm{L}$ used in Table $7-16$. Their relative value $\left(C / C_{0}\right)$ of $0.103 \%$ and $1.03 \%$ of the feed PCE concentration might be adequate for this study to estimate the mass transfer zone. Table 7-16 also shows that a longer mass transfer zone was formed in the carbon column with a higher rate flow. This could be seen by looking up Figure 7-45 that the breakthrough curve with a higher flow rate corresponds to a smaller solution volume at a certain $\mathrm{C}_{\mathrm{b}}$.

Table 7-16 Mass transfer zone for breakthrough curves for 60\% IPA solution

\begin{tabular}{|c|c|c|c|c|c|c|c|}
\hline $\begin{array}{l}\text { Flow } \\
\text { rate }\end{array}$ & $C_{b}$ & $\mathrm{C}_{b} / \mathrm{C}_{0}$ & $\begin{array}{l}\text { Length } 0 \\
\text { column }\end{array}$ & $\begin{array}{l}\text { Length of } \\
\text { MTZ }\end{array}$ & $\begin{array}{l}\text { IPA recovery } \\
\text { capacity }\end{array}$ & $q_{e} @$ EP & $\mathrm{q}_{\mathrm{e}}{ }^{*}$ \\
\hline$(\mathrm{ml} / \mathrm{min})$ & $(\mathrm{mg} / \mathrm{L})$ & $(\%)$ & (cm) & (cm) & $(\mathrm{L} / \mathrm{lb})$ & $(\%)$ & $(\%)$ \\
\hline \multirow[b]{2}{*}{1} & 100 & 0.103 & \multirow{4}{*}{8} & 7.82 & 0.18 & 68 & 70 \\
\hline & 1000 & 1.031 & & 4.88 & 2.39 & 68 & 70 \\
\hline \multirow[b]{2}{*}{4} & 100 & 0.103 & & 15 & 0.034 & 61 & 70 \\
\hline & 1000 & 1.034 & & 14 & 0.40 & 61 & 70 \\
\hline
\end{tabular}


The experimental results show that the mass transfer zone were shorter than the carbon bed for the low flow rate column and longer than the carbon bed for the high rate flow. This reason for this is that the PCE has more time to diffuse into carbon relative to the bulk movement of fluid. Because the column bed with a low rate flow meets the criterion for a typical carbon column configuration that a carbon bed needs to contain at least one mass transfer zone. Thus the results from this test is applicable to the future scale-up bed operation. In the column with a high flow rate, a possible inadequate time appears for the carbon to adsorb the PCE molecules and to form a complete mass transfer zone. Thus the effluent from this column reached the breakpoint much quicker and a lower carbon adsorption capacity of $61 \%$ for this flow than that of $68 \%$ for the low rate flow was observed. The results from this study may not be applicable to the future scale-up bed operation. However, these two $\mathrm{q}_{\mathrm{e}} \mathrm{s}$ indicate that the overall adsorption capacity of the activated carbon for PCE from such a high IPA (60\%) solution is very high, compared to the $\mathrm{q}_{\mathrm{e}}$ of $5 \%$ obtained from a real activated carbon adsorber operated in IBM, Essex Junction, Vermont (Cambio, 1998). However, an initial PCE concentration of less than $5 \mathrm{mg} / \mathrm{L}$ in the solution involved in this operation is a major factor for this low $q_{e}$ of $5 \%$.

The $\mathrm{q}_{\mathrm{e}}{ }^{*}$ of $70 \%$ is slightly higher than the $\mathrm{q}_{\mathrm{e}}$ for the two experiments. This indicates that even the high rate of $6 \mathrm{ml} / \mathrm{min}$ used is small and that allows a "true" adsorption equilibrium in the column to arrive. Thus a $\mathrm{q}_{\mathrm{e}}$ close to $\mathrm{q}_{\mathrm{e}}{ }^{*}$ was observed.

The general alcohol recovery capacity of a $60 \%$ IPA solution is less than $1 \mathrm{~L}$ per pound activated carbon, which is very low. This low capacity might imply that carbon adsorption is not an economical means to recover alcohol from a solution with a high PCE concentration, such as $100,000 \mathrm{mg} / \mathrm{L}$. If the allowable $\mathrm{C}_{\mathrm{b}}$ has a more stringent value of $10 \mathrm{mg} / \mathrm{L}$, typical PCE concentration in the contaminated ground water, the amount of alcohol solution, which could be recovered by this $8 \mathrm{~cm}$ long activated carbon column bed, would be almost zero based on the experimental results. The reason for this low capacity probably lies in the fact that there is a tremendous amount of PCE particles in the solution that demands a huge amount of adsorption sites on the surface of the activated carbon. Thus the amount of activated carbon needed is very large if used to recover a certain amount of such a solution. Other methods, such as water dilution may be considered to deal with this type of solution. Addition of water to such a solution may drop the PCE concentration dramatically due to the much smaller PCE solubility in a lower IPA solution. Then the diluted solution could be overflowed and treated with activated carbon.

The column experiments with a $30 \%$ IPA solution were conducted at two flow rates, $2.4 \mathrm{ml} / \mathrm{min}$ and $6 \mathrm{ml} / \mathrm{min}$, and three influent concentrations, of about 640,710 and $328 \mathrm{mg} / \mathrm{L}$, respectively. The breakthrough curves were plotted and are shown in Figure 7-46, which shows that influent concentration $\mathrm{C} / \mathrm{C}_{0}$ for all of the three cases generally remains constant throughout the experiments. The three breakthrough curves have a typical s-shape, although a part of the breakthrough curve from the experiment with $\mathrm{C}_{0}$ of $640 \mathrm{mg} / \mathrm{L}$ and flow rate of $2.4 \mathrm{~mL} / \mathrm{min}$ has an irregular shape at high effluent concentration zone. The reason for this is not clear, although it is possible that some unaccountable losses of PCE in the effluent could have occurred. 
The solution volume at $\mathrm{C} / \mathrm{C}_{0}=0.5$ and 1.0 are estimated and listed in Table 7-17, which shows that the points of $C / C_{0}=0.5$ appear almost at the same place for the experiments with similar feed concentration. This phenomenon agrees with the typical feature of the breakthrough curve. The point of $\mathrm{C} / \mathrm{C}_{0}=0.5$ has been delayed for the case with a low feed PCE concentration and a low flow rate. This is because a larger volume of the low concentration flow could be treated by the same amount of the activated carbon.

Table 7-17 Solution volume at the $\mathrm{C} / \mathrm{C}_{0}=0.5$ and 1.0

\begin{tabular}{|c|c|c|c|c|}
\hline Effluent Curve & $\mathrm{C}_{0}$ & $\begin{array}{c}\text { Flow rate } \\
(\mathrm{q})(\mathrm{ml} / \mathrm{min})\end{array}$ & $\begin{array}{c}\text { Volume at } \\
\mathrm{C} / \mathrm{C}_{0}=0.5(\mathrm{ml} / \mathrm{g})\end{array}$ & $\begin{array}{c}\text { Volume at } \\
\mathrm{C} / \mathrm{C}_{0}=1.0(\mathrm{ml} / \mathrm{g})\end{array}$ \\
\hline In diamond & 710 & 6 & 500 & 800 \\
\hline In square & 640 & 2.4 & 520 & 900 \\
\hline In triangle & 328 & 2.4 & 840 & 1050 \\
\hline
\end{tabular}

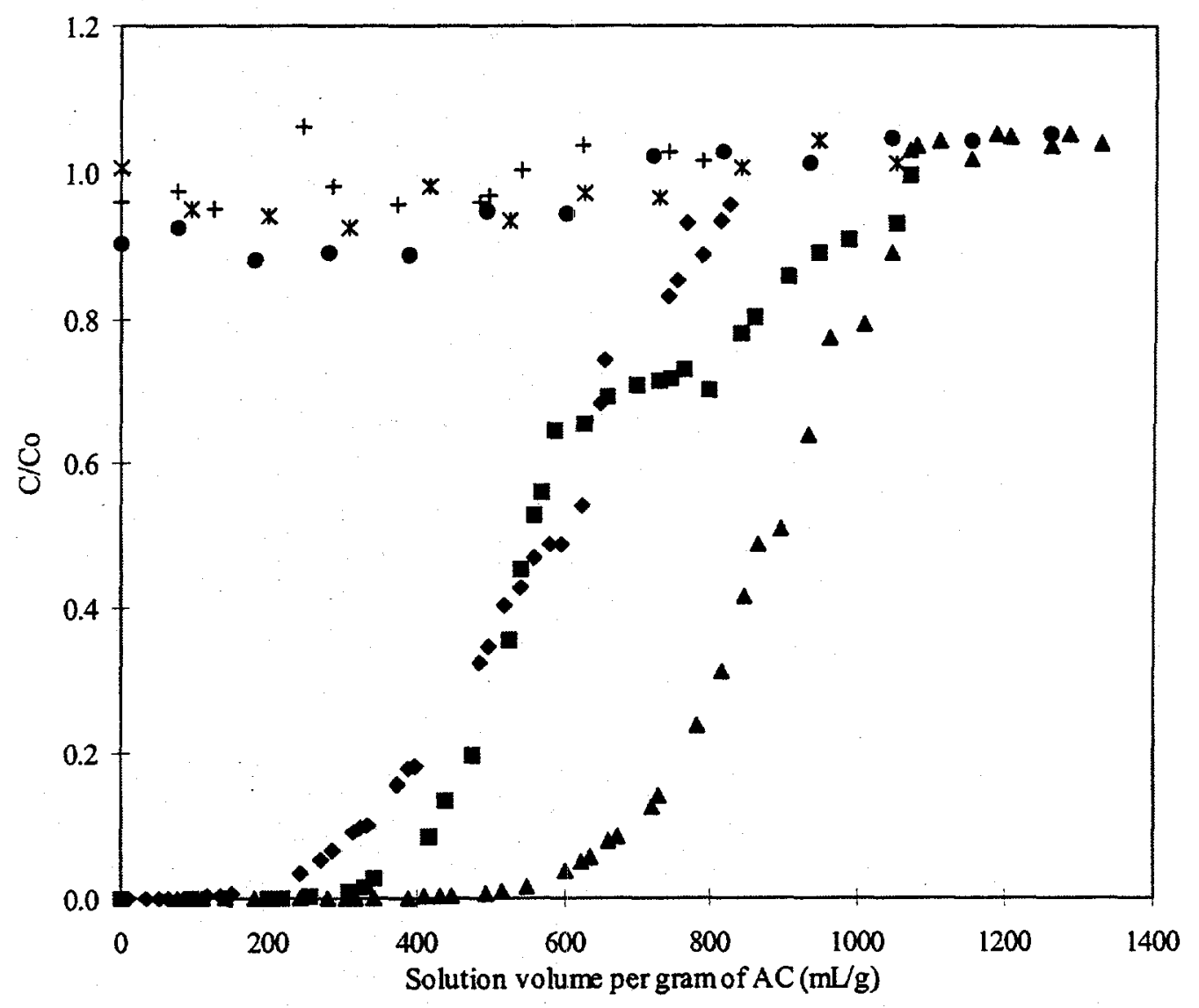

- Effluent, $710 \mathrm{mg} / \mathrm{L}, 6 \mathrm{~mL} / \mathrm{min} \quad$ Effluent, $640 \mathrm{mg} / \mathrm{L}, 2.4 \mathrm{~mL} / \mathrm{min} \triangle \triangle$ Effluent, $328 \mathrm{mg} / 2.4 \mathrm{~mL} / \mathrm{min}$ + Influent, $710 \mathrm{mg} / \mathrm{L}, 6 \mathrm{~mL} / \mathrm{min} \quad *$ Influent, $640 \mathrm{mg} / \mathrm{L}, 2.4 \mathrm{~mL} / \mathrm{min} \quad$ Influent, $328 \mathrm{mg} / 2.4 \mathrm{~mL} / \mathrm{min}$

Figure 7-46 Breakthrough curves for 30\% IPA case

The breakthrough curve at the low rate flow has a steeper slope than does the breakthrough curve at the high rate flow. This feature agrees with the general trend of the breakthrough curve; that is 
the higher the flow rate or superficial velocity of the solution passing through the column bed, the flatter the breakthrough curve appears. However, there is some portion of the curve for the solution with feed concentration of $640 \mathrm{mg} / \mathrm{L}$ at high effluent concentration zone. The reasons could be the errors involved in the experiment or the flow rate variation resulting from the irregular pumping rate.

The length of the mass transfer zones for all the three cases were calculated and are listed in Table 7-18, in which the $\mathrm{C}_{\mathrm{b}}, \mathrm{q}_{\mathrm{e}} @ \mathrm{EP}$ and $\mathrm{q}_{\mathrm{e}}{ }^{*}$ have the same meanings as described for the case of $60 \%$ IPA case. As shown in Table 7-18, the alcohol recovery capacity and mass transfer zone in the carbon bed are affected by the breakpoint concentration $\mathrm{C}_{b}$ and flow rate, which is the same as observed in the experiments for $60 \%$ IPA case.

The carbon bed in the two low flow rate experiments are longer than the calculated mass transfer zone. Therefore these two carbon beds exerted their full adsorption capacities, and the results from these experiments are applicable to the scale-up adsorber. Because the carbon bed is shorter by $20 \%$ than the mass transfer zone for the highest rate flow of $6 \mathrm{ml} / \mathrm{min}$, some adjustment might need to made to the results from this carbon column test if applied to the future scale-up adsorber.

Table 7-18 Mass transfer zone for 30\% IPA solution

\begin{tabular}{|c|c|c|c|c|c|c|c|c|}
\hline $\begin{array}{l}\text { Flow } \\
\text { rate } \\
\end{array}$ & Feed C & $C_{b}$ & $\mathrm{C}_{\mathrm{b}} / \mathrm{C}_{0}$ & $\begin{array}{l}\text { Length of } \\
\text { column }\end{array}$ & $\begin{array}{c}\text { Length of } \\
\text { MTZ }\end{array}$ & $\begin{array}{c}\text { IPA recovery } \\
\text { capacity }\end{array}$ & $\mathrm{q}_{\mathrm{e}} @ \mathrm{EP}$ & $\mathrm{q}{ }^{*}$ \\
\hline$(\mathrm{ml} / \mathrm{min})$ & $(\mathrm{mg} / \mathrm{L})$ & $(\mathrm{mg} / \mathrm{L})$ & $(\%)$ & (cm) & $(\mathrm{cm})$ & $(\mathrm{L} / \mathrm{lb})$ & $(\%)$ & $(\%)$ \\
\hline 2.4 & 328 & 10 & 1.563 & \multirow{3}{*}{8} & 4.64 & 133 & 25.8 & 30 \\
\hline 2.4 & 640 & 10 & 1.563 & & 8.00 & 43 & 37 & 46 \\
\hline 6 & 710 & 10 & 1.408 & & 9.6 & 24 & 26.1 & 49 \\
\hline
\end{tabular}

The alcohol recovery capacities of above $24 \mathrm{~L}$ of alcohol IPA per pound of activated carbon shown in Table 7-18 indicates that carbon adsorption could be an effective and economical way of recovering IPA from a PCE contaminated solution.

Table 7-18 shows that the $q_{e}$ has a value above $25.8 \%$, which is a very high adsorption value. It is also found that the value in $q_{e}$ is proportion to the feed concentration. However, the $q_{e}$ for the highest feed concentration of $710 \mathrm{mg} / \mathrm{L}$ is quite lower than that for the solution with a lower feed concentration of $640 \mathrm{mg} / \mathrm{L}$ and is almost close to the solution with a lowest solution of $328 \mathrm{mg} / \mathrm{L}$. The reason for this is due to fact that no complete mass transfer zone was formed in this column bed. This phenomenon also demonstrates quantitatively how important for a carbon bed to contain a entire mass transfer zone.

Table 7-18 also shows that $\mathrm{q}_{e}{ }^{*}$ is greater than the respective $\mathrm{q}_{\mathrm{e}}$. This makes sense based on Cooper and Alley (1990). 
Compared with the $60 \%$ IPA case, the $30 \%$ IPA case has a lower $\mathrm{q}_{\mathrm{e}}$ because it has a much lower feed concentration. It could be found that the ratio of about 2 in $\mathrm{q}_{\mathrm{e}}$ of the $60 \%$ IPA solution to $30 \%$ IPA solution was much lower than the ratio of about 150 in the initial PCE concentrations for the two solutions. This feature indicates that the IPA concentration has a negative effect on the PCE adsorption onto activated carbon, which is the same as that observed in the batch isotherm studies.

Similar to the way introduced previously, the volume of solution treated by $34 \mathrm{~g}$ activated carbon in batch reactors were estimated and listed in Table 7-18. The $\mathrm{q}_{\mathrm{e}}$ was calculated by assuming that all PCE in this treated solution was adsorbed onto carbon.

The IPA concentration was tracked throughout one experiment for a $30 \%$ IPA case. The results are shown in Figure 7-47. The results show that: (1) The average concentration of the influent and effluent is quite close to the feed IPA concentration of about of $237,000 \mathrm{mg} / \mathrm{L}$ of a $30 \%$ IPA solution. The closeness in influent and effluent concentrations to the feed concentration indicates that there was no noticeable change in IPA concentration as a result of activated carbon adsorption; (2) The influent and effluent IPA concentrations reach the feed IPA concentration almost immediately after starting the experiment, which indicates that the IPA adsorption onto the carbon was weak and could be neglected. As a result, it could be predicted that IPA could be recovered effectively by activated carbon adsorption from PCE contaminated solution.

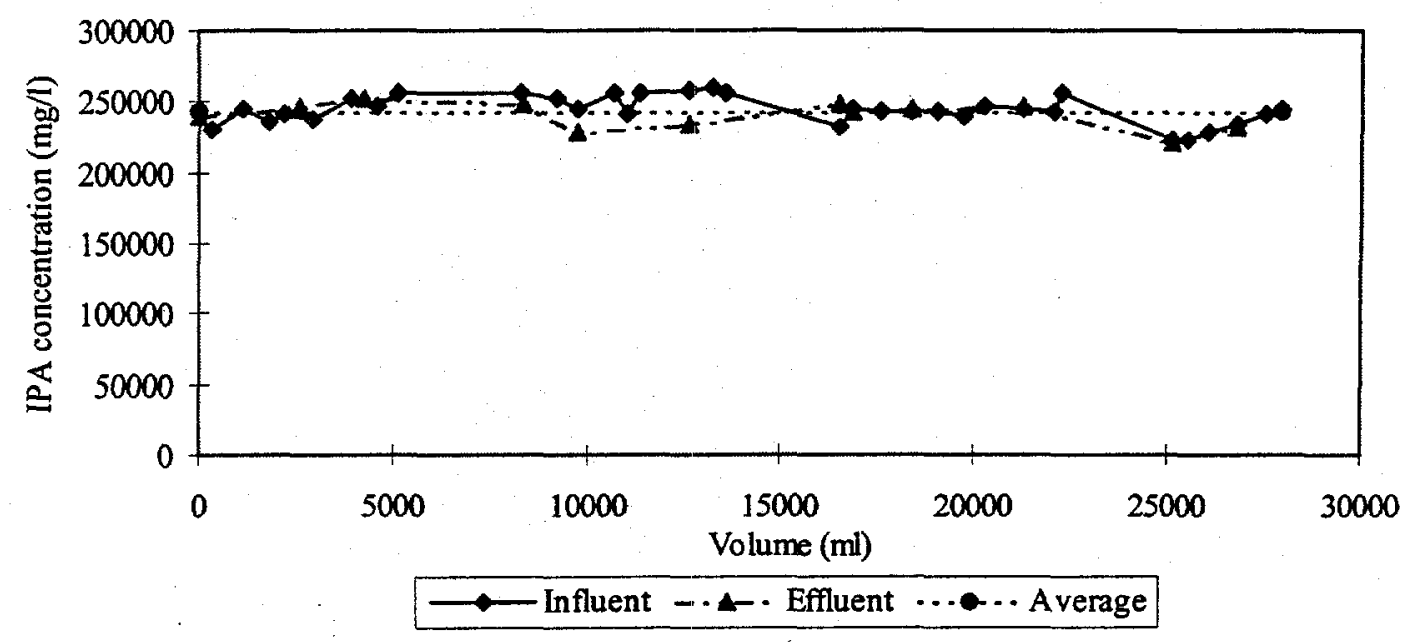

Figure 7-47 IPA concentration profile

The experimental results show that IPA recovery capacity by activated carbon adsorption is very low from a solution of $60 \%$ IPA solution. with a very high PCE concentration of $100,000 \mathrm{mg} / \mathrm{L}$, and that other methods need to be considered to separate IPA from such a PCE contaminated solution. Therefore this economical consideration briefly focuses on the activated carbon recovery of IPA from $30 \%$ IPA solution.

The carbon capacity for the recovery of IPA was used to estimate the cost for the carbon and the savings by reuse of IPA. The cost for the scale-up carbon column adsorber and the cost for conventional treatment of the alcohol flushing extraction solution composed of IPA, water and 
PCE was also estimated based on the information obtained by surveying recent market. Thereafter cost savings from IPA recovery and reuse using activated carbon over the conventional treatment method was calculated. This cost savings was used to evaluate the overall feasibility of the carbon to recover and reuse IPA for alcohol flushing the PCE contaminated sites.

Due to the lack of the knowledge in the yielding rate of the flushing extraction solution from alcohol remediation practice, the economical consideration of this technology was briefly conducted based on the following assumptions:

Items for cost consideration are listed in Table 7-19. Items (4), (5), and (6) in Table 7-19 are quoted from the information provided by Calgon Corporation, Pittsburgh, PA. This cost is estimated for a formal adsorber and its operation, thus it is overestimate for a much simpler apparatus needed for treatment of the solution from a site alcohol flushing remediation.

Table 7-19 Major cost items

\begin{tabular}{|c|c|l|}
\hline Number & & \\
\hline 1 & + & Cost for purchase of IPA \\
\hline 2 & + & Cost for disposal of waste in conventional way \\
\hline 3 & - & Cost for activated carbon \\
\hline 4 & - & Cost for equipment (Capital cost) \\
\hline 5 & - & Annual operating cost \\
\hline 6 & - & Maintenance (5\% of the cost for equipment) \\
\hline
\end{tabular}

Cost savings from the alcohol recovery and reuse was calculated by Equation:

$$
\text { Cost savings }=(1)+(2)-(3)-(4)-(5)-(6)
$$

Where: Items (1) to (6) are defined in Table 7-19.

Price for equipment and materials, which are listed in Table 7-20.

Table 7-20 Price information

\begin{tabular}{|l|c|l|}
\hline \multicolumn{1}{|c|}{ Item } & Price (\$) & \\
\hline Equipment & $285 / \mathrm{gpm}$ & \\
\cline { 1 - 2 } Annual Operating Cost & $17 / \mathrm{gpm}$ & Calgon Corporation, Pittsburgh, PA \\
\hline Activated Carbon & $2.4 / \mathrm{lb}$ & \\
\hline IPA & $1 / \mathrm{L}$ & \\
\cline { 1 - 2 }
\end{tabular}




\begin{tabular}{|l|c|l|}
\hline $\begin{array}{l}\text { Conventional disposal } \\
\text { of PCE solution if not } \\
\text { recovered using carbon }\end{array}$ & $\begin{array}{c}0.5 / \mathrm{L} \\
\text { Heritage Environmental, Inc., Burlington, } \\
\text { Vermont (1998) }\end{array}$ \\
\hline
\end{tabular}

The flow rate was estimated to be 300gallons/per minute (gpm),

(1) The operation period was considered to be 2 years,

(2) Cost for activated carbon was determined based on the column test results.

The cost for the amount of IPA for the initiation of alcohol flushing remediation process was not taken into consideration. The mass of contaminant PCE was used as an independent variable to conduct the calculation. A 30\% IPA solution was considered. The detailed calculation is described as follows:

The mass of PCE was assumed first, then the demand of IPA for flushing this amount of PCE and the total flushing extraction solution were determined. The mass of the activated carbon needed was calculated based on the flushing extraction solution size and the contaminant treatment capacity of the activated carbon, which was determined in the column tests. With the known value of the variables listed above, the cost or savings for each variable was calculated. Finally Equation (7) was applied to find the respective and resultant cost savings. A critical amount of PCE mass at which the cost savings is equal to zero was also determined. Based on the economical consideration, the economical feasibility or soundness of this method was announced.

Based on the above price discussion, the capital cost, maintenance and operating fee were estimated as follows:

(1) Capital cost: $\$ 85,500$ this is for a large scale permanent system (55 gallon drums of carbon are available for small systems)

(2) Maintenance ( $5 \%$ of capital cost): $\$ 8,600$

(3) Operating fee: $\$ 10,200$

Sum of the above three items: $\$ 104,300$

The cost for IPA and the conventional disposal of the alcohol flushing extraction solution are proportional to the amount of PCE to be removed from the ground by the IPA flushing solution. The economical consideration of this IPA recovery and reuse using activated carbon adsorption was conducted as follows.

The mass of PCE was used as the key variable to conduct this economical consideration. The minimum IPA recovery capacity of 24 liters IPA per pound activated carbon revealed by the column tests and a PCE concentration of $710 \mathrm{mg} / \mathrm{L}$ in solution was used for this consideration. The cost savings resulting from activated carbon adsorption was calculated and is listed in Table $7-21$.

Table 7-21 Cost savings

\begin{tabular}{|c|c|c|c|c|c|c|c|c|c|}
\hline PCE (lb) & PCE (mg) & $\begin{array}{c}\text { Waste } \\
\text { Volume(L) }\end{array}$ & IPA (L) & S for IPA & \$ for waste & $\begin{array}{c}\text { Carbon } \\
\text { (lb) }\end{array}$ & $\begin{array}{c}\text { \$ for } \\
\text { carbon }\end{array}$ & $\begin{array}{c}\text { \$ for } \\
\text { Equip. etc. }\end{array}$ & \$ Savings \\
\hline 10 & $4.54 \mathrm{E}+06$ & 6394 & 1918 & 1918 & 3197 & 87 & 209 & 108300 & -103394 \\
\hline
\end{tabular}




\begin{tabular}{|c|c|c|c|c|c|c|c|c|c|}
\hline 50 & $2.27 \mathrm{E}+07$ & 31972 & 9592 & 9592 & 15986 & 436 & 1046 & 108300 & -83769 \\
\hline 100 & $4.54 \mathrm{E}+07$ & 63944 & 19183 & 19183 & 31972 & 872 & 2093 & 108300 & -59238 \\
\hline 220.8 & $1.00 \mathrm{E}+08$ & 141188 & 42356 & 42356 & 70594 & 1925 & 4621 & 108300 & 29 \\
\hline 1000 & $4.54 \mathrm{E}+08$ & 639436.6 & 191831 & 191831 & 319718 & 8720 & 20927 & 108300 & 382322 \\
\hline 10000 & $4.54 \mathrm{E}+09$ & 6394366 & $2 \mathrm{E}+06$ & $2 \mathrm{E}+06$ & 3197183 & 87196 & 209270 & 108300 & 4797923 \\
\hline
\end{tabular}

Table 7-21 shows that the cost for equipment is offset by the savings in cost from the reuse of IPA and conventional disposal of IPA flushing extraction solution when 221 pounds is to be removed from the ground by the IPA solution. The volume of 221 pounds PCE is 12 gallons, which is very tiny in quantity compared with the estimation of several million gallons of PCE contaminant at SRS. Therefore activated carbon adsorption to recover and reuse alcohol is definitely cost-effective and promising. At lower PCE recovery rates 55 gallon drums of activated carbon could be used which would put the capital cost at virtually zero and a considerable cost savings would also be realized. The analysis above was for a large system like one that might be employed at SRS.

Based on the research by Taylor et al. (1998), the research by Pennell et al. (1996) and the telephone communication with Linnemeyer (1998), the scenario of the surfactant flushing method is assumed as follows:

Contaminant: PCE and its concentration averages at $800 \mathrm{mg} / \mathrm{L}$ in solution

Surfactant solution: Surfactant: $\$ 1.25 / \mathrm{lb}$ and $8 \%$ by the weight of the solution

IPA: $\$ 3.75 / \mathrm{L}$ and $8 \%$ by the weight of the solution

$\mathrm{NaCl}: \$ 1 / \mathrm{lb}$ and $15000 \mathrm{mg} / \mathrm{L}$

Surfactant recovery: not considered

The cost for surfactant flushing remediation was estimated and tabulated in Table 7-22. The comparison of the two methods was conducted and is listed Table 7-23. Table 7-23 shows that when the amount of PCE contaminant reaches above the quantity of 374 pounds, the alcohol flushing and alcohol recovery and reuse method is prior to the surfactant flushing method from the economical point of view.

Table 7-22 Cost estimation for surfactant flushing remediation

\begin{tabular}{|c|c|c|c|c|c|c|}
\hline $\begin{array}{c}\text { PCE } \\
(\mathrm{lb})\end{array}$ & $\begin{array}{c}\text { Mixture } \\
(\mathrm{L}) \\
(800 \mathrm{mg} / \mathrm{L})\end{array}$ & $\begin{array}{c}\text { Mixture (lb) } \\
\left(1 / 454 \mathrm{lb} / \mathrm{cm}^{3}\right)\end{array}$ & $\begin{array}{c}\text { Surfactant (lb) } \\
(8 \% \text { by weight) }\end{array}$ & $\begin{array}{c}\text { IPA (L) } \\
(8 \% \text { by } \\
\text { weight })\end{array}$ & $\begin{array}{c}\text { NaCl (lb) } \\
(15000 \mathrm{mg} / \mathrm{L})\end{array}$ & $\begin{array}{c}\text { Cost } \\
(\$)\end{array}$ \\
\hline 1 & 567.5 & 1250 & 100 & 57 & 19 & 201 \\
\hline 10 & 5675 & 12500 & 1000 & 573 & 188 & 2011 \\
\hline 100 & 56750 & 125000 & 10000 & 5732 & 1875 & 20107 \\
\hline 1000 & 567500 & 1250000 & 100000 & 57323 & 18750 & 201073 \\
\hline 10000 & 5675000 & 12500000 & 1000000 & 573232 & 187500 & 2010732 \\
\hline 100000 & 56750000 & 125000000 & 10000000 & 5732323 & 1875000 & 20107323 \\
\hline
\end{tabular}


Table 7-23 Comparison between the alcohol flushing and the surfactant flushing

\begin{tabular}{|c|c|c|c|}
\hline PCE (lb) & $\begin{array}{c}\text { Savings for } \\
\text { alcohol (\$) }\end{array}$ & $\begin{array}{c}\text { Cost for } \\
\text { surfactant } \\
(\$)\end{array}$ & $\begin{array}{c}\text { Savings of } \\
\text { alcohol over } \\
\text { surfactant(\$) }\end{array}$ \\
\hline 10 & -103394 & 2011 & -105405 \\
\hline 50 & -83769 & 10054 & -93823 \\
\hline 100 & -59238 & 20107 & -79345 \\
\hline 374 & 75193 & 75201 & -9 \\
\hline 1000 & 382322 & 201073 & 181249 \\
\hline
\end{tabular}

\subsection{Summary}

The main objective of this research was to investigate the separation of IPA from chlorinated compound contaminated solution at various IPA concentrations using activated carbon adsorption. Batch and column studies were used for this investigation. PCE, IPA and Filtrosorb400 activated carbon were used as the representatives for the contaminant, alcohol and activated carbon to conduct the experiments. The technical and economical feasibility of recovery and reuse of alcohol using activated carbon for alcohol flushing remediation of the chlorinated compounds contaminated ground water and sites was also investigated.

The batch and column studies show that the presence of IPA in solution definitely complicates the adsorption of PCE onto activated carbon primary by enhancing the PCE solubility in the solution. The general parameters of the adsorption of PCE from an IPA and water system by activated carbon are summarized below:

1. An increase in IPA concentration decreases the adsorption capacity of activated carbon for PCE or TCE from an alcohol and water solution.

2. The activated carbon dose needs to be in a certain range in order to have high adsorption efficiency and economical utilization of activated carbon adsorption capacity.

3. The linear Freundlich isotherm is applied to model the experimental data and the results show that the linear Freundlich isotherm is valid over the adsorption systems if the activated carbon dose is in a certain range associated with the IPA concentration and initial PCE concentration of the solution.

4. Even though IPA increases PCE solubility and decreases the adsorption capacity, the higher solubility resulting from the high IPA concentration enables the solution to dissolve more PCE. As a result, a high PCE concentration would be obtained and the higher PCE concentration somewhat enhances the adsorption capacity.

5. An appropriate activated carbon needs to be selected for a specific contaminant in order to have an overall high adsorption efficacy and efficiency. For adsorption of PCE from an IPA and water system, Filtrosorb-400 activated carbon is a good adsorbent.

The column experimental results also show that the activated carbon adsorption to recover and reuse alcohol has merits of reliability, simplicity and low cost of the operation. High alcohol 
recovery capacity and a reduction of subsequent waste problems are also likely. The breakthrough curve of an adsorption column is not only subjected to the influent flow rate but maybe also to the solute concentration as well as the adsorption intensity which occurs in the carbon column.

Due to the fact that the activated carbon in the column is almost constantly in contact with the fresh solution, a higher adsorption capacity of the activated carbon would be reached than that would in a batch carbon reactor. It is one of the technical advantages of the column bed configuration for treatment of solution. It was estimated that the cost for the column adsorber recovery system and the associated operating fee could be saved by reuse of IPA needed to flush and remediate a 221 pounds of $P C E$ at a site. With the consideration of several million pounds of PCE at SRS, more IPA could be recovered using activated carbon. The recovered IPA could be reused for flushing the site and as a result, the cost and waste of this remediation strategy would be minimized.

\subsection{References}

Cambio, R. E-mail Message about the Carbon Adsorber in IBM, Essex Junction, Vermont, 1998.

Chemical and Engineering News, 22, December 1996.

Cheremisinoff, P. N., and Morresi, A. C., Carbon Adsorption Applications, edited in Carbon Adsorption Handbook by Cheremisinoff, P. N. and Ellerbusch, F., Ann Arbor Science Publishers INC., Ann Arbor, Michigan, 1978.

Cookson, J. T. Jr., Adsorption Mechanisms: The Chemistry of Organic Adsorption on Activated Carbon, edited in Carbon Adsorption Handbook by Cheremisinoff, P. N. and Ellerbusch, F., Ann Arbor Science Publishers INC., Ann Arbor, Michigan, 1978.

Cooper, C. D. and Alley, F. C., Air Pollution Control, Waveland Press, Inc., Project Heights, Illinois, 1990.

Crank J., The Mathematics of Diffusion, Claredon Press, London, 1956.

Fuller, L. Defining Product Properties, Clagon Carbon Corporation, Pittsburgh, PA, 1998.

Hansen, R. S., and Craig, R. P., "The Adsorption of Aliphatic Alcohol and Acids from Aqueous Solutions by Nonporous Carbons", J. Phys. Chem., 58, 211, 1954.

Hassler J. W., Purification with Activated Carbon, Chemical Publishing CO., INC., New York, 1974. 
Hayden, N. J., Diebold, J., and Noyes G., "Phase Behavior of Chlorinated Solvent/Water/Alcohol Mixtures with Application to Alcohol Flushing", Accepted Journal of Chemical and Engineering Data, 1998.

Homes, H. N. and Mckelvey, J. B., "The Reversal of Traube's Rule of Adsorption", J. Phys. Chem., 32, 1522-1523, 1928.

Kleinbaum, D. G., Kupper, L. L., and Muller, K. E., Applied Regression Analysis and Other Multivariable Methods, Second Edition, Duxbury Press, 266-267, 1988.

Lin, S. H. and Hsu, F. M., "Liquid-Phase Adsorption of Organic Compounds by Granular Activated Carbon and Activated Carbon Fiber", Ind. Eng. Chem. Res., 34, 2110-2116, 1995

Linnemeyer, H. C. from GeoEngineering Services, Duke Engineering \& Services, Austin, TX 7858, Telephone Communication about the Surfactant Flushing, 1998.

Looney, B. B., Rossabi, J. and Tuck, D. M., "Assessing DNAPL contamination, A/M-Area, Savannah River Site: Phase 1 Results". Westinghouse Savannah River Company, Aiken, South Carolina, 1992.

Luehrs, D. C., Hickey, J. P., Nilsen, P. E., Godbole, K. A., and Rogers, T. N., "Linear Solvation Energy Relationship of the Limiting Partition Coefficient of Organic Solutes Between Water and Activated Carbon", Environmental Science and Technology, Vol., 30, No. 1, 1996.

Nakhla, G. F. and Suidan, M. T., "Evaluation of Competitive Adsorption in Anaerobic GAC Reactors", Journal of Environmental Engineering, October 1995.

Pennell, K. D., Pope, G. A., and Abriola, L. M., "Influence of Viscous and Buoyancy Forces on the Mobilization of Residual Tetrachloroethylene During Surfactant Flushing", Environmental Science and Technology Vol. 30, No. 4, 1328 - 1335,1996.

Perry, E. S. and Weissberger, A. (Editor), Dissolution, Second Completely Revised and Augmented Edition, Interscience Publishers, a Division of Jobn Wiley \& Sons, New York, London, Sydney, 1995.

Rodrigue-Reinoso, F., Molina-Sabio, M. and Muñecas, M. A., "Effect of Microporosity and Oxygen Surface Groups of Activated Carbon in the Adsorption of Molecules of Different Polarity", American Chemical and Society, 1992.

Sawyer, C. N., McCarty, P. L. and Parkin, G. F., Chemistry for Environmental Engineering, Schaum Division, McGraw-Hill, Inc., 1221 Avenue of the Americas, New York, NY 10020, 1994. 
Solley, W. B., Merk, C. F. and Pierce, R. R., Estimated use of water in the United States in 1985, U. S. Geological Survey Circular 1004, p82, 1998.

Tessmer, C. H., Vidic, B. D. and Uranowski, L. J., "Impact of Oxygen- Containing Surface Functional Groups on Activated Carbon Adsorption of Phenols", Environmental Science \& Technology, Vol. 31, No. 7, p1872-1878, 1997.

Taylor, T. P., and Pennell, K. D., "Surfactant Enhanced Recovery of Tetrachloroethylene from Porous Media", EnvironMEET'98.

Tchobanoglous, G., Schroeder, E. D., Water Quality, Addison-Wesley Publishing Company, 1987.

U. S. EPA, Drinking Water Standards and Health Goals, Federal Register, July 8, 1987 and Federal Register, January 30, 1991.

US EPA, "Granular Activated Carbon Treatment", Engineering Bulletin, EPA/540/2-91/024, October 1991.

US EPA, Safe Drinking Water Act Amendments of 1986, PL 99-339, 1986.

Verschueren, K., Handbook of Environmental Data on Organic Chemicals, Second Edition, Van Nostrand Reinhold, New York, 1983.

Vidic, R. D., Suidan, M. T., Sorial, G. A., Brenner, R. C., "Molecular Oxygen and the Adsorption of the Phenol --- Effect of Functional Groups", Water Environment Research, Volume 65, Number 2, 1993.

Vidic, R. D., Sorial, G. A., Papadims, S. P., and Suidan, M. T., "GAC Adsorption of a Mixture of Natural Organic Matter and VOCs in the Presence and Absence of Molecular Oxygen", American Water Works Association Annual Conference, Philadelphia, PA, June, 1991.

Weber W. J. Jr., Physicochemical Processes for Water Quality Control, John Wiley \& Sons, New York, 1972.

Weber, W. J. Jr., and Bernardin, F. E., Jr., Removal of Organic Substances from Municipal Wastewaters by Physicochemical Processes, edited by Bernard B. Berger in Control of Organic Substances in Water and Wastewater, EPA-600, April 1983.

Weber, W. J. Jr., Carter, M. C., Olmstead, K. P., and Katz,. L. E., "Evaluation of the MIDDAS System for Designing GAC Adsorbors", United States Environmental protection Agency Research and Development, EPA/600/s2-91/048, December 1991. 
Yeh, H. C. and W. E. Kastenberg, W. E., "Health risk assessment of biodegradable volatile organic chemicals: A case ștudy of PCE, TCE, DCE and VC", Journal of Hazardous Materials, 27 (1991) 111-126, 1991.

\section{Appendix A Statistical Analysis of the Isotherm Parameters}

The purpose of this statistical analysis was to determine if the scatter in the data was due to experimental variability or a true difference due to the different contact time used. The linear regression parameters (intercept of $\log \left(\mathrm{K}_{\mathrm{F}}\right)$ and slope of $1 / \mathrm{n}$ ) over all adsorption data sets were used to conduct this analysis. The analysis follows the method listed from page of 266 to 267 (Kleinbaum et al., 1988).

For $M$ data set and $F$ data set of one IPA concentration case, the intercepts are $\log \left(K_{F}\right)_{M}$ and $\log \left(\mathrm{K}_{\mathrm{F}}\right)_{\mathrm{F}}$, and the slopes are $(1 / \mathrm{n})_{\mathrm{M}}$ and $(1 / \mathrm{n})_{\mathrm{F}}$. Based on the two raw adsorption data sets, the test statistics $T_{1}$ and $T_{0}$ were calculated and $P$-value associated with the number of the data of the two data sets was determined by consulting Table A-2 (Kleinbaum et al., 1988). The analysis was conducted stepwise. The criteria for the statistical analysis are listed in Table App.-1. The confidence interval for determination of $\mathrm{P}$ value is $95 \%$.

Table App.-1 Criteria for statistical analysis

\begin{tabular}{|c|c|c|}
\hline Condition & Conclusion & Implication \\
\hline $\mid \mathrm{T}_{d} \geq \mathrm{P}$ & $\log \left(\mathrm{K}_{\mathrm{F}}\right)_{\mathrm{M}} \neq \log \left(\mathrm{K}_{\mathrm{F}}\right)_{\mathrm{F}}$ & Nonexistence of statistical identity in adsorption capacity \\
\hline$\left|\mathrm{T}_{0}\right|<\mathrm{P}$ & $\log \left(\mathrm{K}_{\mathrm{F}}\right)_{\mathrm{M}}=\log \left(\mathrm{K}_{\mathrm{F}}\right)_{\mathrm{F}}$ & Existence of statistical identity in adsorption capacity \\
\hline$\left|\mathrm{T}_{1}\right| \geq \mathrm{P}$ & $(1 / \mathrm{n})_{\mathrm{M}} \neq(1 / \mathrm{n})_{\mathrm{F}}$ & Nonexistence of statistical identity in adsorption intensity \\
\hline$\left|\mathrm{T}_{1}\right|<\mathrm{P}$ & $(1 / \mathrm{n})_{\mathrm{M}}=(1 / \mathrm{n})_{\mathrm{F}}$ & Existence of statistical identity in adsorption intensity \\
\hline
\end{tabular}

If there is an existence in both adsorption capacity and the adsorption intensity, a conclusion would be made that the two contact times of the two experiments $\mathrm{M}$ and $\mathrm{F}$ are statically the same in terms of the arrival of adsorption equilibrium.

The statistical analysis results for investigating the effect of contact time are listed in Table App.2 , and the statistical analysis results for comparison among the adsorption data for $60 \%$ IPA concentration solutions are listed in Table App.-3.

Table App.-2 Statistical analysis results for different contact time

\begin{tabular}{|c|c|c|c|c|}
\hline $\begin{array}{l}\text { IPA } \\
(\%)\end{array}$ & Try & $\begin{array}{l}\text { Contact } \\
\text { Time }\end{array}$ & $\left|\begin{array}{c}\mathrm{C}_{0} \\
(\mathrm{mg} / \mathrm{L})\end{array}\right|$ & Statistical Analysis Results \\
\hline \multirow{4}{*}{0} & 1 & $28 \mathrm{hr}$ & 100 & \multirow{4}{*}{$\begin{array}{l}\mathrm{K}_{F_{1}}=\mathrm{K}_{\mathrm{F} 2} ; \mathrm{K}_{-\mathrm{F} 1}=\mathrm{K}_{\mathrm{F} 3} ; \mathrm{K}_{\mathrm{F} 1}>\mathrm{K}_{\mathrm{F} 4} 1 / \mathrm{n}_{1}<1 / \mathrm{n}_{2} ; 1 / \mathrm{n}_{1}=1 / \mathrm{n}_{3} ; 1 / \mathrm{n}_{1}<1 / \mathrm{n}_{4} \\
\mathrm{~K}_{\mathrm{F} 2}=\mathrm{K}_{\mathrm{F} 3} ; \mathrm{K}_{\mathrm{F} 2}=\mathrm{K}_{\mathrm{F} 4}: 1 / \mathrm{n}_{2}=1 / \mathrm{n}_{3} ; 1 / \mathrm{n}_{2}=1 / \mathrm{n}_{4} \\
\mathrm{~K}_{\mathrm{F}}>\mathrm{K}_{\mathrm{F} 4}: 1 / \mathrm{n}_{3}=1 / \mathrm{n}_{4}\end{array}$} \\
\hline & 2 & $12 \mathrm{~d}$ & 70 & \\
\hline & 3 & $22 \mathrm{~d}$ & 110 & \\
\hline & 4 & $30 \mathrm{~d}$ & 65 & \\
\hline \multirow[b]{2}{*}{15} & 1 & $28 \mathrm{hr}$ & 240 & \multirow{2}{*}{$\begin{array}{l}\mathrm{K}_{\mathrm{F} 1}=\mathrm{K}_{\mathrm{F} 2} ; \mathrm{K}_{\mathrm{F} 1}=\mathrm{K}_{\mathrm{F} 3}: 1 / \mathrm{n}_{1}=1 / \mathrm{n}_{2} ; 1 / \mathrm{n}_{1}=1 / \mathrm{n}_{3} \\
\mathrm{~K}_{\mathrm{F} \mathrm{K} 2}=\mathrm{K}_{\mathrm{F} \mathrm{K} 3}: 1 / \mathrm{n}_{\mathrm{K} 2}=1 / \mathrm{n}_{\mathrm{K} 3}\end{array}$} \\
\hline & 2 & $12 \mathrm{~d}$ & 160 & \\
\hline
\end{tabular}




\begin{tabular}{|c|c|c|c|l|}
\cline { 2 - 4 } & 3 & $30 \mathrm{~d}$ & 160 & \\
\hline \multirow{3}{*}{30} & 1 & $40 \mathrm{hr}$ & 1000 & \multicolumn{1}{|c|}{$\mathrm{K}_{\mathrm{F} 1}=\mathrm{K}_{\mathrm{F}_{2}} ; \mathrm{K}_{\mathrm{F} 1}=\mathrm{K}_{\mathrm{F} 3}: 1 / \mathrm{n}_{1}=1 / \mathrm{n}_{2}: 1 / \mathrm{n}_{1}=1 / \mathrm{n}_{3}$} \\
\cline { 2 - 4 } & 2 & $12 \mathrm{~d}$ & 950 & $\mathrm{~K}_{\mathrm{F} 2}=\mathrm{K}_{\mathrm{F} 3}: 1 / \mathrm{n}_{2}=1 / \mathrm{n}_{3}$ \\
\cline { 2 - 4 } & 3 & $30 \mathrm{~d}$ & 760 & \\
\hline \multirow{3}{*}{60} & 1 & $40 \mathrm{hr}$ & 99000 & $\mathrm{~K}_{\mathrm{F} 1}<\mathrm{K}_{\mathrm{F}_{2}} ; \mathrm{K}_{\mathrm{F} 1}=\mathrm{K}_{\mathrm{F} 3}: 1 / \mathrm{n}_{1}>\mathrm{n}_{\mathrm{M} 2} ; 1 / \mathrm{n}_{1}=1 / \mathrm{n}_{3}$ \\
\cline { 2 - 5 } & 2 & $12 \mathrm{~d}$ & $1 \mathrm{E}+05$ & $\mathrm{~K}_{\mathrm{F} 2}=\mathrm{K}_{\mathrm{F} 3}: 1 / \mathrm{n}_{2}=1 / \mathrm{n}_{3}$ \\
\cline { 2 - 5 } & 3 & $30 \mathrm{~d}$ & $1 \mathrm{E}+05$ & \\
\hline
\end{tabular}

Table App.-2 shows that: (1) there is an existence of statistical identity in both $\mathrm{K}_{\mathrm{F}}\left(\mathrm{Log}\left(\mathrm{K}_{\mathrm{F}}\right)\right.$ and $1 / \mathrm{n}$ generally for $0 \%$ IPA case and for $15 \%$ and $30 \%$ IPA cases. (2) This is partial existence of statistical identity in $\mathrm{K}_{\mathrm{F}}\left(\log \left(\mathrm{K}_{\mathrm{F}}\right)\right.$ and $1 / \mathrm{n}$ for $60 \%$ IPA case.

Table App.-3 Statistical analysis results for all data for $60 \%$ IPA solutions

\begin{tabular}{|c|c|l|}
\hline Try & $\mathrm{C}_{0}(\mathrm{mg} / \mathrm{L})$ & \multicolumn{1}{|c|}{ Statistical Analysis Results } \\
\hline 1 & 1000 & $\mathrm{~K}_{\mathrm{F} 1}=\mathrm{K}_{\mathrm{F}_{2}} ; \mathrm{K}_{\mathrm{F}_{1}}<\mathrm{K}_{\mathrm{F}_{3}: 1 / \mathrm{n}_{1}=1 / \mathrm{n}_{2} ; 1 / \mathrm{n}_{1}>1 / \mathrm{n}_{3}}$ \\
\hline 2 & 10000 & $\mathrm{~K}_{\mathrm{F}_{2}<\mathrm{K}_{\mathrm{F}}: 1 / \mathrm{n}_{2}>1 / \mathrm{n}_{3}}$ \\
\hline 3 & 100000 & \\
\hline
\end{tabular}

Table App.-3 shows that the adsorption parameters are statistical identical for solutions with an initial PCE concentration not greater than $10,000 \mathrm{mg} / \mathrm{L}$. The $\mathrm{K}_{\mathrm{F}}$ and $1 / \mathrm{n}$ over all three tests in solutions with an initial PCE concentration about $100,000 \mathrm{mg} / \mathrm{L}$ were calculated. The statistical comparison between these two parameters and those for solutions with initial concentrations of 1000 and $10000 \mathrm{mg} / \mathrm{L}$ was also conducted. The conclusion is similar to that discussed above. 


\section{$8 \quad$ Process Feasibility \\ 8.1 Introduction}

Numerous laboratory, modeling and field studies of in-situ flushing technologies for remediation of NAPLs have been reported in the literature. The Ground Water Remediation Technologies Analysis Center (GWRTAC) maintains a database of in-situ flushing studies both on-going and completed, including field studies. GWRTAC (1998) summarizes 84 case studies and found that $24 \%$ were laboratory/bench scale, $50 \%$ were pilot/field demonstration and $26 \%$ were full-scale/commercial flushing projects. As of November 1998, 57\% have been completed, $25 \%$ are in-progress, and the remaining are in various stages of design. A summary of the field-scale projects is provided in Table 1 (GWRTAC 1998). Of these field studies, several that have been reported in the literature were reviewed and are presented in this section. A variety of flushing solutions, including surfactants, cosolvents and a combination of surfactants and cosolvents have been investigated in recent years. Of the 84 studies reviewed by GWRTAC (1998), 9\% were cosolvents, $54 \%$ were surfactants, $17 \%$ were water and the remaining $20 \%$ were other methods. The design of the field studies are generally based on laboratory and modeling studies. Sabatini et al. (1997), for example, investigated the use of surfactants to enhance remediation using an vertical circulation well. According to Sabatini et al. (1997), laboratory experiments and model simulations were crucial for obtaining regulatory approval to conduct the field test.

This section presents a review of various field studies of cosolvent and surfactant flushing at various stages of implementation. In addition, a simple groundwater model was developed using the Princeton Transport Code (PTC) and used to compare various flushing scenarios for an in-situ flushing scheme.

\subsection{Field Studies}

Field application of in-situ flushing has only been on-going for the past decade and has included surfactants, cosolvents, foams or combinations of these. One of the earliest in-situ field studies considered surfactant flushing of soils contaminated with oils and PCBs. It was conducted in the early 1990s by Abdul et al. (1992) and Abdul and Ang (1994). Within the past few years several more field applications have been conducted on LNAPLs and DNAPLs in the saturated zone. Table 8.2 contains a summary of these studies reviewed including site location, NAPL of concern, flushing details, and flush efficiency. Table 8.3 provides a plan view of the pumping scenarios used in the various studies.

Two recent in-situ cosolvent flushing field applications using ethanol as the cosolvent to remove PCE have been conducted by researchers at the University of Florida (Annable 1999). At the time of this report, however, the details of these projects had not been published and are not include. However, the results showed that alcohol flushing is very effective at removing PCE and that it offers a simple and effective means of removing DNAPL from the saturated zone (Annable 1999).

A summary of several of the in-situ flushing research studies reviewed is provided below. These 


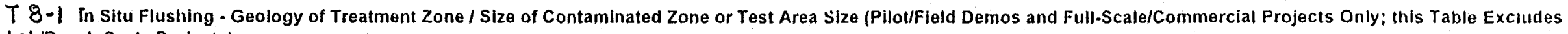
Lab/Bench-Scale Projects)

Total Number of Case Studies $=64$

\begin{tabular}{|c|c|c|c|c|c|c|c|}
\hline $\begin{array}{c}\text { GWRTAC } \\
\text { ID } \\
\end{array}$ & $\begin{array}{c}\text { Project } \\
\text { Name } \\
\end{array}$ & $\begin{array}{c}\text { Geology of Maln Target } \\
\text { Treatment Zone } \\
\end{array}$ & $\begin{array}{c}\text { Geology of Target Treatment } \\
\text { Zone Clarification }\end{array}$ & $\begin{array}{c}\begin{array}{c}\text { Volume of } \\
\text { Contaminated } \\
\text { Soll (Vadose Zone) }\end{array} \\
\end{array}$ & $\begin{array}{c}\text { Maximum Areal } \\
\text { Extont of Main } \\
\text { Ground-water } \\
\text { Contaminant Plume }\end{array}$ & $\begin{array}{c}\text { Pore Volume } \\
\text { Contained in } \\
\text { Main Ground-water } \\
\text { Contaminant Plume }\end{array}$ & $\begin{array}{c}\text { Scale of } \\
\text { Project }\end{array}$ \\
\hline FLSH0066 & Aluminum Company Site & $\begin{array}{l}\text { Unconsol. Sedș. - Poonly Sorred } \\
\text { Predom. Fine-Grained }\end{array}$ & Helerogeneous, silty backfill & $1,000 \mathrm{~m} 3$ (about $1,300 \mathrm{yd} 3$ ) & & & $\begin{array}{l}\text { Full- } \\
\text { Scale/Commercial }\end{array}$ \\
\hline FLSH0O02 & $\begin{array}{l}\text { Bog Ureek Farm, Howell } \\
\text { Township. NJ }\end{array}$ & Unspecified & & & & & $\begin{array}{l}\text { Full- } \\
\text { Scale/Commercial }\end{array}$ \\
\hline FLSH0039 & Camp Lejeune & $\begin{array}{l}\text { Unconsol. Seds. - Predom. Well Sorted } \\
\text { Sand/Gravel }\end{array}$ & $\begin{array}{l}\text { Fine to very fine sand underlain by clay } \\
\text { aquilard }\end{array}$ & & & $\begin{array}{l}4.800 \text { gallon swept poref } \\
\text { volume in lest zone } \\
\text { (contained } 87 \text { gal } \\
\text { ONAPL before SEAR) }\end{array}$ & $\begin{array}{l}\text { Piloufield } \\
\text { Demonstration }\end{array}$ \\
\hline FLSHO003 & $\begin{array}{l}\text { Canadian AFB Borden, Allision. } \\
\text { Ontario, Canada }\end{array}$ & $\begin{array}{l}\text { Unconsol. Seds. - Predom. Well Sorled } \\
\text { Sand/Gravel }\end{array}$ & $\begin{array}{l}\text { Unconsolidaled sand aquifer, St. } \\
\text { Lawrence Lowlands - beach sand } \\
\text { deposit undertain by glacial till }\end{array}$ & & $\begin{array}{l}9.8 \times 9.8(001(96 \mathrm{ft} 2) \\
\text { lest cell }\end{array}$ & 2,400 gallions & $\begin{array}{l}\text { Pilolifield } \\
\text { Demonsiration }\end{array}$ \\
\hline FLSH0047 & $\begin{array}{l}\text { Cape Canaveral Lodge } \\
\text { Complex } 34\end{array}$ & $\begin{array}{l}\text { Unconsol. Seds. - Poonly Sorted } \\
\text { Predom. Coarse Grained }\end{array}$ & Barrier island sediments & & $50 \times 75$ fool lesl area & & $\begin{array}{l}\text { Pilou/field } \\
\text { Demonsiration }\end{array}$ \\
\hline FLSHOOO4 & Chem-Dyne, Hamillon, $\mathrm{OH}$ & $\begin{array}{l}\text { Unconsol. Seds. - Poorly Sorted } \\
\text { Predom. Coarse Grained }\end{array}$ & & & & & $\begin{array}{l}\text { Full- } \\
\text { Scale/Commercial }\end{array}$ \\
\hline FLSH0077 & $\begin{array}{l}\text { Coasi Guard Facility, Elizabein } \\
\text { Cily, NC }\end{array}$ & Unspecified & NA (Lab Sludy) & & & & $\begin{array}{l}\text { Laboralory/Bench } \\
\text { Scale }\end{array}$ \\
\hline FLSHOO2O & Confidential & $\begin{array}{l}\text { Unconsol. Seds. - Poorly Sorted } \\
\text { Predom. Coarse Grained }\end{array}$ & $\begin{array}{l}\text { Coastal Plain - The Surfical Aquiler } \\
\text { (silty fine sand) }\end{array}$ & & . & & $\begin{array}{l}\text { Full- } \\
\text { Scale/Commercial }\end{array}$ \\
\hline FLSHO00S & $\begin{array}{l}\text { Cornell University - Surfactant } \\
\text { Enhance Biodegradation }\end{array}$ & Unconsol. Seds. - Predom. Clay/Sill & & & & & $\begin{array}{l}\text { LaboralorylBench } \\
\text { Scale }\end{array}$ \\
\hline FLSH0006 & $\begin{array}{l}\text { Corpus Christi Dupont, Corpus } \\
\text { Christi, TX } \\
\end{array}$ & $\begin{array}{l}\text { Unconsol. Seds. - Predom. Well Sorted } \\
\text { SandiGravel }\end{array}$ & $\begin{array}{l}\text { Gulf Coast, well-sorled fine sand } \\
\text { inierbedded wilh clay }\end{array}$ & & $\begin{array}{l}25 \times 35 \text { fool }(87512) \\
\text { lest area }\end{array}$ & & $\begin{array}{l}\text { Pilou/Field } \\
\text { Demonstration }\end{array}$ \\
\hline FLSH0007 & $\begin{array}{l}\text { Cross Brothers Pall, Pembroke, } \\
\text { il }\end{array}$ & $\begin{array}{l}\begin{array}{l}\text { Unconsol. Seds. - Predom. Well Sorled } \\
\text { Sand/Gravel }\end{array} \\
\end{array}$ & $\begin{array}{l}\text { Very well sorted tine-grained sand } \\
\text { underlain by clay confining layer al } 45 \text {. } \\
50 \text { leel bgs }\end{array}$ & $\begin{array}{l}4 \text { lo } 6 \text { acre extent source } \\
\text { area }\end{array}$ & & & $\begin{array}{l}\text { Full- } \\
\text { Scale/Conmercial }\end{array}$ \\
\hline FLSH0062 & $\begin{array}{l}\text { Dover AFB. Dover. DE } \\
\text { (Cosolven! Mobilizalion) }\end{array}$ & $\begin{array}{l}\text { Unconsul. Seds. - Poorly Sorted } \\
\text { Predom. Coarse Grained }\end{array}$ & $\begin{array}{l}\text { Lynch Heights and Columbia Fms. } \\
\text { uncontined aquifer - silty, poorly soned } \\
\text { sands, polential for discontinuous clay } \\
\text { lenses and gravely sand lenses }\end{array}$ & : & & & \begin{tabular}{|l} 
Pilot/field \\
Demonstration
\end{tabular} \\
\hline FLSH0063 & $\begin{array}{l}\text { Oover AFB. Dover, DE } \\
\text { (Macromolecular Solubilization) }\end{array}$ & $\begin{array}{l}\text { Unconsol. Seds. - Poorly Sorted } \\
\text { Predom. Coarse Grained }\end{array}$ & $\begin{array}{l}\text { Lynch Heights and Columbia Fms. } \\
\text { unconfined aquifer - silty, poorly sorted } \\
\text { sands, potential for discontinuous clay } \\
\text { lenses and gravely sand lenses }\end{array}$ & 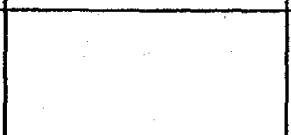 & & & $\begin{array}{l}\text { Pilou/field } \\
\text { Demonstration }\end{array}$ \\
\hline FLSH0064 & $\begin{array}{l}\text { Dover AFB, Dover, DE (Single- } \\
\text { phase Microemulsion) }\end{array}$ & $\begin{array}{l}\text { Unconsol. Seds. - Poorly Sorted } \\
\text { Predom Coarse Grained }\end{array}$ & $\begin{array}{l}\text { Lynch Heights and Columbia Fms } \\
\text { unconfined aquifer - silly, poarly sorted } \\
\text { sands, potential for disconlinuous clay } \\
\text { lenses and gravely sand lenses }\end{array}$ & & & & $\begin{array}{l}\text { Pilouffield } \\
\text { Demonstration }\end{array}$ \\
\hline FLSHO061 & $\begin{array}{l}\text { Oover AFB, Dover. DE (Test } \\
\text { Cell 2. Surlaclant Solubilization }\end{array}$ & $\begin{array}{l}\text { Unconsol. Seds. - Poorly Sorled } \\
\text { Predom Coarse Grained }\end{array}$ & $\begin{array}{l}\text { Lynch Heights and Columbia Fms. } \\
\text { unconfined aquiler - silty, poorly sorled } \\
\text { sands, potential for disconthnuous clay } \\
\text { lenses and gravely sand lenses }\end{array}$ & & & & $\begin{array}{l}\text { Piloufield } \\
\text { Demonstration }\end{array}$ \\
\hline
\end{tabular}




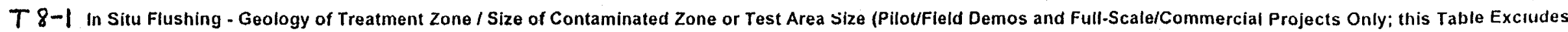
Lab/Bench-Scale Projects)

Total Number of Case Studies $=64$

\begin{tabular}{|c|c|c|c|c|c|c|c|}
\hline $\begin{array}{l}\text { GWRTAC } \\
\text { ID }\end{array}$ & $\begin{array}{l}\text { Project } \\
\text { Name }\end{array}$ & $\begin{array}{l}\text { Geology of Main Target } \\
\text { Treatment Zone }\end{array}$ & $\begin{array}{c}\text { Goology of Target Treatment } \\
\text { Zone Clariflcation }\end{array}$ & $\begin{array}{c}\text { Volume of } \\
\text { Contaminated } \\
\text { Soll (Vadose Zone) }\end{array}$ & \begin{tabular}{|c|} 
Maximum Areal \\
Extent of Main \\
Ground-water \\
Contaminant Plume
\end{tabular} & \begin{tabular}{|c|} 
Pore Volume \\
Contained in \\
Main Ground-water \\
Contaminant Plume \\
\end{tabular} & $\begin{array}{l}\text { Scale of } \\
\text { Project }\end{array}$ \\
\hline FLSHO0Og & \begin{tabular}{|l} 
Daver AFB, Dover, DE (TesI \\
Cell 3. Cosolvent Solubilization)
\end{tabular} & $\begin{array}{l}\text { Unconsol. Seds. Poorly Sorled } \\
\text { Predom. Coarse Grained }\end{array}$ & $\begin{array}{l}\text { Lynch Heights and Columbia fms. } \\
\text { unconfined aquifer - silty, poorly sorted } \\
\text { sands, potential for discontinuous clay } \\
\text { lenses and gravely sand lenses }\end{array}$ & & & & $\begin{array}{l}\text { Pilol/field } \\
\text { Demonstration }\end{array}$ \\
\hline FLSH0010 & \begin{tabular}{|l} 
Eckentelder, Inc. - Surlactant \\
Recycling
\end{tabular} & Unspecitied & Lab siudy-soil lest bed & & & & \begin{tabular}{|l|} 
Pitotfifield \\
Demonsiration
\end{tabular} \\
\hline FLSHOO73 & \begin{tabular}{|l|}
$\begin{array}{l}\text { Envirogen - Effects of Emulsion } \\
\text { Viscosity }\end{array}$ \\
\end{tabular} & Unspecified & NA (Lab Study) & & & & $\begin{array}{l}\text { Laboralory/Bench } \\
\text { Scale }\end{array}$ \\
\hline FLSH 0072 & \begin{tabular}{|l|} 
Envirogen - Surfaclant \\
Foam/Ejoaugmentalion
\end{tabular} & Unspecified & NA (Lab Study) & & & & $\begin{array}{l}\text { Laboratory/Bench } \\
\text { Scale }\end{array}$ \\
\hline FLSHO011 & $\begin{array}{l}\text { Estrie Region Machine Shop. } \\
\text { Quebec, CA }\end{array}$ & $\begin{array}{l}\text { Unconsol. Seds. - Predom. Well Sorted } \\
\text { Sand/Gravel }\end{array}$ & $\begin{array}{l}\text { St. Lawrence Lowlands - Fine sand witt } \\
10-12 \% \text { silt }\end{array}$ & $1,800 \mathrm{~m}^{3}$ (aboul $\left.63,575 \mathrm{fl2}\right)$ & & & $\begin{array}{l}\text { Full- } \\
\text { Scale/Commercial }\end{array}$ \\
\hline FLSH0021 & $\begin{array}{l}\text { Former Dry Cleaning Facilly, } \\
\text { Jacksonville, FL. }\end{array}$ & $\begin{array}{l}\text { Unconsol. Seds. - Predom. Well Sorled } \\
\text { Sand/Gravel }\end{array}$ & Sandy alluvium & & & & $\begin{array}{l}\text { Pitot/Field } \\
\text { Demonstration }\end{array}$ \\
\hline FLSH0059 & \begin{tabular}{|l|}
$\begin{array}{l}\text { Fredricksburg, VA Wood } \\
\text { Trealing Slle }\end{array}$ \\
\end{tabular} & \begin{tabular}{|l|} 
Unconsol. Seds- - Poorly Sorted \\
Predom. Fine-Grained
\end{tabular} & $\begin{array}{l}\text { Clay-sill alluvium, cresole perched on } \\
\text { fiay tenses }\end{array}$ & & & & $\begin{array}{l}\text { Piloulfield } \\
\text { Demonstration }\end{array}$ \\
\hline FLSH0012 & $\begin{array}{l}\text { General Motors NAO Research } \\
\text { \& Development Center. } \\
\text { Warren, MI }\end{array}$ & $\begin{array}{l}\text { Unconsol. Seds. - Interbedded } \\
\text { Sediments }\end{array}$ & $\begin{array}{l}\text { Central Glacialed Plains - } 10 \text { to } 13 \text { feet } \\
\text { of sandy fill overtying fine-grained } \\
\text { alluvial and sandy glacial outwash }\end{array}$ & $\begin{array}{l}10 \text { tool diameler } \times 5 \text { fool } \\
\text { deep lesi plot }\end{array}$ & & & $\begin{array}{l}\text { Piloufield } \\
\text { Demionstration }\end{array}$ \\
\hline FLSH0084 & \begin{tabular}{|l|} 
Georessources - Aquifer \\
Washing by Micellar Solutions
\end{tabular} & Unspecified & NA (Lab Sludy) & & & & $\begin{array}{l}\text { Laboratory/Bench } \\
\text { Scale }\end{array}$ \\
\hline FLSH0013 & $\begin{array}{l}\text { GHEA Associales, New Jersey } \\
\text { Operating Facilly of Major U.S. } \\
\text { Corporation }\end{array}$ & Unconsol. Seds. - Predom. Clay/Silt & Clayey soils & & & & $\begin{array}{l}\text { Pilot/field } \\
\text { Demonstration }\end{array}$ \\
\hline FLSHOO14 & $\begin{array}{l}\text { Goose Farm. Plumsled Twp. } \\
\text { NJ }\end{array}$ & $\begin{array}{l}\text { Unconsol. Seds. - Poonly Sorled } \\
\text { Predonl. Coarse Grained }\end{array}$ & Coastal Plain - Kirkwood Aquiler & & & & $\begin{array}{l}\text { Fult- } \\
\text { Scale/Commercial }\end{array}$ \\
\hline FLSHOOSO & Gull Power - Lymn Haven, FL & $\begin{array}{l}\text { Unconisol. Seds. - Poorly Sorled } \\
\text { Predom Coarso Grained }\end{array}$ & $\begin{array}{l}\begin{array}{l}\text { Coastal Plain - The Surfical Aquiler } \\
\text { (silty line sand) }\end{array} \\
\end{array}$ & & & & $\begin{array}{l}\text { Full- } \\
\text { Scale/Commercial }\end{array}$ \\
\hline FLSH0058 & Hialeah Counly, FL & Bedrock - Carbonate - Karst & $\begin{array}{l}\text { SE Coastal Plain Cly org rich sand over } \\
\text { solution cavily limestone }\end{array}$ & & & & $\begin{array}{l}\text { Pitol/Field } \\
\text { Demonstration }\end{array}$ \\
\hline FLSH0056 & \begin{tabular}{|l|} 
Hill Air Force Base (Cell 4, OU \\
1 - Cyclodextrin Solubilization)
\end{tabular} & $\begin{array}{l}\text { Unconsol. Seds. - Poorly Sorted } \\
\text { Predom. Coarse Grained }\end{array}$ & $\begin{array}{l}\text { Great Basin - Poorly sorled alluvial } \\
\text { sand and gravel undentain by lacustrian } \\
\text { clay }\end{array}$ & & $\begin{array}{l}3 \times 5 \mathrm{~m}(9.8 \times 16.4 \mathrm{ft}) \text { or } \\
\text { about } 160 \mathrm{t2} 2 \text { lest cell }\end{array}$ & 1.000 to 2,500 gallons & $\begin{array}{l}\text { Pilloufifield } \\
\text { Demonstration }\end{array}$ \\
\hline FLSHOO55 & $\begin{array}{l}\text { Hill Air Force Base (OU 2- } \\
\text { Foam Flood) }\end{array}$ & $\begin{array}{l}\text { Unconsol. Seds. - Poorly Sorted } \\
\text { Predom. Coarse Grained }\end{array}$ & $\begin{array}{l}\text { Greal Basin - Poorly sonted alluvial } \\
\text { sand and gravel underlain by lacustrian } \\
\text { clay }\end{array}$ & & & & $\begin{array}{l}\text { Piloufiela } \\
\text { Demonstration }\end{array}$ \\
\hline FLSH0057 & $\begin{array}{l}\text { Hill Air Force Base (OU2 1/5 } \\
\text { Full Scale Surfactant Flood) }\end{array}$ & $\begin{array}{l}\text { Unconsol. Seds. Poorly Sorlod } \\
\text { Predom. Coarse Grained }\end{array}$ & $\begin{array}{l}\text { Great Basin - Poorly sorted alluvial } \\
\text { sand and gravel underlain by lacustrian } \\
\text { clay }\end{array}$ & & & & $\begin{array}{l}\text { Full- } \\
\text { Scale/Commercral }\end{array}$ \\
\hline FLSH0015 & $\begin{array}{l}\text { Hill Air Force Base, UT (Cell 3, } \\
\text { Ou1 - Cosolvent Mobilization) }\end{array}$ & $\begin{array}{l}\text { Unconsol. Seds. - Poorly Sorled } \\
\text { Predom. Coarse Grained }\end{array}$ & $\begin{array}{l}\text { Great Basin-Poorly sorted alluvial sand } \\
\text { and gravel underiain by lacustrian clay }\end{array}$ & & $\begin{array}{l}3 \times 5 \mathrm{~m}(9.8 \times 16.4 \mathrm{fl} \text { or } \\
\text { about } 160 \mathrm{nI} 2) \text { test cell }\end{array}$ & 1,500 gallons & $\begin{array}{l}\text { Plot/Field } \\
\text { Demorstration }\end{array}$ \\
\hline FLSH0016 & $\begin{array}{l}\text { Hill Air Force Base, UT (Cell 5. } \\
\text { OU1 - Surtactant Mobilization) }\end{array}$ & $\begin{array}{l}\text { Unconsol. Seds. - Poorly Sorted } \\
\text { Predoin. Coarse Grained }\end{array}$ & $\begin{array}{l}\text { Greal Basin - Poorly sorted alluvial } \\
\text { sand and gravel underlain by lacustran' } \\
\text { clay }\end{array}$ & & $\begin{array}{l}3 \times 5 \mathrm{~m}(9.8 \times 16.411 \text { or } \\
\text { aboul } 160 \text { 112) lest cell }\end{array}$ & 1,500 to 2,500 gallons & $\begin{array}{l}\text { Piloufield } \\
\text { Demonstration }\end{array}$ \\
\hline FLSHO017 & $\begin{array}{l}\text { Hill Air Force Base, Ur (Cell } 6 . \\
\text { OU1-Surlactant Solubilization) }\end{array}$ & $\begin{array}{l}\text { Unconsol. Seds. - Poorly Sorted } \\
\text { Piedom. Coarse Grained }\end{array}$ & $\begin{array}{l}\text { Greal Basin - Poorly sorled alluvial } \\
\text { sand and gravel underlain by lacustrian }\end{array}$ & & $\begin{array}{l}3 \times 5 \mathrm{~m}(9.8 \times 16.4 \mathrm{flor} \\
\text { aboul } 160 \mathrm{ft} 2) \text { lest cell }\end{array}$ & & $\begin{array}{l}\text { Piloufield } \\
\text { Demansiration }\end{array}$ \\
\hline
\end{tabular}




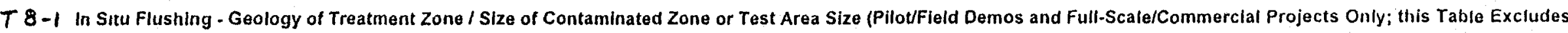
Lab/Bench-Scale Projects)

Total Number of Case Studies $=64$

\begin{tabular}{|c|c|c|c|c|c|c|c|}
\hline $\begin{array}{l}\text { GWRTAC } \\
\text { ID }\end{array}$ & $\begin{array}{l}\text { Project } \\
\text { Name }\end{array}$ & $\begin{array}{l}\text { Goology of Main Target } \\
\text { Treatment Zone }\end{array}$ & $\begin{array}{l}\text { Geology of Target Treatment } \\
\text { Zone Clarification }\end{array}$ & $\begin{array}{l}\text { Volume of } \\
\text { Contaminated } \\
\text { Soll (Vadose Zone) }\end{array}$ & \begin{tabular}{|c|} 
Maximum Areal \\
Extent of Main \\
Ground-water \\
Contaminant Plume
\end{tabular} & $\begin{array}{c}\text { Pore Volume } \\
\text { Contained in } \\
\text { Main Ground-water } \\
\text { Contaminant Plume }\end{array}$ & $\begin{array}{l}\text { Scale of } \\
\text { Project }\end{array}$ \\
\hline FLSH0018 & $\begin{array}{l}\text { Hill Air Force Base, UT (Cell } 8 . \\
\text { Ou1 - SurfactanUCosolvent } \\
\text { Snílubilizalion) }\end{array}$ & $\begin{array}{l}\text { Unconsol. Seds. - Poorly Sorted } \\
\text { Predom. Coarse Grained }\end{array}$ & $\begin{array}{l}\text { Greal Basin - Poorly sorled alluvial } \\
\text { sand and gravel underlain by lacustrian } \\
\text { clay }\end{array}$ & & $\begin{array}{l}3 \times 5 \mathrm{~m}(9.8 \times 16.4 \mathrm{fl} \text { af } \\
\text { about } 160 \mathrm{fl}) \text { lest cell }\end{array}$ & 1,000 to 2,500 gallons & $\begin{array}{l}\text { Pinolfirield } \\
\text { Demonstration }\end{array}$ \\
\hline FLSH0019 & $\begin{array}{l}\text { Hill Air Force Base. UT (OU2 - } \\
\text { Micellar Flood) }\end{array}$ & $\begin{array}{l}\text { Unconsol. Seds. - Poorly Sorled } \\
\text { Predom. Coarse Grained }\end{array}$ & $\begin{array}{l}\text { Greal Basin-Poorly sorted alluvial } \\
\text { sand and gravel underlain by lacustrian } \\
\text { clay }\end{array}$ & & \begin{tabular}{|l|}
$6 \times 9 \mathrm{~m}(19.7 \times 29.5 \mathrm{il}$ \\
about $580 \mathrm{ft} 2)$ lest area
\end{tabular} & 15,000 gallons & $\begin{array}{l}\text { Pilouf Field } \\
\text { Demonstration }\end{array}$ \\
\hline FLSH0022 & $\begin{array}{l}\text { Hill Air Force Base, UT (Test1. } \\
\text { OU1 - Cosolvent Solubilization) }\end{array}$ & $\begin{array}{l}\text { Unconsol. Seds. - Poorly Sorled } \\
\text { Predom. Coarse Grained }\end{array}$ & $\begin{array}{l}\text { Great Basin - Poorly sorted alluviat } \\
\text { sand and gravel underlain by lacustrian } \\
\text { clay }\end{array}$ & & $\begin{array}{l}3 \times 5 \mathrm{~m}(9.8 \times 16.4 \mathrm{ht} \text { or } \\
\text { aboul } 160 \mathrm{il} 2) \text { lest cell }\end{array}$ & $\begin{array}{l}4.000 \text { L L (aboul } 1,057 \\
\text { gal) }\end{array}$ & $\begin{array}{l}\text { Pilot/freld } \\
\text { Demonstration }\end{array}$ \\
\hline FLSH0023 & $\begin{array}{l}\text { Hooker ChemicallRuco } \\
\text { Polymer, NY }\end{array}$ & $\begin{array}{l}\text { Unconsol. Seds. - Predom, Well Sorled } \\
\text { Sand/Gravel }\end{array}$ & Upper Glacial Formalion & & & & $\begin{array}{l}\text { Full- } \\
\text { Scate/Commercial }\end{array}$ \\
\hline FLSH0024 & $\begin{array}{l}\text { Howard University. } \\
\text { Washington, DC - Trealment of } \\
\text { PCBs with Surfactants }\end{array}$ & Unspecified & Lab sludy & & & & $\begin{array}{l}\text { Laboratory/Bench } \\
\text { Scale }\end{array}$ \\
\hline FLSH0025 & JADCO-Hughes, Belmonl, NC & Unspecified & & & & & \begin{tabular}{|l|} 
Pitol/field \\
Demonsiration
\end{tabular} \\
\hline FLSHOOO1 & $\begin{array}{l}\text { Koppers Co. Inc.. Seaboard } \\
\text { Plant, Kearny. NJ }\end{array}$ & $\begin{array}{l}\text { Unconsol. Seds. - Poonly Sorted } \\
\text { Predom. Coarse Grained }\end{array}$ & Fill unit & & & & $\begin{array}{l}\text { Piloulfield } \\
\text { Demonsiration }\end{array}$ \\
\hline FLSH0O26. & $\begin{array}{l}\text { Laramie, WY, Private wood } \\
\text { trealing site }\end{array}$ & $\begin{array}{l}\text { Unconsol. Seds. - Poorly Sorted } \\
\text { Predom. Coarse Grained }\end{array}$ & $\begin{array}{l}\text { Gradualed from fine sands, silts and } \\
\text { clays at surface to coarse sands and } \\
\text { fine gravels at base }\end{array}$ & $\begin{array}{l}>500,000 \text { yd } 3 \text { lotal }(27 \times 27 \\
\text { lest cell and } 130 \times 130 \text { II tes } \\
\text { cell) }\end{array}$ & $\begin{array}{l}(27 \times 27 \mathrm{fl}(729 \mathrm{fl}) \text { lest } \\
\text { cell and } 130 \times 130 \mathrm{ft} \\
(16.900 \mathrm{fl} 2) \text { lest cell) }\end{array}$ & $\begin{array}{l}5.000 \text { gallons within } 27 \\
\times 27 \text { fl test cell }\end{array}$ & $\begin{array}{l}\text { Pilou/field } \\
\text { Demonstration }\end{array}$ \\
\hline FLSHOO27 & Lee Chemical, Liberly, MO & $\begin{array}{l}\text { Unconsol. Seds - Predom, Well Sorled } \\
\text { Sand/Gravel }\end{array}$ & $\begin{array}{l}\text { a Missouri River bottom alluvial } \\
\text { sediments }\end{array}$ & & & . & $\begin{array}{l}\text { Full- } \\
\text { Scale/Conmercial }\end{array}$ \\
\hline FLSH0028 & Lipari Landfilt, Pitman, $N\rfloor$ & Unspectied & Cohansay Aquifer & & & & $\begin{array}{l}\text { Full- } \\
\text { Scale/Conmercial }\end{array}$ \\
\hline FLSH0071 & $\begin{array}{l}\text { Louisiana State Universily - } \\
\text { Colloidal Gas Aphron from } \\
\text { Naturally-Derived Surfaclant }\end{array}$ & Unspecilied & NA (Lab Study) & & & & $\begin{array}{l}\text { Laboralory/Bench } \\
\text { Scale }\end{array}$ \\
\hline FLSH0029 & \begin{tabular}{|l|} 
Michigan Stale Universily- \\
Surfactant Research and Soils \\
Modification with Surfactants
\end{tabular} & Unspecified & Lab studies & & & & $\begin{array}{l}\text { Laboratory/Bench } \\
\text { Scale }\end{array}$ \\
\hline FLSHOO30 & $\begin{array}{l}\text { Montana Pole \& Trealing. Bulle } \\
\text { MT }\end{array}$ & $\begin{array}{l}\text { Unconsol. Seds. - Predom. Well Sorted } \\
\text { Sand/Gravel }\end{array}$ & Sandy alluvial malerial & $\begin{array}{l}200,000 \text { ydu total site } \\
(50,000 \text { lo } 60,000 \text { yd } 3 \text { may } \\
\text { be remediated via in silu } \\
\text { flushing) }\end{array}$ & & 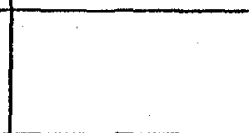 & 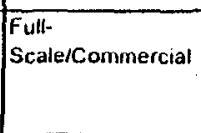 \\
\hline FLSH0070 & $\begin{array}{l}\text { National Tsing Hua University- } \\
\text { Effects of Surfactants of } \\
\text { Sorption/Micellar Solubilization }\end{array}$ & Unspecified & NA (Lab Study) & & & & $\begin{array}{l}\text { LaboratorylBench } \\
\text { Scale }\end{array}$ \\
\hline FLSH0060 & $\begin{array}{l}\text { National Water Res Inst, Env } \\
\text { Canada - Humic Acid Flushing }\end{array}$ & Unspecilied & Mediurn to coarse-graned sand & & & & $\begin{array}{l}\text { Laboratory/gench } \\
\text { Scale }\end{array}$ \\
\hline FLSH0031 & Ninith Avenue Dump. Gary, IN & $\begin{array}{l}\text { Unconsol. Seds. - Poorly Sorted } \\
\text { Predom. Coarse Grained }\end{array}$ & $\begin{array}{l}\text { Sand/wasle (Mostly sand bul mixed } \\
\text { with debris (limbers, elc.). Originally } \\
\text { was welland (Dune and Swale) } \\
\text { formation particular to northern indiana }\end{array}$ & & & & $\begin{array}{l}\text { Fuil- } \\
\text { Scale/Commercial }\end{array}$ \\
\hline
\end{tabular}




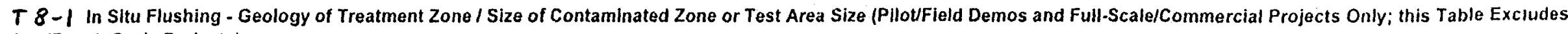
Lab/Bench-Scale Projects)

Total Number of Case Studies $=6$

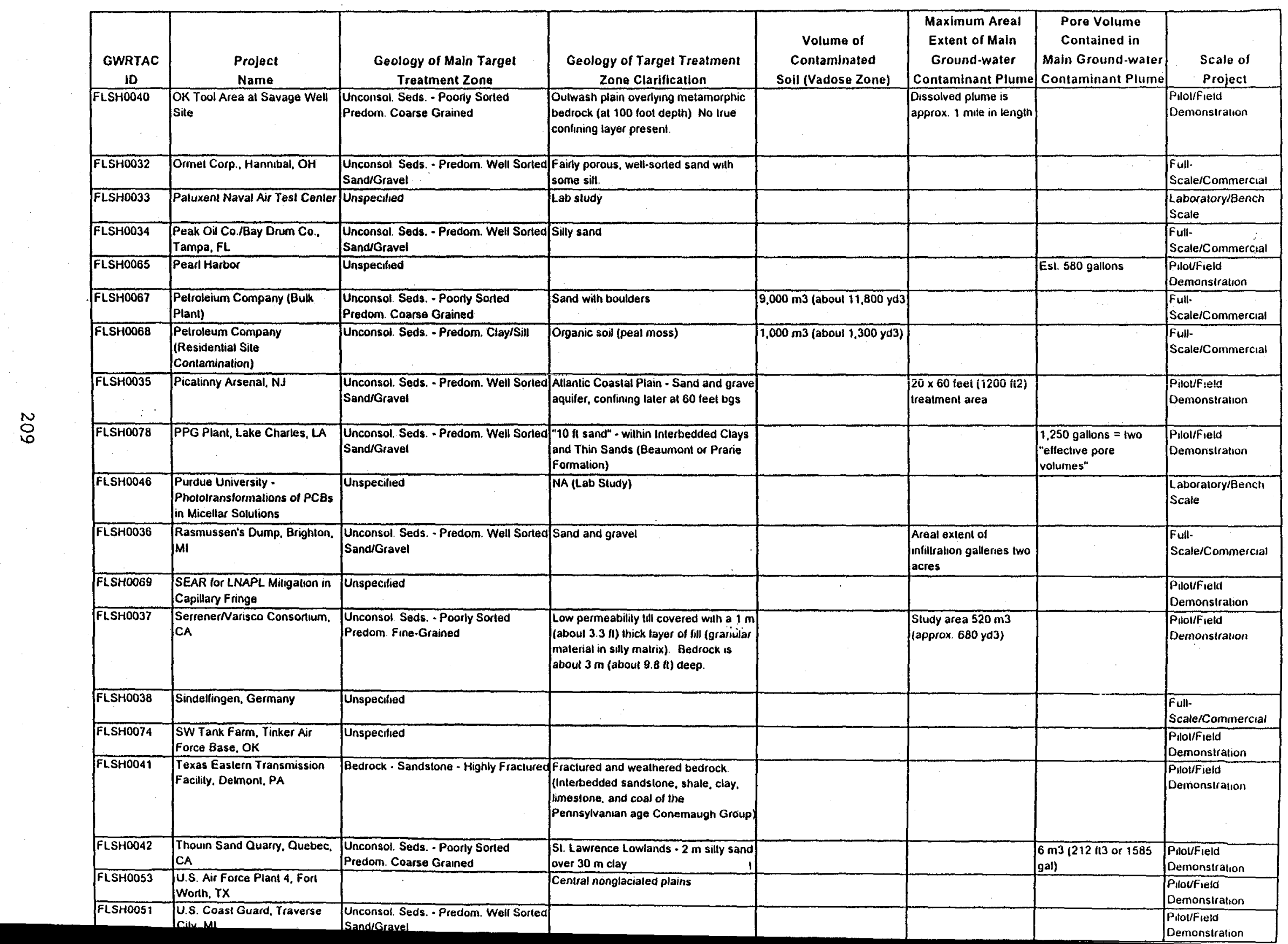




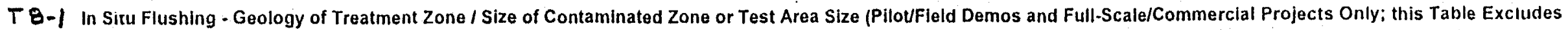
Lab/Bench-Scale Projects)

Total Number of Case Studies $=64$

\begin{tabular}{|c|c|c|c|c|c|c|c|}
\hline $\begin{array}{l}\text { GWRTAC } \\
\text { ID }\end{array}$ & $\begin{array}{l}\text { Project } \\
\text { Name }\end{array}$ & $\begin{array}{l}\text { Geology of Main Target } \\
\text { Treatment Zone }\end{array}$ & $\begin{array}{c}\text { Geology of Target Treatment } \\
\text { Zone Clarification }\end{array}$ & $\begin{array}{c}\text { Volume of } \\
\text { Contaminated } \\
\text { Soil (Vadose Zone) }\end{array}$ & $\begin{array}{c}\text { Maximum Areal } \\
\text { Extent of Maln } \\
\text { Ground-water } \\
\text { Contaminant Plume }\end{array}$ & $\begin{array}{c}\text { Pore Volume } \\
\text { Contained in } \\
\text { Main Ground-water } \\
\text { Contaminant Plume }\end{array}$ & $\begin{array}{l}\text { Scale of } \\
\text { Project }\end{array}$ \\
\hline FLSH0054 & $\begin{array}{l}\text { U.S. DOE Gaseous Diffusion } \\
\text { PInt, Paducah. KY }\end{array}$ & $\begin{array}{l}\text { Unconsal. Seds. - Predom. Well Sorted } \\
\text { Sand/Gravel }\end{array}$ & $\begin{array}{l}\text { Appalachian Plateau Gravelly Fine to } \\
\text { Medium Sand }\end{array}$ & & (19 & & $\begin{array}{l}\text { Pitloufield } \\
\text { Demonstration }\end{array}$ \\
\hline$\longdiv { \text { FLSH0075 } }$ & $\begin{array}{l}\text { U.S. DOE Paducah Gaseous } \\
\text { Diffusion Plant }\end{array}$ & \begin{tabular}{|l|} 
Unspecilied \\
\end{tabular} & & & & & $\begin{array}{l}\text { Laboralory/Bench } \\
\text { Scale }\end{array}$ \\
\hline FLSHOOOB & $\begin{array}{l}\text { U.S. DOE Portsmoulh Gaseous } \\
\text { Diffusion Sile. Pikelon. OH }\end{array}$ & $\begin{array}{l}\text { Uncoissol. Seds. - Predom. Well Sorted } \\
\text { SandiGravel }\end{array}$ & $\begin{array}{l}\text { Nonglaciated Central Plain - Basal } \\
\text { alluvial gravel (Gallia Formation) }\end{array}$ & & $16.4 \times 6.6$ leel $(108 \mathrm{fi2})$ & 3,700 gallons & $\begin{array}{l}\text { Piloufield } \\
\text { Demonsiration }\end{array}$ \\
\hline FL.SH0043 & $\begin{array}{l}\text { Umalitla Army Depol } \\
\text { (Lagoons). Hermiston, OR }\end{array}$ & $\begin{array}{l}\text { Unconsol. Seds. - Predom. Weil Sorled } \\
\text { Sand/Gravel }\end{array}$ & $\begin{array}{l}\text { Sandy alluvium w/ low organic content } \\
\text { overlying solid basall contining layer }\end{array}$ & $48,400 \mathrm{yd}$ & 350 acres & & $\begin{array}{l}\text { Full- } \\
\text { Scale/Conmercial }\end{array}$ \\
\hline FLSH0044 & $\begin{array}{l}\text { United Chrome Products, } \\
\text { Corvallis, OR }\end{array}$ & Unconsol. Seds. - Predom. Clay/Sill & $\begin{array}{l}\text { Upper waler-bear zone (sill), over silly } \\
\text { clay }\end{array}$ & & & $2.6 \mathrm{MM}$ gallons & \begin{tabular}{|l|} 
Pilotifield \\
Dermonstration
\end{tabular} \\
\hline FLSH0083 & $\begin{array}{l}\text { University of Arizona - } \mathrm{HC} \\
\text { Solubilzation and Mobilization } \\
\text { by Biosurfactant }\end{array}$ & Unspecified & NA (Lab Sludy) & & & & $\begin{array}{l}\text { Laboralory/Bench } \\
\text { Scale }\end{array}$ \\
\hline FLSH0082 & $\begin{array}{l}\text { Universily of Florida - Two- } \\
\text { Dimensional flow Experiments } \\
\text { and Simulations }\end{array}$ & Unspecified & NA (Lâb Sludy) & & & & $\begin{array}{l}\text { Laboratory/Bench } \\
\text { Scale }\end{array}$ \\
\hline FLSH0081 & $\begin{array}{l}\text { Universily of Ilinois - impact of } \\
\text { Surtactant of Configuration of } \\
\text { HC Lens }\end{array}$ & Unspecified & NA (Lab Sludy) & & $\because$ & & $\begin{array}{l}\text { Laboratory/Bench } \\
\text { Scale }\end{array}$ \\
\hline FLSH0052 & Universily of Michigan & Unspecilied & & & & & $\begin{array}{l}\text { Laboratory/Bench } \\
\text { Scale }\end{array}$ \\
\hline FLSHOOAS & $\begin{array}{l}\text { University of Michigan - } \\
\text { Surfactant Remediation ol } \\
\text { NAPL.Contaminated Aquifers }\end{array}$ & Unspecilied & & & & & $\begin{array}{l}\text { Laboralory/Bench } \\
\text { Scale }\end{array}$ \\
\hline FLSH0079 & $\begin{array}{l}\text { University of North Carolina - } \\
\text { Bank Fommation in Surfactant } \\
\text { Remediation of DNAPL }\end{array}$ & Unspecified & NA (Lab Study) & & & & $\begin{array}{l}\text { Laboralory/Bench } \\
\text { Scale }\end{array}$ \\
\hline FLSH0080 & $\begin{array}{l}\text { University of Oklahoma: } \\
\text { Remediation of Chlorinaled } \\
\text { Solvents Using Edible } \\
\text { Surfactancls }\end{array}$ & Unspecilied & NA (Lab Sludy) & . & & & $\begin{array}{l}\text { Laboralory/Bench } \\
\text { Scale. }\end{array}$ \\
\hline FLSH0078 & UST Sile, Shawnee. OK & Unspecilied & & & & & $\begin{array}{l}\begin{array}{l}\text { Pliotfield } \\
\text { Demonstration }\end{array} \\
\end{array}$ \\
\hline FLSH0048 & $\begin{array}{l}\text { Vineland Chemical, Vineland. } \\
\text { Nu }\end{array}$ & $\begin{array}{l}\text { Unconsal Seds - Predom. Well Sorted } \\
\text { Sand/Gravel }\end{array}$ & $\begin{array}{l}\text { Medium to fine sand with low organic } \\
\text { content }\end{array}$ & $126.000 \mathrm{yd} 3$ & & & $\begin{array}{l}\text { Full- } \\
\text { Scale/Commercial }\end{array}$ \\
\hline FLSHOO49 & $\begin{array}{l}\text { Volk Air National Guard Base. } \\
\text { WI }\end{array}$ & Unspecilied & Sand (Soll beneath fire training pii) & & & & $\begin{array}{l}\text { Pilou/field } \\
\text { Demonstration }\end{array}$ \\
\hline
\end{tabular}


T $8-2$. Literature Review of In-Situ Flushing Filed Studies

\begin{tabular}{|c|c|c|c|c|c|c|c|}
\hline $\begin{array}{l}\text { Author(s) and/or } \\
\text { P.I.(s) }\end{array}$ & Site Location & Contaminants & $\begin{array}{l}\text { Target } \\
\text { Media }\end{array}$ & $\begin{array}{l}\text { Flushing } \\
\text { Solution }\end{array}$ & Flow Rates & $\begin{array}{c}\text { Duration of } \\
\text { Test }\end{array}$ & \% Recovery \\
\hline $\begin{array}{l}\text { Sabatini et al } \\
\text { (1997) }\end{array}$ & $\begin{array}{l}\text { Coast Guard } \\
\text { Station, } \\
\text { Traverse City, } \\
\text { MI }\end{array}$ & $\begin{array}{l}\text { PCE, TCE and } \\
\text { recalcitrant jet } \\
\text { fuel } \\
\text { components }\end{array}$ & $\begin{array}{l}\text { Soil, Ground- } \\
\text { water }\end{array}$ & $\begin{array}{l}\text { Surfactant } \\
\text { (Dowfax 8390) }\end{array}$ & $1 \mathrm{gpm}(\mathrm{VCW})$ & 20 hours & $95 \%$ surfactant \\
\hline $\begin{array}{l}\text { McRay et al } \\
(1998)\end{array}$ & $\begin{array}{l}\text { OU 1, Hill Air } \\
\text { Force Base, UT }\end{array}$ & LNAPL & $\begin{array}{l}\text { Soil, Ground- } \\
\text { water }\end{array}$ & $\begin{array}{l}\text { Cyclodextrin } \\
(10 \% w t)\end{array}$ & $6450 \mathrm{~L} / \mathrm{d}$ & $\begin{array}{l}12 \text { days ( } w / 1 \\
\text { day rest at } 10 \\
\text { days) }\end{array}$ & $\begin{array}{l}41-44 \% \text { NAPL, } 100+1- \\
5 \% \text { cyclodextrin }\end{array}$ \\
\hline $\begin{array}{l}\text { Hirasaki et al } \\
\text { (1997) }\end{array}$ & $\begin{array}{l}\text { OU 2, Hill Air } \\
\text { Force Base, UT }\end{array}$ & $\begin{array}{l}\text { Multi- } \\
\text { component } \\
\text { DNAPL }\end{array}$ & $\begin{array}{l}\text { Soil, Ground- } \\
\text { water }\end{array}$ & $\begin{array}{l}\text { Foam/ } \\
\text { Surfactant }\end{array}$ & $\begin{array}{l}\text { ln=2 gpm } \times 3 \text { wells, } \\
\text { hc- } 1=3 \mathrm{gpm}, \mathrm{hc}-2=1 \\
\text { gpm, Out }=3.3 \mathrm{gpm} \times \\
3 \text { wells }\end{array}$ & 3.2 dilys & $\begin{array}{l}99 \% \text { surfictant, } 96 \% \\
\mathrm{NaCl}, 100++\% \\
\text { DNAPL }\end{array}$ \\
\hline Jawitz et al (1998) & $\begin{array}{l}\text { Hill Air Force } \\
\text { Base, UT }\end{array}$ & $\begin{array}{l}\text { Complex } \\
\text { NAPL }\end{array}$ & $\begin{array}{l}\text { Soil, Ground- } \\
\text { water }\end{array}$ & $\begin{array}{l}\text { Surfactant/ } \\
\text { Cosolvent }\end{array}$ & $3000 \mathrm{~L} / \mathrm{d}$ & 18 dilys & $\sim 70 \%$ NAPL \\
\hline $\begin{array}{l}\text { Londergarden et } \\
\text { al (1997) }\end{array}$ & $\begin{array}{l}\text { OU 2, Hill Air } \\
\text { Force Base, UT }\end{array}$ & $\begin{array}{l}\text { Chlorinated } \\
\text { Solvents, } \\
\text { Petroleum } \\
\text { Hydrocarbons }\end{array}$ & $\begin{array}{l}\text { Soil, Ground- } \\
\text { water }\end{array}$ & $\begin{array}{l}\text { Surfactant/ } \\
\text { Cosolvent/ } \\
\text { Salinily } \\
\text { (Electrolyte) }\end{array}$ & $\begin{array}{l}\text { (I) } 2 \mathrm{gpm} \\
\text { (II) } 2.5 \mathrm{gpm}\end{array}$ & $\begin{array}{l}\text { (I) } 0.6 \text { days } \\
\text { (II) } 3.4 \text { days }\end{array}$ & $\begin{array}{l}\text { (I) } 71 \% \text { surfactant, } \\
85 \% \text { tracers, } \sim 55 \% \\
\text { DNAPL } \\
\text { (II) } \sim 40 \% \text { original } \\
\text { (pre-(I)) DNAPL }\end{array}$ \\
\hline $\begin{array}{l}\text { Fountain et al } \\
(1997)\end{array}$ & $\begin{array}{l}\text { Canadian AFB } \\
\text { Borden }\end{array}$ & $\mathrm{PCE}$ & $\begin{array}{l}\text { Ground- } \\
\text { water } \\
\end{array}$ & Surfactant Blend & inconsistent & 6 months & $\begin{array}{l}\text {-80\% residual } \\
\text { DNAPL }\end{array}$ \\
\hline $\begin{array}{l}\text { Fountain et al } \\
\text { (1997) }\end{array}$ & $\begin{array}{l}\text { DuPont, Corpus } \\
\text { Christi, TX } \\
\end{array}$ & $\begin{array}{l}\text { Carbon } \\
\text { Tetrachloride }\end{array}$ & $\begin{array}{l}\text { Soil, Ground- } \\
\text { water }\end{array}$ & $\begin{array}{l}\text { Biodegradiable } \\
\text { Surfactants } \\
\end{array}$ & inconsistent & $10 \mathrm{PV}$ & $-100 \%$ \\
\hline $\begin{array}{l}\text { Abdul et al (1992, } \\
\text { 1994) }\end{array}$ & $\begin{array}{l}\text { General } \\
\text { Motors, } \\
\text { Warren, MI } \\
\end{array}$ & PCBs, Oils & Soil & $\begin{array}{l}0.75 \text { wt } \% \\
\text { surfactant }\end{array}$ & $\begin{array}{l}\text { (I) and (II) In }=77 \\
\text { gpd, out }=157 \text { gpd }\end{array}$ & $\begin{array}{l}\text { (I) } 70 \text { dalys } \\
\text { (II) } 90 \text { dalys }\end{array}$ & $\begin{array}{l}\text { (I) } 7 \% \\
\text { (II) } 19 \%\end{array}$ \\
\hline
\end{tabular}


T 8-3 Spatial Depiction of In-Situ Flushing Fired Studies

\begin{tabular}{|c|c|c|}
\hline $\begin{array}{c}\text { Author(s) } \\
\text { and/or P.I.(s) }\end{array}$ & Site Location & $\begin{array}{c}\text { Plan View } \\
(\mathrm{I}=\text { Injection Well, } \mathrm{E}=\text { Extraction Well, } \\
\mathrm{HC}=\text { Hydraulic Control Well (injection)) }\end{array}$ \\
\hline $\begin{array}{l}\text { Sabatini et al } \\
\text { (1997) }\end{array}$ & $\begin{array}{l}\text { Coast Guard } \\
\text { Station, } \\
\text { Traverse City, } \\
\text { MI }\end{array}$ & $\begin{array}{r}\text { Vertical (irculation ivell } \\
\text { (not modeled) }\end{array}$ \\
\hline $\begin{array}{l}\text { McRay et al } \\
\text { (1998) }\end{array}$ & $\begin{array}{l}\text { OU 1, Hill Air } \\
\text { Force Base, } \\
\text { UT }\end{array}$ & $3 m \begin{array}{ll}I & E \\
I & E \\
I & E \\
\end{array}$ \\
\hline $\begin{array}{l}\text { Hirasaki et al } \\
\text { (1997) }\end{array}$ & $\begin{array}{l}\text { OU 2, Hill Air } \\
\text { Force Base, } \\
\text { UT }\end{array}$ & 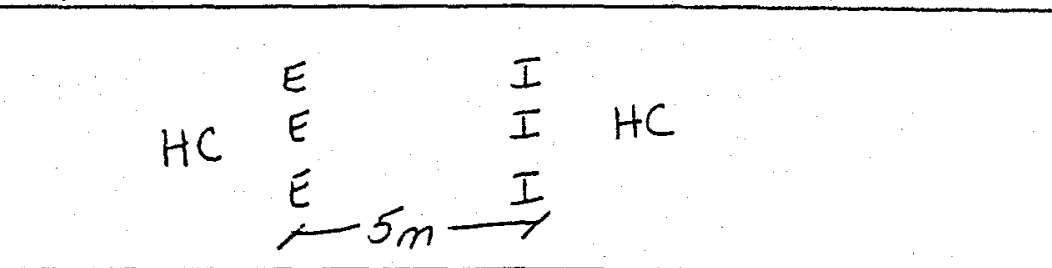 \\
\hline $\begin{array}{l}\text { Jawitz et al } \\
\text { (1998) }\end{array}$ & $\begin{array}{l}\text { Hill Air Force } \\
\text { Base, UT }\end{array}$ & $\xi^{\text {sheetpling - }}$ \\
\hline $\begin{array}{l}\text { Londergarden } \\
\text { et al (1997) }\end{array}$ & $\begin{array}{l}\text { OU 2, Hill Air } \\
\text { Force Base, } \\
\text { UT }\end{array}$ & $\begin{array}{lll}I & I C & I+ \\
E & E & E \underset{T}{T}\end{array}$ \\
\hline $\begin{array}{l}\text { Fountain et al } \\
\text { (1997) }\end{array}$ & $\begin{array}{l}\text { Canadian AFB } \\
\text { Borden }\end{array}$ & $\int_{\operatorname{sm}}\left[\begin{array}{ll}\frac{E}{I} & E \\
\frac{E}{I} & \frac{E}{E} \\
\frac{E}{I} & \text { sheet piling }\end{array}\right.$ \\
\hline $\begin{array}{l}\text { Fountain et al } \\
\text { (1997) }\end{array}$ & $\begin{array}{l}\text { DuPont, } \\
\text { Corpus } \\
\text { Christi, TX }\end{array}$ & $4 m\left\{\begin{array}{l}I E^{I} I^{I} I \\
I\end{array}\right.$ \\
\hline $\begin{array}{l}\text { Abdul et al } \\
(1992,1994)\end{array}$ & $\begin{array}{l}\text { General } \\
\text { Motors, } \\
\text { Warren, MI }\end{array}$ & $\begin{array}{l}\text { single extraction } \\
\text { well } \\
\text { (not moreled) }\end{array}$ \\
\hline
\end{tabular}


studies were chosen because they had an interesting remediation set up, similar contaminants or similar flushing solutions.

Sabatini et al. (1997)

The researchers in this field trial used a vertical circulation well (VCW) for injection and extraction. The location of the trial was in the shallow, unconfined aquifer of the Coast Guard Station in Traverse City, Michigan. This system was tested for its efficacy in controlling the chemical extractants added to the subsurface. The objective of the study was to evaluate the behavior of the flushing solution, 3.6 wt. \% Dowfax 8390 surfactant, and to recover $100 \%$ of the surfactant. The surfactant, Dowfax 8390 was supplied by Dow Chemical Company and is classified as a hexadecyl (C16) diphenyl oxide disulfonate (DPDS). A secondary objective was to demonstrate enhanced removal of PCE through the use of this in-situ flushing technique. The injection rate was approximately 1 gallon per minute (gpm) with a total of 540 gallons injected. Five hours after surfactant injection had begun, fresh water was pumped into the two nearest down gradient monitoring wells to for a hydraulic ridge. Once injection was complete (after approximately 20 hours), fresh water was pumped through the VCW. The results of the study showed that there was nearly $95 \%$ surfactant recovery with little retardation but a high degree of dilution.

The researchers were able to make the following recommendations based on this field demonstration.

1. Characterization of flow regime and contaminant distribution is essential.

a. Laboratory batch and column studies using native materials to assess fate and transport mechanisms were extremely valuable.

b. Laboratory data may be used in a computer model, one as simple as a conservative travel time model or one using more complex multi-dimensional transport equations to estimate flow paths and contaminant distribution.

2. Complete recovery of the flushing solution is not possible but appropriate hydraulic controls can aid in approaching this goal. Therefore, any risks associated with the unrecoverable mass need to be addressed.

3. Facility infrastructure was invaluable to the successful implementation of the remediation scheme. When selecting a site for field trials specific consideration should be given to shelter, power, water, heat and location of ancillary services.

\section{McCray and Brusseau (1998)}

This field trial involved the use of cyclodextrin, a sugar, as the active component of the flushing solution, creating what is known as a complex sugar flush (CSF). Using sugar addressed two issues, additional chemicals pumped into the ground and uncontrolled mobilization, which are often considered disadvantages for in-situ flushing techniques to remove DNAPLs. The innocuous nature of the sugar alleviates the concern of unrecoverable mass and with regard to migration of the contaminant, the cyclodextrin solution operates primarily by increasing its solubility without significantly altering interfacial tension. 
The target compound at the site, Operable Unit 1 at Hill Air Force Base, Utah, was a multicomponent LNAPL. The LNAPL was found in the unsaturated zone and upper portion of the saturated zone due to smearing caused by seasonal fluctuations in the groundwater elevation. The study was restricted to an area within a hydraulically controlled enclosed cell, $3 \mathrm{~m} \times 5 \mathrm{~m}$ to reduce the possibility of migration. Four injection wells and three extraction well were employed. The cyclodextrin was shown to increase the solubility of the more hydrophobic components to a greater extent compared to the less hydrophobic compounds. However, the less hydrophobic components had a greater relative mass removed during the test due to their higher initial solubilities.

The hydraulic conductivity in the saturated zone was $4.5 \times 10^{-2} \mathrm{~cm} / \mathrm{s}$ and the effective porosity was approximately $20 \%$. A confining layer of clay was located $8-8.5$ meters below ground surface (bgs) and its hydraulic conductivity was measured to be between $10^{-5}$ to $10^{-4} \mathrm{~cm} / \mathrm{s}$ in the horizontal direction but only $10^{-7} \mathrm{~cm} / \mathrm{s}$ in the vertical direction. To begin the test, 19 pore volumes (PV) of water were injected and pumped from the cell. A flushing solution containing $10 \mathrm{wt} \%$ cyclodextrin was then injected for 8 PV yielding a total of $64500 \mathrm{~L}$ over 10 days. After the 10 days of CSF, the researchers paused the system for 1 day to evaluate mass transfer limitations followed by 2 final days of CSF. Finally, $5 \mathrm{PV}$ of water was flushed through the cell. The results showed that approximately $41-44 \%$ of the initial NAPL and $100 \pm 5 \%$ of the cyclodextrin were recovered.

\section{Hirasaki (1997)}

This field study was conducted at Hill Air Force Base, Utah. The specific location at the base was Operable Unit 2 where the subsurface consists of a heterogeneous alluvial aquifer contaminated with a residual of a multi-component DNAPL. A combination of a surfactant solution and foam were injected through 3 injection wells and 3 extraction wells. Hydraulic control wells were placed upgradient and downgradient of the flushed zone and were used to reduce migration of the flushing solution and the contaminant. The foam was injected into areas of high permeability for the purpose of diverting the surfactant into the lower permeability areas, where the DNAPL was located. A simple technique of increasing the injection pressure was used to produce the foam at a single well. Each well was used at two hour intervals for foam injection on a rotating basis.

An initial partitioning interwell tracer tests (PITT) indicated that there was $21 \pm 7$ gallons of DNAPL within the test area. A solution of $3.5 \mathrm{wt} \%$ surfactant was injected for 3.2 days at $1 \mathrm{PV} /$ day. $\mathrm{NaCl}$ was also injected to condition the groundwater and optimize conditions for dissolution. The injection wells operated at 2gpm each while the upgradient hydraulic control well (HC 2) injected $1 \mathrm{gpm}$ and the down gradient hydraulic control well ( $\mathrm{HC} 1$ ) injected $3 \mathrm{gpm}$. Each extraction well removed $3.3 \mathrm{gpm}$ from the aquifer to balance the total in and out of the test area at $10 \mathrm{gpm}$. The foam reduced the swept volume of the flushing solution by an estimated $50 \%$. Water flooding was employed subsequent to the surfactant flush to help remove any residual surfactant, $\mathrm{NaCl}$ and foam. When completed, $99 \%$ of the surfactant and $96 \% \mathrm{NaCl}$ were recovered. The amount of DNAPL removed (37 gallons) was greater than the initial measured volume. This discrepancy was explained by the likelihood that DNAPL migrated into the test area from beyond the injection wells. Despite this apparent influx of DNAPL, a final PITT indicated that there was only 1.6 to 2.6 gallons 
remaining in the subsurface within the test zone.

Jawitz et al. (1998)

The test site for this field demonstration was Hill Air Force Base, Utah. The NAPL of concern was an extremely complex, multiple component NAPL. A Winsor type 1 alcohol/surfactant mixture was employed as the flushing solution. Solubilization was the primary mode of NAPL removal due to the formation of a single phase microemulsion between the contaminants and flushing solution. The solution was composed of $5.5 \mathrm{wt} \%$ surfactant and alcohol in water. Soil cores, PITTs and NAPL constituent breakthrough curves (BTC) were used to evaluate initial and final conditions at the test site. The researchers employed sheetpiles to construct a hydraulically isolated test cell $2.8 \mathrm{~m} \mathrm{x} 4.6$ $\mathrm{m}$. The sheetpiles were driven into the clay confining layer approximately $3.7 \mathrm{~m}$ below ground surface (bgs). Three extraction wells and four injection wells were located within the cell. The effective pore volume of the media within the cell was measured to be $6.9 \mathrm{~kL}$ with a porosity of $14 \%$ and a hydraulic conductivity of $0.1 \mathrm{~cm} / \mathrm{s}$. Saturation and spatial distribution of the contaminants were characterized with tracers.

Lab work indicated that five pore volumes were required to elute all available NAPL from the soil. In the field, this figure was nearly doubled by the researchers to account for higher hydrodynamic dispersion due to field scale heterogeneities. The flushing scheme involved 9 PV of precursor solution (surfactant, alcohol and water) followed by one pore volume of surfactant only and finally 6.5 PV of water. The results from the pre- and post-flush PITTs indicated that $72 \%$ of the NAPL was removed. The BTC, run after the flush, showed that 55 to $75 \%$ of the NAPL was removed when the pre-flush PITT was used for the initial condition and $60-175 \%$ removal using the soil core data for initial conditions. The soil core data have extremely large standard deviations and therefore the PITT was emphasized in reporting pre-flush conditions.

\section{Londergan et al. (1997)}

This field trial involved the use of surfactants in the flushing solution. The location of the trial was Hill Air Force Base, Utah, and specifically Operable Unit 2 . The hydraulic conductivity at the site was measured between $10^{-3}$ and $10^{-2} \mathrm{~cm} / \mathrm{s}$ with the water table at 25 feet bgs. The aquifer was approximately 19 feet bgs and there was a zone of residual DNAPL 4 feet thick at the bottom of the aquifer ( $42-46$ feet bgs) where saturation averaged $22 \%$. The injection and extraction wells were drilled into the zone of contamination.

Phase I of the project involved a preliminary PITT to establish the initial DNAPL volume (346 gallons). The DNAPL consisted of approximately $70 \%$ trichloroethene, $10 \%$ tetrachloroethene, $10 \%$ 1,1,1-trichloroethane, and $10 \%$ other constituents. A pilot scale flood was employed during this phase to evaluate the efficacy of the surfactant mixture and insure that the hydraulic conductivity remained relatively constant over the course of the test and to assess the treatability of the effluent. The flushing solution was composed of $7.7 \mathrm{wt} \%$ surfactant (sodium dihexyl sulfosuccinate) and was injected at an average rate of $2 \mathrm{gpm}$ for 0.6 days. The total flow rate for the extraction wells 
averaged $7.8 \mathrm{gpm}$. The hydraulic control well operated at $6 \mathrm{gpm}$ during this phase. The flushing solution was followed by 8.5 day flood of water only. The volume of contaminant removed during the pilot scale flood was 185 gallons and was composed of both free phase and dissolved phase DNAPL. Only $71 \%$ of the surfactant and $85 \%$ of the tracers were removed. These low figures were explained by the possibility that high level of dilution caused the surfactant and tracer concentrations to be below equipment detection limits.

Phase II of the project also employed a preliminary PITT which indicated that 148 gallons of DNAPL remained in the aquifer. The flushing solution used in this phase was composed of $7.75 \mathrm{wt}$ $\%$ surfactant (sodium dihexyl sulfosuccinate) and $4.47 \mathrm{wt} \%$ alcohol (isopropanol). A concentration of $7000 \mathrm{ppm}$ of $\mathrm{NaCl}$ was also used to precondition the groundwater to maximize the potential for dilution. The flushing mixture was injected for 3.4 days at a rate of $2.5 \mathrm{gpm}$. The hydraulic control well maintained an average rate of $7.0 \mathrm{gpm}$ and a total of $9.3 \mathrm{gpm}$ was extracted during this phase. The flushing solution was followed by 11 days of water only. The final results indicated that $98.5 \%$ of the DNAPL was removed and only 5 gallons remained in the subsurface within the test area.

The major problem encountered during this field trial was a build up of IPA in the air stripper used to treat the effluent. This build up produced operating temperatures that were too high for optimal stripper performance. There was also a build up of sediment in the treatment system. It was believed that the sediment was mobilized by the addition of sodium into the flushing solution. The system was not designed with and therefore did not contain any filters to prevent the sediment clogging problem.

\section{Fountain (1997)}

This paper compared two field trials and each employed solubilization as its contaminant removal approach. The first site described in this paper was the Canadian Forces Base Borden site operated by the University of Waterloo Centre for Groundwater research. The aquifer in the test area was nearly isotropic, composed of generally homogeneous media with a relatively high hydraulic conductivity. The aquifer was contaminated with tetrachloroethylene (PCE), a single component DNAPL. These conditions made the field situation close to ideal.

The hydraulically isolated test cell was constructed using sheet piles driven $4 \mathrm{~m}$ into the underlying clay unit. The cell was $3 \mathrm{m \times 3} \mathrm{m}$ in plan view with a secondary $5 \mathrm{~m} \times 5 \mathrm{~m}$ cell. The wells were contained in the inner cell and consisted of 5 injection and 5 extraction wells. The water level was kept near the ground surface and a controlled release of PCE was initiated. A total of $231 \mathrm{~L}$ of PCE was injected into the test cell. The top meter was excavated to evaluate the PCE distribution and subsequently replaced with bentonite to cap the bottom $3 \mathrm{~m}$. The mobile DNAPL was extracted using a water flood with a hydraulic gradient of 0.3 . Approximately $50 \%$ of the initial release was removed in the excavation of the top meter and during the water flood.

Once a residual was established, the surfactant flushing solution (1:1 mixture of nonylphenol ethoxylate and phosphated nonylphenol ethoxylate) was pumped through the cell for 14 pore 
volumes for a total of 130,000 L from November 10, 1990 to May 30, 1991. Pumping was not continuous because the system required constant attention, therefore it was shut down if an operator was unavailable. A $2 \%$ surfactant solution was injected into the aquifer, extracted at the extraction wells and passed through an air stripper. The treated effluent was reused by adding surfactant to a concentration of $2 \%$ and then re-injecting the solution into the aquifer. Therefore, the system was run as a continuous loop.

The solubility limit of the PCE in solution was determined in the lab to be approximately 11,000 $\mathrm{mg} / \mathrm{L}$. Some of the effluent samples were higher than $10,000 \mathrm{mg} / \mathrm{L} \mathrm{PCE}$, illustrating the ability to use laboratory obtained parameters in the field. A water flood followed the surfactant circulation. A total of $65 \mathrm{~L}$ of PCE was removed as dissolved phase during the flush portion of this trial. Data from this site illustrated that the limiting factor in cleanup was the solubilizing capability of the surfactant. The surfactants chosen for the Borden site degraded significantly during the 6 months after pumping from an assumed combination of hydrolysis and biodegradation. Both of these results emphasize careful selection of surfactants for in-situ flush strategies.

The second site described in the paper was located in Corpus Christi, Texas at the Du Pont Plant. This was an actual contaminated site as opposed to a controlled release. The DNAPL in the aquifer was composed primarily of carbon tetrachloride. The geology of the test area was a fine-grained, well sorted sand approximately $3.6 \mathrm{~m}$ bgs and $3.5 \mathrm{~m}$ thick. Beneath this sandy layer, a thick clay layer confined the DNAPL. Rather than sheet piling, hydraulic control was used at this site. Originally, the test area was $6 \mathrm{~m} \times 10 \mathrm{~m}$ but after an examination of the contaminant location and groundwater levels, the area was reduced to $6 \mathrm{~m} \times 5 \mathrm{~m}$. Both configurations utilized three injection wells and three extraction wells.

Prior to the site layout adjustment, a flush was conducted in the $6 \mathrm{~m} \times 10 \mathrm{~m}$ area. Four pore volumes $(367,000 \mathrm{~L})$ were injected over a period of 76 days at a $\mathrm{dh} / \mathrm{dl}$ of 1 . Biodegradation problems with the surfactant selected and biofouling difficulties at the injection wells made analysis of this flush difficult. A second flush was performed in the smaller area when 6 PV $(320,000 \mathrm{~L})$ were pumped through the test section at a dh/dl of 2 . Soil cores and monitoring well samples were collected to evaluate the results of the two tests. The hydraulic conductivity for the area was estimated to be 5 $x 10^{-3} \mathrm{~cm} / \mathrm{s}$. The researchers found that the concentration of contaminant in the effluent was well below the solubility limit for the compounds. The intermittent nature of the initial flush did not provide increased concentrations after rest periods indicating that the dissolution was not mass transfer limited. The results of this trial concluded that DNAPL can be effectively removed using a surfactant flush. Virtually all of the DNAPL was removed within the test area after approximately 10 pore volumes of flushing solution. The researchers also found that the rate of DNAPL removal was related to the geology since layers with higher clay contents took longer than layers with lower percentages of clay. It was concluded that along with site heterogeneities, well configuration is important. The flow paths at the Corpus Christi site were non-uniform and the velocity varied in different areas.

Both trials illustrated that the performance in the lab may be directly translated to the field. They 
also identified the evaluation of solubilization and sorption of surfactants onto clay minerals as a critical step toward optimizing surfactant flushing solution performance.

\begin{abstract}
Abdul et al. (1992)
This paper described soil washing using a surfactant solution to remove PCBs and oils. The test plot was 10 feet in diameter and 15 feet deep. Real time data was used to adjust the surfactant application rate to minimize mobilization beyond the capture zone. Samples included both soil cores and recovery well effluent. Over a period of 70 days, 5375 gallons ( 5.7 pore volumes) of a $0.75 \%$ aqueous surfactant (Witconol SN70) solution was injected into the test zone at an average rate of 77 gpd. The amount of leachate recovered totaled 10,981 gallons at an average rate of $157 \mathrm{gpd}$. Mathematical modeling was used to optimize both the injection, to minimize lateral spreading, and the extraction rate, to keep the water table depressed but limit the amount of uncontaminated groundwater intercepted. The initial contaminant amounts in the test area were $15 \mathrm{~kg}$ PCBs and 157 $\mathrm{kg}$ oils. At the end of the test, the researchers determined that they had recovered $1.6 \mathrm{~kg}$ PCBs and 16.9 oils or approximately $10 \%$ of the original mass.
\end{abstract}

\title{
Abdul and Ang (1994)
}

This paper describes Phase II of the project reported in Abdul et al. (1992). The objective of this phase was to confirm technical viability of the technology. The surfactant solution $(0.75 \mathrm{wt} \%)$ was applied with a sprinkler system for 90 days followed by 24 days of water only ( 500 gallons). Both Phase I and II illustrate the utility of lab scale studies. In Phase I the researchers flushed for 5.7 pore volumes and removed $7 \%$ of the contaminants in the laboratory which was close to the $10 \%$ removed in the field scale experiment. In the laboratory, $19 \%$ of the original contaminant mass was removed in Phase II after 8 pore volumes and in the field an 8 pore volume flush extracted $24 \%$ of the initial contaminant mass. Soil cores taken before phases I and II and after phase II were used to quantify mass reduction and evaluate the redistribution of contaminants before and after the surfactant flushes. To quantify the volume required to remove a majority of the contaminant mass, the researchers injected 105 pore volumes in the laboratory and recorded an $85 \%$ reduction in PCBs and oil ( $90 \%$ oil only). Phase II supported the conclusions of Phase I, that in-situ surfactant flushing is a promising strategy for the remediation of site contaminated with NAPLs.

\section{Summary of Field Studies}

The field studies reviewed suggest that in-situ flushing techniques can significantly reduce remediation times. Some important points extracted from the field studies are listed below:

1. The type, concentration, and biochemical properties of the chosen flushing solutions are important.

2. Site characterization is critical to design an effective remediation scheme.

3. Laboratory studies and modeling exercises can aid in the design of an effective flushing remediation scheme.

4. Although heterogeneities can slow down the clean up process, they do not appear to prevent some acceleration in remediation of an entire aquifer (Fountain et al. 1997- 


\section{Corpus Christi).}

Based on these field studies, it seems that both cosolvent flushing and surfactant flushing are technically feasible remediation technologies for the remediation of DNAPL contaminated aquifers. The successes of these studies provide incentive for more widespread use of in-situ flushing technologies. However, besides technical feasibility, implementation of cosolvent flushing is also dependant on economic and regulatory factors.

\subsection{Modeling}

Computer models are useful tools when trying to understand groundwater flow and contaminant fate and transport. When used in conjunction with lab and/or pilot tests, computer models can help design an optimal system allowing quick accurate comparison of different remediation scenarios. Several designs can be compared to one another in minutes rather than the weeks, months or sometimes years required to produce laboratory data. Computer models are not a panacea, however, as their limitations can sometimes outweigh their advantages. Simulation results are only as reliable as their input data and governing equations. Heterogeneities characteristic of most geologic formations can render a model employing average or general input data useless. The output must be kept in context with the results of physical experiments. The most value can be gained from these tools when there is a continual exchange between computer and physical models.

The modeling program used during the investigation for this section was the Princeton Transport Code (PTC), a three dimensional flow and contaminant transport simulator (Badu et al.,1997). The code was first developed at Princeton University, Princeton, New Jersey, and was written in FORTRAN-77. The user interface for PTC is MeshMaker (ARGUSOne, 1997). The MeshMaker PTC interface was developed to provide the user with a relatively simple way to define a problem that can be solved by the more powerful and complex PTC code. The general program, MeshMaker, was inspired by the need to have a blank canvas to perform various complex mathematical modeling procedures and is used to create the finite difference grids and finite element meshes graphically. PTC uses these meshes or grids and the different variables or properties assigned to each element or node within Meshmaker. The user allocates aquifer and contaminant characteristics to individual nodes, regions or globally (to the entire mesh). The characteristics that are input into the program include permeability, concentration, boundary conditions such as flow rate and head and initial conditions for flow and/or concentration. MeshMaker allows the import of digitized maps for direct input into the model as well as other types of graphical information, which can be placed in the background and referred to as needed during the modeling process. The software allows for more than one layer in the cross-section making it three-dimensional. The finite difference method of analyses is employed in the $\mathrm{x}$ and $\mathrm{y}$ planes while the finite element method is used for the $z$ plane. The preceding description briefly discusses the intricacies of PTC and Meshmaker but for further information and detail please refer to Badu et al. (1997). 


\section{Methods for modeling study}

The goal of this portion of the study was to evaluate and compare published in-situ flushing scenarios with alternative designs. This was accomplished given a single set of input parameters (site characteristics) from a hypothetical site that was not generated by any actual field data but rather represents an ideal and relatively simple, homogenous situation. A hypothetical site was used to try to equally compare previously used remediation schemes (well configurations) with each other and with our ideas.

Our standard site employed two homogenous, anisotropic geologic layers having different values of hydraulic conductivity $(\mathrm{K})$. The lateral $\mathrm{K}$ value for the upper level was $17.28 \mathrm{~m} / \mathrm{d}\left(10^{-2} \mathrm{~cm} / \mathrm{s}\right)$ and for the lower level it was an order of magnitude less at $1.728 \mathrm{~m} / \mathrm{d}\left(10^{-3} \mathrm{~cm} / \mathrm{s}\right)$. The vertical hydraulic conductivity for each layer was assumed to be $10 \%$ of the lateral value, a common characteristic of in-situ porous media. These particular values were used because they are similar to those of the soils used in the alcohol flushing experiments conducted in the laboratory study part of this research.

The well configurations and the pumping rates were taken directly from the literature for use within the model. The wells screened in the bottom layer only. Each layer was $25 \mathrm{~m}$ thick for the sites employing sheetpiling and $5 \mathrm{~m}$ for the others. The layer depths were different because the scenarios employing sheetpiling experienced flow significantly above ground level. The size of the test cell and the pumping scheme were taken directly from the literature, however, and the researchers did not report such difficulties. This indicates that the geology of the standard site is quite different from that of the actual field study. Increasing the depth of the layers and sheetpiling remedied this problem. The media within the model does not possess any heterogeneities which means that the results of the simulations do not adequately describe the behavior of the groundwater if there were any anomalies in either of the strata (lenses of more or less porous media, boulders with cracks or fissures, etc.).

The success and accuracy of a computer model designed to generate actual past, present and/or future conditions, depend heavily on the quality of the data from the field or laboratory experiment upon which it is based. For this section, the soil types, stratigraphy, hydraulic conductivities, hydraulic gradient, porosity, dispersivity and hydraulic gradient are important input parameters that must reflect experimental data for the PTC model to be useful. The field studies explored for this evaluation did not provide this information in the literature.

The simulations were run for the published scenarios in order to compare the well configurations. The results of these modeling efforts reveal that the top layer experienced generally even gradients or no gradients without any apparent influence from the wells. The lower layers were influenced, however. The discrepancy between the upper and lower layer was caused by the different values of hydraulic conductivity, both lateral and vertical, entered for each layer. Also the wells were only screened in the lower layer. The ability of water to move between the two layers is limited by the $10^{-4}$ magnitude vertical hydraulic conductivity of the lower layer. For the purposes of this section, the focus will be upon the lower layers since the contours illustrated within them result directly from 
the pumping scheme. Also, given a site with more conductive layers, these same general results are expected for the aquifer as a whole. A break down of the results follows. Please refer to the previous section (Field Studies) for a more in-depth discussion of the background for each study.

\section{Model Results}

Scenario 1 (Fountain (1997)- Borden):

The output for this scenario is illustrated in Figure 8.1. On each of the output graphs there are vertical and horizontal scales in meters. The total depth of the aquifer in this simulation is $50 \mathrm{~m}$. In this figure it is shown that the injection wells are pumping at a head only slightly above the extraction wells. A larger difference is not necessary because the pumping rates are the same and there is not a hydraulic gradient to compete with the flow direction. The sheetpiling constrains the flow so that it travels only from the injection wells to the extraction wells. Within the model, all sides of the field study were deemed 'no flow' boundaries. This designation does not allow for possible by-passing above or below the sheets or for any leakage into or out of the test cell.

Scenario 2 (Hirasaki et al. (1997)):

The output for this scenario is shown in Figure 8.2. Hydraulic control (HC) wells are employed in this simulation. The upstream $\mathrm{HC}$ appears to be of limited help keeping the injected fluid from migrating off-site. The downstream HC seems to work as it was intended, to ensure capture, but does not appear to be necessary given the set up used in the model. The extraction wells have an adequate radius of influence as determined from the figure indicating that they have the capacity to recover most, if not all, of the injected solution. Perhaps the upstream HC well would serve the configuration better if it were at a higher pumping rate since it seems to be overcome by the injection wells here.

Scenario 3 (Jawitz et al. (1998)):

The results of this simulation are illustrated in Figure 8.3. This output is of another setup employing sheetpiling ( $50 \mathrm{~m}$ deep) for hydraulic control of the study. The results are quite similar to those for Scenario 1 where the injected fluid is forced to flow directly into the extraction wells exclusively. The pump rates are much lower here than in any of the other studies evaluated, most likely due to the geology of the site used in the original experiment.

Scenario 4 (Londergan et al. (1997):

The output for this scenario is shown in Figure 8.4. This design employs hydraulic control wells to limit off-site migration of injected solution and contaminant. The upstream $\mathrm{HC}$ appears to work but as stated for Scenario 2, it does not seem to be necessary given the contour lines illustrated in the figure. The capture efficiency with and without the $\mathrm{HC}$ would likely be high either way. The money saved by not employing $\mathrm{HC}$ could perhaps be used more effectively elsewhere in the project.

Scenario 5 (McCray and Brusseau (1998)):

The results of this simulation are illustrated in Figure 8.5. The output of this scenario is roughly the same as that for Scenario 3. The differences are that this design uses 3 extraction wells rather than 


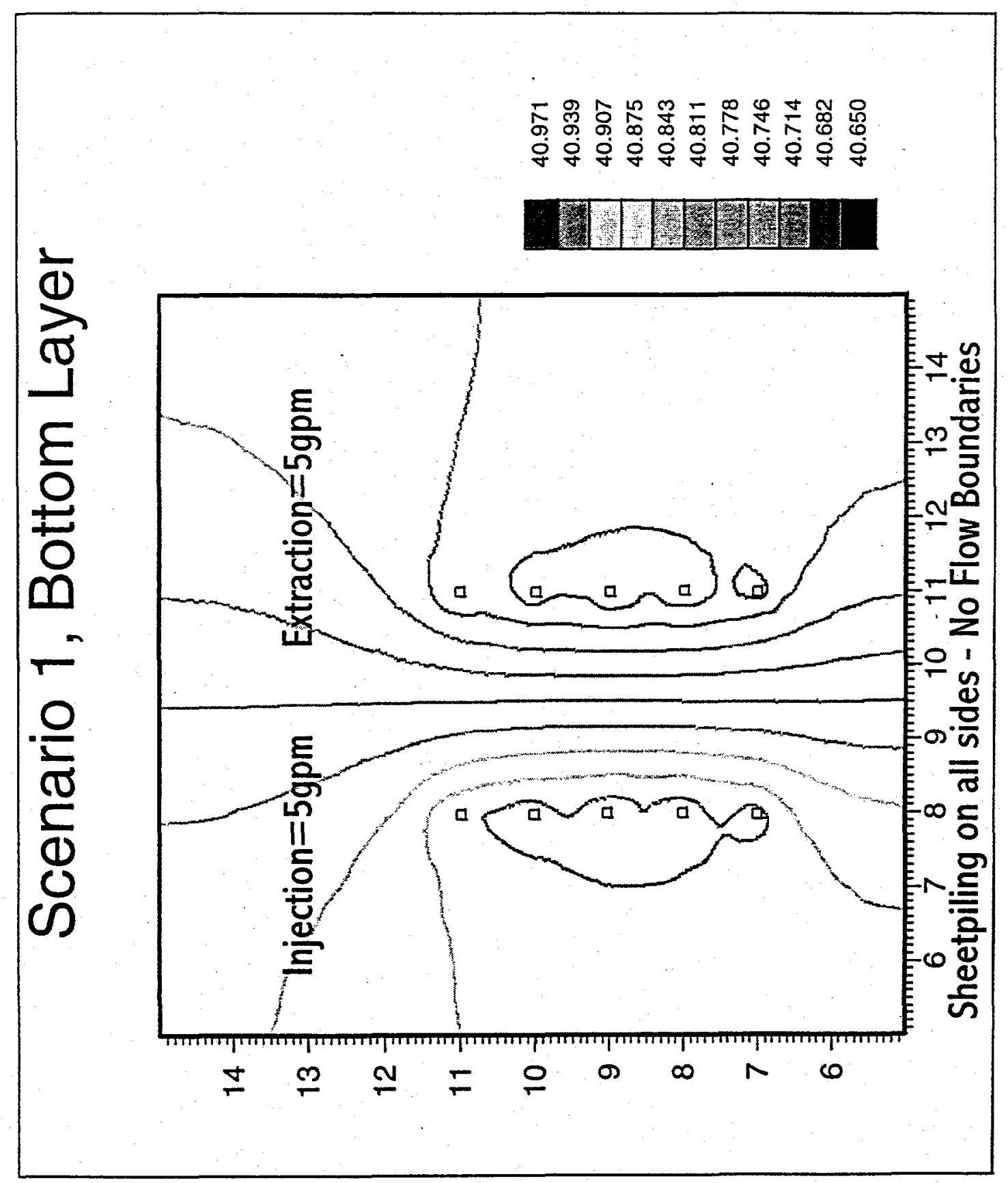

Figure 8.1 


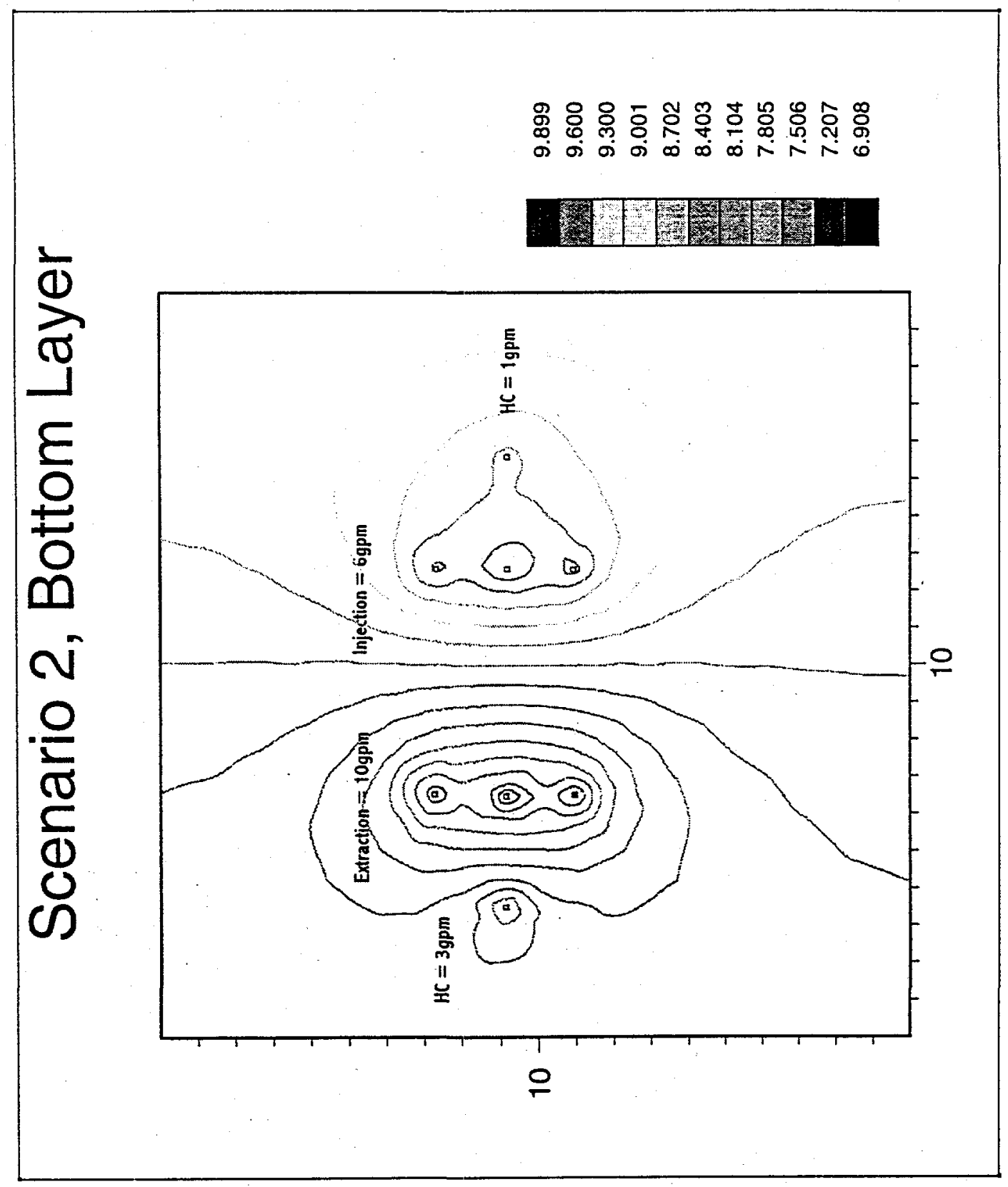

Figure 8.2 


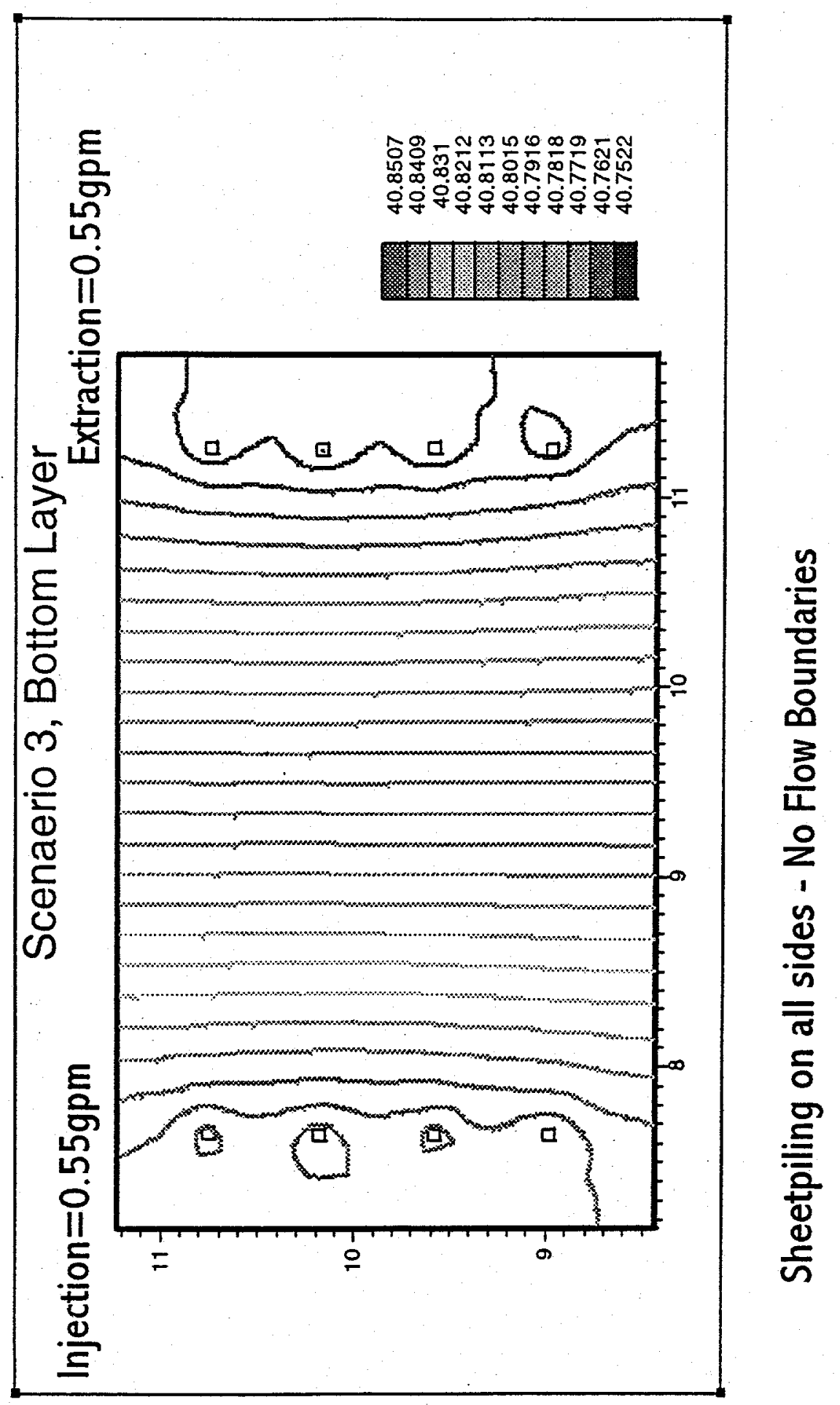

Figure 8.3 


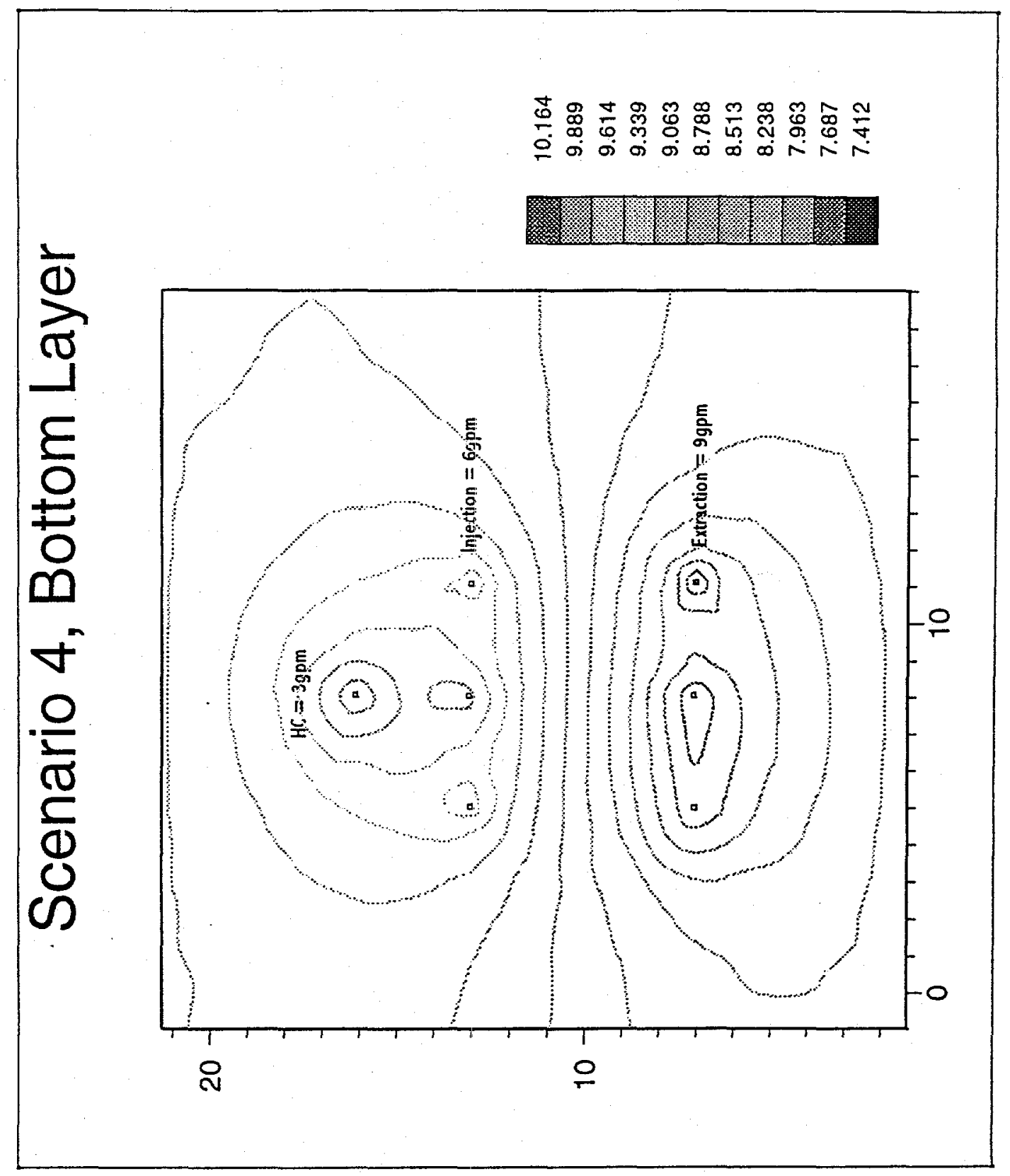

Figure 8.4 


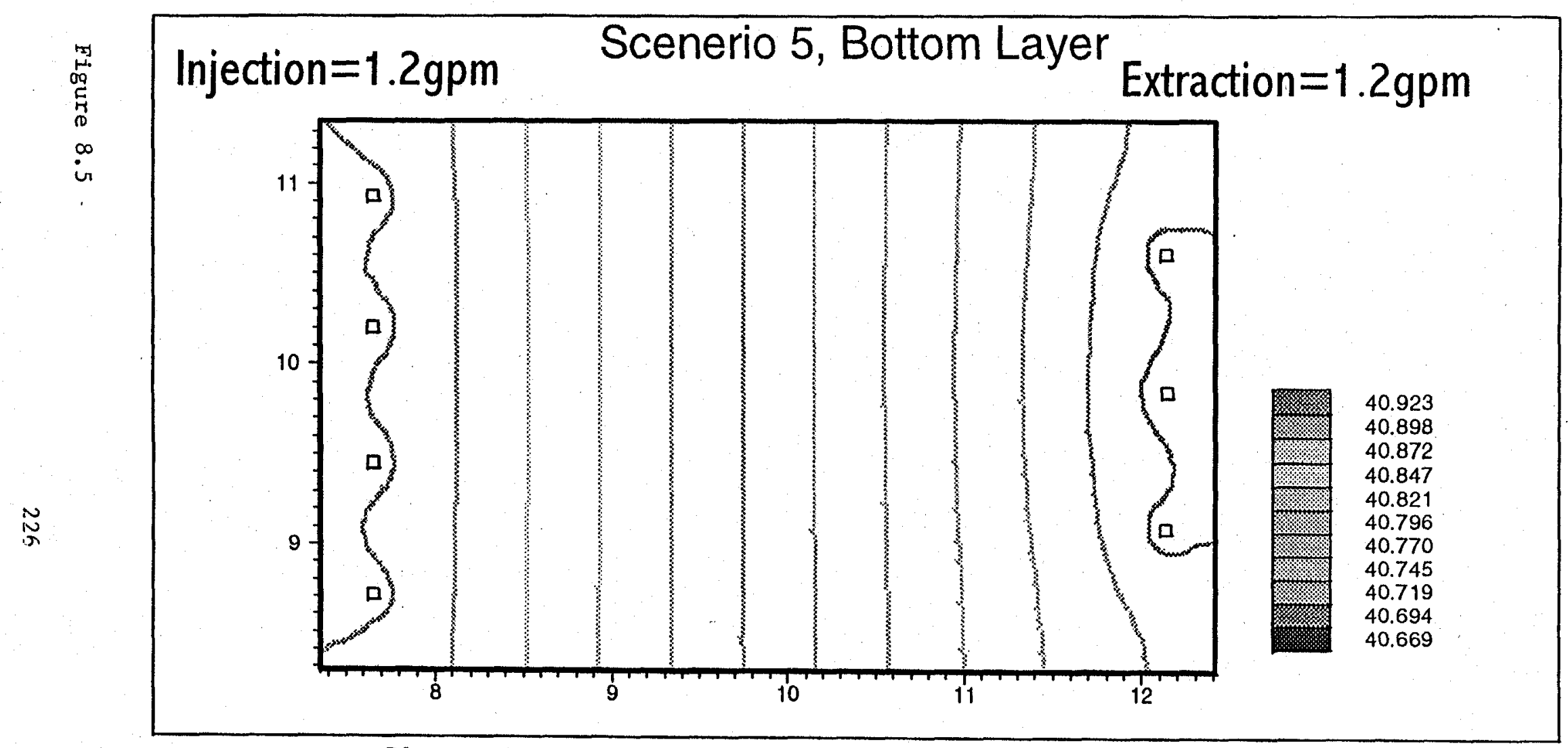

Sheetpiling on all sides - No Flow Boundaries 
four and the pumping rates are approximately twice as much as those for Scenario 3 . The reduction in extraction wells does not appear to make a difference in the capture efficiency since the researchers used sheetpiling around the study area. The problem associated with using fewer extraction wells is the possibility of forming dead zones or pockets of solution and contaminant that remain caught in a corner of the test cell and are not extracted.

Scenario 6 (Fountain (1997)):

The output for this scenario is illustrated in Figures 8.6 and 8.7. The results in Figure 8.6 are for a change in head across the site of $0.5 \mathrm{~m}$ while the change is only $0.1 \mathrm{~m}$ in Figure 8.7 . With $0.5 \mathrm{~m}$ as the change in head, there does not appear to be a significant amount of capture but once the value is changed more of the injected fluid is able to reach the extraction wells. The poor design illustrated in Figure 6 compared to the more promising shown in Figure 8.7 illustrates the importance of incorporating the proper input values into the model. The soils in the lower layer of the model are similar to those at the actual site with regard to hydraulic conductivity and the low pumping rates indicate that there is not a significant gradient at the test site but other than that, there is no other information available. As discussed in a later section, the groundwater flow direction can significantly change the results of these simulations and if the direction were left to right instead of top to bottom, the output for this scenario would be quite different.

\section{Alternative Scenarios}

In an effort to evaluate different well configurations for a given hydrogeologic condition, five designs, each slightly different from those found in the literature, were constructed and tested within PTC. Table 8.4 describes the well configuration for each scenario. All had the following components in common:

- 2 layers of sand, with each layer $5 \mathrm{~m}$ thick

- bottom layer hydraulic conductivity $=1.728 \mathrm{~m} / \mathrm{d}\left(10^{-3} \mathrm{~cm} / \mathrm{s}\right)$ with vertical hydraulic conductivity $=0.1728 \mathrm{~m} / \mathrm{d}\left(10^{-2} \mathrm{~cm} / \mathrm{s}\right)$

- top layer hydraulic conductivity $=17.28 \mathrm{~m} / \mathrm{d}\left(10^{-2} \mathrm{~cm} / \mathrm{s}\right)$ with vertical hydraulic conductivity $=1.728 \mathrm{~m} / \mathrm{d}\left(10^{-2} \mathrm{~cm} / \mathrm{s}\right)$

- constant head boundaries on two opposite sides and no flow boundaries at the other two sides

- pumping rates (combined): injection $=5 \mathrm{gpm}$, extraction $=6 \mathrm{gpm}$ 


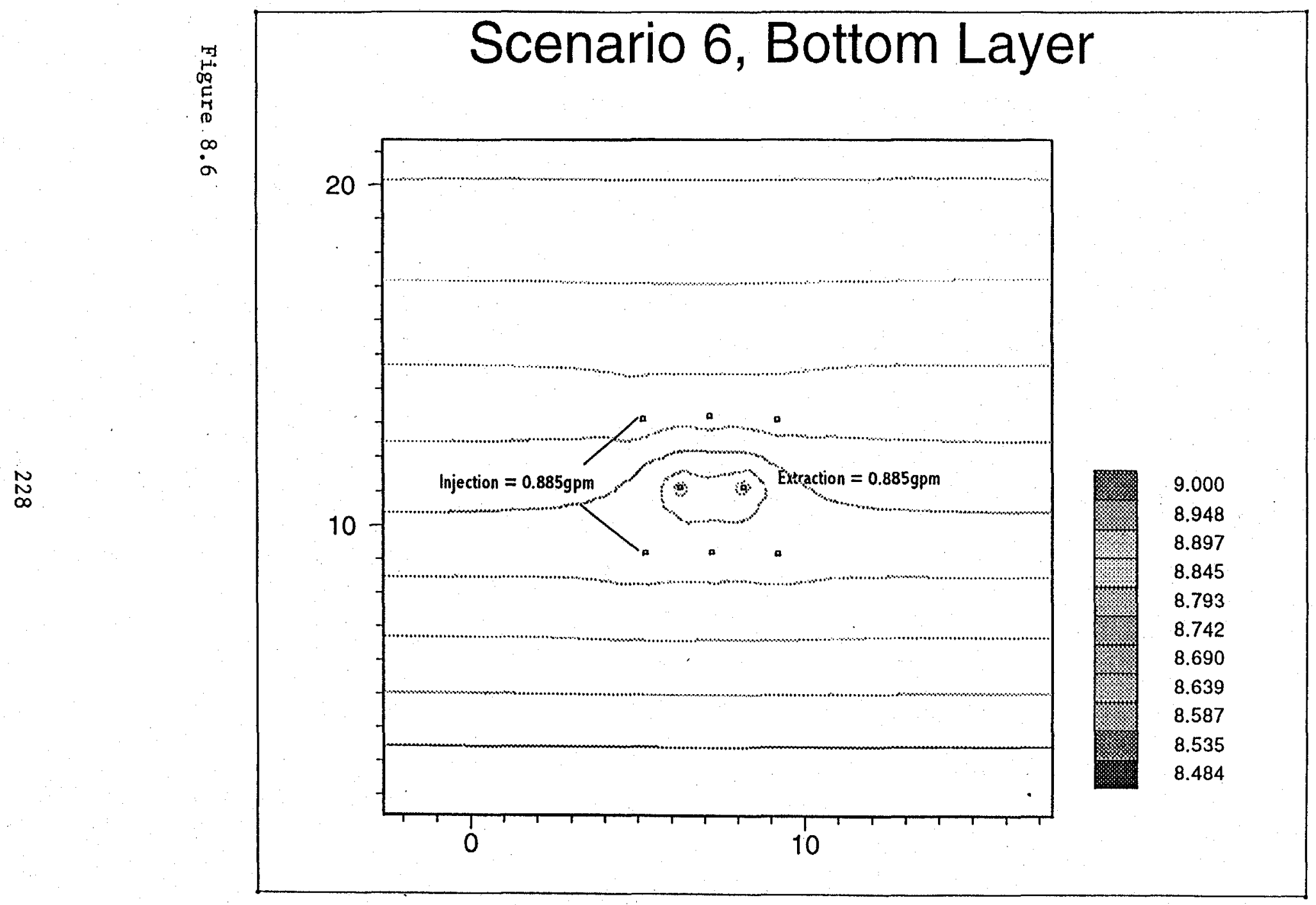




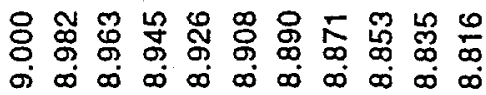

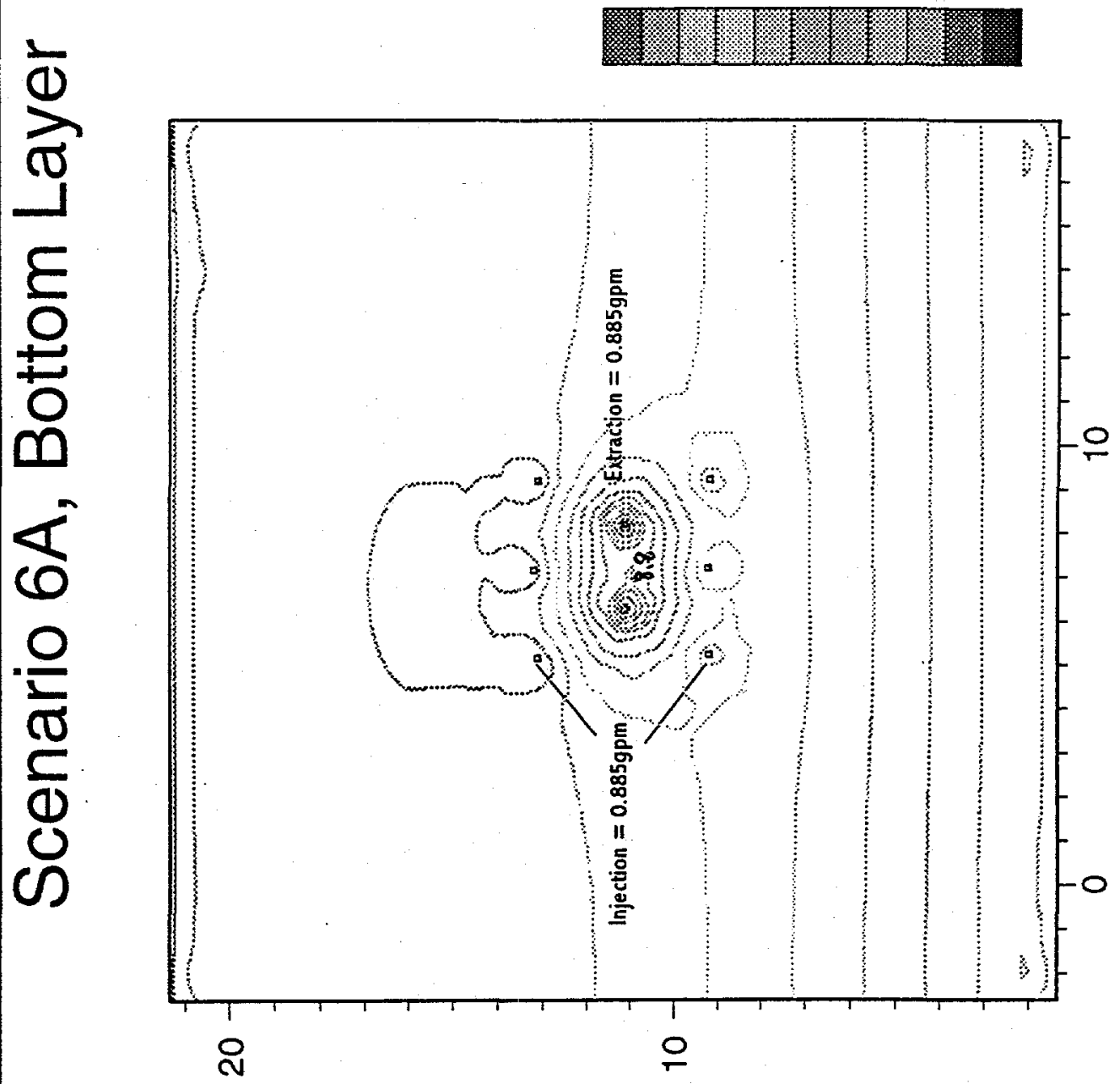

Figure 8.7 
Table 8.4: Alternative Remedial Designs

\begin{tabular}{|c|c|c|c|c|}
\hline Design 1 & Design 2 & Design 3 & Design 4 & Design 5 \\
\hline $\begin{array}{c}5 \text { wells: } 1 \\
\text { extraction }(E), \\
4 \text { injection }(I)\end{array}$ & $\begin{array}{c}5 \text { wells: } 3 \\
\text { extraction (E), } \\
2 \text { injection (I) }\end{array}$ & $\begin{array}{c}8 \text { wells: } 2 \\
\text { extraction (E), } \\
6 \text { injection (I) }\end{array}$ & $\begin{array}{c}8 \text { wells: } 2 \\
\text { extraction (E), } \\
6 \text { injection (I) }\end{array}$ & $\begin{array}{c}8 \text { wells: } 2 \\
\text { extraction }(E), \\
6 \text { injection }(I)\end{array}$ \\
\hline Plan View & Plan View & Plan View & Plan View & Plan View \\
\hline 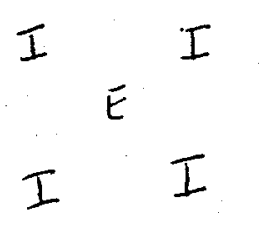 & $\begin{array}{c}E E E \\
I I\end{array}$ & $\begin{array}{l}I \begin{array}{l}I \\
E\end{array} \\
I E I \\
I\end{array}$ & $\begin{array}{lll}I & E & I \\
I & E & I \\
I & & I\end{array}$ & $I E^{I} E^{I} I$ \\
\hline
\end{tabular}

\begin{abstract}
Alternative Scenario Model Results
With the alternative designs, as illustrated with the simulation results of the literature-derived scenarios, the pumping scheme did not appear to influence the top layers. Again, this is due to the different hydraulic conductivity values (both lateral and vertical) of the two layers in the system and the fact that the wells are only screened in the lower aquifer. As with the published scenarios, the lower layer results are considered generally reliable for the scope of this paper; to compare difference scenarios given a set of constant site parameters. The results for the lower layers are discussed below.
\end{abstract}

Design 1, '5-Spot':

The output for this setup is shown in Figure 8.8. This design appears to be effective for the site selected, as determined by its deep cone of depression and large percentage of injected fluid being captured at the extraction wells. This specific injection/extraction approach is commonly used in the oil industry during enhanced oil recovery.

\title{
Design 2:
}

The output for this model run is illustrated in Figure 8.9. This setup captures most of the injected fluid with very little migration off-site. This is the only design, both from the literature and this group of alternatives, employing more extraction wells than injection wells.

Design 3, 'Oval':

The results of this simulation are revealed in Figure 8.10. There appear to be some downstream losses with this design. The contour lines here and in Figure 8.8 show the difference in having an injection/extraction ration of $3: 1$ versus $4: 1$. The pumps are not working nearly as hard in this oval design as they were in the 5-spot. The efficiency of this setup may be improved if oriented lengthwise along the groundwater flow direction. The designs having injection wells on both the upstream and downstream sides of the extraction wells, such as this, would likely perform better at sites with low hydraulic gradients to limit migration off-site due to downstream injection. 


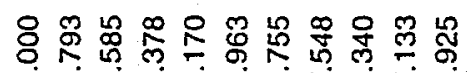

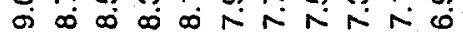
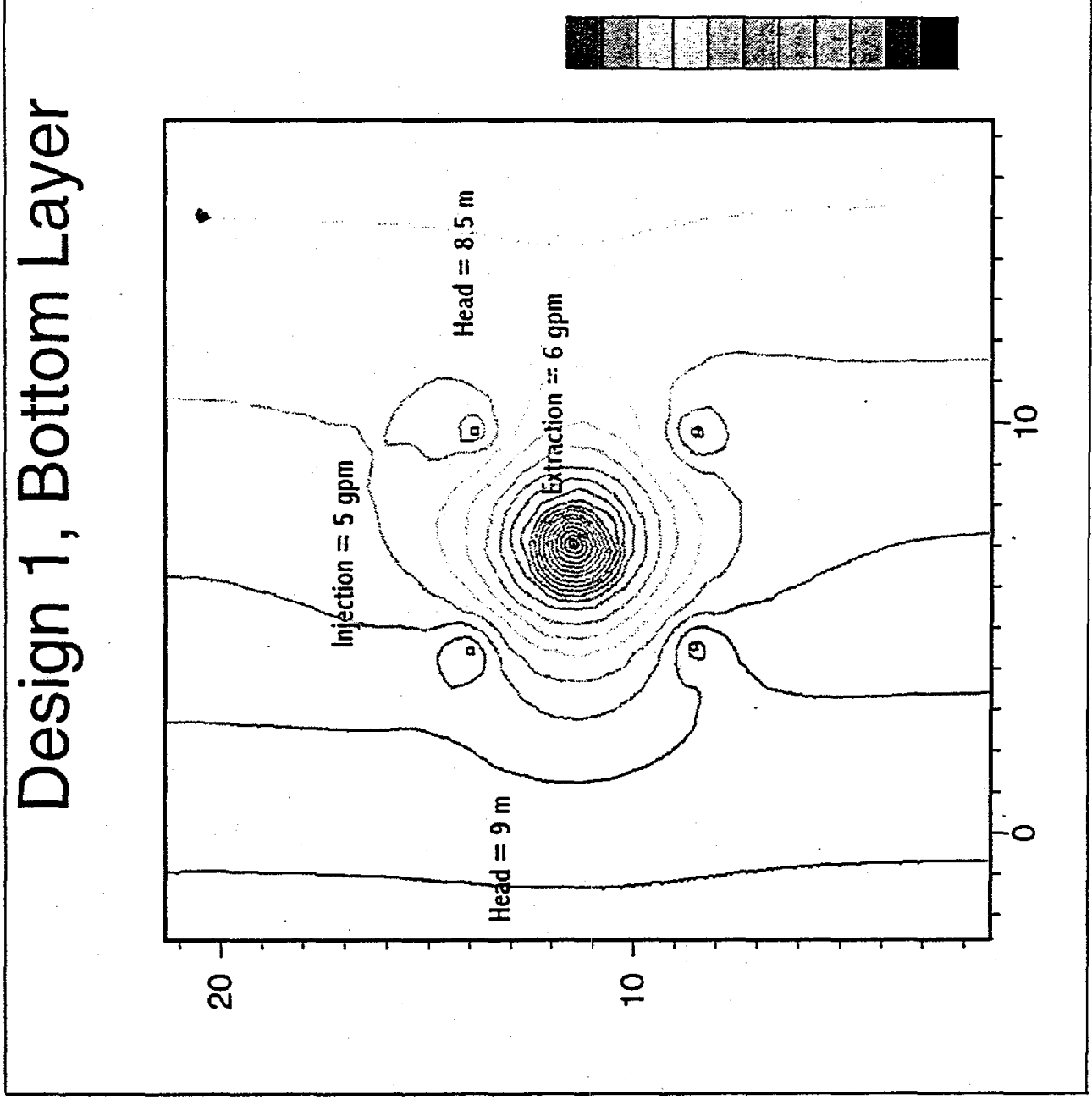

Figure 8.9 


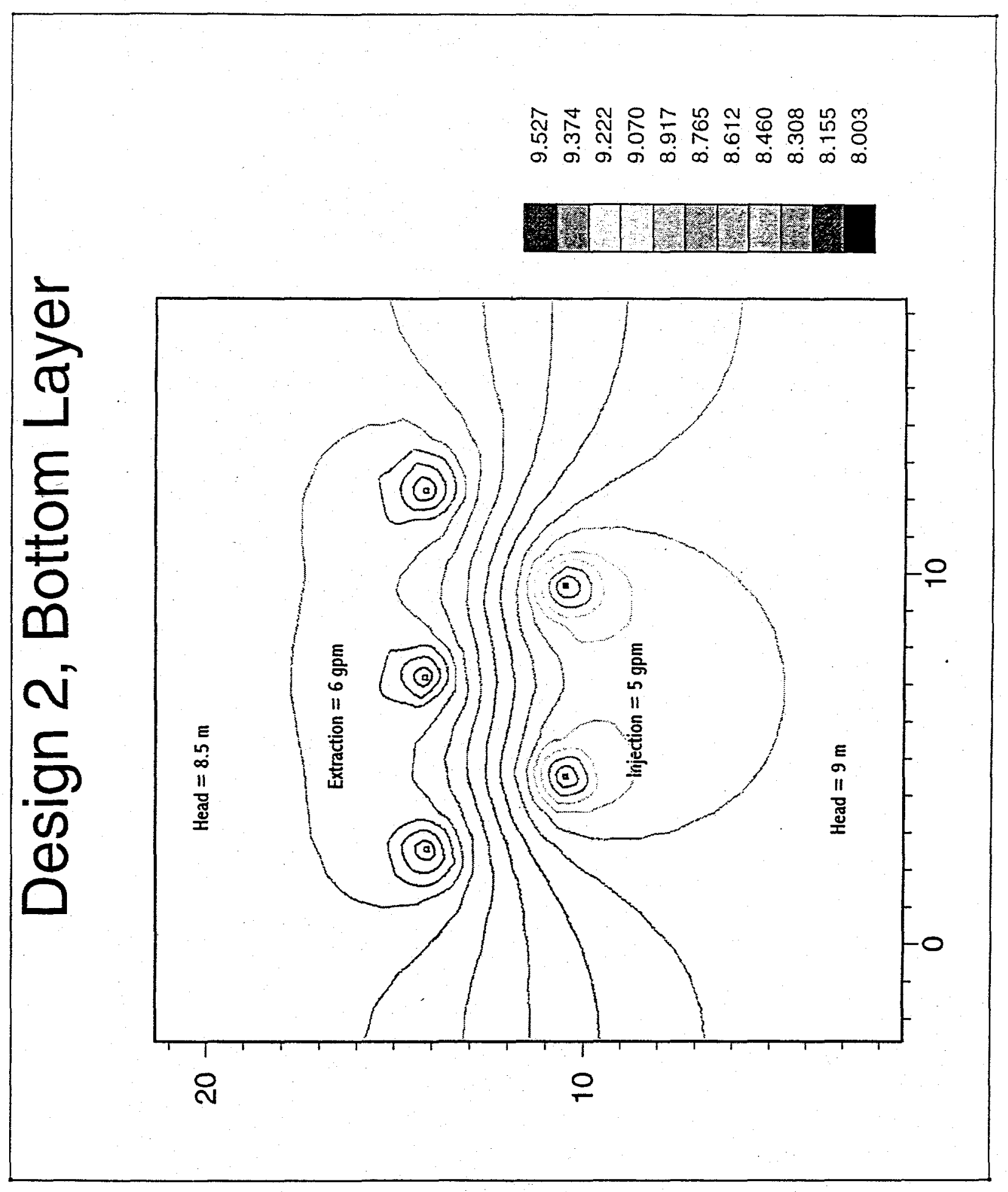

Figure 8.9 


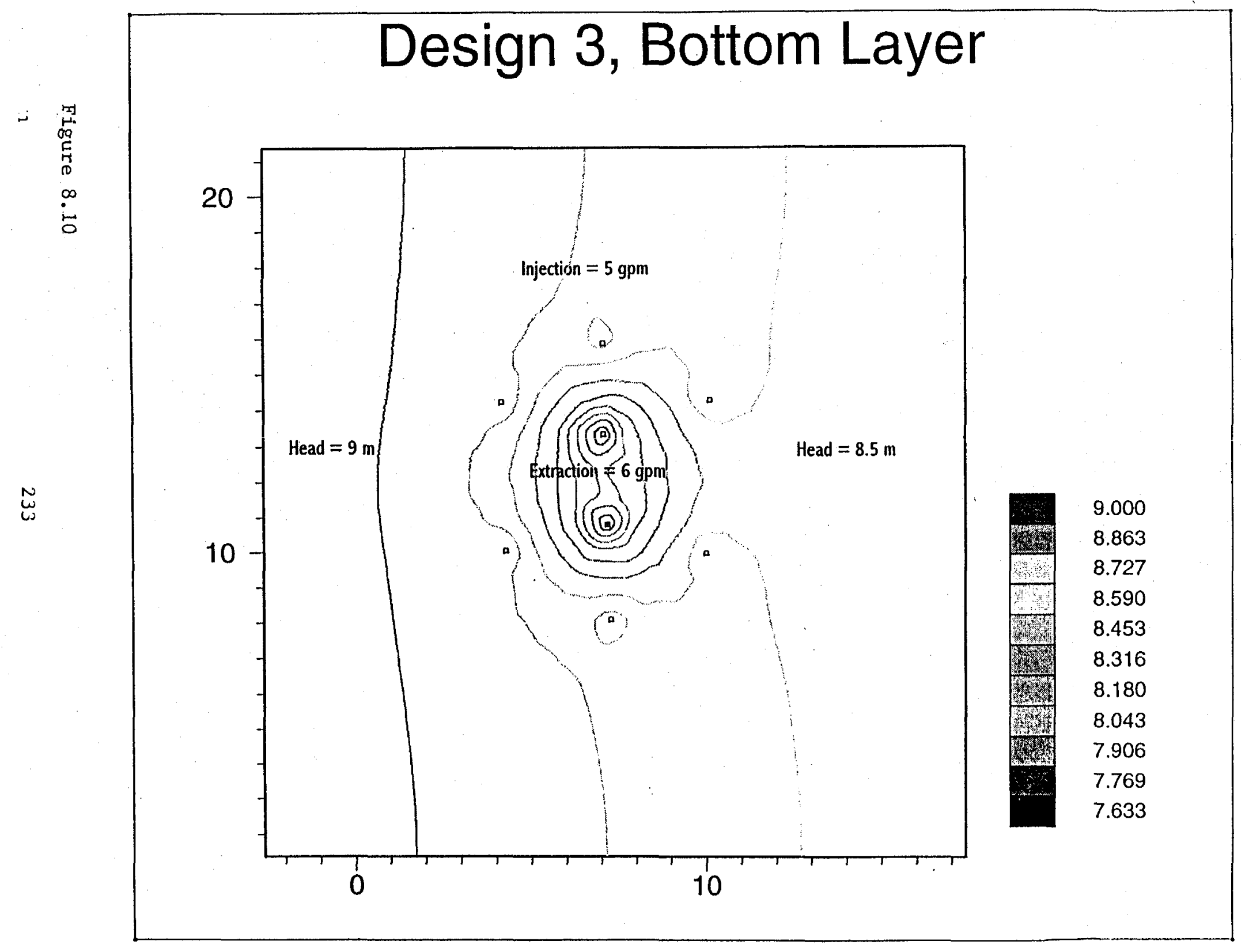




\section{Design 4:}

Figure 8.11 shows the outcome of the modeled scenario. This Figure and the following, Figure 8.12, are basically the same, just oriented differently to the groundwater flow direction. The results of this design are that there are significant losses due to the downstream injection wells. As mentioned previously, a location with a shallow hydraulic gradient may be more appropriate for this design. Other options include lowering the rates of the downstream injection wells and/or increasing the rates at the extraction wells.

\section{Design 5, 'Dual 5-Spot':}

The output for this run is illustrated in Figure 8.12. This orientation, when compared to that in Figure 8.11 (Design 4) appears to be more effective with much less off-site migration.

The computer model was completed for each of the new designs, compared to one another as well as to the published scenarios. In using the model, it is evident that proper soil and groundwater characteristics must be known for a site for it to be properly simulated. It is difficult to compare the new configurations with the published designs in any detail due to the limited information in the literature for necessary for accurate modeling. Yet, general comparisons can be made. It appears that hydraulic control, whether using sheetpiling or wells, is not normally necessary. Proper well placement and pumping schemes should limit any off-site migration. The key is gathering sufficient data for the development of an appropriate design for the site conditions and contaminant characteristics. A computer model can be a tremendous enhancement in that the designer can run a number of simulations over a range of values to help produce an effective and efficient plan.

Comparisons made between the two 5-well configurations, Designs 1 and 2 (Figures 8.8 and 8.9), revealed that hydraulic control wells are not needed for this standard site, that each setup is able to capture most, if not all of the injected fluid. The large cone of depression in Figure 8.8 (Design 1) is due to the extraction well working to pull fluid from 4 injection wells. The cones in Figure 9 are much smaller since there are three working on two injection wells. Of the two, Design 2 seems to be the better from an economic standpoint, with regard to mechanical load on the wells, and from a capture standpoint. The quality that may make the 5 -spot more desirable is that it can be built upon in any direction depending on plume migration.

The three 8-well scenarios, Designs 3, 4 and 5 (Figures 8.10, 8.11 and 8.12) were compared to one another. The highest capture efficiency appears to occur with the dual 5-spot (Design 5, Figure 8.12). This is the most desirable of the three for the standard site used in the model with the oval setup next. Design 4 (Figure 8.11) should not be used on this hypothetical site due to the significant loss of injected fluid. A 5-well design would obviously be less expensive to construct but such cost savings would not materialize if it were an adequate. For the standard site employed for this model and out of all alternative designs, Design 2 seems to be the most appropriate because of its high capture efficiency economic benefit of having more than one extraction well. However, the best of the 8-well designs, Design 5, is simply an expanded 5-spot (Design 1). In the case where expansion of the flushing study is likely, it would be prudent to begin with Design 1 rather than Design 2. 


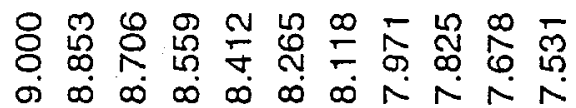
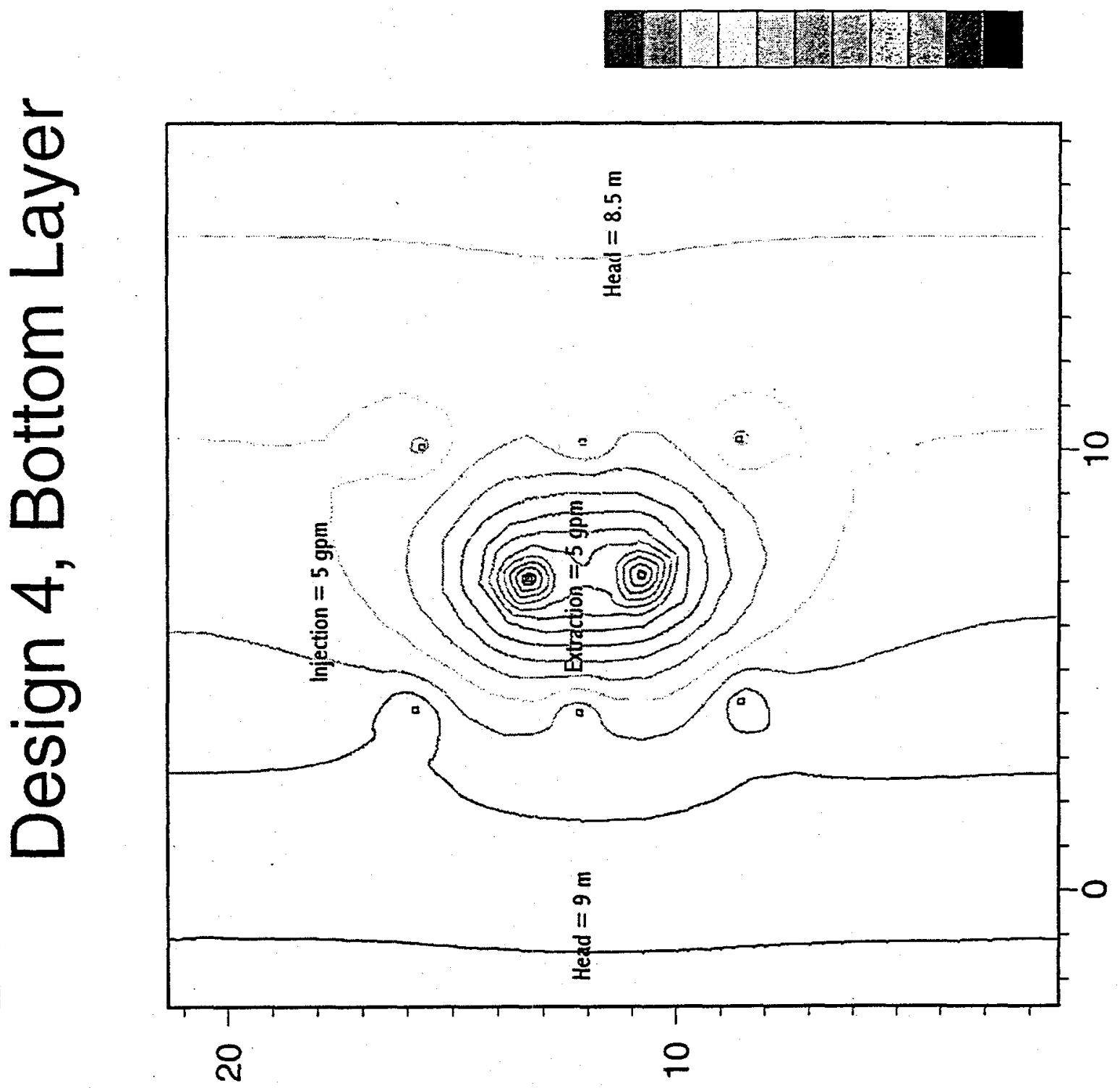

Figure 8.11 


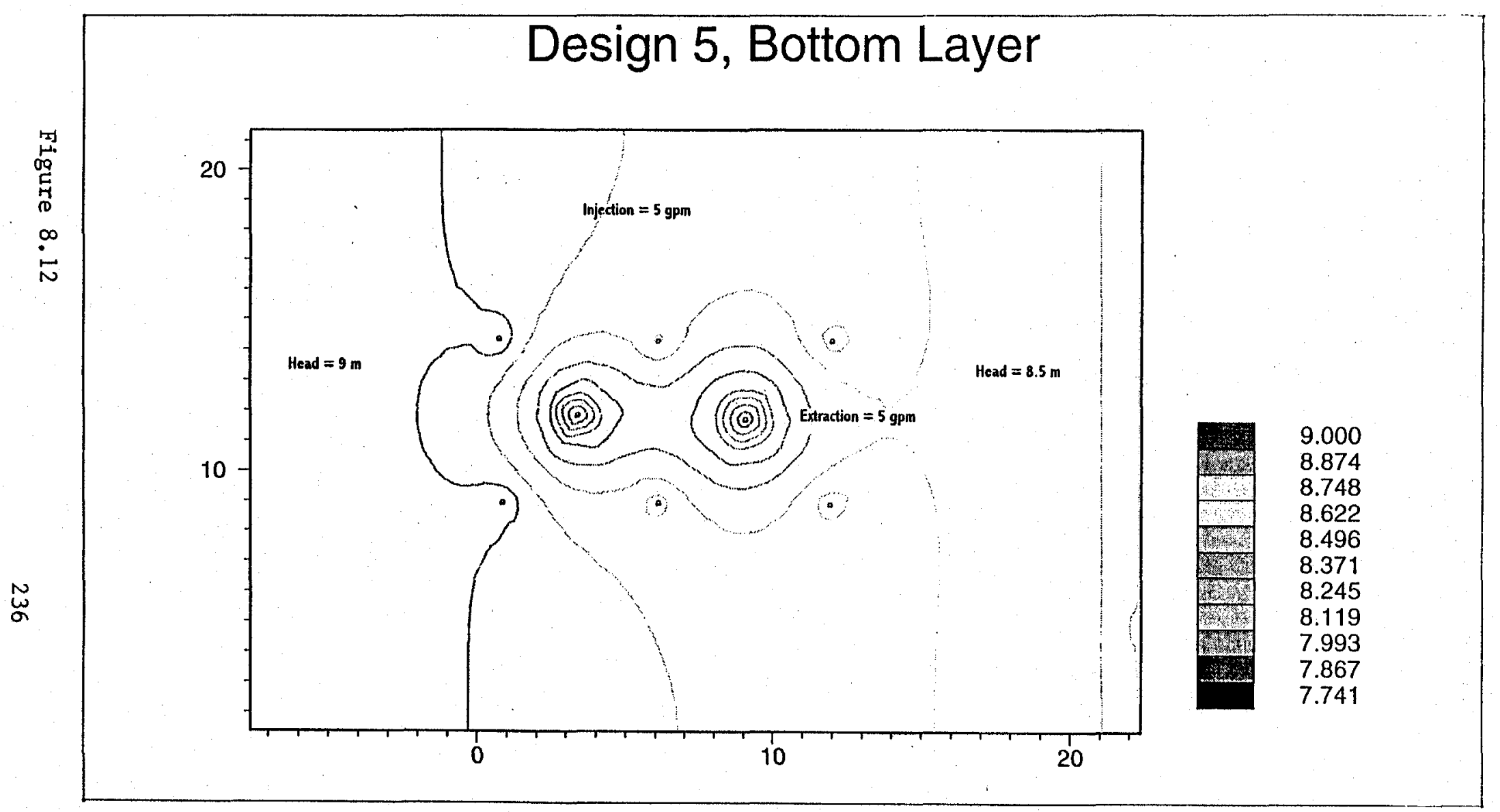




\subsection{Summary}

Computer modeling can significantly enhance the design process for in-situ flushing studies. Used correctly, they can decrease the time and the costs associated with developing an efficient strategy for the site and contaminant in question. It is important to be aware of the fact that appropriate input values are essential for adequate representation of the geologic and hydrologic characteristics of the site. A continual information exchange between the field and/or laboratory results and the modeler will yield the most efficient design outcome. The program used in the modeling process should also be understood with regard to its abilities and perhaps more importantly, its limitations. Models can be powerful tools, to be used in conjunction with field work and professional judgment, but must be employed within their range of its applicability.

The computer model used for evaluating the field studies from the literature and five alternatives to those published designs was the Princeton Transport Code (PTC). The program is a threedimensional flow and transport simulator with MeshMaker as the user interface. The outcome of the simulations revealed that more information is required to model the published data adequately. To simulate and evaluate the published scenarios using the model, it is necessary to collect the relevant geologic and hydrologic details of the particular sites at which it was employed. However, the model was useful in the evaluation of the alternative designs. From the alternatives, it was found that for a standard site a system of two upstream injection wells and three downstream extraction wells is the most effective with regard to recovery of the injected fluid. If expansion of the remedial design is likely then a 5-spot configuration would be the most appropriate due to the ease at which it can be built upon.

\subsection{Economic Feasibility}

Many factors need to be considered to assess the economic feasibility of cosolvent flushing. Although extensive site characterization is necessary for this technology, it is assumed that much characterization is already completed at sites or would also be necessary for other in-situ remediation technologies. The following is a general cost comparison of a conventional pump and treat system with a hypothetical cosolvent flush at a DNAPL-contaminated manufacturing site in Vermont.

The simple cost comparison of a long term pump and treat operation (at a local manufacturing facility in Vermont) is compared to a shorter pump and treat scheme with a hypothetical alcohol flushing system added in Table 8.5 .

Table 8.5. Comparison of long-term pump and treat system (at a local manufacturing facility) with a shorter pump and treat system with additional alcohol flushing for this same site.

\begin{tabular}{lllll}
\hline \#Wells & $\begin{array}{l}\text { Time } \\
\text { of Trtmt. }\end{array}$ & $\begin{array}{l}\text { Cost of Wells } \\
\text { Installation }\end{array}$ & $\begin{array}{l}\text { Recovery } \\
\text { System Cost }\end{array}$ & $\begin{array}{l}\text { O\&M Cos s : P u m p, } \\
\text { Treat, Sampling, Sal. }\end{array}$ \\
& &
\end{tabular}

Pump and Treat Alone 


$\begin{array}{lll} & \$ 600,000(37 \text { shallow wells, } \\ & \left.20-40^{\prime} \text { plus piping }\right) \quad \$ 500,000 \\ 40 & \$ 200,000(3 \text { bedrock wells }) \\ (20 \mathrm{yr} \text { life }) & \frac{\$ 800,000 \text { total }}{20}\end{array}$

Initial Capital Costs: $\$ 1.3$ million

Total Cost of Project in Today's Dollars (Capital, O\&M): $\$ 0.8 \mathrm{~m}(\mathrm{x} 3)+\$ 0.5 \mathrm{~m}(\mathrm{x} 3)+$ $\$ 1.5 \mathrm{~m}(\mathrm{x} 50)=\$ 78.9$ million

Pump and Treat Plus Alcohol Flushing

Pump and Treat (as above)

$40 \quad 15 \mathrm{yrs} \quad \$ 600,000$

$\$ 500,000 \quad \$ 1,500,000 / \mathrm{yr}$

Alcohol Flushing System

20

$1-2$ yrs $\$ 300,000$

$\$ 300,000$

$+\$ 50,000$ (IPA)

Initial Capital Costs: $\$ 1.85$ million

Total Cost of Project in Today's Dollars (Capital, O\&M): $\$ 1.1 \mathrm{~m}+\$ 0.85 \mathrm{~m}+\$ 1.5 \mathrm{~m}(\mathrm{x} 15)$

$+\$ 0.5 \mathrm{~m}(\mathrm{x} 2)=\$ 25.45$ million

Approximately, forty wells have been installed at this Vermont site for plume containment; 37 shallow wells at 20 to 40 feet and 3 bedrock aquifer wells at about $400 \mathrm{ft}$. The costs for the initial installation of the wells and recovery system as well as yearly operation and maintenance costs have been estimated by the site engineer and are shown in Table 8.5. The operation and maintenance costs include pumping, recovery system maintenance, hazardous waste disposal, sampling, salaries for 2.5 site personnel related to remediation, and other standard O\&M costs. The design life for the wells and recovery system is twenty years, therefore, additional capital costs for replacement of wells and the treatment system have been added. Estimates of cleanup time vary from 10-15 years at the chemical distribution center $(C D C)$ and fire training areas to several hundred years in the area under one of the main manufacturing buildings. An optimistic pump and treat time of 50 years has been used in this cost analysis.

There are believed to be two to three source areas (high DNAPL concentrations in soil, and some separate phase during initial pumping) that would be amenable to alcohol flushing near the main manufacturing building. The hypothetical alcohol flushing system would be used in these locations. The shorter pump and treat scenario (with alcohol flushing) assumes that the alcohol flushing would remove the DNAPL source but a small amount of PCE would remain at levels equivalent to those currently found at the $\mathrm{CDC}$ and, therefore, 10 - 15 years of pump and treat may still be needed at this location. A 10-15 year follow up is a worst case scenario. 
The cost comparison (Table 8.5) raises a very important issue; that the capital costs are generally insignificant for a lengthy pump and treat operation as compared to operation and maintenance costs. Any substantial reduction in overall pump and treat time through source removal, therefore, will dramatically reduce the overall cost of the project. For example, the initial capital costs for the site used in Table 8.5, is about the same as the operation and maintenance costs for one year. In terms of present dollars, the total cost of this pump and treat system for 50 years of operation is about 80 million dollars. Cutting down the time of pumping and treating to 15 years, as an estimate of what the additional alcohol flushing system might do, results in a total present day cost of about 25 million dollars. In this case, the initial capital costs for the system with alcohol flushing, would not be that much more since the source areas are located at about $20-40 \mathrm{ft}$ and some of the pump and treat system features would only need to be expanded and modified for the alcohol flushing system.

The above cost analysis is very general and does not contain all the complexity necessary for a thorough cost analysis. A complete cost analysis would be necessary for each site and each specific system designed for that site and was beyond the scope of this project. For example, one viable method to reduce the recovery system cost in a cosolvent flushing system is to reuse the alcohol solution. Experiments have demonstrated that activated carbon treatment removes the majority of dissolved contaminate (PCE, in this case) without being greatly affected by the presence of alcohol (IPA, in this case) in the aqueous solution. This type of treatment is quite standard for water but some understanding of the concentration of PCE and alcohol on recovery would need to be used in designing the system. Results of our research presented in Chapter 7 were provided to Michael Annable at the University of Florida and were applied to their test site in Dover Air Force Base (Hayden et al. 1999). This recovery system and other specifics of a remediation design lend to the complexity of accurate, general cost analyses for field projects. In addition, the limited number of sites where cost information has been gathered and reported make it difficult to accurately determine general costs for in-situ flushing technologies.

Because so many of the field studies conducted to date have been for research and demonstration purposes, little has been reported in the literature regarding cost analysis of in-situ flushing remediation. However, a November 1998 Ground Water Remediation Technologies Analysis Center (GWRTAC) report provides a compilation of economic information from those sites where economics has been considered and is summarized in Table 8.6..

\subsection{Regulatory Feasibility}

In-situ flushing generally uses injection wells to deliver the flushing solution to the contaminated aquifer. Injection wells are regulated by the Underground Injection Control (UIC) program under the Federal Safe Drinking Water Act. An EPA report (March 1996) indicated that 35 States have completely or partially delegated UIC programs. In the remaining 15 States the UIC programs are administered by EPA regional offices. While the federal UIC program does not require a permit for or inhibit use of injection wells, delegated State programs may do both. Summarizing from the conclusions of the EPA report, the use of injectants for in-situ aquifer remediation appears possible in most states. No state has a direct regulatory prohibition of such remediation technologies. Only 
a few states have rejected most or all of the proposals received based on policy or technical merits. Also, most states rely on the technical merits of the proposal and decide on issuance of a permit or project approval on a case-by-case basis. Many of the sites where in-situ flushing has been demonstrated are located on military bases and are associated with research institutions with partial involvement by the environmental remediation industry. It should be noted that at the time this EPA report was written, 15 states had not received any in-situ flushing remediation proposals to review.

Many state regulators have had little or no exposure to the risks and benefits of in-situ flushing techniques and may be hesitant to implement such measures at appropriate sites. However, many states do have the regulatory system (UIC programs) that allow for underground injection for the purposes of aquifer remediation. With these programs in place it may be possible to obtain permits for underground injection. Education and understanding of the remediation process will be necessary before many state regulators understand the benefits of this type of underground injection.

The other major obstacle to implementation of in-situ flushing techniques is the lack of control that these technologies appear to provide. Many researchers fear that mobilization of DNAPLs will be severe enough to cause the NAPL to travel to unaffected portions of the aquifer and perhaps contaminate yet unaffected sensitive receptors. Mobilization has not been characterized well enough to discount this possibility. However, some two dimensional tank experiments conducted in our laboratory suggested that although mobilization can be noted during an alcohol flush, mobilized portions of the DNAPL (PCE in this case) only moved a few centimeters. The tank contained approximately $15 \mathrm{~cm}$ of saturated aquifer and was comprised of various layers. The mobilization that was noted did not cross into other layers and seemed to move only a few pore lengths. This suggested that although portions of the DNAPL may move they are likely to become re-trapped. Field situations may be different, and problems adjusting scale from this type of two dimensional bench scale system to the field make it impossible to determine how DNAPLs will respond in the field. However, this idea of pore scale movement and re-trappment may also occur at the field scale. It is interesting to note that none of the field studies reviewed seemed concerned with mobilization and none of them reported "losing" a significant portion of the DNAPL. However, before regulatory acceptance of in-situ flushing as a viable remediation technique, additional field work and additional characterization of physical phenomena such as mobilization at the field scale may be necessary.

Another obstacle that may be difficult to overcome is public acceptance of introducing more anthropogenic substances to the subsurface. Here, as with regulators, education and proving the success of this technology at test sites will be critical to overcoming this obstacle. Also, the public needs to understand that conventional pump and treat remediation systems are highly ineffective when trying to remediate aquifers contaminated with DNAPLs. With no other alternatives, approval of more innovative technologies at highly contaminated sites is necessary before these technologies can be applied to sites where groundwater reuse is the ultimate goal. 


\subsection{References}

Abdul, A.S., T.L. Gibson, C.C. Ang, J.C. Smith and R.E. Sobczynski. 1992. In Situ Surfactant Washing of Polychlorinated Biphenyls and Oils from a Contaminated Site. Groundwater. 30(2): 219-231.

Abdul, A.S. and C.C. Ang. 1994. In Situ Surfactant Washing of Polychlorinated Biphenyls and Oils from a Contaminated Site: Phase II Pilot Study. Groundwater. 32(5): 727-734.

Annable, M.D. 1999. Personal communication.

Babu D.K., G.F.Pinder, A. Niemi, D.P.Ahlfeld, S.A.Stothoff, "Chemical Transport by ThreeDimensional Groundwater Flows, PTC", Princeton University, 84-WR-3, 1997.

Fountain, J.C., R.C. Starr, T. Middleton, M. Beikirch, C. Taylor and D. Hodge. 1996. A Controlled Field test of Surfactant-Enhanced Aquifer Remediation. Groundwater. 34(5): 910-916.

Fountain, J.C. 1997. The role of field trials in development and feasibility assessment of surfactantenhanced aquifer remediation. Water Environment Research. 69(2): 188-195.

Hayden, N.J., Zhou, H., Annable, M.D., and Brooks, M.C. 1999. Alcohol recovery from alcohol/water/chlorinated solvent mixtures using activated carbon. Presented at the American Geophysical Union Spring Meeting in Boston, MA.

Hirasaki, G.J., C.A. Miller, R. Szafranski, J.B. Lawson, H. Meinardus, M.Jin, J.T. Londergan, R.E. Jackson, G.A. Pope and W.H. Wade. 1997. Society of Petroleum Engineers. SPE 39292. Presented at the 1997 SPE Annual Technical Conference and Exhibition. San Antonio. 5-8 October, $1997.16 \mathrm{pp}$.

Jawitz, J.W., M.D. Annable, P.S.C. Rao and R.D. Rhue. 1998. Field Implementation of a Winsor Type I Surfactant/Alcohol Mixture for an In Situ Solubilization of a Complex LNAPL as a Single-Phase Microemulsion. Environ. Sci. Tech. 32(4):523-530.

Knox, R.C. D.A Sabatini, J.H. Harwell, R.E. Brown, C.C. West, F. Blaha and C. Griffin. 1997. Surfactant Remediation Field Demonstration Using a Vertical Circulation Well. Groundwater. 35(6): 948-953.

Londergan, J.T., G.A. Pope, C.L. Brown and H.W. Meinardus. 1997. Surfactant Enhanced Remediation of an Alluvial Aquifer Contaminated with DNAPL. Presented at the 1997 Petroleum Hydrocarbons and Organic Chemicals in Groundwater Prevention, Detection andRemediation Conference. Houston. 12-14 November, 1997. 13 pp.

McCray, J.E. and M.L. Brusseau. 1998. Cyclodextrin-Enhanced In Situ Flushing of MultipleComponent Immiscible Organic Liquid Contamination at the Field Scale: Mass Removal Effectiveness. Environ. Sci. Tech. 32(9): 1285-1293.

Sabatini, D.A., R.C. Knox, J.H. Harwell, T. Soerens, L.Chen, R.E. Brown and C.C. West. 1997. Design of a Surfactant Remediation Field Demonstration Based on Laboratory and Modeling Studies. Groundwater. 35(6): 954-963. 
The results of this research coupled with results of others presented in the previous section indicate that alcohol flushing is a viable technique for many sites contaminated with DNAPLs. Results of alcohol reuse also indicate that alcohol flushing may be cost effective. Alcohol flushing offers a remediation option for DNAPLs located deep within the saturated zone which are difficult if not impossible to remove by current remediation strategies, thus greatly expediting restoration time, reducing total remediation cost and reducing human and environmental risk.

Several alcohol flushing demonstration studies have been implemented in the past few years. These include one at Hill Air Force Base, a dry cleaner in Florida and Dover Air Force base. All of these have been successful in enhancing the removal of the DNAPL. Recently at Dover Air Force Base, eight pore volumes of alcohol removed approximately $65 \%$ of the amount that had been intentionally spilled at the spot (Brooks et al. 1999). Eight pore volumes refers to the overall pore volume of the test cell. It does not refer to individual zones of low permeable media. Annable (1999) expressed concern that they could not remove all of what was spilled within 8 pore volumes. Our research presented in this report suggests that a flush of eight pore volumes would not sufficiently address the low permeable layers and areas where DNAPL may also reside. This is an important point that must be considered when applying this technology. Zones of varying permeability within an aquifer will always exist within a natural aquifer. To successfully remove the DNAPL from these zones several pore volumes of flushing solution must be swept through them. This may mean that dozens of pore volumes are swept through the more permeable zones. Our research shows that the alcohol solution can be recycled which makes a flushing strategy that may takes months to complete economically feasible.

A longer term cosolvent flushing study with alcohol recycling would seem a logical next choice for the demonstration of this technology at a site where media layering exists. Alcohol offers several unique advantages over surfactants and should be given greater attention in the coming year. Specifically it is much less complicated than surfactants in terms of the chemistry involved. While surfactants can be tailor made for any situation, most environmental applications do not require this level of complexity. Surfactants can also interact with the aquifer material further complicating the remediation. Surfactants also appear to be more difficult to reuse and thus may be more costly in the long run. The reason for the slightly greater interest in surfactants over alcohols, appears to be a carry over from the oil industry and not necessarily due to their greater applicability.

Specific design recommendations for an alcohol flushing system are beyond the scope of this report and depend on the specific site, however, some general recommendations can be made. In a highly layered system, a lower flow rate would be desired to enhance mass transfer of contaminants from lower permeable regions to the more permeable regions thus enhancing the efficiency of the system. However, a high enough flow rate is required to ensure that hydraulic control could be maintained throughout the site. While ethanol has been the alcohol of choice due to regulatory concerns, IPA is more effective at enhancing solubility and is also less expensive. Application of IPA in a field demonstration would seem the next logical choice in the application of this technology. Due to the 
pumping of groundwater with the alcohol solution some dilution will occur so a system that can recover or reconstitute the alcohol for reinjection should also be considered.

In evaluating the application of this technology some effort must be made to determine the permeability variations within the media tested and appropriate calculations performed to determine if these low permeable zones have been swept with the alcohol solution. In systems with extremely low permeable zones, such as clay, the time to achieve one pore volume may not be feasible. The time to sweep these should be determined for areas above these low permeable layers which may have larger saturations of DNAPL. Because of the density difference between the flushing solution and the initial groundwater present in the pores, some attention also needs to be given to density override issues. Adjusting the density of the alcohol solution to approximate water may need to be considered.

Alcohol flushing has recently been shown to be a viable technique for DNAPL remediation at some sites. A key issue in the application of this technology to additional sites, including sites which may have considerable layering of the aquifer materials, is recognizing that it will take time for the flushing solution to access the least permeable layers. This may mean a considerable number of pore volumes of solution to be flushed through the media. The ability to reuse the alcohol solution in these situations promises to enhance the economic attractiveness of this technology.

\subsection{References}

Annable, M.D. 1999. Personal communication.

Brooks, M.C., Annable, M.D., Rao, P.S., Hatfield, K., Wood, A.L. and Enfield, C.G. 1999. A pilotscale demonstration of DNAPL remediation by cosolvent flushing. Presented at the American Geophysical Union Spring Meeting, Boston, MA. 